\title{
Luminescent Water-Soluble Oxygen-Sensor Films and Their Application in Pressure Sensitive Paints
}

\author{
by
}

Arezoo Habibagahi, M.Sc.

\author{
A Thesis submitted to the Faculty of Graduate Studies \\ in partial fulfillment of the requirements for the degree of \\ Doctor of Philosophy \\ Carleton University \\ Ottawa, Canada \\ July 2010
}

Copyright (C) 2010, Arezoo Habibagahi 


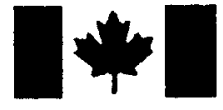

Library and Archives

Canada

Published Heritage

Branch

395 Wellington Street

Ottawa ON K1A ON4

Canada
Bibliothèque et

Archives Canada

Direction du

Patrimoine de l'édition

395 , rue Wellington

Ottawa ON K1A ON4

Canada
Your file Votre référence
ISBN: $978-0-494-69920-1$
Our file Notre référence
ISBN: $978-0-494-69920-1$
NOTICE:

The author has granted a nonexclusive license allowing Library and Archives Canada to reproduce, publish, archive, preserve, conserve, communicate to the public by telecommunication or on the Internet, loan, distribute and sell theses worldwide, for commercial or noncommercial purposes, in microform, paper, electronic and/or any other formats.

The author retains copyright ownership and moral rights in this thesis. Neither the thesis nor substantial extracts from it may be printed or otherwise reproduced without the author's permission.
AVIS:

L'auteur a accordé une licence non exclusive permettant à la Bibliothèque et Archives Canada de reproduire, publier, archiver, sauvegarder, conserver, transmettre au public par télécommunication ou par l'Internet, prêter, distribuer et vendre des thèses partout dans le monde, à des fins commerciales ou autres, sur support microforme, papier, électronique et/ou autres formats.

L'auteur conserve la propriété du droit d'auteur et des droits moraux qui protège cette thèse. $\mathrm{Ni}$ la thèse ni des extraits substantiels de celle-ci ne doivent être imprimés ou autrement reproduits sans son autorisation.
In compliance with the Canadian Privacy Act some supporting forms may have been removed from this thesis.

While these forms may be included in the document page count, their removal does not represent any loss of content from the thesis.
Conformément à la loi canadienne sur la protection de la vie privée, quelques formulaires secondaires ont été enlevés de cette thèse.

Bien que ces formulaires aient inclus dans la pagination, il n'y aura aucun contenu manquant.

\section{Canadä}


This page has been removed for privacy reasons. 


\begin{abstract}
Luminescent water-soluble oxygen sensors have been prepared by bonding iridium complexes to inexpensive water-soluble polymers, such as poly(ethylene glycol) methyl ether-aldehyde (PEGME-Aldehyde) or Silamine D208-EDA. The luminescent iridium complexes, $\quad\left[\operatorname{Ir}(\mathrm{fppy})_{2}(t-\mathrm{Bu}-\mathrm{iCN})_{2}\right]\left[\mathrm{CF}_{3} \mathrm{SO}_{3}\right], \quad\left[\operatorname{Ir}\left(\mathrm{C}_{6}\right)_{2}\right.$ (acacacid) $]$, $\left[\operatorname{Ir}(\text { ppy })_{2}(\right.$ amppy $\left.) C l\right], \quad\left[\operatorname{Ir}(\text { ppy })_{2}(\right.$ phendiald $\left.)\right]\left[\mathrm{PF}_{6}\right], \quad$ where $\quad$ fppy $\quad=\quad 4-(2-$ pyridyl)benzaldehyde, $t$ - $\mathrm{Bu}-\mathrm{iCN}=$ tert-butyl isocyanide, $\mathrm{C}_{6}=3$-(2'-benzothiazolyl)-7$\mathrm{N}, \mathrm{N}$-diethylaminocoumarin, acacacid $=4,6$-dioxoheptanoic acid, ppy $=2$ phenylpyridine, amppy $=5$-aminomethyl-2-phenylpyridine, and phendiald $=1,10$ phenanthroline-2,9-dicarboxaldehyde, have been synthesized and characterized by nuclear magnetic resonance, absorption and emission spectroscopies. These complexes along with $\left[\operatorname{Ir}(\operatorname{tpy})_{2}(t-\mathrm{Bu}-\mathrm{iCN})_{2}\right]\left[\mathrm{CF}_{3} \mathrm{SO}_{3}\right]$ and $\left[\operatorname{Ir}(\mathrm{ppy})_{2}(\mathrm{fppy})\right]$, where tpy $=2-(p-$ tolyl)pyridine, were dispersed in or covalently attached to functionalized water-soluble polymers by reductive amination or coupling reactions. Aqueous blends of these watersoluble luminophores with colloidal microcrystalline cellulose (MC) were used to prepare oxygen sensor films, otherwise known as pressure sensitive paints (PSPs). Oxygen sensitivities of these PSPs were determined as a function of blended MC and obeyed Stern-Volmer kinetics. These novel materials offer an environmentally friendly alternative to non-aqueous PSP formulations and provide improved workplace safety, and lower contamination.
\end{abstract}

Oxygen quenching sensitivity data (slope of a linear fit to the modified SternVolmer plot) for water-soluble luminophores composed of Silamine-attached $\left[\operatorname{Ir}(f \mathrm{ppy})_{2}(t\right.$ - 
$\left.\mathrm{Bu}-\mathrm{iCN})_{2}\right]\left[\mathrm{CF}_{3} \mathrm{SO}_{3}\right]$ and $\left[\operatorname{Ir}(\mathrm{ppy})_{2}(\mathrm{fppy})\right]$ are between 0.4 and 0.5 , which are appropriate for pressure sensitive paint applications. Investigation of luminescence microscopy and solid state lifetime measurements revealed that the preferential binding of the luminophore to $\mathrm{MC}$ increased its luminescence lifetime and was primarily responsible for the improved oxygen quenching sensitivity of PSP films that include MC.

The temperature dependence of luminescence was examined between 10 to 40 ${ }^{\circ} \mathrm{C}$ for a number of PSPs and gave temperature sensitivities (percent emission intensity loss per degree) of between $-0.8 \%$ and $-1.6 \%{ }^{\circ} \mathrm{C}^{-1}$ at 1 bar atmospheric pressure. 


\section{Acknowledgements}

I would first and foremost like to gratefully acknowledge the invaluable supervision of Dr. Robert J. Crutchley during this work.

I would also like to thank Dr. Glenn Yap for the X-ray crystallography, Dr. Youssef Mébarki for help with the oxygen sensitivity calibrations, Dr. Wayne Z.Y. Wang

for the use of his equipment for acquiring the steady-state emission spectrum, Dr. Carlos Monreal for luminescence microscopy, and Dr. J. C. Scaiano and Michel Grenier for assistance in collecting lifetime data.

Financial support by Carleton University and Ontario Graduate Scholarships in Science and Technology (OGSST) is appreciated.

A special thanks to my lab members Sana Sultan, Mohommad M. R. Choudhuri, and Farah Sabrin, with whom I enjoyed working in the lab. Thanks to Keith Bourque for his help with acquiring NMR data. Thanks to Jim Logan for updating our computers. Thank you, Tony O'Neil, for help in instrument lab. Thanks to Susaritha Chandrabose, Tanya Rudd, and Peter Mosher for all the help at Stores.

The everlasting devotion and encouragement of my father, and memory of my mother who is not with me today, made this possible.

My greatest thanks go to my caring husband, Mohammad R. Nikseresht, and our son, Ali. This would have never been possible without your love and inspiration. 


\section{Table of Contents}

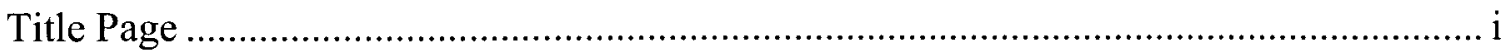

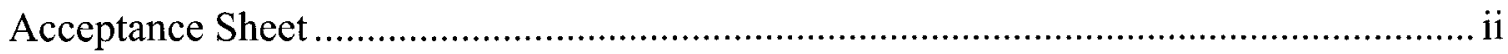

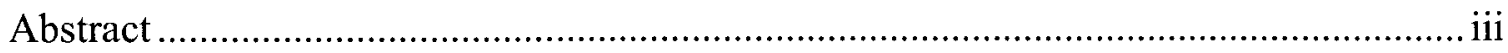

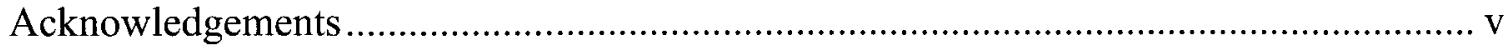

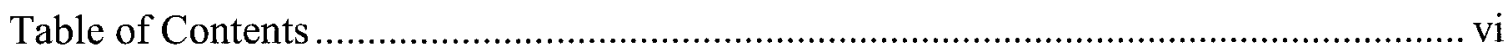

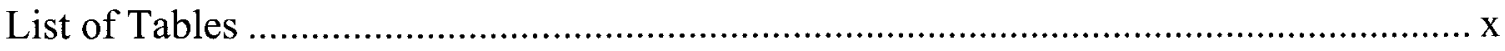

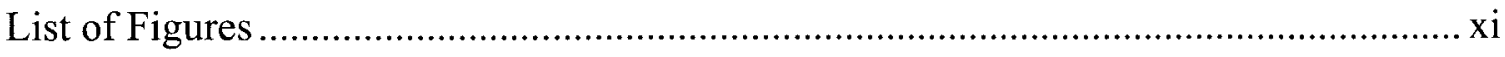

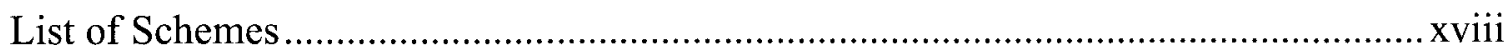

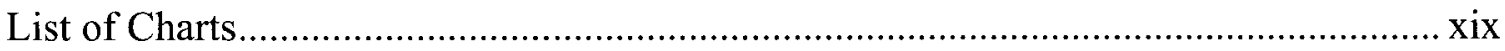

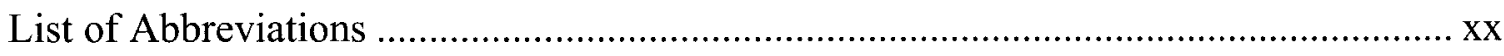

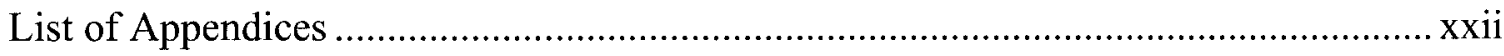

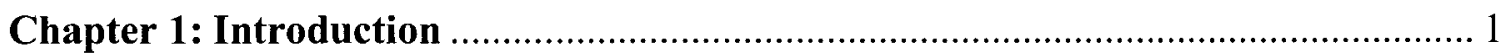

$1.1 \quad$ Optical Oxygen Sensors............................................................................. 1

1.2 Photophysical Properties of Oxygen Sensor Luminophores ................................ 2

1.3 Energy Transfer Mechanisms and Luminescence Quenching ................................5

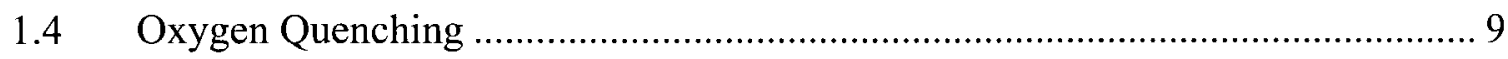

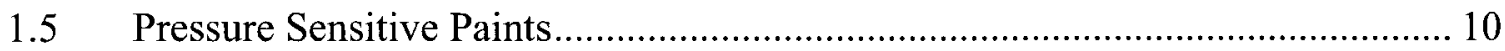

1.6 Materials for Optical Oxygen Sensors ………….......................................... 12

1.6.1 Luminescent Probes for PSPs ………......................................................... 13

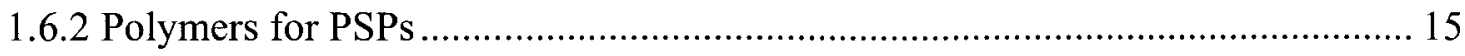


1.6.3 Electrochemical Adsorption of Luminophores

1.7 Water-Soluble Pressure Sensitive Paints ....................................................... 18

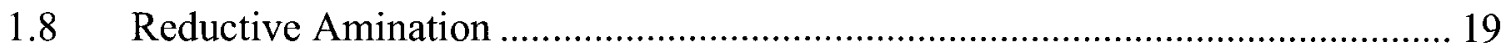

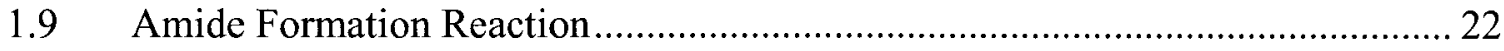

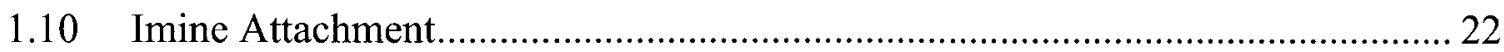

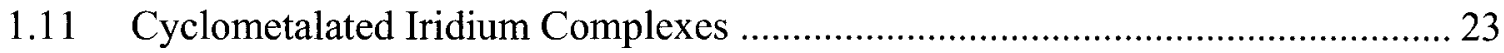

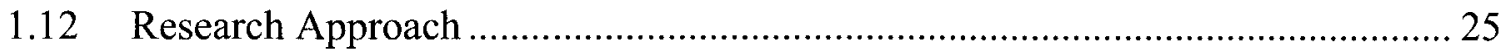

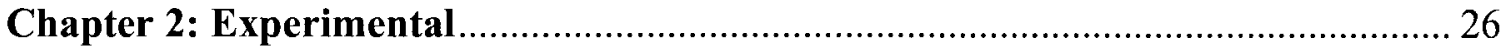

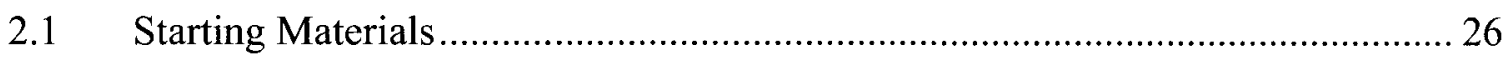

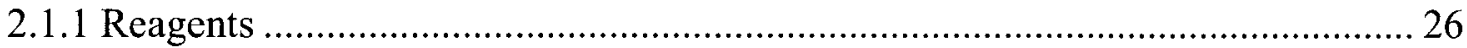

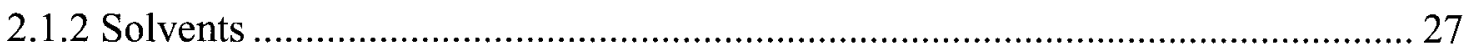

2.1.3 Compressed Gas ................................................................................. 28

2.1.4 Ion Exchange Resin, Drying Agent, and PSP Additive ............................... 28

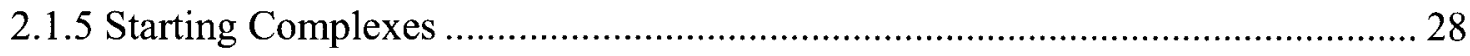

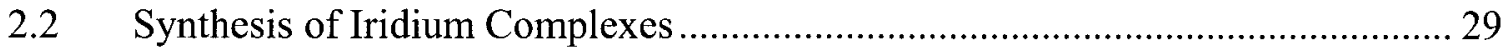

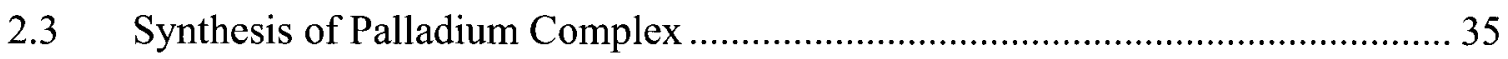

2.4 Synthesis of Functionalized Water-Soluble Polymer ...................................... 36

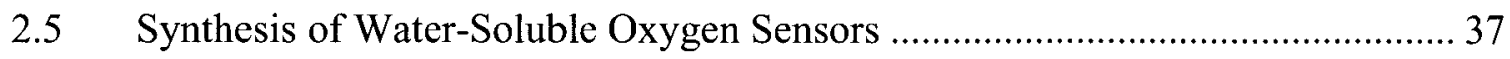

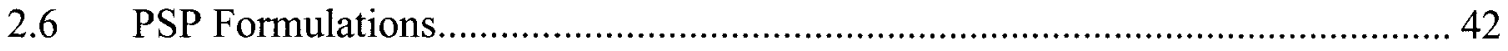

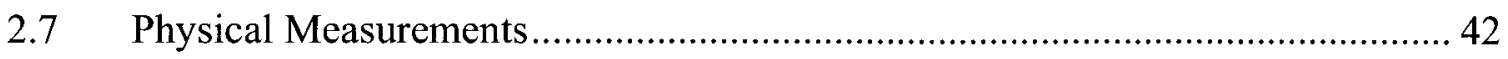

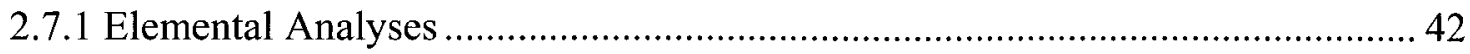

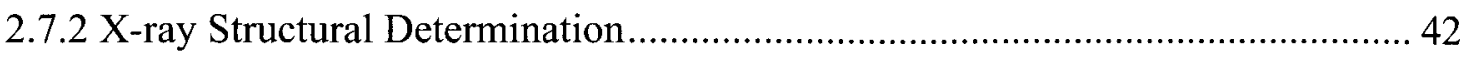




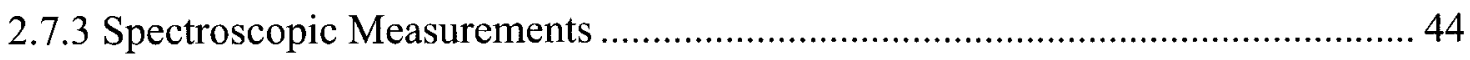

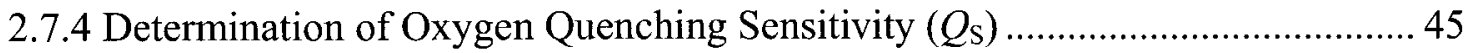

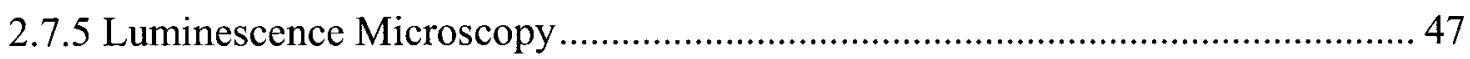

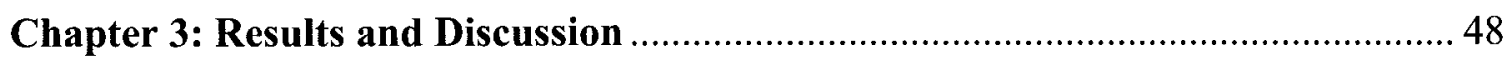

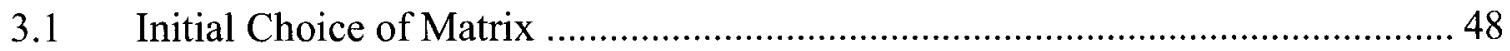

3.2 Initial Choice of Luminophore

3.3 Characterization of Initial Pressure Sensitive Paint (PSP) Films ......................... 49

3.4 Synthesis of Water-Soluble Oxygen Sensor 8 ……........................................ 52

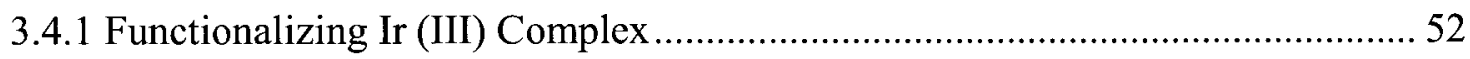

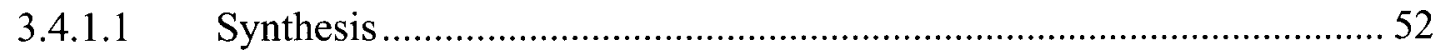

3.4.1.2 X-ray Crystallography ................................................................. 54

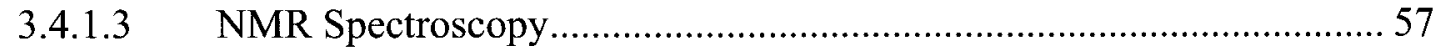

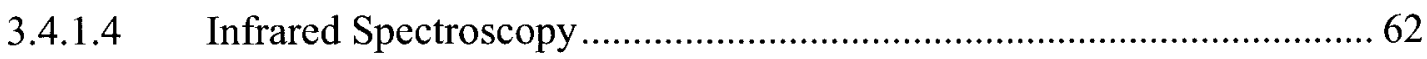

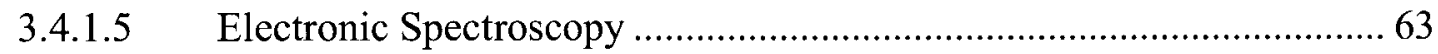

3.4.2 Synthesis of Water-Soluble Oxygen Sensor 8 by Reductive Attachment of 2 to Silamine D208-EDA and Spectroscopic Characterization...........................................6 65

3.5 Synthesis of Water-Soluble Oxygen Sensor $11 \ldots \ldots \ldots \ldots \ldots \ldots \ldots \ldots \ldots \ldots \ldots \ldots \ldots \ldots \ldots \ldots . . .78$

3.5.1 Copper-Catalyzed Oxidation of Poly(ethylene glycol) Methyl Ether (PEGME) to

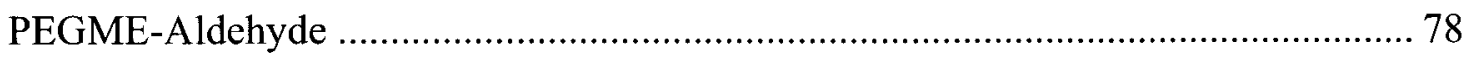

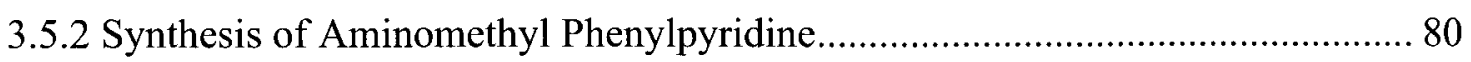

3.5.3 Coupling of Amppy to PEGME-Aldehyde and Spectroscopic Characterization. 85 
3.5.4 PEGME Attached Ir-Amppy Dimer Synthesis and Spectroscopic Characterization

3.5.5 Synthesis of Water-Soluble Oxygen Sensor 11 - PEGME Attached [Ir(amppy $)_{2}(t-$

$\left.\mathrm{Bu}-\mathrm{iCN})_{2}\right]\left[\mathrm{CF}_{3} \mathrm{SO}_{3}\right]-$ Synthesis and Spectroscopic Characterization ...................... 90

3.6 Characterization of Pressure Sensitive Paint (PSP) Films of 2, 8, and 11 ......... 91

3.7 Other Functionalized Ir (III) Complexes …........................................ 102

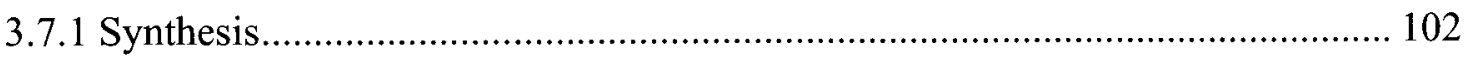

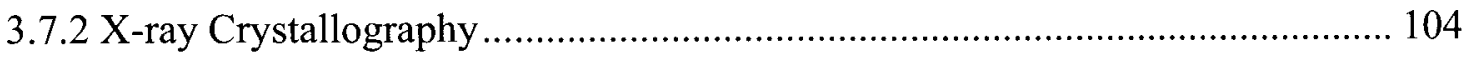

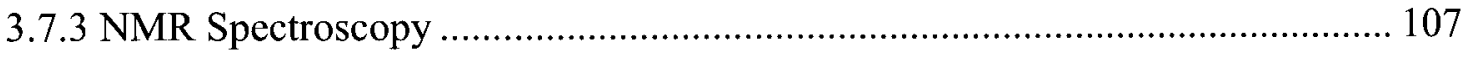

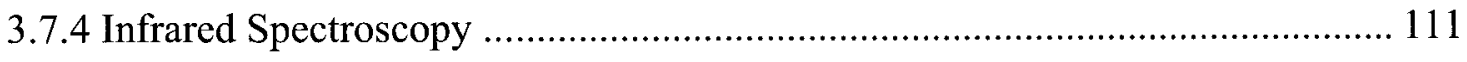

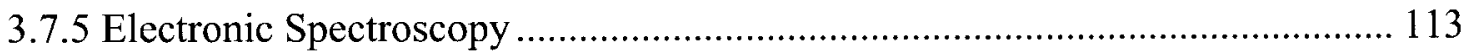

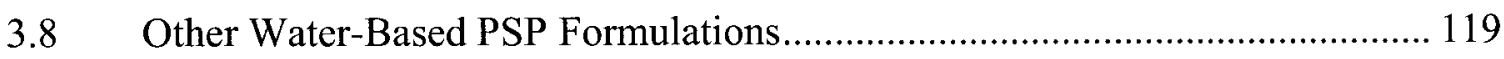

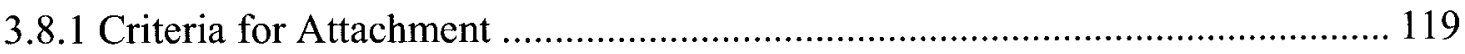

3.8.2 Synthesis of 9 by Coupling of $\mathbf{3}$ to Silamine D208-EDA and Spectroscopic

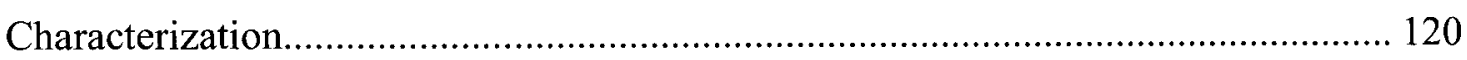

3.8.3 Preparation and Spectroscopic Characterization of 10............................... 122

3.8.4 Synthesis of $\mathbf{1 2}$ by Coupling of $\mathbf{5}$ to Silamine D208-EDA and Spectroscopic

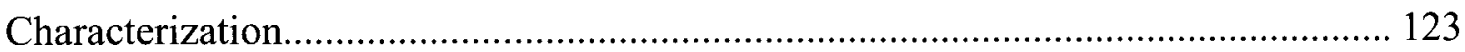

3.8.5 Synthesis of $\mathbf{1 3}$ by Reductive Attachment of $\mathbf{5}$ to Silamine D208-EDA and

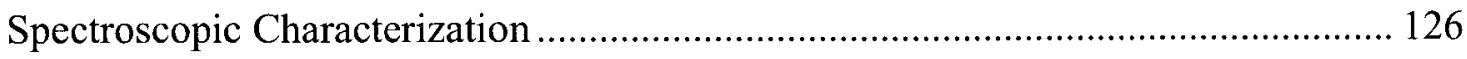

3.8.6 Synthesis of $\mathbf{1 4}$ by Reductive Attachment of $\mathbf{6}$ to Silamine D208-EDA and

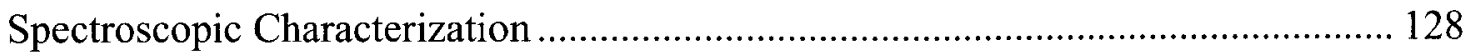


3.8.7 Synthesis of 15 by Coupling of 1-Pyrenebutyric Acid (PBA) to Silamine D208EDA and Spectroscopic Characterization .............................................................. 131

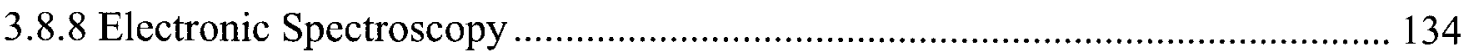

3.8.9 Characterization of Pressure Sensitive Paint (PSP) Films ................................ 142

3.8.9.1 PSP 9

3.8.9.2 PSP 10

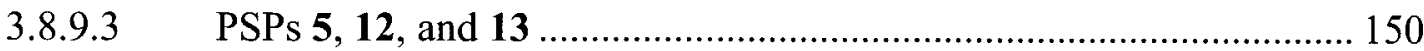

3.8.9.4 PSP 14

3.8.9.5 PSP 15

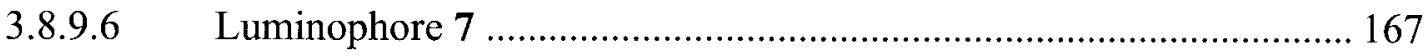

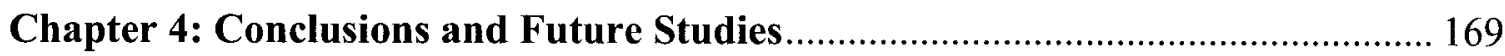

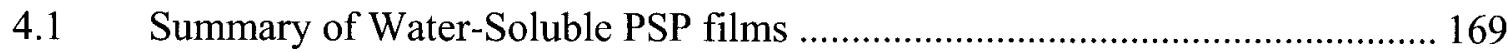

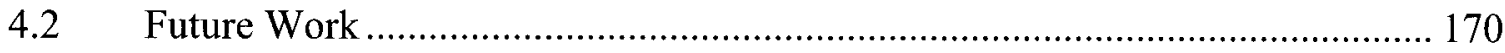

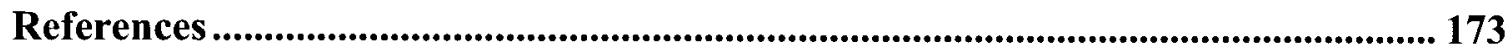

\section{List of Tables}

Table 3.1: PSP films oxygen sensitivity data for 1................................................... 51

Table 3.2: X-ray Crystallographic Data and Refinement Details for 2 $\cdot 0.25\left(\mathrm{CH}_{2} \mathrm{Cl}_{2}\right) \ldots . .56$

Table 3.3: Selected Bond Lengths $(\AA)$ and Angles $\left(^{\circ}\right)$ for $2 \cdot 0.25\left(\mathrm{CH}_{2} \mathrm{Cl}_{2}\right) \ldots \ldots \ldots \ldots \ldots \ldots . . . .56$

Table 3.4: PSP films oxygen sensitivity data for $1,2,8$, and 11 .................................. 96 
Table 3.5: Luminescence lifetime data in $\mu$ s (with pre-exponential factors in parentheses) of 8 in Silamine:MC films 99

Table 3.6: X-ray Crystallographic Data and Refinement Details for $4 \cdot \mathrm{CH}_{2} \mathrm{Cl}_{2} \ldots \ldots \ldots . .106$

Table 3.7: Selected Bond Lengths $(\AA)$ and Angles $\left(^{\circ}\right)$ for $4 \cdot \mathrm{CH}_{2} \mathrm{Cl}_{2} \ldots \ldots \ldots \ldots \ldots \ldots \ldots \ldots . . . . . . . . . . .106$

Table 3.8: Solution absorption and emission data for complexes 1-6 ...................... 115

Table 3.9: Luminescence quantum yields and lifetimes for complexes 1-7 ................ 119

Table 3.10: Solution absorption and emission data for 8-15 ............................. 136

Table 3.11: Luminescence quantum yields and lifetimes for 8-15 ......................... 136

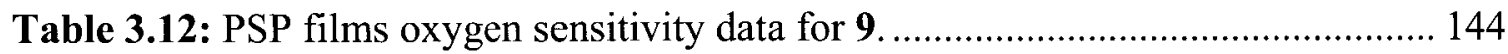

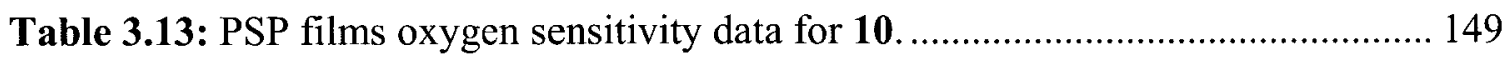

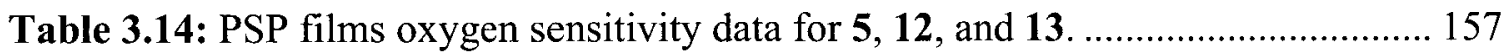

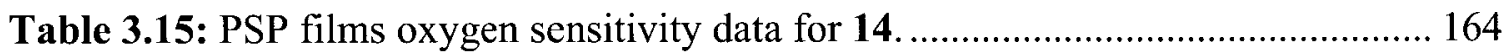

\section{List of Figures}

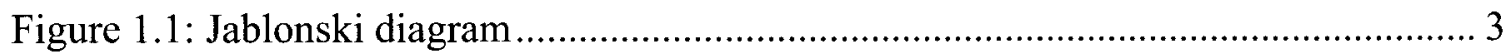

Figure 1.2: Dexter energy transfer, where $\mathrm{D}$ is the donor and $\mathrm{A}$ is the acceptor molecule. 6 Figure 1.3: Schematic picture of pressure sensitive paint coated model surface. 11

Figure 3.1: Effect of pressure on thin film luminescence of 1 in PDMS-based copolymer. The decrease of intensity with increasing the air pressure is displayed. Spectra were acquired at 1.3 mbar, 0.34 bar, 0.68 bar, 1 bar, 1.36 bar, 1.70 bar, 2.04 bar, 2.38 bar, 2.72 bar, and 3.06 bar. Background correction has been performed.

Figure 3.2: Stern-Volmer plots of 1 in PEG, ; in 1:1 PEG: MC, •; and in 1:9 PEG: MC,

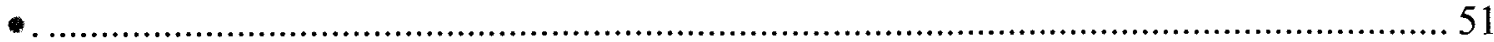


Figure 3.3: ORTEP of $2 \cdot 0.25\left(\mathrm{CH}_{2} \mathrm{Cl}_{2}\right)$ with ellipsoids at $30 \%$. The alternate disordered contribution, second symmetrically unique ion pair, triflate counter ion, methylene chloride solvent molecule, and hydrogen atoms are omitted for clarity.

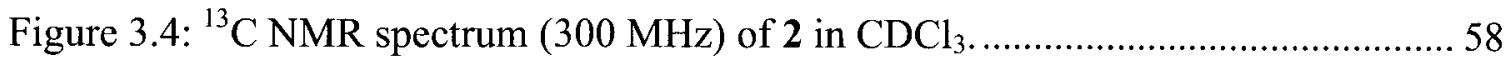

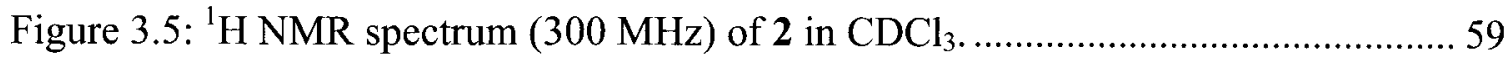

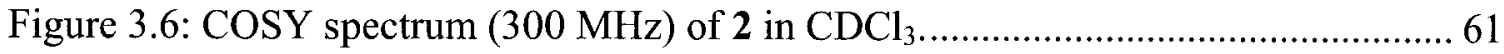

Figure 3.7: Simplified representation of COSY spectrum of 2 in $\mathrm{CDCl}_{3} \ldots \ldots \ldots \ldots \ldots \ldots \ldots . . . . . . . .61$

Figure 3.8: Infrared spectrum (KBr pellet) of 2 with $v(\mathrm{C} \equiv \mathrm{N})$ at $2177 \mathrm{~cm}^{-1} \ldots \ldots \ldots \ldots \ldots \ldots . . . .62$

Figure 3.9: Solution electronic absorption and emission spectra of 2. The absorption spectrum was acquired in $\mathrm{CH}_{3} \mathrm{CN}$ and the emission spectrum was acquired in $\mathrm{CH}_{2} \mathrm{Cl}_{2} . .64$

Figure 3.10: ${ }^{1} \mathrm{H}$ NMR spectrum $(300 \mathrm{MHz})$ of Silamine D208-EDA in $\mathrm{CDCl}_{3} \ldots \ldots \ldots \ldots .67$

Figure 3.11: ${ }^{1} \mathrm{H}$ NMR spectrum $(300 \mathrm{MHz})$ of 2 dispersed in Silamine D208-EDA in

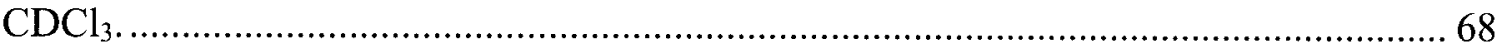

Figure 3.12: Aromatic range of ${ }^{1} \mathrm{H}$ NMR spectrum (300 MHz) of 2 dispersed in Silamine in $\mathrm{CDCl}_{3}$. Note the aldehyde proton chemical shift at $9.58 \mathrm{ppm}$

Figure 3.13: ${ }^{1} \mathrm{H}$ NMR spectrum (300 MHz) of 8 in $\mathrm{CDCl}_{3}$. The loss of the aldehyde peak due to the condensation reaction is labeled............................................................... 70

Figure 3.14: Infrared spectra (thin films) of 2 in Silamine (top) and 8 (bottom) displaying no aldehyde $\mathrm{C}=\mathrm{O}$ band in the polymer bound luminophore. 71

Figure 3.15: Solution electronic absorption and emission spectra of 8 . The absorption spectrum was acquired in $\mathrm{CH}_{3} \mathrm{CN}$ and the emission spectrum was acquired in $\mathrm{CH}_{2} \mathrm{Cl}_{2}$. For 8, the extinction coefficient at $\lambda=255 \mathrm{~nm}$ was assumed to be identical with that of 1 at $260 \mathrm{~nm}$

Figure 3.16: Qualitative solution emission spectra of 1 (red), 2 (blue), and 8 (black) in $\mathrm{CH}_{3} \mathrm{CN}$.

Figure 3.17: Effect of matrix on thin film luminescence of 1. Matrices compositions are: 9:1 Silamine: MC (black), 1:2 Silamine: MC (red), and 1:5 Silamine: MC (green). For each formulation, the spectrum at $14.7 \mathrm{psi}$ is shown, which is normalized to its maximum emission intensity .75

Figure 3.18: Effect of matrix on thin film luminescence of 2. Matrices compositions are: 9:1 Silamine: MC (black), 5:1 Silamine: MC (red), 1:1 Silamine: MC (green), 1:2 
Silamine: MC (yellow), and 1:5 Silamine: MC (blue). For each formulation, the spectrum at $14.7 \mathrm{psi}$ is shown, which is normalized to its maximum emission intensity.

Figure 3.19: Effect of matrix on thin film luminescence of 8. Matrices compositions are: 5:1 Silamine: MC (black), 1:1 Silamine: MC (red), and 1:5 Silamine: MC (green). For each formulation, the spectrum at $14.7 \mathrm{psi}$ is shown, which is normalized to its maximum emission intensity.

Figure 3.20: Infrared spectrum (thin film) of PEGME-Aldehyde with $v(\mathrm{C}=\mathrm{O})$ at $1719 \mathrm{~cm}^{-}$ 1

Figure 3.21: ${ }^{1} \mathrm{H}$ NMR spectrum $(300 \mathrm{MHz})$ of amppy in $\mathrm{CDCl}_{3}$. 83

Figure 3.22: Infrared spectrum ( $\mathrm{KBr}$ pellet) of amppy with $v(\mathrm{~N}-\mathrm{H})$ at 3437,3269 , and $1602 \mathrm{~cm}^{-1}$.

Figure 3.23: Electron-Impact mass spectrum of amppy with the molecular ion peak at 181 $\mathrm{m} / \mathrm{z}$.

Figure 3.24: Aromatic region of the ${ }^{1} \mathrm{H}$ NMR spectrum (300 MHz) of PEGME-Aldehyde in $\mathrm{D}_{2} \mathrm{O}$ showing the aldehyde chemical shift at $9.6 \mathrm{ppm}$. 86

Figure 3.25: Aromatic region of the ${ }^{1} \mathrm{H}$ NMR spectrum (300 MHz) of PEGME-attached amppy in $\mathrm{D}_{2} \mathrm{O}$ showing amppy chemical shifts.

Figure 3.26: ${ }^{1} \mathrm{H}$ NMR spectrum (300 MHz) of PEGME-attached Ir-amppy dimer in $\mathrm{D}_{2} \mathrm{O}$.

Figure 3.27: Solution electronic absorption and emission spectra of PEGME attached Iramppy dimer. Both spectra were acquired in $\mathrm{CH}_{2} \mathrm{Cl}_{2}$. The extinction coefficient was assumed to be identical with that of $\left[\operatorname{Ir}(\text { ppy })_{2} \mathrm{Cl}\right]_{2}$.

Figure 3.28: Solution electronic absorption and emission spectra of 11. The absorption spectrum was acquired in $\mathrm{CH}_{3} \mathrm{CN}$ and the emission spectrum was acquired in $\mathrm{CH}_{2} \mathrm{Cl}_{2}$. For 11, the extinction coefficient at $\lambda=253 \mathrm{~nm}$ was assumed to be identical with that of 1 at $260 \mathrm{~nm}$......

Figure 3.29: Effect of pressure on thin film luminescence of $\mathbf{2}$ in 1:1 Silamine: MC. The decrease of intensity with increasing the air pressure is displayed. Spectra were acquired at $1.3 \mathrm{mbar}, 0.34$ bar, $0.68 \mathrm{bar}, 1 \mathrm{bar}, 1.36 \mathrm{bar}, 1.70 \mathrm{bar}, 2.04 \mathrm{bar}, 2.38 \mathrm{bar}, 2.72 \mathrm{bar}$, and 3.06 bar. Background correction has been performed.

Figure 3.30: Effect of pressure on thin film luminescence of 8 in 1:1 Silamine: MC. The decrease of intensity with increasing the air pressure is displayed. Spectra were acquired at $1.3 \mathrm{mbar}, 0.34 \mathrm{bar}, 0.68 \mathrm{bar}, 1 \mathrm{bar}, 1.36 \mathrm{bar}, 1.70 \mathrm{bar}, 2.04 \mathrm{bar}, 2.38 \mathrm{bar}, 2.72 \mathrm{bar}$, and 3.06 bar. Background correction has been performed. 
Figure 3.31: Effect of pressure on thin film luminescence of 11 in 4:1 PEG: MC. The decrease of intensity with increasing the air pressure is displayed. Spectra were acquired at 1.3 mbar, 0.34 bar, 0.68 bar, 1 bar, 1.36 bar, 1.70 bar, 2.04 bar, 2.38 bar, 2.72 bar, and 3.06 bar. Background correction has been performed. 94

Figure 3.32: Stern-Volmer plots of 1 in 9:1 Silamine: MC, ; 1 in 1:1 Silamine: MC, $\bullet \mathbf{2}$ in 9:1 Silamine: $\mathrm{MC}, \bullet ; 2$ in 1:1 Silamine: $\mathrm{MC}, \quad ; \mathbf{8}$ in 9:1 Silamine: $\mathrm{MC}, \bullet ; \mathbf{8}$ in $1: 1$ Silamine: $\mathrm{MC}, \bullet ; 11$ in 4:1 PEG: MC, $\bullet$ 95

Figure 3.33: Luminescence microscopy of films of $\mathbf{8}$ performed under ambient conditions: A, 9:1 Silamine:MC; B, 5:1 Silamine:MC; C, 1:1 Silamine:MC; D, 1:2.3 Silamine:MC; E, 1:5 Silamine:MC. The scale in red is $10 \mu \mathrm{m}$.

Figure 3.34: Temperature versus emission intensity ratio $\left(\mathrm{I}_{\text {ref }}\right.$ is the intensity at $\left.10^{\circ} \mathrm{C}\right)$ of 1 in 1:1 Silamine: $M C$ : vacuum $\Delta$ and $P=1$ bar $\bullet, 2$ in 1:1 Silamine: $M C$ : vacuum $\bullet$ and $\mathrm{P}=1$ bar $\boldsymbol{\nabla}$, and 8 in 1:1 Silamine: $\mathrm{MC}$ : vacuum and $\mathrm{P}=1$ bar 100

Figure 3.35: ORTEP of $\mathbf{4} \cdot \mathrm{CH}_{2} \mathrm{Cl}_{2}$. The methylene chloride solvent molecule and aromatic-ring hydrogen atoms are omitted for clarity.

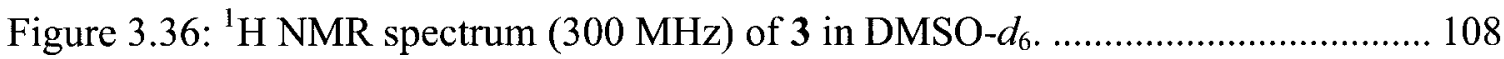

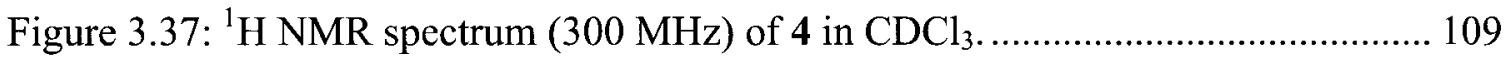

Figure 3.38: ${ }^{1} \mathrm{H}$ NMR spectrum $(300 \mathrm{MHz})$ of 6 in acetone- $d_{6}$. The aldehyde resonance is labelled. 110

Figure 3.39: Infrared spectrum (KBr pellet) of 3 with $v(C=O)$ at 1680 and $1607 \mathrm{~cm}^{-1} .111$

Figure 3.40: Infrared spectrum (KBr pellet) of 4 with $v(\mathrm{~N}-\mathrm{H})$ at $3281 \mathrm{~cm}^{-1}$. 112

Figure 3.41: Infrared spectrum (KBr pellet) of 6 with $v(C=O)$ at $1729 \mathrm{~cm}^{-1}$ 113

Figure 3.42: Solution electronic absorption and emission spectra of 3. Both the absorption and emission spectra were acquired in $\mathrm{CH}_{2} \mathrm{Cl}_{2}$. 116

Figure 3.43: Solution electronic absorption and emission spectra of 4. Both the absorption and emission spectra were acquired in $\mathrm{CH}_{2} \mathrm{Cl}_{2}$ 117

Figure 3.44: Solution electronic absorption and emission spectra of 6 . Both the absorption and emission spectra were acquired in $\mathrm{CH}_{2} \mathrm{Cl}_{2}$. 118

Figure 3.45: Solution electronic absorption and emission spectra of 9. Both spectra were acquired in $\mathrm{CH}_{2} \mathrm{Cl}_{2}$. For 9 , the extinction coefficient at $\lambda=445 \mathrm{~nm}$ was assumed to be identical with that of 3 at $447 \mathrm{~nm}$. See Table 3.8 data. 
Figure 3.46: Solution electronic absorption and emission spectra of 10. Both spectra were acquired in $\mathrm{CH}_{2} \mathrm{Cl}_{2}$

Figure 3.47: Solution electronic absorption and emission spectra of 12. Both spectra were acquired in $\mathrm{CH}_{2} \mathrm{Cl}_{2}$. For 12 , the extinction coefficient at $\lambda=285 \mathrm{~nm}$ was assumed to be identical with that of $\mathbf{5}$ at $290 \mathrm{~nm}$.

Figure 3.48: Solution electronic absorption and emission spectra of 13. Both spectra were acquired in $\mathrm{CH}_{2} \mathrm{Cl}_{2}$. For 13 , the extinction coefficient at $\lambda=282 \mathrm{~nm}$ was assumed to be identical with that of 5 at $290 \mathrm{~nm}$.

Figure 3.49: Solution electronic absorption and emission spectra of 14. Both spectra were acquired in $\mathrm{CH}_{2} \mathrm{Cl}_{2}$. For 14, the extinction coefficient at $\lambda=269 \mathrm{~nm}$ was assumed to be identical with that of 6 at $257 \mathrm{~nm}$. See Table 3.8 data.

Figure 3.50: Solution electronic absorption and emission spectra of 15. Both spectra were acquired in $\mathrm{CH}_{2} \mathrm{Cl}_{2}$. For 15 , the extinction coefficient at $\lambda=344 \mathrm{~nm}$ was assumed to be identical with that of PBA at $341 \mathrm{~nm}$.

Figure 3.51: Effect of matrix on thin film luminescence of 9. Matrices compositions are: Silamine (black), 1:1 Silamine: MC (red), and 1:5 Silamine: MC (green). For each formulation, the spectrum at $14.7 \mathrm{psi}$ is shown, which is normalized to its maximum emission intensity.....

Figure 3.52: Effect of matrix on thin film luminescence of 10. Matrices compositions are: Silube (black), 5:1 Silube: MC (red), 1:1 Silube: MC (green), and 1:5 Silube: MC (yellow). For each formulation, the spectrum at $14.7 \mathrm{psi}$ is shown, which is normalized to its maximum emission intensity.

Figure 3.53: Effect of matrix on thin film luminescence of 12. Matrices compositions are: 2.3:1 Silamine: MC (black), 1:1 Silamine: MC (red), and 1:9 Silamine: MC (green). For each formulation, the spectrum at $14.7 \mathrm{psi}$ is shown, which is normalized to its maximum emission intensity

Figure 3.54: Effect of matrix on thin film luminescence of 13. Matrices compositions are: 2.3:1 Silamine: MC (black), 1:1 Silamine: MC (red), and 1:9 Silamine: MC (green). For each formulation, the spectrum at $14.7 \mathrm{psi}$ is shown, which is normalized to its maximum emission intensity. 140

Figure 3.55: Effect of matrix on thin film luminescence of 14. Matrices compositions are: 5:1 Silamine: MC (black), 1:1 Silamine: MC (red), and 1:2.3 Silamine: MC (green). For each formulation, the spectrum at $14.7 \mathrm{psi}$ is shown, which is normalized to its maximum emission intensity 141

Figure 3.56: Effect of pressure on thin film luminescence of 9 in Silamine. The decrease of intensity with increasing the air pressure is displayed. Spectra were acquired at 1.3 
mbar, 0.34 bar, 0.68 bar, 1 bar, 1.36 bar, 1.70 bar, 2.04 bar, 2.38 bar, 2.72 bar, and 3.06 bar. Background correction has been performed.

Figure 3.57: Effect of pressure on thin film luminescence of 9 in 1:1 Silamine: MC. The decrease of intensity with increasing the air pressure is displayed. Spectra were acquired at $1.3 \mathrm{mbar}, 0.34 \mathrm{bar}, 0.68 \mathrm{bar}, 1 \mathrm{bar}, 1.36 \mathrm{bar}, 1.70 \mathrm{bar}, 2.04 \mathrm{bar}, 2.38 \mathrm{bar}, 2.72 \mathrm{bar}$, and 3.06 bar. Background correction has been performed.

Figure 3.58: Stern-Volmer plots of $\mathbf{9}$ in Silamine, $\bullet$; in 1:1 Silamine: MC, $\bullet$; and in 1:5 Silamine: MC,

Figure 3.59: Effect of pressure on thin film luminescence of $\mathbf{1 0}$ in Silube. The decrease of intensity with increasing the air pressure is displayed. Spectra were acquired at $1.3 \mathrm{mbar}$, 0.34 bar, 0.68 bar, 1 bar, 1.36 bar, 1.70 bar, 2.04 bar, 2.38 bar, 2.72 bar, and 3.06 bar. Background correction has been performed.

Figure 3.60: Effect of pressure on thin film luminescence of 10 in 5:1 Silube: MC. The decrease of intensity with increasing the air pressure is displayed. Spectra were acquired at $1.3 \mathrm{mbar}, 0.34$ bar, 0.68 bar, 1 bar, 1.36 bar, 1.70 bar, 2.04 bar, 2.38 bar, 2.72 bar, and 3.06 bar. Background correction has been performed. 146

Figure 3.61: Effect of pressure on thin film luminescence of 10 in 1:1 Silube: MC. The decrease of intensity with increasing the air pressure is displayed. Spectra were acquired at $1.3 \mathrm{mbar}, 0.34$ bar, $0.68 \mathrm{bar}, 1 \mathrm{bar}, 1.36 \mathrm{bar}, 1.70 \mathrm{bar}, 2.04 \mathrm{bar}, 2.38 \mathrm{bar}, 2.72 \mathrm{bar}$, and 3.06 bar. Background correction has been performed.

Figure 3.62: Effect of pressure on thin film luminescence of 10 in 1:5 Silube: MC. The decrease of intensity with increasing the air pressure is displayed. Spectra were acquired at 1.3 mbar, 0.34 bar, 0.68 bar, 1 bar, 1.36 bar, 1.70 bar, 2.04 bar, 2.38 bar, 2.72 bar, and 3.06 bar. Background correction has been performed. 148

Figure 3.63: Stern-Volmer plots of $\mathbf{1 0}$ in Silube, $\bullet$; in 5:1 Silube: MC, $\bullet$; in 1:1 Silube: $\mathrm{MC}, \bullet$; and in 1:5 Silube: $\mathrm{MC}$,

Figure 3.64: Effect of pressure on thin film luminescence of $\mathbf{5}$ in 2.3:1 Silamine: MC. The decrease of intensity with increasing the air pressure is displayed. Spectra were acquired at $1.3 \mathrm{mbar}, 0.34$ bar, $0.68 \mathrm{bar}, 1 \mathrm{bar}, 1.36 \mathrm{bar}, 1.70 \mathrm{bar}, 2.04 \mathrm{bar}, 2.38 \mathrm{bar}, 2.72 \mathrm{bar}$, and 3.06 bar. Background correction has been performed.

Figure 3.65: Effect of pressure on thin film luminescence of 5 in 1:1 Silamine: MC. The decrease of intensity with increasing the air pressure is displayed. Spectra were acquired at $1.3 \mathrm{mbar}, 0.34$ bar, 0.68 bar, 1 bar, 1.36 bar, 1.70 bar, 2.04 bar, 2.38 bar, $2.72 \mathrm{bar}$, and 3.06 bar. Background correction has been performed.

Figure 3.66: Effect of pressure on thin film luminescence of 12 in 2.3:1 Silamine: MC. The decrease of intensity with increasing the air pressure is displayed. Spectra were 
acquired at 1.3 mbar, 0.34 bar, 0.68 bar, 1 bar, 1.36 bar, 1.70 bar, 2.04 bar, 2.38 bar, 2.72 bar, and 3.06 bar. Background correction has been performed.

Figure 3.67: Effect of pressure on thin film luminescence of 13 in 2.3:1 Silamine: MC. The decrease of intensity with increasing the air pressure is displayed. Spectra were acquired at 1.3 mbar, 0.34 bar, 0.68 bar, 1 bar, 1.36 bar, 1.70 bar, 2.04 bar, 2.38 bar, 2.72 bar, and 3.06 bar. Background correction has been performed.

Figure 3.68: Effect of pressure on thin film luminescence of 13 in 1:1 Silamine: MC. The decrease of intensity with increasing the air pressure is displayed. Spectra were acquired at 1.3 mbar, 0.34 bar, 0.68 bar, 1 bar, 1.36 bar, 1.70 bar, 2.04 bar, 2.38 bar, 2.72 bar, and 3.06 bar. Background correction has been performed.

Figure 3.69: Effect of pressure on thin film luminescence of 13 in 1:9 Silamine: MC. The decrease of intensity with increasing the air pressure is displayed. Spectra were acquired at 1.3 mbar, 0.34 bar, 0.68 bar, 1 bar, 1.36 bar, 1.70 bar, 2.04 bar, 2.38 bar, 2.72 bar, and 3.06 bar. Background correction has been performed.

Figure 3.70: Stern-Volmer plots of $\mathbf{5}$ in 2.3:1 Silamine: MC, $\bullet$; 5 in 1:1 Silamine: $\mathrm{MC}$, $\bullet$ 12 in 2.3:1 Silamine: $\mathrm{MC}, \boldsymbol{1 2}$ in 1:1 Silamine: $\mathrm{MC}, \quad ; 13$ in 2.3:1 Silamine: $\mathrm{MC}, \bullet ; 13$ in 1:1 Silamine: $\mathrm{MC}$,

Figure 3.71: Luminescence microscopy of films of 13 performed under ambient conditions: A, 9:1 Silamine: MC; B, 5:1 Silamine: MC; C, 1:1 Silamine: MC; D, 1:5 Silamine: MC. The scale in red is $10 \mu \mathrm{m}$.

Figure 3.72: Temperature versus emission intensity ratio $\left(\mathrm{I}_{\mathrm{ref}}\right.$ is the intensity at $\left.10^{\circ} \mathrm{C}\right)$ of 5 in 2.3:1 Silamine: MC: 1 bar $\bullet 13$ in 2.3:1 Silamine: MC: 1 bar 160

Figure 3.73: Effect of pressure on thin film luminescence of 14 in 5:1 Silamine: MC. The decrease of intensity with increasing the air pressure is displayed. Spectra were acquired at 1.3 mbar, 0.34 bar, 0.68 bar, 1 bar, 1.36 bar, 1.70 bar, 2.04 bar, 2.38 bar, 2.72 bar, and 3.06 bar. Background correction has been performed. 161

Figure 3.74: Effect of pressure on thin film luminescence of 14 in 1:1 Silamine: MC. The decrease of intensity with increasing the air pressure is displayed. Spectra were acquired at 1.3 mbar, 0.34 bar, 0.68 bar, 1 bar, 1.36 bar, 1.70 bar, 2.04 bar, 2.38 bar, 2.72 bar, and 3.06 bar. Background correction has been performed.

Figure 3.75: Effect of pressure on thin film luminescence of 14 in 1:2.3 Silamine: MC. The decrease of intensity with increasing the air pressure is displayed. Spectra were acquired at 1.3 mbar, 0.34 bar, 0.68 bar, 1 bar, 1.36 bar, 1.70 bar, 2.04 bar, 2.38 bar, 2.72 bar, and 3.06 bar. Background correction has been performed.

Figure 3.76: Stern-Volmer plots of 14 in 5:1 Silamine: MC, $\bullet$; 14 in 1:1 Silamine: MC, 
Figure 3.77: Temperature versus emission intensity ratio $\left(\mathrm{I}_{\text {ref }}\right.$ is the intensity at $\left.10^{\circ} \mathrm{C}\right)$ of 14 in 5:1 Silamine: $M C$ : vacuum

Figure 3.78: Effect of pressure on thin film luminescence of 15 in 9:1 Silamine: MC. The decrease of intensity with increasing the air pressure is displayed. Spectra were acquired at 1.3 mbar (black) and 1 bar (red)...

Figure 3.79: Effect of pressure on thin film luminescence of 7 in 9:1 PDMS-based

Copolymer: MC. Spectra were acquired at 1.3 mbar (black) and 1 bar (red). 168

\section{List of Schemes}

Scheme 1.1: Schematic explanation of dynamic quenching. 6

Scheme 1.2: Schematic explanation of static quenching. 7

Scheme 1.3: Reductive amination reaction between a carbonyl functionalized

luminophore (L) and water-soluble amine-functionalized polymer Silamine. 20

Scheme 1.4: Reductive amination reaction between a double carbonyl functionalized

luminophore (L) and two cross-linked water-soluble amine-functionalized polymer

Silamine.

Scheme 1.5: Amide formation reaction between a carboxyl and an amine. 22

Scheme 1.6: Imine attachment reaction between an aldehyde and an amine. 23

Scheme 3.1: Synthesis of starting functionalized iridium dimer. 53

Scheme 3.2: Synthesis of 2 53

Scheme 3.3: Reductive amination of 2 with Silamine D208-EDA to make 8 65

Scheme 3.4: Mechanism of copper-catalyzed oxidation of PEGME to PEGME-

Aldehyde, $\mathrm{m}=\mathrm{n}-1$

Scheme 3.5: Preparation of amppy by Suzuki coupling reaction. 81

Scheme 3.6: Intermediates in amppy preparation by Suzuki coupling reaction. 82 
Scheme 3.7: Coupling reaction of amppy with PEGME-Aldehyde............................. 85

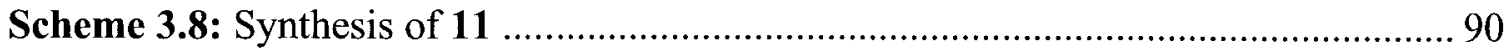

Scheme 3.9: Synthesis of starting iridium dimers............................................. 102

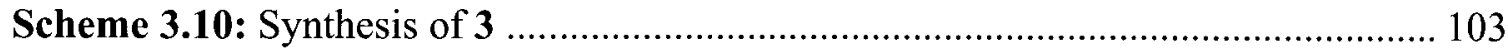

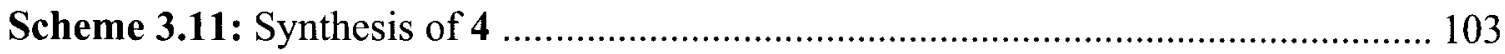

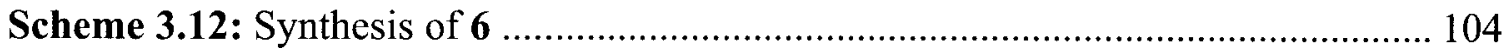

Scheme 3.13: Dispersing 4 in Silube CS-1 to make 10. .......................................... 122

Scheme 3.14: Coupling reaction of 5 with Silamine D208-EDA to make 12.............. 124

Scheme 3.15: Reductive amination of 5 with Silamine D208-EDA to make 13 .......... 127

Scheme 3.16: Reductive amination of 6 with Silamine D208-EDA to make 14.......... 129

Scheme 3.17: Coupling reaction of PBA with Silamine to make 15........................ 132

\section{List of Charts}

Chart 1: Structure of $\left[\operatorname{Ir}(\text { fppy })_{2}(t-\mathrm{Bu}-\mathrm{iCN})_{2}\right]\left[\mathrm{CF}_{3} \mathrm{SO}_{3}\right], 2$, with the numbering used in

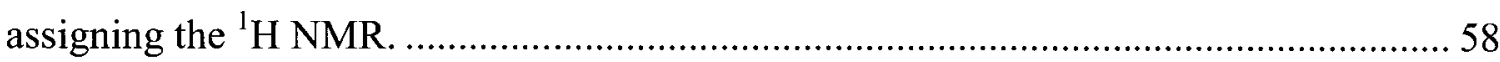

Chart 2: Structure of $\left[\operatorname{Ir}\left(\mathrm{C}_{6}\right)_{2}\right.$ (acacacid) $], 3$, with the numbering used in assigning the ${ }^{1} \mathrm{H}$ NMR. 108

Chart 3: Structure of $\left[\operatorname{Ir}(\mathrm{ppy})_{2}(\right.$ phendiald $\left.)\right]\left[\mathrm{PF}_{6}\right], \mathbf{6}$ 110 


\section{List of Abbreviations}

acac

acacacid

AIBN

amppy

bpy

$\mathrm{C}_{6}$

$\mathrm{CN}-t-\mathrm{Bu}$

COSY

DCC

DEAD

$\mathrm{dpp}$

$\Phi$

FIB

fppy

$\mathrm{H}_{2}$ TMpyp-4

HOMO

$k_{l}$

$k_{n r}$

$k_{q}$

$K_{S V}$
Acetylacetonate

4,6-Dioxoheptanoic acid

Azobisisobutyronitrile

5-Aminomethyl-2-phenylpyridine

2,2'-Bipyridine

3-(2'-Benzothiazolyl)-7-N,N-diethylaminocoumarin

tert-butyl isocyanide

Correlation spectroscopy

$\mathrm{N}, \mathrm{N}$ '-dicyclohexylcarbodiimide

Diethylazodicarboxylate

diphenylphenanthroline

Luminescence quantum yield

Poly(hexafluoroisopropyl-co-heptafluoro- $n$-butyl

methacrylate)

4-(2-Pyridyl)benzaldehyde

5,10,15,20-tetrakis(1-methyl-4-pyridyl)-21H,23H-porphine

Highest occupied molecular orbital

Radiative decay (luminescence) rate

Non-radiative decay rate

Bimolecular quenching constant

Stern-Volmer quenching constant 


\begin{tabular}{|c|c|}
\hline LEC & Light-emitting electrochemical cell \\
\hline LUMO & Lowest unoccupied molecular orbital \\
\hline $\mathrm{MC}$ & Microcrystalline cellulose \\
\hline MLCT & Metal-to-ligand charge-transfer \\
\hline OEP & Octaethylporphine \\
\hline OEPK & Octaethylporphyrin ketones \\
\hline OLED & Organic light-emitting diode \\
\hline OTf & Trifluoromethanesulfonate \\
\hline PBA & 1-Pyrenebutyric acid \\
\hline PDMS & Poly(dimethylsiloxane) \\
\hline PDMS-based copolymer & $\begin{array}{l}\text { Dimethylsiloxane-( } 60 \% \text { propylene oxide- } 40 \% \text { ethylene } \\
\text { oxide) block polymer }\end{array}$ \\
\hline PEGME & Poly(ethylene glycol) methyl ether \\
\hline phendiald & 1,10-Phenanthroline-2,9-dicarboxaldehyde \\
\hline ppy & 2-Phenylpyridine \\
\hline PSP & Pressure sensitive paint \\
\hline PVA & Polyvinylalcohol \\
\hline Qs & Oxygen quenching sensitivity \\
\hline$\tau$ & Luminescence lifetime \\
\hline TFPP & Tetrakis(pentafluorophenyl) porphyrin \\
\hline $\operatorname{Tg}$ & Glass transition temperature \\
\hline TMpyp-4 & 5,10,15,20-Tetrakis(1-methyl-4-pyridyl)-porphine \\
\hline TMS & Tetramethylsilane \\
\hline
\end{tabular}


TMSP

tpy
1-Trimethylsilyl-1-propyne

2-(p-Tolyl)pyridine

\section{List of Appendices}

Appendix A: Crystallographic Data..................................... 188 


\section{Chapter 1}

\section{Introduction}

\subsection{Optical Oxygen Sensors}

Various oxygen measurement techniques have been described in the literature including amperometric, ${ }^{1}$ paramagnetic, ${ }^{2}$ thermoluminescence, ${ }^{3}$ and chemiluminescence ${ }^{4}$ methods. Clark electrodes offer a standard and trusted technique for oxygen measurement. The method is based on the reduction of oxygen at the cathode. However, parameters affecting diffusion of oxygen, and electrical interference can lead to inaccuracy in the measurements. Furthermore, since oxygen is consumed, the method can be inappropriate for a number of practical applications. Another disadvantage of these sensors is their limited lifetime due to the consumption of the electrolyte by the oxygen being reduced. Therefore, improvement of novel non-destructive oxygen measurement methods is of considerable interest. Paramagnetic sensors exploit the fundamental magnetic field distortion by oxygen, and are applied in gas analysis. ${ }^{2}$ One of the current techniques in the field is optical oxygen sensors. Reduction of luminescence intensity of organic luminophores in presence of oxygen was first reported and the mechanism of emission loss explained in $1935 .^{5}$ Research in optical oxygen sensors has found application in a variety of fields including in vivo imaging, ${ }^{6}$ aerodynamics, ${ }^{7}$ environmental analysis, ${ }^{8}$ analytical chemistry, ${ }^{9}$ food industry, ${ }^{10}$ and biochemistry. ${ }^{11}$ Data 
are sent out as emitted light signals in these sensors. Optical oxygen sensors are required to be low-cost and easy to apply with no electrical interference.

\subsection{Photophysical Properties of Oxygen Sensor Luminophores}

Spontaneous emission of light or photoluminescence is generated in particular compounds after excitation, as illustrated in the Jablonski diagram (Figure 1.1). Vertical lines symbolize that molecular nuclei are stationary during electronic transitions. Absorption of light with appropriate wavelength results in several radiative and nonradiative processes. Usually the luminophore is excited to a higher vibrational level of an electronic singlet excited state, S1. Relaxation to the lowest vibrational level may happen by internal conversion. Once the excited molecule restores to the ground state, it emits a longer wavelength light compared to the initial light. A short-lived emission from a singlet (spin-paired electrons) excited state is fluorescence. Because there is no change in spin multiplicity in transition from the excited singlet state to the ground state, the lifetime of an excited singlet state and consequently the decay time of spin-allowed fluorescence are normally short $\left(10^{-9}\right.$ to $\left.10^{-7} \mathrm{~s}\right)$. Intersystem crossing from the singlet excited state to the triplet state can also take place. The transition from the first excited triplet state to the ground state has a low probability, as long-lived phosphorescence $\left(10^{-5}\right.$ to $10 \mathrm{~s})^{12}$ is associated with a change in electron spin. The presence of an atom with a high atomic number can enhance the rate of the spin-forbidden transitions by "heavy atom effect" which is due to spin-orbit coupling of the excited molecule. 
Phosphorescence appears at longer wavelengths compared with fluorescence, which is due to the relative energies of the excited states.

The energy difference between absorption and emission is called Stokes shift. It occurs when excited vibrational levels of the excited state is populated. Internal conversion to the zeroth vibrational level of the excited state decreases the energy of the excited state and so emission occurs at significantly lower energies than absorption.

An excited molecule may also undergo non-radiative decay from an excited state to a ground state which is basically the conversion of electronic excitation into vibrational and rotational energies in the molecule or its environment. The probability of intramolecular energy transfer between two electronic states is inversely proportional to the energy gap, $\Delta E$, between the two states (energy gap law). Therefore, the non-radiative rate constant is reduced by the size of the energy gap between the two states.

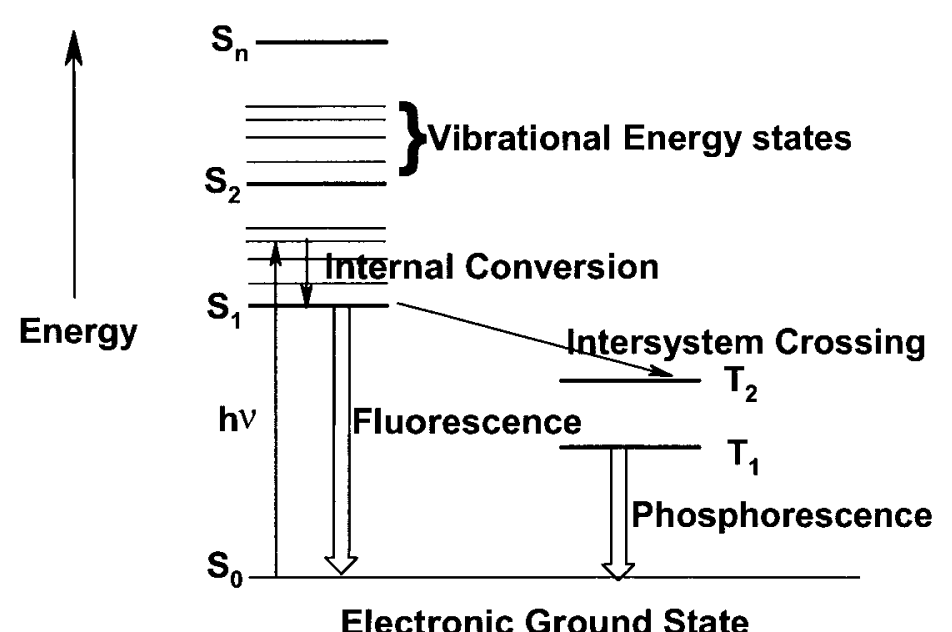

Figure 1.1: Jablonski diagram 
The average time the molecule remains in its excited state prior to emitting a photon is termed luminescence lifetime which is an important parameter for sensor applications. The excited state de-population rate is the sum of all radiative and nonradiative processes and the luminescence lifetime is the inverse of the decay rate:

$$
\tau=\frac{1}{k_{l}+k_{n r}}
$$

where $k_{l}$ is the radiative decay (luminescence) rate and $k_{n r}$ is the non-radiative decay rate. The efficiency of the luminescence is shown by the luminescence quantum yield, which is the ratio of the number of photons emitted to the number of photons absorbed.

$$
\Phi=\frac{\text { Numberof photons emitted }}{\text { Numberof photons absorbed }}
$$

The luminescence quantum yield can be determined by the excited state decay rates as well:

$$
\Phi=\frac{k_{l}}{\sum_{i} k_{i}}
$$

where $k_{l}$ is the radiative decay (luminescence) rate and $k_{i}$ is any radiative or non-radiative excited state decay rate. 


\subsection{Energy Transfer Mechanisms and Luminescence Quenching}

Theodor Förster proposed a mechanism for energy transfer between different molecules. ${ }^{13}$ Initially, the light is absorbed by the luminophore. Due to internal conversion, this "donor" luminophore relaxes to an excited electronic state that can resonance with an excited state of an "acceptor" molecule. In this mechanism, energy transfer is determined by three parameters. Overlapping of the luminescence spectrum of the donor and the absorption spectrum of the acceptor, termed as Förster's overlap integral, is one of the factors controlling the probability of energy transfer. Another parameter is the distance between the donor and the acceptor molecules, since the interaction between molecules is a dipole-dipole interaction. The third factor is the orientation of the dipoles of the two molecules. The rate of energy transfer is represented in terms of an effective distance, $R_{0}$, the distance between the donor and the acceptor $(r)$, and the luminescence lifetime of the donor $\left(\tau_{l}\right)$ :

$$
k_{E T}=\frac{1}{\tau_{l}}\left(\frac{R_{0}}{r}\right)^{6}
$$

Another energy transfer mechanism is the Dexter mechanism. ${ }^{14}$ As is shown in Figure 1.2, when the interaction energy between the two molecules is high and the molecules are in close proximity, it is possible that the excited electron is exchanged between the donor and acceptor molecules. The relative energies of the HOMO and LUMO of the two molecules are shown in Figure 1.2. 


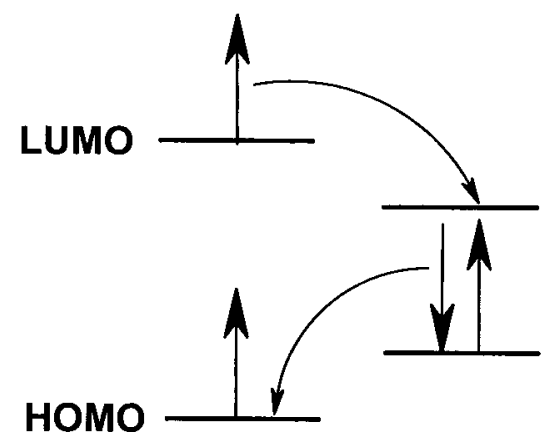

${ }^{3} \mathbf{D}^{*}$
A

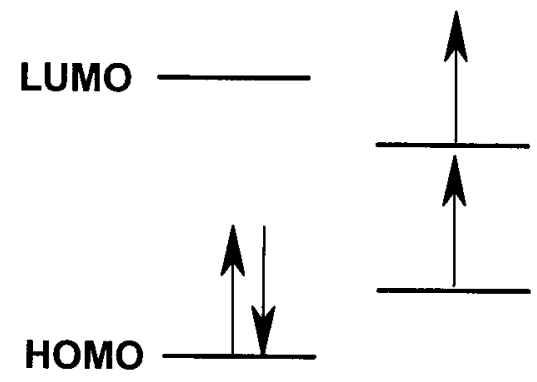

D

Figure 1.2: Dexter energy transfer, where $\mathrm{D}$ is the donor and $\mathrm{A}$ is the acceptor molecule.

Excited state quenching can be collisional and static. When energy transfer from the excited luminophore to the quencher occurs through molecular collisions, it is called collisional quenching. As shown in Scheme 1.1, the luminophore is relaxed to its ground state by a non-radiative decay process and both the luminescence quantum yield and lifetime decrease consequently. ${ }^{15}$

$\mathbf{D} \stackrel{\mathbf{h} v}{\longrightarrow} \mathrm{D}^{*} \underset{-\mathbf{Q}^{*}}{\longrightarrow} \mathrm{D}$

Scheme 1.1: Schematic explanation of dynamic quenching

In static quenching a non-luminescent complex is formed between the luminophore and the quencher. Scheme 1.2 describes the static quenching process. In this case, the luminescence lifetime of the uncomplexed luminophore is unchanged. 


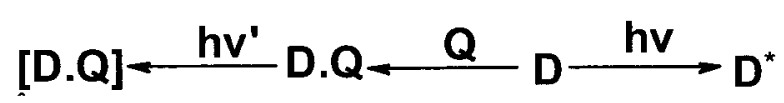

\section{Non-luminescent}

Scheme 1.2: Schematic explanation of static quenching.

Long-lived triplet state excited molecules are principally subject to interactions with other molecules. Diffusion-limited collisional oxygen quenching is described by the Stern-Volmer relationship, in which both luminescence lifetime and intensity are applicable.

$$
\frac{I_{0}}{I}=\frac{\tau_{0}}{\tau}=1+k_{q} \tau_{0}\left[O_{2}\right]=1+K_{S V}\left(p_{O_{2}}\right)
$$

where $I_{0}$ and $I$ are the luminescence intensities, and $\tau_{0}$ and $\tau$ are the lifetimes in the absence and presence of oxygen, respectively, $k_{q}$ is the bimolecular quenching constant, and $K_{S V}$ is the Stern-Volmer constant. $k_{q}$ is described by a modified Smoluchowski equation $^{16}$ :

$$
k_{q}=4 \pi N_{P}\left(D_{A}+D_{B}\right) \times 10^{3}
$$

where $N$ is Avogadro's number, $p$ is a factor representing the probability of a collision resulting in quenching and relates to the radius of interaction between the donor (luminophore) and the acceptor (oxygen), and $D_{A}$ and $D_{B}$ are the diffusion coefficients of the donor and acceptor, respectively. Normally, $k_{q}$ is considered constant under defined experimental conditions.

In describing static quenching, the complex formation equilibrium constant for the non-luminescent complex is taken into account: 


$$
K_{s}=\frac{[D . Q]}{[D][Q]}
$$

where $[D . Q],[D]$, and $[Q]$ are the complex, luminophore, and quencher concentrations, respectively. The luminescence intensity is dependent on the uncomplexed luminophore concentration $([D])$. Considering:

$$
[D . Q]=\left[D_{0}\right]-[D]
$$

where $\left[D_{0}\right]$ is the total concentration of luminophore, and replacing it into equation (1.7) results in:

$$
K_{s}=\frac{\left[D_{0}\right]}{[D][Q]}-\frac{1}{[Q]}
$$

Substituting luminophore concentrations with luminescence intensities gives:

$$
\frac{I_{0}}{I}=1+K_{s}[Q]
$$

Equation (1.10) has the same form as that of equation (1.5) which is defined for collisional quenching.

For quenching by oxygen, a single luminophore environment results in a linear plot of $I_{0} / I$ or $\tau_{0} / \tau$ vs. $\left[O_{2}\right]$. When the luminophore happens to be in multiple quenching environments, curvature may appear in the Stern-Volmer plot. Luminophores in the most accessible environments are easily quenched at low pressures, while those luminophores in less accessible environments will be quenched at higher pressures. Each environment therefore has its own quenching dependence $\left(\mathrm{K}_{\mathrm{SV}}\right)$ and so curvature in the plot results. A model explaining multiple quenching accessibilities can be utilized (equation 1.11). ${ }^{15,17}$

$$
\frac{I}{I_{0}}=\frac{\tau}{\tau_{0}}=\frac{1}{\frac{f_{1}}{1+K_{S V 1}\left[O_{2}\right]}+\frac{f_{2}}{1+K_{S V 2}\left[O_{2}\right]}+\frac{f_{3}}{1+K_{S V 3}\left[O_{2}\right]}+\ldots}
$$


where $f_{1}, f_{2}, f_{3} \ldots$ are the fractions of total unquenched luminescence intensity from different environments and $K_{S V I}, K_{S V 2}, K_{S V 3} \ldots$ are the Stern-Volmer constants for those environments. An adequate approximation would be taking only two terms in equation 1.11, representing double quenching in heterogeneous polymers, called a two-site model. $^{15,17}$

\subsection{Oxygen Quenching}

Gijzeman et al. ${ }^{18}$ described the mechanism of oxygen quenching of the aromatic excited triplet state, in which the relative contributions of the two mechanisms depend on the solvent. Studies on oxygen quenching of the excited metal to ligand charge transfer states of luminescent complexes of ruthenium (II), osmium (II), and iridium (III) assigned the process to energy transfer. ${ }^{19}$ However, the electron transfer process was found possible in which the quenching rate constants inversely correlate with the excited state energy, and correlate with the oxidation potentials of the complexes. ${ }^{19}$

The Stern-Volmer relationship (equation 1.5) can be utilized to correlate luminescence intensity with oxygen pressure in oxygen quenching sensors. However, calibrations at zero oxygen pressure, especially in wind tunnel tests, are not practical. Provided that Henry's law is applicable to oxygen concentration in pressure sensitive materials (equation 1.12, where $p$ is the partial pressure of oxygen, $c$ is molar concentration of oxygen, and $k$ is the Henry's law constant), it is expected that the oxygen concentration will change with global air pressure. 


$$
P=k c
$$

In order to make the Stern-Volmer relation more practical, it is possible to substitute oxygen concentration with air pressure and do reference measurements at 1 atm pressure. This will yield equation (1.13):

$$
\frac{I_{r e f}}{I}=\frac{\tau_{r e f}}{\tau}=C+Q_{s} \frac{P}{P_{r e f}}
$$

where $P_{\text {ref }}$ is the reference pressure (generally $1 \mathrm{~atm}$ ) at which the luminescent compound's intensity is $I_{\mathrm{ref}}$ and $C$ is normally $1-Q s$ if temperature is constant. Oxygen quenching sensitivity $(Q s)$ is obtained from the slope of a linear fit to the modified SternVolmer plot (equation 1.13). However, since the pressure sensitive method (section 1.5) is applied to solid phases, deviations from linearity can happen (see eqn 1.11).

\subsection{Pressure Sensitive Paints}

In the field of aerodynamics, conventional pressure distribution measurement requires the use of pressure taps or transducers. The primary disadvantage of this technique is that since the pressure taps are placed at separate spots on the model, pressure data are only provided at those points. In addition, minimization of flow intrusion by taps and transducers requires considerable time and expense when installing these devices. In the pressure sensitive paint (PSP) technique, an oxygen-sensor luminophore is incorporated in paint formulations whose photophysical properties change with changing air pressure. ${ }^{7 \mathrm{~b}, 20}$ PSPs have several advantages over discrete pressure taps 
including high spatial resolution, less time consumption, surface-flow visualization, simplicity and versatility. ${ }^{21}$ This technique is shown in Figure 1.3.

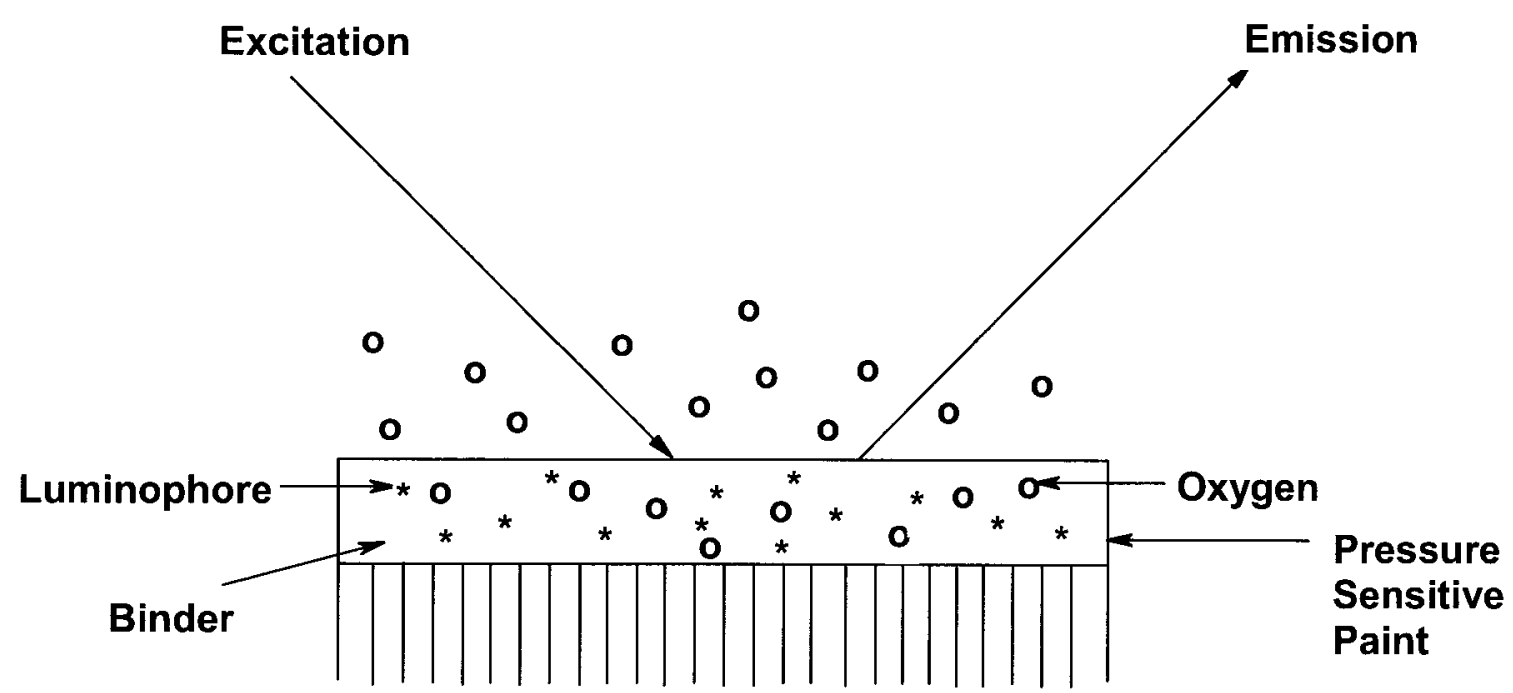

Model Surface

Figure 1.3: Schematic picture of pressure sensitive paint coated model surface.

It is possible to design oxygen sensors for specific practical applications. ${ }^{22}$ Since highly pressure sensitive sensors with large values of $Q s$ cause large decrease in intensity for a pressure increase, detection difficulties may arise. For pressure sensitive paints used in the range of $0-1 \mathrm{~atm}$, values of $0.4-0.8$ for $Q s$ result in good accuracy. ${ }^{23}$

Ideally, a PSP would only respond to oxygen concentration. However, temperature affects the luminescence of PSPs by increasing the non-radiative decay rate of the luminophore. It can also change the oxygen permeability of the binder according to equation (1.14): 


$$
P=P_{0} \exp \left(\frac{-E_{P}}{R T}\right)
$$

where $P$ is the permeability coefficient, $P_{0}$ is a pre-exponential factor, $E_{P}$ the activation energy of permeation, $R$ the gas constant, and $T$ the temperature in Kelvin. It is desirable for the permeability coefficient of PSP binders to be largely independent of temperature. A possible solution is a dual luminophore PSP formulation which contains an oxygen sensitive luminophore and a temperature sensitive luminophore that is not quenched by oxygen. This formulation can provide temperature versus pressure data that can be used to correct for the temperature sensitivity of the luminophore being quenched by oxygen. $^{20 u-v, 24,21 a}$

\subsection{Materials for Optical Oxygen Sensors}

Several requirements are needed to be met by luminophores before being used in sensors. These include practical excitation wavelengths, large molar absorption coefficients, photostability, long lifetimes, and high quantum yields. Polymers are required to dissolve the luminophore and have good mechanical properties and high oxygen permeabilities.

Thickness of films of soft and deformable polymers would change under changing pressure, leading to unreliable data. Because of this, polymers possessing the mechanical properties of environmental stress crack resistance, resistance to flow, and low compressibility are optimal for PSP applications. 
Oxygen permeation through flawless polymeric matrix happens by successive steps of dissolution of oxygen and diffusion of the dissolved oxygen. The permeation rate is determined by oxygen permeability coefficient $P$ which is calculated as

$$
P=D S
$$

where $D$ and $S$ are the oxygen diffusion constant and oxygen solubility coefficient, respectively.

\subsubsection{Luminescent Probes for PSPs}

Not many luminescent compounds are appropriate for pressure sensitive paint applications, since they are expected to meet several conditions including: photostability, long lifetime (for improved quenching efficiency), large Stokes shift, high emission quantum yield (in order to increase ease of measurement), and stability against singlet oxygen. In addition, visible excitation would eliminate safety concerns. Practically, there is no perfectly appropriate luminescent compound for all PSP calibrations.

Luminophores utilized in PSPs include organic dyes, ${ }^{25}$ polycylic aromatic hydrocarbons, ${ }^{26}$ and organometallic compounds. ${ }^{27}$ Organic dyes usually suffer from short excited state lifetimes.

Polycyclic aromatic hydrocarbons are quenched by oxygen and have been utilized in optical oxygen sensors. As an example, pyrene with an excited-state lifetime of $400 \mathrm{~ns}$ has been studied for oxygen sensing. However, its photostability limits the 
applicability ${ }^{26 c,}{ }^{28}$ Associating a polymer ligated cobaltporphyrin with 1-pyrenebutyric acid, a dual-mode and non-Stern-Volmer oxygen pressure sensitive film has been developed. ${ }^{29}$ Another example of polycyclic aromatic hydrocarbons is decacyclene as an oxygen sensor luminophore for which a blue excitation can be used. ${ }^{26 g}$

Organometallic compounds demonstrate large Stokes shifts and efficient and long-lived emissions and are often utilized as optical oxygen pressure sensors. Varying the metallic centre or ligands, a library of compounds with different properties can be prepared. Basically, the energy and characteristics of the lowest excited state define the luminophore's properties, since emission is generated from this level. ${ }^{30}$ For efficient energy transfer to oxygen, the proper energy for the lowest excited state is $\geq 95 \mathrm{kJmol}^{-1}$. Because d-d states of the metallic centre are not luminescent, the lowest excited state should be a charge-transfer or a ligand $\pi-\pi^{*}$. Also, in order to avoid thermal population of the d-d states and rapid decay and large temperature sensitivity as a consequence, they should be well separated from the emitting level.

Transition metal polypyridyl compounds of ruthenium, rhenium, osmium, and iridium and metalloporphines of platinum and palladium are especially used in oxygen sensors. One of the extensively used polypyridyl complexes utilized in the field is $\mathrm{Ru}(\mathrm{dpp})_{3}(\mathrm{dpp}=$ diphenylphenanthroline $) .{ }^{27 \mathrm{a}}$ The photophysical properties have been increased by expanded conjugation of the diimine ligand (from bipyridine to phenanthroline to diphenylphenanthroline). A thermally populated d-d state in ruthenium complexes, especially $\mathrm{Ru}(\mathrm{bpy})_{3}{ }^{2+}$ results in deactivation and temperature sensitivity consequences ${ }^{30}$ More photostable osmium polypyridyl complexes with excitation in the red region of visible spectrum are reported to show similar sensitivities as ruthenium 
complexes. ${ }^{27 b}$ Normally, ionic transition metal polypyridyl compounds are insoluble in organic polymers. However, intensely luminescent iridium complexes are soluble in organic polymers (due to similar polarities) and show long lifetimes (order of $\mu \mathrm{s}$ ) and high quantum yields. Therefore, iridium complexes are desirable candidates as oxygen sensors. $^{27 \mathrm{c}-\mathrm{f}, 27 \mathrm{i}-\mathrm{j}, 31}$

Platinum and palladium porphyrins display strong room temperature luminescence with long lifetimes (order of hundreds of $\mu \mathrm{s}$ ) and high quantum yields. ${ }^{27 a}$ PtOEP has been used in several optical oxygen sensor studies in which it displayed photobleaching at continuous illumination conditions. PtTFPP (Pt(II) tetrakis(pentafluorophenyl) porphyrin) shows enhanced stability due to the presence of electron-withdrawing fluorine atoms. ${ }^{27 \mathrm{~h}}$ Platinum and palladium octacthylporphyrin ketones (OEPK) are reported as photostable optical sensors. ${ }^{27 \mathrm{~g}, \mathrm{i}}$

Other luminescent transition metal complexes investigated for oxygen sensing applications include compounds based on copper, ${ }^{32}$ zirconium, ${ }^{33}$ lanthanides,${ }^{34}$ and gold. $^{35}$

\subsubsection{Polymers for PSPs}

Binders' properties are considerably important in oxygen sensors. Normally, a good binder for luminescent probe immobilization should be easy to prepare, photostable, mechanically strong, soluble in an appropriate solvent, stable against singlet oxygen, have appropriate oxygen permeability, dissolve the luminophore well, and have little or no impact on photophysical properties of the luminescent probes. 
Generally, luminophores are immobilized in binders by covalent attachment or embedding technology. Covalent attachment prevents luminophore aggregation (and the subsequent self-quenching) due to poor solubility. Furthermore, it can control luminophore distribution between the different components of the paint. ${ }^{36,7 \mathrm{c}}$ Ruthenium complexes have been covalently attached to polythionylphosphazenes and silicon-dioxide polymers $^{36 c,} 37$ Derosa et al. synthesized polymer-bound iridium complexes by hydrosilation reaction. ${ }^{7 c}{ }^{36 \mathrm{~d}}$ Attachment of palladium or platinum porphyrin to poly(trimethylsilylpropyne) has also been studied. ${ }^{38}$

Organic binders are commonly used in PSP application. ${ }^{39}$ Normally, the luminophore is dispersed in a polymeric solution. Different organic polymers have been considered in oxygen sensors owing to their distinctive oxygen permeabilities. ${ }^{40}$ Cellulose derivatives, fluoropolymers, silicone polymers and organic glassy polymers including polystyrene and poly(methylmethacrylate) can be named. ${ }^{26 g}$ Cellulose derivatives such as ethyl cellulose and cellulose acetate produce mechanically strong films. Introducing a plasticizer to cellulose derivatives provides highly oxygen permeable films. ${ }^{31 d}$ Fluoropolymers such as poly(hexafluoroisopropyl-co-heptafluoro- $n$-butyl methacrylate) (known as FIB) and poly(2,2,2-trifluoroethyl-co-isobutyl methacrylate) are photostable oxygen permeable polymers. ${ }^{41}$ The American standard polymeric binder in PSPs, FIB, necessitates hazardous solvent ( $\alpha, \alpha, \alpha$-trifluorotoluene) application. ${ }^{42}$ Poly(dimethylsiloxane)s (PDMS) have large oxygen permeabilities, ${ }^{43}$ but they suffer from poor mechanical properties. Cross-linking would improve mechanical properties, but may result in different luminophore environments due to film non-uniformity. ${ }^{44}$ Furthermore, because of high hydrophobicity of these polymers, aggregate and 
microcrystallite formation in them have been reported. ${ }^{45}$ Poly(1-trimethylsily-1-propyne) (poly(TMSP)) is reported to have higher gas permeability compared with PDMS while producing durable porous films. ${ }^{46}$ Organic glassy polymers have lower oxygen permeabilities compared with fluoropolymers or silicon polymers but provide good mechanical properties. ${ }^{39}$

Avnir et al. immobilized rhodomine $6 \mathrm{G}$ into an organic ceramic presenting the sol-gel method. ${ }^{47}$ This method has applications in oxygen sensor technology, however, the films were brittle and inadequate for oxygen diffusion due to the high polarity of the film. ${ }^{48}$

Organic-inorganic hybrid films are used in oxygen sensors, ${ }^{48 a,}{ }^{49}$ which are grouped into two types: type I in which van der Waals forces or hydrogen bonds exist between organic and inorganic molecules, and type II in which covalent bonds exist between organic and inorganic parts. ${ }^{50}$ Commonly, inorganic particles improve the mechanical properties of the polymeric film, and increase the surface area. ${ }^{17 e, 51}$ Metal oxides including $\mathrm{Al}_{2} \mathrm{O}_{3}, \mathrm{Fe}_{2} \mathrm{O}_{3}, \mathrm{Sb}_{2} \mathrm{O}_{3}$, and $\mathrm{ZrO}_{2}$ have been dispersed on the surface of cellulose and its derivatives. ${ }^{52}$ Nanostructured materials such as aluminum oxide embedded into polyvinylalcohol (PVA) were also studied as possible matrices for organometallic compounds. ${ }^{51,53}$ Consistent films of ormosil have been prepared, in which organic parts are assembled in silicon-oxide networks. ${ }^{54}\left[\mathrm{Ru}(\mathrm{dpp})_{3}\right]^{2+}$ immobilized in fluorinated xerogels consisting of n-propyltrimethoxysilicane and 3,3,3trifluoropropylmethoxysilane has been investigated and detected as highly oxygen sensitive sensors. ${ }^{55}$ The films are even, uniform, and durable. 


\subsubsection{Electrochemical Adsorption of Luminophores}

Binders increase the response time by decreasing the luminophore's access to oxygen. A method has been developed to electrochemically anodize aluminum surfaces and omit binders in order to decrease the response time. A uniform porous surface is produced which adsorbs the luminophore. In this method, the luminescence lifetime of the luminophore defines the response time. ${ }^{20 t, 56}$ However, this method is only applicable to aluminum surfaces and some luminophores are not easily adsorbed.

\subsection{Water-Soluble Pressure Sensitive Paints}

PSP formulations in current use are based in organic solvents and their application and recycling engender workplace safety and contamination concerns. These concerns could be eliminated by using an entirely aqueous PSP formulation that possesses acceptable performance compared to commercial PSPs. Furthermore, the surface of the paint will be smooth after evaporation of the relatively high boiling water.

Oglesby and Ingram developed a water-based PSP that incorporated non-volatile organic solvents, an emulsion polymer and a platinum porphyrin luminescent compound. $^{57}$

Luminescent iridium complexes have been used in PSPs because of their large quantum yields of emission under room temperature and normal pressure, and efficient quenching of the photoexcited triplet state by molecular oxygen. However, their solubility is poor in aqueous solution. The poor solubility can be overcome by covalent 
attachment to a water-soluble polymer. It can also prevent aggregation or leaching of the luminophore. Polydimethylsiloxane is used in oxygen sensors ${ }^{44,58}$ as a well known oxygen permeable polymer, the mechanical properties of which need to be improved for practical applications. Polyethylene glycol is a polar rubbery polymer with oxygen permeability of $0.5 \times 10^{-13} \mathrm{~cm}^{3} \mathrm{~cm} / \mathrm{cm}^{2}$.S.Pa in the semi-crystalline and $6.1 \times 10^{-13}$ $\mathrm{cm}^{3} \mathrm{~cm} / \mathrm{cm}^{2} . \mathrm{S} . \mathrm{Pa}$ in the amorphous phase (at $35^{\circ} \mathrm{C} ; \mathrm{MW}=$ approx. $1,000,000$ ), which is independent of pressure. ${ }^{59}$ It has been used in cellulose-based films as a plasticizer to change barrier and physical properties, thus making more flexible films. ${ }^{60}$

\subsection{Reductive Amination}

Reductive amination of carbonyl compounds became an important and practical Rout in synthesis of amines, after the presentation of a method using cyanoborohydride, ${ }^{61}$ for in situ reductive amination reaction. However, this method has usage difficulties due to the toxicity of the reagent.

In situ reductive amination with methanolic pyridine-borane ${ }^{62}$ presents a conveniently mild process for covalent attachment of carbonyl functionalized and amine functionalized compounds. Pyridine-borane is commercially available, relatively stable in air, and easy to use. Molecular sieves are utilized in situ to imine formation to absorb water. It has been reported ${ }^{62}$ that the intermediate imine is reduced during the reaction, not the acidic workup.

Inexpensive water-soluble amine-functionalized siloxane polymer, Silamine, ${ }^{63}$ is commercially available, to which a carbonyl functionalized luminophore can be attached. 
Also, poly(ethylene glycol) methyl ether (PEGME) can be oxidized to PEGME-aldehyde by a copper-catalyzed procedure under mild conditions. ${ }^{64}$ In this approach an amine functionalized luminophore will provide the appropriate reaction site. Reductive amination reaction between a carbonyl functionalized luminophore and Silamine is demonstrated in Scheme 1.3.<smiles>CC(C)(O[Si](C)(C)C)[Si](C)(C)O[Si](C)(C)O[Si](C)(C)O[Si](C)(C)C</smiles>

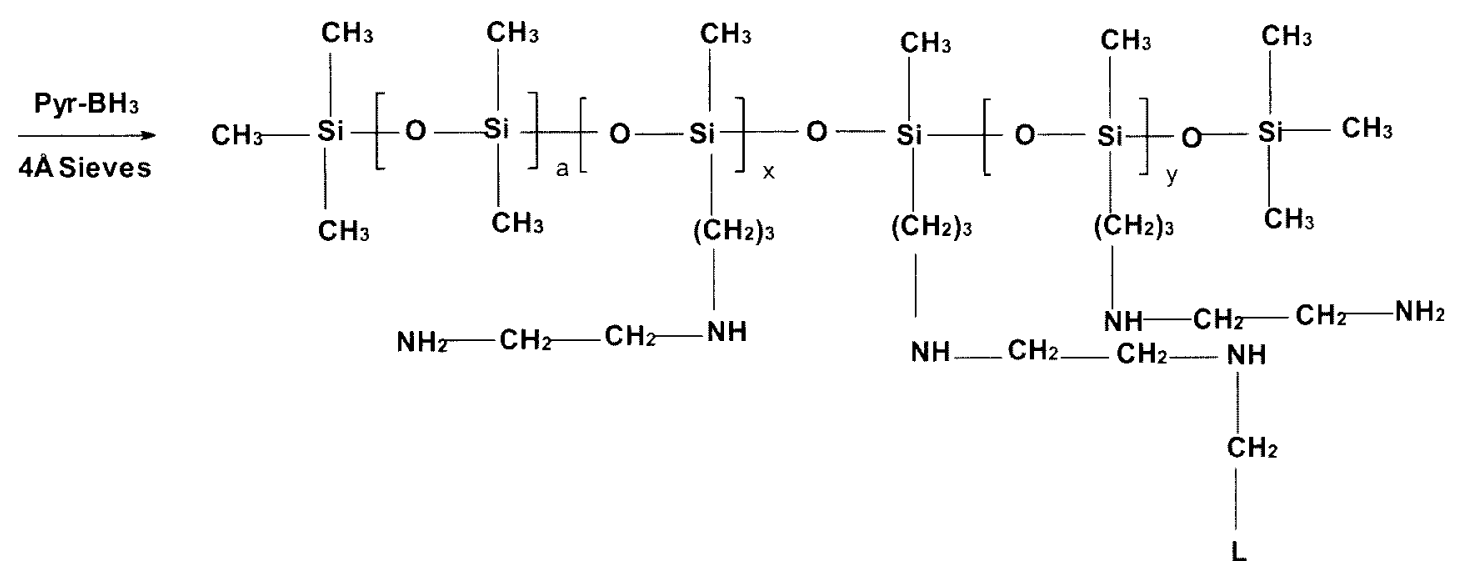

Scheme 1.3: Reductive amination reaction between a carbonyl functionalized luminophore (L) and water-soluble amine-functionalized polymer Silamine.

In case of double-functionalized luminophores, the attachment can be of crosslinked Silamine chains, as shown in Scheme 1.4. 
<smiles>C[Si](C)(C)O[Si](C)(C)O[Si](C)(C)O[Si](C)(C)CNCCN</smiles>

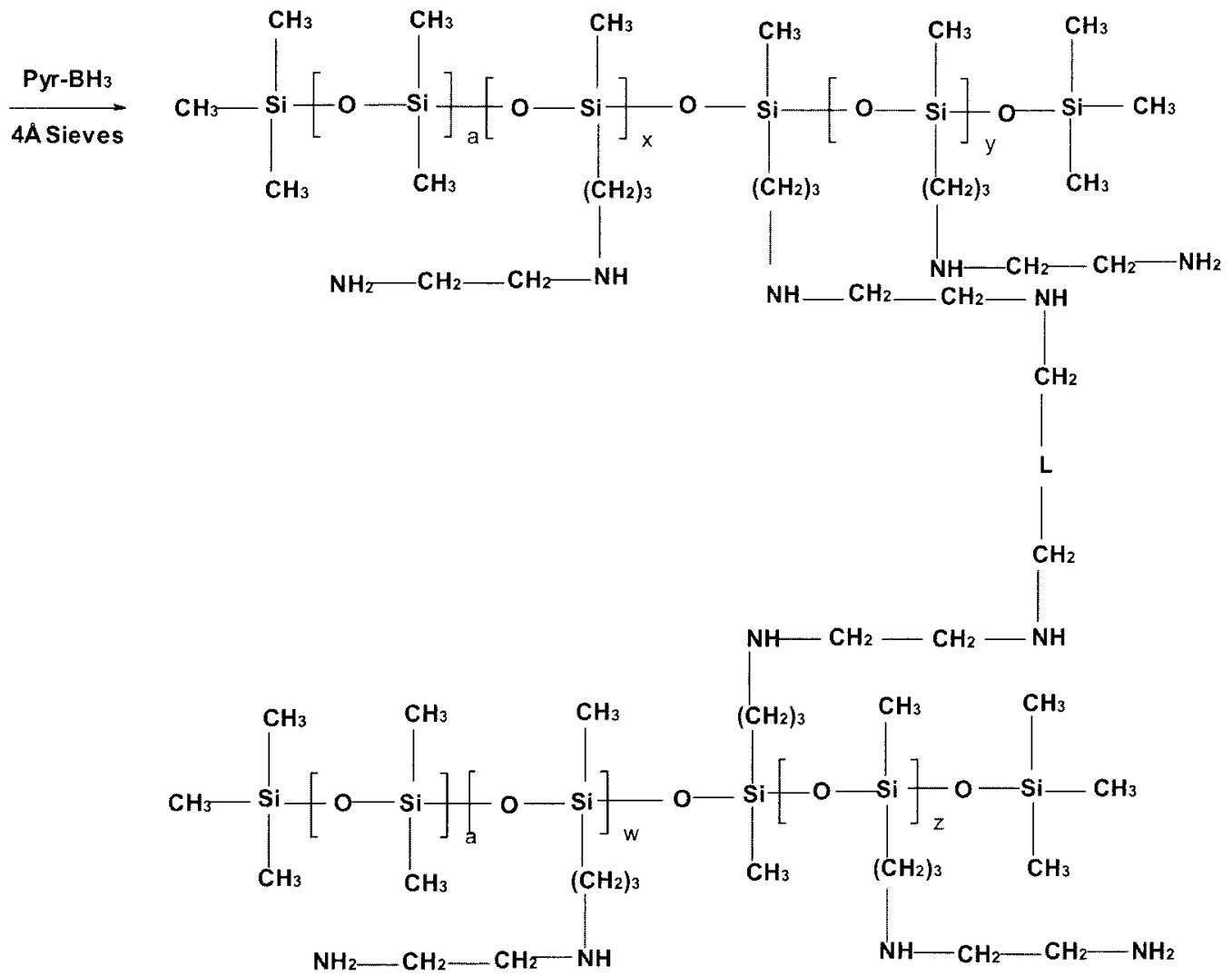

Scheme 1.4: Reductive amination reaction between a double carbonyl functionalized luminophore (L) and two cross-linked water-soluble amine-functionalized polymer Silamine.

Using mono-functionalized luminophores can prevent this problem. However, as long as the solubility of the product in water is satisfactory, this would not affect the practicality of the method. 


\subsection{Amide Formation Reaction}

Utilizing the coupling agent dicyclohexylcarbodiimide ${ }^{65}$ introduced an effective

method for peptide or in general, amide bond formation. In this method, a carboxyl and an amino functional group couple in high yield at room temperature. Furthermore, it can be conducted in aqueous solution, due to insensitivity to water. $\mathrm{N}, \mathrm{N}^{\prime}$-dicyclohexylurea is the by-product which is simply separated, since it has a very low solubility in water and most organic solvents.

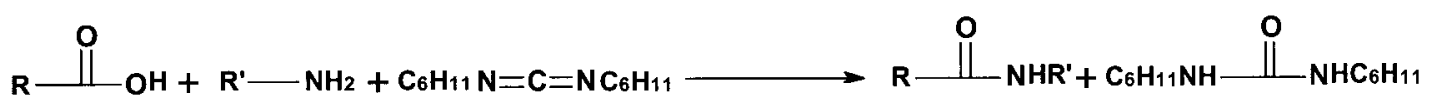

Scheme 1.5: Amide formation reaction between a carboxyl and an amine.

\subsection{Imine Attachment}

Another reaction that can be used in covalent attachment of luminophores to polymers is imine formation reaction between carbonyl and amine functionalized moieties. ${ }^{66}$ Normally, this method is acid-catalyzed, in which the reactants are refluxed, in the presence of an azeotroping reagent if needed. Imines can be produced quantitatively from highly reactive aromatic aldehydes with no need to eliminate the byproduct water during the reaction. 


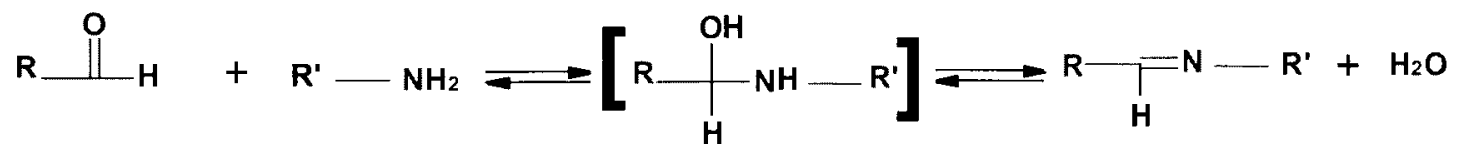

Scheme 1.6: Imine attachment reaction between an aldehyde and an amine.

Carbonyl functionalized iridium complexes have been utilized to cross-link Lalanine, avidin, and biotin, ${ }^{67}$ as well as to covalently attach to amine-terminated polymers. $^{68}$

\subsection{Cyclometalated Iridium Complexes}

Luminescent iridium complexes possess high quantum yields due to small nonradiative decay rates. The excited state of these complexes is ligand-based and, by varying the substituents on the ligands, the emission wavelengths can be systematically varied. Cyclometalated iridium complexes are neutral or ionic compounds with strong photophysical properties, which have been investigated in different studies, including biological labeling, ${ }^{67 a, 69}$ sensors, ${ }^{7 \mathrm{c}, 27 \mathrm{c}, 27 \mathrm{j}, 70}$ light-emitting electrochemical cells (LECs), ${ }^{71}$ organic light-emitting diodes (OLEDs), ${ }^{72}$ and sensitizers of luminescence. ${ }^{73}$ Examples of iridium-complex applications in the field of sensors are homocysteine, metal cations, and oxygen sensors. The coordinating arrangement around the Ir atom contains disposition of a 5 or 6 membered metallocycle, with 2-phenylpyridine (ppy) being the most common ligand. Facial $\operatorname{Ir}(\text { ppy })_{3}$ was the first iridium complex studied as an oxygen sensor in polystyrene. $^{27 d}$ The conventional synthesis of an $\operatorname{Ir}(\mathrm{III})$ complex is performed by a twostep method. In the first step a chloro bridged dinuclear $\operatorname{Ir}(\mathrm{III})$ dimer is prepared. ${ }^{72 \mathrm{c},}{ }^{74} \mathrm{By}$ substituting the chloro ligands with a third ligand (usually bidentate), the luminescent 
compound forms. Most of the cyclometalating ligands have one neutral and one anionic coordinating site, e.g. in ppy, the phenyl ring provides the anionic site. The $\mathrm{Ir}-\mathrm{C}$ bond between the $\operatorname{Ir}(\mathrm{III})$ metal and ppy-based ligands is strong, comparable to covalent bonds. Therefore, these complexes are thermally stable, while having electronic interactions between orbitals of iridium centre and the ligands.

Basically, four transitions can be observed in these complexes: singlet and triplet metal-to-ligand charge transfer ( ${ }^{1} \mathrm{MLCT}$ and $\left.{ }^{3} \mathrm{MLCT}\right)$ and singlet and triplet ligandcentered $\left({ }^{1} \mathrm{LC}\right.$ and $\left.{ }^{3} \mathrm{LC}\right)$ transitions, with energetic order of: ${ }^{1} \mathrm{LC}>{ }^{1} \mathrm{MLCT}>{ }^{3} \mathrm{MLCT} \geq$ ${ }^{3} \mathrm{LC}$. Generally, it is believed that the phosphorescent state is a mixed state of MLCT and LC transition states, originated from spin-orbit coupling. ${ }^{75}$

There is efficient energy transfer from the long-lived triplet excited state of luminescent iridium(III) complexes to the triplet ground state of molecular oxygen, leading to luminescence quenching and singlet oxygen production. ${ }^{36 \mathrm{~d}}$ Therefore, these compounds can be used as oxygen sensors in different areas. ${ }^{7 c, 43 a, 53,76}$.

In order to acquire solid state sensors, it is essential for the iridium luminophore to be compatible with the matrix. ${ }^{70 b,} 77$ Fluorinated polyacrylates, PDMS, poly(thionylphosphazene)s, and polystyrene have been used as the matrix. ${ }^{58,78}$ Covalent attachment of these luminophores to the polymeric binder has been the favorable method. DeRosa et al. have described the synthesis and characterization of polymer bound luminescent iridium complexes. ${ }^{36 \mathrm{~d}, 36 \mathrm{e}, 58}$ Employing polystyrene led to desirable oxygen sensors with good mechanical properties. 


\subsection{Research Approach}

In this project, completely water-soluble pressure sensitive paints will be synthesized and characterized as environmentally friendly alternatives to non-aqueous paint formulations, in order to improve workplace safety, and lower contamination.

Inexpensive water-soluble polymers will be utilized in developing appropriate matrices for dissolving luminophores. Providing spray-able formulations with good mechanical properties as well as high oxygen permeabilities will be the challenges to overcome.

Novel functionalized luminescent iridium complexes will be used, in order to benefit from their large quantum yields of emission and efficient quenching of the excited triplet state by molecular oxygen. The poor solubility of the complexes will be overcome by their covalent attachment to water-soluble polymers.

Producing water-soluble luminescent polymers by covalent attachment to commercially available or synthesized functionalized water-soluble polymers will be investigated. Reductive amination, amide formation, and imine attachment reactions will be studied as practical covalent bonding methods.

Photophysical properties, oxygen quenching sensitivity, and matrix composition impact on the sensitivity of the formulations will be explored. 


\section{Chapter 2}

\section{Experimental}

\subsection{Starting Materials}

\subsubsection{Reagents}

Iridium (III) chloride hydrate (Pressure Chemical), 4-(2-pyridyl)benzaldehyde (fppy) $\quad(\geq 97 \%, \quad$ Aldrich), 2-phenylpyridine $\quad$ (ppy) $\quad(98 \%, \quad$ Aldrich $), \quad$ silver trifluoromethanesulfonate (AgOTf) $(99+\%$, Aldrich), tert-butyl isocyanide (CN-t-Bu) ( $\geq 97 \%$, Aldrich), 4,6-Dioxoheptanoic acid (acacacid) (Aldrich), triethylamine ( $\geq 99.5 \%$, Fluka), hydrochloric acid (ACS Reagent grade, Anachemia), 5-aminomethyl-2-chloro pyridine (97\%, Aldrich), acetylchloride (98\%, Aldrich), phenylboronic acid (95\%, Aldrich), tetrakis (triphenylphosphine) palladium (0) (99\%, Aldrich), sodium carbonate (ACS Reagent grade, $\geq 99.5 \%$, Aldrich), sodium hydroxide (lab grade, Anachemia), potassium hexafluorophosphate $(98 \%$, Aldrich), 5,10,15,20-tetrakis(1-methyl-4pyridyl)-21H,23H-porphine,tetra-p-tosylate $\quad$ salt $\quad\left(\left[\mathrm{H}_{2} \text { TMpyp-4][OTs }\right]_{4} \quad\right.$ (Aldrich), palladium chloride (dry, Fisher), sodium tetrafluoroborate ( $\geq 97 \%$, Aldrich), ammonium hexafluorophosphate (95+\%, Aldrich), copper (I) chloride (ACS Reagent grade, $\geq 90 \%$, Aldrich), 1,10-phenanthroline (99+\%, Aldrich), potassium carbonate (ACS Reagent grade, $\geq 99 \%$, Aldrich), diethyl azodicarboxylate (Aldrich), poly(ethylene glycol) methyl ether-550 ( $\mathrm{M}_{\mathrm{n}} \sim 550$, Aldrich), pyridine-borane (Aldrich), N,N'-dicyclohexylcarbodiimide 
(99\%, Aldrich), Silube CS-1 (MW 3000, acid value 44-52, Siltech Corp.), polyethylene glycol (PEG) (average $\mathrm{M}_{\mathrm{n}}$ Ca. 200; Aldrich), (hydroxypropyl) methyl cellulose (Aldrich), dimethylsiloxane-(60\% propylene oxide- $40 \%$ ethylene oxide) block polymer (PDMSbased copolymer) (MW 30,000, Gelest), and 1-pyrenebutyric acid (97\%, Aldrich) were used as received without further purification. Silamine D208 EDA (MW 2475, amine value 30 , Siltech Corp.) was heated to $80^{\circ} \mathrm{C}$ under vacuum for 30 minutes before use in reductive attachment reaction. 1,10-Phenanthroline-2,9-dicarboxaldehyde was prepared according to Chandler. ${ }^{79}$

\subsubsection{Solvents}

Dichloromethane (Technical, ACP chemicals) was dried over calcium hydride (90-95\%, Aldrich) and distilled and stored under argon before use. Methanol (HPLC Grade, J. T. Baker), diethyl ether (Anhydrous ACS, Fisher Scientific), hexanes (Spectro grade, Caledon), tetrahydrofuran (Certified, Fisher Scientific), ethanol (Anhydrous, Commercial Alcohols), ethyl acetate (Reagent grade, Caledon), methyl tert-butyl ether (Reagent grade, Aldrich), N,N'-dimethylformamide (Reagent grade, Caledon), chloroform (Reagent grade, Caledon), and toluene (Reagent grade, Caledon) were used as received. Acetone (ACS Reagent grade, Anachemia) was distilled before use. 2Ethoxyethanol (ReagentPlus ${ }^{\mathrm{TM}}$, Aldrich) was distilled and dried over $\mathrm{MgSO}_{4}(\geq 97 \%$, Aldrich). For absorption and emission spectroscopies, acetonitrile (Spectrograde, Fisher Scientific) and dichloromethane (Spectro grade, Caledon) were used as received. For NMR spectroscopy, chloroform- $d$ (99.8 atom $\% \mathrm{D}, \mathrm{CDN}$ Isotopes), dimethyl- $d_{6}$ sulfoxide 
(99.9 atom \% D, CDN Isotopes), deuterium oxide (99.9 atom \% D, CDN Isotopes), acetone- $d_{6}(99.9$ atom \% D, CDN Isotopes), and tetramethylsilane $(99.9+\%$, Aldrich) were used as received.

\subsubsection{Compressed Gas}

Argon (ultra high purity, Praxair) was used as received.

\subsubsection{Ion Exchange Resin, Drying Agent, and PSP Additive}

Dowex $^{\circledR} 1-\mathrm{X} 10$ anion exchanger ionic form $\mathrm{Cl}^{-}(200-400$ mesh, J. T. Baker Chemical), $4 \AA$ molecular sieves (activated, Aldrich), and Cellulose (colloidal microcrystalline, Aldrich) were used as received.

\subsubsection{Starting Complexes}

Starting materials $\left[\operatorname{Ir}(\mathrm{fppy})_{2} \mathrm{Cl}\right]_{2},\left[\operatorname{Ir}\left(\mathrm{C}_{6}\right)_{2} \mathrm{Cl}\right]_{2}$, and $\left[\operatorname{Ir}(\mathrm{ppy})_{2} \mathrm{Cl}\right]_{2}$ were synthesized using the methods of Lo, ${ }^{67 \mathrm{a}}$ Thompson, ${ }^{72 \mathrm{c}}$ and Watts and Nonoyama. ${ }^{74}$ 


\subsection{Synthesis of Iridium Complexes}

\section{$\left[\operatorname{Ir}(\operatorname{tpy})_{2}(t-\mathrm{Bu}-\mathrm{iCN})_{2}\right]\left[\mathrm{CF}_{3} \mathrm{SO}_{3}\right], 1$}

$\left[\operatorname{Ir}(\mathrm{tpy})_{2}(t-\mathrm{Bu}-\mathrm{iCN})_{2}\right]\left[\mathrm{CF}_{3} \mathrm{SO}_{3}\right]$ was prepared according to $\mathrm{Li}$ et al. ${ }^{75}$

\section{$\left[\operatorname{Ir}(f p p y)_{2}(t-\mathrm{Bu}-\mathrm{iCN})_{2}\right]\left[\mathrm{CF}_{3} \mathrm{SO}_{3}\right], 2$}

$\left[\operatorname{Ir}(\mathrm{fppy})_{2} \mathrm{Cl}\right]_{2}\left(0.14 \mathrm{~g}, \quad 0.12 \mathrm{mmol}\right.$, F.W. $\left.1184.15 \mathrm{~g} . \mathrm{mol}^{-1}\right)$ was dissolved in dichloromethane $(15 \mathrm{~mL})$ and a solution of AgOTf $(0.07 \mathrm{~g}, 0.27 \mathrm{mmol}, \mathrm{F} . \mathrm{W} .256 .94$ g. $\left.\mathrm{mol}^{-1}\right)$ in methanol $(15 \mathrm{~mL})$ was added and the resultant solution was stirred for 1 hour and then filtered. To the orange filtrate was added $\mathrm{CN}-t-\mathrm{Bu}(0.25 \mathrm{~g}, 3.0 \mathrm{mmol}, \mathrm{F} . \mathrm{W}$. 83.13 g.mol ${ }^{-1}$ ) and the resultant solution was stirred for 2 days at room temperature. The reaction mixture was evaporated to dryness under vacuum and the yellow product was filtered and grown as crystals by ether diffusion into a dichloromethane solution of it. $\left[\operatorname{Ir}(\mathrm{fppy})_{2}(\mathrm{t}-\mathrm{Bu}-\mathrm{iCN})_{2}\right]\left[\mathrm{CF}_{3} \mathrm{SO}_{3}\right]\left(0.15 \mathrm{~g}, 0.17 \mathrm{mmol}, 72 \%\right.$ yield, F.W. $\left.871.95 \mathrm{~g} \cdot \mathrm{mol}^{-1}\right)$

Elemental analysis as the monosolvated $\mathrm{C}_{35} \mathrm{H}_{34} \mathrm{IrN}_{4} \mathrm{O}_{5} \mathrm{~F}_{3} \mathrm{~S} \cdot \mathrm{CH}_{2} \mathrm{Cl}_{2} \mathrm{Calcd}(\%)$ : $\mathrm{C}$ 45.19, H 3.79, N 5.86. Found: C 45.13, H 3.78, N 5.74.

${ }^{1} \mathrm{H}$ NMR ( $\mathrm{CDCl}_{3}, 300 \mathrm{MHz}$ ): 9.70 (Ald., 1H, s), 9.20 (N6, 1H, d, $5.7 \mathrm{~Hz}$ ), 8.13 (N3,4, 2H, m), $7.82(\mathrm{P} 3,1 \mathrm{H}, \mathrm{d}, 8.0 \mathrm{~Hz}), 7.69(\mathrm{~N} 5,1 \mathrm{H}, \mathrm{td}, 6.3 \mathrm{~Hz}), 7.53(\mathrm{P} 4,1 \mathrm{H}, \mathrm{dd}, 8.0$ $\mathrm{Hz}), 6.61(\mathrm{P} 6,1 \mathrm{H}, \mathrm{s}), 1.37(\mathrm{t}-\mathrm{Bu}, 9 \mathrm{H}, \mathrm{s}) \mathrm{ppm}$. UV-vis $\left(\mathrm{CH}_{3} \mathrm{CN}\right) \varepsilon_{231}\left(3.07 \times 10^{4}\right), \varepsilon_{258}$ $\left(2.51 \times 10^{4}\right), \varepsilon_{276(\text { Shoulder })}\left(2.24 \times 10^{4}\right), \varepsilon_{306}\left(2.42 \times 10^{4}\right), \varepsilon_{316}\left(2.64 \times 10^{4}\right), \varepsilon_{353}\left(6.92 \times 10^{3}\right.$ L.mol $\left.{ }^{-1} \cdot \mathrm{cm}^{-1}\right)$. 


\section{$\left[\operatorname{Ir}\left(\mathbf{C}_{6}\right)_{2}\right.$ (acacacid) $], 3$}

$\left[\operatorname{Ir}\left(\mathrm{C}_{6}\right)_{2} \mathrm{Cl}\right]_{2}\left(0.15 \mathrm{~g}, 0.13 \mathrm{mmol}, \quad\right.$ F.W. $\left.1154.20 \mathrm{~g} . \mathrm{mol}^{-1}\right)$ was dissolved in dichloromethane $(25 \mathrm{~mL})$ and a solution of AgOTf $(0.07 \mathrm{~g}, 0.31 \mathrm{mmol}$, F.W. 256.06 g.mol $\left.{ }^{-1}\right)$ in methanol $(5 \mathrm{~mL})$ was added and refluxed under argon for 2 hours. The cloudy orange solution was cooled to room temperature and gravity filtered to remove $\mathrm{AgCl}$. 4,6Dioxoheptanoic acid (acacacid) (0.05 g, $0.32 \mathrm{mmol}$, F.W. 158.16 g.mol $\left.{ }^{-1}\right)$ and triethylamine $(0.7 \mathrm{~mL})$ were added to the filtrate. The solution was stirred overnight at room temperature under argon. After solvent-stripping to dryness, the orange solid was precipitated out of a dichloromethane solution using hexanes. It was then dissolved in tetrahydrofuran $(10 \mathrm{~mL})$ and hydrochloric acid solution $(1.27 \mathrm{~mL}, 0.01 \mathrm{M})$ was added. The organic solvent was evaporated followed by introducing dichloromethane $(20 \mathrm{~mL})$. The solution was washed with distilled water and the bright orange organic layer was separated. The reaction mixture was then evaporated to dryness and the orange product was recrystallized by addition of hexanes to a concentrated dichloromethane solution of it. $\left[\operatorname{Ir}\left(\mathrm{C}_{6}\right)_{2}\right.$ (acacacid)] $\left(0.10 \mathrm{~g}, 0.10 \mathrm{mmol}, 38 \%\right.$ yield, F.W. $1048.23{\mathrm{~g} . \mathrm{mol}^{-1}}^{-1})$

Elemental analysis as the semihydrate $\mathrm{C}_{47} \mathrm{H}_{43} \mathrm{IrN}_{4} \mathrm{O}_{8} \mathrm{~S}_{2} \cdot 0.5 \mathrm{H}_{2} \mathrm{O}$ Calcd (\%): C 53.40, H 4.19, N 5.30. Found: C 53.41, H 4.05, N 5.26.

${ }^{1} \mathrm{H}$ NMR (DMSO-d $\left.6,300 \mathrm{MHz}\right): 12.02\left(\mathrm{ac}_{5}, \mathrm{bs}, 1 \mathrm{H}\right), 8.15(\mathrm{~m}, 2 \mathrm{H}), 7.39(\mathrm{~m}, 6 \mathrm{H})$, $6.35(\mathrm{~m}, 2 \mathrm{H}), 6.00(\mathrm{~m}, 4 \mathrm{H}), 4.98\left(\mathrm{ac}_{2}, \mathrm{~s}, 1 \mathrm{H}\right), 3.26\left(\mathrm{C6}_{\text {ethylene }}, \mathrm{q}, 8 \mathrm{H}, 6.8 \mathrm{~Hz}\right), 2.22(\mathrm{~m}$, 2H), $2.08(\mathrm{~m}, 2 \mathrm{H}), 1.69\left(\mathrm{ac}_{1}, \mathrm{~s}, 3 \mathrm{H}\right), 0.94\left(\mathrm{C6}_{\text {methyl }}, \mathrm{q}, 12 \mathrm{H}, 6.9 \mathrm{~Hz}\right) \mathrm{ppm}$. UV-vis $\left(\mathrm{CH}_{2} \mathrm{Cl}_{2}\right) \varepsilon_{447}\left(6.51 \times 10^{4}\right), \varepsilon_{474}\left(7.54 \times 10^{4} \mathrm{~L} \cdot \mathrm{mol}^{-1} \cdot \mathrm{cm}^{-1}\right)$. 


\section{5-Aminomethyl-2-phenylpyridine amppy}

To a solution of 5-aminomethyl-2-chloropyridine (1.0 g, 7.01 mmol, F.W. 142.59 $\left.\mathrm{gmol}^{-1}\right)$ in dichloromethane $(15 \mathrm{~mL})$ at $0^{\circ} \mathrm{C}$ was added triethylamine $(1.1 \mathrm{~mL}, 7.71$ $\mathrm{mmol})$ followed by acetylchloride $(0.55 \mathrm{~mL}, 7.71 \mathrm{mmol})$. The reaction was allowed to warm to room temperature and was stirred overnight. It was poured into water $(15 \mathrm{~mL})$ and the layers were separated. The organic layer was treated with charcoal $(0.8 \mathrm{~g})$, heated to reflux, filtered over Celite, and concentrated to an oil. The oil was dissolved in dichloromethane and crystallized by addition of diethylether. The solids were filtered to afford 5-acetadimomethyl-2-chloro pyridine (1.14 g, 6.18 mmol, 88\% yield, F.W. 184.63 $\left.\mathrm{gmol}^{-1}\right)$

${ }^{1} \mathrm{H} \mathrm{NMR}\left(\mathrm{CDCl}_{3}, 300 \mathrm{MHz}\right): 8.31(1 \mathrm{H}, \mathrm{d}, 2.1 \mathrm{~Hz}), 7.65(1 \mathrm{H}, \mathrm{dd}, 8.3 \mathrm{~Hz}, 2.7 \mathrm{~Hz})$, $7.31(1 \mathrm{H}, \mathrm{d}, 8.4 \mathrm{~Hz}), 6.0(1 \mathrm{H}, \mathrm{bs}), 4.44(2 \mathrm{H}, \mathrm{d}, 6.0 \mathrm{~Hz}), 2.06(3 \mathrm{H}, \mathrm{s})$, FTIR (KBr pellet): $v(\mathrm{~N}-\mathrm{H})_{\text {acetamide }} 3281 \mathrm{~cm}^{-1}, v(\mathrm{C}=\mathrm{O})_{\text {acetamide }} 1649 \mathrm{~cm}^{-1}, \mathrm{MS}(\mathrm{EI}) \mathrm{M}^{+}=184$

5-acetadimomethyl-2-chloro pyridine $\left(0.34 \mathrm{~g}, 1.84 \mathrm{mmol}, \mathrm{F} . \mathrm{W} .184 .63 \mathrm{gmol}^{-1}\right)$ and $\mathrm{PhB}(\mathrm{OH})_{2}\left(0.34 \mathrm{~g}, 2.79 \mathrm{mmol}, \mathrm{F} . \mathrm{W} .121 .93 \mathrm{gmol}^{-1}\right)$ were dissolved in a mixture of toluene $(25 \mathrm{~mL})$ and ethanol $(10 \mathrm{~mL})$. The mixture was flushed with argon three times and stirred at room temperature for 10 minutes. Then $\mathrm{Pd}\left(\mathrm{PPh}_{3}\right)_{4}(0.11 \mathrm{~g}, 0.095 \mathrm{mmol}$, F.W. 1,155.58 $\mathrm{gmol}^{-1}$ ) was added. It was stirred for 15 minutes followed by addition of a solution of $\mathrm{Na}_{2} \mathrm{CO}_{3}\left(0.39 \mathrm{~g}, 3.68 \mathrm{mmol}, \mathrm{F} . \mathrm{W} .105 .99 \mathrm{gmol}^{-1}\right)$ in water $(10 \mathrm{~mL})$. The reaction mixture was then degassed twice more and refluxed overnight. It was cooled to room temperature, filtered, and the layers were separated. The aqueous layer was 
extracted with ethyl acetate, and the organic extracts were combined and evaporated to dryness. The crude solid was dissolved in methanol $(30 \mathrm{~mL})$, filtered, and concentrated hydrochloric acid $(0.6 \mathrm{~mL})$ was added. The solution was concentrated to a low volume, washed with tetrahydrofuran, and dried. The product was then triturated in tetrahydrofuran and filtered to afford 5-acetadimomethyl-2-phenyl pyridine hydrochloride (0.39 g, $1.30 \mathrm{mmol}, 71 \%$ yield, F.W. $\left.299.20 \mathrm{gmol}^{-1}\right)$.

${ }^{1} \mathrm{H}$ NMR (DMSO- $\left.d_{6}, 400 \mathrm{MHz}\right): 8.73(1 \mathrm{H}, \mathrm{s}), 8.31(2 \mathrm{H}, \mathrm{m}), 8.11(2 \mathrm{H}, \mathrm{m}), 7.62$ $(3 \mathrm{H}, \mathrm{m}), 5.56(3 \mathrm{H}, \mathrm{bs}), 4.44(2 \mathrm{H}, \mathrm{m}), 1.92(3 \mathrm{H}, \mathrm{s})$, FTIR $(\mathrm{KBr}$ pellet $): v(\mathrm{~N}-\mathrm{H})_{\text {acetamide }}$ $3362 \mathrm{~cm}^{-1}, \mathrm{v}(\mathrm{C}=\mathrm{O})_{\text {acetamide }} 1649 \mathrm{~cm}^{-1}, \mathrm{MS}(\mathrm{EI}) \mathrm{M}^{+}=226$

To a solution of 5-acetadimomethyl-2-phenyl pyridine hydrochloride $(0.14 \mathrm{~g}, 0.53$ mmol, F.W. 262.74 gmol $\left.^{-1}\right)$ in methanol $(5 \mathrm{~mL})$ was added concentrated hydrochloric acid $(2.5 \mathrm{~mL})$. The reaction mixture was heated to reflux overnight and concentrated to a low volume. Tetrahydrofuran $(50 \mathrm{~mL})$ was added and the white precipitate was filtered to afford 5-aminomethyl-2-phenyl pyridine hydrochloride $(0.12 \mathrm{~g}, 0.47 \mathrm{mmol}, 89 \%$ yield, F.W. $257.16 \mathrm{gmol}^{-1}$ ).

${ }^{1} \mathrm{H}$ NMR (D $\left.2 \mathrm{O}, 300 \mathrm{MHz}\right): 8.80(1 \mathrm{H}, \mathrm{s}), 8.52(1 \mathrm{H}, \mathrm{dd}, 8.5 \mathrm{~Hz}, 1.8 \mathrm{~Hz}), 8.24(1 \mathrm{H}$, $\mathrm{d}, 8.5 \mathrm{~Hz}), 7.82(2 \mathrm{H}, \mathrm{m}), 7.61(3 \mathrm{H}, \mathrm{m}), 4.37(2 \mathrm{H}, \mathrm{s})$ FTIR (KBr pellet): $v(\mathrm{~N}-\mathrm{H})_{\text {ammonium }}$ $2855 \mathrm{~cm}^{-1}, \mathrm{MS}(\mathrm{EI}) \mathrm{M}^{+}=184$

5-aminomethyl-2-phenyl pyridine hydrochloride $(0.61 \mathrm{~g}, 2.80 \mathrm{mmol}, \mathrm{F} . \mathrm{W} .257 .16$ $\mathrm{gmol}^{-1}$ ) was dissolved in water $(25 \mathrm{~mL})$ and filtered. The $\mathrm{pH}$ was adjusted to $\sim 12$ with concentrated aqueous sodium hydroxide. It was then diluted with methyl tert-butyl ether 
$(25 \mathrm{~mL})$. The organic layer was separated to afford 5-aminomethyl-2-phenyl pyridine (0.31 g, $1.68 \mathrm{mmol}, 60 \%$ yield, F.W. $\left.184.24 \mathrm{gmol}^{-1}\right)$.

${ }^{1} \mathrm{H} \mathrm{NMR}\left(\mathrm{CDCl}_{3}, 300 \mathrm{MHz}\right): 8.66(1 \mathrm{H}, \mathrm{d}, 1.1 \mathrm{~Hz}), 8.00(2 \mathrm{H}, \mathrm{m}), 7.76(2 \mathrm{H}, \mathrm{m})$, $7.50(3 \mathrm{H}, \mathrm{m}), 3.97(2 \mathrm{H}, \mathrm{s}), 1.48(2 \mathrm{H}, \mathrm{s})$ FTIR $\left(\mathrm{KBr}\right.$ pellet): $v(\mathrm{~N}-\mathrm{H})_{\text {amine }} 3437$ and 3269 $\mathrm{cm}^{-1}, v(\mathrm{~N}-\mathrm{H})_{\text {amine }} 1602 \mathrm{~cm}^{-1}$, MS (EI) $\mathrm{M}^{+}=184$

\section{$\left[\operatorname{Ir}(\text { ppy })_{2}\right.$ (amppy)Cl], 4}

$\left[\operatorname{Ir}(\mathrm{ppy})_{2} \mathrm{Cl}\right]_{2} \quad\left(0.5 \mathrm{~g}, \quad 0.47 \mathrm{mmol}, \quad\right.$ F.W. $\left.1072.11 \mathrm{gmol}^{-1}\right)$ was dissolved in dichloromethane $(40 \mathrm{~mL})$ and a solution of AgOTf $(0.24 \mathrm{~g}, 0.94 \mathrm{mmol}, \mathrm{F} . W .256 .06$ g. $\left.\mathrm{mol}^{-1}\right)$ in methanol $(10 \mathrm{~mL})$ was added and the resultant solution was refluxed under argon for 2 hours. The cloudy yellow solution was cooled to room temperature and gravity filtered to remove AgCl. Amppy (0.22g, $1.2 \mathrm{mmol}$, F.W. $\left.184.24 \mathrm{gmol}^{-1}\right)$ and triethylamine $(0.3 \mathrm{~mL})$ were added and the resultant solution was stirred overnight at room temperature under argon. The reaction mixture was evaporated to dryness and the yellow product was grown as diamond shaped crystals by hexanes diffusion into a dichloromethane solution of it. [Ir(ppy) $)_{2}$ (amppy)Cl] $(0.53 \mathrm{~g}, 0.74 \mathrm{mmol}, 79 \%$ yield, F.W. 720.30 g.mol $\left.{ }^{-1}\right)$

Elemental analysis as the monosolvated $\mathrm{C}_{34} \mathrm{H}_{28} \mathrm{IrN}{ }_{4} \mathrm{Cl} \cdot \mathrm{CH}_{2} \mathrm{Cl}_{2}$ Calcd (\%): C 52.21, H 3.76, N 6.96. Found: C 51.88, H 3.58, N 7.05

${ }^{1} \mathrm{H} \mathrm{NMR}\left(\mathrm{CDCl}_{3}, 300 \mathrm{MHz}\right): 9.93$ (d, 1H, $\left.5.6 \mathrm{~Hz}\right), 8.54$ (d, 1H, $\left.5.5 \mathrm{~Hz}\right), 8.19$ (d, 1H, $1.7 \mathrm{~Hz}), 7.81(\mathrm{~m}, 6 \mathrm{H}), 7.44(\mathrm{~m}, 8 \mathrm{H}), 7.19(\mathrm{~m}, 1 \mathrm{H}), 6.79(\mathrm{~m}, 4 \mathrm{H}), 6.34(\mathrm{~d}, 1 \mathrm{H}, 7.5$ $\mathrm{Hz}), 6.17(\mathrm{dd}, 1 \mathrm{H}, 7.5$ and $0.69 \mathrm{~Hz}), 3.36(\mathrm{~m}, 2 \mathrm{H}), 1.62(\mathrm{~s}, 2 \mathrm{H}) \mathrm{ppm}$. UV-vis $\left(\mathrm{CH}_{2} \mathrm{Cl}_{2}\right)$ $\varepsilon_{259}\left(3.38 \times 10^{4}\right), \varepsilon_{392}\left(1.78 \times 10^{3} \mathrm{~L} \cdot \mathrm{mol}^{-1} \cdot \mathrm{cm}^{-1}\right), \varepsilon_{431}\left(1.31 \times 10^{3} \mathrm{~L} \cdot \mathrm{mol}^{-1} \cdot \mathrm{cm}^{-1}\right)$. 


\section{$\left[\operatorname{Ir}(\text { ppy })_{2}(\mathrm{fppy})\right], 5$}

$\left[\operatorname{Ir}(\mathrm{ppy})_{2}(\mathrm{fppy})\right]$ was prepared according to Evans et al. ${ }^{68}$

\section{$\left[\operatorname{Ir}(\text { ppy) })_{2}\right.$ (phendiald)][PF 6$], 6$}

$\left[\operatorname{Ir}(\text { ppy })_{2} \mathrm{Cl}\right]_{2}\left(0.11 \mathrm{~g}, 0.10 \mathrm{mmol}\right.$, F.W. 1072.11 g.mol $\left.{ }^{-1}\right)$ was dissolved in methanol/dichloromethane $(30 \mathrm{~mL}, \quad 1: 1 \quad \mathrm{v} / \mathrm{v})$ and 1,10-phenanthroline-2,9dicarboxaldehyde $\left(0.05\right.$ g, $0.21 \mathrm{mmol}$, F.W. 236.23 g.mol $\left.{ }^{-1}\right)$ was added and the resultant solution was stirred at reflux under argon for 4 hours. The orange solution was cooled to room temperature and $\mathrm{KPF}_{6}\left(0.037 \mathrm{~g}, 0.20 \mathrm{mmol}, \mathrm{F} . \mathrm{W} .184 .07 \mathrm{~g} . \mathrm{mol}^{-1}\right)$ was added. It was then evaporated to dryness and the orange product was recrystallized by addition of hexanes to a concentrated dichloromethane solution of it. $\left[\operatorname{Ir}(\mathrm{ppy})_{2}\right.$ (phendiald)][PF 6$](0.15$ g, $0.17 \mathrm{mmol}, 85 \%$ yield, F.W. $881.80 \mathrm{~g} \cdot \mathrm{mol}^{-1}$ )

${ }^{1} \mathrm{H}$ NMR (acetone- $d_{6}, 300 \mathrm{MHz}$ ): 9.70 (Ald., $\left.2 \mathrm{H}, \mathrm{s}\right), 9.11(\mathrm{~m}, 2 \mathrm{H}), 8.51(\mathrm{~m}, 2 \mathrm{H})$, $8.37(\mathrm{~d}, 2 \mathrm{H}, 8.3 \mathrm{~Hz}), 8.21(\mathrm{~m}, 2 \mathrm{H}), 7.93(\mathrm{~m}, 6 \mathrm{H}), 6.98(\mathrm{~m}, 4 \mathrm{H}), 6.86(\mathrm{dd}, 2 \mathrm{H}, 15.7$ and $8.2 \mathrm{~Hz}), 6.27(\mathrm{~m}, 2 \mathrm{H}) \mathrm{ppm}$. UV-vis $\left(\mathrm{CH}_{2} \mathrm{Cl}_{2}\right) \varepsilon_{257}\left(3.75 \times 10^{4}\right), \varepsilon_{293}\left(4.55 \times 10^{4}\right), \varepsilon_{384}(8.53$ $\left.\times 10^{3} \mathrm{~L} \cdot \mathrm{mol}^{-1} \cdot \mathrm{cm}^{-1}\right), \varepsilon_{508}\left(1.28 \times 10^{3}\right)$. 


\subsection{Synthesis of Palladium Complex}

\section{[PdTMpyp-4][PF $\left.]_{6}\right]_{4}, 7$}

The synthesis was carried out following a modified procedure of Pasternack ${ }^{80}$ as follows:

N,N'-Dimethylformamide $(5 \mathrm{~mL})$ was brought to reflux and $5,10,15,20$ tetrakis(1-methyl-4-pyridyl)-21H,23H-porphine,tetra-p-tosylate salt $(0.05 \mathrm{~g}, 0.04 \mathrm{mmol}$, F.W. 1363.63 g.mol ${ }^{-1}$ ) was added. The resultant mixture was stirred for 1 minute in order to allow for complete dissolution and then palladium chloride $(0.007 \mathrm{~g}, 0.04 \mathrm{mmol}, \mathrm{F}$.W. $177.33 \mathrm{~g} \cdot \mathrm{mol}^{-1}$ ) was added. The resultant solution was stirred at reflux overnight after which it was evaporated to dryness and then dissolved in water $(15 \mathrm{~mL})$. The deep redorange solution was filtered through Celite and $\mathrm{NaBF}_{4}(1.6 \mathrm{~g}, 0.01 \mathrm{~mol}, \mathrm{~F} . \mathrm{W} .109 .79$ g. $\mathrm{mol}^{-1}$ ) was added to the filtrate. The resultant mixture was centrifuged (2000 rpm, 15 minutes) and the resultant red solid was suspended in water with anion exchange resin Dowex $^{\circledR} 1-\mathrm{X} 10$ in the chloride form $(0.22 \mathrm{~g})$. The mixture was stirred for 1 hour and was then filtered to remove the anion exchange resin. $\mathrm{NH}_{4} \mathrm{PF}_{6}(0.96 \mathrm{~g}, 5.9 \mathrm{mmol}, \mathrm{F} . \mathrm{W} .163$ g. $\mathrm{mol}^{-1}$ ) was added to the filtrate to precipitate the product which was then dissolved by addition of adequate amount of acetone. The purple product was acquired by slow evaporation of the solvent. It was filtered, washed with methanol and ether, and dried in vacuo. [PdTMpyp-4][PF 6$]_{4}\left(0.034 \mathrm{~g}, 0.025 \mathrm{mmol}, 62 \%\right.$ yield, F.W. $1363.10{\mathrm{~g} . \mathrm{mol}^{-1}}^{-1})$

Elemental analysis as $\mathrm{C}_{44} \mathrm{H}_{36} \mathrm{~F}_{24} \mathrm{~N}_{8} \mathrm{P}_{4} \mathrm{Pd}$ Calcd (\%): C 38.77, H 2.66, N 8.22. Found: C 38.88, H 2.78, N 7.83. 
UV-vis absorption data was matched with reference 81.

\subsection{Synthesis of Functionalized Water-Soluble Polymer}

\section{i) Copper-Catalyzed Oxidation of Poly(Ethylene Glycol) Methyl Ether (PEGME) to PEGME-Aldehyde}

$\mathrm{CuCl}\left(0.05 \mathrm{~g}, 0.51 \mathrm{mmol}, \mathrm{F} . \mathrm{W} .99 .00 \mathrm{gmol}^{-1}\right)$ and 1,10-phenanthroline $(0.09 \mathrm{~g}$, $0.50 \mathrm{mmol}$, F.W. $\left.180.21 \mathrm{gmol}^{-1}\right)$, and $\mathrm{K}_{2} \mathrm{CO}_{3}\left(2.75 \mathrm{~g}, 0.02 \mathrm{~mol}\right.$, F.W. $\left.138.21 \mathrm{gmol}^{-1}\right)$ were added to toluene $(80 \mathrm{~mL})$ and the mixture was stirred for 30 minutes at room temperature. Diethylazodicarboxylate (DEAD) $\left(0.09 \mathrm{~g}, 0.52 \mathrm{mmol}, \quad\right.$ F.W. $\left.174.15 \mathrm{gmol}^{-1}\right)$ and poly(ethylene glycol) methyl ether-550 (11g, 0.02 mol, typical $\left.M_{n} 550\right)$ were added successively, and the mixture was heated for 3 hours on an oil bath at $80^{\circ} \mathrm{C}$ while air was gently bubbled through the reaction mixture. The catalyst was then filtered and the product was precipitated by addition of anhydrous diethyl ether followed by leaving in freezer, filtering and washing with cold anhydrous diethyl ether $\left(\sim-6^{\circ} \mathrm{C}\right)$. It was then dried and PEGME-Aldehyde was isolated as a colorless semisolid.

IR $(\mathrm{KBr}$ pellet $): \mathrm{v}(\mathrm{C}=\mathrm{O})_{\text {aldehyde }} 1719 \mathrm{~cm}^{-1}, \mathrm{v}(\mathrm{C}-\mathrm{H})_{\text {aldehyde }} 2724 \mathrm{~cm}^{-1}$ 


\subsection{Synthesis of Water-Soluble Oxygen Sensors}

\section{i) Reductive Attachment of 2 to Silamine D208-EDA to Synthesize 8}

To Silamine D208-EDA (1.5 g, $0.6 \mathrm{mmol}$, pre-dried by heating at $80{ }^{\circ} \mathrm{C}$ under vacuum for 30 minutes) was added dichloromethane $(24 \mathrm{~mL})$. The solution was degassed with argon for 10 minutes and then $0.53 \mathrm{~g}$ of activated $4 \AA$ molecular sieves and $2(0.09$ $\mathrm{g}, 0.1 \mathrm{mmol})$ were added followed by pyridine-borane $(0.01 \mathrm{~mL}, 0.099 \mathrm{mmol})$. After the solution was stirred at room temperature under argon for 16 hours, methanol $(10 \mathrm{~mL})$ was added and the resultant solution was treated with $6 \mathrm{~N} \mathrm{HCl}(10 \mathrm{~mL})$ for 15 minutes, and then $\mathrm{pH}$ was adjusted to 12 using concentrated aqueous $\mathrm{NaOH}$ solution. $\mathrm{NaCl}$ was precipitated by addition of diethyl ether $(100 \mathrm{~mL})$ and filtered off. The filtrate was solvent stripped and the residue dissolved in a small volume of acetone which was filtered sequentially through $1.0 \mu \mathrm{m}$ and $0.45 \mu \mathrm{m}$ Gelman acrodiscs. The filtered solution was added dropwise to rapidly stirred hexanes yielding a clear, yellow polymer fluid.

\section{ii) Covalent Attachment of 3 to Silamine D208-EDA to Synthesize 9}

To Silamine D208-EDA (1.0 g, $0.4 \mathrm{mmol})$ was added ethyl acetate $(5 \mathrm{~mL}) .3$ $(0.07 \mathrm{~g}, 0.07 \mathrm{mmol})$ dissolved in ethyl acetate $(5 \mathrm{~mL})$ was added followed by $\mathrm{N}, \mathrm{N}$ '-

dicyclohexylcarbodiimide $\left(0.04 \mathrm{~g}, 0.19 \mathrm{mmol}, \mathrm{F} . \mathrm{W} .206 .33 \mathrm{gmol}^{-1}\right)$ dissolved in ethyl acetate $(1 \mathrm{~mL})$. After the solution was stirred at room temperature for 6 hours, it was solvent stripped and the residue dissolved in a small volume of water which was filtered 
sequentially through $1.0 \mu \mathrm{m}$ and $0.45 \mu \mathrm{m}$ Gelman acrodiscs. A clear, orange polymer fluid was obtained after solvent stripping.

\section{iii) Dispersion of 4 in Silube CS-1 to Produce 10}

To Silube CS-1 (0.3 g, $0.1 \mathrm{mmol})$ was added ethyl acetate $(1 \mathrm{~mL}) .4(0.02 \mathrm{~g}, 0.03$ $\mathrm{mmol})$ dissolved in ethyl acetate $(3 \mathrm{~mL})$ was added and the solution was stirred at room temperature for 6 hours. It was then solvent stripped and the residue dissolved in a small volume of water which was filtered sequentially through $1.0 \mu \mathrm{m}$ and $0.45 \mu \mathrm{m}$ Gelman acrodiscs. A clear, yellow polymer fluid was obtained after solvent stripping.

\section{iv) Covalent Attachment of Amppy to PEGME-Aldehyde}

Amppy (0.33g, $1.79 \mathrm{mmol}$, F.W. $\left.184.24 \mathrm{gmol}^{-1}\right)$ was dissolved in chloroform (10 $\mathrm{mL})$ and added to a solution of PEGME-Aldehyde (1.0g, $1.82 \mathrm{mmol}$, typical $\left.\mathrm{M}_{\mathrm{n}} 550\right)$ in chloroform $(10 \mathrm{~mL})$. The resultant solution was stirred at reflux under argon for 2.5 days. It was then solvent stripped, dissolved in water, filtered through $1.0 \mu \mathrm{m}$ and $0.45 \mu \mathrm{m}$ Gelman Acrodiscs ${ }^{\mathrm{TM}}$, and evaporated.

\section{v) Synthesis of PEGME Attached Ir-amppy Dimer}

Iridium trichloride hydrate $\left(0.13 \mathrm{~g}, 0.44 \mathrm{mmol}, \mathrm{F} . \mathrm{W} .298 .58 \mathrm{gmol}^{-1}\right)$ was combined with PEGME-amppy $(1.0 \mathrm{~g}, \sim 0.87 \mathrm{mmol}$ amppy), dissolved in a mixture of 2- 
ethoxyethanol $(10 \mathrm{~mL})$ and water $(5 \mathrm{~mL})$, and refluxed for 24 hours under argon. The deep orange solution was filtered and the filtrate was evaporated to dryness. It was then dissolved in methanol $(30 \mathrm{~mL})$, filtered, and used in the next step.

vi) Synthesis of PEGME Attached $\left[\operatorname{Ir}(\operatorname{amppy})_{2}(\mathrm{t}-\mathrm{Bu}-\mathrm{iCN})_{2}\right]\left[\mathrm{CF}_{3} \mathrm{SO}_{3}\right], 11$

To the methanolic solution of PEGME attached Ir-amppy dimer acquired from the previous step was added AgOTf (0.13g, $0.51 \mathrm{mmol}$, F.W. $256.94{\mathrm{~g} . \mathrm{mol}^{-1}}^{-1})$ to yield a cream-colored slurry. After the slurry was stirred for 1 hour, it was filtered and $\mathrm{CN}-t-\mathrm{Bu}$ (0.25 g, $3.0 \mathrm{mmol}$, F.W. 83.13 g. $\left.\mathrm{mol}^{-1}\right)$ and dichloromethane $(20 \mathrm{~mL})$ were added to it. The resultant solution was stirred for 2 days at room temperature. The reaction mixture was evaporated to dryness under vacuum and the residue was dissolved in acetone, filtered through $1.0 \mu \mathrm{m}$ and $0.45 \mu \mathrm{m}$ Gelman Acrodiscs ${ }^{\mathrm{TM}}$, and evaporated. The filtered solution was added dropwise to rapidly stirred hexanes, yielding a clear, yellow polymer fluid.

\section{vii) Covalent Attachment of 5 to Silamine D208-EDA to Synthesize 12}

A solution of 5 ( $34 \mathrm{mg}, 0.05 \mathrm{mmol}$, F.W. 682.80 g. $\left.\mathrm{mol}^{-1}\right)$ in dichloromethane $(5$ $\mathrm{mL})$ was added to a solution of Silamine D208-EDA $(0.25 \mathrm{~g}, 0.10 \mathrm{mmol}, \mathrm{M} . \mathrm{W} .2400)$ in dichloromethane $(10 \mathrm{~mL})$. The resultant solution was stirred at reflux under argon for 2 days. It was solvent stripped to dryness and the product was dissolved in acetone $(5 \mathrm{~mL})$ 
and filtered through $1.0 \mu \mathrm{m}$ and $0.45 \mu \mathrm{m}$ Gelman Acrodiscs ${ }^{\mathrm{TM}}$. The filtered solution was added dropwise to rapidly stirred hexanes, yielding a clear, yellow polymer fluid.

\section{viii) Reductive Attachment of 5 to Silamine D208-EDA to Synthesize 13}

To Silamine D208-EDA (1.5g, $0.6 \mathrm{mmol}, \mathrm{M} . \mathrm{W} .2400$, pre-dried by heating at 80 ${ }^{\circ} \mathrm{C}$ under vacuum for 30 minutes $)$ was added dichloromethane $(24 \mathrm{~mL})$. The solution was degassed with argon for 10 minutes and then $0.53 \mathrm{~g}$ of activated $4 \AA$ molecular sieves and $5\left(0.08 \mathrm{~g}, 0.12 \mathrm{mmol}\right.$, F.W. $\left.682.80 \mathrm{~g} \cdot \mathrm{mol}^{-1}\right)$ were added followed by pyridine-borane $(0.01 \mathrm{~mL}, 0.099 \mathrm{mmol})$. After the solution was stirred at room temperature under argon for 16 hours, methanol $(10 \mathrm{~mL})$ was added and the resultant solution was treated with $6 \mathrm{~N}$ $\mathrm{HCl}(10 \mathrm{~mL})$ for 15 minutes, and then $\mathrm{pH}$ was adjusted to 12 using concentrated aqueous $\mathrm{NaOH}$ solution. $\mathrm{NaCl}$ was precipitated by addition of diethyl ether $(100 \mathrm{~mL})$ and filtered off. The filtrate was solvent stripped and the residue dissolved in a small volume of acetone which was filtered sequentially through $1.0 \mu \mathrm{m}$ and $0.45 \mu \mathrm{m}$ Gelman Acrodiscs. The filtered solution was added dropwise to rapidly stirred hexanes, yielding a clear, yellow polymer fluid.

\section{ix) Reductive Attachment of 6 to Silamine D208-EDA to Synthesize 14}

To Silamine D208-EDA $\left(1.5 \mathrm{~g}, 0.6 \mathrm{mmol}\right.$, pre-dried by heating at $80{ }^{\circ} \mathrm{C}$ under vacuum for 30 minutes) was added dichloromethane $(24 \mathrm{~mL})$. The solution was degassed with argon for 10 minutes and then $0.53 \mathrm{~g}$ of activated $4 \AA$ molecular sieves and $6(0.09$ 
$\mathrm{g}, 0.1 \mathrm{mmol})$ were added followed by pyridine-borane $(0.01 \mathrm{~mL}, 0.099 \mathrm{mmol})$. After the solution was stirred at room temperature under argon for 16 hours, methanol $(10 \mathrm{~mL})$ was added and the resultant solution was treated with $6 \mathrm{~N} \mathrm{HCl}(10 \mathrm{~mL})$ for 15 minutes, and then $\mathrm{pH}$ was adjusted to 12 using concentrated aqueous $\mathrm{NaOH}$ solution. $\mathrm{NaCl}$ was precipitated by addition of diethyl ether $(100 \mathrm{~mL})$ and filtered off. The filtrate was solvent stripped and the residue dissolved in a small volume of acetone which was filtered sequentially through $1.0 \mu \mathrm{m}$ and $0.45 \mu \mathrm{m}$ Gelman acrodiscs. The filtered solution was added dropwise to rapidly stirred hexanes, yielding a clear, orange polymer fluid.

x) Covalent Attachment of 1-Pyrenebutyric Acid (PBA) to Silamine D208-EDA to Synthesize 15

To Silamine D208-EDA (1.0 g, $0.4 \mathrm{mmol})$ was added ethyl acetate $(5 \mathrm{~mL})$. PBA $\left(0.001 \mathrm{~g}, 0.003 \mathrm{mmol}\right.$, F.W. $\left.288.35 \mathrm{gmol}^{-1}\right)$ dissolved in ethyl acetate $(5 \mathrm{~mL})$ was added followed by N,N'-dicyclohexylcarbodiimide $\left(0.04 \mathrm{~g}, 0.19 \mathrm{mmol}\right.$, F.W. $\left.206.33 \mathrm{gmol}^{-1}\right)$ dissolved in ethyl acetate $(1 \mathrm{~mL})$. After the solution was stirred at room temperature for 6 hours, it was solvent stripped and the residue dissolved in a small volume of water which was filtered sequentially through $1.0 \mu \mathrm{m}$ and $0.45 \mu \mathrm{m}$ Gelman acrodiscs. A clear polymer fluid was obtained after solvent stripping. 


\subsection{PSP Formulations}

For each PSP formulation, a solution of dye in $\mathrm{MeOH}$ or $\mathrm{H}_{2} \mathrm{O}$ was added to another solution that was prepared by mixing water-soluble polymer in $\mathrm{H}_{2} \mathrm{O}$. The combined solutions were filtered through a $1.0 \mu \mathrm{m}$ Gelman Acrodisc and then mixed with microcrystalline cellulose in $\mathrm{H}_{2} \mathrm{O}$.

\subsection{Physical Measurements}

\subsubsection{Elemental Analyses}

Elemental analyses were performed by Canadian Microanalytical Service, Ltd. of Delta, BC, Canada.

\subsubsection{X-ray Structural Determination}

The x-ray structures of 2 and 4 were performed by Dr. Glenn P. A. Yap at the University of Delaware. The following experimental description was written by Dr. Yap.

For $\mathbf{2 \cdot 0 . 2 5}\left(\mathrm{CH}_{2} \mathrm{Cl}_{2}\right)$ : A crystal was mounted using viscous oil onto a glass fiber and cooled to the data collection temperature. Data were collected on a Brüker-AXS APEX CCD diffractometer with graphite-monochromated Mo-K $\alpha$ radiation $(\lambda=0.71073$ $\AA$ ). Unit cell parameters were obtained from 60 data frames, $0.3^{\circ} \omega$, from three different sections of the Ewald sphere. No symmetry higher than triclinic was observed and 
solution in the centrosymmetric space group option yielded chemically reasonable and computationally stable results of refinement. The data set was treated with SADABS absorption corrections based on redundant multiscan data. ${ }^{82}$ The structure was solved using direct methods and refined with full-matrix, least-squares procedures on $F^{2}$. Two symmetrically unique but chemically equivalent ion pairs are located in the asymmetric unit. One disordered methylene chloride molecule of crystallization per unit cell was located away from the ion pairs. The disordered triflate anions and methylene chloride molecule were treated as diffused contributions (Squeeze/Platon). ${ }^{83}$ Two $t$-Bu-iCN ligands (one on each cation) and one fppy ligand were located disordered in two positions with refined site occupancies of $65 / 35,80 / 20$ and 54/46, respectively. The structure displays disorder that cannot be satisfactorily modeled because of the subtle differences $(<1 \AA)$ in atomic positions leading to larger than ideal $U e q_{\max } / U e q_{\min }$ ranges, low C-C precision and Hirshfeld test failures. All bonds were restrained as rigid bonds with common $U_{i j}$ values within standard deviation. All non-hydrogen atoms were refined with anisotropic displacement parameters. All hydrogen atoms were treated as idealized contributions. Structure factors are contained in the SHELXTL 6.12 program library. ${ }^{82 \mathrm{a}}$ The structure has been deposited at the Cambridge Crystallographic Data Centre under CCDC 717607.

For 4: A data crystal was selected and mounted on a glass fiber using viscous oil flash-cooled to the data collection temperature. Data were collected on a Brüker-AXS APEX CCD diffractometer with graphite-monochromated Mo-K $\alpha$ radiation $(\lambda=0.71073$ $\AA$ ). Unit cell parameters were obtained from 60 data frames, $0.3^{\circ} \omega$, from three different sections of the Ewald sphere. The systematic absences in the data and the unit cell 
parameters were uniquely consistent to the reported space group. The data-sets were treated with SADABS absorption corrections based on redundant multiscan data. ${ }^{82 \mathrm{~b}}$ The structures were solved using direct methods and refined with full-matrix, least-squares procedures on $F^{2}$. A methylene chloride solvent molecule was found cocrystallized. The hydrogen atoms on the amine nitrogen atom were located from the electron density difference map and refined with $\mathrm{N}-\mathrm{H}$ distance $=0.95 \AA$ and with $U_{i s o}=1.2 U_{i j}$ of the nitrogen atom. All non-hydrogen atoms were refined with anisotropic displacement parameters. All other hydrogen atoms were treated as idealized contributions. Atomic scattering factors are contained in the SHELXTL 6.12 program library. ${ }^{82 a}$

\subsubsection{Spectroscopic Measurements}

All 1-D and 2-D NMR spectra were performed on a Bruker 300 spectrometer at ambient temperatures using NORELL XR-55 sample tubes and were referenced to TMS.

Absorption spectra were acquired on a Varian Cary 5 UV-vis-NIR spectrophotometer at ambient temperatures using $1.000 \mathrm{~cm}$ path length quartz cells

(Wilmad). Scans were performed at $500 \mathrm{~nm} / \mathrm{min}$ vs. solvent backgrounds, with a spectral bandwidth of $2.00 \mathrm{~nm}$, a signal averaging time of $0.033 \mathrm{~s}$, and a data interval of 1.000 nm.

Steady-state emission spectra were acquired on a Shimadzu RF-1501 spectrofluorophotometer at ambient temperatures using quartz fluorimeter cells (Wilmad) of $1.000 \mathrm{~cm}$ path length. 
To obtain time-resolved fluorescence decays, the samples were excited with the third harmonic of a Continuum Surelite $2 \mathrm{Nd}$ :YAG laser generating pulses at $355 \mathrm{~nm}$ of 6 ns duration and $15 \mathrm{~mJ}$ energy. The signals from the monochromator/photomultiplier system were initially captured by a Tetronix TDS 2012 oscilloscope and transferred to a Macintosh PC clone computer with software LFP V3.0 developed in the LabVIEW environment from Luzchem. A total of 10 shots were averaged to obtain the decay traces. All solution lifetime experiments were done in static $1.0 \times 1.0 \mathrm{~cm}^{2}$ fused silica cuvettes, following purging with inert gas for $30 \mathrm{~min}$. Each thin-film lifetime measurement was acquired on a plain microscope slide (Globe Scientific Inc.), sprayed with the paint using a conventional airbrush and mounted in a pressure chamber described elsewhere. ${ }^{36 \mathrm{~d}, \mathrm{e}}$

For the quantum yield determinations, the samples were optically matched to solutions of Coumarin 1 (Laser grade, Fisher) in ethanol $(\varphi=0.73)^{48}$ at room temperature using a Shimadzu RF-1501 spectrofluorophotometer. Quartz fluorimeter cells (Wilmad) of $1.000 \mathrm{~cm}$ path length were used in quantum yield measurements.

Electron Impact Mass Spectrometry (EI-MS) was performed at the University of Ottawa Mass Spectrometry centre.

Infrared spectroscopy was performed on a Varian 1000-FT-IR spectrometer as $\mathrm{KBr}$ pellets. Data was analyzed using Varian resolutions 4.1.0.101 software.

\subsubsection{Determination of Oxygen Quenching Sensitivity $\left(Q_{\mathrm{S}}\right)$}

The oxygen sensitive paints were calibrated. Each PSP formulation was applied to a primed aluminum plate with a Tristar Starpoxy fluid resistant white epoxy primer 
(DHMS C4.01 Ty3) using a conventional airbrush and compressed argon as the propellant. It was then mounted in a pressure chamber described elsewhere. ${ }^{36 \mathrm{~d}, ~ e}$ The average film thickness was estimated to be $2 \pm 1 \mu \mathrm{m}$ by a Positector 6000 model thickness gauge. Excitation in the UV was provided by a Hamamatsu Lightningcure LC5 200W (model L8333 Hg-Xe) source by a $10 \mathrm{~m} \times 8 \mathrm{~mm}$ Oriel UV-vis liquid light guide. Several optical filters in parallel were placed inside the lamp, before the light guide, to block wavelengths greater than $400 \mathrm{~nm}$ (Rapp Optoelectronic UV-1 and Hamamatsu A7028-05 filter). Excitation in the blue was provided by a Photonics Research Systems PRS100B blue LED lamp coupled with a 420-500 bandpass filter (03-FIV-28, Melles Griot). Thin-film emission spectra were collected using an Acton Research Corporation SpectruMM CCD detection system. A fiber optic lightguide (LG-455-020), equipped with a Kodak Wratten Gelatin 3 filter absorbing ultraviolet radiations passed the emission through a SpectraPro-150 imaging dual grating monochromator/spectrograph onto a 16bit Hamamatsu $1024 \times 256 \mathrm{CCD}$. Following the dark current subtraction, the spectra were normalized by the maximum intensity at atmospheric pressure to produce the spectra variations with pressure. Data for the Stern-Volmer plots were obtained by integrating the spectra over wavelengths between 460 and $560 \mathrm{~nm}$. These measurements were taken at $24{ }^{\circ} \mathrm{C}$, between 0.007 and 45 psi. A Scanivalve Corp. pressure calibrator/controller and a thermoelectric cooler coupled to a temperature controller were used to control pressure and temperature, respectively. Measurements at the reference pressure of $14.7 \mathrm{psi}$ were taken two times during the calibration in order to estimate the photostability of the paints. The excitation light source stability was monitored with a temperature controlled Oriel 7123 photodiode. 
The decrease in emission intensity with increasing air pressure relative to the emission intensity at $1 \mathrm{~atm}$ pressure was treated according to Stern-Volmer kinetics and modified Stern-Volmer plots were acquired. Oxygen quenching sensitivity $\left(Q_{s}\right)$ data were calculated from linear fits to the Stern-Volmer plots with $r^{2}>0.95$. Multiple quenching processes arising from heterogeneous luminophore environments could result in curvature in the Stern-Volmer plots of thin films. In these cases criteria for acceptable range of $P / P_{\text {ref }}$ was considered as $\mathrm{r}^{2}>0.95$. Measurements were repeated at least three times and the error in reported $Q_{\mathrm{s}}$ data was estimated at \pm 0.01 .

\subsubsection{Luminescence Microscopy}

All microscopy was performed at Agriculture Canada. PSP films were sprayed on plain microscope slides (Globe Scientific Inc.). The luminescence images were observed in air using a confocal microscope Carl Zeiss LSM510 Meta (405 nm, 42.6\% laser intensity) with an ECPlan-Neofluar 40x/1.30 Oil Dic M27 objective exciting at $405 \mathrm{~nm}$ and collecting image data at $505 \mathrm{~nm}$. The observed images were analyzed with LSM510 V.4.0 SP2. 


\section{Chapter 3}

\section{Results and Discussion}

\subsection{Initial Choice of Matrix}

The first challenge in this project was acquiring water-soluble matrices that dissolve luminophores and have good mechanical properties and high oxygen permeabilities. To start, a copolymerization reaction was developed in order to produce copolymers of water-soluble poly(N-vinyl pyrrolidone) or poly(N,N-dimethylacrylamide) and oxygen permeable poly(2, 2, 2-trifluoroethyl methacrylate). While some watersoluble copolymers were successfully prepared via radical polymerization using AIBN as the initiator, the synthesis was not pursued due to low oxygen permeabilities and practical problems in sprayed films of them. The problems occurred during pressure sensitivity calibrations, when the films cracked under changing pressures. Therefore, commercially available polyethylene glycol (PEG) (average $\mathrm{M}_{\mathrm{n}}$ Ca. 200), (hydroxypropyl) methyl cellulose, and a water-soluble copolymer of the well known oxygen permeable polymer, polydimethylsiloxane (dimethylsiloxane-(60\% propylene oxide- $40 \%$ ethylene oxide) block polymer (PDMS-based copolymer) (MW 30,000) were utilized as the initial matrices to develop water-based PSP formulations. The mechanical properties of these polymers need to be improved for practical applications. To compensate for the poor mechanical properties of the water-soluble polymers, microcrystalline cellulose (MC) was included in the formulations. MC was decided on, since it is a semicrystalline 
polymer with a low chemical reactivity and has been used widely as binder in tableting technology in order to improve compactibility.

\subsection{Initial Choice of Luminophore}

Luminescent iridium complexes have been used in PSPs because of their large quantum yields of emission under room temperature and normal pressure, and efficient quenching of the photoexcited triplet state by molecular oxygen. The first iridium complex studied in water-based PSP formulations was the charged complex 1 which is soluble in water-methanol mixtures containing up to 75 volume percent of water.

\subsection{Characterization of Initial Pressure Sensitive Paint (PSP) Films}

The pressure-dependent film emission spectra of $\mathbf{1}$ in different matrices were studied. Figure 3.1 shows a representative example. Films of 1 displayed an intense yellow-white luminescence with UV irradiation under low pressure conditions. The decrease in emission intensity with increasing air pressure relative to the emission intensity at $1 \mathrm{~atm}$ pressure can be treated according to Stern-Volmer kinetics (See Experimental) and Figure 3.2 shows modified Stern-Volmer plots of 1 in three PEGbased formulations.

Oxygen quenching sensitivity $\left(Q_{\mathrm{s}}\right)$ data for various thin films were calculated from linear fits to the Stern-Volmer plots with $r^{2}>0.95$ and have been placed in Table 3.1. Multiple quenching processes arising from heterogeneous luminophore 
environments could result in curvature in the Stern-Volmer plots of thin films. In these cases appropriate linear fits with $r^{2}>0.95$ were not acquired. Constrained pressure ranges presenting linear fits to the plots were chosen to find $Q_{\mathrm{s}}$ data.

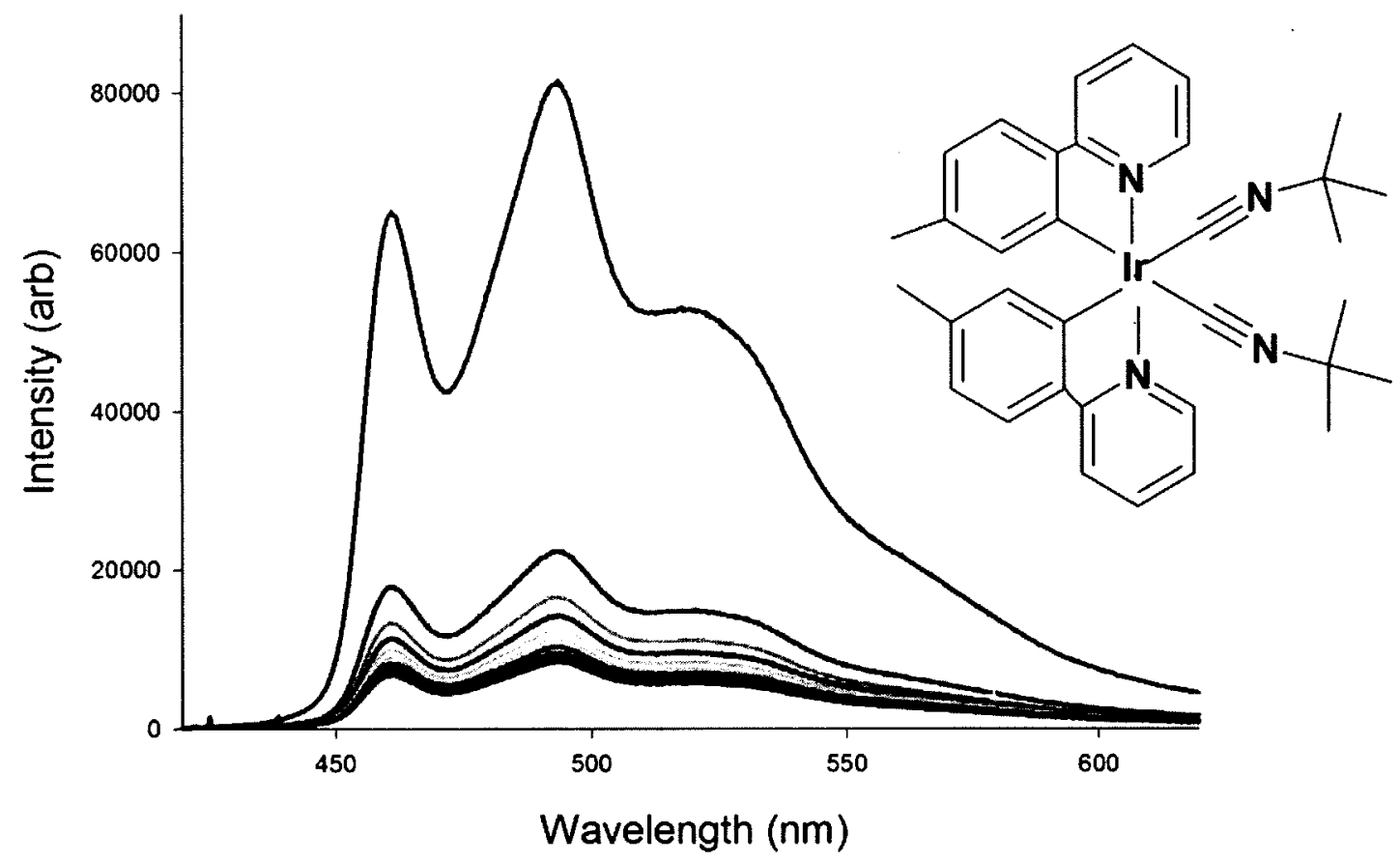

Figure 3.1: Effect of pressure on thin film luminescence of 1 in PDMS-based copolymer. The decrease of intensity with increasing the air pressure is displayed. Spectra were acquired at 1.3 mbar (black), 0.34 bar (red), 0.68 bar (green), 1 bar (purple), 1.36 bar (blue), 1.70 bar (grey), 2.04 bar (dark red), 2.38 bar (dark green), 2.72 bar (dark blue), and 3.06 bar (dark purple). Background correction has been performed. 


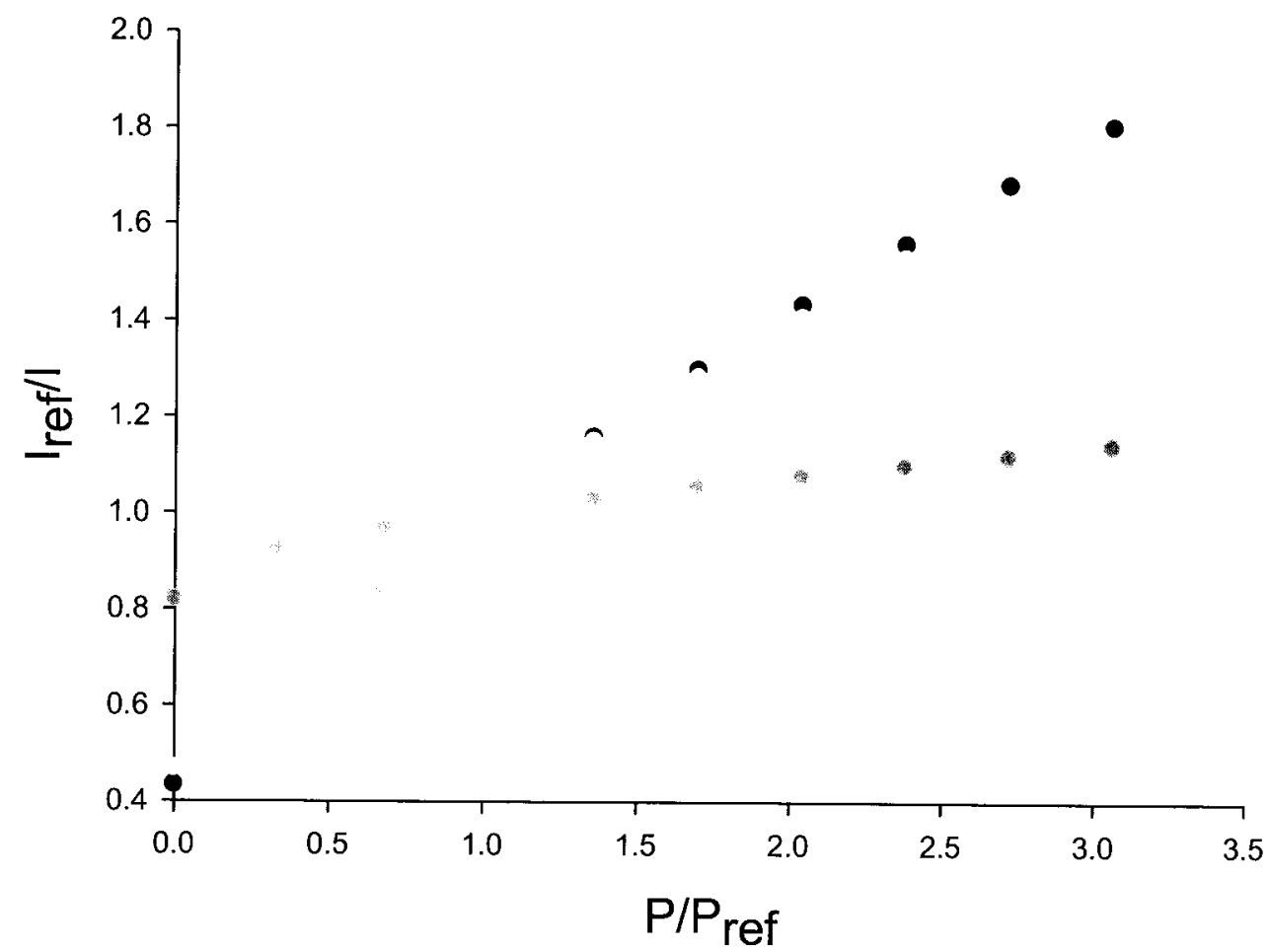

Figure 3.2: Stern-Volmer plots of 1 in PEG, ; in 1:1 PEG: MC, •; and in 1:9 PEG: MC,

Table 3.1: PSP films oxygen sensitivity data for $\mathbf{1}$.

\begin{tabular}{|c|c|c|}
\hline Luminophore & Matrix & $\boldsymbol{Q}_{\mathbf{s}}\left(\mathbf{r}^{2}\right)$ \\
\hline $\mathbf{1}$ & (Hydroxypropyl) Methyl Cellulose $^{\mathrm{a}}$ & $0.38(0.987)^{\mathrm{a}}$ \\
& PEG & $0.41(0.991)^{\mathrm{a}}$ \\
& 1:1 PEG: MC & $0.43(0.990)^{\mathrm{a}}$ \\
& 1:9 PEG: MC & $0.08(0.985)^{\mathrm{b}}$ \\
& PDMS-based Copolymer & $0.36(0.983)^{\mathrm{b}}$ \\
& $1: 1$ PDMS-Cop.: MC & $0.45(0.991)^{\mathrm{b}}$ \\
& 1 :9 PDMS-Cop.: MC & $0.31(0.987)^{\mathrm{c}}$ \\
\hline
\end{tabular}

${ }^{\mathrm{a}} 0.007-45 \mathrm{psi} ;{ }^{\mathrm{b}} 5-45 \mathrm{psi} ;{ }^{\mathrm{c}} 10-45 \mathrm{psi}$

Oxygen sensitivity as well as mechanical properties of the film is increased as a consequence of the blending of $\mathrm{MC}$ with luminophores compared with the film composed 
of the polymer with low glass transition temperature. It is suggested that this effect originates from a decrease in the luminophore's freedom of motion and consequentially an increase in its luminescence lifetime. Moreover, the preferential binding of luminophore to $\mathrm{MC}$ could lead to de-aggregation and a decrease in self-quenching.

\subsection{Synthesis of Water-Soluble Oxygen Sensor 8}

\subsubsection{Functionalizing Ir (III) Complex}

In order to overcome the poor solubility of complex 1 in aqueous solutions, the covalent attachment of the analogous functionalized complex $\mathbf{2}$ to water soluble polymers was investigated.

\subsubsection{Synthesis}

Starting material $\left[\operatorname{Ir}(\mathrm{fppy})_{2} \mathrm{Cl}\right]_{2}$ (Scheme 3.1) was prepared according to Lo. ${ }^{67 \mathrm{a}}$ 

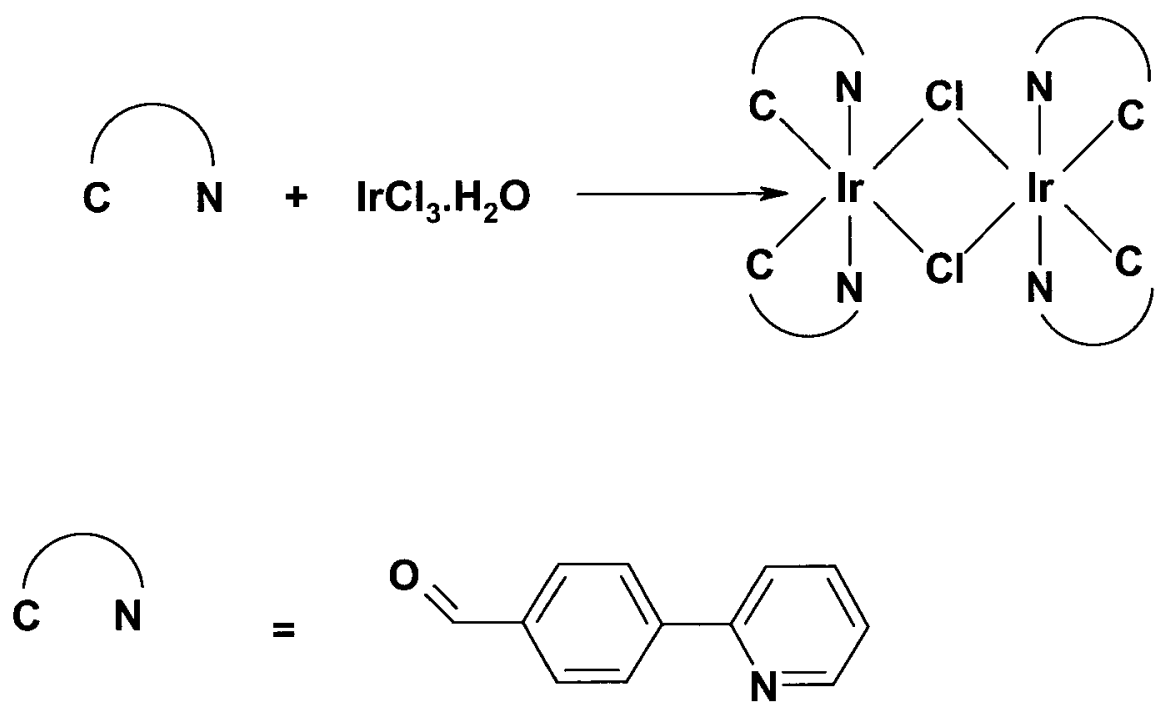

Scheme 3.1: Synthesis of starting functionalized iridium dimer.

Complex 2, $\left[\mathrm{Ir}(\mathrm{fppy})_{2}(t-\mathrm{Bu}-\mathrm{iCN})_{2}\right]\left[\mathrm{CF}_{3} \mathrm{SO}_{3}\right]$ (fppy = 4-(2-pyridyl)benzaldehyde, and $t$-Bu-iCN $=$ tert-butyl isocyanide), was synthesized in a high yield $(72 \%)$ by cleaving the iridium dimer $\left[\operatorname{Ir}(\mathrm{fppy})_{2} \mathrm{Cl}\right]_{2}$ with silver triflate followed by coordination of the tert-butyl isocyanide ligand (shown in Scheme 3.2).
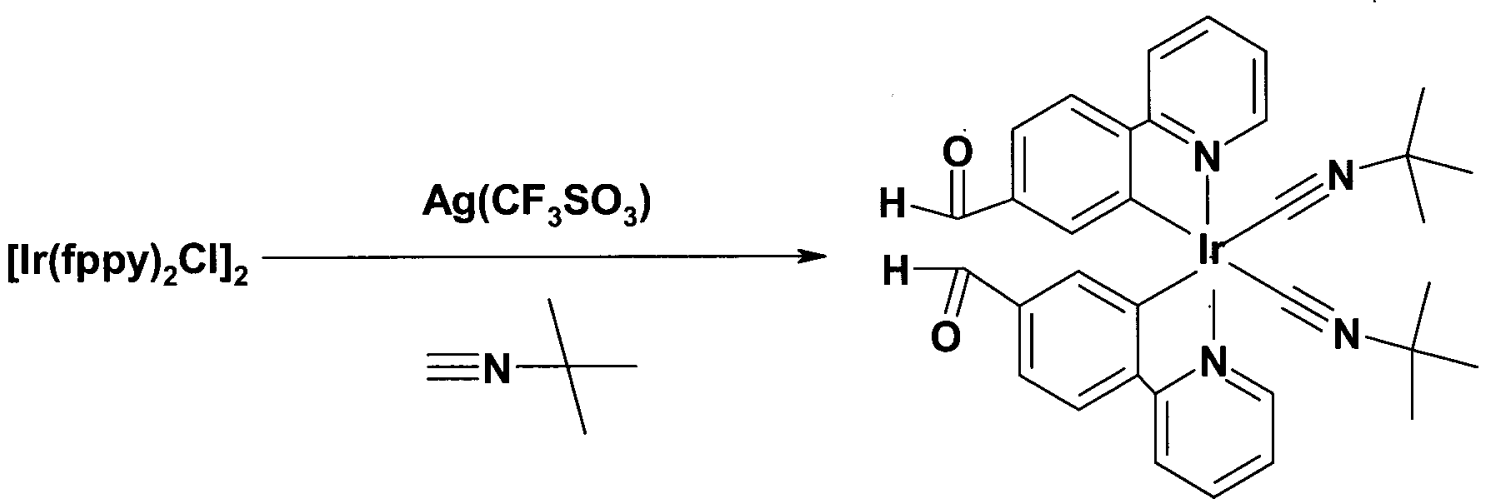

Scheme 3.2: Synthesis of 2 
The pyridylbenzaldehyde ligands provide the reactive sites for the reductive amination reaction.

\subsubsection{X-ray Crystallography}

Structure of 2 was confirmed by X-ray crystallography on its single crystals grown from dichloromethane solutions by diffusion of ether. It was shown that two symmetrically unique but chemically equivalent ion pairs are located in the asymmetric unit of 2. The ORTEP drawing of $2 \cdot 0.25\left(\mathrm{CH}_{2} \mathrm{Cl}_{2}\right)$ is shown in Figures 3.3. Crystallographic data and some selected bond lengths and angles are given in Tables 3.2-3.3. Full data of atomic coordinates, bond lengths and angles for the complex can be found in Appendix A. 


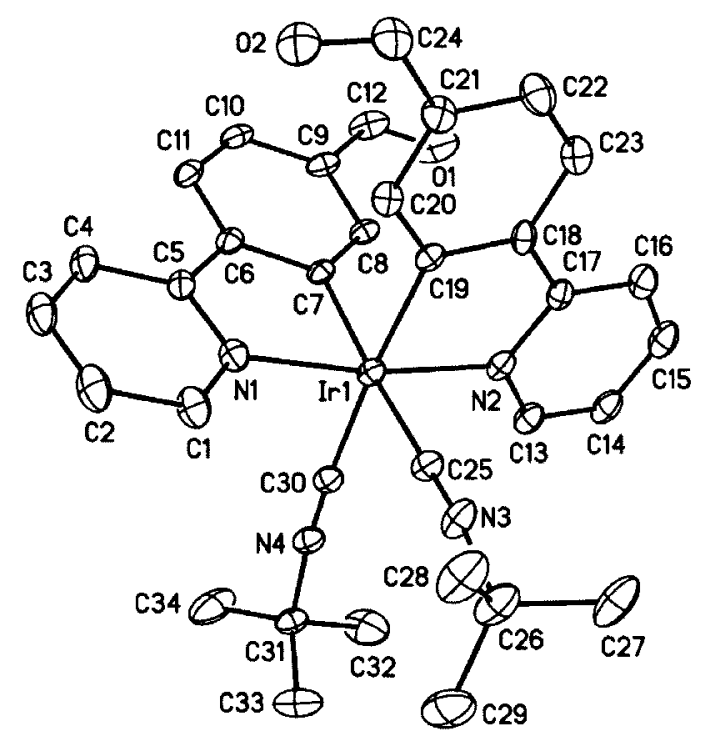

Figure 3.3: ORTEP of $2 \cdot 0.25\left(\mathrm{CH}_{2} \mathrm{Cl}_{2}\right)$ with ellipsoids at $30 \%$. The alternate disordered contribution, second symmetrically unique ion pair, triflate counter ion, methylene chloride solvent molecule, and hydrogen atoms are omitted for clarity. 
Table 3.2: X-ray Crystallographic Data and Refinement Details for $2 \cdot 0.25\left(\mathrm{CH}_{2} \mathrm{Cl}_{2}\right)$.

$$
\text { Empirical formula } \quad \mathrm{C}_{35} \mathrm{H}_{34} \mathrm{~F}_{3} \mathrm{IrN}_{4} \mathrm{O}_{3} \mathrm{~S} \cdot 0.25\left(\mathrm{CH}_{2} \mathrm{Cl}_{2}\right)
$$

$\mathrm{M}_{\mathrm{r}}$

Cryst syst

Space group

unit cell dimensions

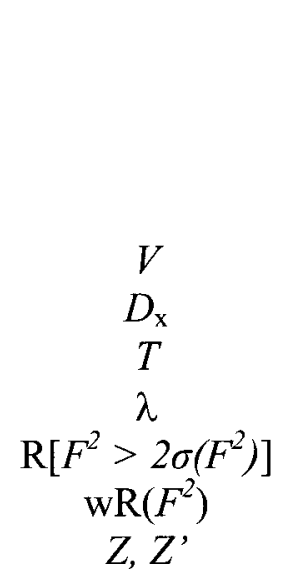

$\mu$
861.15

Triclinic

$$
P-1
$$

$a=15.164(5) \AA$

$b=17.083(6) \AA$

$c=17.242(6) \AA$

$\alpha=117.569(5)^{\circ}$

$\beta=104.876(5)^{\circ}$

$\gamma=100.075(5)^{\circ}$

$3595(2) \AA^{3}$

$1.591 \mathrm{~g} \mathrm{~cm}^{-3}$

$120(2) \mathrm{K}$

$0.71073 \AA$

0.0498

0.1343

4,2

$3.864 \mathrm{~mm}^{-1}$

Table 3.3: Selected Bond Lengths $(\AA)$ and Angles $\left(^{\circ}\right)$ for $2 \cdot 0.25\left(\mathrm{CH}_{2} \mathrm{Cl}_{2}\right){ }^{\text {a }}$

\begin{tabular}{llcc}
\hline Ir1-N1 & $2.049(5), 2.039(10)$ & $\mathrm{N} 1-\mathrm{Ir} 1-\mathrm{N} 2$ & $170.7(2), 165.2(6)$ \\
$\mathrm{Ir} 1-\mathrm{N} 2$ & $2.047(5), 2.051(5)$ & $\mathrm{C} 25-\mathrm{Ir} 1-\mathrm{C} 30$ & $91.9(4), 89.1(3)$ \\
$\mathrm{Ir} 1-\mathrm{C} 7$ & $2.046(6), 2.057(11)$ & $\mathrm{C} 7-\mathrm{Ir} 1-\mathrm{N} 1$ & $80.1(2), 80.0(5)$ \\
$\mathrm{Ir} 1-\mathrm{C} 25$ & $2.037(8), 2.062(6)$ & $\mathrm{C} 19-\mathrm{Ir} 1-\mathrm{N} 2$ & $80.3(2), 79.9(2)$ \\
$\mathrm{C} 12-\mathrm{O} 1$ & $1.147(9), 1.148(14)$ & $\mathrm{C} 25-\mathrm{Ir} 1-\mathrm{C} 30$ & $91.94(4), 89.1(3)$ \\
$\mathrm{C} 24-\mathrm{O} 2$ & $1.202(8), 1.193(7)$ & $\mathrm{N} 3-\mathrm{C} 25$ & $1.138(10), 1.157(8)$ \\
$\mathrm{N} 3-\mathrm{C} 26$ & $1.515(13), 1.457(10)$ & $\mathrm{N} 4-\mathrm{C} 30$ & $1.164(8), 1.118(8)$ \\
$\mathrm{N} 4-\mathrm{C} 31$ & $1.487(9), 1.464(8)$ &
\end{tabular}


In $2 \cdot 0.25\left(\mathrm{CH}_{2} \mathrm{Cl}_{2}\right)$, iridium occupies a distorted octahedral coordination sphere of four carbon and two nitrogen donor atoms in which tert-butyl isocyanide ligands are cis to each other. In this stereochemistry, the bidentate pyridylbenzaldehyde ligands are expected to be coordinated to iridium with pyridine nitrogens trans to each other because that is the geometry of the reagent dimer complex. ${ }^{84}$ This is confirmed in Figure 3.3 and by the Ir-N bond lengths (Table 3.3) which are similar to those of analogous complexes possessing the bis(pyridylbenzaldehyde)iridium fragment. ${ }^{83}$, ${ }^{85}$ Figure 3.3 also shows that the tert-butyl isocyanide ligands are slightly bent with Ir1-C30-N4 and C30-N4-C31 bond angles of $176.8(6)^{\circ}$ and $173.2(7)^{\circ}$ respectively. This is similar to that observed in crystal structures of other tert-butyl isocyanide iridium complexes. ${ }^{86}$

\subsubsection{NMR Spectroscopy}

The successful synthesis of $\mathbf{2}$ was confirmed by displaying characteristic resonances of tert-butyl protons of the ligand in the proton NMR spectrum. Moreover, the ${ }^{13} \mathrm{C}$ NMR (Figure 3.4) spectrum of 2 showed all twelve aromatic and aldehyde carbon peaks between 120 to $195 \mathrm{ppm}$ as well as the characteristic quaternary carbon signal of tert-butyl at $59 \mathrm{ppm}$. 


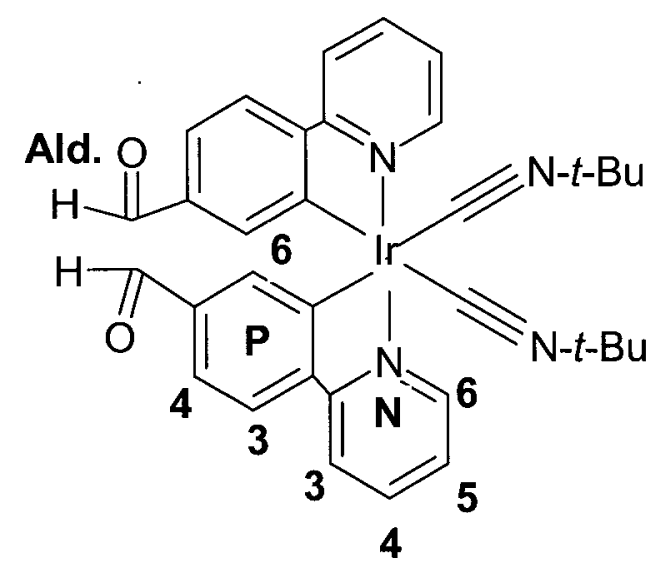

Chart 1: Structure of $\left[\operatorname{Ir}(\mathrm{fppy})_{2}(t-\mathrm{Bu}-\mathrm{iCN})_{2}\right]\left[\mathrm{CF}_{3} \mathrm{SO}_{3}\right], 2$, with the numbering used in assigning the ${ }^{1} \mathrm{H}$ NMR.

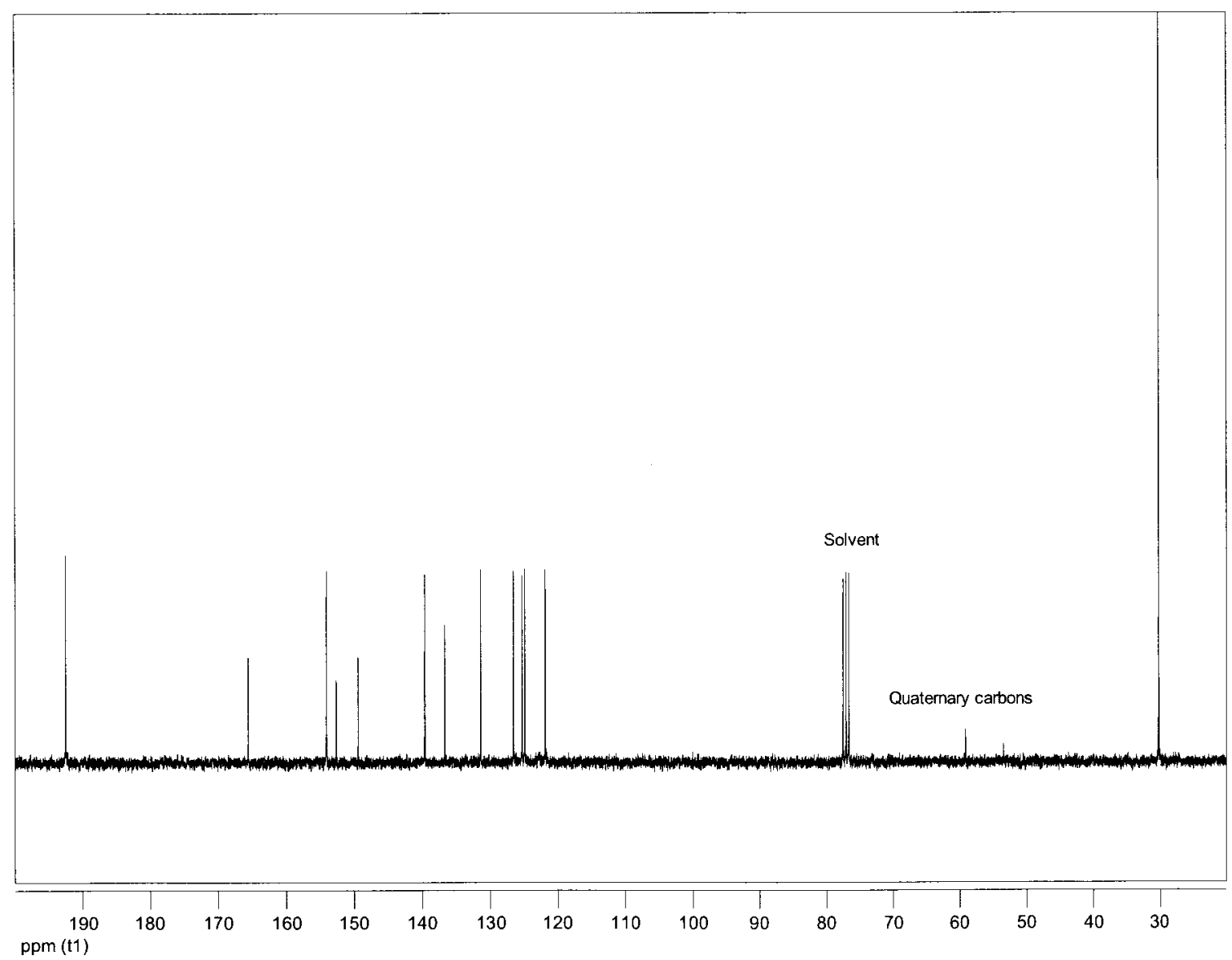

Figure 3.4: ${ }^{13} \mathrm{C}$ NMR spectrum $(300 \mathrm{MHz})$ of 2 in $\mathrm{CDCl}_{3}$. 
In order to assign hydrogens of pyridyl benzaldehyde ligands, 1-D and 2-D techniques of ${ }^{1} \mathrm{H}$ NMR (Figure 3.5) and COSY (correlation spectroscopy, Figure 3.6) were used.

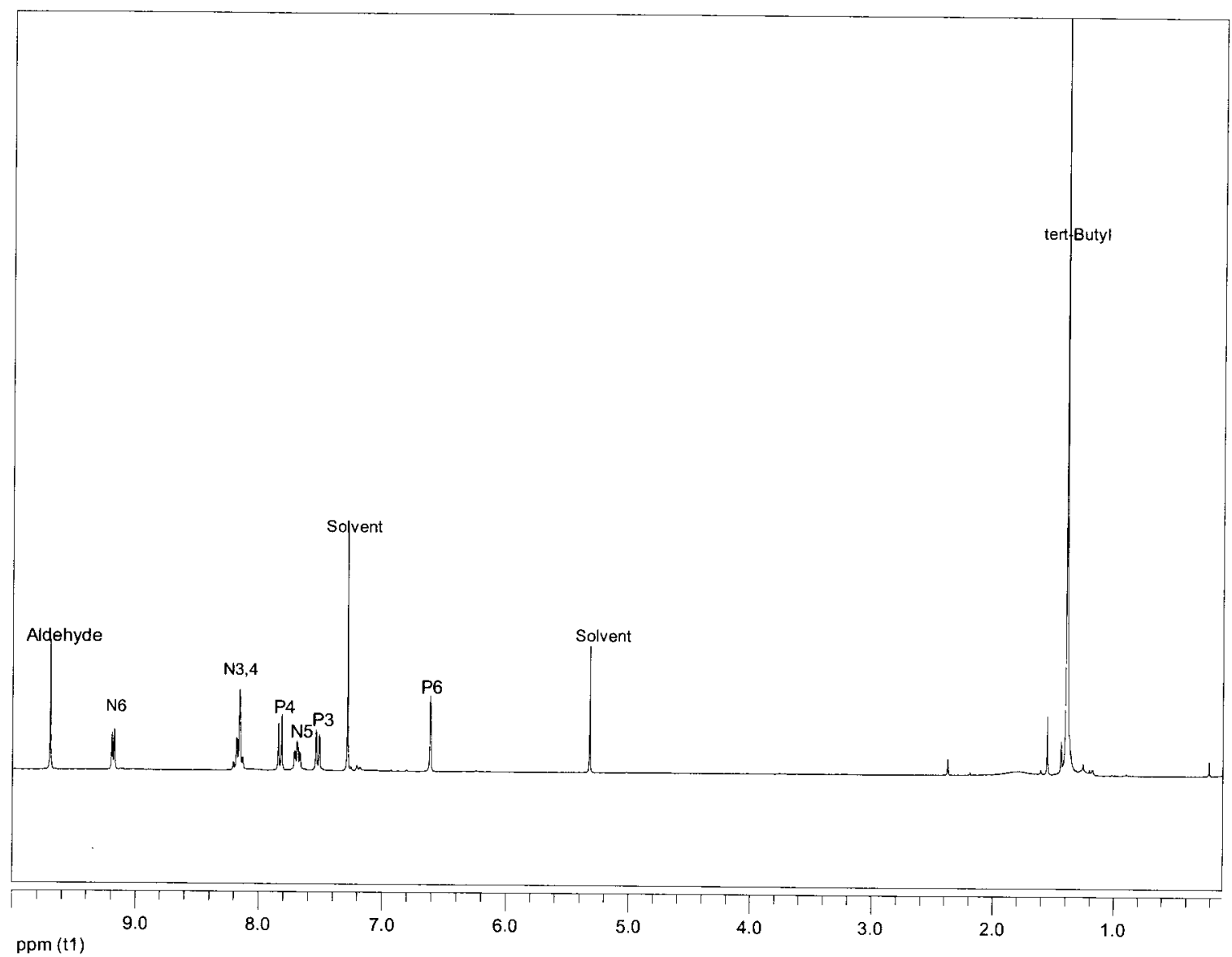

Figure 3.5: ${ }^{1} \mathrm{H}$ NMR spectrum $(300 \mathrm{MHz})$ of 2 in $\mathrm{CDCl}_{3}$.

The COSY spectrum of $\mathbf{2}$ is shown in Figure 3.6. Spatially, the P6 proton of $\mathbf{2}$ is isolated from other protons by at least four bonds and appears as a singlet. No other aromatic hydrogen signals are easily identifiable based on multiplicity. To further interpret this COSY spectrum, it is necessary to analyze a model of the molecule graphically and determine possible interactions between protons. For example, the P4 
proton has a potential interaction with $\mathrm{P} 3$ over three bonds. The expected result is a doublet for P4. Mutual interactions are between P3 and P4, N3 and N4, N4 and N5, and N5 and N6.

The aromatic region of proton spectrum in Figure 3.5 is grouped into six regions. In order to simplify the discussion, letters are used to represent each region. For example "A" refers to the singlet at $6.61 \mathrm{ppm}$, " $\mathrm{B}$ " refers to the peaks centered at $7.53 \mathrm{ppm}$ and so on. Utilizing this method, a simplified COSY spectrum can be created as shown in Figure 3.7. The goal is to assign each region to a specific proton. Region $\mathrm{C}$ can be assigned to N4 or N5 due to interactions with two other groups of peaks E and F. Since assigning region $\mathrm{F}$ to $\mathrm{N} 6$ is more reasonable than $\mathrm{N} 3$, the $\mathrm{C}$ area resonances are therefore assigned to N5 proton. It follows that regions $\mathrm{B}$ and $\mathrm{D}$ must be due to the P3 and P4 protons, respectively. 


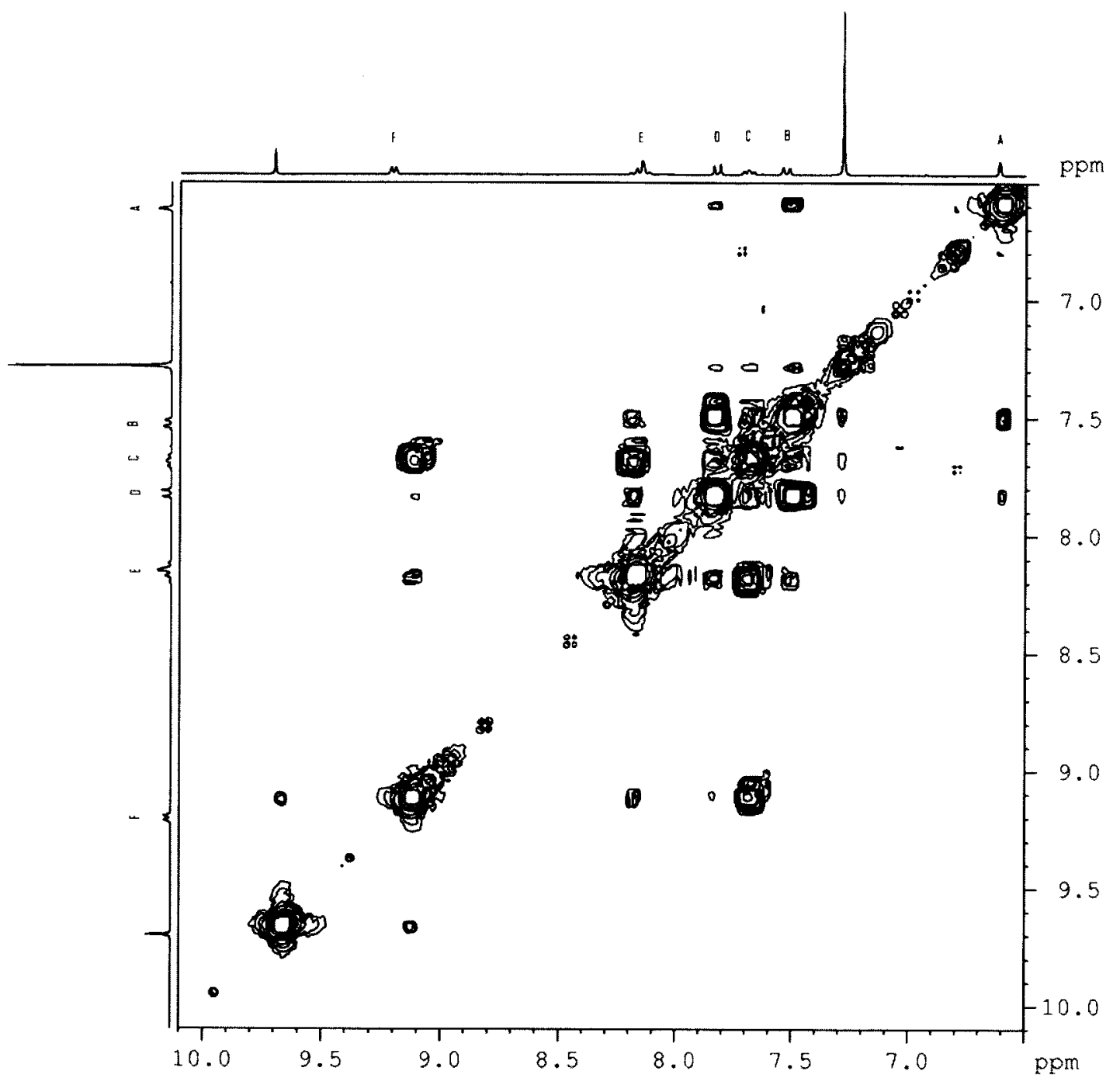

Figure 3.6: COSY spectrum $(300 \mathrm{MHz})$ of 2 in $\mathrm{CDCl}_{3}$.

\begin{tabular}{|c|c|c|c|c|c|c|}
\hline & $\mathrm{F}(9.20)$ & $\mathrm{E}(8.13)$ & $\mathrm{D}(7.82)$ & $\mathrm{C}(7.69)$ & $\mathrm{B}(7.53)$ & $\mathrm{A}(6.61)$ \\
\hline $\mathrm{A}(6.61)$ & & & & & & $\mathrm{AA}$ \\
\hline $\mathrm{B}(7.53)$ & & & $\mathrm{BD}$ & & $\mathrm{BB}$ & \\
\hline $\mathrm{C}(7.69)$ & $\mathrm{CF}$ & $\mathrm{CE}$ & & $\mathrm{CC}$ & & \\
\hline $\mathrm{D}(7.82)$ & & & $\mathrm{DD}$ & & $\mathrm{DB}$ & \\
\hline $\mathrm{E}(8.13)$ & & $\mathrm{EE}$ & & $\mathrm{EC}$ & & \\
\hline F (9.20) & $\mathrm{FF}$ & & & FC & & \\
\hline
\end{tabular}

Figure 3.7: Simplified representation of COSY spectrum of 2 in $\mathrm{CDCl}_{3}$. 


\subsubsection{Infrared Spectroscopy}

The infrared spectrum of complex $\mathbf{2}$ is shown in Figure 3.8 in which the $v(\mathrm{C} \equiv \mathrm{N})_{\text {isocyanide }}$ is seen at $2177 \mathrm{~cm}^{-1}$.

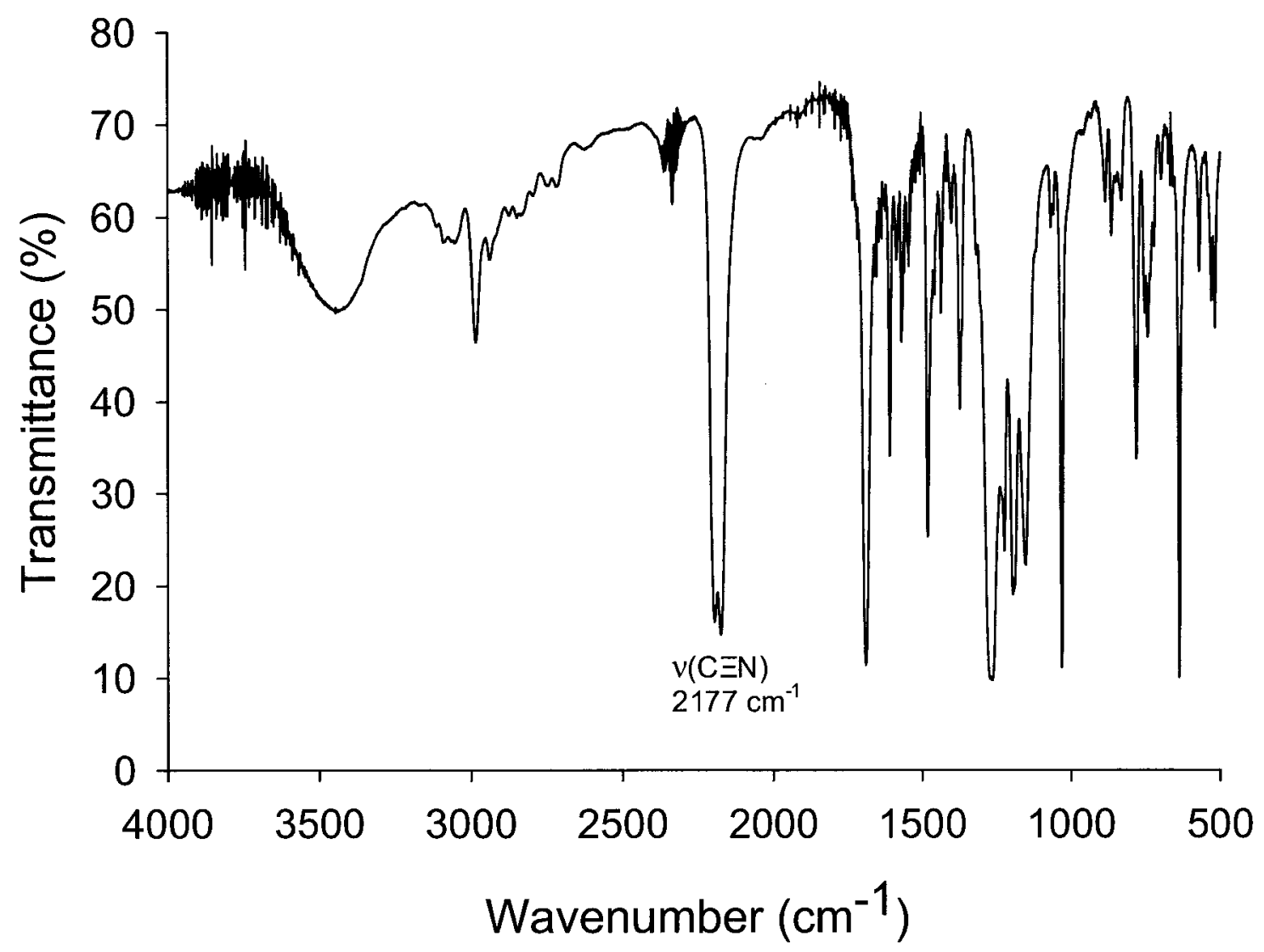

Figure 3.8: Infrared spectrum $\left(\mathrm{KBr}\right.$ pellet) of 2 with $v(\mathrm{C} \equiv \mathrm{N})$ at $2177 \mathrm{~cm}^{-1}$. 


\subsubsection{Electronic Spectroscopy}

The room-temperature quantitative UV-Vis and emission spectra have been collected for $\mathbf{2}$ and are shown in Figure 3.9. Assignments are made by comparing the absorption spectrum of $\mathbf{2}$ with analogous complexes. ${ }^{67 a, 75}$

Three characteristic types of well-resolved absorption bands are observed in $\mathbf{2}$. Intense absorption bands below $320 \mathrm{~nm}\left(\varepsilon \sim 3.0 \times 10^{4} \mathrm{~cm}^{-1} \mathrm{M}^{-1}\right)$ are assigned to allowed $\pi-\pi^{*}$ transitions of the fppy ligand. ${ }^{67 \mathrm{a}}$ The weaker band appearing at $353 \mathrm{~nm}(\varepsilon=6920$ $\mathrm{cm}^{-1} \mathrm{M}^{-1}$ ) can be assigned to $\mathrm{Ir} \rightarrow$ fppy charge-transfer transitions. ${ }^{72 k, 84}$ The aldehyde group of the fppy ligand appears to decrease the extinction coefficient of the MLCT band and to shift it to longer wavelengths compared to the MLCT band of its nonfunctionalized analogue $1 .^{75}$ The decrease in MLCT intensity reflects a decrease in metal-ligand coupling ${ }^{87}$ and the shift to longer wavelengths, particularly for emission, is consistent with a stabilization of the $\pi^{*}$ orbitals of fppy by a conjugated electronwithdrawing substituent. The emission band shows a vibronic progression that has been previously analyzed for a series of $\operatorname{Ir}(\text { tolylpyridine })_{2}$ complexes, assuming a dominant vibrational stretch. ${ }^{84}$ The relative intensities of these vibronic components is evidently substituent dependent as shown by the emission spectrum of $\mathbf{2}$, compared with $\mathbf{1}$ and $\mathbf{8}$ (2 attached to Silamine) (see section 3.4.2), but this was not investigated further. 


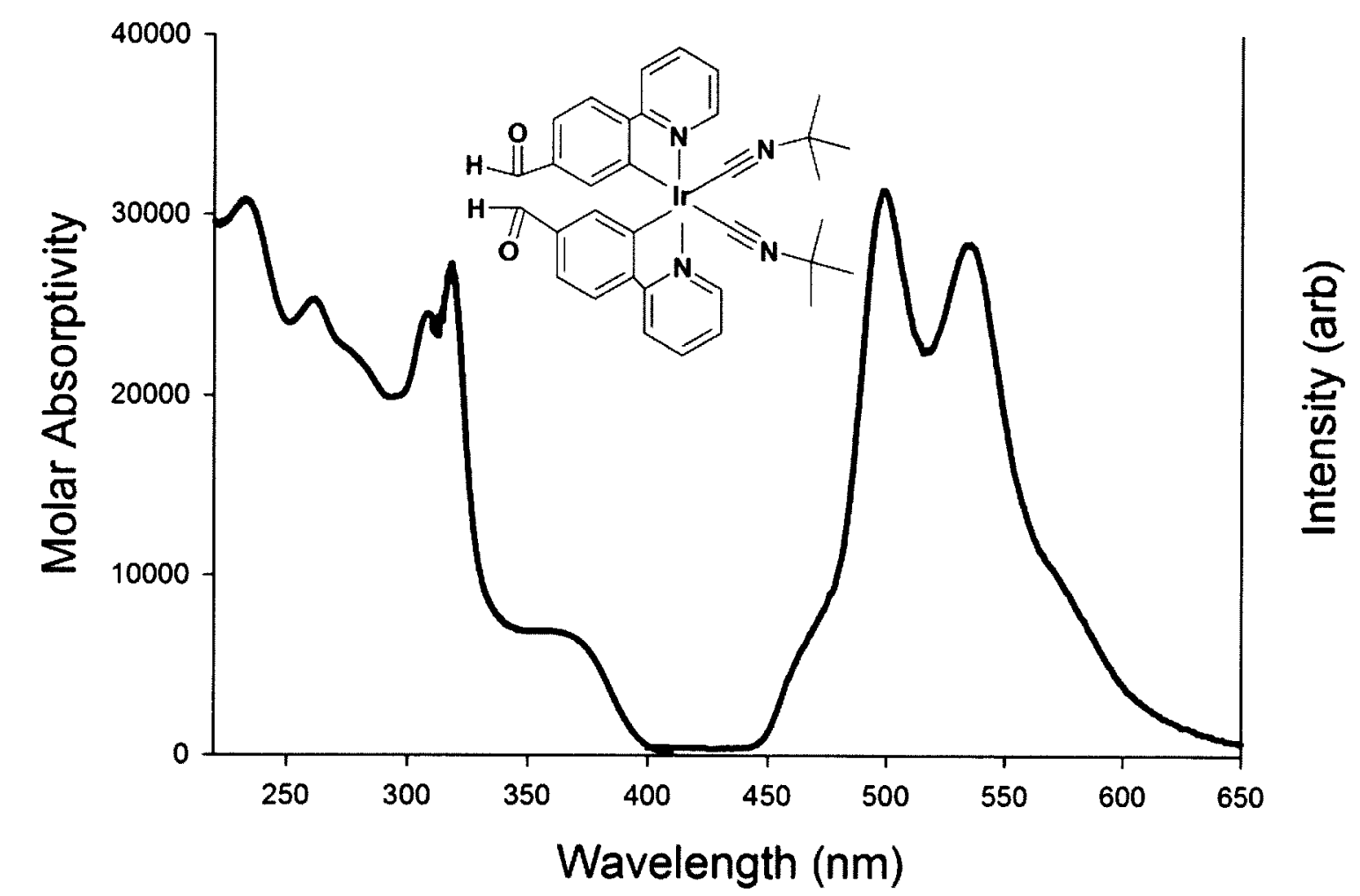

Figure 3.9: Solution electronic absorption and emission spectra of 2 . The absorption spectrum was acquired in $\mathrm{CH}_{3} \mathrm{CN}$ and the emission spectrum was acquired in $\mathrm{CH}_{2} \mathrm{Cl}_{2}$.

Complex 2 is strongly luminescent (quantum yield $\phi=0.35$ ) with a long luminescence lifetime $(\tau=17.3 \mu \mathrm{s})$ at room temperature. Emission is suggested to be originating from a triplet excited state, based on the long lifetime and similarity of quantum yield to that of $f a c-\operatorname{Ir}(t p y)_{3}$ reported in the literature. ${ }^{75}$ In addition, it benefits from a large Stokes shift $\left(7100 \mathrm{~cm}^{-1}\right)$. 
3.4.2 Synthesis of Water-Soluble Oxygen Sensor 8 by Reductive Attachment of 2 to Silamine D208-EDA and Spectroscopic Characterization

Reductive amination reaction between $\mathbf{2}$ and the water-soluble aminefunctionalized Silamine D208-EDA gave the luminophore-functionalized Silamine, 8, as shown in Scheme 3.3.

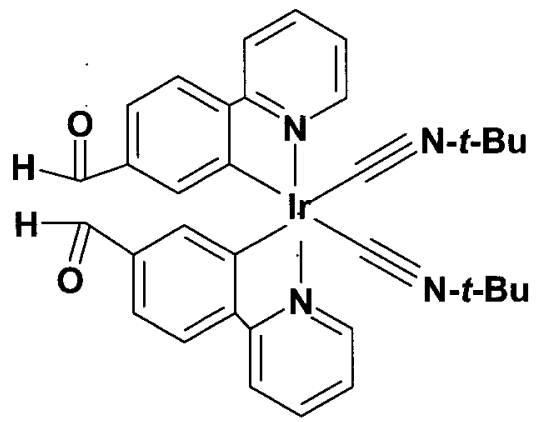<smiles>C[Si](C)(C)O[Si](C)(C)O[Si](C)(C)O[Si](C)(C)O[Si](C)(C)C</smiles>

2<smiles></smiles>

8<smiles>[R]=C[Si](C)(O[Si](C)(C)[Si](C)(C)O[Si](C)(C)[Si](C)(C)O[Si](C)(C)C)[Si](C)(C)[Si](C)(C)[Si](C)(C)O[Si](C)(C)C</smiles>

$$
\mathrm{NH}_{2}-\mathrm{CH}_{2}-\mathrm{CH}_{2}-\mathrm{NH} \quad \mathrm{NH}-\mathrm{CH}_{2}-\mathrm{CH}_{2}-\mathrm{NH}
$$

Scheme 3.3: Reductive amination of 2 with Silamine D208-EDA to make 8. 
The yield of the reaction in Scheme 3.3 was estimated by assuming that the electronic absorption properties of the iridium complex in $\mathbf{8}$ were the same as those of free complex 1. The electronic spectrum of $\mathbf{8}$ was used to calculate attachment yield considering 8 as a solution of 1 in Silamine. Thus, a quantitative solution of $\mathbf{8}$ in $\mathrm{CH}_{2} \mathrm{Cl}_{2}$ showed a $\pi-\pi^{*}$ absorption band at $263 \mathrm{~nm}$ that was assumed to possess the same extinction coefficient as the $\pi-\pi^{*}$ absorption band of $1\left(\lambda=260 \mathrm{~nm}, \varepsilon=3.35 \times 10^{4} \mathrm{M}^{-1}\right.$ $\mathrm{cm}^{-1}$ ). Using the molar absorptivity of the $\pi-\pi^{*}$ transition of $\mathbf{1}$ as the Silamine-attached luminophore model complex, the actual concentration of luminophore was determined. The calculated concentration of iridium complex in $\mathbf{8}$ indicated quantitative attachment of 2 to Silamine.

The attachment of $\mathbf{2}$ shown in Scheme 3.3 is of cross-linked Silamine chains. However, it is also possible for 2 to bind to a single Silamine chain by one or two methyl-amine links. 2 dispersed in Silamine shows an aldehyde proton chemical shift in the ${ }^{1} \mathrm{H}$ NMR at approx. $9.58 \mathrm{ppm}$ (Figure 3.12). There was no evidence of unreacted aldehyde in the ${ }^{1} \mathrm{H}$ NMR of 8 (see Figures 3.10-3.13), which may indicate a complete condensation reaction or reduction of the aldehyde group by pyridine-borane. Unfortunately, ${ }^{1} \mathrm{H}$ NMR chemical shift of phenylpyridine protons overlaps with an imine proton that may be present. This, together with the large molecular weight of $\mathbf{8}$, makes an assignment of the chemical shift of an imine proton ambiguous. Nevertheless, the similarity of $\mathbf{8}$ and 1's photophysical properties and electronic absorption spectroscopy (see Figure 3.15, compare with reference 75) suggests that imine linkages were reduced to methyl-amines by pyridine- borane. This was desired in order to closely 
match the electronic and photophysical properties of the Silamine bound iridium complex 8 to those of complex 1 .

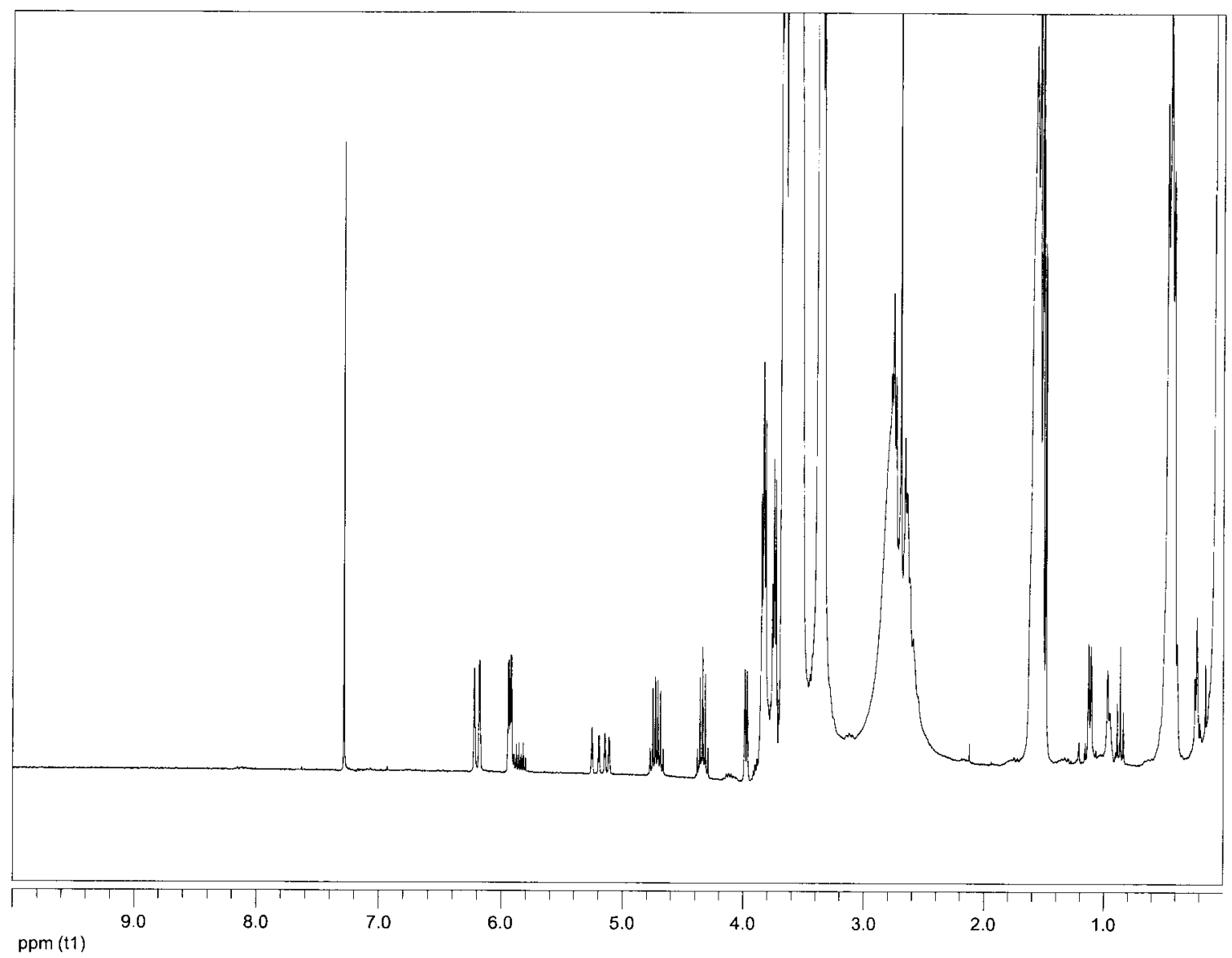

Figure 3.10: ${ }^{1} \mathrm{H}$ NMR spectrum $(300 \mathrm{MHz})$ of Silamine D208-EDA in $\mathrm{CDCl}_{3}$. 


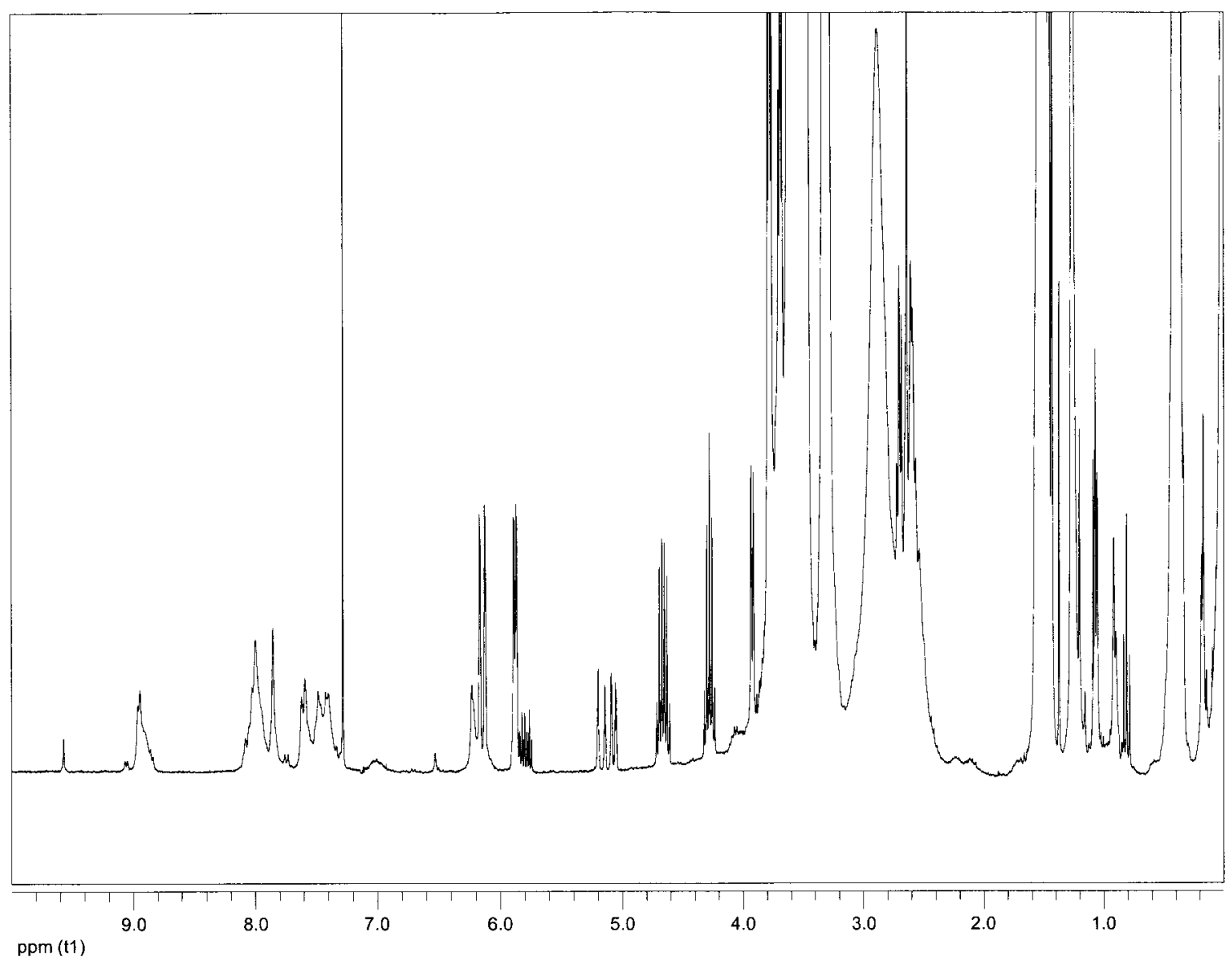

Figure 3.11: ${ }^{1} \mathrm{H}$ NMR spectrum $(300 \mathrm{MHz})$ of 2 dispersed in Silamine D208-EDA in $\mathrm{CDCl}_{3}$. 


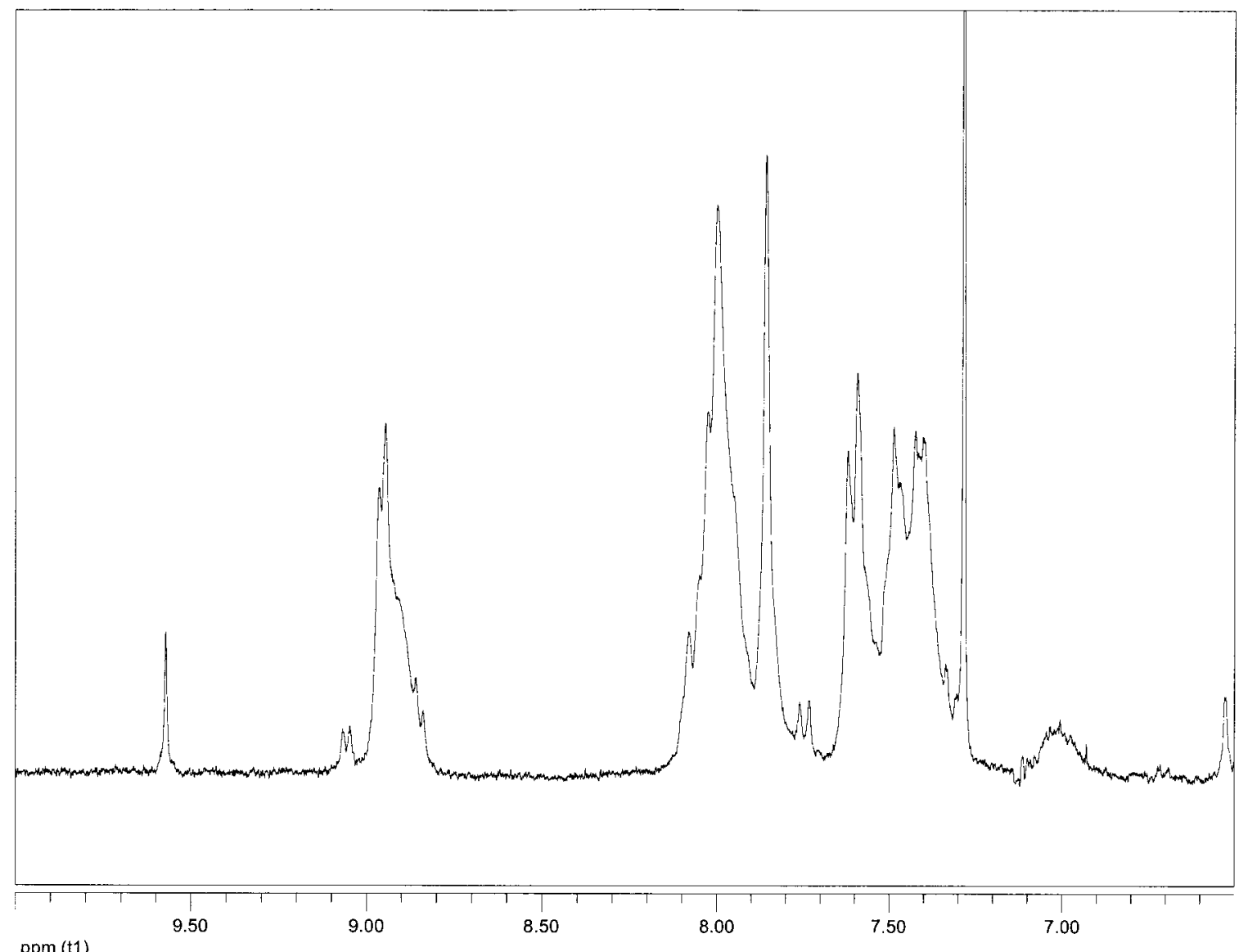

Figure 3.12: Aromatic range of ${ }^{1} \mathrm{H}$ NMR spectrum $(300 \mathrm{MHz})$ of 2 dispersed in Silamine in $\mathrm{CDCl}_{3}$. Note the aldehyde proton chemical shift at $9.58 \mathrm{ppm}$ 


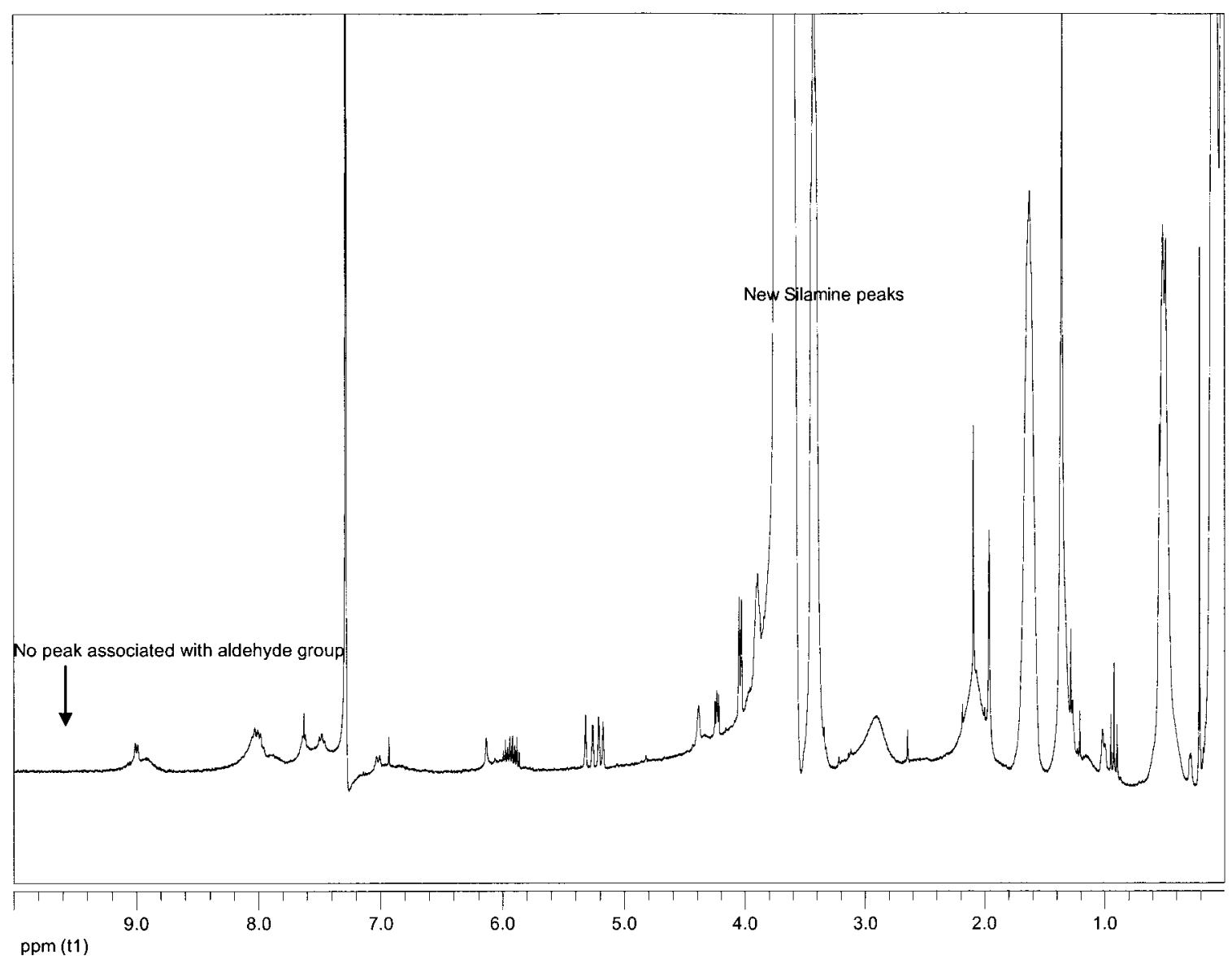

Figure 3.13: ${ }^{1} \mathrm{H}$ NMR spectrum $(300 \mathrm{MHz})$ of 8 in $\mathrm{CDCl}_{3}$. The loss of the aldehyde peak due to the condensation reaction is labeled.

Luminophore attachment in $\mathbf{8}$ was proved by using IR spectroscopy as well. The IR spectrum of the iridium complex 2 in Silamine shows $v(C=O)$ as a strong band at $1686 \mathrm{~cm}^{-1}$, whereas 8 displays no aldehyde $\mathrm{C}=\mathrm{O}$ band (see Figure 3.14). Complete reaction with Silamine gives rise to the disappearance of the $v(C=O)$ in the IR spectrum of 8 , while bands at $1609,1591,1564$, and $1454 \mathrm{~cm}^{-1}$, corresponding to cyclometalating ligands $\mathrm{C}=\mathrm{C}$ and $\mathrm{C}=\mathrm{N}$ vibrational stretches, are retained. 

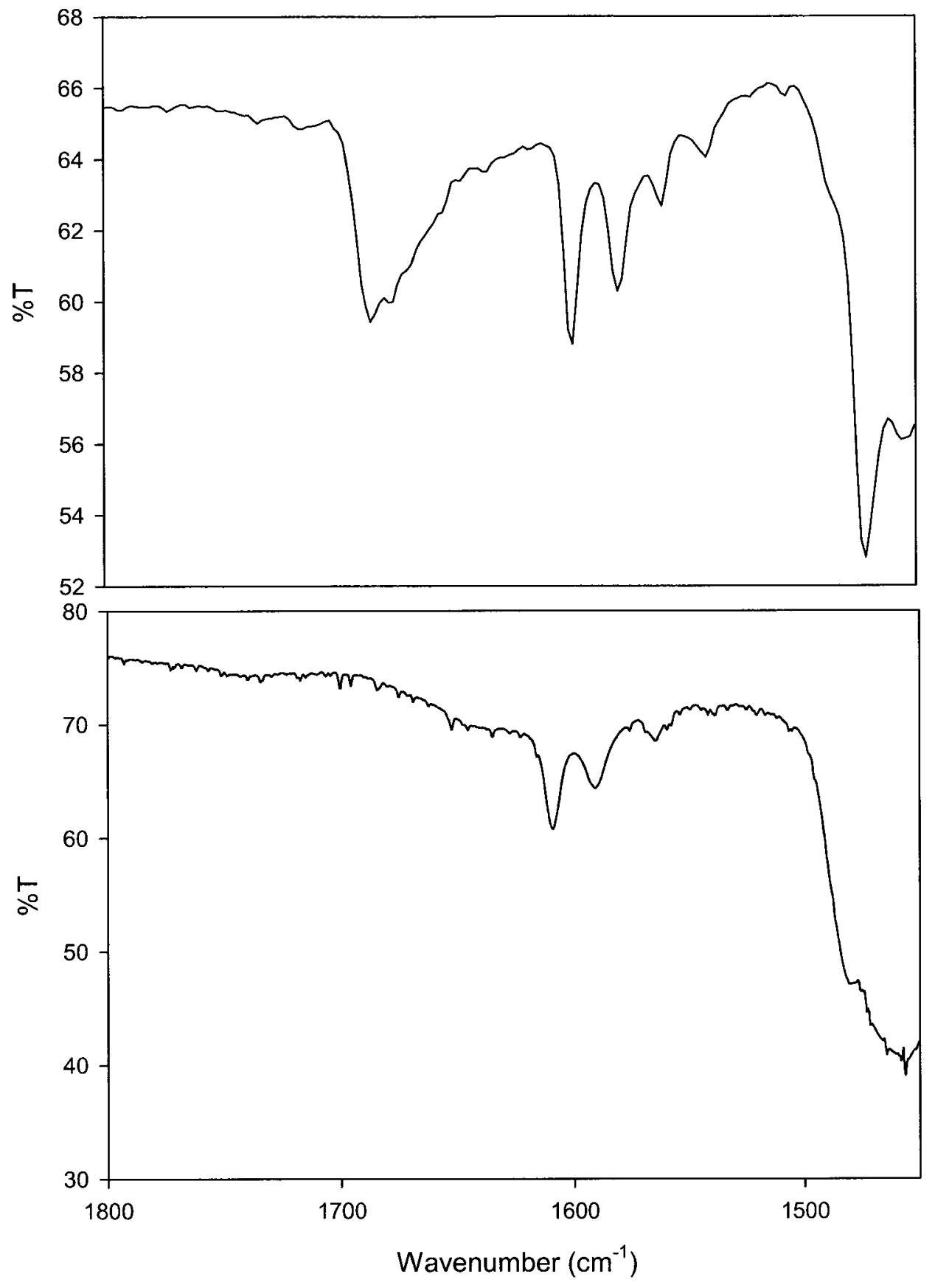

Figure 3.14: Infrared spectra (thin films) of 2 in Silamine (top) and 8 (bottom) displaying no aldehyde $\mathrm{C}=\mathrm{O}$ band in the polymer bound luminophore.

Room temperature absorption and emission spectra data for $\mathbf{8}$ are shown in Figure 3.15 , which are very similar to those of 1 . Three characteristic absorption bands 
are observed; the band at $\lambda=258 \mathrm{~nm}$ is assigned to fppy ligand $\pi-\pi^{*}$ transitions, whereas the band $\lambda=367 \mathrm{~nm}$ is assigned to iridium to fppy metal-to-ligand charge-transfer (MLCT) transitions. ${ }^{67 \mathrm{a}, 72 \mathrm{k}, 84}$
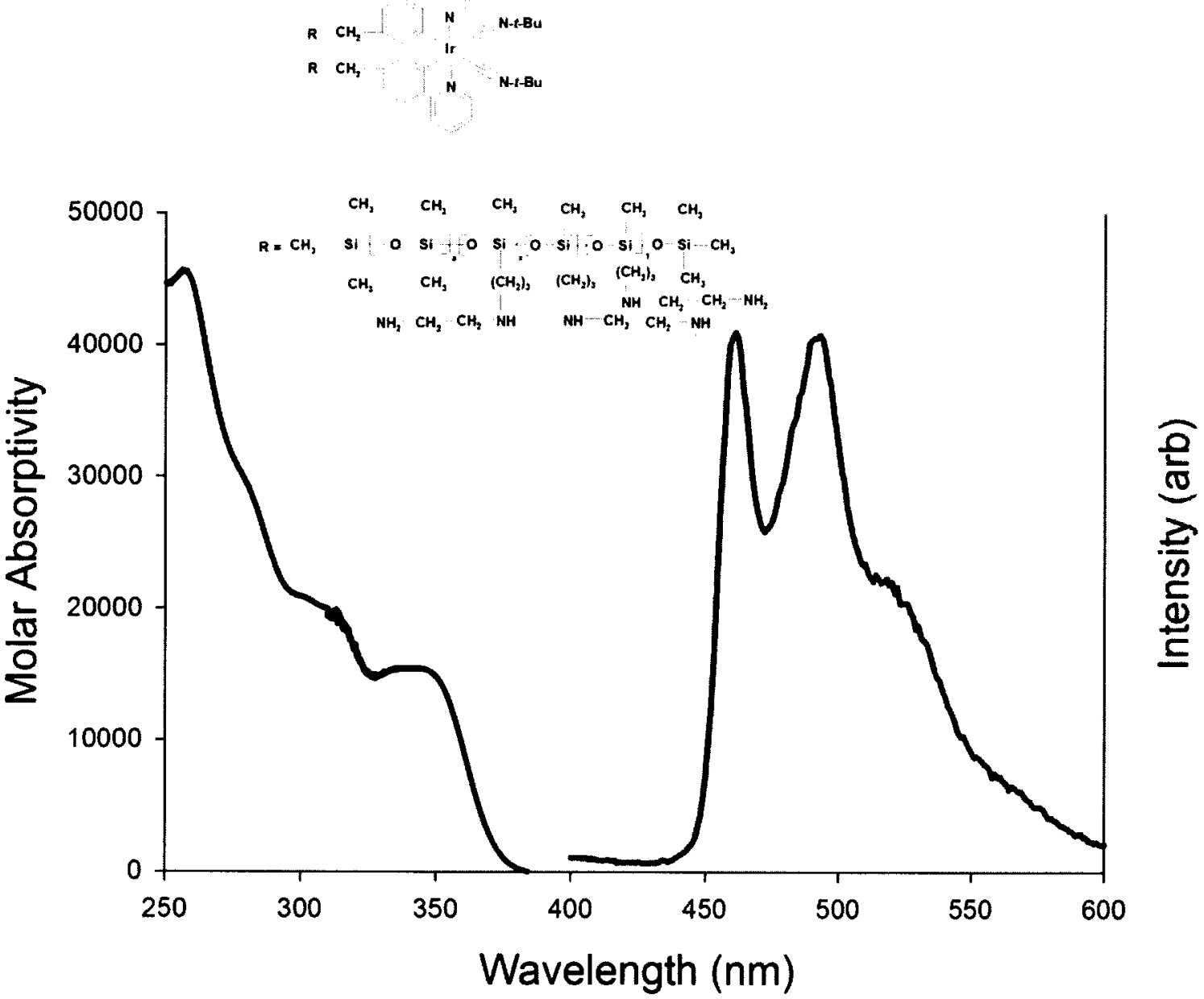

Figure 3.15: Solution electronic absorption and emission spectra of 8 . The absorption spectrum was acquired in $\mathrm{CH}_{3} \mathrm{CN}$ and the emission spectrum was acquired in $\mathrm{CH}_{2} \mathrm{Cl}_{2}$. For 8 , the extinction coefficient at $\lambda=255 \mathrm{~nm}$ was assumed to be identical with that of 1 at $260 \mathrm{~nm}^{75}$ 
The relative intensities of vibronic components of the emission band is shown to be substituent dependent (see Figure 3.16), however any additional analysis was not performed.

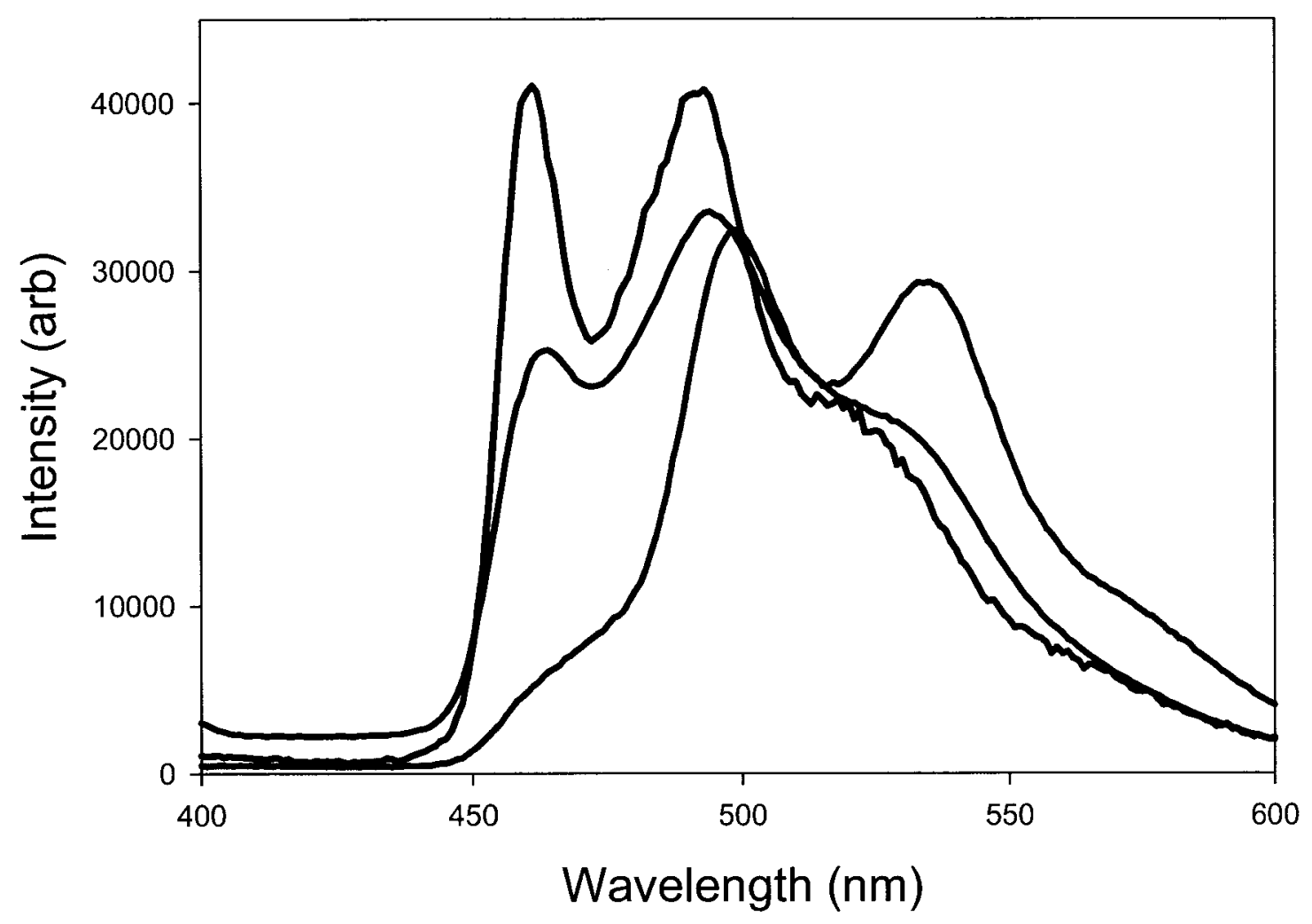

Figure 3.16: Qualitative solution emission spectra of 1 (red), 2 (blue), and 8 (black) in $\mathrm{CH}_{3} \mathrm{CN}$.

Bonding with Silamine slightly perturbs the photophysical properties of the luminophore. The excited MLCT state for $\mathbf{8}$ has a smaller emission quantum efficiency ( 0.23$)$ but a longer lifetime ( $20.6 \mu \mathrm{s})$ compared to those of $\mathbf{2}(\phi=0.35$ and $\tau=17.3 \mu \mathrm{s})$.

Figures 3.17-3.19 give the emission spectra of thin films of luminophores 1, 2, and 8. It appears that in case of $\mathbf{1}$ and $\mathbf{2}$, the emission spectra depend highly on the thin film formulation. Due to low mechanical properties of Silamine D208-EDA, MC was 
blended with PSPs before spraying them into films. The emission band shapes are more or less similar and the shift of the maxima in $\mathbf{2}$ relative to $\mathbf{1}$ and $\mathbf{8}$ has been decreased compared to what was seen in solution emission spectra. There are two kinds of matrix effects on emission spectra. Effects arising from the refractive index and dielectric constant are general effects which are essentially defined by the Lippert equation. ${ }^{88}$ Effects resulting from specific chemical interactions between the luminophore and matrix are specific matrix effects. ${ }^{88}$ Also, the shift can be attributed to aggregate formation due to a higher solubility of the luminophore in MC. As is displayed in Figure 3.18, it is proposed that once $\mathrm{MC}$ proportion in the film formulation is increased, the luminophore distributes in MC. Increasing MC concentration in the film, sharpens the low-energy shoulder of the emission spectrum. The emission spectra of thin films of 1 in $9: 1$ Silamine: MC, 1:2 Silamine: MC, and 1:5 Silamine: MC matrices also exhibit this effect (Figure 3.17). As illustrated in Figure 3.19, the thin film emission spectra of $\mathbf{8}$ show small changes by increasing MC proportion. 


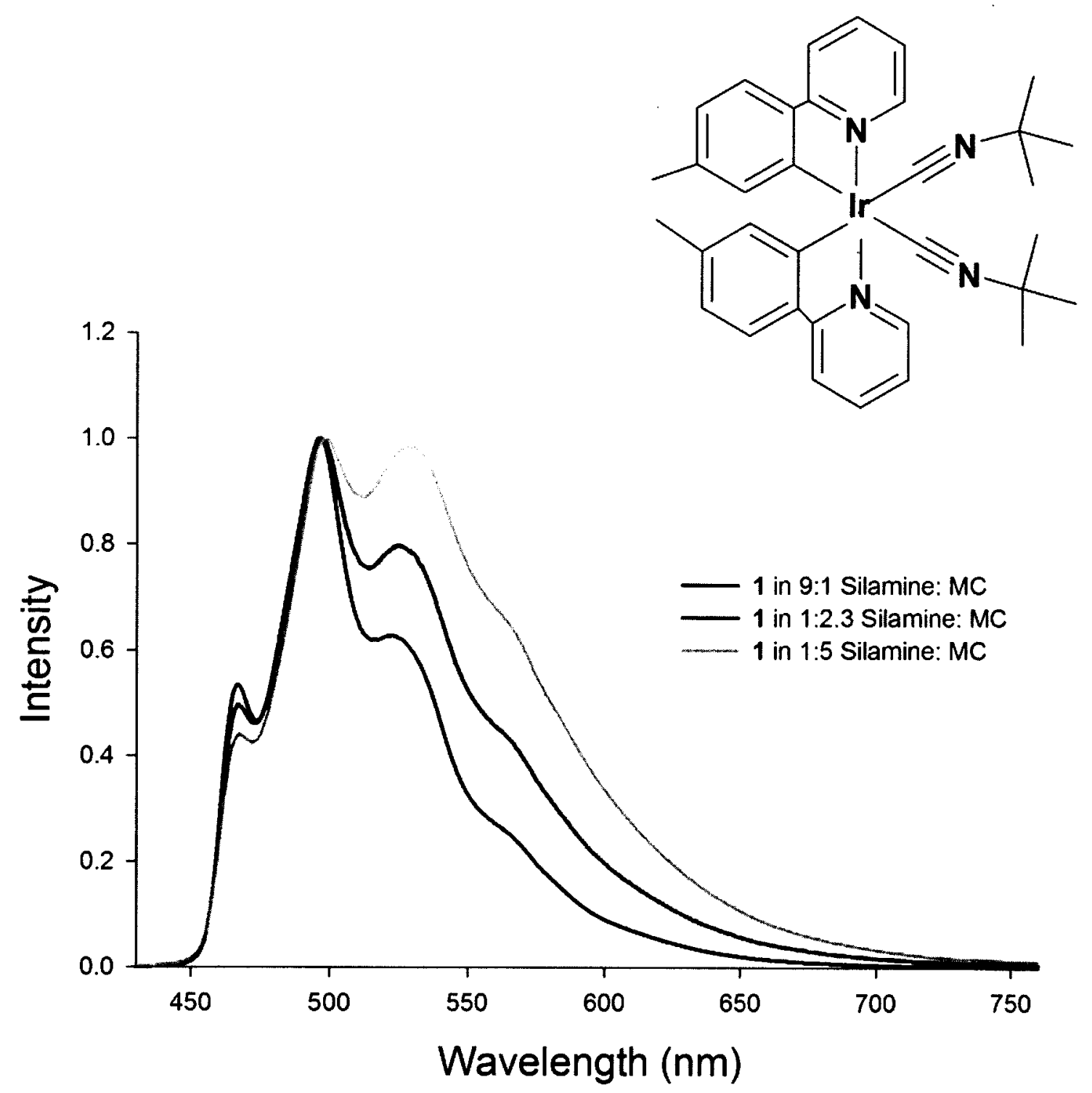

Figure 3.17: Effect of matrix on thin film luminescence of 1. Matrices compositions are: 9:1 Silamine: MC (black), 1:2 Silamine: MC (red), and 1:5 Silamine: MC (green). For each formulation, the spectrum at $14.7 \mathrm{psi}$ is shown, which is normalized to its maximum emission intensity. 


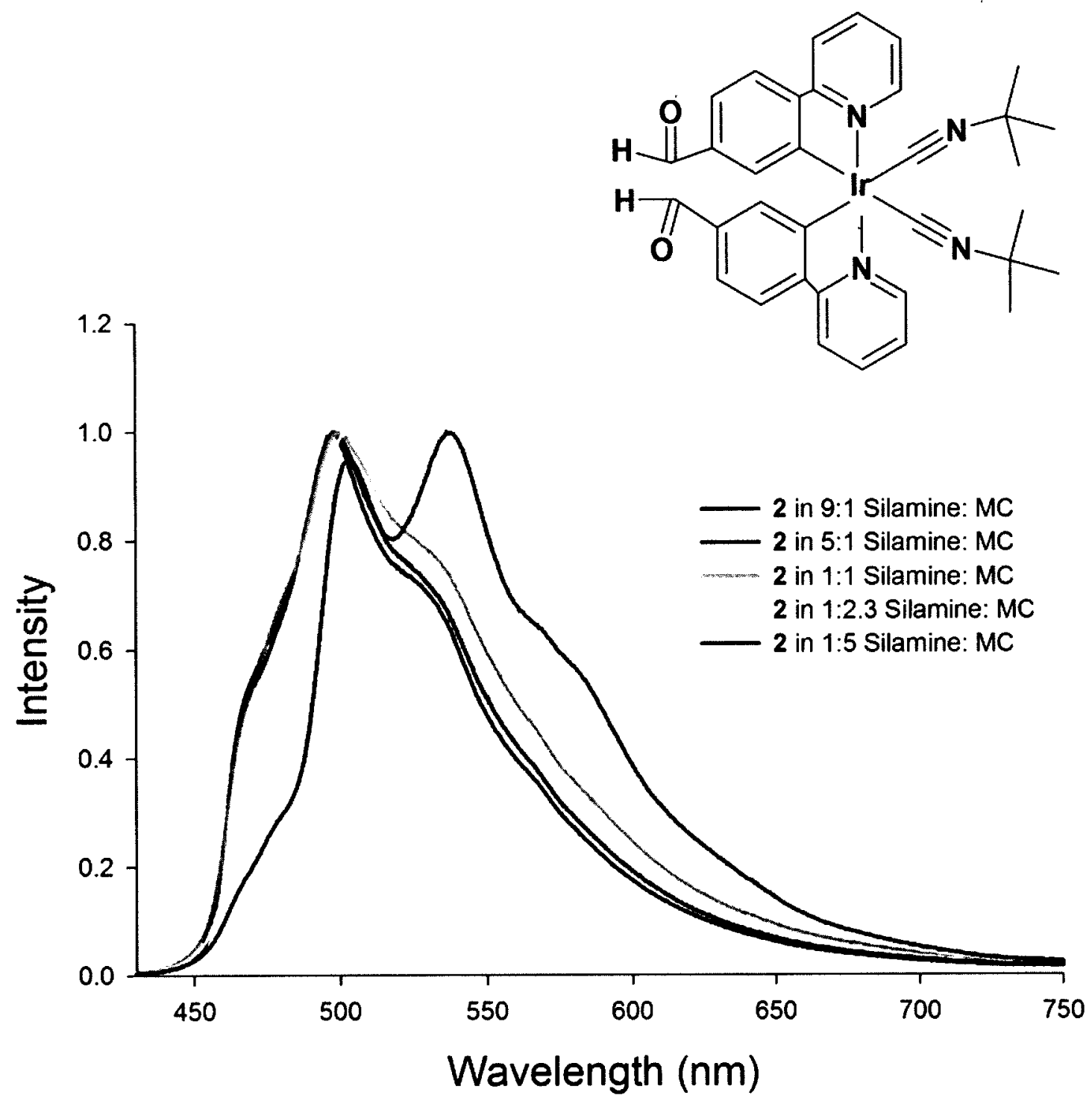

Figure 3.18: Effect of matrix on thin film luminescence of 2. Matrices compositions are: 9:1 Silamine: MC (black), 5:1 Silamine: MC (red), 1:1 Silamine: MC (green), 1:2 Silamine: MC (yellow), and 1:5 Silamine: MC (blue). For each formulation, the spectrum at 14.7 psi is shown, which is normalized to its maximum emission intensity. 

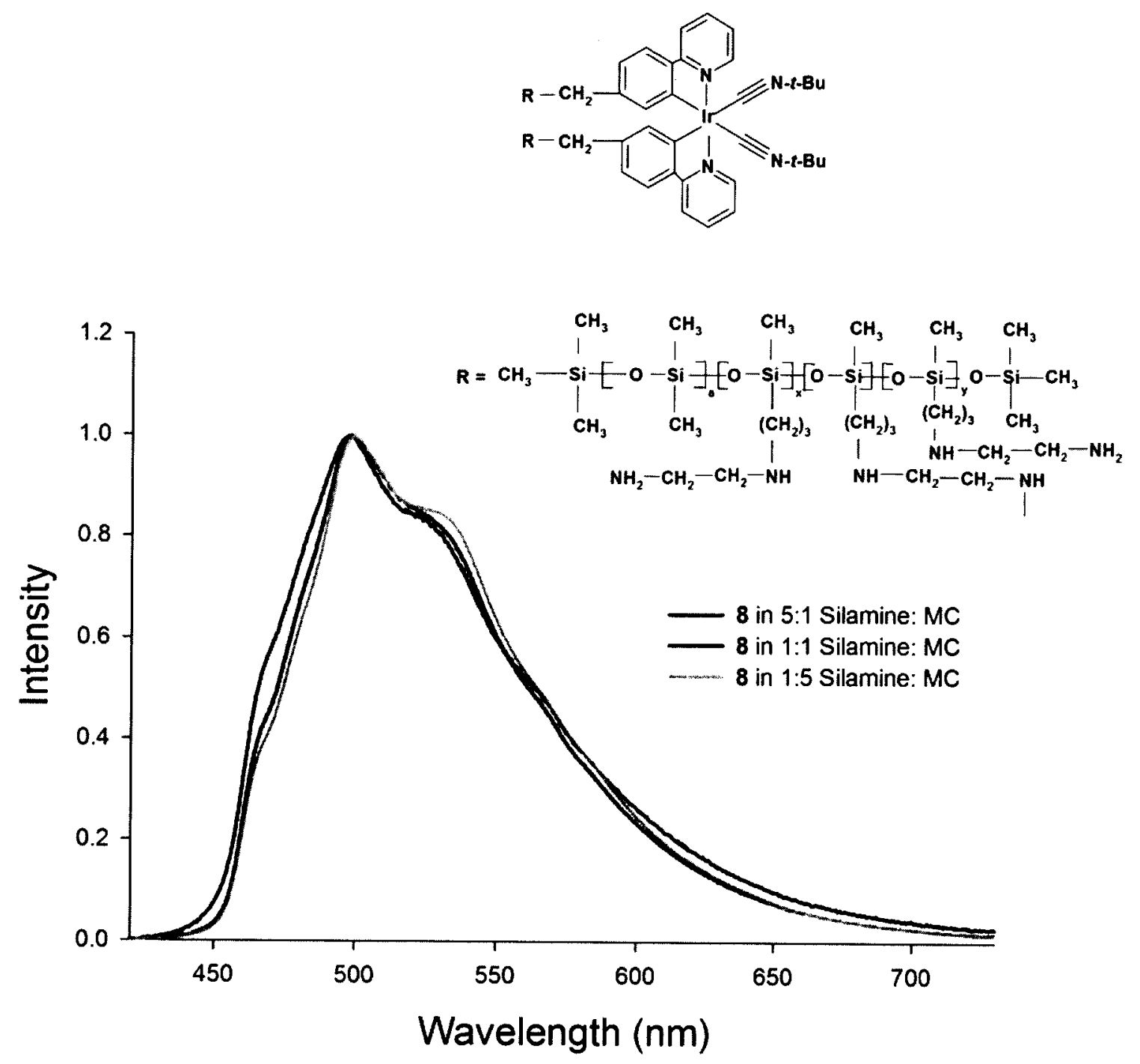

Figure 3.19: Effect of matrix on thin film luminescence of 8. Matrices compositions are: 5:1 Silamine: MC (black), 1:1 Silamine: MC (red), and 1:5 Silamine: MC (green). For each formulation, the spectrum at $14.7 \mathrm{psi}$ is shown, which is normalized to its maximum emission intensity. 


\subsection{Synthesis of Water-Soluble Oxygen Sensor 11}

\subsubsection{Copper-Catalyzed Oxidation of Poly(ethylene glycol) Methyl Ether (PEGME) to PEGME-Aldehyde}

In order to oxidize PEGME into PEGME-Aldehyde a practical copper-catalyzed procedure under mild conditions, invented by Markó et al. ${ }^{64}$ was followed. Air was used in the catalytic method in which water is the only generated by-product. To increase the lifetime of the catalyst and the reaction rate, diethylazodicarboxylate was utilized. Insoluble $\mathrm{K}_{2} \mathrm{CO}_{3}$ adsorbs the heterogeneous catalyst. It is suggested that initially an alkoxide-bound azo-copper complex is produced which is transformed to a carbonylbound hydrazino-copper complex by a hydrogen-transfer reaction (Scheme 3.4). A binuclear copper (II) peroxide is then formed due to reaction with oxygen which is converted to a hydroxyl $\mathrm{Cu}(\mathrm{I})$ complex after homolytic cleavage and hydrogen atom abstraction from the hydrazine. The catalyst is restored by exchange between PEGME and the $\mathrm{OH}$ ligand, releasing a water molecule. 


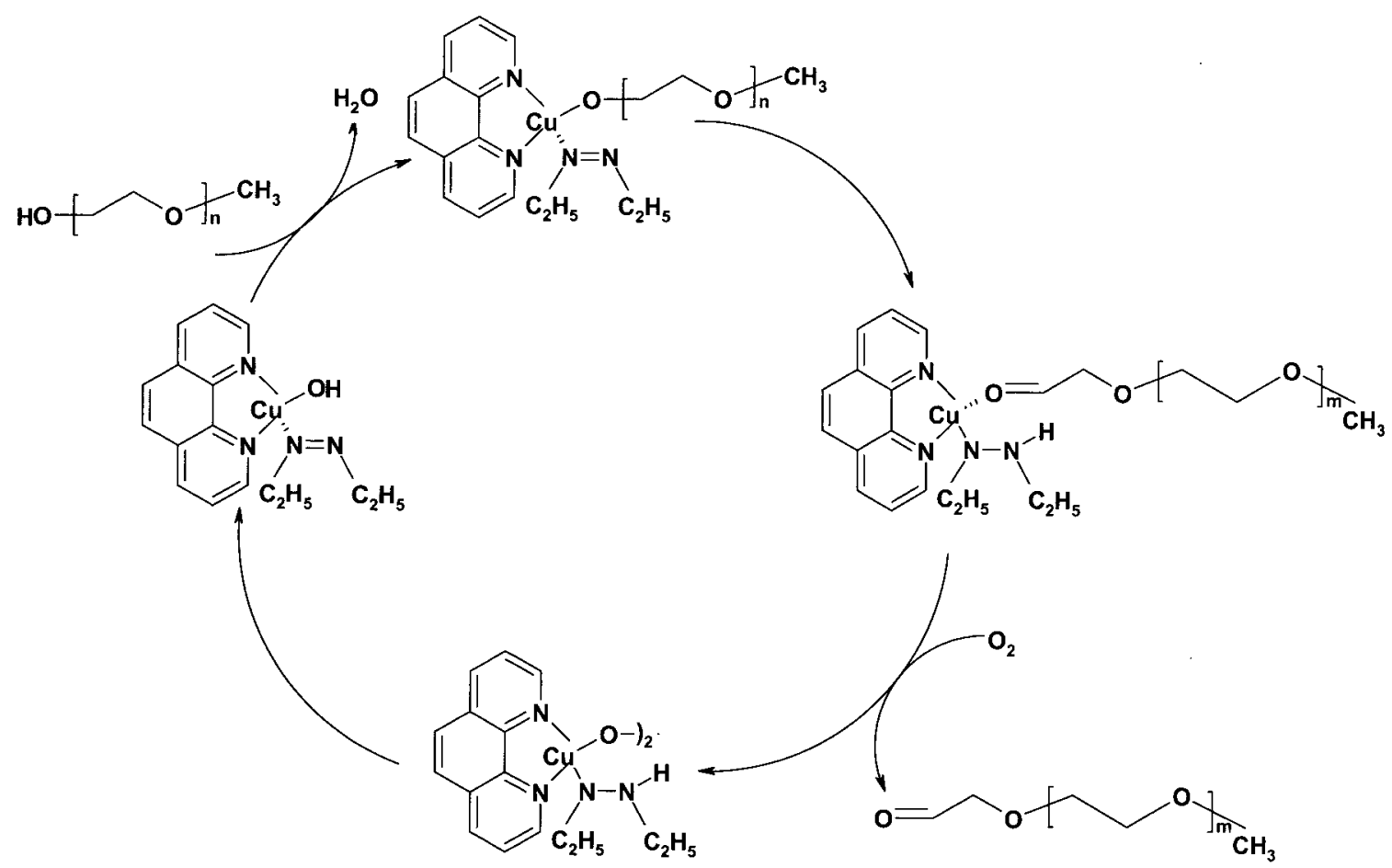

Scheme 3.4: Mechanism of copper-catalyzed oxidation of PEGME to PEGMEAldehyde, $\mathrm{m}=\mathrm{n}-1$

In PEGME-Aldehyde infrared spectrum, the $v(\mathrm{C}=\mathrm{O})$ aldehyde is seen at $1719 \mathrm{~cm}^{-1}$ (Figure 3.20), indicating the successful oxidation. 


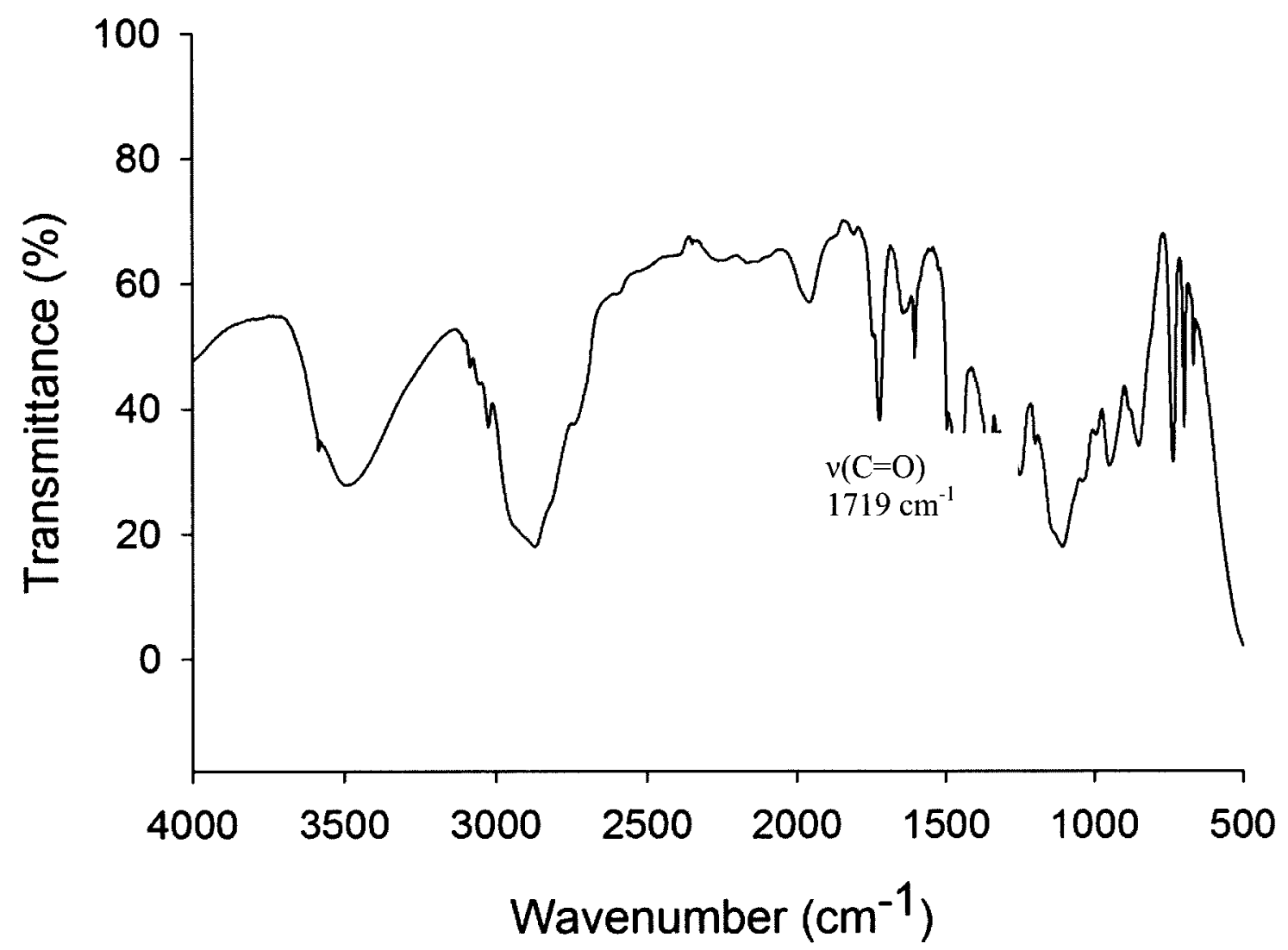

Figure 3.20: Infrared spectrum (thin film) of PEGME-Aldehyde with $v(C=O)$ at 1719 $\mathrm{cm}^{-1}$.

\subsubsection{Synthesis of Aminomethyl Phenylpyridine}

Aminomethyl phenylpyridine (amppy) was synthesized by a Suzuki coupling reaction as shown in Scheme 3.5. 5-aminomethyl-2-chloro pyridine was first protected as the acetamide then the palladium mediated coupling proceeded efficiently with subsequent cleavage of the acetamide using $\mathrm{HCl}^{89}$

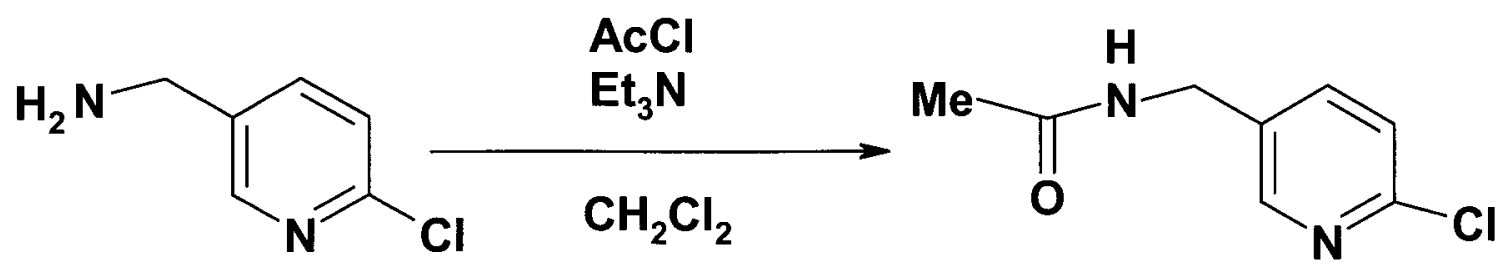




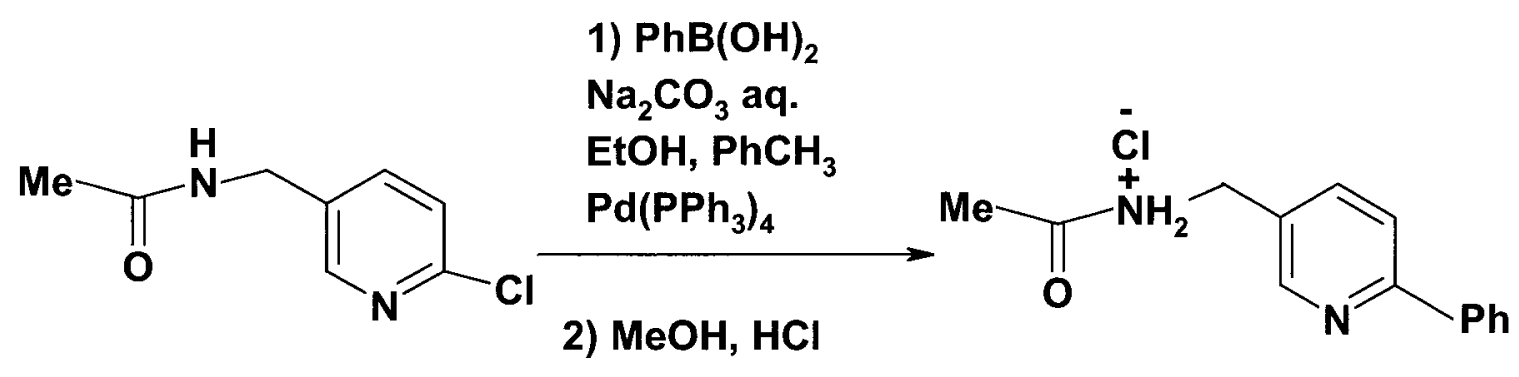<smiles>CC(=O)[NH2+]Cc1ccc(-c2ccccc2)nc1</smiles>

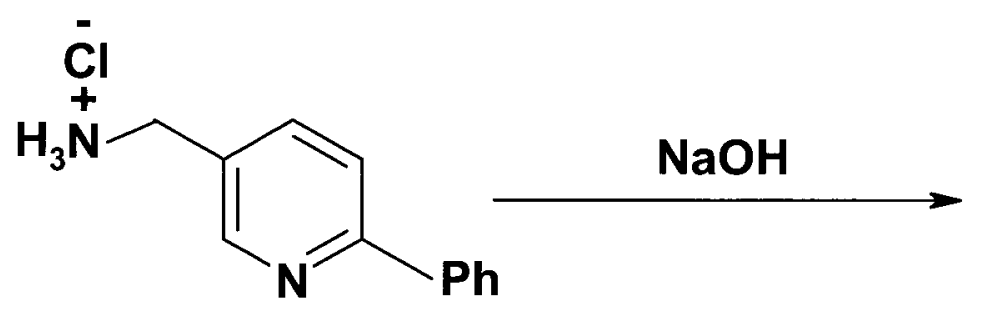

Scheme 3.5: Preparation of amppy by Suzuki coupling reaction.

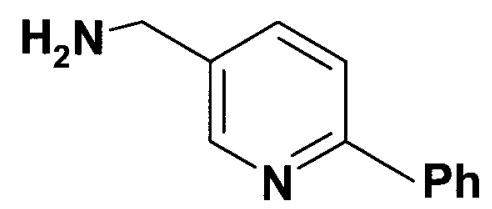

amppy

Oxidative addition of the acetadimomethyl pyridine chloride to the palladium $(0)$ complex generates a palladium (II) intermediate. This then undergoes a transmetallation with phenylboronic acid, expelling the product by reductive elimination and regenerating the palladium (0) catalyst. Sodium carbonate is the base used in order to accelerate the transmetallation step. 

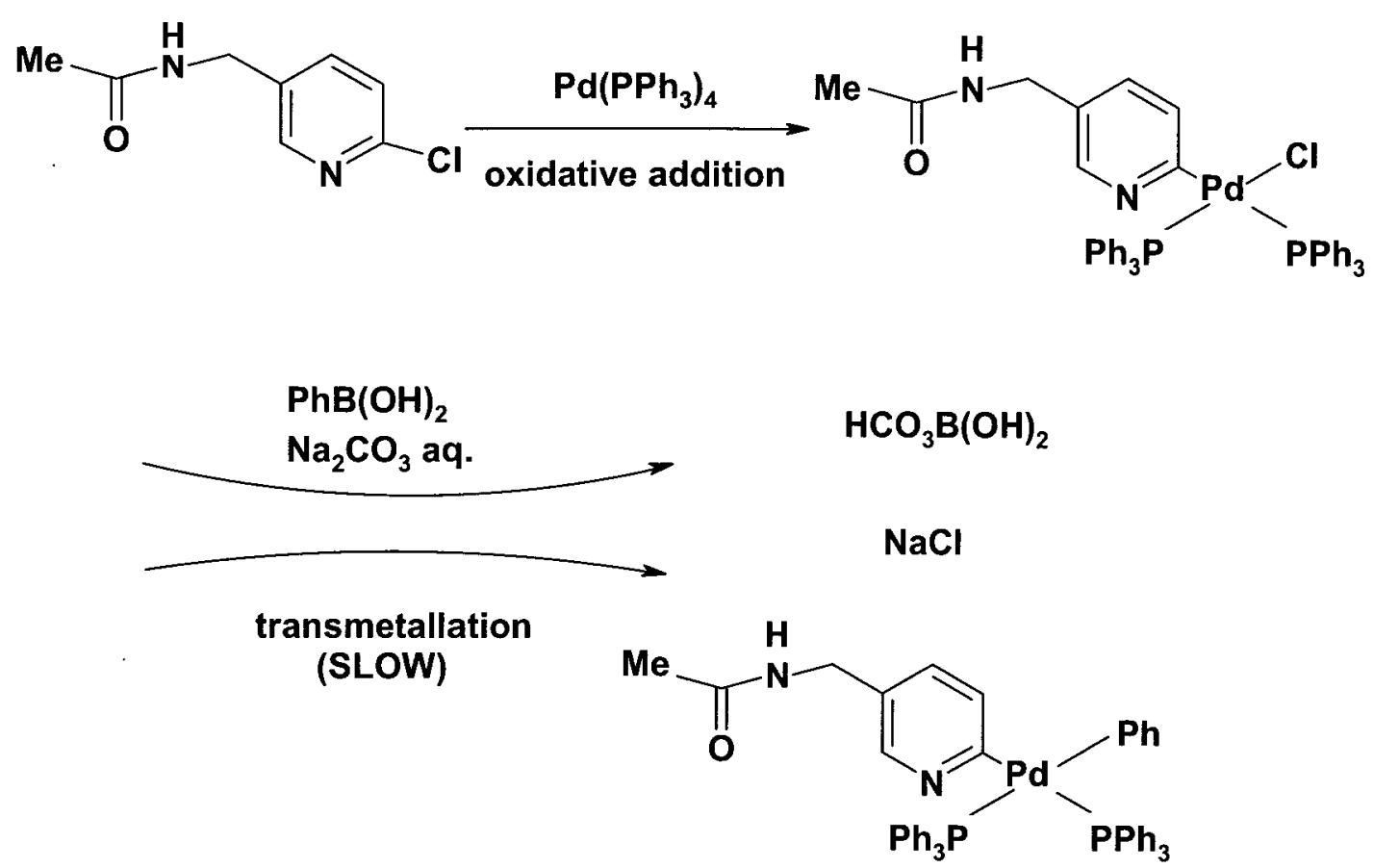

Scheme 3.6: Intermediates in amppy preparation by Suzuki coupling reaction.

${ }^{1} \mathrm{H}$ NMR, IR, and mass spectrometry confirmed successful synthesis of amppy. Chemical shifts and integration of the ${ }^{1} \mathrm{H}$ NMR spectrum of amppy (Figure 3.21 ) were appropriate for the phenyl and pyridyl protons. The expected singlet methylene and broad amine protons peaks were clearly visible. The infrared spectrum of the ligand showed representative absorbances of the $v(\mathrm{~N}-\mathrm{H})_{\text {amine }}$ at 3437,3269 , and $1602 \mathrm{~cm}^{-1}$. Mass spectrum confirmed the molecular weight of amppy with a molecular ion peak at $184 \mathrm{~m} / \mathrm{z}$. 


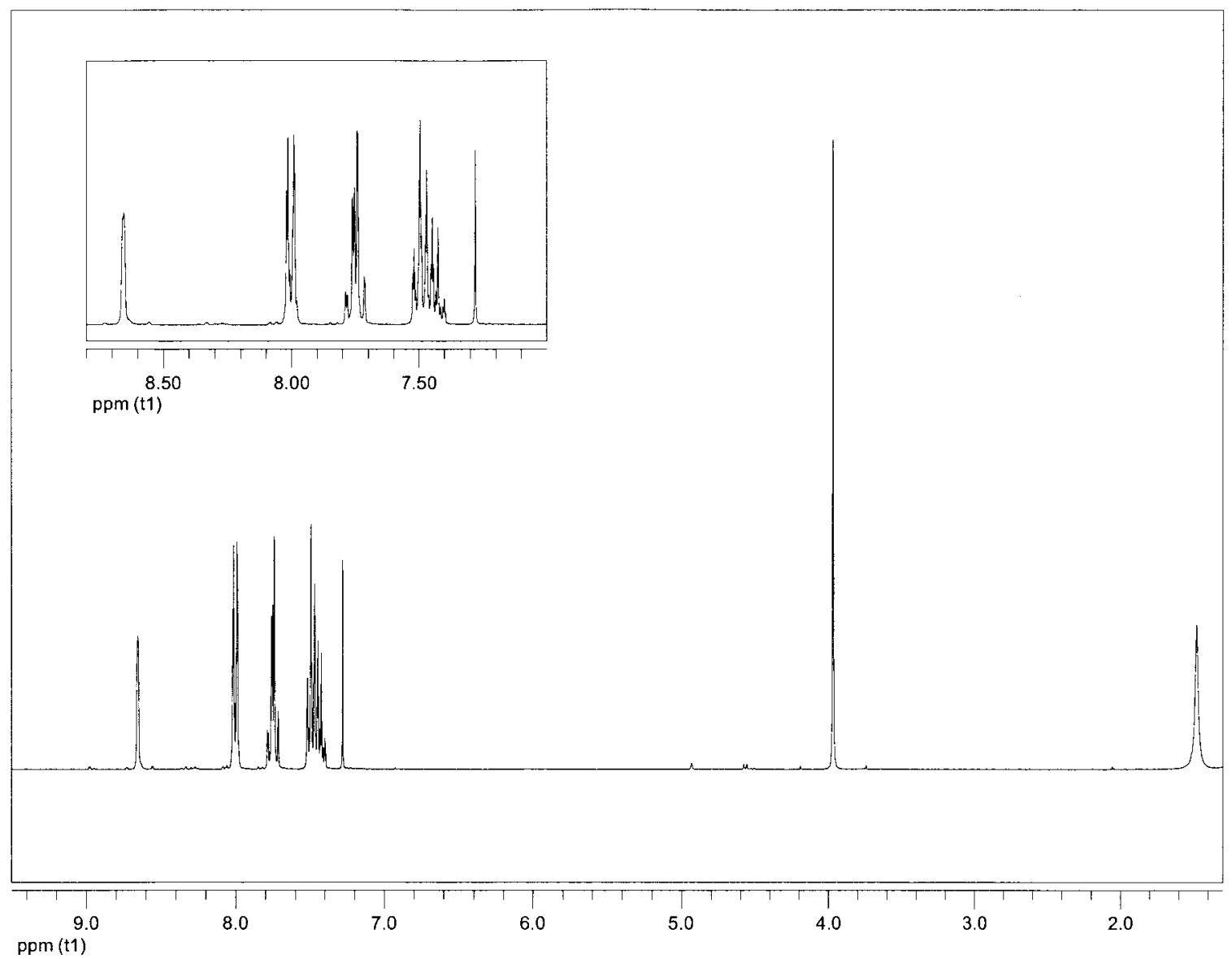

Figure 3.21: ${ }^{1} \mathrm{H}$ NMR spectrum (300 MHz) of amppy in $\mathrm{CDCl}_{3}$. 


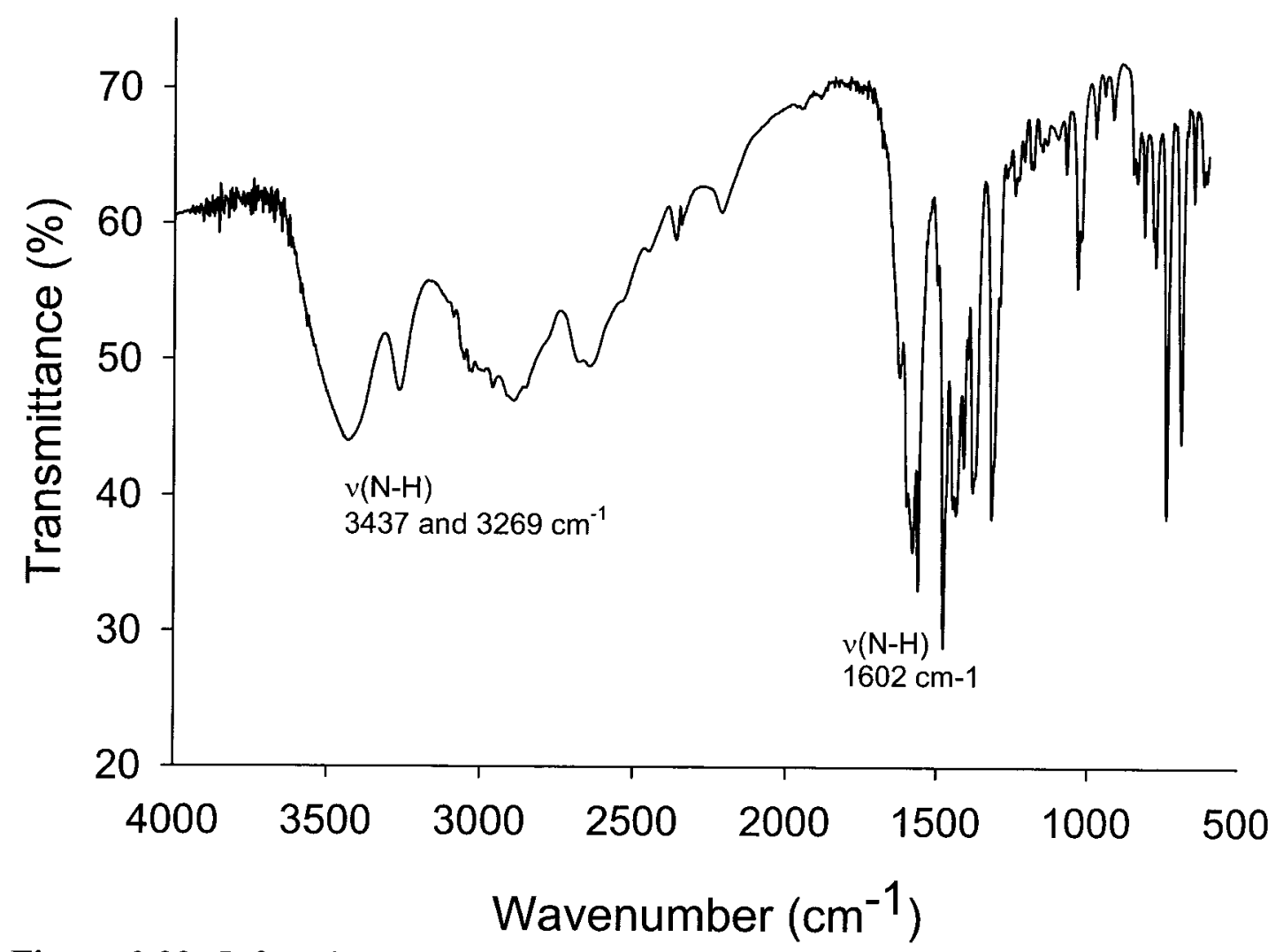

Figure 3.22: Infrared spectrum (KBr pellet) of amppy with $v(\mathrm{~N}-\mathrm{H})$ at 3437,3269 , and $1602 \mathrm{~cm}^{-1}$. 


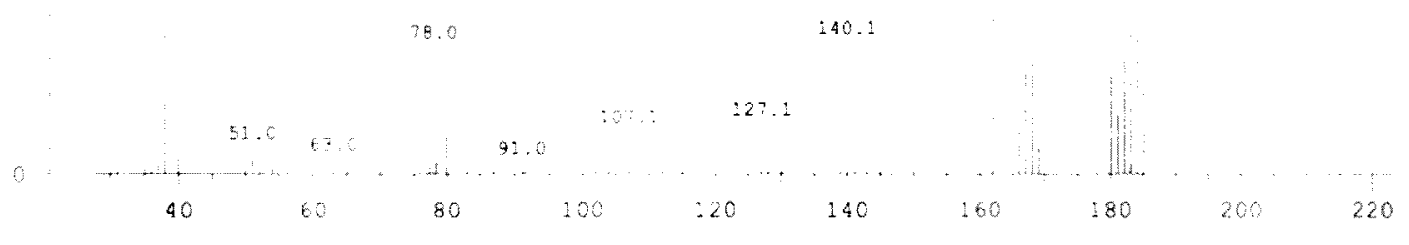

Figure 3.23: Electron-Impact mass spectrum of amppy with the molecular ion peak at $181 \mathrm{~m} / \mathrm{z}$.

\subsubsection{Coupling of Amppy to PEGME-Aldehyde and Spectroscopic Characterization}

In order to study amppy as a functionalized ligand, it was bound to PEGMEAldehyde as illustrated in Scheme 3.7. An imine is yielded by the nucleophilic addition of the amine to the aldehyde group followed by an elimination of water.<smiles>C[14CH2]OCCOCC=NCc1ccc(-c2ccccc2)nc1</smiles>

Scheme 3.7: Coupling reaction of amppy with PEGME-Aldehyde 
The aromatic region of the ${ }^{1} \mathrm{H}$ NMR spectrum of PEGME-Aldehyde and PEGME-attached amppy is illustrated in Figures 3.24-25. Whereas PEGME-Aldehyde shows an aldehyde peak at $9.6 \mathrm{ppm}$, PEGME-attached amppy shows peaks as follows: $8.69(1 \mathrm{H}, \mathrm{d}, 1.8 \mathrm{~Hz}), 8.27(1 \mathrm{H}, \mathrm{dd}, 2.1,8.4 \mathrm{~Hz}), 8.07(1 \mathrm{H}, \mathrm{d}, 8.4 \mathrm{~Hz}), 7.85$ (2H, dd, 3.0 , $6.7 \mathrm{~Hz}), 7.56(3 \mathrm{H}, \mathrm{m})$.

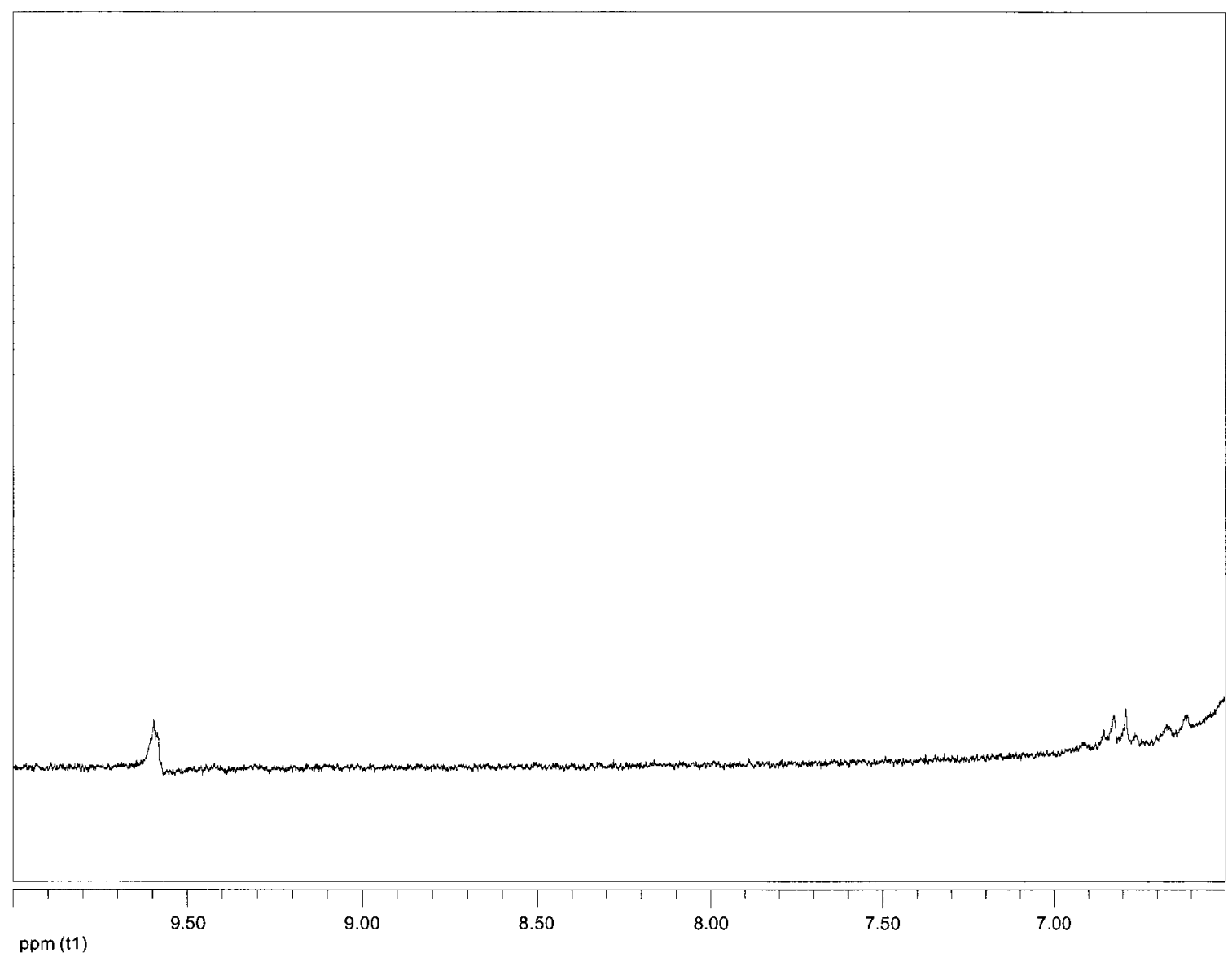

Figure 3.24: Aromatic region of the ${ }^{1} \mathrm{H}$ NMR spectrum (300 MHz) of PEGMEAldehyde in $\mathrm{D}_{2} \mathrm{O}$ showing the aldehyde chemical shift at $9.6 \mathrm{ppm}$. 


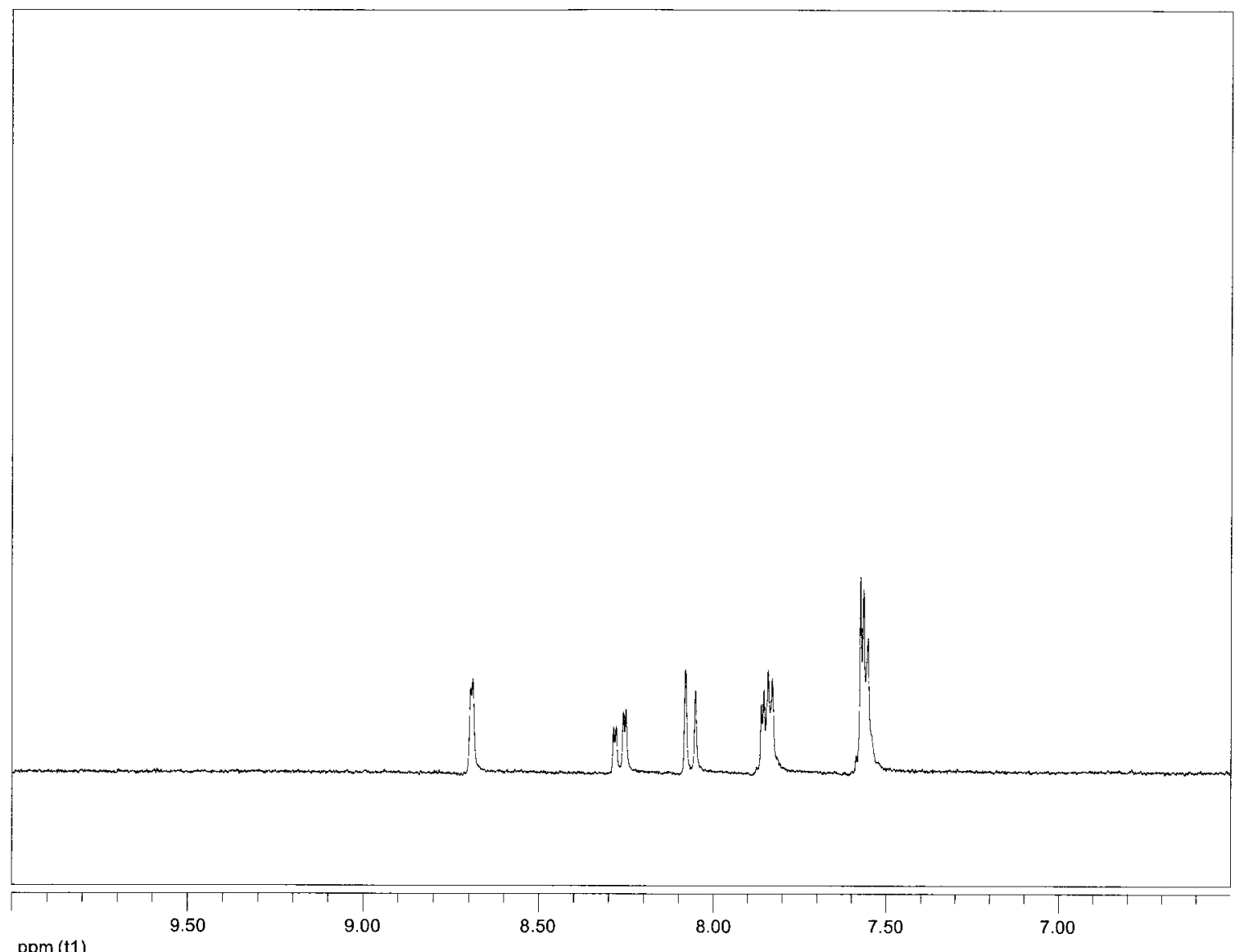

Figure 3.25: Aromatic region of the ${ }^{1} \mathrm{H}$ NMR spectrum (300 MHz) of PEGME-attached amppy in $\mathrm{D}_{2} \mathrm{O}$ showing amppy chemical shifts.

The calculated concentration of amppy in PEGME-attached amppy (see section 3.4.2) revealed quantitative attachment of amppy to PEGME-Aldehyde.

\subsubsection{PEGME Attached Ir-Amppy Dimer Synthesis and Spectroscopic Characterization}

PEGME-amppy is metallated easily using a method similar to Watts and Nonoyama ${ }^{74}$ reactions. It reacts with iridium trichloride to give the PEGME attached Ir- 
amppy dimer characterized by spectroscopic methods. This iridium dimer is an important reagent polymer that could lead to an entire family of water-based PSP formulations.

Proton NMR data for the PEGME attached amppy dimer of $\operatorname{Ir}(\mathrm{III})$ in $\mathrm{D}_{2} \mathrm{O}$ is shown in Figure 3.26. The non-quantitative aromatic region peaks suggests that the environment is not homogeneous.

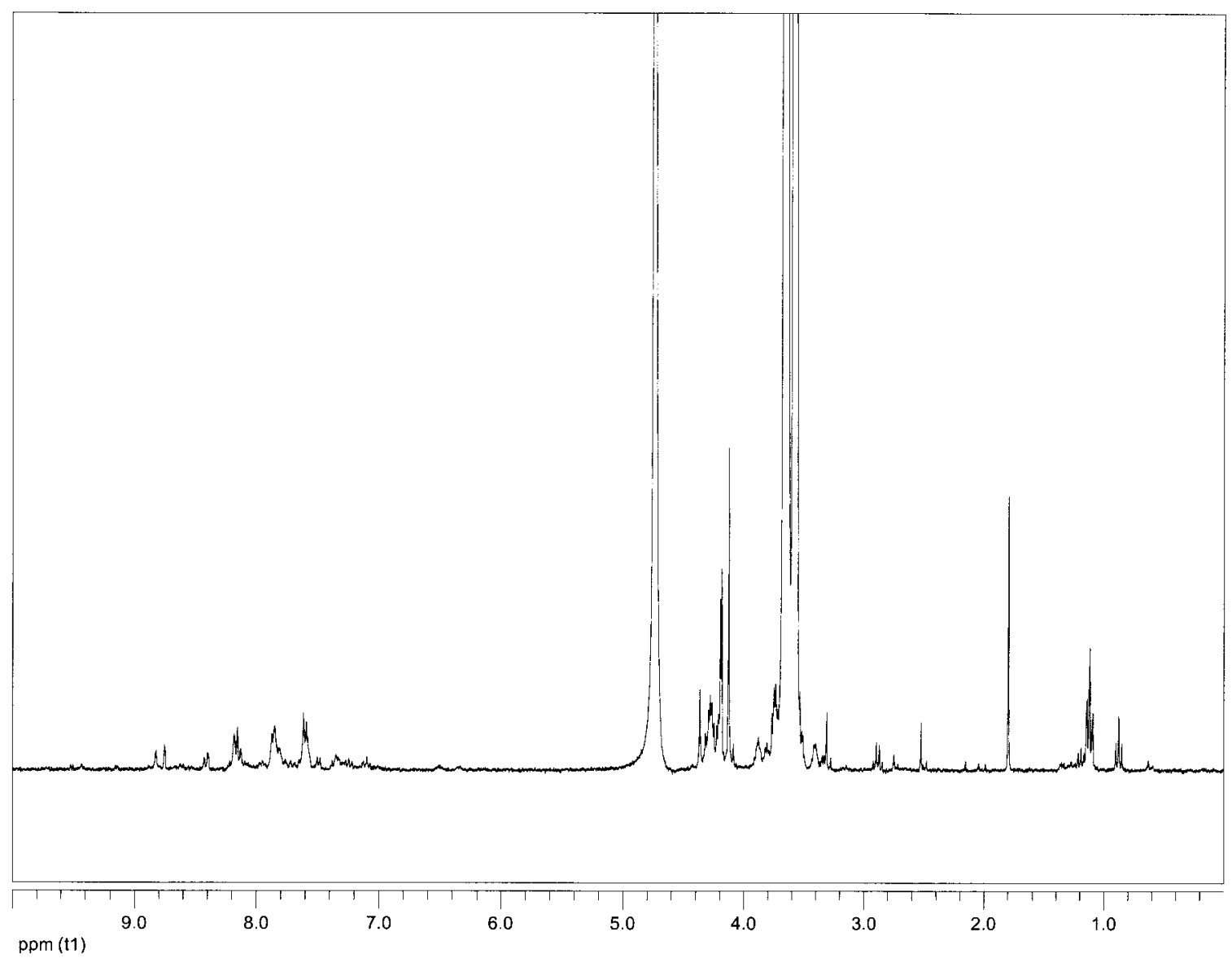

Figure 3.26: ${ }^{1} \mathrm{H}$ NMR spectrum $(300 \mathrm{MHz})$ of PEGME-attached Ir-amppy dimer in $\mathrm{D}_{2} \mathrm{O}$.

The absorption and luminescence spectra of PEGME attached Ir-amppy dimer are shown in Figure 3.27. The intense ultraviolet absorption band observed at $279 \mathrm{~nm}$ is 
assigned to an intraligand transition. Several transitions between 300 and $500 \mathrm{~nm}$ can be assigned to MLCT transitions. It displays a relatively intense emission at $460 \mathrm{~nm}$.

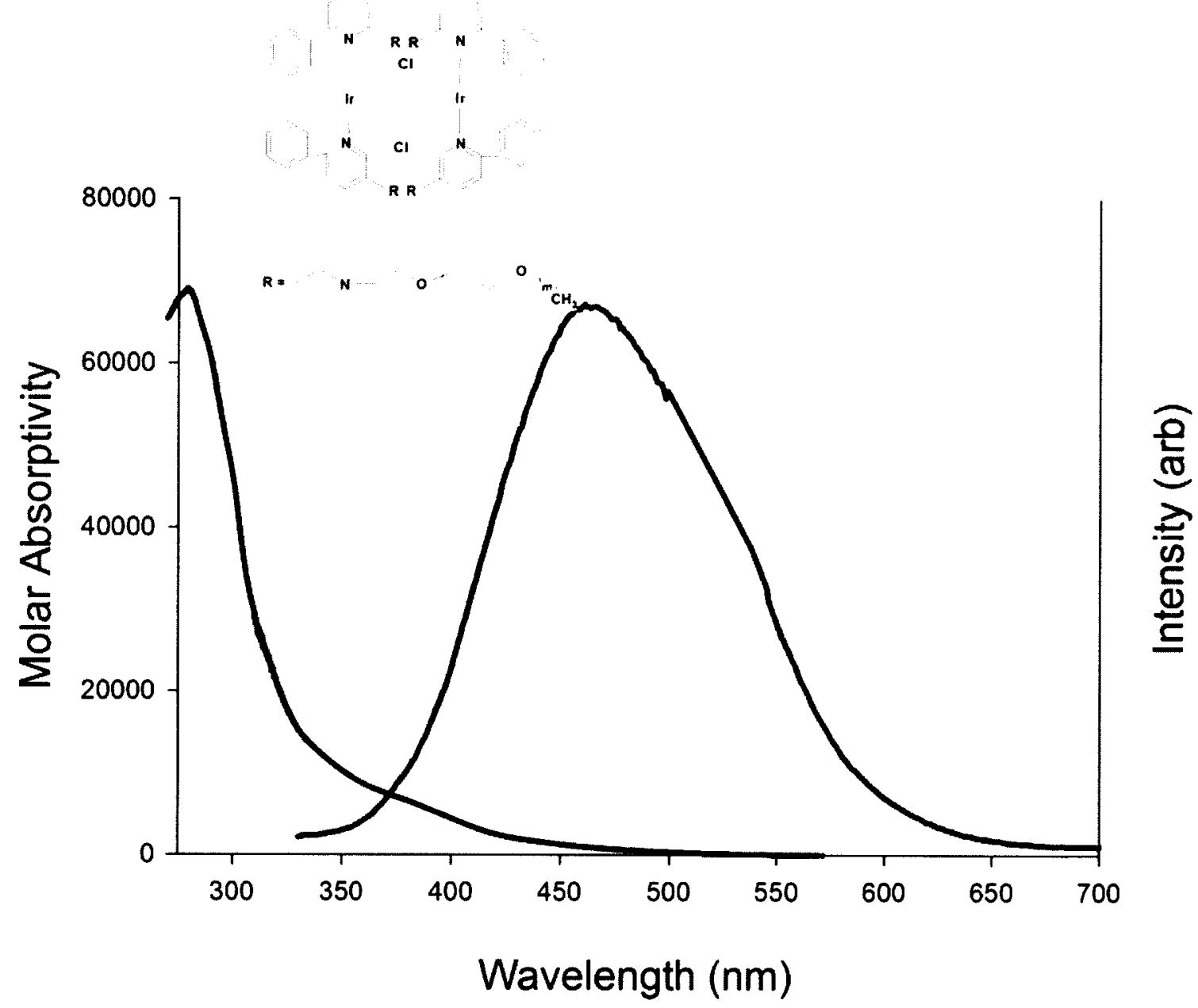

Figure 3.27: Solution electronic absorption and emission spectra of PEGME attached Iramppy dimer. Both spectra were acquired in $\mathrm{CH}_{2} \mathrm{Cl}_{2}$. The extinction coefficient was assumed to be identical with that of $\left[\operatorname{Ir}(\mathrm{ppy})_{2} \mathrm{Cl}\right]_{2}$. 


\subsubsection{Synthesis of Water-Soluble Oxygen Sensor 11 - PEGME Attached $\left[\operatorname{Ir}(\operatorname{amppy})_{2}(\mathrm{t}-\mathrm{Bu}-\mathrm{iCN})_{2}\right]\left[\mathrm{CF}_{3} \mathrm{SO}_{3}\right]$ - Synthesis and Spectroscopic Characterization}

The PEGME attached $\left[\operatorname{Ir}(\operatorname{amppy})_{2}(\mathrm{t}-\mathrm{Bu}-\mathrm{iCN})_{2}\right]\left[\mathrm{CF}_{3} \mathrm{SO}_{3}\right]$ complex was prepared from PEGME attached amppy dimer by chloride abstraction with AgOTf followed by complexation with CN-t-Bu (shown in Scheme 3.8).
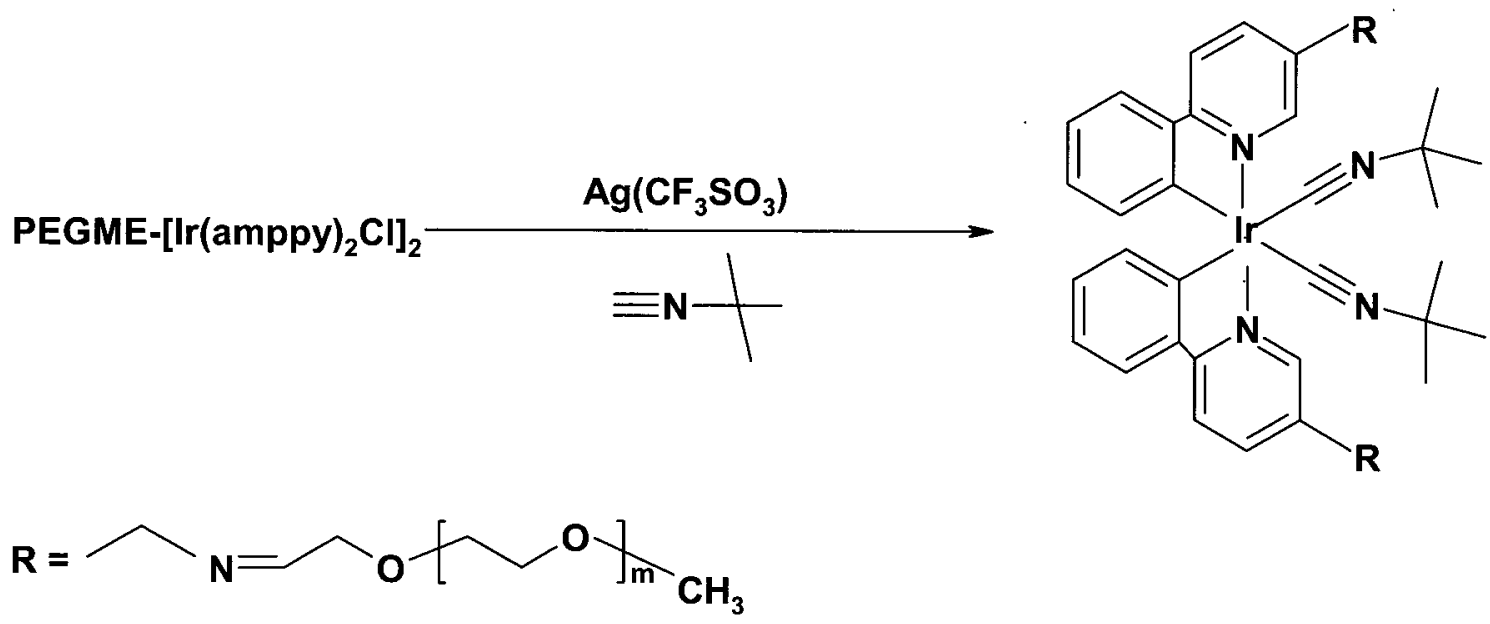

Scheme 3.8: Synthesis of 11

The room temperature absorption and emission spectra displayed by $\mathbf{1 1}$ is shown in Figure 3.28. The attachment of the complex to PEGME results in little change in the photophysical properties of the $\left[\operatorname{Ir}(\operatorname{amppy})_{2}(\mathrm{t}-\mathrm{Bu}-\mathrm{iCN})_{2}\right]\left[\mathrm{CF}_{3} \mathrm{SO}_{3}\right]$ complex relative to 8 . The three typical absorption bands are found as amppy ligand $\pi-\pi^{*}$ transition band at $\lambda=$ $253 \mathrm{~nm}$, and iridium to amppy metal-to-ligand charge-transfer (MLCT) transition band at $\lambda=371 \mathrm{~nm}$. 


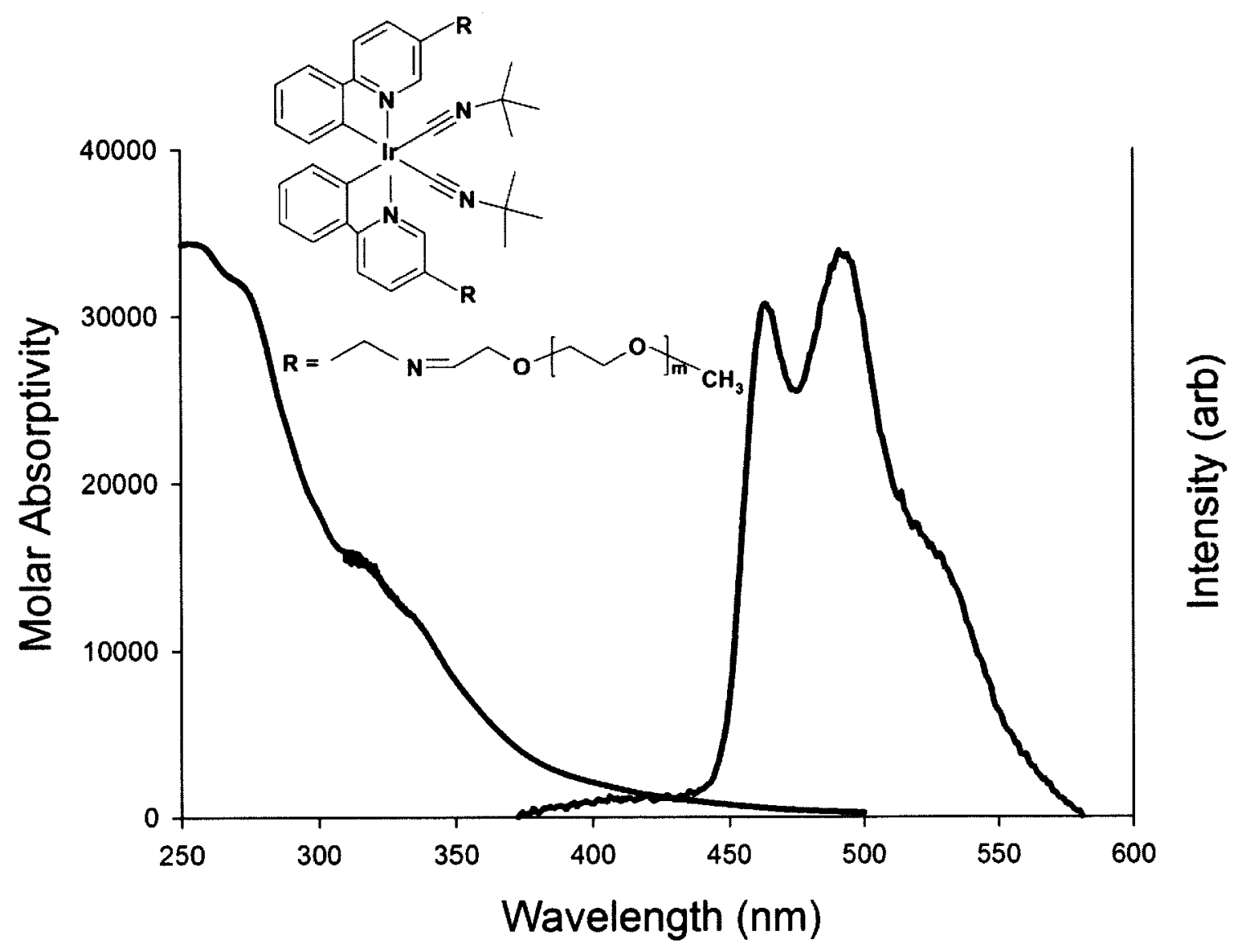

Figure 3.28: Solution electronic absorption and emission spectra of 11. The absorption spectrum was acquired in $\mathrm{CH}_{3} \mathrm{CN}$ and the emission spectrum was acquired in $\mathrm{CH}_{2} \mathrm{Cl}_{2}$. For 11, the extinction coefficient at $\lambda=253 \mathrm{~nm}$ was assumed to be identical with that of 1 at $260 \mathrm{~nm}^{75}$

\subsection{Characterization of Pressure Sensitive Paint (PSP) Films of 2, 8, and 11}

Among PSPs incorporating 2, 8, and 11, the completely water-soluble film formulations are those of $\mathbf{8}$ and 11. To compensate for the poor mechanical properties of Silamine D208-EDA, microcrystalline cellulose (MC) was included in the formulations. The pressure-dependent film emission spectra of $\mathbf{2 , 8}$, and 11 in different matrices were studied and are represented in Figures 3.29-3.31. Films of 8 displayed an intense yellowwhite luminescence with UV irradiation under low pressure conditions, while films of 2 
showed a noticeably reduced luminescence despite having the same concentration of luminophore and a higher emission quantum yield. This suggests that $\mathbf{2}$ aggregates and self-quenches in the film formulations used in this study. Various thin films oxygen sensitivities were measured and compared in Table 3.4. It is evident that the oxygen sensitivity of emission intensity is linear in the pressure range of 0.007-45 psi (Figure 3.32). Figure 3.32 shows modified Stern-Volmer plots of $\mathbf{1}, \mathbf{2}$, and $\mathbf{8}$ in two Silamine:MC formulations.

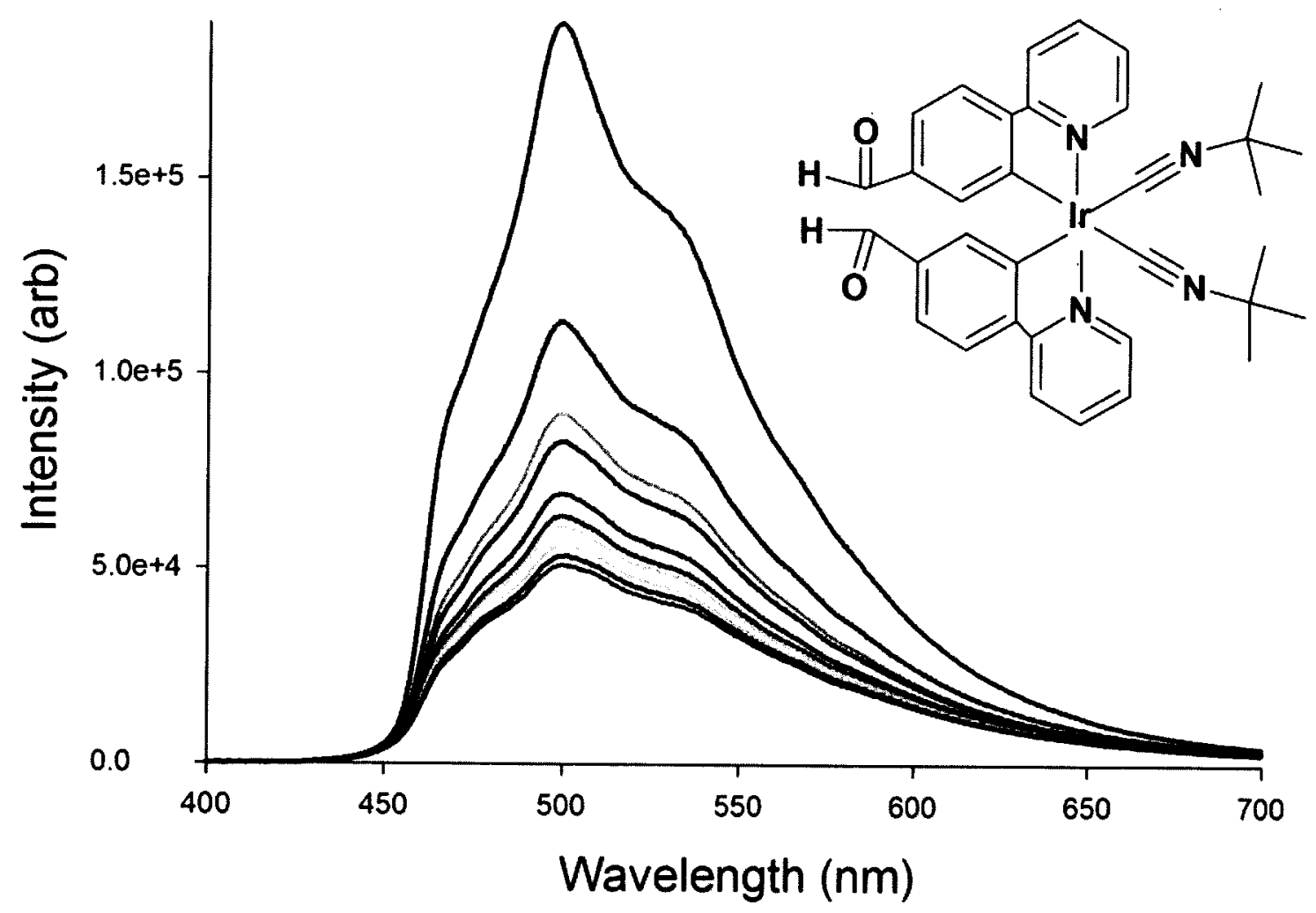

Figure 3.29: Effect of pressure on thin film luminescence of 2 in 1:1 Silamine: MC. The decrease of intensity with increasing the air pressure is displayed. Spectra were acquired at 1.3 mbar, 0.34 bar, 0.68 bar, 1 bar, 1.36 bar, 1.70 bar, 2.04 bar, 2.38 bar, 2.72 bar, and 3.06 bar. Background correction has been performed. 


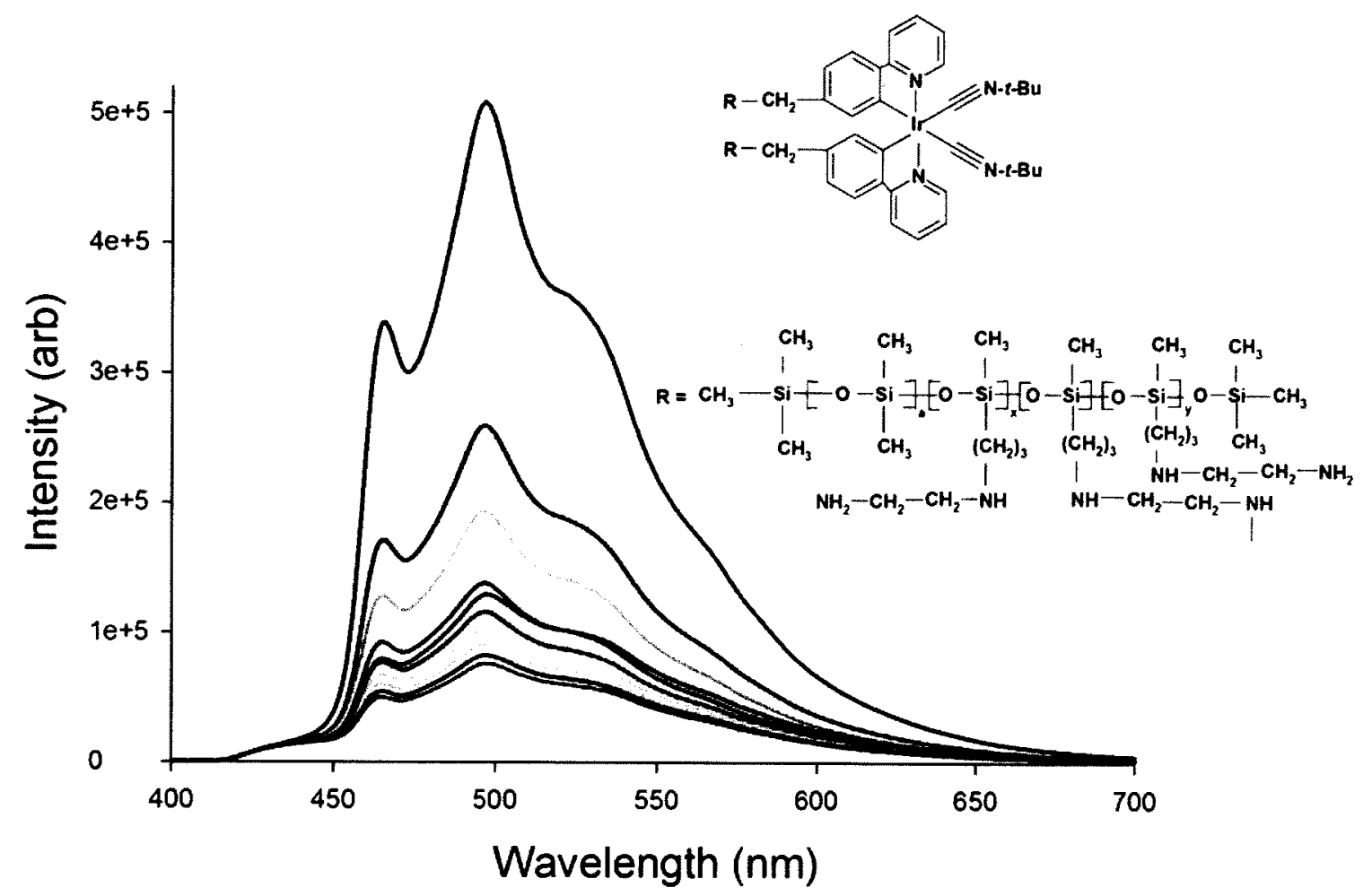

Figure 3.30: Effect of pressure on thin film luminescence of 8 in 1:1 Silamine: MC. The decrease of intensity with increasing the air pressure is displayed. Spectra were acquired at 1.3 mbar, 0.34 bar, 0.68 bar, 1 bar, 1.36 bar, 1.70 bar, 2.04 bar, 2.38 bar, 2.72 bar, and 3.06 bar. Background correction has been performed. 


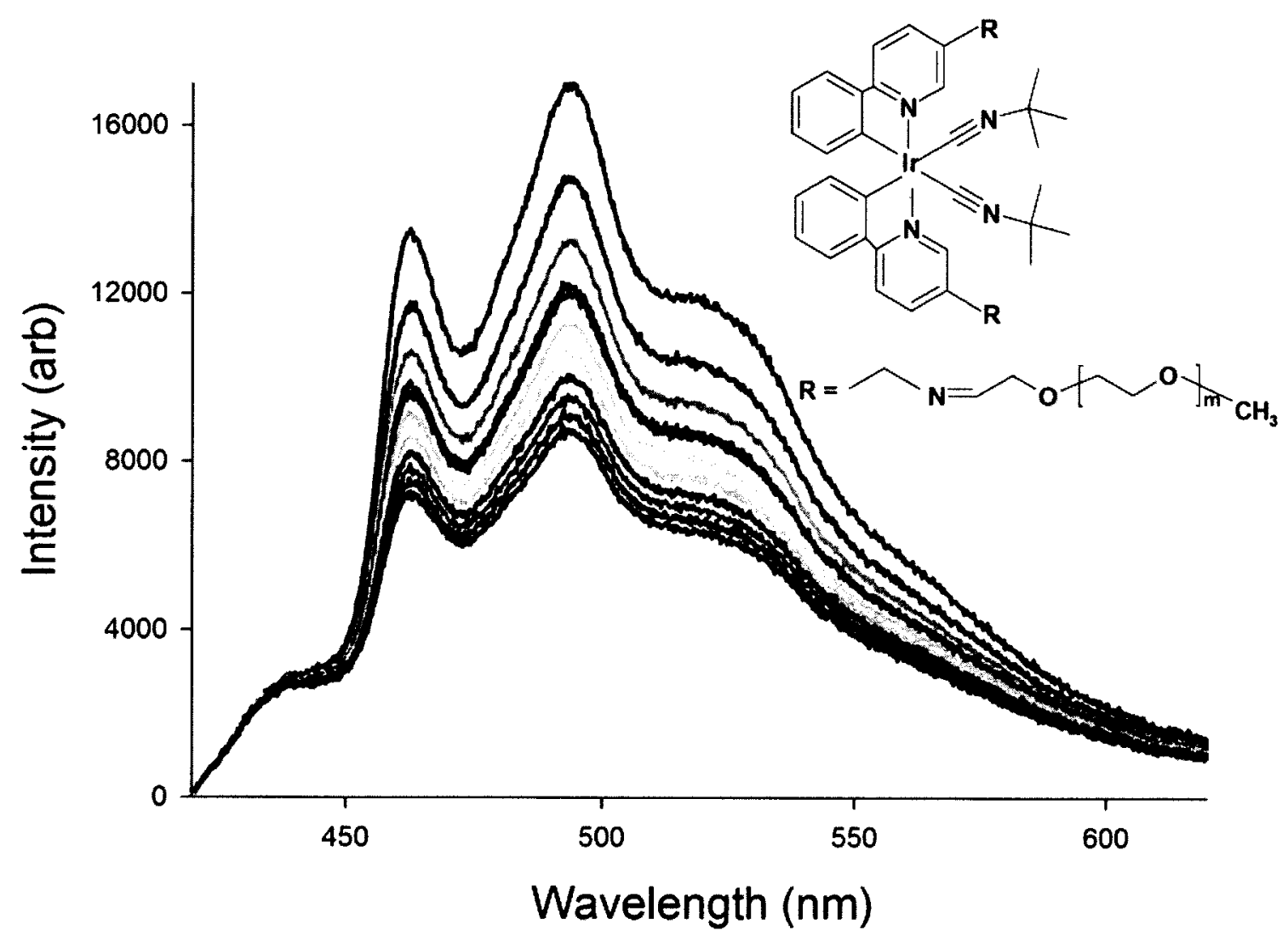

Figure 3.31: Effect of pressure on thin film luminescence of 11 in 4:1 PEG: MC. The decrease of intensity with increasing the air pressure is displayed. Spectra were acquired at 1.3 mbar, 0.34 bar, 0.68 bar, 1 bar, 1.36 bar, 1.70 bar, 2.04 bar, 2.38 bar, 2.72 bar, and 3.06 bar. Background correction has been performed.

Oxygen quenching sensitivity of $\mathbf{8}$ in 1:1 Silamine: MC is the greatest compared with sensitivities of films of 1, 2, or 11, (see Figure 3.32 and Table 3.4). 


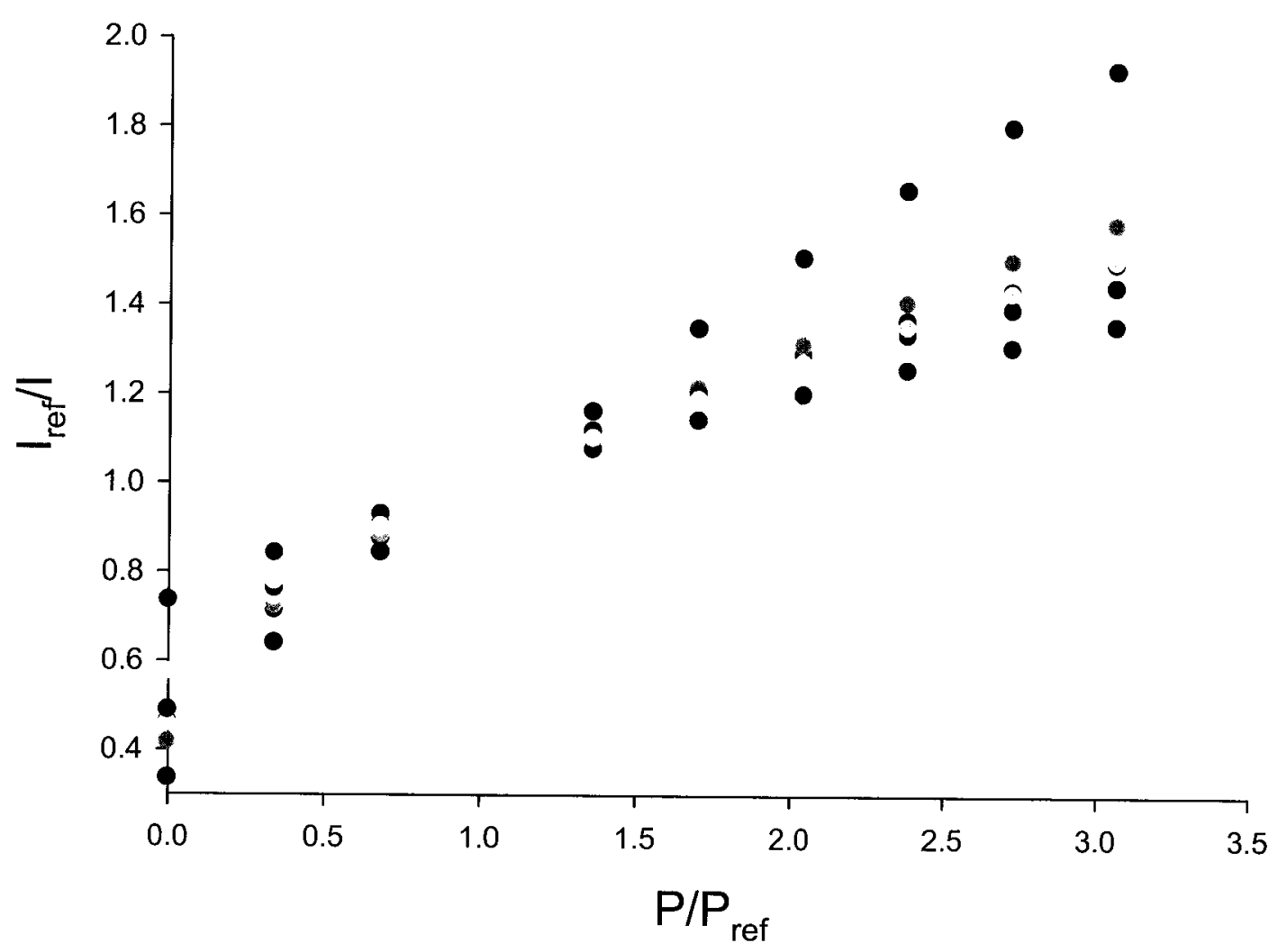

Figure 3.32: Stern-Volmer plots of 1 in 9:1 Silamine: MC, ; 1 in 1:1 Silamine: MC, ; $\mathbf{2}$ in 9:1 Silamine: MC, $\bullet ; 2$ in 1:1 Silamine: MC, ; 8 in 9:1 Silamine: MC, $\bullet ; \mathbf{8}$ in 1:1 Silamine: $\mathrm{MC}, \bullet ; 11$ in 4:1 PEG: $\mathrm{MC}, \bullet$. 
Table 3.4: PSP films oxygen sensitivity data for $\mathbf{1 , 2}, \mathbf{8}$, and 11.

\begin{tabular}{|c|c|c|}
\hline Luminophore & Matrix & $Q_{\mathrm{s}}\left(\mathrm{r}^{2}\right)$ \\
\hline 1 & $\begin{array}{c}\text { 9:1 Silamine: } \mathrm{MC} \\
\text { 5:1 Silamine : MC } \\
\text { 1:1 Silamine: } \mathrm{MC} \\
\text { 1:2.3 Silamine: } \mathrm{MC} \\
\text { 1:5 Silamine: } \mathrm{MC}\end{array}$ & $\begin{array}{l}0.26(0.992)^{b} \\
0.28(0.991)^{b} \\
0.30(0.980)^{b} \\
0.26(0.981)^{b} \\
0.26(0.975)^{b}\end{array}$ \\
\hline 2 & $\begin{array}{c}\text { 9:1 Silamine: } \mathrm{MC} \\
\text { 5:1 Silamine : MC } \\
\text { 1:1 Silamine: } \mathrm{MC} \\
1: 2.3 \text { Silamine: } \mathrm{MC} \\
\text { 1:5 Silamine : MC }\end{array}$ & $\begin{array}{l}0.24(0.973)^{\mathrm{b}} \\
0.25(0.977)^{\mathrm{b}} \\
0.29(0.974)^{\mathrm{b}} \\
0.27(0.970)^{\mathrm{b}} \\
0.24(0.974)^{\mathrm{b}}\end{array}$ \\
\hline 8 & $\begin{array}{c}\text { 9:1 Silamine: } \mathrm{MC} \\
\text { 5:1 Silamine : MC } \\
\text { 1:1 Silamine: } \mathrm{MC} \\
\text { 1:2.3 Silamine: } \mathrm{MC} \\
\text { 1:5 Silamine: } \mathrm{MC}\end{array}$ & $\begin{array}{l}0.28(0.976)^{b} \\
0.28(0.955)^{b} \\
0.50(0.989)^{\mathrm{a}} \\
0.44(0.975)^{\mathrm{a}} \\
0.33(0.982)^{\mathrm{b}}\end{array}$ \\
\hline 11 & 4:1 PEG: MC & $0.20(0.986)^{\mathrm{a}}$ \\
\hline
\end{tabular}

Oxygen sensitivity as well as mechanical properties of the film is increased as a consequence of the blending of $\mathrm{MC}$ with luminophores compared with the film composed of the polymer with low glass transition temperature. It is suggested that this effect originates from a decrease in the luminophores freedom of motion and consequentially an increase in its luminescence lifetime. Moreover, the preferential binding of luminophore to MC could lead to de-aggregation and a decrease in selfquenching.

Increasing the MC content in PSP formulations of $\mathbf{8}$, results in decrease in downward curvature of the Stern-Volmer plots. This is supposed to be owing to the increased rigidity of the matrix and the confined movement of the luminophore molecules. This is evident in Figure 3.32 for 8 in 9:1 Silamine: MC and a 1:1 Silamine: $\mathrm{MC}$ blend. Several low Tg polymer: $\mathrm{MC}$ ratios were used for luminophores in order to 
determine the conditions for optimum PSP performance. As seen from Figure 3.32 and the data in Table 3.4, quenching sensitivity (particularly for 8) is dependent upon microcrystalline cellulose concentration. To help understand this, the luminescence microscopy of films of $\mathbf{8}$ was examined, and the results are shown in Figure 3.33. 


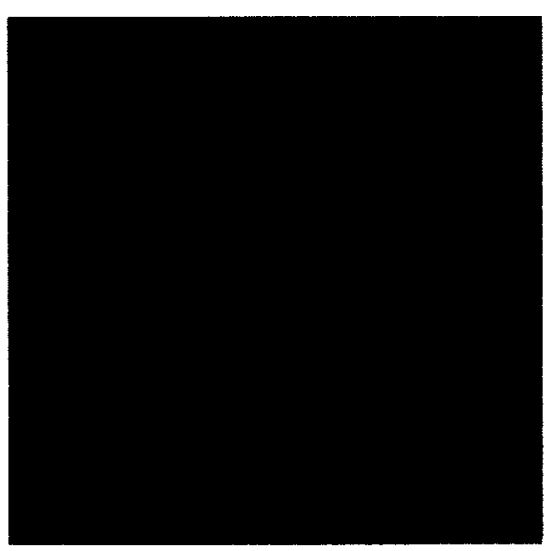

A

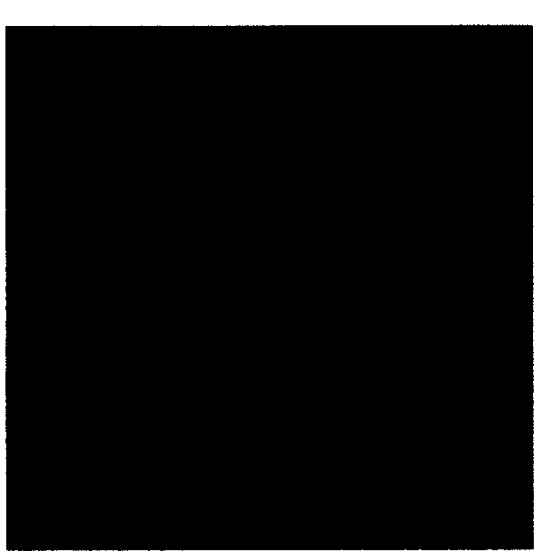

C

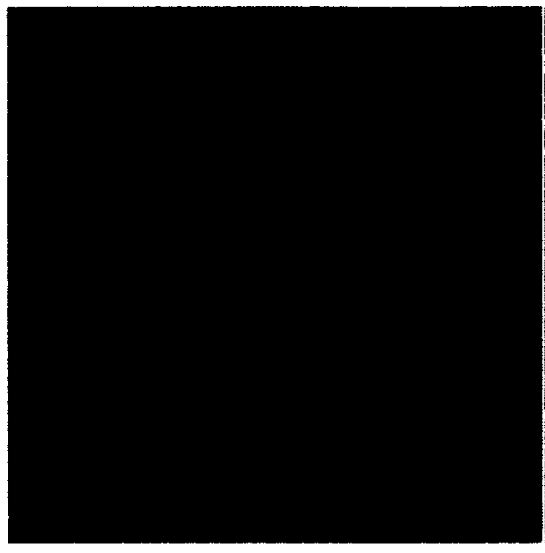

B

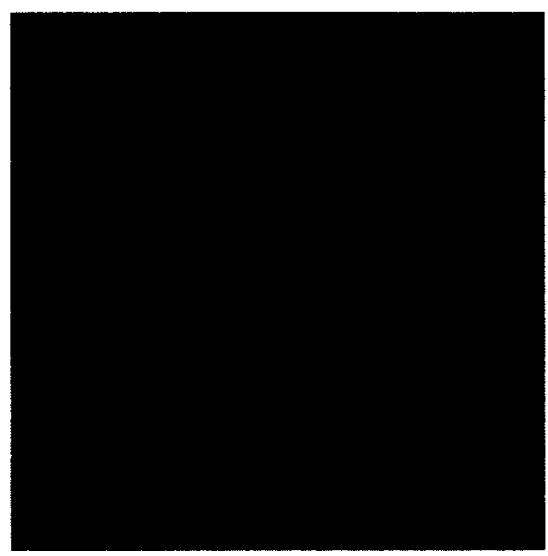

D

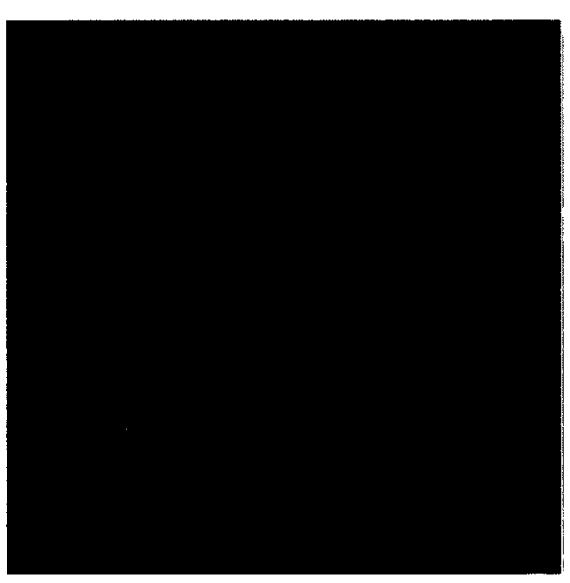

E

Figure 3.33: Luminescence microscopy of films of 8 performed under ambient conditions: A, 9:1 Silamine:MC; B, 5:1 Silamine:MC; C, 1:1 Silamine:MC; D, 1:2.3 Silamine:MC; E, 1:5 Silamine:MC. The scale in red is $10 \mu \mathrm{m}$. 
In Figure $3.33 \mathrm{~A}$, luminescence microscopy shows that at low concentration of $\mathrm{MC}$, the luminescence from the film is largely dispersed with some local enhancement around particles of MC. This localization of luminescence around MC particles becomes even more obvious in Figure $3.33 \mathrm{C}$, the film that gives the best oxygen sensor performance. In Figure $3.33 \mathrm{E}$, the film that has the greatest concentration of $\mathrm{MC}$, an overall loss of luminescence intensity has obviously occurred which is attributed to light scattering by MC particles but there is still evidence of luminophore association with MC. It is suggested that the positively charged iridium luminophore in $\mathbf{8}$ is electrostatically attracted to the polar hydroxyl groups on the surface of MC.

Lifetime measurements of 9:1, 1:1 and 1:5 Silamine:MC films of 8 , under ambient pressure and vacuum, are compiled in Table 3.5. Emission decay was fitted with a double exponential assuming two luminophore environments: 8 in Silamine polymer and $\mathbf{8}$ associated with the MC surface. The data show an increase in lifetime with increasing $\mathrm{MC}$ fraction which can be due to a decrease in non- radiative decay of the excited molecule.

Table 3.5: Luminescence lifetime data in $\mu$ s (with pre-exponential factors in parentheses) of 8 in Silamine:MC films

\begin{tabular}{|c|c|c|c|c|c|c|}
\hline & \multicolumn{3}{|c|}{ Vacuum } & \multicolumn{3}{c|}{ Ambient pressure } \\
\hline $\begin{array}{c}\text { Silamine:MC } \\
\text { film }\end{array}$ & $\tau_{1}$ & $\tau_{2}$ & $\mathrm{R}^{2}$ & $\tau_{1}$ & $\tau_{2}$ & $\mathrm{R}^{2}$ \\
\hline $9: 1$ & $0.5(0.7)$ & $5.9(0.3)$ & 0.990 & $0.3(0.9)$ & $4.9(0.1)$ & 0.986 \\
\hline $1: 1$ & $0.6(0.8)$ & $17.6(0.2)$ & 0.995 & $0.6(0.7)$ & $12.9(0.3)$ & 0.997 \\
\hline $1: 5$ & $3.2(0.8)$ & $25.8(0.2)$ & 0.993 & $0.5(0.9)$ & $15.3(0.1)$ & 0.984 \\
\hline
\end{tabular}

Quenching sensitivity $\mathrm{Q}_{\mathrm{s}}$ is dependent upon both lifetime and the quenching rate constant $\mathrm{k}_{\mathrm{q}}$ (Eqn 1.5 and 1.13) and so the observation of an optimum $\mathrm{Q}_{\mathrm{s}}$ value (see Table 
3.4) with MC fraction suggests that the quenching rate constant, or more specifically the diffusion of oxygen, must decrease with increasing fraction of MC. The optimum PSP formulation in Table 3.4 is $\mathbf{8}$ in 1:1 Silamine:MC which gave an oxygen sensitivity of 0.50 , with good linear behavior in a pressure range of 0.007 to $45 \mathrm{psi}$.

The temperature dependence of emission intensity of $\mathbf{1 ,} 2$ and $\mathbf{8}$ in 1:1 Silamine:MC films were examined in the temperature range of 10 to $40{ }^{\circ} \mathrm{C}$ under vacuum and 1 bar atmospheric pressure. The emission intensities were normalized to that at $10{ }^{\circ} \mathrm{C}$ and the results plotted in Figure 3.34.

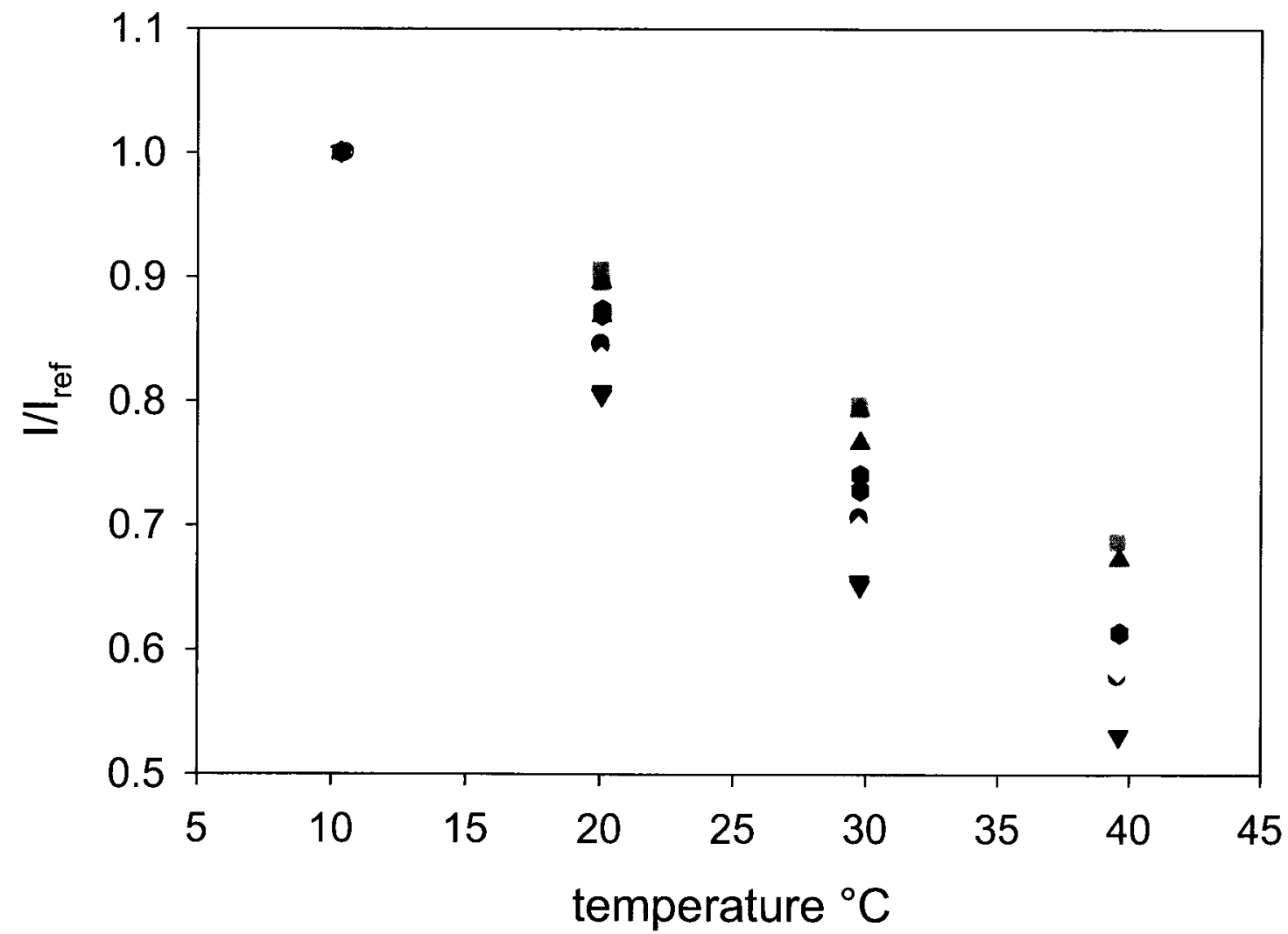

Figure 3.34: Temperature versus emission intensity ratio $\left(\mathrm{I}_{\mathrm{ref}}\right.$ is the intensity at $\left.10^{\circ} \mathrm{C}\right)$ of 1 in 1:1 Silamine: $\mathrm{MC}$ : vacuum $\boldsymbol{\Delta}$ and $\mathrm{P}=1 \mathrm{bar} \bullet, \mathbf{2}$ in 1:1 Silamine: $\mathrm{MC}$ : vacuum $\bullet$ and $\mathrm{P}=1 \mathrm{bar} \boldsymbol{\nabla}$, and 8 in 1:1 Silamine: $\mathrm{MC}$ : vacuum $\bullet$ and $\mathrm{P}=1 \mathrm{bar}$. 
In Figure 3.34, the data for the temperature dependence of emission gave good linear least-squares fits passing through or at least closely approaching the normalization value at $10{ }^{\circ} \mathrm{C}$. The temperature sensitivities (percent intensity loss per degree) of the films were determined to be $-1.1 \%$ and $-1.3 \%{ }^{\circ} \mathrm{C}^{-1}$ for $1,-1.5 \%$ and $-1.6 \%{ }^{\circ} \mathrm{C}^{-1}$ for 2 , and $-1.1 \%$ and $-1.4 \%{ }^{\circ} \mathrm{C}^{-1}$ for 8 at vacuum and 1 bar atmospheric pressure, respectively. The increase in temperature sensitivity at 1 bar atmospheric pressure relative to that at vacuum can be ascribed to the temperature dependence of bimolecular oxygen quenching and is expected but the significant temperature sensitivities of the films of $\mathbf{1 , 2}$ and $\mathbf{8}$ in vacuum are not. Luminescent decay of $\operatorname{Ir}\left(\mathrm{C}_{\mathrm{s}}\right)(\mathrm{acac})_{2}$, where $\mathrm{C}_{\mathrm{s}}$ is 3-benzothiazol-2-yl)-7(diethylamino)-coumarin and acac is acetylacetonate, showed very little dependence on temperature from 1 to $50{ }^{\circ} \mathrm{C}$ in vacuum. ${ }^{36 \mathrm{~d}}$ Similarly, PSP's using osmium complex luminophores have shown a range of temperature sensitivities varying from -0.11 to -1.00 $\%{ }^{\circ} \mathrm{C}^{-1}$ in vacuum ${ }^{20 \mathrm{c}}$ which suggests that the nature of the coordination sphere plays a critical role in determining temperature sensitivity. In any case, the temperature sensitivity of 8 in 1:1 Silamine:MC films must be compensated for to obtain correct barometric data and this can be achieve by using a temperature sensitive calibrant as in a dual-luminophore oxygen and temperature sensor film. ${ }^{20 u}$ 


\subsection{Other Functionalized Ir (III) Complexes}

\subsubsection{Synthesis}

Starting materials $\left[\operatorname{Ir}\left(\mathrm{C}_{6}\right)_{2} \mathrm{Cl}\right]_{2}$ and $\left[\operatorname{Ir}(\mathrm{ppy})_{2} \mathrm{Cl}\right]_{2}$ (Scheme 3.9) were prepared according to Thompson, ${ }^{72 \mathrm{c}}$ and Watts and Nonoyama. ${ }^{74}$
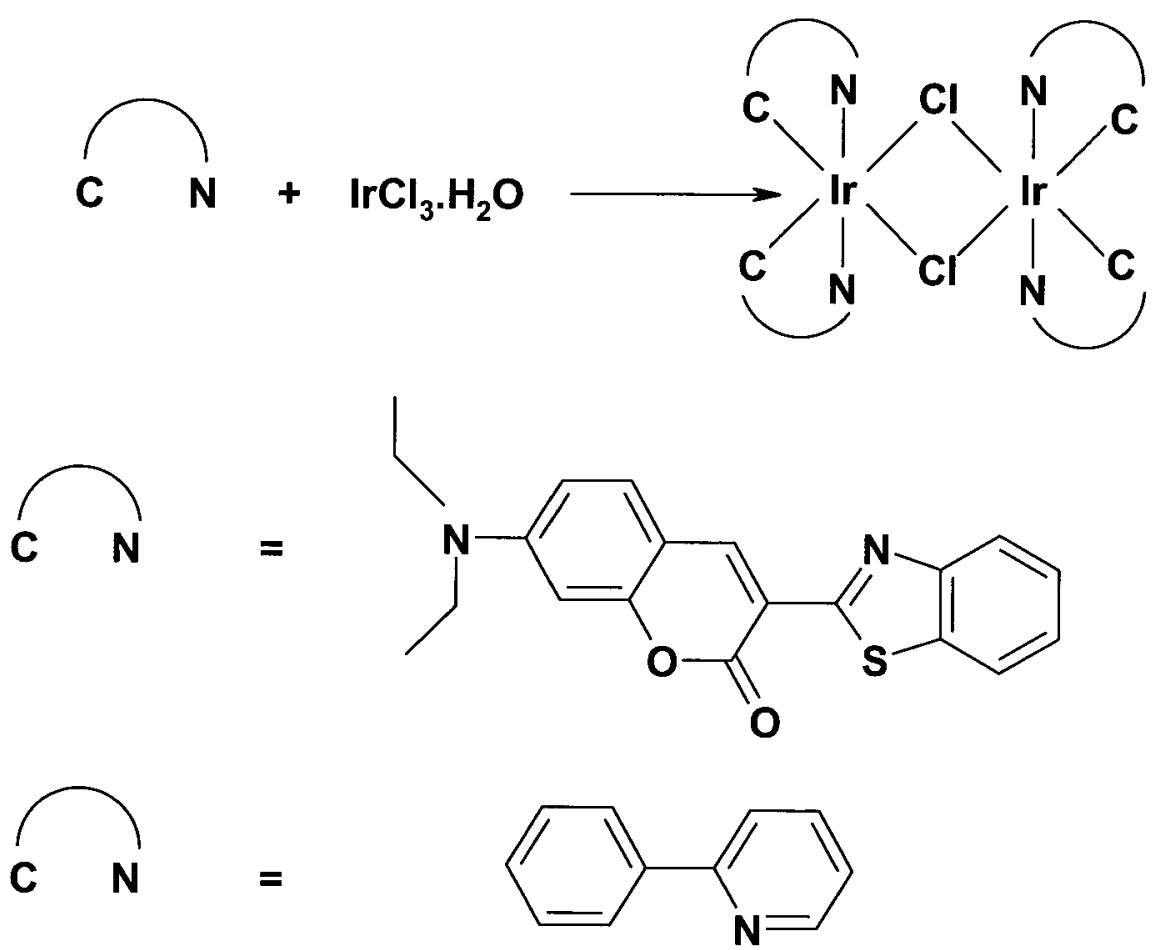

Scheme 3.9: Synthesis of starting iridium dimers.

Commercially unavailable ligand 1,10-phenanthroline-2,9-dicarboxaldehyde was prepared according to Chandler. ${ }^{79}$ 
Synthesis of iridium complexes involved breaking of starting dimers using silver triflate (except for 6) followed by coordination of the second ligand (shown in Schemes $3.10,3.11$, and 3.12).

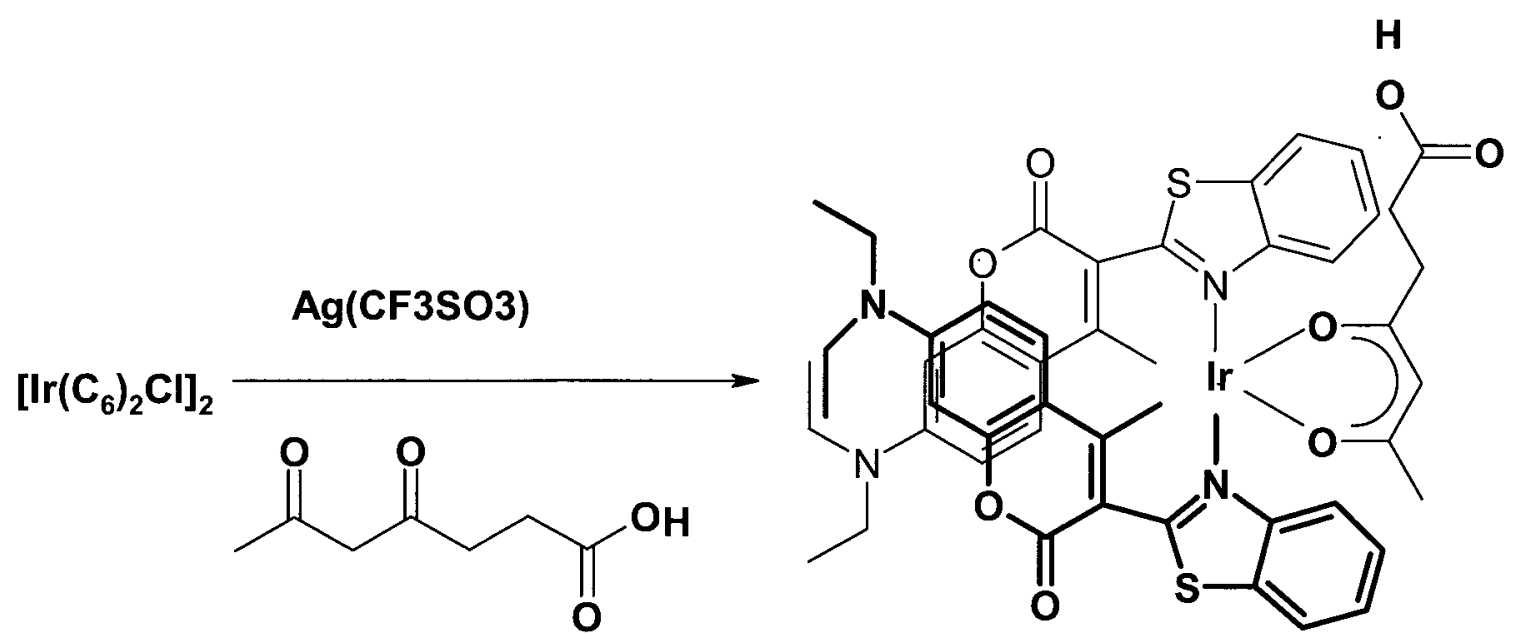

Scheme 3.10: Synthesis of 3
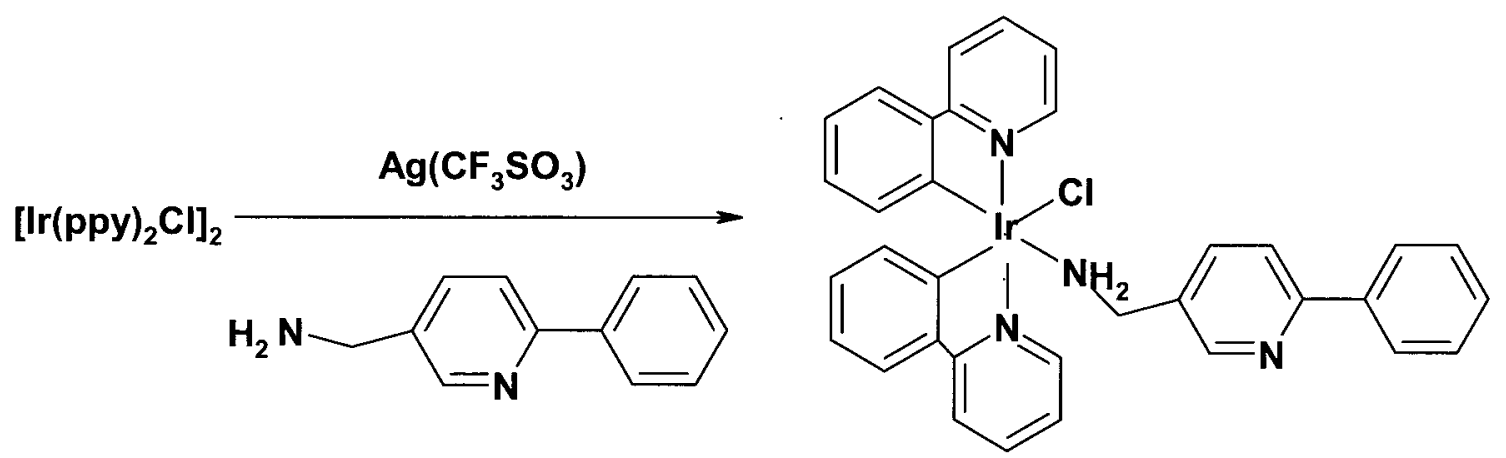

Scheme 3.11: Synthesis of 4 


\section{$\left[\operatorname{lr}(\mathrm{ppy})_{2} \mathrm{Cl}\right]_{2}$}
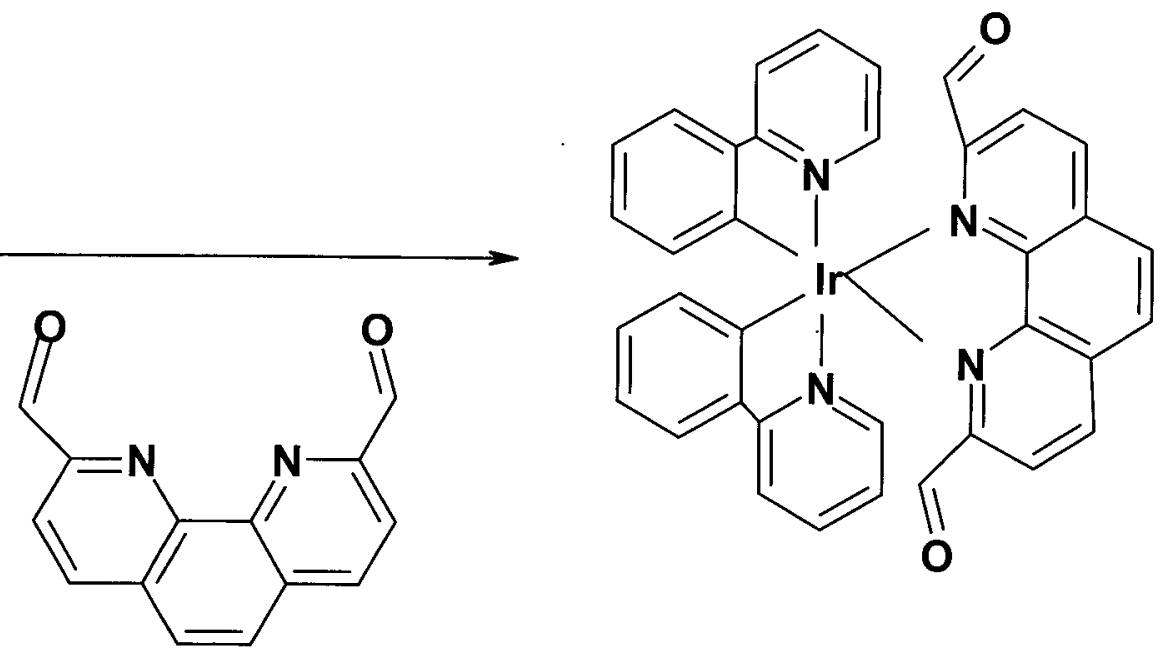

Scheme 3.12: Synthesis of 6

Complex 3 was synthesized from $\left[\operatorname{Ir}\left(\mathrm{C}_{6}\right)_{2} \mathrm{Cl}\right]_{2}$ using silver triflate to cleave the iridium dimer, and the acid functionalized ligand used was 4,6-dioxoheptanoic acid (acacacid). An acceptable yield was obtained (38\%).

The non-ionic complex 4 was synthesized from $\left[\operatorname{Ir}(\mathrm{ppy})_{2} \mathrm{Cl}\right]_{2}$ at room temperature using silver triflate to cleave the chloride bridged dimer. ${ }^{90}$ The amine group of amppy coordinates to iridium preventing the cyclometalation of phenylpyridine. A high yield (79\%) was obtained.

6 was synthesized from $\left[\operatorname{Ir}(\mathrm{ppy})_{2} \mathrm{Cl}\right]_{2}$ and 1,10-phenanthroline-2,9dicarboxaldehyde in a high yield (85\%).

\subsubsection{X-ray Crystallography}

Structure of $\mathbf{4}$ was confirmed by X-ray crystallography on its single crystals grown from dichloromethane solutions by diffusion of hexanes. The ORTEP drawing of 
4. $\mathrm{CH}_{2} \mathrm{Cl}_{2}$ is shown in Figure 3.35. Crystallographic data and some selected bond lengths and angles are given in Tables 3.6-3.7. Full data of atomic coordinates, bond lengths and angles for the complex can be found in Appendix A.

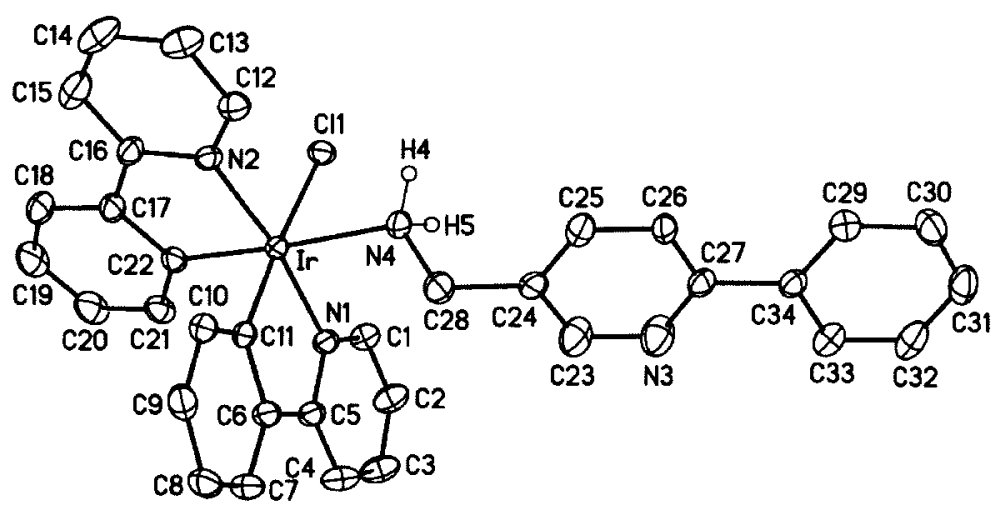

Figure 3.35: ORTEP of $4 \cdot \mathrm{CH}_{2} \mathrm{Cl}_{2}$. The methylene chloride solvent molecule and aromatic-ring hydrogen atoms are omitted for clarity. 
Table 3.6: X-ray Crystallographic Data and Refinement Details for $4 \cdot \mathrm{CH}_{2} \mathrm{Cl}_{2}$.

Empirical formula $\quad \mathrm{C}_{34} \mathrm{H}_{28} \mathrm{IrN}_{4} \mathrm{Cl} \cdot \mathrm{CH}_{2} \mathrm{Cl}_{2}$

$\mathrm{M}_{\mathrm{r}}$

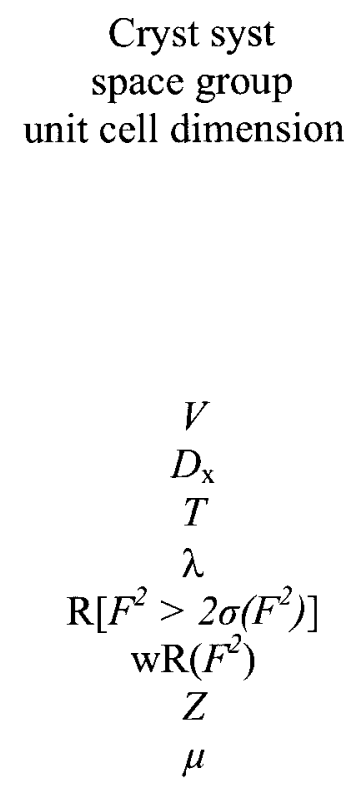

805.18

$$
\begin{aligned}
& \text { Monoclinic } \\
& P 2(1) / c
\end{aligned}
$$$$
a=10.4363(15) \AA
$$$$
b=25.923(4) \AA
$$$$
c=11.7427(17) \AA
$$$$
\alpha=90.00^{\circ}
$$$$
\beta=100.315(2)^{\circ}
$$$$
\gamma=90.00^{\circ}
$$

$3125.5(8) \AA^{3}$

$1.711 \mathrm{~g} \mathrm{~cm}^{-3}$

$200(2) \mathrm{K}$

$0.71073 \AA$

0.0305

0.0684

4

$4.560 \mathrm{~mm}^{-1}$

Table 3.7: Selected Bond Lengths $(\AA)$ and Angles $\left(^{\circ}\right.$ ) for $4 \cdot \mathrm{CH}_{2} \mathrm{Cl}_{2}$.

$\begin{array}{cccc}\mathrm{Ir} 1-\mathrm{N} 1 & 2.040(3) & \mathrm{N} 4-\mathrm{Ir} 1-\mathrm{Cl} & 82.56(9) \\ \mathrm{Ir} 1-\mathrm{N} 2 & 2.047(3) & \mathrm{C} 28-\mathrm{N} 4-\mathrm{Ir} 1 & 119.0(2) \\ \mathrm{Ir} 1-\mathrm{C} 11 & 1.994(3) & \mathrm{C} 11-\mathrm{Ir} 1-\mathrm{N} 1 & 80.62(13) \\ \mathrm{Ir} 1-\mathrm{C} 22 & 1.997(3) & \mathrm{C} 22-\mathrm{Ir} 1-\mathrm{N} 2 & 80.27(14) \\ \mathrm{Ir} 1-\mathrm{Cl1} & 2.4894(9) & \mathrm{C} 25-\mathrm{Ir} 1-\mathrm{C} 30 & 91.94(4), 89.1(3) \\ \mathrm{Ir} 1-\mathrm{N} 4 & 2.222(3) & & \end{array}$

In $4 \cdot \mathrm{CH}_{2} \mathrm{Cl}_{2}$ iridium is octahedrally coordinated with one chlorine, two carbon, and three nitrogen donor atoms in which aminomethyl phenylpyridine and chlorine ligands are cis to each other. The Ir-N and Ir-C bond lengths (Table 3.7) are similar to those of analogous complexes possessing the bis(phenylpyridine)iridium fragment. ${ }^{84,91}$ Being the amppy nitrogen trans to the anionic phenyl ligand and not part of a 
metallocycle, the amppy Ir-N bond length is greater than the ppy Ir-N bonds. It is longer by ca. $0.09 \AA$ than that of an analogous complex possessing ppy ligands with nitrogens trans to carbon atoms. ${ }^{91}$

\subsubsection{NMR Spectroscopy}

The successful syntheses of the iridium complexes were confirmed by displaying characteristic resonances of the ligand in the proton NMR spectrum. These characteristic chemical shifts are new methyl, methylene, and carboxylic acid protons for $\mathbf{3}$, a more complicated spectrum in the aromatic region, and new amine and methylene protons for 4, and aldehyde protons for 6 .

In the NMR spectrum of $\mathbf{3}$, the carboxylic acid group (5, Chart 2) displays a downfield broad peak of an integration of one (12.02 ppm). 1 and 2 protons are singlets found at $1.69 \mathrm{ppm}$ and $4.98 \mathrm{ppm}$, respectively and 3 and 4 protons are found as multiplets at 2.22 and $2.08 \mathrm{ppm}$. The aromatic region shows four multiplet resonances for C6 ligands. C6 ethyls appear at 3.27 and $0.94 \mathrm{ppm}$. 


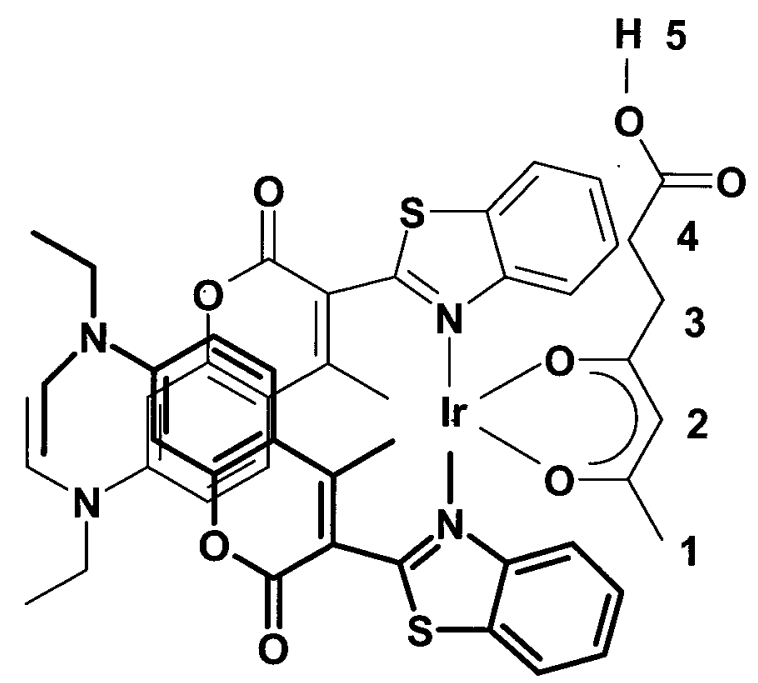

Chart 2: Structure of $\left[\operatorname{Ir}\left(\mathrm{C}_{6}\right)_{2}\right.$ (acacacid) $], \mathbf{3}$, with the numbering used in assigning the ${ }^{1} \mathrm{H}$ NMR.

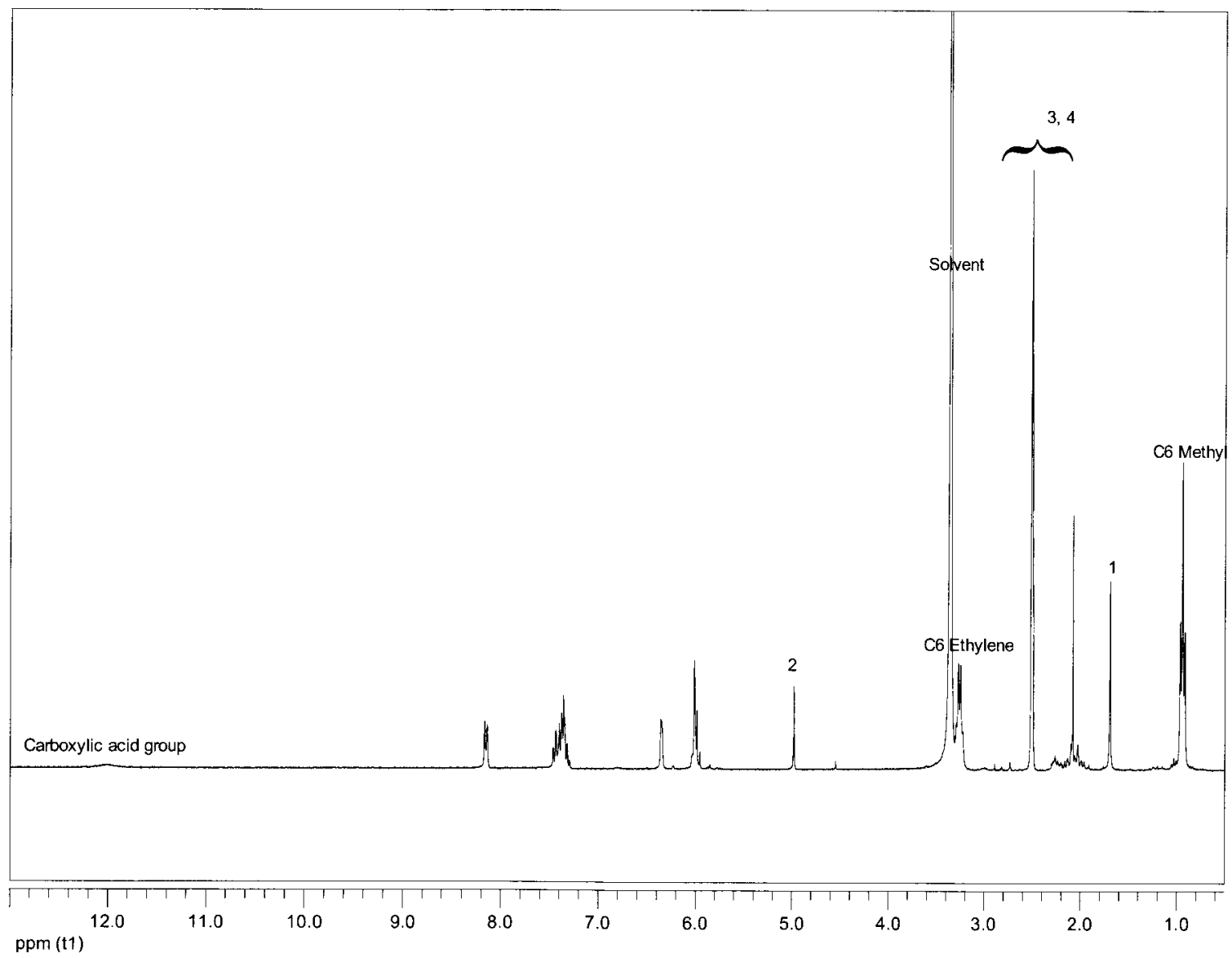

Figure 3.36: ${ }^{1} \mathrm{H}$ NMR spectrum $(300 \mathrm{MHz})$ of 3 in DMSO- $d_{6}$. 
The splitting pattern of the ${ }^{1} \mathrm{H}$ NMR of $\mathbf{4}$ exhibits complexity in the phenyl and pyridyl region.

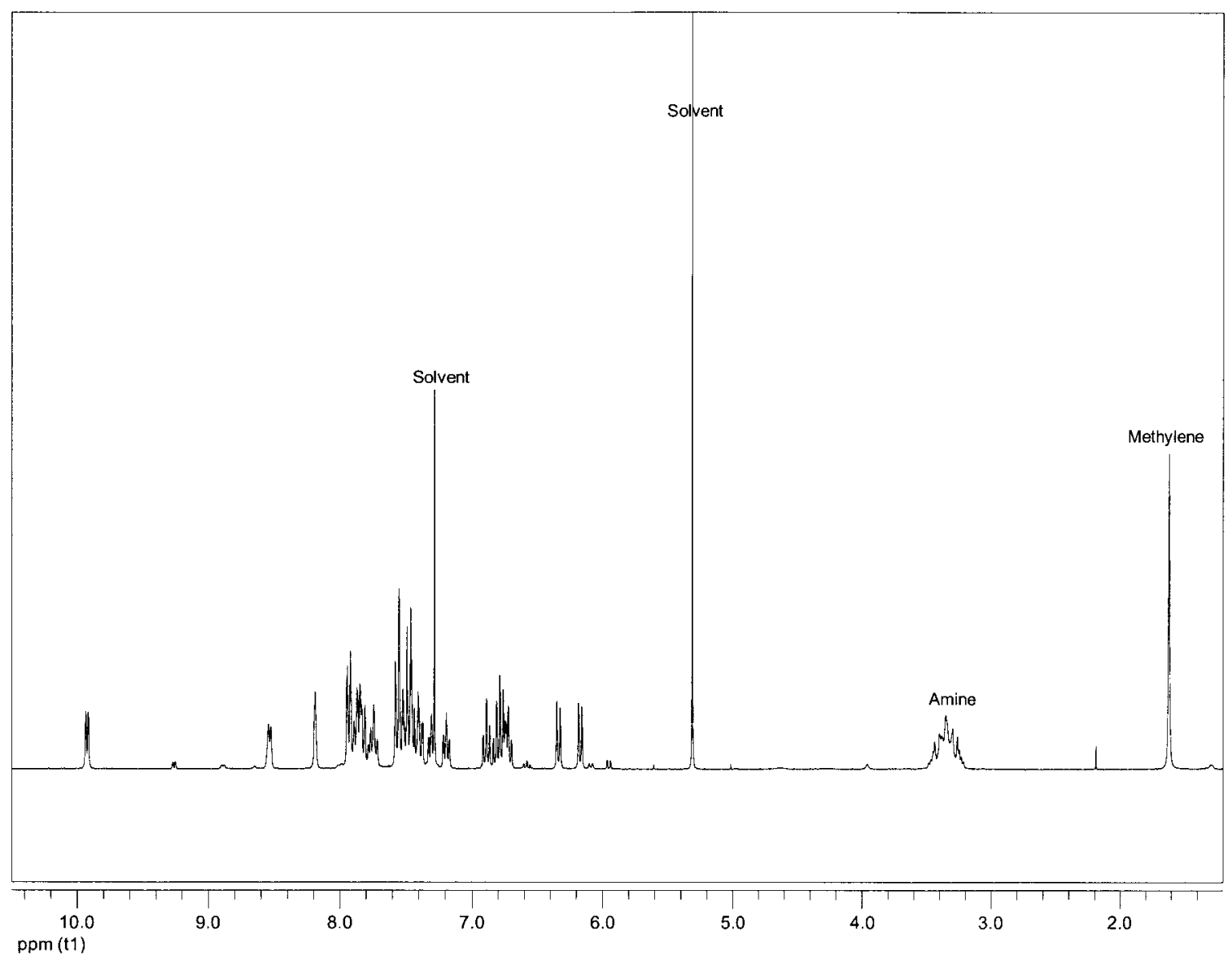

Figure 3.37: ${ }^{1} \mathrm{H}$ NMR spectrum $(300 \mathrm{MHz})$ of 4 in $\mathrm{CDCl}_{3}$.

The aldehyde protons of $\mathbf{6}$ display a peak at the expected chemical shift $(9.70$ ppm). 


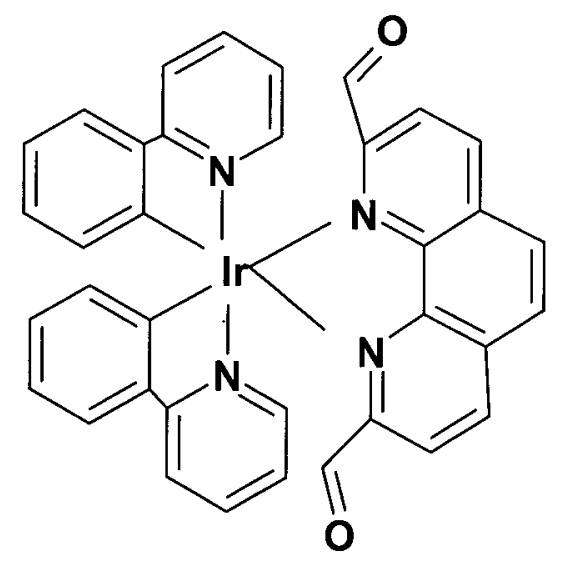

Chart 3: Structure of $\left[\operatorname{Ir}(\text { ppy })_{2}(\right.$ phendiald $\left.)\right]\left[\mathrm{PF}_{6}\right], 6$.

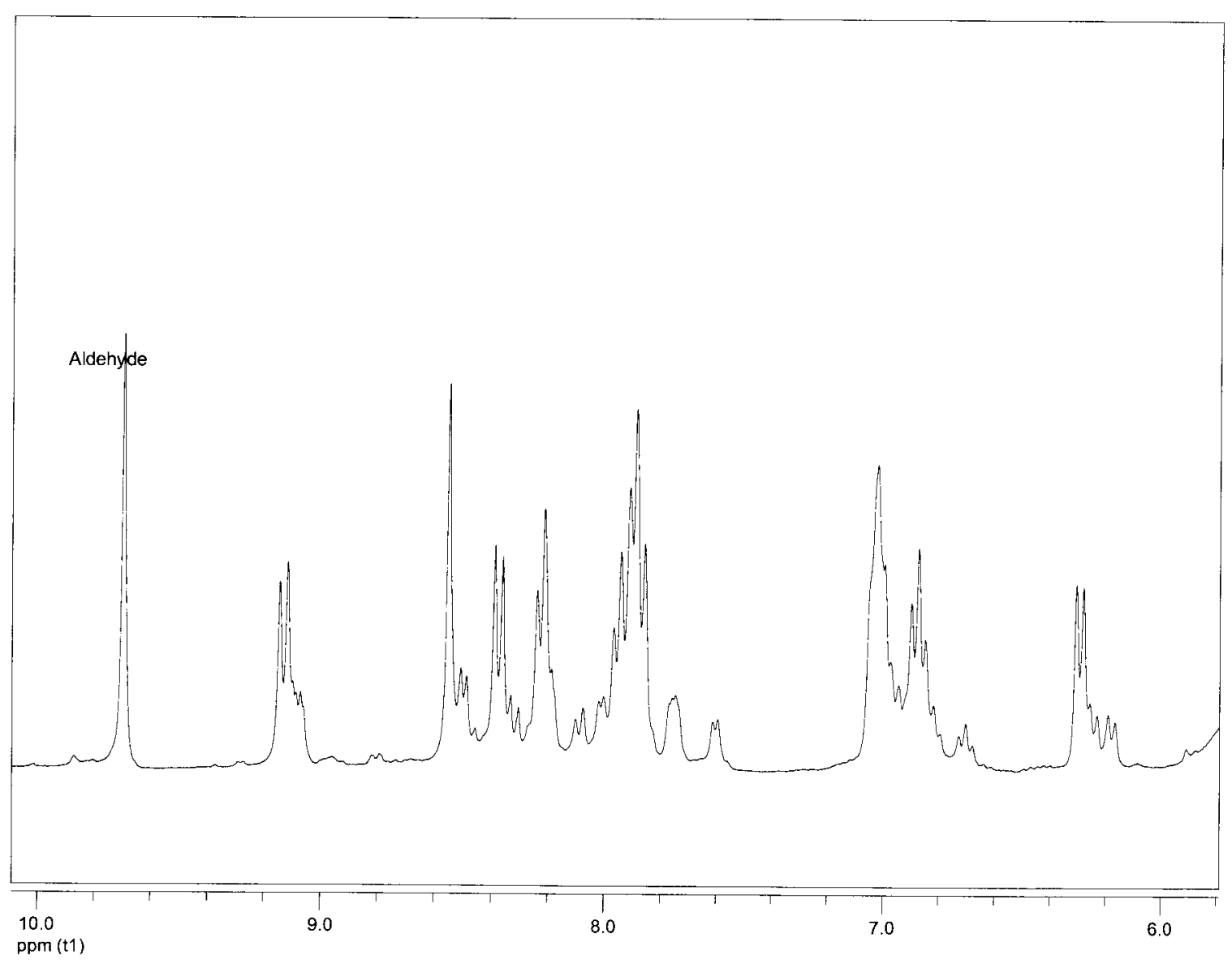

Figure 3.38: ${ }^{1} \mathrm{H}$ NMR spectrum $(300 \mathrm{MHz})$ of 6 in acetone- $d_{6}$. The aldehyde resonance is labelled. 


\subsubsection{Infrared Spectroscopy}

The infrared spectra of complexes 3, 4, and $\mathbf{6}$ are shown in Figures 3.39-3.41, respectively. For 3, Figure 3.39, shows two $v(C=O)_{\text {acacacid }}$ stretches appear at $1680 \mathrm{~cm}^{-1}$ (lactone) and $1607 \mathrm{~cm}^{-1}$ (carboxylic acid). For 4, Figure 3.40 , the $v(\mathrm{~N}-\mathrm{H})_{\text {amine }}$ is seen at $3281 \mathrm{~cm}^{-1}$. Also, in 6 a v $(\mathrm{C}=\mathrm{O})_{\text {phendiald }}$ stretch is shown at $1729 \mathrm{~cm}^{-1}$.

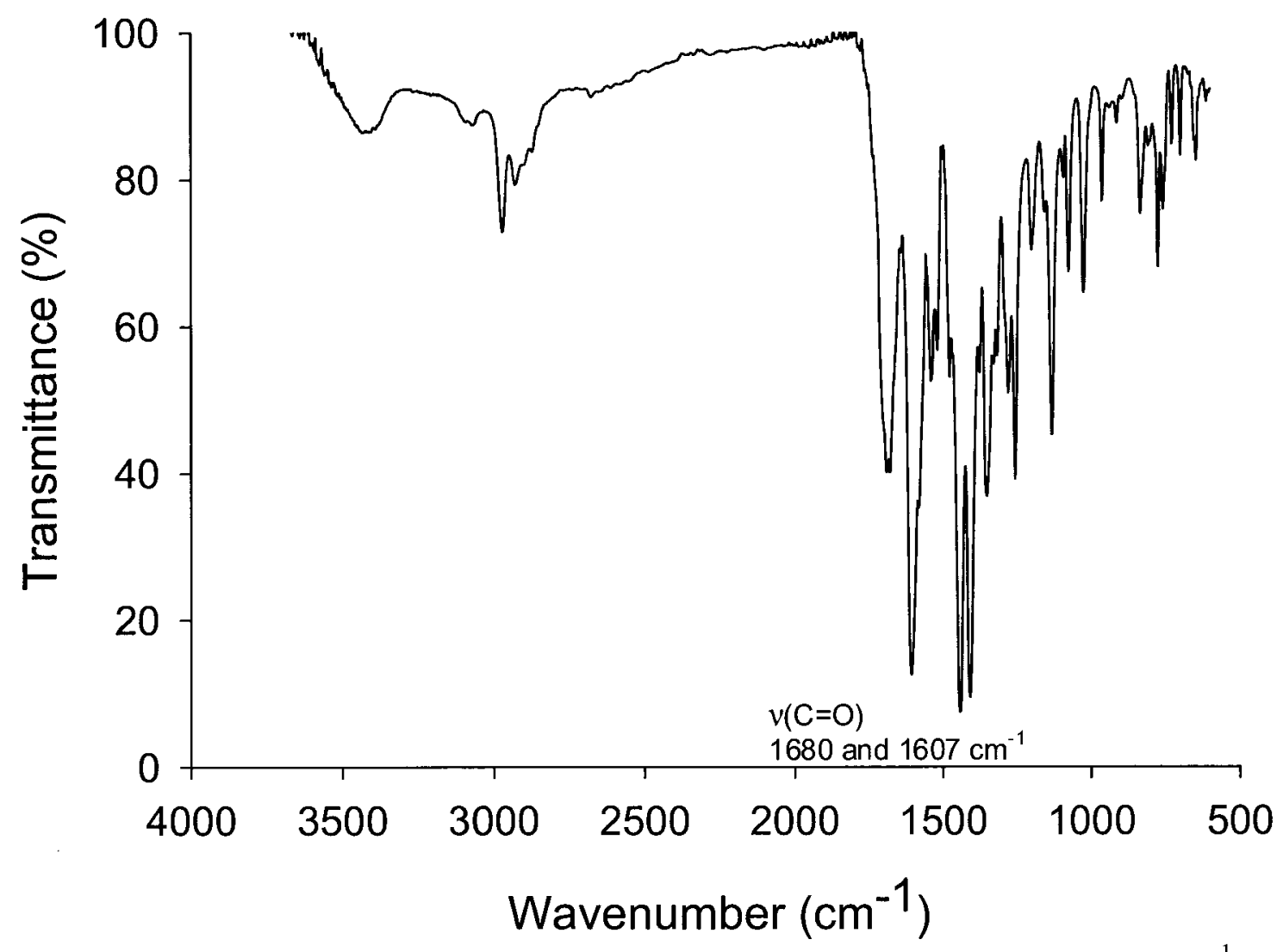

Figure 3.39: Infrared spectrum (KBr pellet) of 3 with $v(C=O)$ at 1680 and $1607 \mathrm{~cm}^{-1}$. 


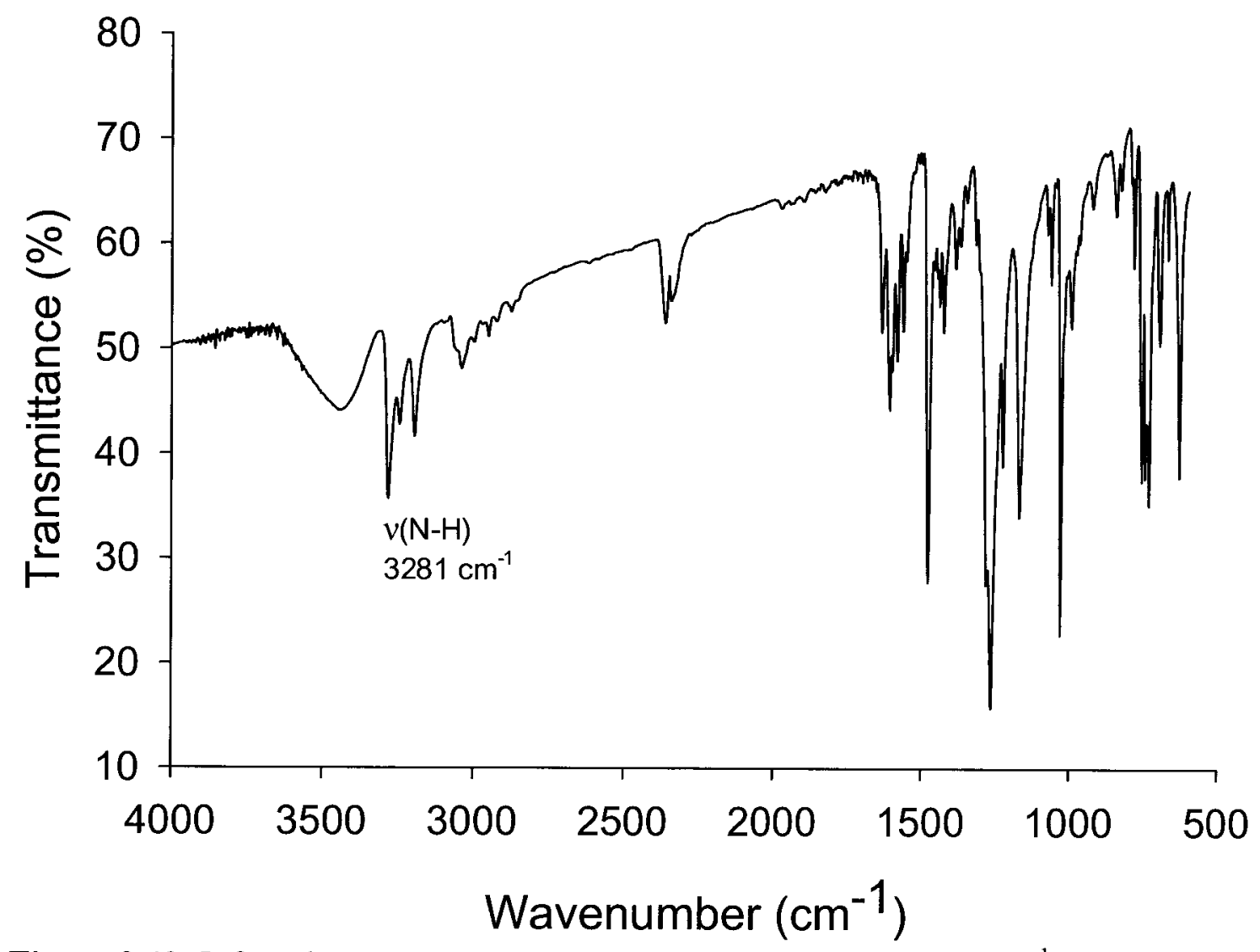

Figure 3.40: Infrared spectrum (KBr pellet) of 4 with $v(\mathrm{~N}-\mathrm{H})$ at $3281 \mathrm{~cm}^{-1}$. 


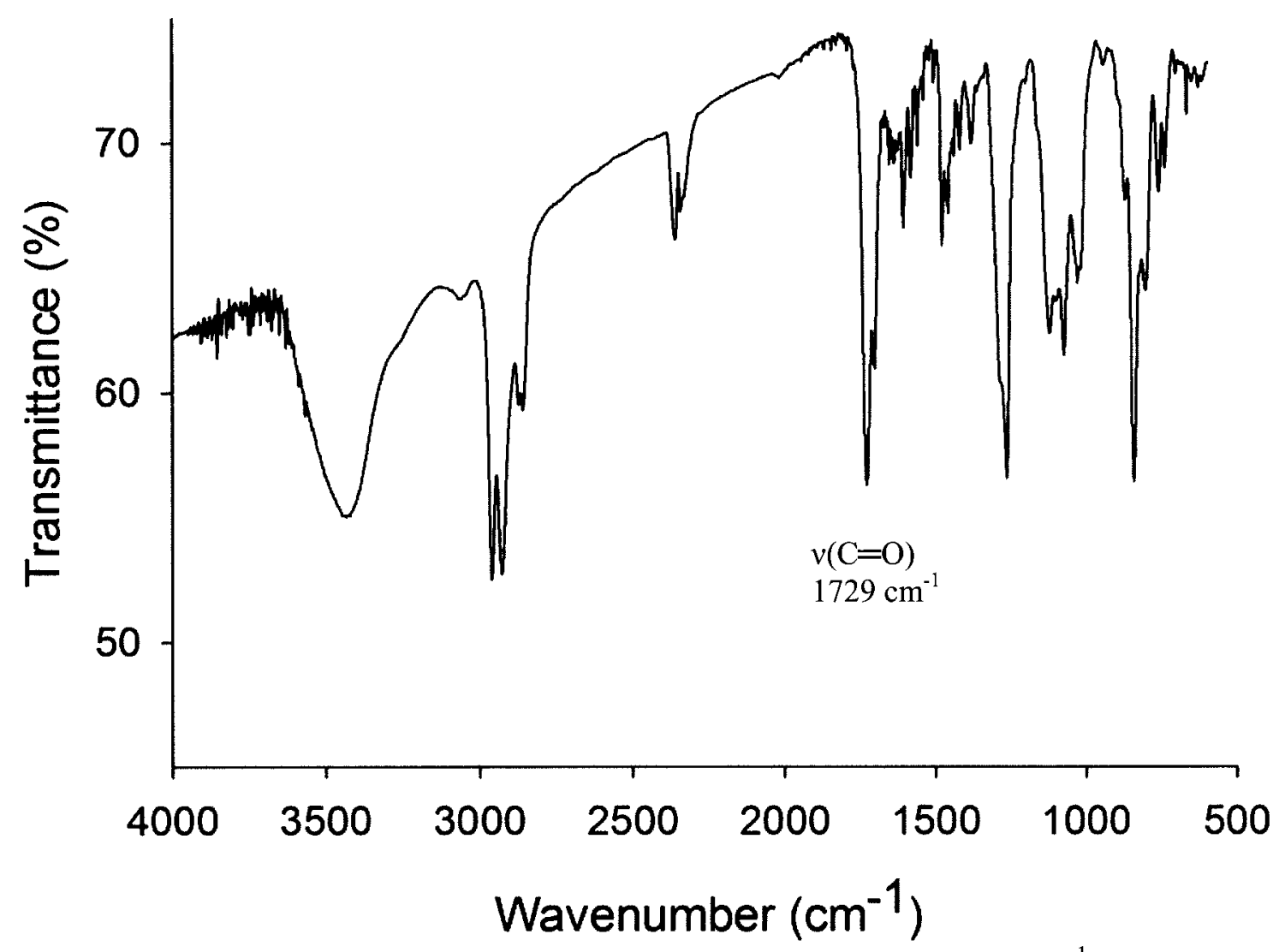

Figure 3.41: Infrared spectrum (KBr pellet) of 6 with $v(C=O)$ at $1729 \mathrm{~cm}^{-1}$.

\subsubsection{Electronic Spectroscopy}

The room-temperature quantitative UV-Vis and emission spectra have been collected for all the complexes and are shown in Figures 3.42-3.44. All spectroscopic data are given in Table 3.8 and assignments made by comparing the absorption spectra with those of analogous complexes. ${ }^{36 e, 67 a, 72 c, 92} 4$, and 6 exhibit ultraviolet intense absorption bands below $300 \mathrm{~nm}\left(\varepsilon \sim 3.0-4.0 \times 10^{4} \mathrm{~cm}^{-1} \mathrm{M}^{-1}\right)$ which are assigned to allowed $\pi-\pi^{*}$ transitions of the aromatic ligands. Weaker bands located at longer 
wavelengths can be assigned to MLCT transitions consisting of ${ }^{1}$ MLCT and ${ }^{3} \mathrm{MLCT}$ transitions.

Two intense $\pi-\pi^{*}$ transitions of the C6 ligand are observed in the absorbance spectrum of complex 3. C6 is a common fluorescent laser dye with a green fluorescence in solution and high quantum efficiency at room temperature. It does not show any detectable phosphorescence. ${ }^{72 \mathrm{c}}$ The emission maximum appears at $500 \mathrm{~nm}$ which shifts to $570 \mathrm{~nm}$ in 3 as a result of coordination to Ir, which is similar to the nonfunctionalized complex, (C6 $)_{2} \operatorname{Ir}(\mathrm{acac})$. This is an indication of intersystem crossing prompted by the vicinity of the heavy Ir atom. ${ }^{72 c} \mathrm{~A}$ vibronic fine structure is also shown in the structured luminescent spectrum of $\mathbf{3}$, thus the emission is from a ligand-based excited state.

The absorption spectrum of 4 exhibits intense phenyl- and pyridyl-based $\pi-\pi^{*}$ band at $259 \mathrm{~nm}$ with ${ }^{1}$ MLCT transitions at $392 \mathrm{~nm}$ and ${ }^{3}$ MLCT transitions at $431 \mathrm{~nm}$. No peak is observed at wavelengths longer than $470 \mathrm{~nm}$. The solution emission spectrum of 4 shows a single broad band with a maximum at $531 \mathrm{~nm}$ and no clear lowenergy shoulder; hence the emission is from a mainly MLCT-based excited state. ${ }^{91}$

Complex 6 exhibits intense high-energy absorption bands at $257 \mathrm{~nm}(\varepsilon=3.75 \times$ $\left.10^{4} \mathrm{~cm}^{-1} \mathrm{M}^{-1}\right)$ and $293 \mathrm{~nm}\left(\varepsilon=4.55 \times 10^{4} \mathrm{~cm}^{-1} \mathrm{M}^{-1}\right)$ and a weaker broad absorption band at $384 \mathrm{~nm}$ in the electronic absorbance spectrum, which are assigned to intraligand $\pi-\pi^{*}$ and spin-allowed MLCT transitions, respectively. A weaker absorption tail is also displayed around 473-555 $\mathrm{nm}$ in the spectrum, characteristic of spin-forbidden MLCT transitions. It shows an intense, broad, structureless orange-yellow luminescence with maximum at $584 \mathrm{~nm}$ in dichloromethane solution at room temperature. Based on 
literature,$^{93}$ the emission is assigned to be originating from a charge-transfer triplet excited state involving low-lying $\pi^{*}$ orbitals of the coordinated phendiald ligand as the acceptor orbital. ${ }^{92}$

Table 3.8: Solution absorption and emission data for complexes 1-6

\begin{tabular}{|c|c|c|c|c|c|c|}
\hline & $1^{\mathrm{c}}$ & 2 & 3 & 4 & $5^{f}$ & 6 \\
\hline $\begin{array}{c}\lambda_{\max } \\
\pi-\pi^{*} \\
(\mathrm{~nm})(\varepsilon)^{\mathrm{a}}\end{array}$ & $\begin{array}{c}260 \\
\left(3.35 \times 10^{4}\right)\end{array}$ & $\begin{array}{c}258^{\mathrm{d}} \\
\left(2.51 \times 10^{4}\right)\end{array}$ & $\begin{array}{c}447^{\mathrm{e}} \\
\left(6.51 \times 10^{4}\right)\end{array}$ & $\begin{array}{c}259^{\mathrm{e}} \\
\left(3.38 \times 10^{4}\right)\end{array}$ & $\begin{array}{c}290^{\mathrm{g}} \\
\left(3.60 \times 10^{4}\right)\end{array}$ & $\begin{array}{c}257^{\mathrm{e}} \\
\left(3.75 \times 10^{4}\right)\end{array}$ \\
\hline $\begin{array}{c}\lambda_{\max } \\
\mathrm{MLCT} \\
(\mathrm{nm})(\varepsilon)\end{array}$ & $\begin{array}{c}348 \\
\left(1.21 \times 10^{4}\right)\end{array}$ & $\begin{array}{c}367^{\mathrm{d}} \\
\left(6.92 \times 10^{3}\right)\end{array}$ & $\begin{array}{c}474^{\mathrm{e}} \\
\left(7.54 \times 10^{4}\right)\end{array}$ & $\begin{array}{c}392^{\mathrm{e}} \\
\left(1.78 \times 10^{3}\right) \\
431^{\mathrm{e}} \\
\left(1.31 \times 10^{3}\right)\end{array}$ & $\begin{array}{c}379^{\mathrm{g}} \\
\left(7.40 \times 10^{3}\right)^{\mathrm{h}} \\
454^{\mathrm{g}} \\
\left(2.60 \times 10^{3}\right)\end{array}$ & $\begin{array}{c}384^{\mathrm{e}} \\
\left(8.53 \times 10^{3}\right) \\
508^{\mathrm{e}} \\
\left(1.28 \times 10^{3}\right)\end{array}$ \\
\hline $\begin{array}{c}\lambda_{\max } \\
\text { emission } \\
(\mathrm{nm})\end{array}$ & 458 & $\begin{array}{l}496^{\mathrm{e}} \\
534^{\mathrm{e}}\end{array}$ & $570^{\mathrm{e}}$ & $531^{\mathrm{e}}$ & $510^{g}$ & $584^{\mathrm{e}}$ \\
\hline $\begin{array}{c}\lambda_{\max } \\
\text { emission } \\
(\mathrm{nm})^{\mathrm{b}}\end{array}$ & & $573^{e}$ & $619^{\mathrm{e}}$ & & $540^{\mathrm{g}}$ & \\
\hline
\end{tabular}

${ }^{\mathrm{a}}$ units of $\mathrm{M}^{-1} \mathrm{~cm}^{-1},{ }^{\mathrm{b}}$ low-energy shoulder, ${ }^{\mathrm{c}}$ from reference $75,{ }^{\mathrm{d}} \mathrm{CH}_{3} \mathrm{CN},{ }^{\mathrm{e}} \mathrm{CH}_{2} \mathrm{Cl}_{2},{ }^{\mathrm{f}}$ from reference $68,{ }^{\mathrm{g}}$ toluene, ${ }^{\mathrm{h}}$ broad 


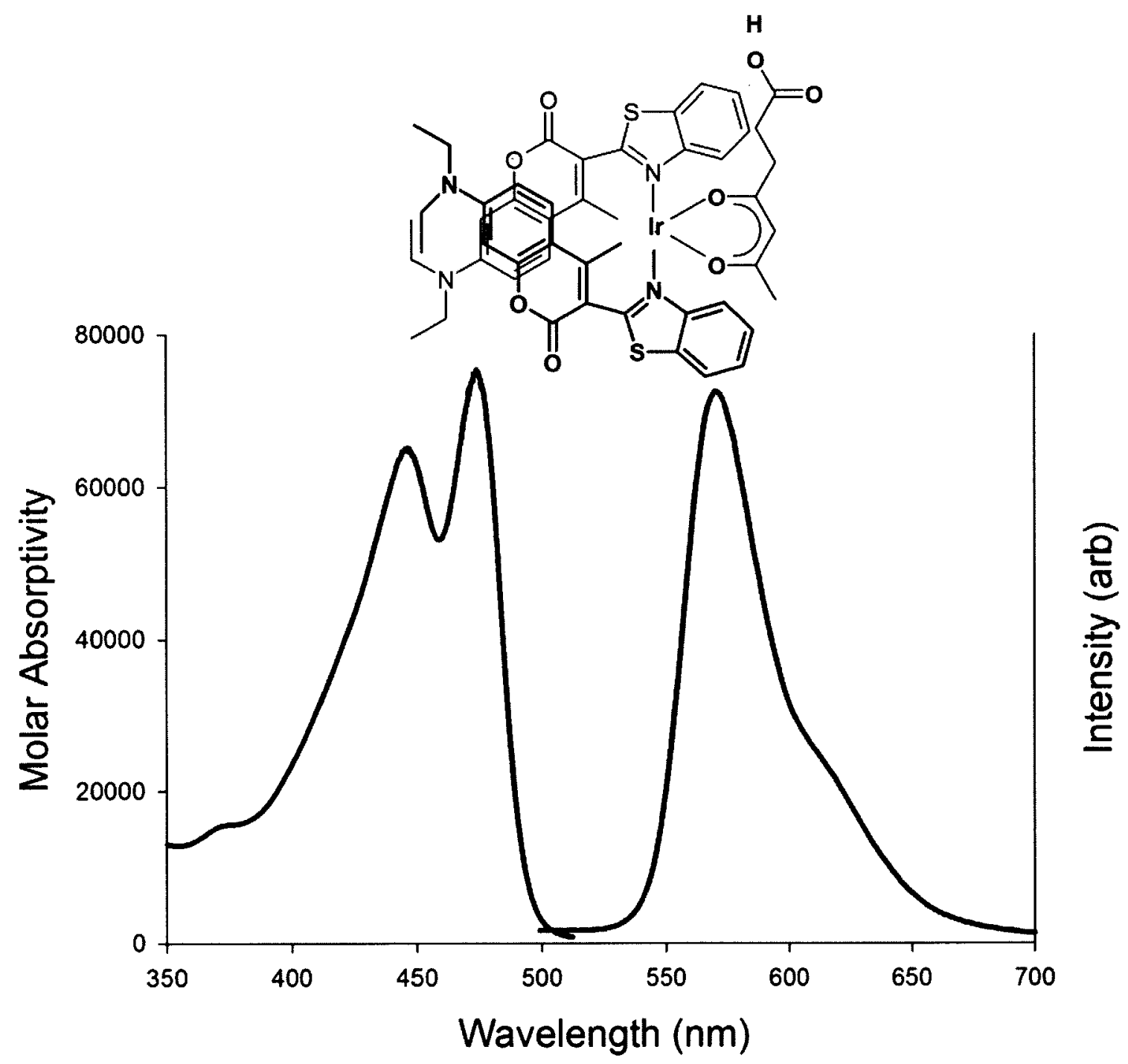

Figure 3.42: Solution electronic absorption and emission spectra of 3. Both the absorption and emission spectra were acquired in $\mathrm{CH}_{2} \mathrm{Cl}_{2}$. 


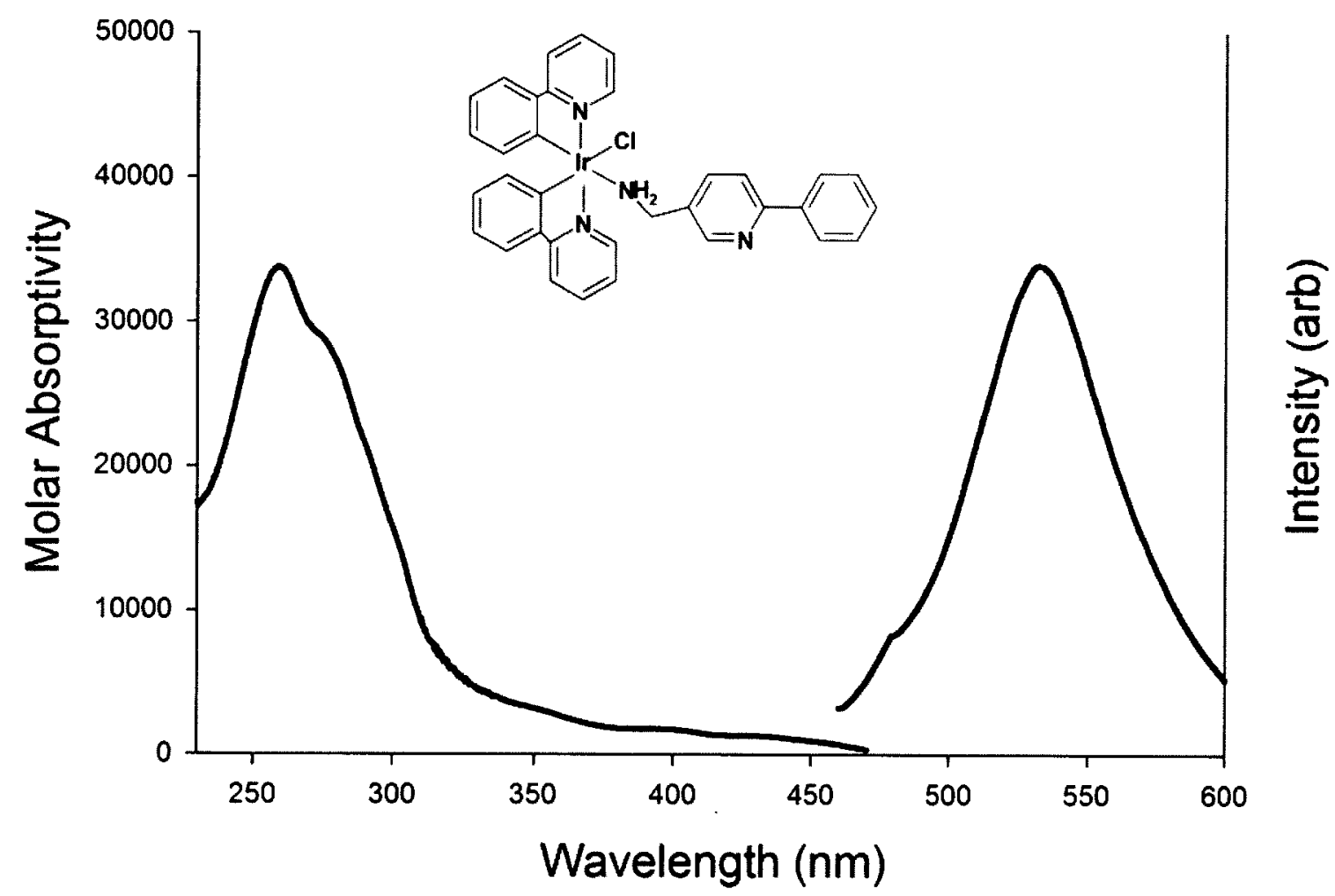

Figure 3.43: Solution electronic absorption and emission spectra of 4 . Both the absorption and emission spectra were acquired in $\mathrm{CH}_{2} \mathrm{Cl}_{2}$. 


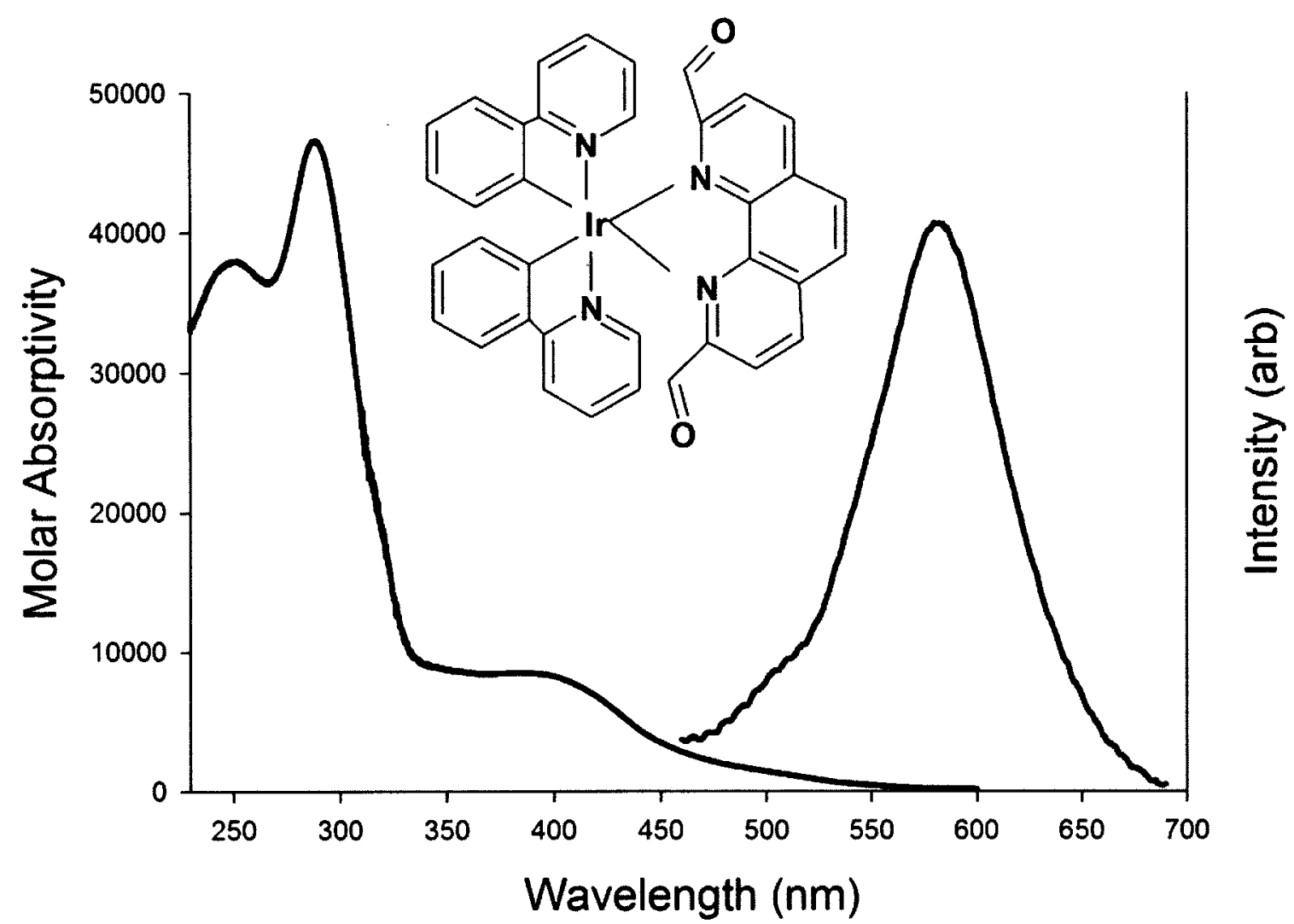

Figure 3.44: Solution electronic absorption and emission spectra of 6 . Both the absorption and emission spectra were acquired in $\mathrm{CH}_{2} \mathrm{Cl}_{2}$.

Luminescence lifetime and quantum yields for complexes 1-6 are tabulated in Table 3.9. Complex 3 has an emission quantum yield of 0.01 with $1.76 \mu$ s lifetime. The phosphorescent transition quantum yield is different from that of the fluorescent transition of C6 ligand. Higher non-radiative rate constants can be resulted from excited state reactions of the carboxylic acid group. Complex 6 has a luminescence lifetime of $2.3 \mu \mathrm{s}$ at room temperature. Complexes $\mathbf{2}$ and $\mathbf{3}$ were found to be the most favorable luminophores, according to the lifetime and quantum yield data. Among all complexes, 2 has the longest lifetime and highest emission quantum yield. For $\mathbf{3}$, its strong absorbance in the visible region means that efficient excitation is possible using longwavelength radiation. 
Table 3.9: Luminescence quantum yields and lifetimes for complexes 1-7

\begin{tabular}{|c|c|c|c|c|c|c|c|}
\hline & $\mathbf{1}^{\mathbf{a}}$ & $\mathbf{2}$ & $\mathbf{3}$ & $\mathbf{4}$ & $\mathbf{5}^{\mathbf{b}}$ & $\mathbf{6}$ & $\mathbf{7}^{\mathbf{c}}$ \\
\hline$\phi$ & 0.28 & 0.35 & 0.01 & 0.004 & 0.05 & 0.007 & 0.006 \\
\hline$\tau(\mu \mathrm{s})$ & 35.6 & 17.3 & 1.76 & 0.20 & 0.9 & 2.3 & 144 \\
\hline
\end{tabular}

${ }^{\mathrm{a}}$ from reference $75,{ }^{\mathrm{b}}$ from reference $68,{ }^{\mathrm{c}}$ from reference 81

\subsection{Other Water-Based PSP Formulations}

\subsubsection{Criteria for Attachment}

Comparing water-soluble polymer bound oxygen sensors with dispersions of the luminophores in water-soluble polymers, the altered behavior proved the bindings between luminophores and polymers. For example, polymer bound luminophores are water-soluble, whereas trying to dissolve polymer dispersed luminophores in water, crystallized the luminophores out of solutions. Moreover, electronic absorption and emission, and oxygen sensitivity of the polymer bound sensors would be different from dispersions. 


\subsubsection{Synthesis of 9 by Coupling of 3 to Silamine D208-EDA and Spectroscopic Characterization}

The covalent attachment of complex 3 to Silamine D208-EDA occurred at room temperature by the use of coupling agent dicyclohexylcarbodiimide. ${ }^{65}$ The coupling agent has been often used in peptide synthesis ${ }^{94}$ and its mechanism is shown below:

Step 1

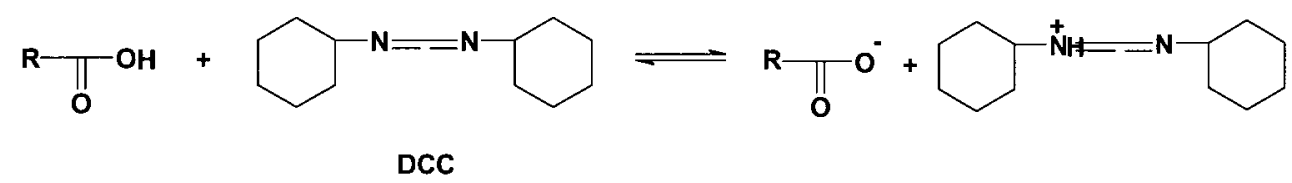

Step 2<smiles></smiles><smiles>[R]C(=O)O/C(=N/C1CCCCC1)NC1CCCCC1</smiles>

Step 3
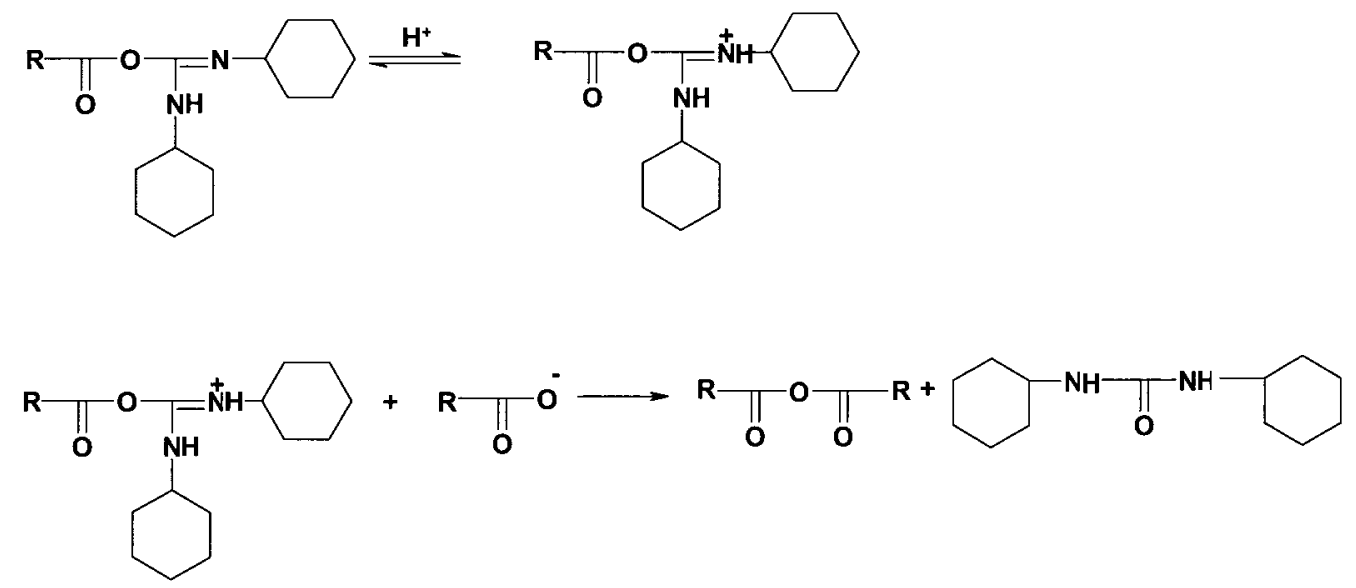

Step 5

$$
\mathrm{R}-\prod_{\mathrm{O}} \mathrm{O}-\prod_{\mathrm{O}} \mathrm{R}+\mathrm{R}^{\prime} \mathrm{NH}_{2} \longrightarrow \mathrm{R} \prod_{\mathrm{O}} \mathrm{NHR}^{\prime}+\mathrm{R} \prod_{\mathrm{O}} \mathrm{OH}
$$

The acid anhydride of $\mathbf{3}$, as shown in Step 5 reacts with the amine groups of the Silamine polymer, giving 9. Using the method explained in section 3.4 .2 , the attachment yield of the orange polymer was $83 \%$. 
9 displays room temperature absorption and emission spectra shown in Figure 3.45. The Silamine attachment causes only subtle changes in the photophysical characteristics of the luminophore, suggesting that the carboxylic group of the acacacid does not influence the excited state.
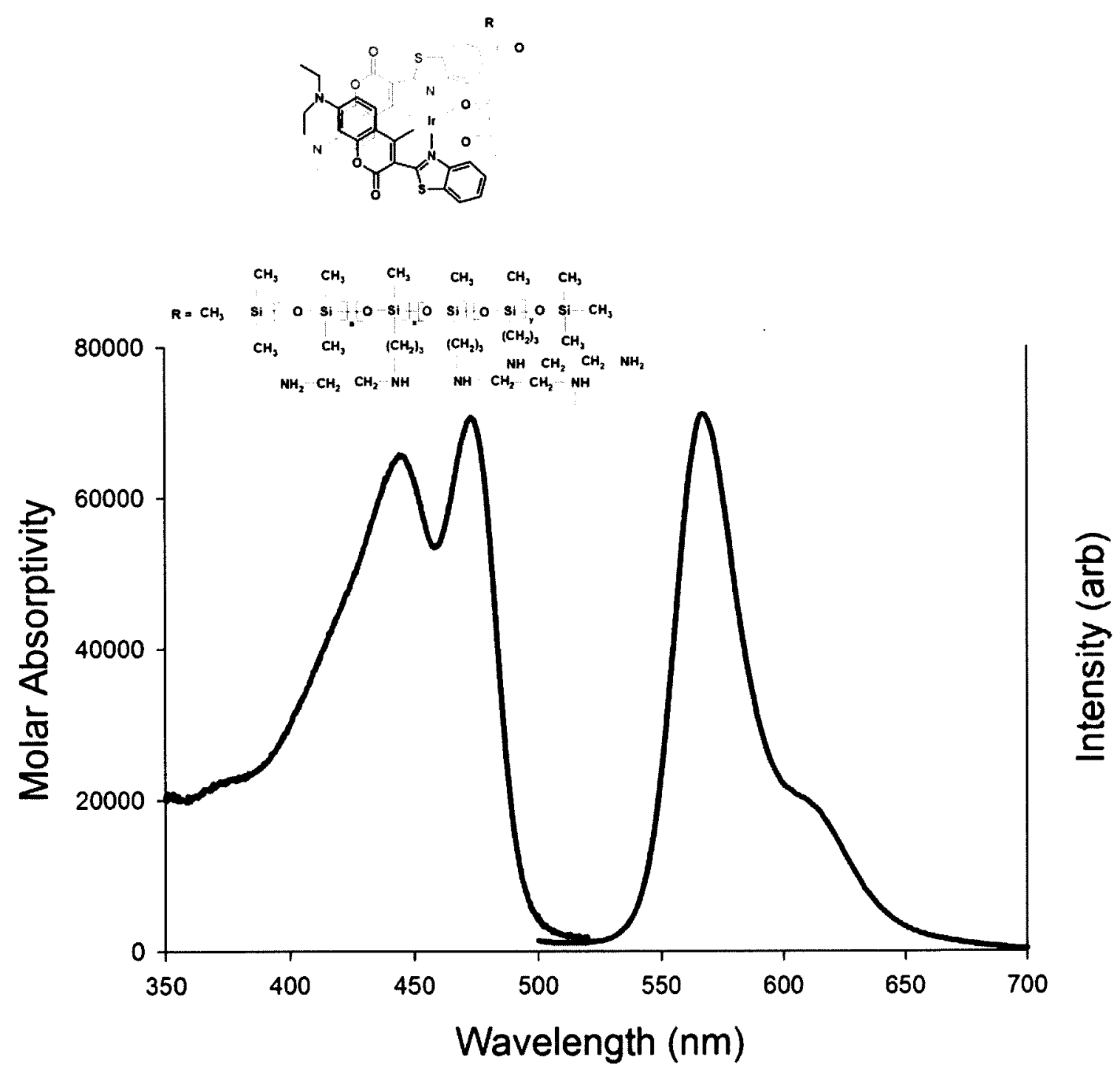

Figure 3.45: Solution electronic absorption and emission spectra of 9. Both spectra were acquired in $\mathrm{CH}_{2} \mathrm{Cl}_{2}$. For 9 , the extinction coefficient at $\lambda=445 \mathrm{~nm}$ was assumed to be identical with that of 3 at $447 \mathrm{~nm}$. See Table 3.8 data. 


\subsubsection{Preparation and Spectroscopic Characterization of 10}

The dispersion of complex 4 in Silube CS-1 was tried in order to develop watersoluble luminophore 10. The formulation of $\mathbf{1 0}$ is shown in Scheme 3.13.

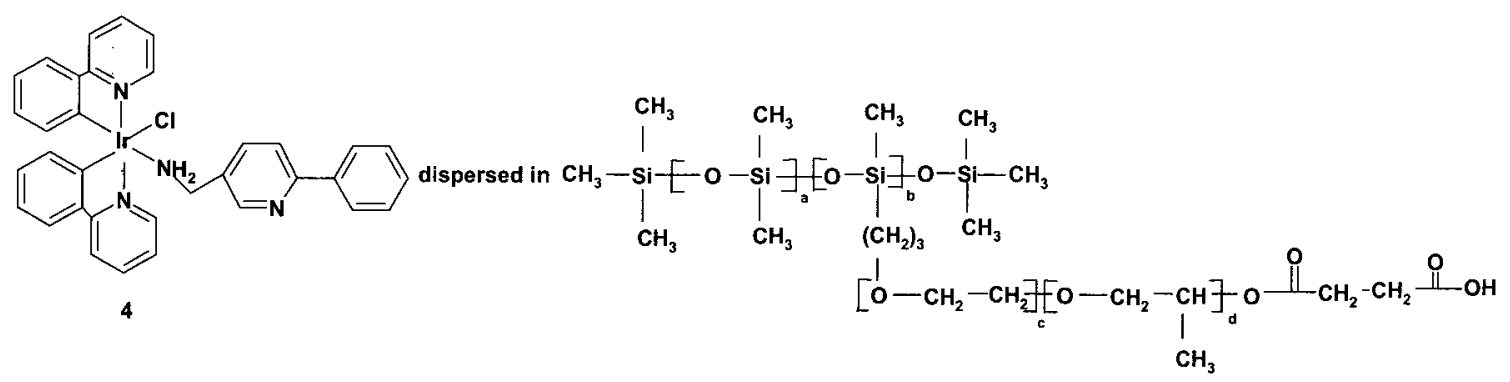

Scheme 3.13: Dispersing 4 in Silube CS-1 to make 10.

The absorption and emission spectra of $\mathbf{1 0}$ are shown in Figure 3.46. The intense absorption band in ultraviolet region at $255 \mathrm{~nm}$ is assigned to intraligand transition while absorption bands at $396 \mathrm{~nm}$ and $457 \mathrm{~nm}$ can be assigned to ${ }^{1}$ MLCT and ${ }^{3}$ MLCT transitions, respectively. A single broad band is observed in the solution emission spectrum of 10 with a maximum at $525 \mathrm{~nm}$. 


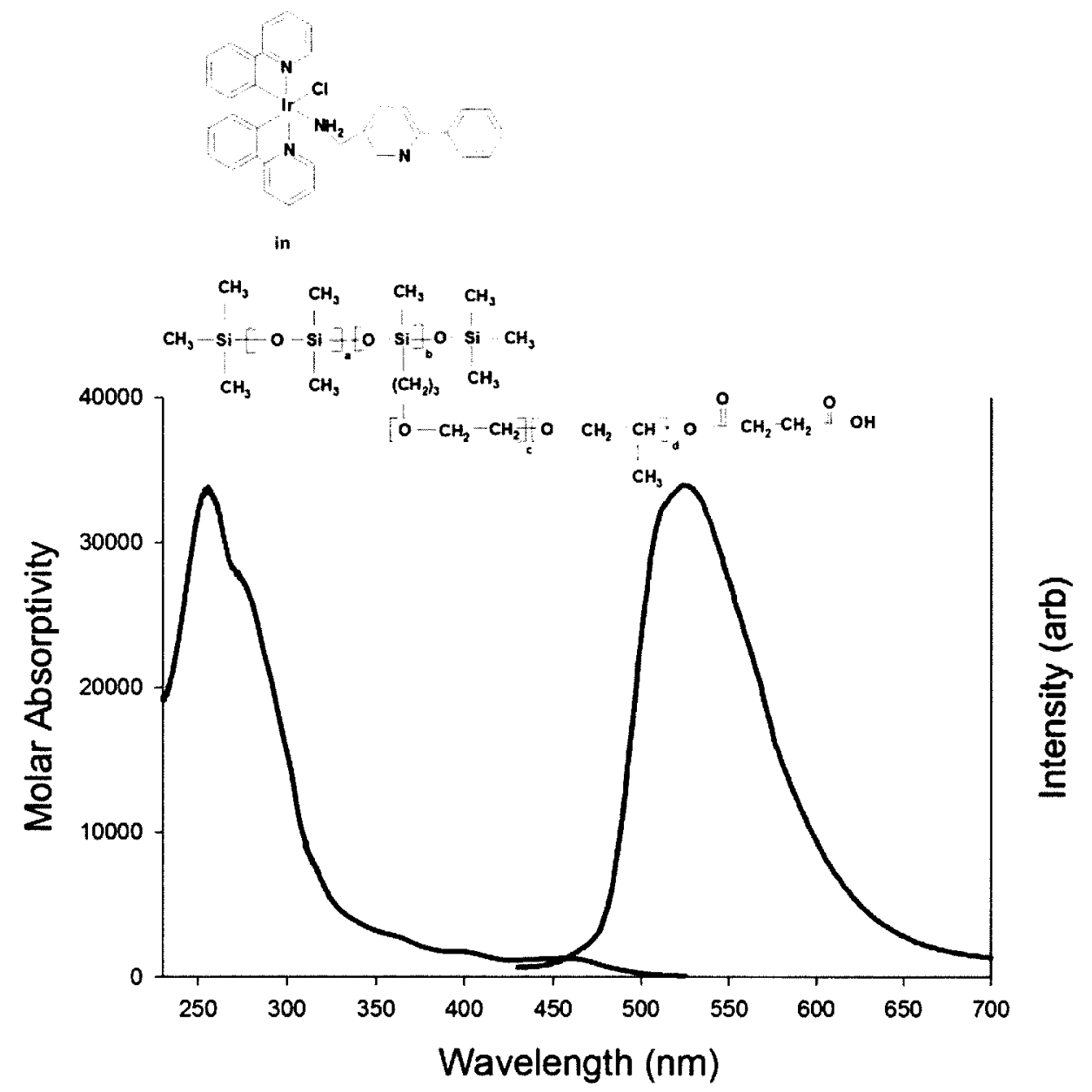

Figure 3.46: Solution electronic absorption and emission spectra of 10. Both spectra were acquired in $\mathrm{CH}_{2} \mathrm{Cl}_{2}$.

\subsubsection{Synthesis of 12 by Coupling of 5 to Silamine D208-EDA and Spectroscopic Characterization}

In order to study 5 in a water-soluble formulation, it was bound to Silamine as illustrated in Scheme 3.14. An imine is formed by the reaction between amine functionalized polymer and the aldehyde group followed by an elimination of water. 
<smiles>C[Si](C)(C)O[Si](C)(C)OC[Si](C)(C)O[Si](C)(C)C[Si](C)(C)O[Si](C)(C)CNCCN</smiles>

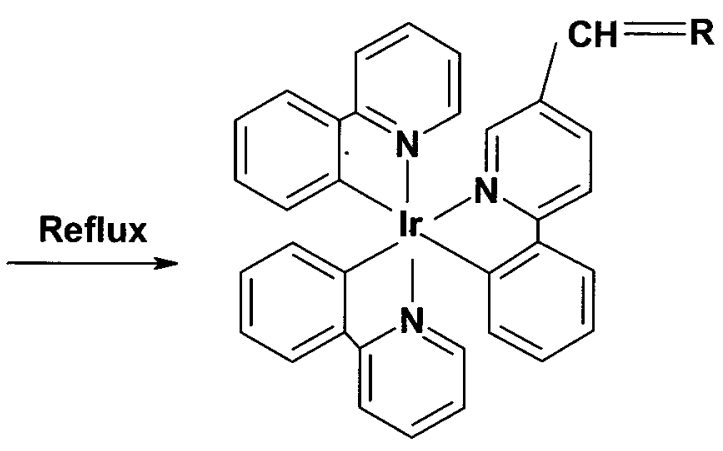

12

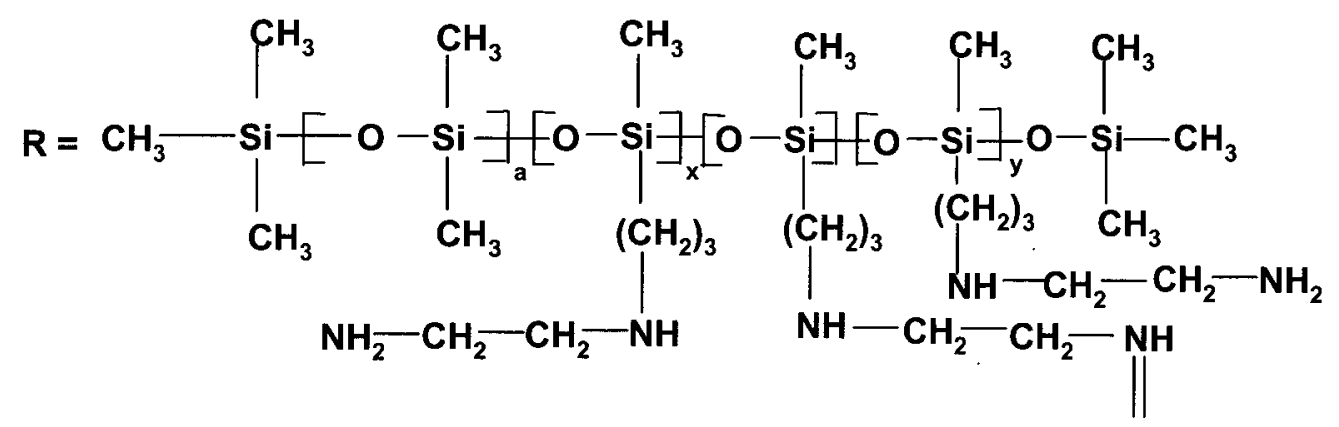

Scheme 3.14: Coupling reaction of 5 with Silamine D208-EDA to make 12.

Attachment yield of the yellow polymer was calculated, (section 3.4.2), to be $97 \%$.

The absorption and emission spectra of 12 are shown in Figure 3.47. The intraligand transition appears as an intense absorption band in ultraviolet region at 285 
$\mathrm{nm}$ while MLCT transitions are shown as transitions between 360 and $490 \mathrm{~nm}$. The emission is displayed at $528 \mathrm{~nm}$.

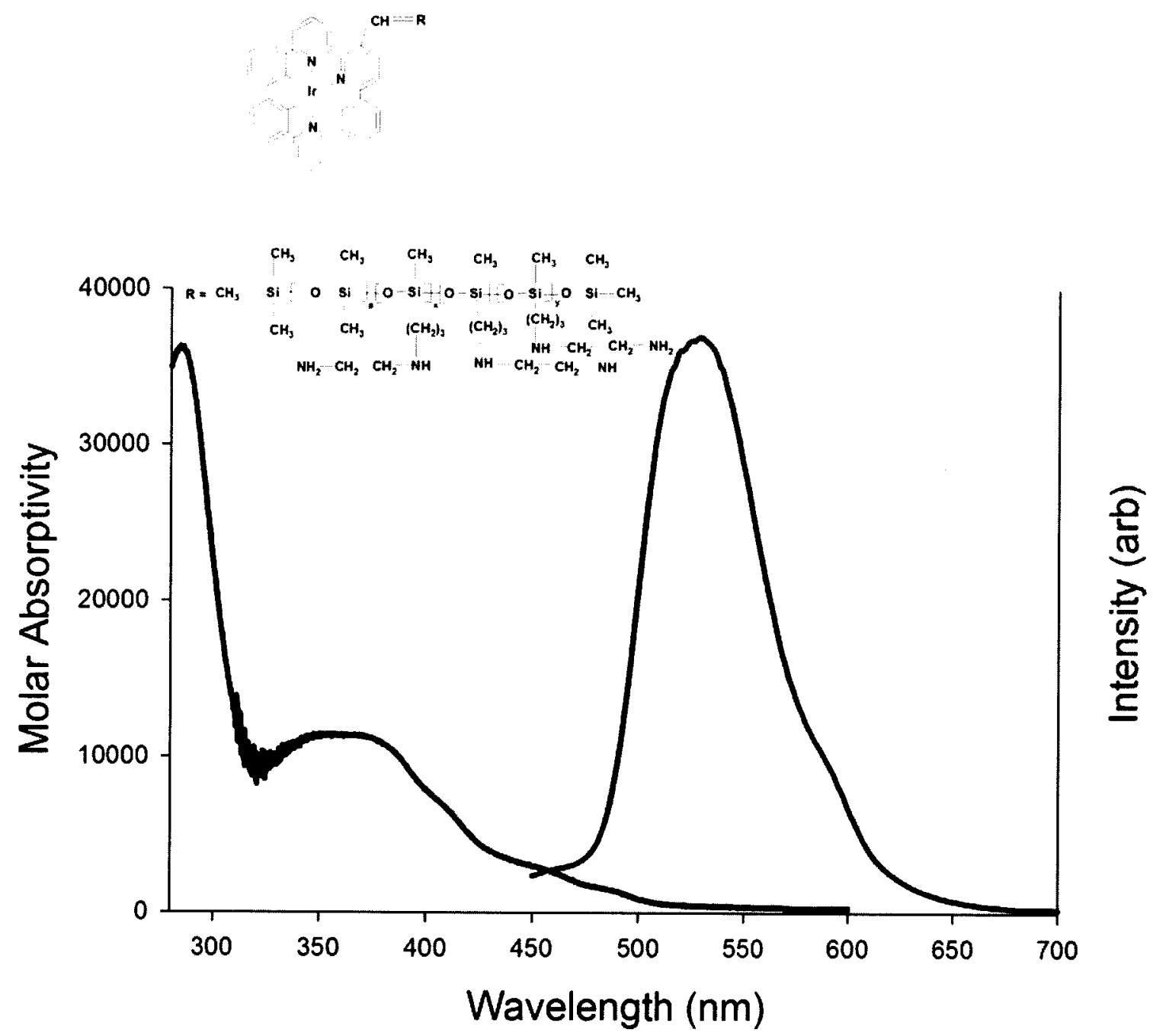

Figure 3.47: Solution electronic absorption and emission spectra of 12. Both spectra were acquired in $\mathrm{CH}_{2} \mathrm{Cl}_{2}$. For 12, the extinction coefficient at $\lambda=285 \mathrm{~nm}$ was assumed to be identical with that of $\mathbf{5}$ at $290 \mathrm{~nm}$. ${ }^{68}$ 
3.8.5 Synthesis of 13 by Reductive Attachment of 5 to Silamine D208-EDA and Spectroscopic Characterization

The luminophore-functionalized Silamine, 13, was produced by reductive amination reaction between $\mathbf{5}$ and the water-soluble amine-functionalized Silamine D208-EDA, as shown in Scheme 3.15. 
<smiles></smiles><smiles>C[Si](C)(C)CO[Si](C)(C)O[Si](C)(C)O[Si](C)(C)CNCCN</smiles>

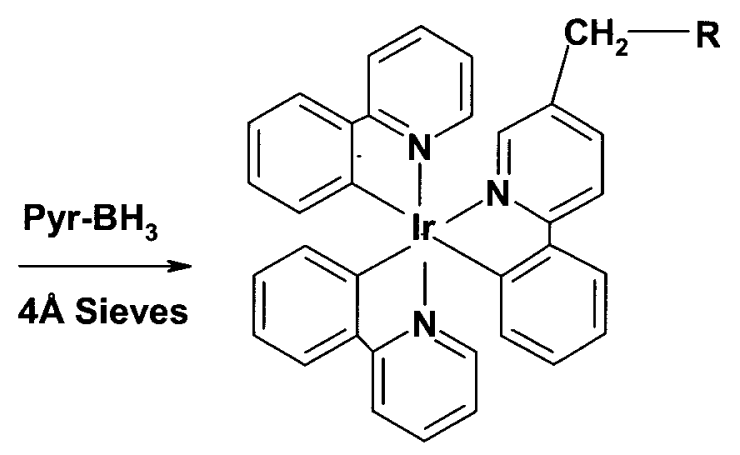

13

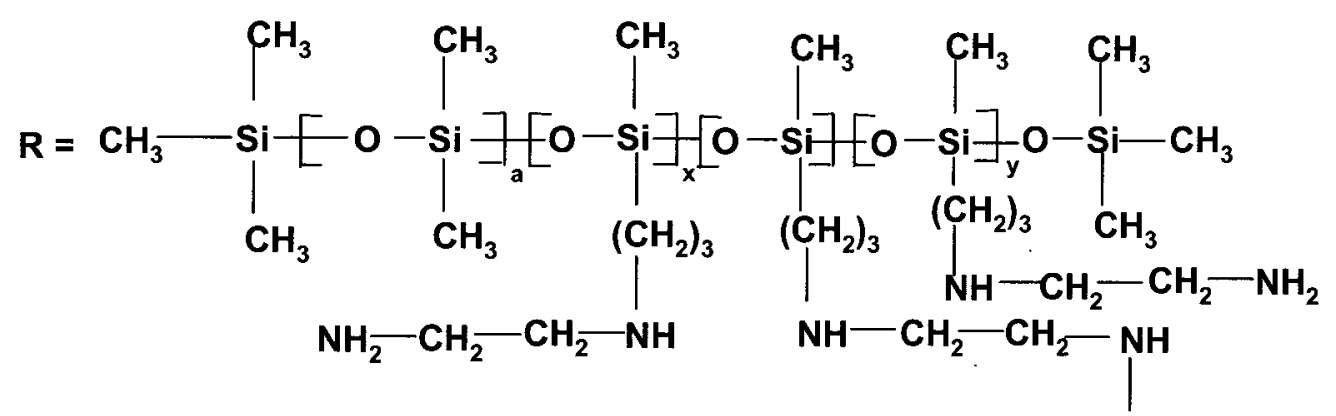

Scheme 3.15: Reductive amination of 5 with Silamine D208-EDA to make 13.

Using the method explained in section 3.4.2, the attachment yield of the yellow polymer was $96 \%$.

The electronic absorption and emission spectra of $\mathbf{1 3}$ are similar to those of $\mathbf{5}^{68}$ and 12 and are shown in Figure 3.48. 


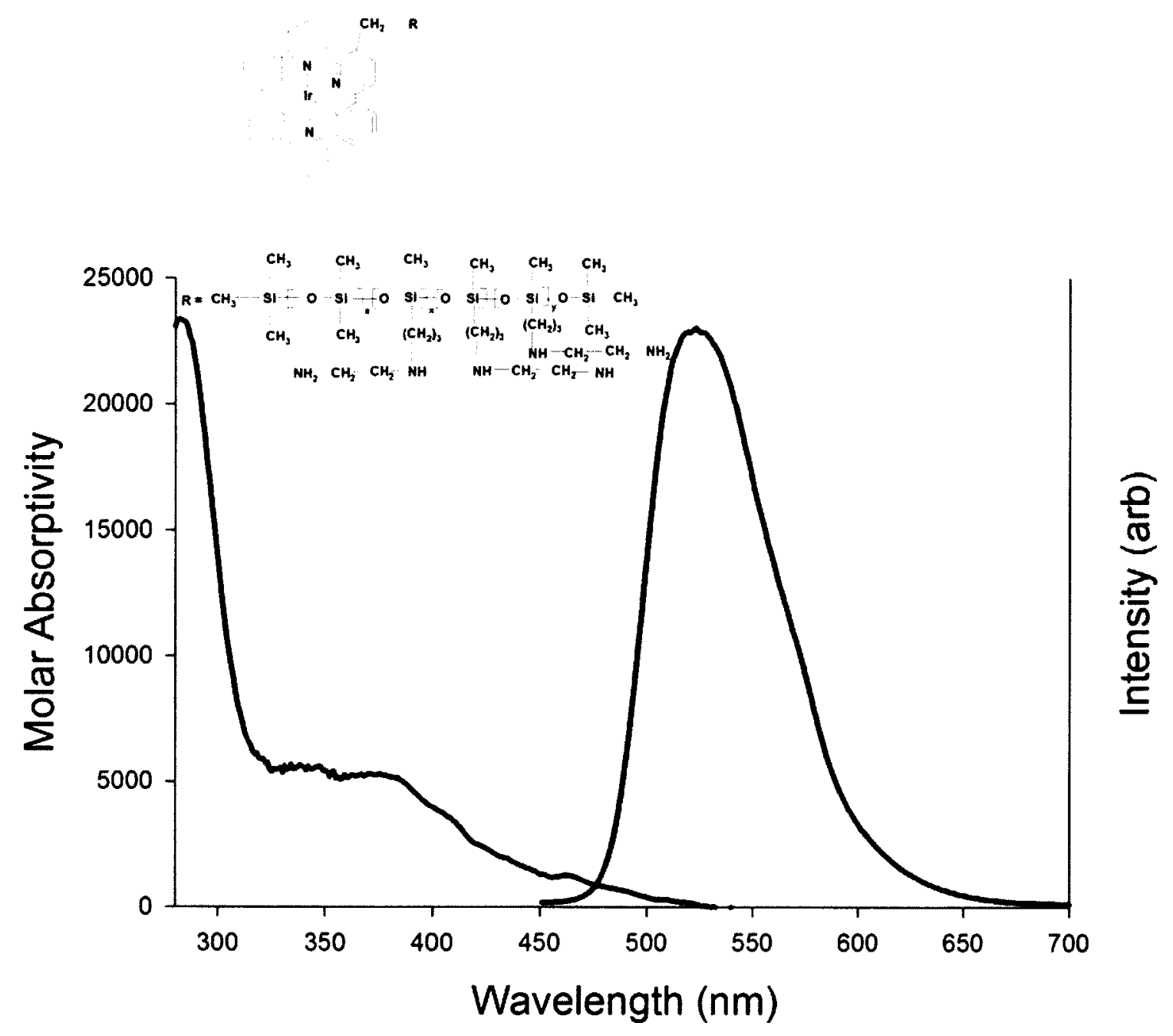

Figure 3.48: Solution electronic absorption and emission spectra of 13. Both spectra were acquired in $\mathrm{CH}_{2} \mathrm{Cl}_{2}$. For 13 , the extinction coefficient at $\lambda=282 \mathrm{~nm}$ was assumed to be identical with that of 5 at $290 \mathrm{~nm}^{68}$

3.8.6 Synthesis of 14 by Reductive Attachment of 6 to Silamine D208-EDA and Spectroscopic Characterization

Reductive amination reaction between 6 and the water-soluble aminefunctionalized Silamine D208-EDA gave the luminophore-functionalized Silamine, 14, as shown in Scheme 3.16. 


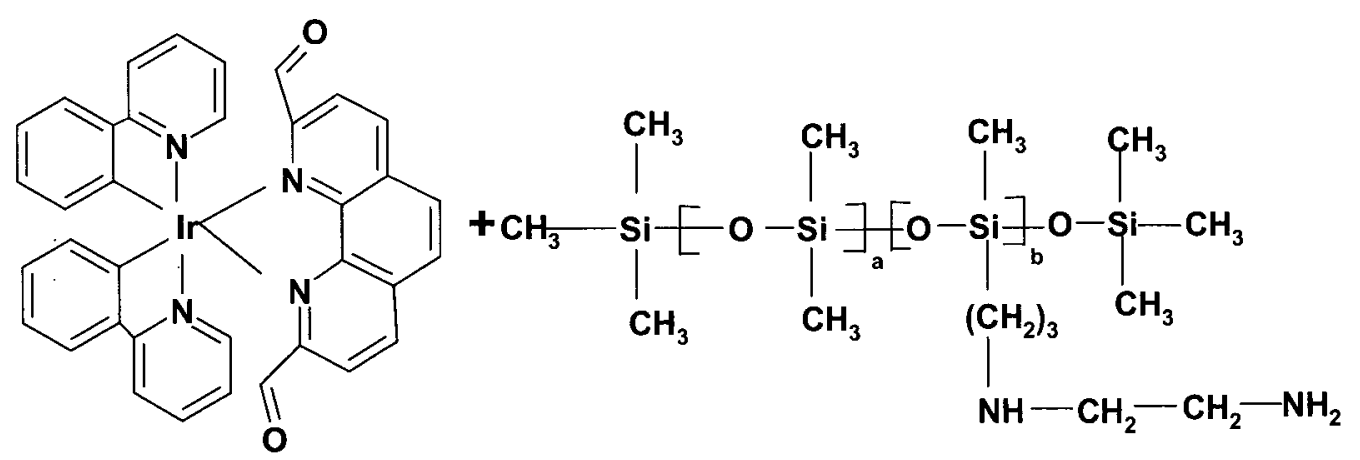<smiles></smiles>

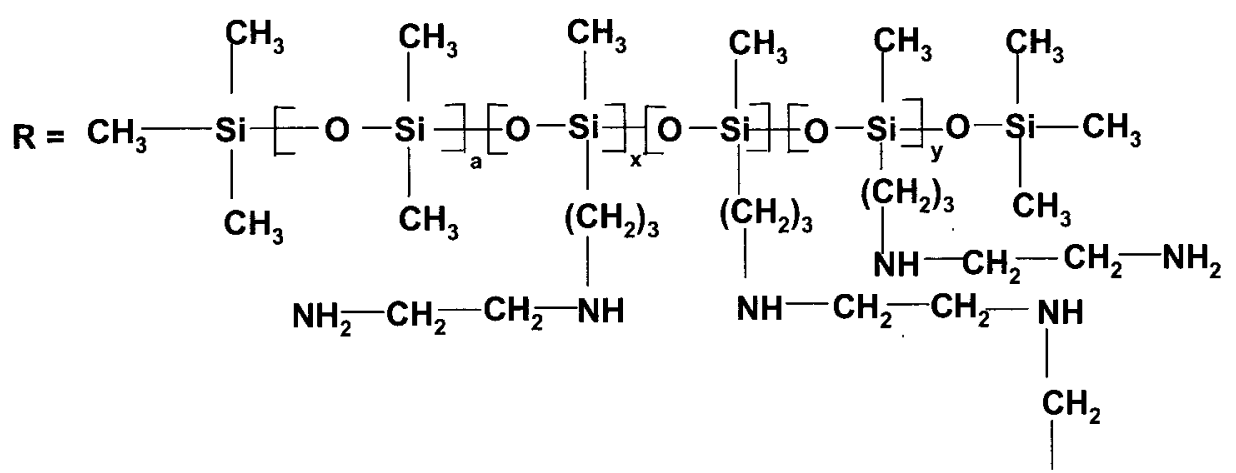<smiles>[O]C=O</smiles>

Scheme 3.16: Reductive amination of 6 with Silamine D208-EDA to make 14.

The attachment yield of the orange polymer was calculated, (see section 3.4 .2 ) to be $63 \%$. 
As shown in Scheme 3.16, it is possible for 6 to cross-link Silamine chains, or bind to a single Silamine chain by one or two methyl-amine links.

Figure 3.49 shows the electronic absorption and emission spectra of 14. This water-soluble polymer-bound luminophore exhibits an intense high-energy absorption band at $269 \mathrm{~nm}$ which is assigned to intraligand $\pi-\pi^{*}$ transitions. Spin-allowed MLCT transitions appear as a weaker absorption shoulder at $400 \mathrm{~nm}$ while spin-forbidden MLCT transitions are displayed as a weaker absorption tail around 478-496 nm. At room temperature, $\mathbf{1 4}$ in dichloromethane solution shows a broad orange-yellow luminescence at $504 \mathrm{~nm}$ with a low-energy shoulder at $527 \mathrm{~nm}$ which is assigned to be originating from a MLCT triplet excited state involving low-lying $\pi^{*}$ orbitals of the coordinated phendiald ligand as the acceptor orbital. ${ }^{92}$ The emission spectrum does not vary when the excitation wavelength is changed in the region above $360 \mathrm{~nm}$, suggesting that only the single, lower energy component is excited at these wavelengths. 


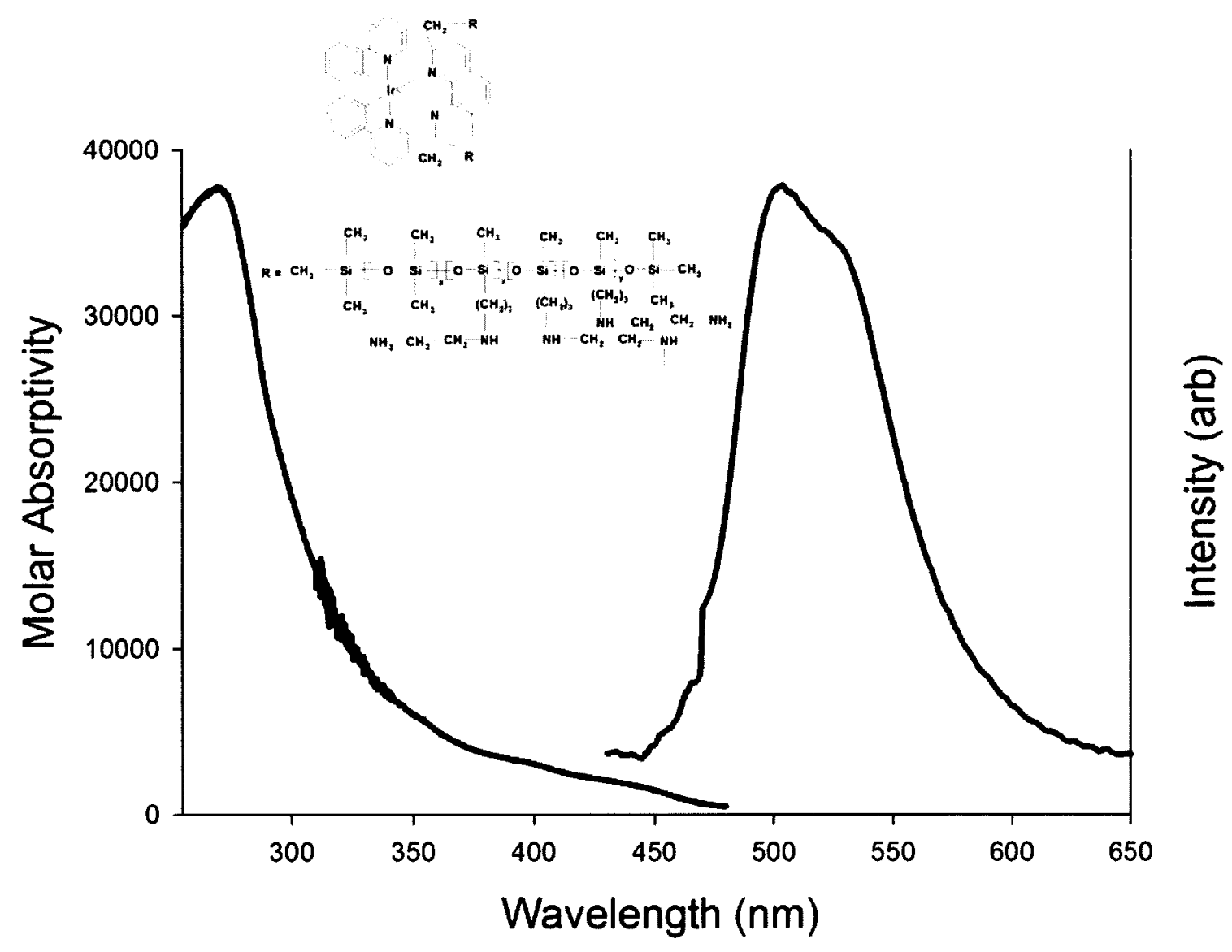

Figure 3.49: Solution electronic absorption and emission spectra of 14. Both spectra were acquired in $\mathrm{CH}_{2} \mathrm{Cl}_{2}$. For 14, the extinction coefficient at $\lambda=269 \mathrm{~nm}$ was assumed to be identical with that of 6 at $257 \mathrm{~nm}$. See Table 3.8 data.

\subsubsection{Synthesis of 15 by Coupling of 1-Pyrenebutyric Acid (PBA) to Silamine D208-}

\section{EDA and Spectroscopic Characterization}

Pyrene-based PSPs have lower temperature sensitivities compared with other PSPs. ${ }^{20 \mathrm{~b}}$ However, pyrene sublimation is a disadvantage. ${ }^{95}$ Since Silamine has a large number of amine functionalities, an appropriate concentration of pyrene can be obtained by coupling it with PBA in order to acquire excimer emission which is more sensitive to oxygen compared with monomer emission. In addition, the sublimation of pyrene is 
prevented by polymer attachment. The coupling reaction was used for acquiring $\mathbf{1 5}$, in the same manner utilized for synthesizing 9, and is shown in Scheme 3.17.
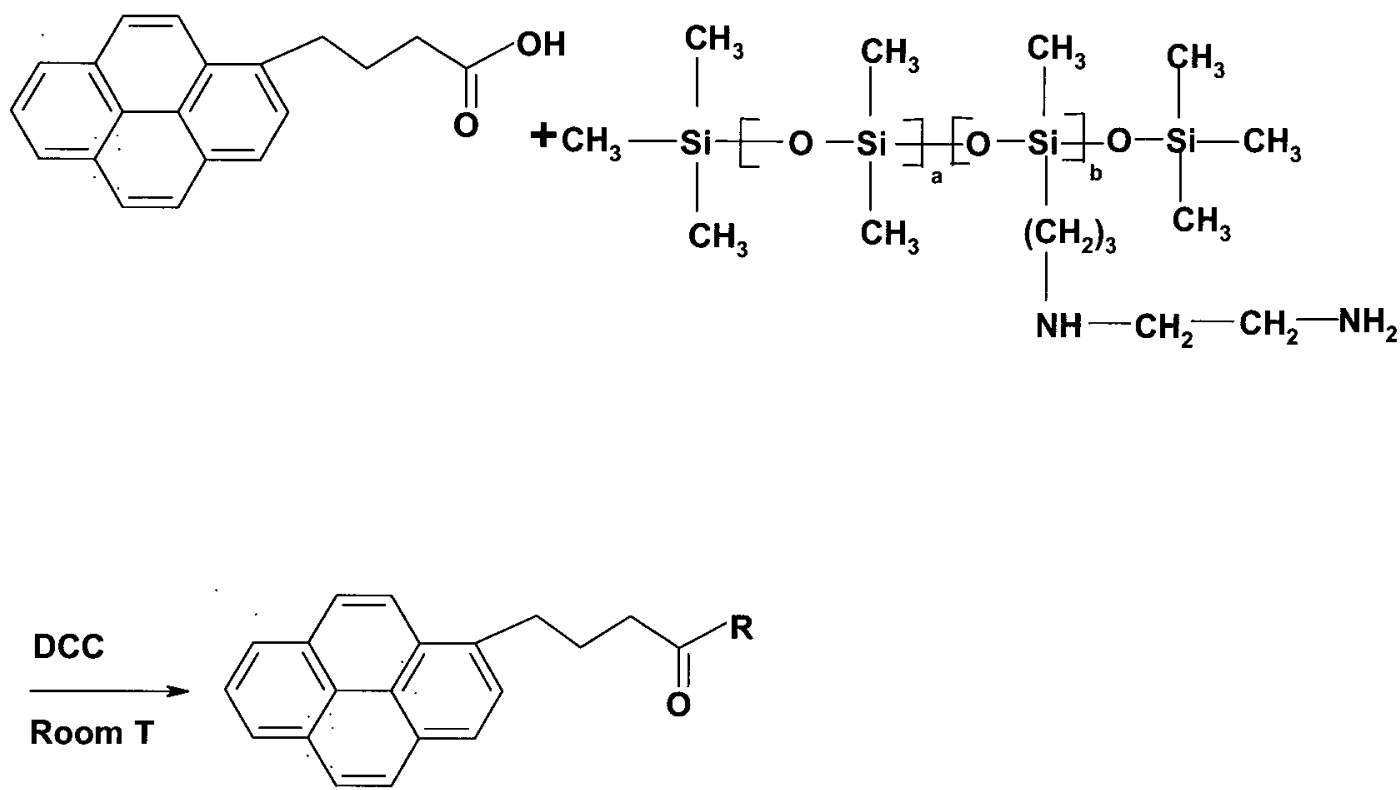

15

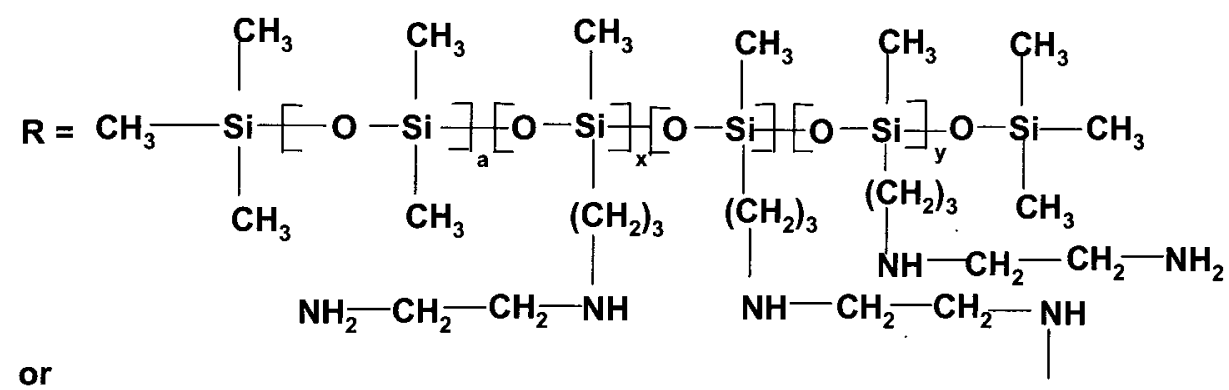

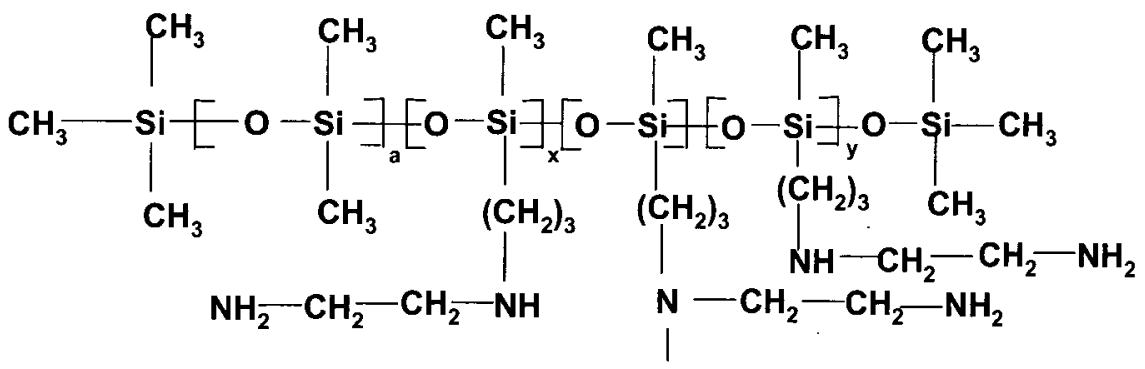

Scheme 3.17: Coupling reaction of PBA with Silamine to make 15. 
Attachment yield of the colourless polymer was calculated, as explained in section 3.4.2. and indicated a quantitative attachment of PBA to Silamine.

The absorption and emission spectra of $\mathbf{1 5}$ are shown in Figure 3.50. The $\alpha$-band appears at $345 \mathrm{~nm}$ and the $p$-bands are shown in ultraviolet region at 267, 277, and 329 nm. 15 shows two characteristic emission peaks: a blue monomer fluorescence in solution at 381 , and $399 \mathrm{~nm}$ and excimer peak at $479 \mathrm{~nm}$. 


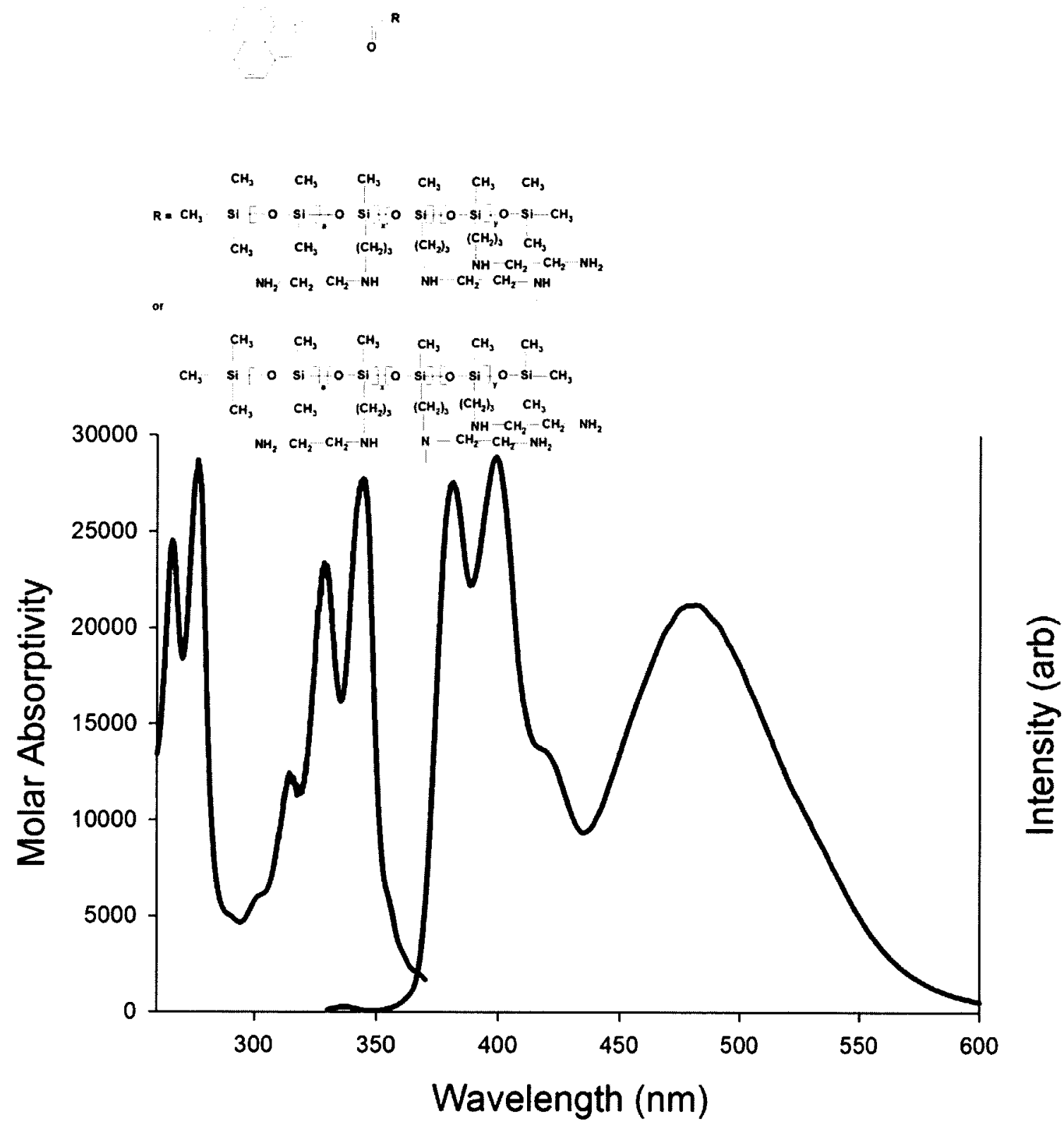

Figure 3.50: Solution electronic absorption and emission spectra of 15. Both spectra were acquired in $\mathrm{CH}_{2} \mathrm{Cl}_{2}$. For 15, the extinction coefficient at $\lambda=344 \mathrm{~nm}$ was assumed to be identical with that of PBA at $341 \mathrm{~nm}^{96}$

\subsubsection{Electronic Spectroscopy}

The room-temperature UV-Vis and emission data of $\mathbf{8 - 1 5}$ are given in Table 3.10 and the luminescence lifetimes and quantum yields for all of luminophores 8-15 are 
listed in Table 3.11. Comparing each polymer bound oxygen sensor with the original dye, (8 and 2,9 and 3,12 and 5,13 and 5, 14 and 6, compare Tables 3.11 and 3.9), reveals that upon attachment to a polymer, each luminophore shows an increase in excited state lifetime. Interactions of excited state with the functional groups can give rise to non-radiative decay which might have been reduced in case of attached dyes. The increase in lifetime can also originate from a decrease in the dyes motion in the polymeric matrix. In 10, the luminophore is not attached to the polymer and in $\mathbf{1 4}$, the presence of the unreacted aldehyde functional groups can still result in non-radiative decay. 


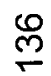

$=$ i i

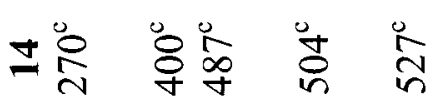

in $\stackrel{t}{0}$

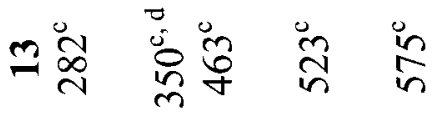

$\pm \quad \begin{array}{ll}0 \\ 0\end{array}$

ฯ

행ㅇㅇ

$\frac{10}{\infty}$

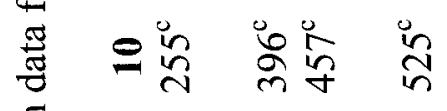

苛

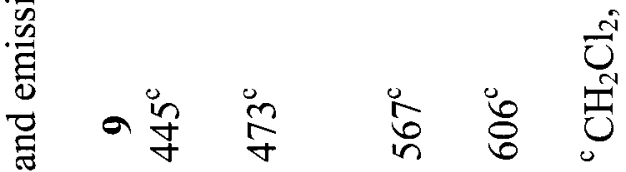

产

营 तेmm

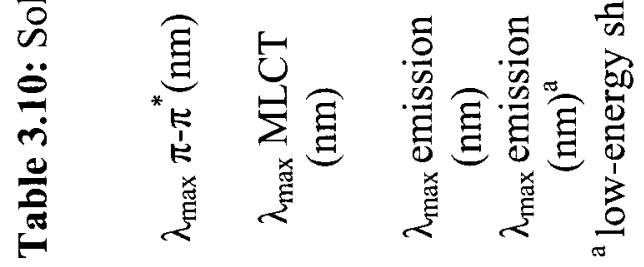

몽

¿্

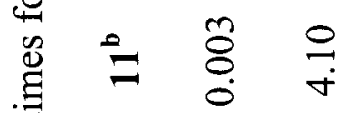

$=\begin{array}{lll} & & 0 \\ 0 & 0 & 0 \\ 0 & 0 \\ 0 & 0 & 0 \\ 0 & 0 & 0 \\ 0 & 0 & 0 \\ 0 & 0 & 0 \\ 0\end{array}$

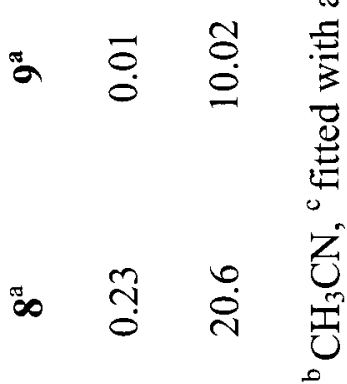

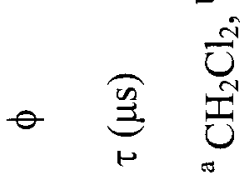


The effect of matrix on thin film luminescence of $\mathbf{9}, \mathbf{1 0}, \mathbf{1 2}, \mathbf{1 3}$, and 14 is presented in Figures 3.51-3.55. Small variation in thin film emission spectrum due to matrix change is displayed.

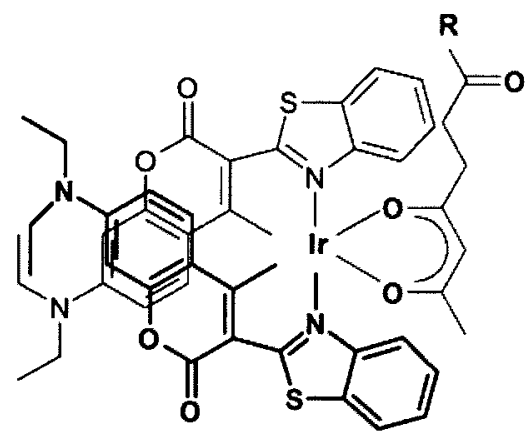

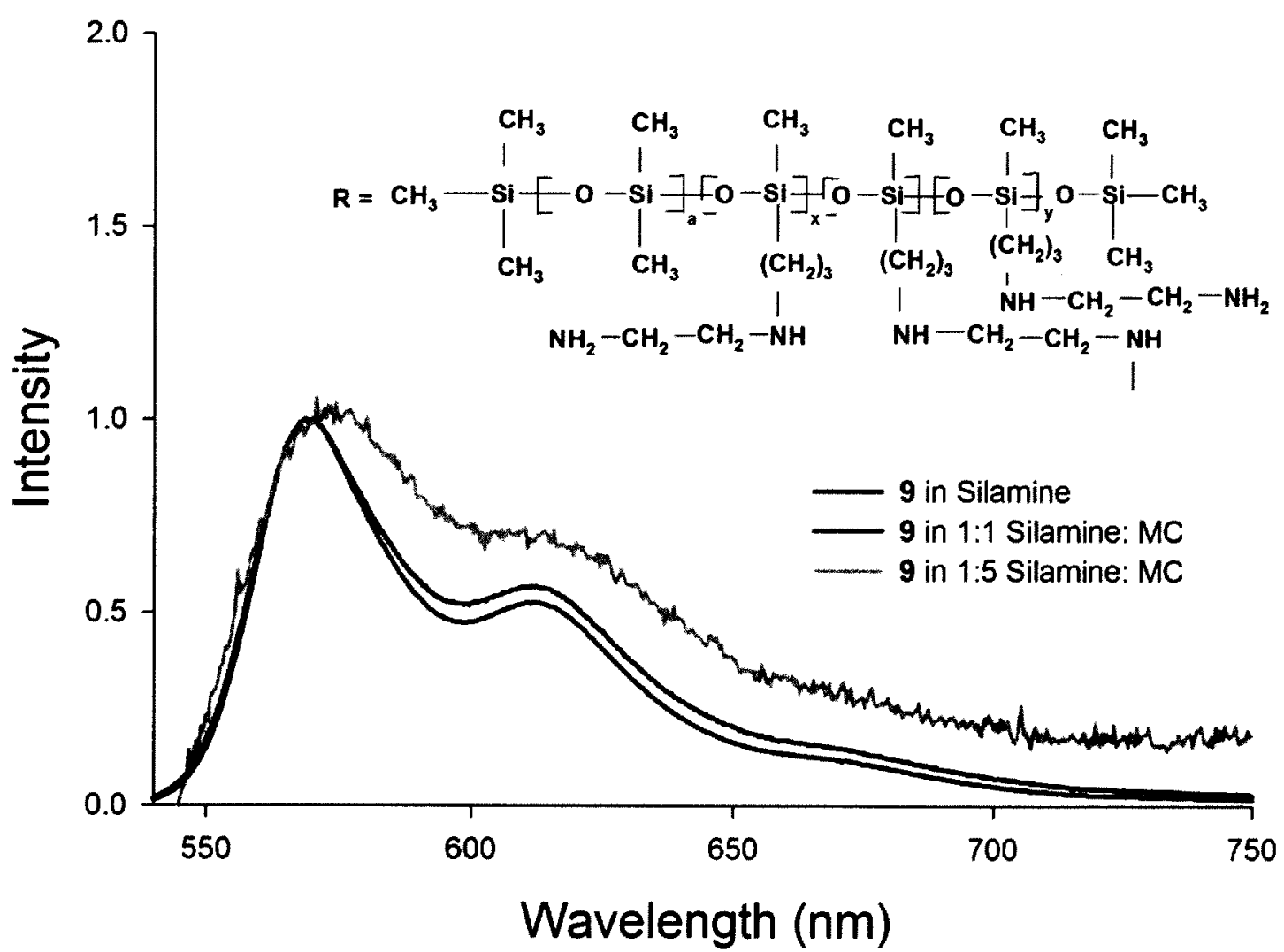

Figure 3.51: Effect of matrix on thin film luminescence of 9. Matrices compositions are: Silamine (black), 1:1 Silamine: MC (red), and 1:5 Silamine: MC (green). For each formulation, the spectrum at $14.7 \mathrm{psi}$ is shown, which is normalized to its maximum emission intensity. 


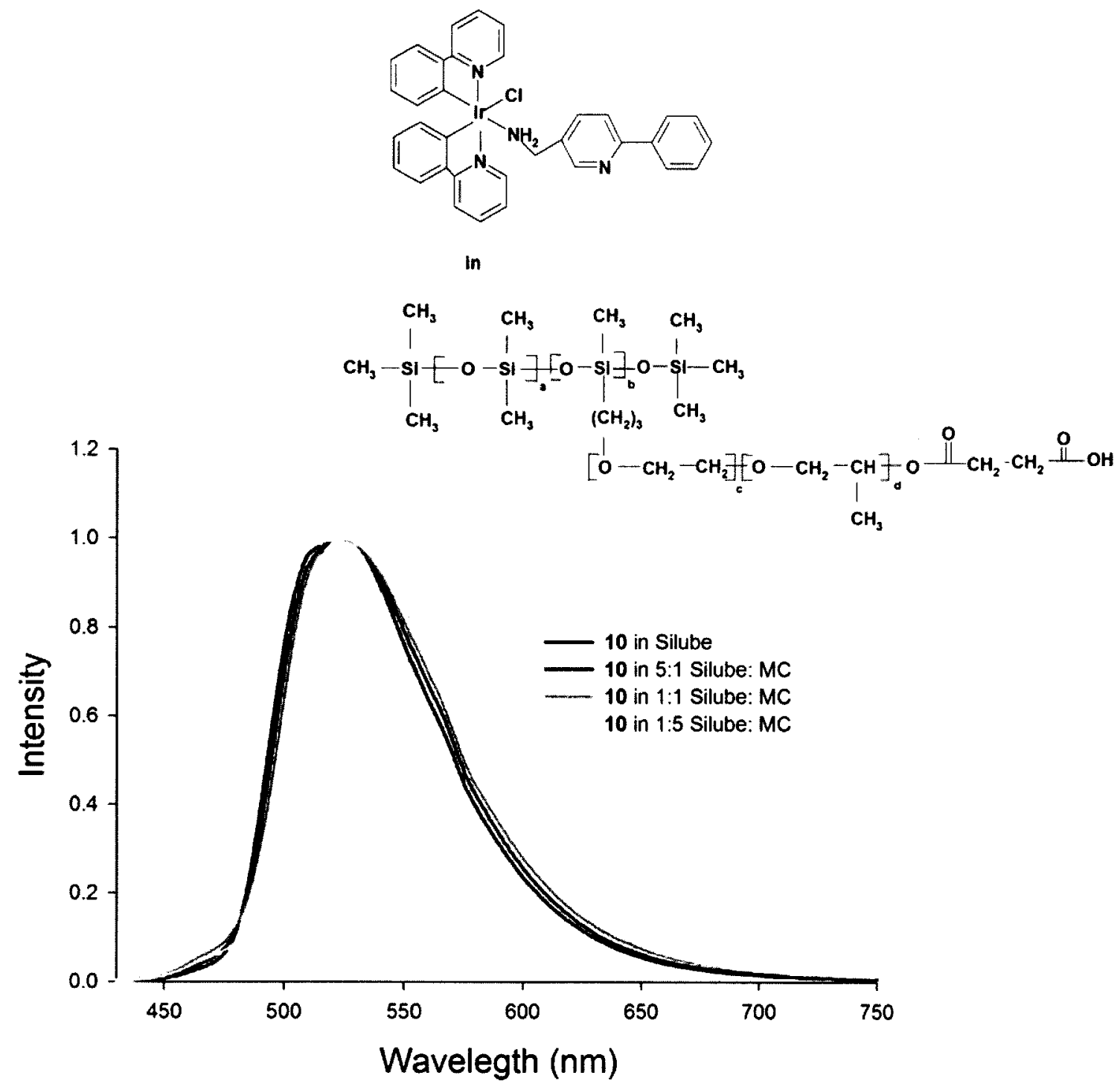

Figure 3.52: Effect of matrix on thin film luminescence of 10. Matrices compositions are: Silube (black), 5:1 Silube: MC (red), 1:1 Silube: MC (green), and 1:5 Silube: MC (yellow). For each formulation, the spectrum at $14.7 \mathrm{psi}$ is shown, which is normalized to its maximum emission intensity. 


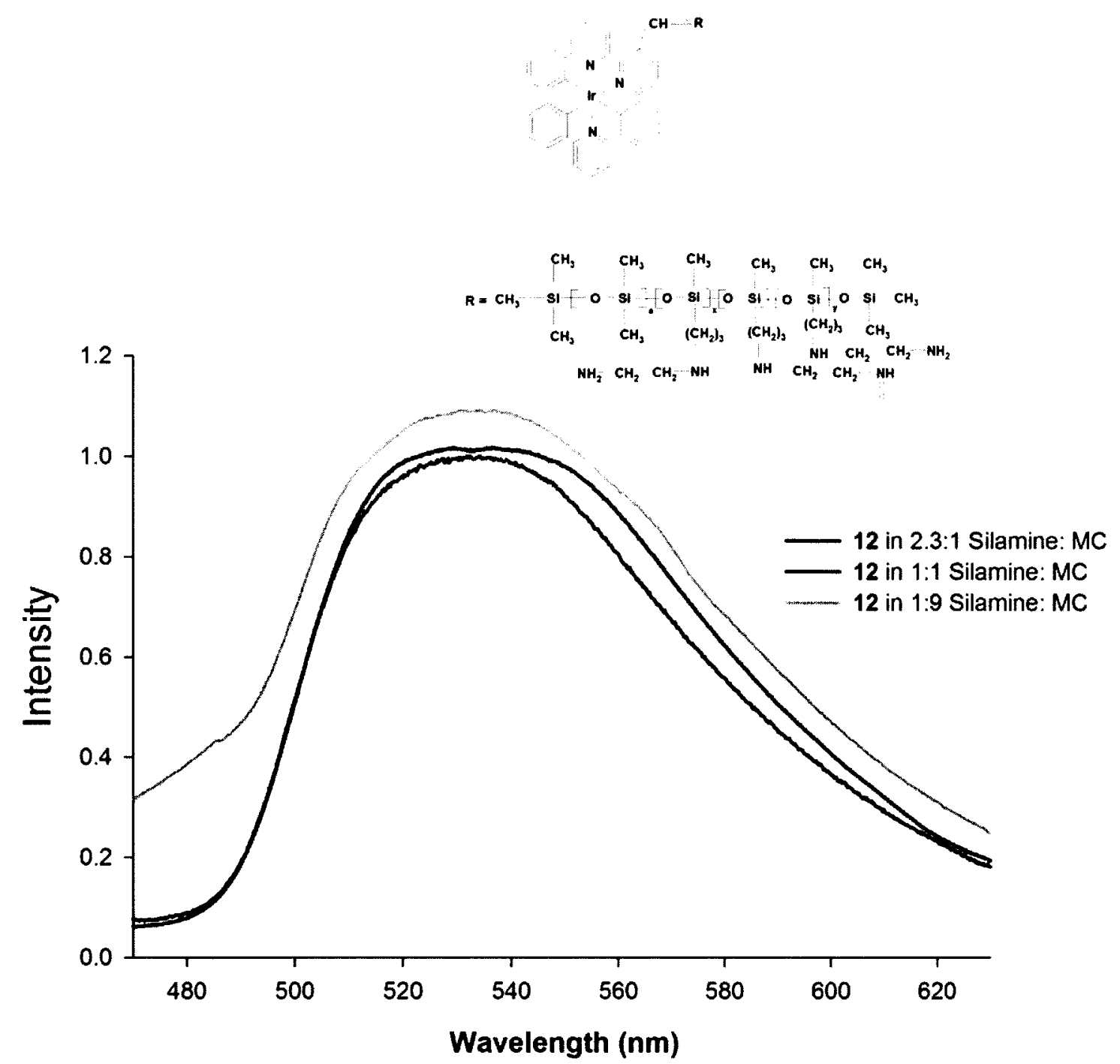

Figure 3.53: Effect of matrix on thin film luminescence of 12. Matrices compositions are: 2.3:1 Silamine: MC (black), 1:1 Silamine: MC (red), and 1:9 Silamine: MC (green). For each formulation, the spectrum at $14.7 \mathrm{psi}$ is shown, which is normalized to its maximum emission intensity. 

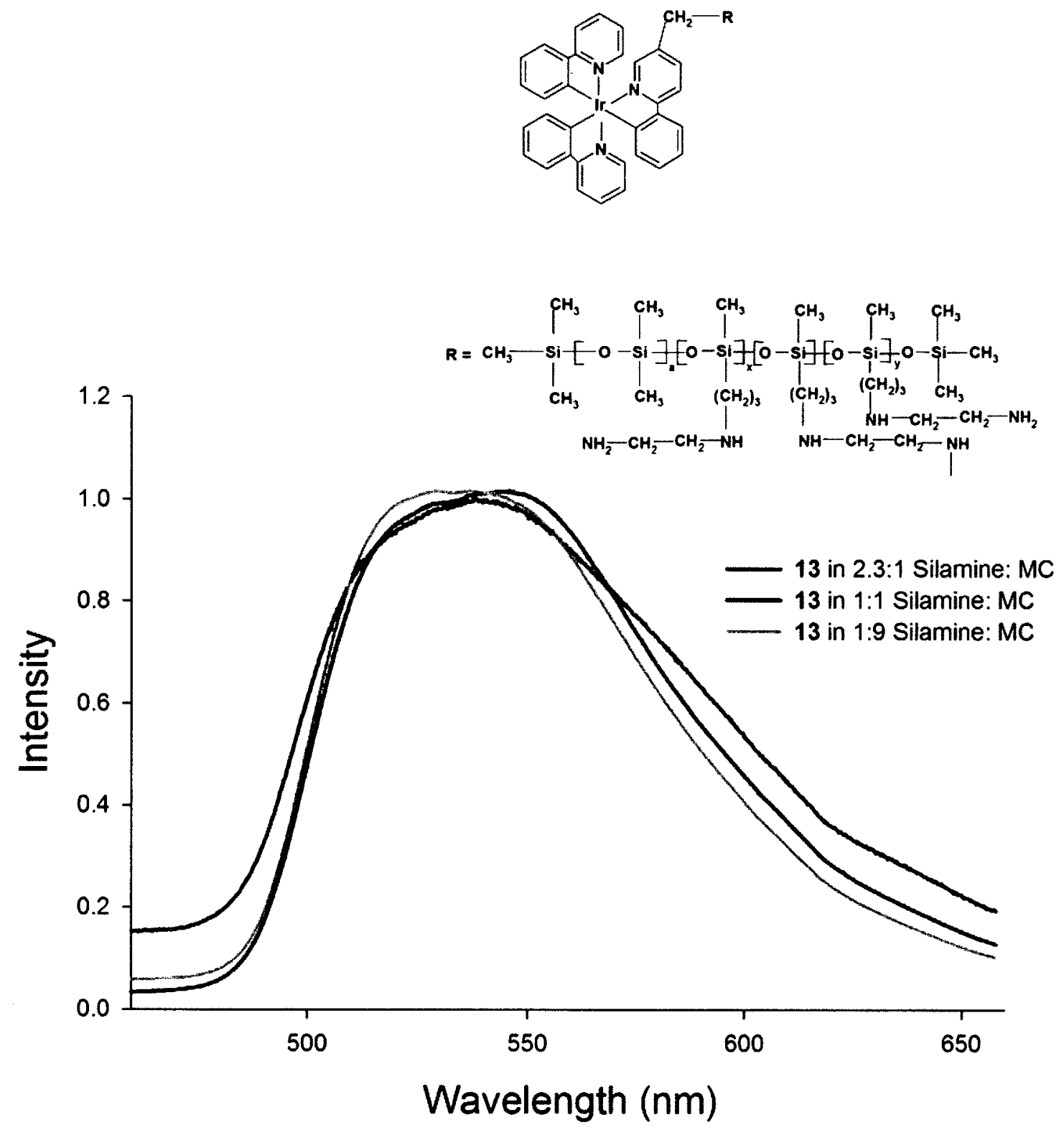

Figure 3.54: Effect of matrix on thin film luminescence of 13. Matrices compositions are: 2.3:1 Silamine: MC (black), 1:1 Silamine: MC (red), and 1:9 Silamine: MC (green). For each formulation, the spectrum at 14.7 psi is shown, which is normalized to its maximum emission intensity. 

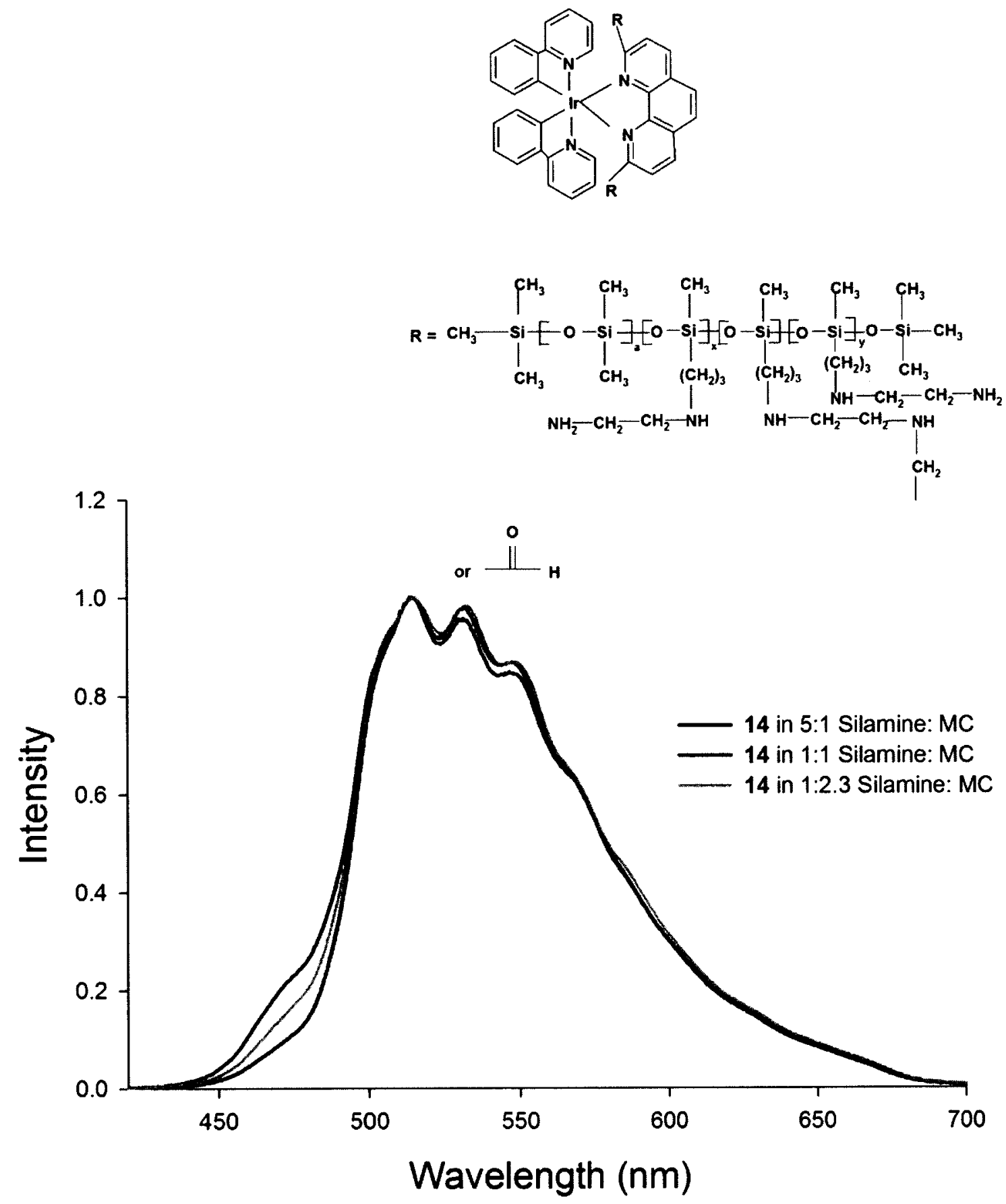

Figure 3.55: Effect of matrix on thin film luminescence of 14. Matrices compositions are: 5:1 Silamine: MC (black), 1:1 Silamine: $M C$ (red), and 1:2.3 Silamine: MC (green). For each formulation, the spectrum at $14.7 \mathrm{psi}$ is shown, which is normalized to its maximum emission intensity. 


\subsubsection{Characterization of Pressure Sensitive Paint (PSP) Films}

\subsubsection{PSP 9}

9 benefits from absorbance in the visible region and efficient excitation using long-wavelength radiation. Results for oxygen sensor 9 are given in Figures 3.56-3.58 and Table 3.12 .

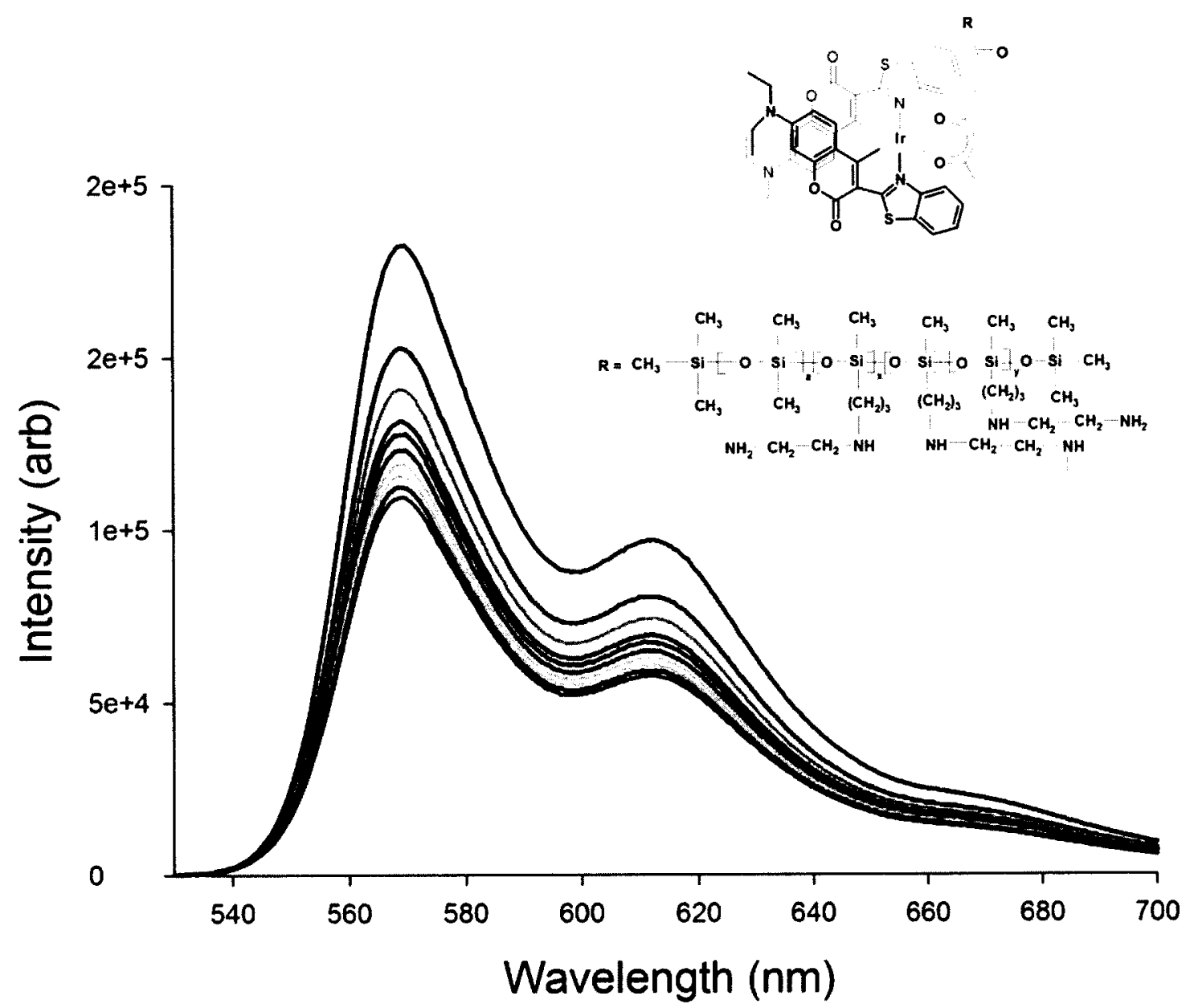

Figure 3.56: Effect of pressure on thin film luminescence of 9 in Silamine. The decrease of intensity with increasing the air pressure is displayed. Spectra were acquired at 1.3 mbar, 0.34 bar, 0.68 bar, 1 bar, 1.36 bar, 1.70 bar, 2.04 bar, 2.38 bar, 2.72 bar, and 3.06 bar. Background correction has been performed. 


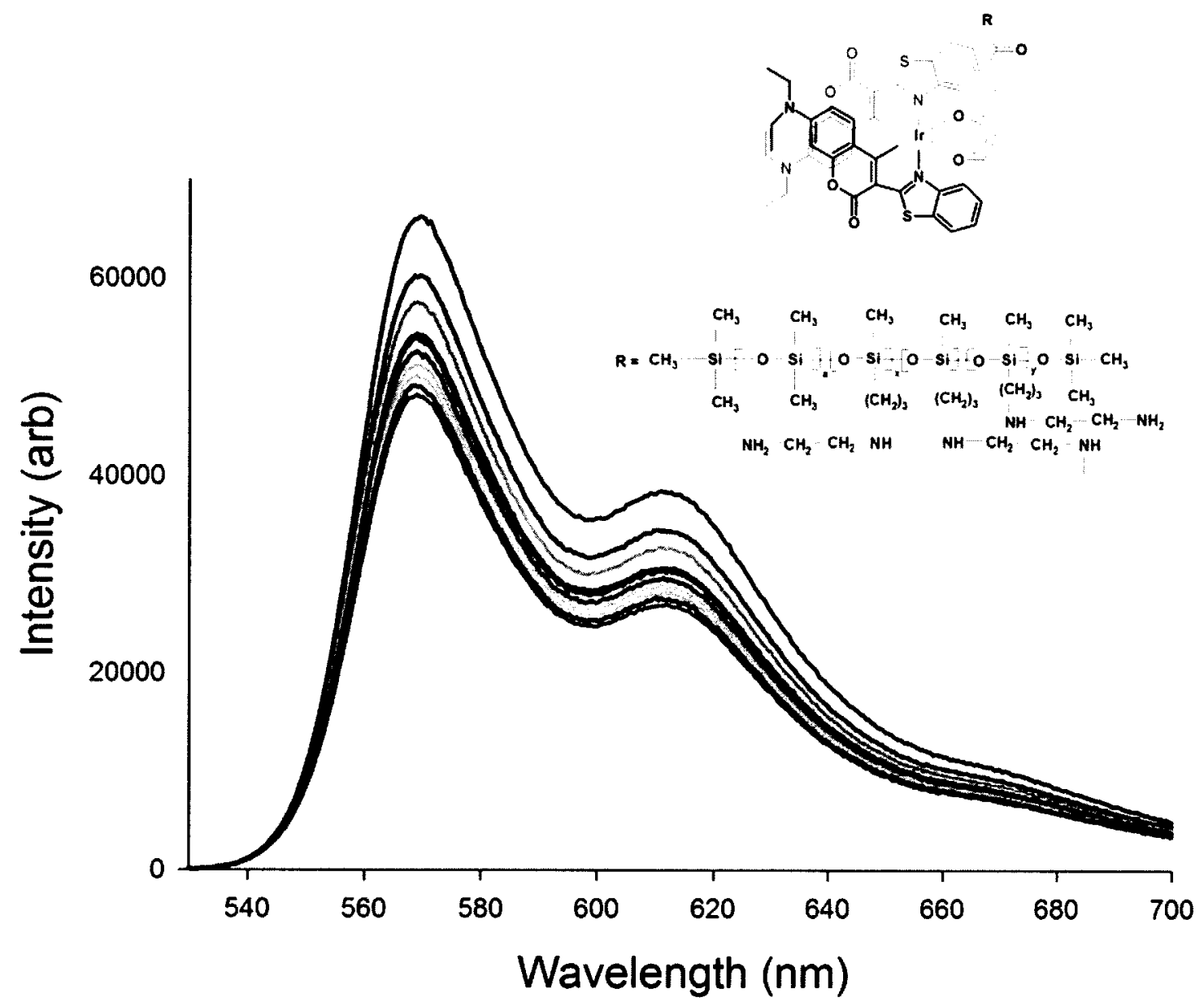

Figure 3.57: Effect of pressure on thin film luminescence of 9 in 1:1 Silamine: MC. The decrease of intensity with increasing the air pressure is displayed. Spectra were acquired at 1.3 mbar, 0.34 bar, 0.68 bar, 1 bar, 1.36 bar, 1.70 bar, 2.04 bar, 2.38 bar, 2.72 bar, and 3.06 bar. Background correction has been performed.

Among three film compositions, oxygen quenching sensitivity of 9 in Silamine is the greatest, which is displayed in the Stern-Volmer plots in Figure 3.58. 


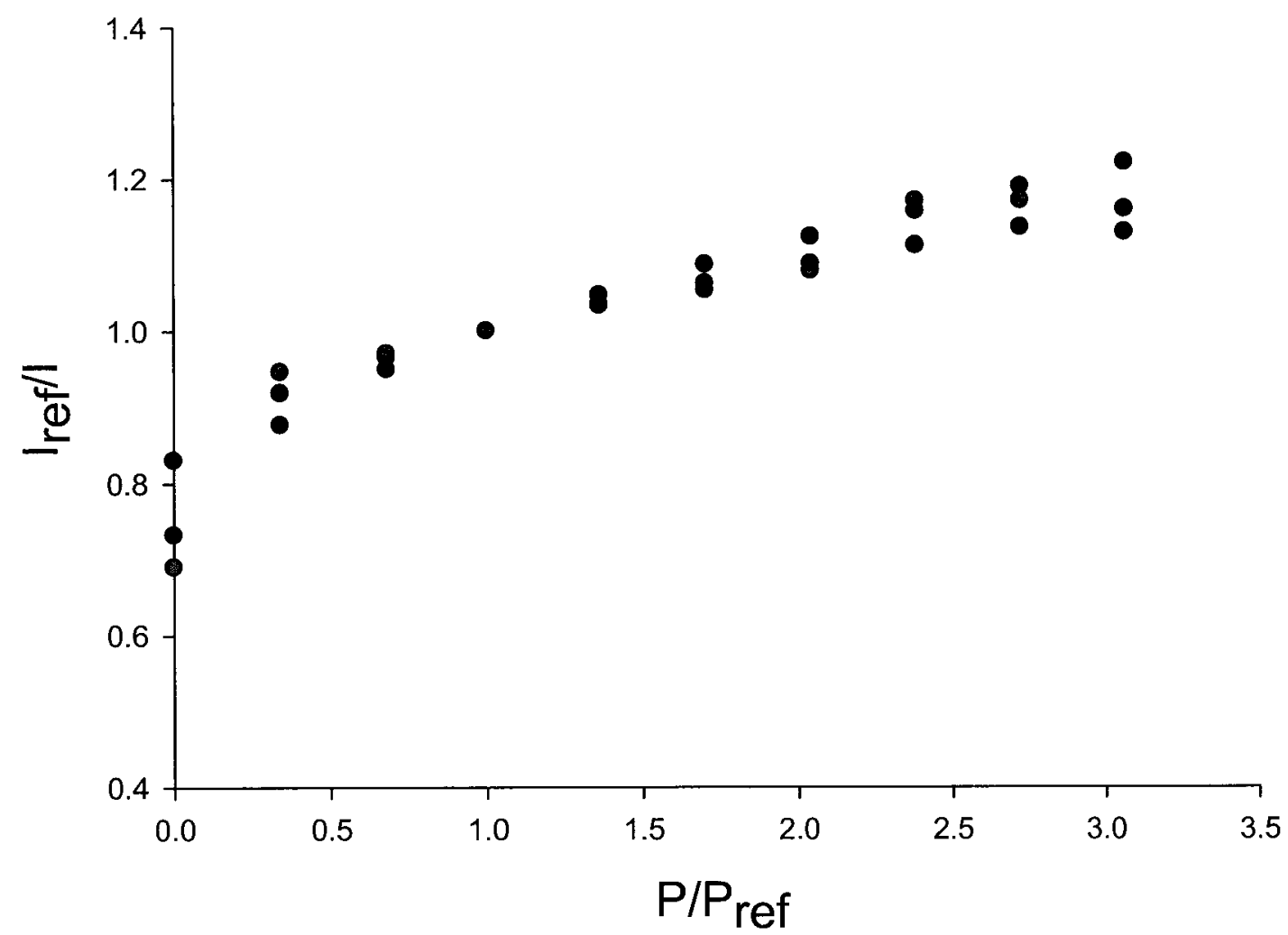

Figure 3.58: Stern-Volmer plots of $\mathbf{9}$ in Silamine, $\bullet$; in 1:1 Silamine: MC, $\bullet$; and in 1:5 Silamine: MC, $\bullet$.

Table 3.12: PSP films oxygen sensitivity data for 9 .

\begin{tabular}{|c|c|c|}
\hline Luminophore & Matrix & $\boldsymbol{Q}_{\mathbf{s}}\left(\mathrm{r}^{2}\right)$ \\
\hline $\mathbf{9}$ & Silamine & $0.12(0.974)^{\mathrm{b}}$ \\
& 1:1 Silamine: MC & $0.08(0.972)^{\mathrm{b}}$ \\
& $1: 5$ Silamine: MC & $0.10(0.957)^{\mathrm{d}}$ \\
\hline
\end{tabular}

${ }^{\mathrm{b}} 5-45 \mathrm{psi} ;{ }^{\mathrm{d}} 5-40 \mathrm{psi}$

\subsubsection{PSP 10}

Oxygen sensitivity calibration results for oxygen sensor films of $\mathbf{1 0}$ are presented in Figures 3.59-3.62 and Table 3.13. 


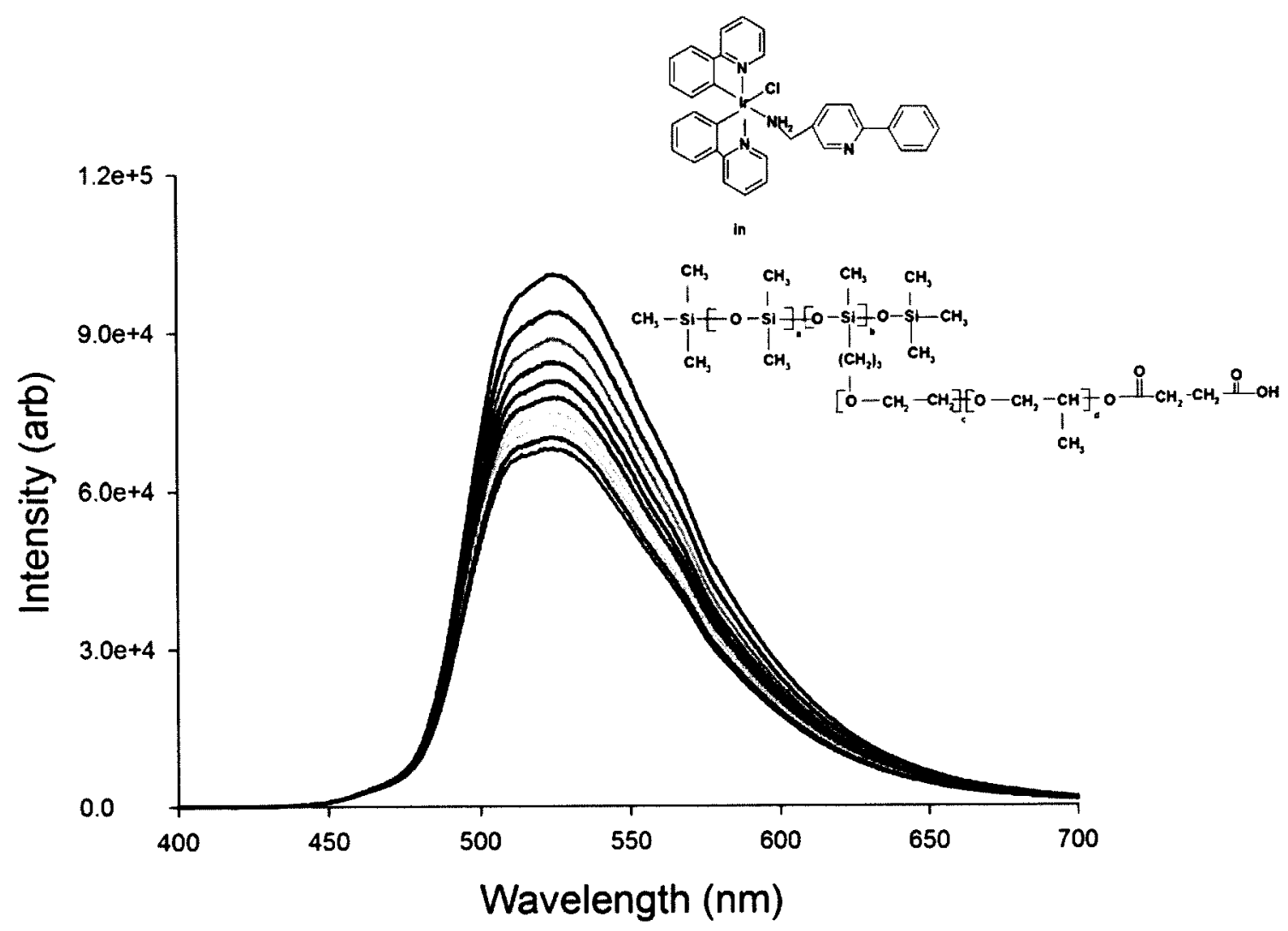

Figure 3.59: Effect of pressure on thin film luminescence of 10 in Silube. The decrease of intensity with increasing the air pressure is displayed. Spectra were acquired at 1.3 mbar, 0.34 bar, 0.68 bar, 1 bar, 1.36 bar, 1.70 bar, 2.04 bar, 2.38 bar, 2.72 bar, and 3.06 bar. Background correction has been performed. 


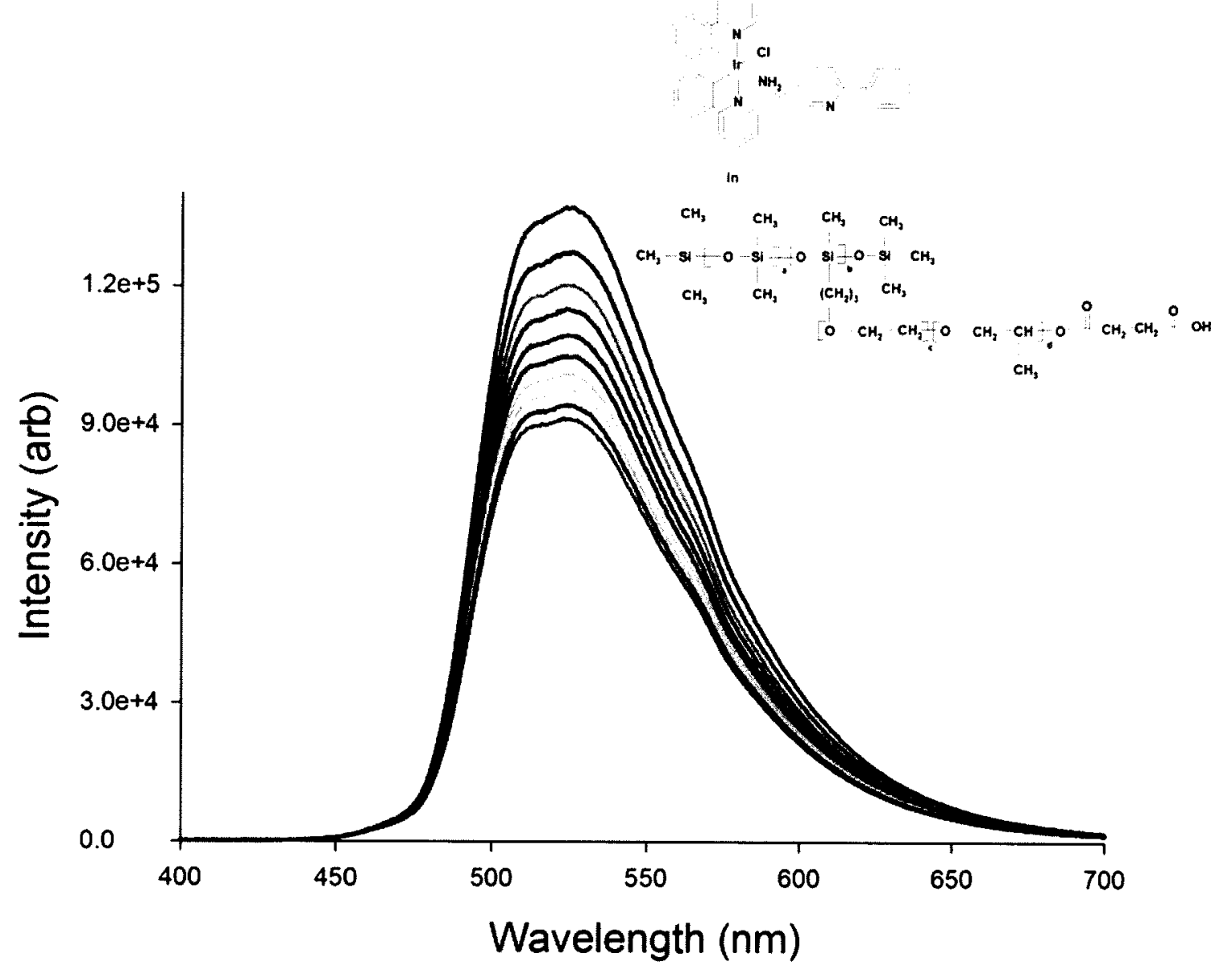

Figure 3.60: Effect of pressure on thin film luminescence of 10 in 5:1 Silube: MC. The decrease of intensity with increasing the air pressure is displayed. Spectra were acquired at 1.3 mbar, 0.34 bar, 0.68 bar, 1 bar, 1.36 bar, 1.70 bar, 2.04 bar, 2.38 bar, 2.72 bar, and 3.06 bar. Background correction has been performed. 


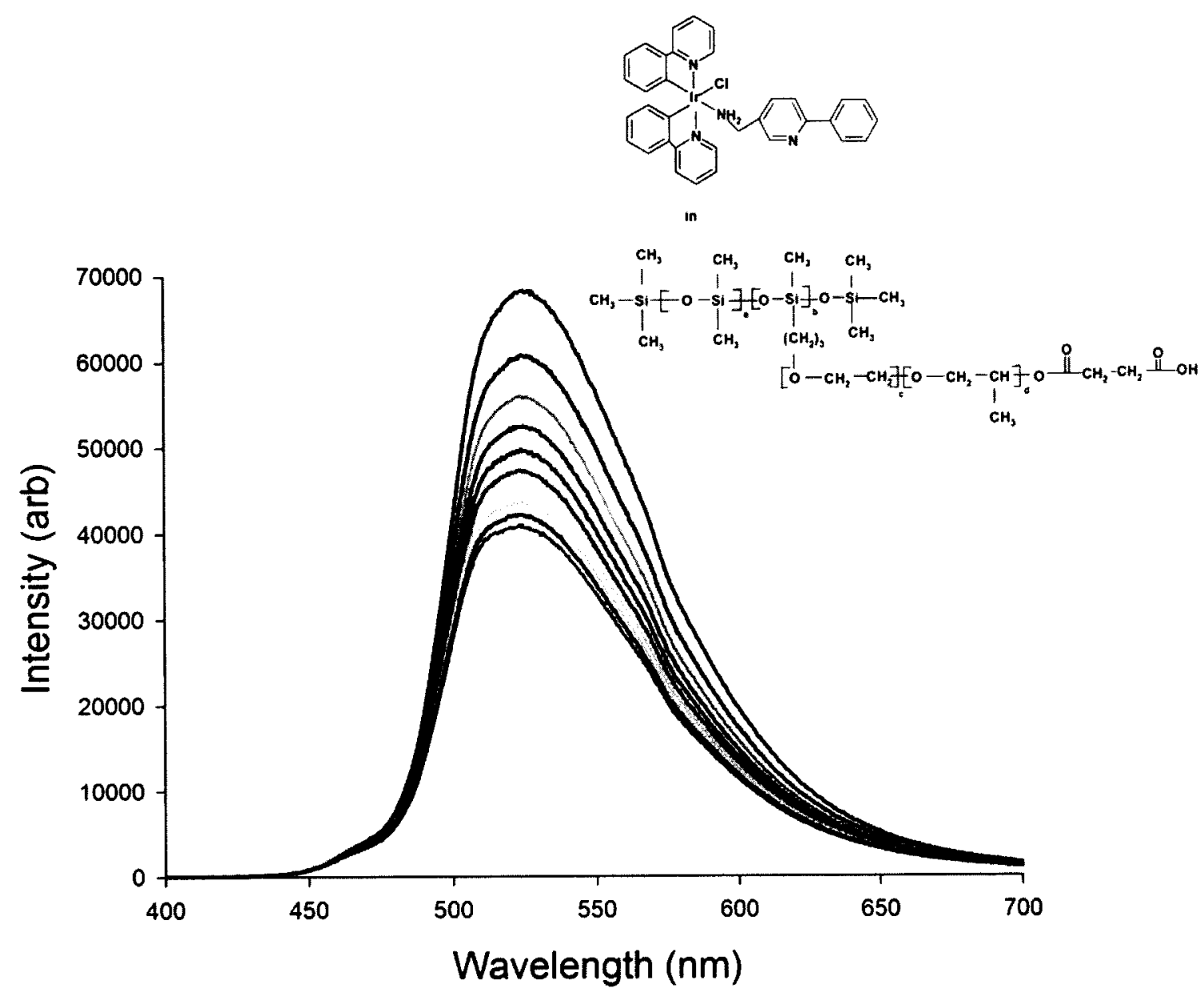

Figure 3.61: Effect of pressure on thin film luminescence of 10 in 1:1 Silube: MC. The decrease of intensity with increasing the air pressure is displayed. Spectra were acquired at 1.3 mbar, 0.34 bar, 0.68 bar, 1 bar, 1.36 bar, 1.70 bar, 2.04 bar, 2.38 bar, 2.72 bar, and 3.06 bar. Background correction has been performed. 

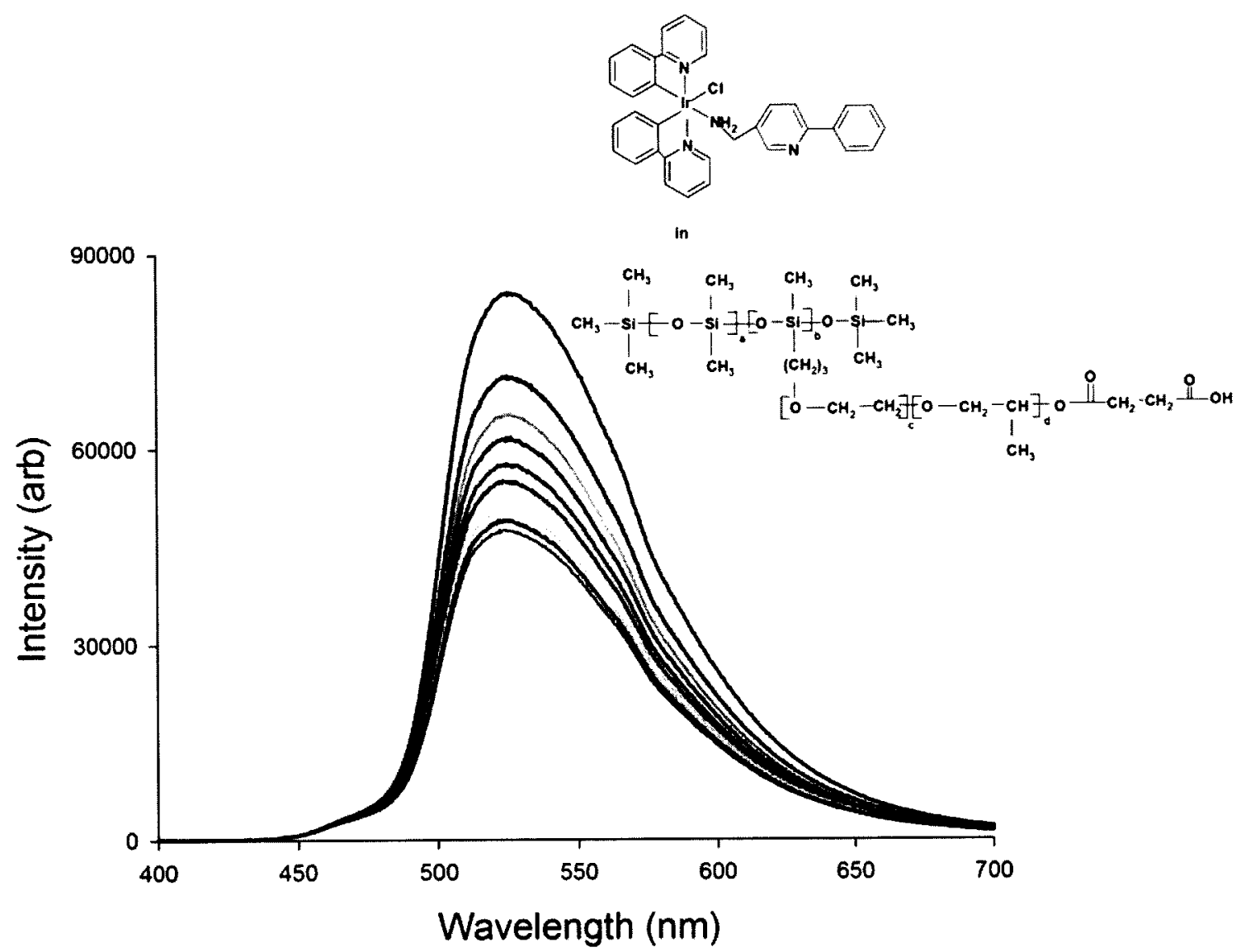

Figure 3.62: Effect of pressure on thin film luminescence of 10 in 1:5 Silube: MC. The decrease of intensity with increasing the air pressure is displayed. Spectra were acquired at 1.3 mbar, 0.34 bar, 0.68 bar, 1 bar, 1.36 bar, 1.70 bar, 2.04 bar, 2.38 bar, 2.72 bar, and 3.06 bar. Background correction has been performed.

Among four film compositions, oxygen quenching sensitivity of $\mathbf{1 0}$ in 1:5 Silube: MC is the greatest, which is displayed in the Stern-Volmer plots in Figure 3.63. 


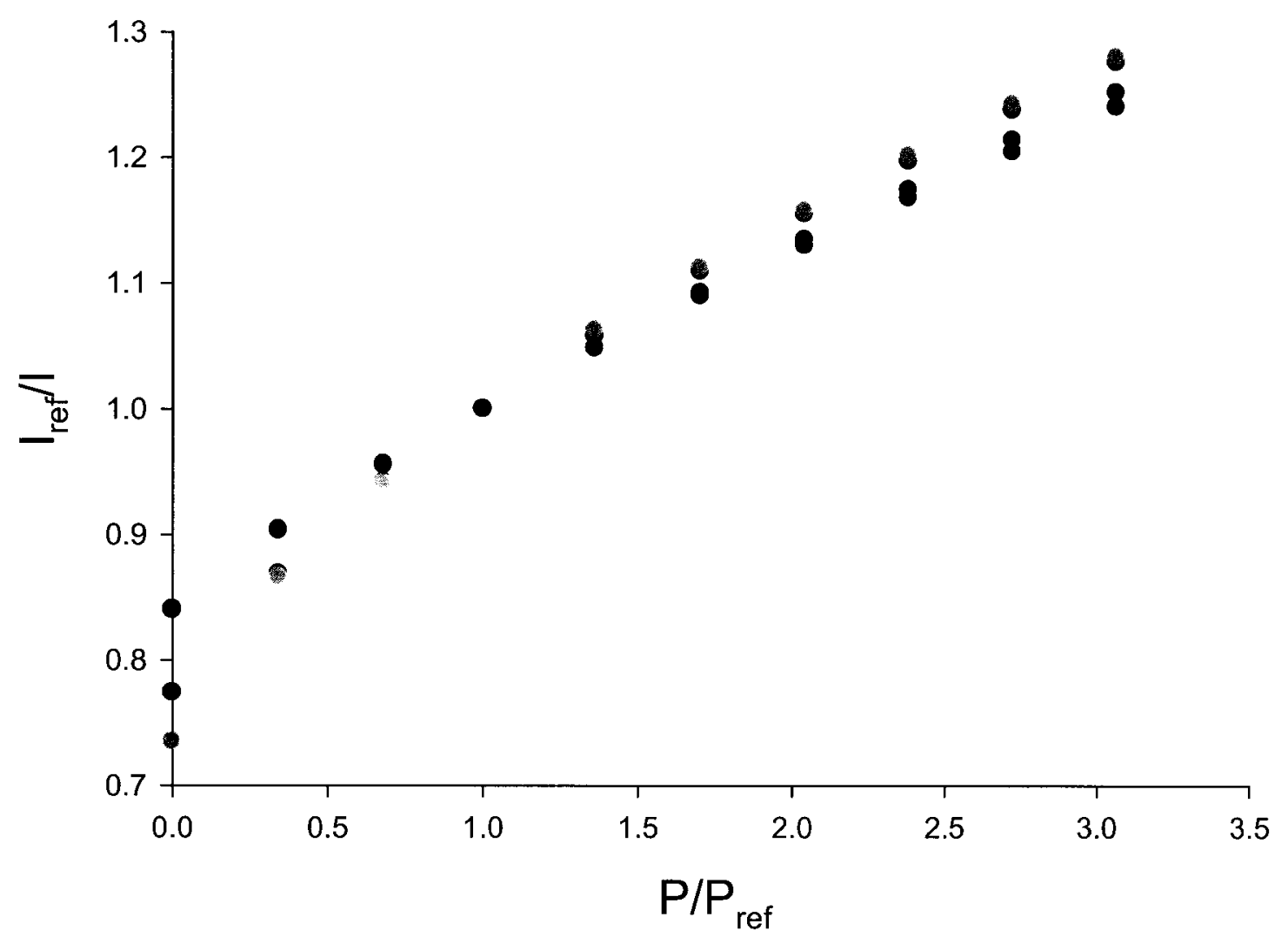

Figure 3.63: Stern-Volmer plots of $\mathbf{1 0}$ in Silube, $\bullet$; in 5:1 Silube: MC, $\bullet$; in 1:1 Silube: $\mathrm{MC}, \bullet$; and in 1:5 Silube: MC,

Table 3.13: PSP films oxygen sensitivity data for 10 .

\begin{tabular}{|c|c|c|}
\hline Luminophore & Matrix & $\boldsymbol{Q}_{\mathbf{s}}\left(\mathbf{r}^{2}\right)$ \\
\hline 10 & Silube & $0.13(0.992)^{\mathrm{a}}$ \\
& 5:1 Silube : MC & $0.13(0.995)^{\mathrm{a}}$ \\
& $1: 1$ Silube: MC & $0.16(0.978)^{\mathrm{a}}$ \\
& $1: 5$ Silube: MC & $0.17(0.964)^{\mathrm{a}}$ \\
\hline
\end{tabular}

${ }^{\mathrm{a}} 0.007-45 \mathrm{psi}$

As displayed in Figure 3.63, all Stern-Volmer plots are fairly linear, an indication of a uniform matrix and little self-aggregation.

Blending $\mathrm{MC}$ with $\mathbf{1 0}$ once again results in increase in both oxygen sensitivity and mechanical properties of the film (Figure 3.63 and the data in Table 3.13). This 
suggests that the lifetime must be increasing with increasing fraction of $\mathrm{MC}$ in case of Silube polymer.

However, the oxygen quenching sensitivity data for $\mathbf{1 0}$ in the above mentioned matrices are inadequate for wind tunnel applications, although larger than those of 9. Nevertheless, coupling reaction offers a suitable procedure for producing polymer bound oxygen sensors. It is worth to perform more research on the synthesis of other aminefunctionalized iridium complexes with favorable lifetimes and quantum yields.

\subsubsection{PSPs 5, 12, and 13}

Oxygen sensitivities of 5, 12 and $\mathbf{1 3}$ were studied in order to compare luminophore linkages. Thin films oxygen sensitivities were measured and compared in Table 3.14.

The pressure-dependent emission spectra of 5, 12, and 13 in different film matrices are shown in Figures 3.64-3.69.

The coupling 5 with Silamine to form 12 resulted in an increase in oxygen quenching sensitivity. According to solution luminescence lifetime data, (Tables 3.9 and 3.11), the extended lifetime of $\mathbf{1 2}$ is known to be responsible for this effect. Films of $\mathbf{1 3}$ displayed increased oxygen quenching sensitivities compared with $\mathbf{5}$ or $\mathbf{1 2}$ as shown by Figure 3.70. 


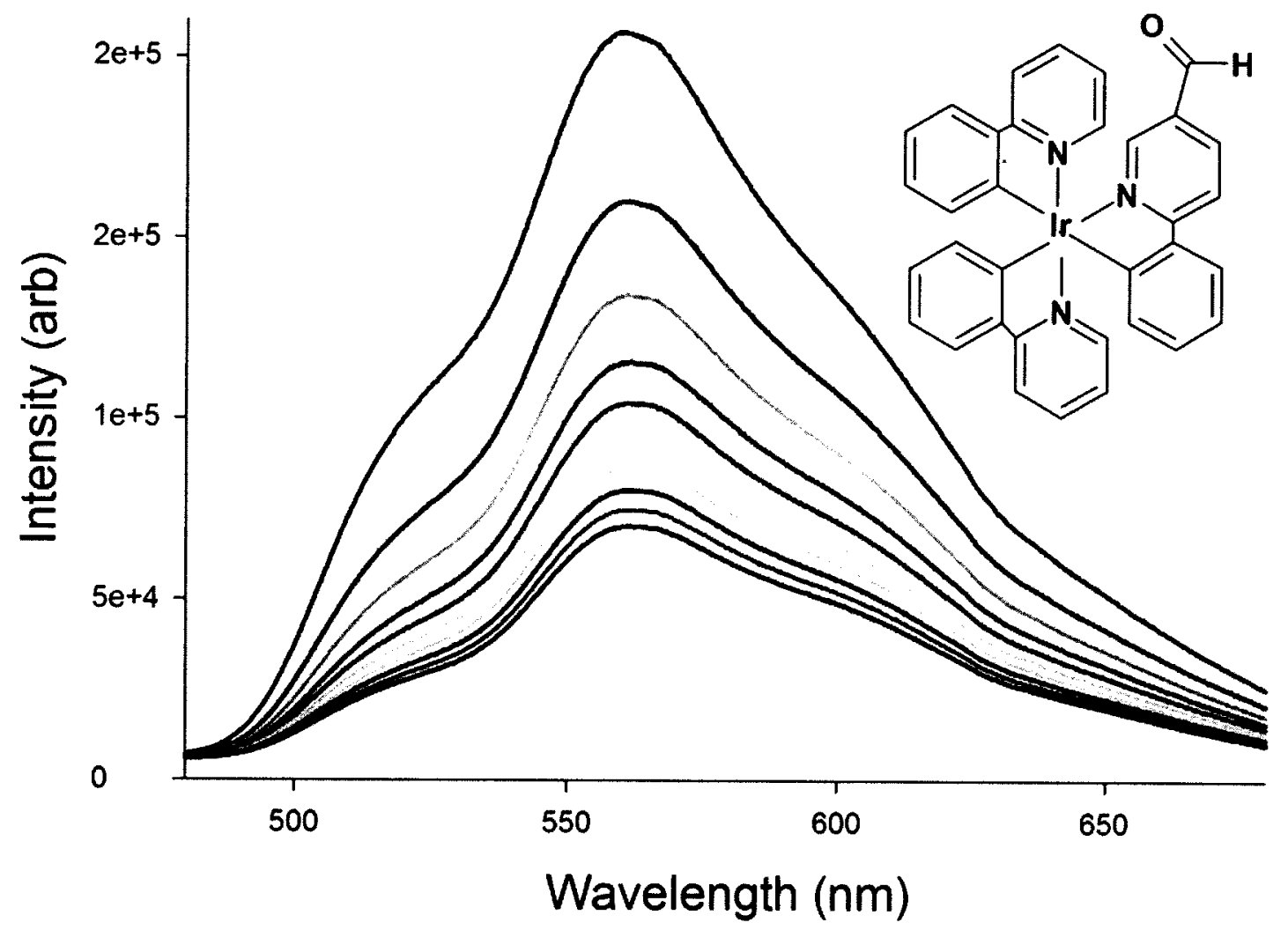

Figure 3.64: Effect of pressure on thin film luminescence of 5 in 2.3:1 Silamine: MC. The decrease of intensity with increasing the air pressure is displayed. Spectra were acquired at 1.3 mbar, 0.34 bar, 0.68 bar, 1 bar, 1.36 bar, 1.70 bar, 2.04 bar, 2.38 bar, 2.72 bar, and 3.06 bar. Background correction has been performed. 


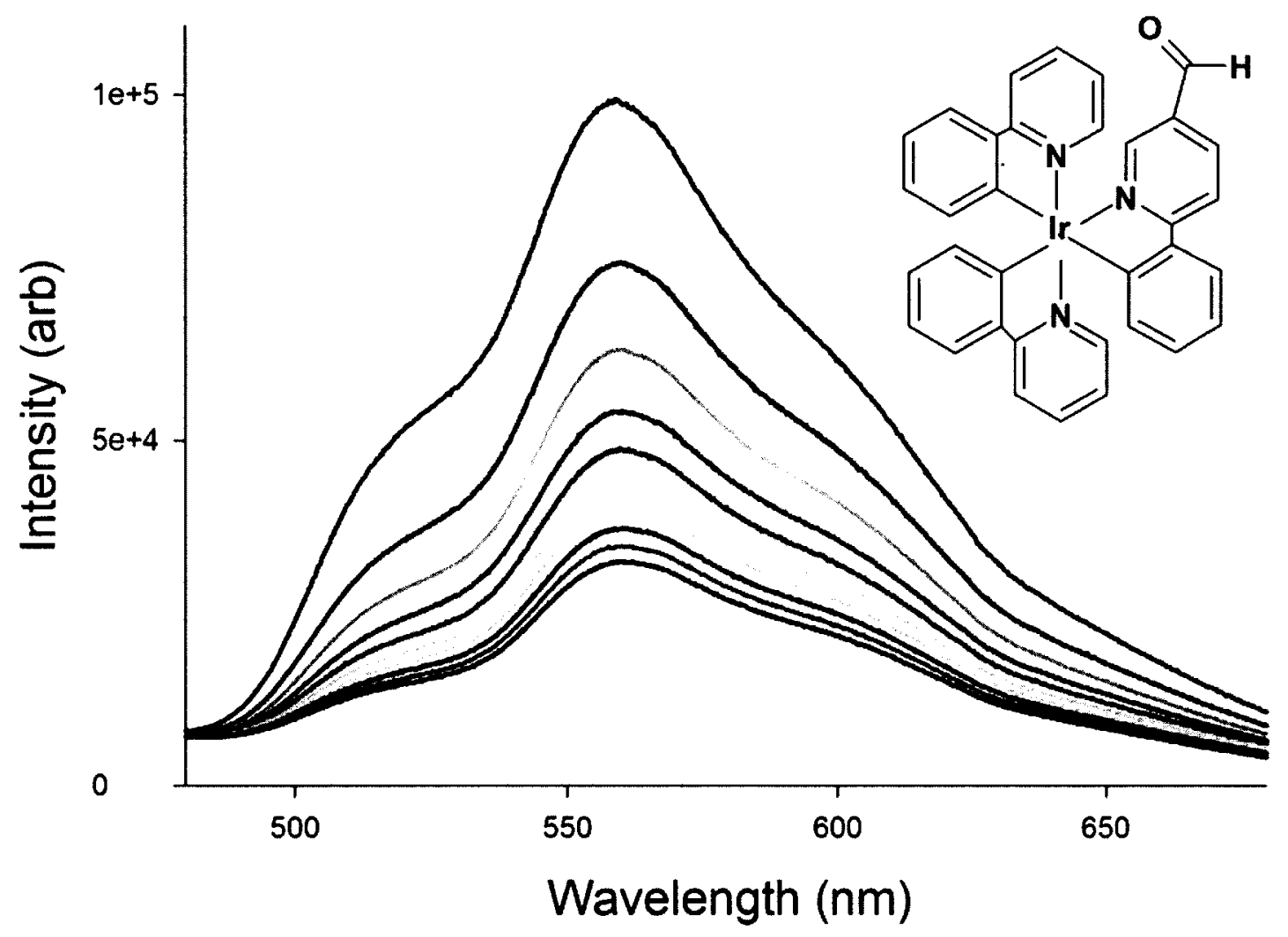

Figure 3.65: Effect of pressure on thin film luminescence of 5 in 1:1 Silamine: MC. The decrease of intensity with increasing the air pressure is displayed. Spectra were acquired at 1.3 mbar, 0.34 bar, 0.68 bar, 1 bar, 1.36 bar, 1.70 bar, 2.04 bar, 2.38 bar, 2.72 bar, and 3.06 bar. Background correction has been performed. 


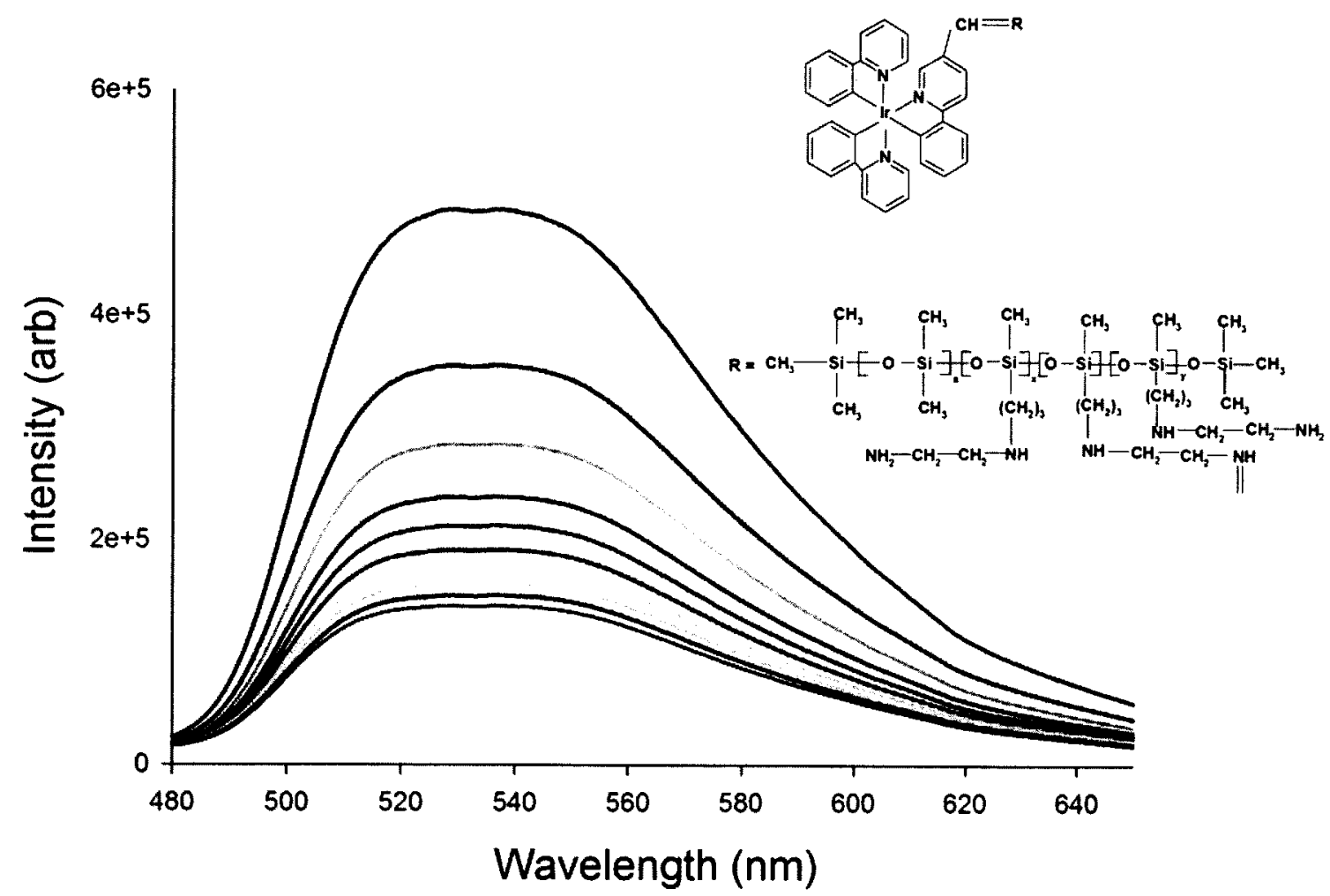

Figure 3.66: Effect of pressure on thin film luminescence of 12 in 2.3:1 Silamine: MC. The decrease of intensity with increasing the air pressure is displayed. Spectra were acquired at 1.3 mbar, 0.34 bar, 0.68 bar, 1 bar, 1.36 bar, 1.70 bar, 2.04 bar, 2.38 bar, 2.72 bar, and 3.06 bar. Background correction has been performed. 


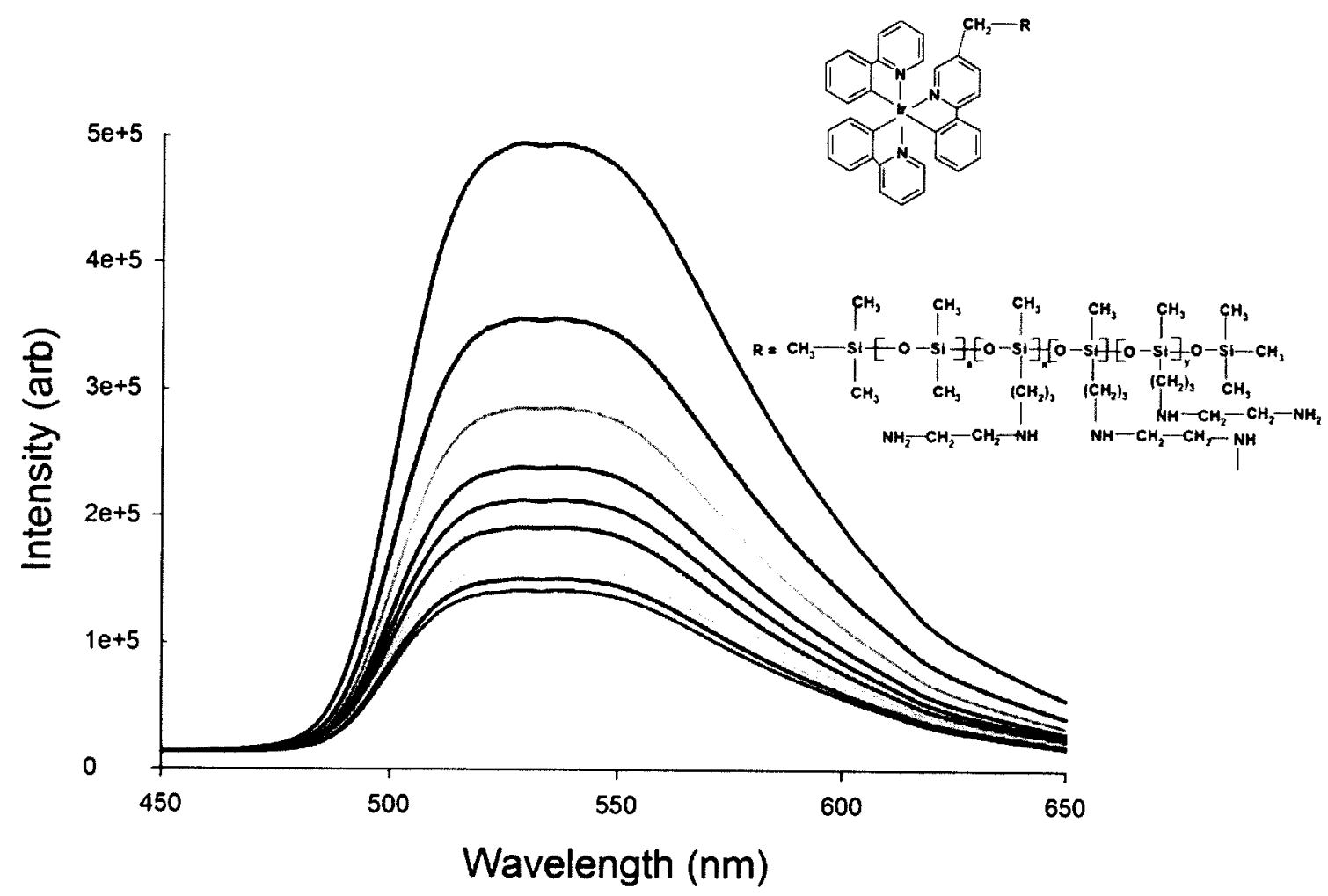

Figure 3.67: Effect of pressure on thin film luminescence of 13 in 2.3:1 Silamine: MC. The decrease of intensity with increasing the air pressure is displayed. Spectra were acquired at 1.3 mbar, 0.34 bar, 0.68 bar, 1 bar, 1.36 bar, 1.70 bar, 2.04 bar, 2.38 bar, 2.72 bar, and 3.06 bar. Background correction has been performed. 


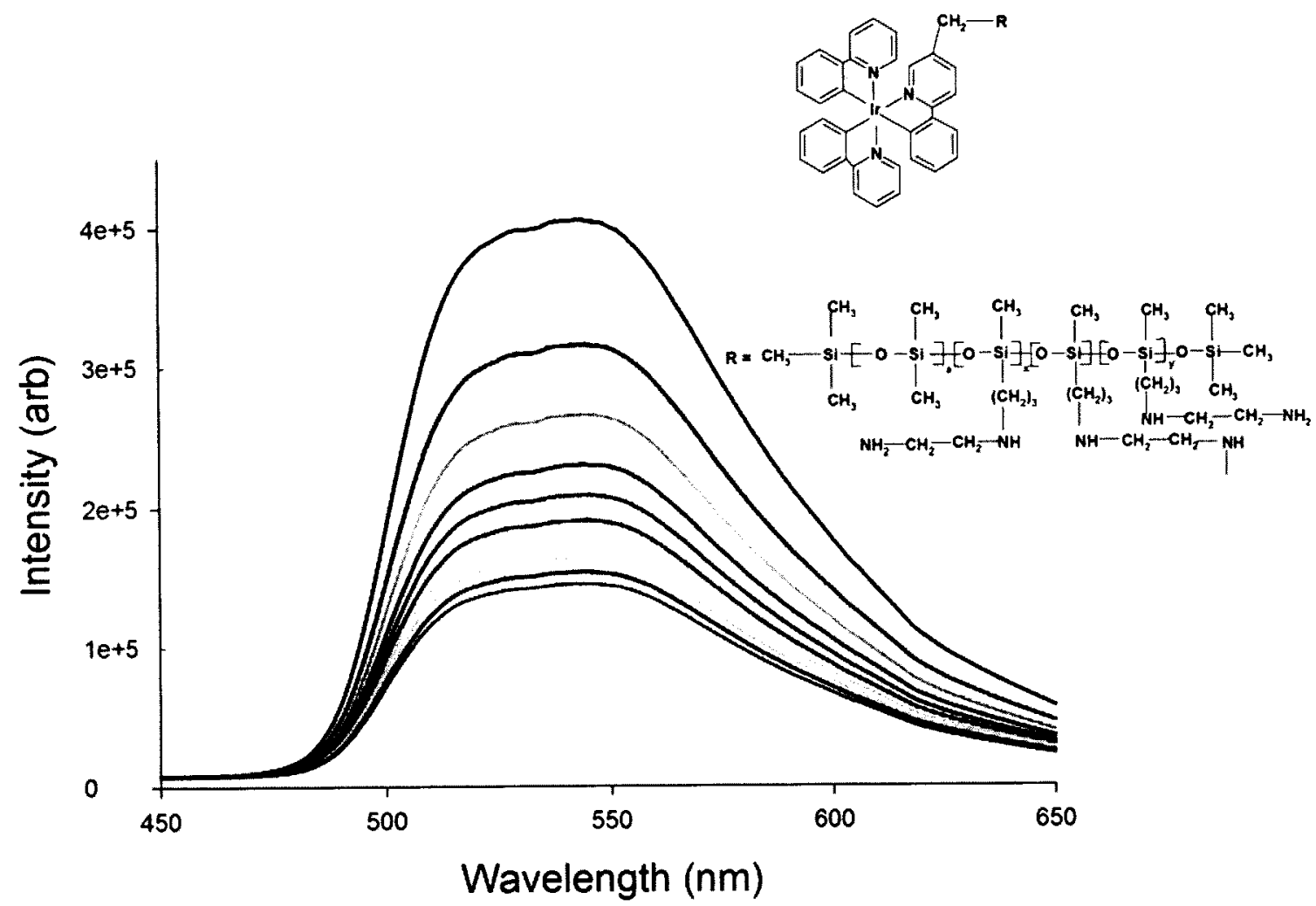

Figure 3.68: Effect of pressure on thin film luminescence of 13 in 1:1 Silamine: MC. The decrease of intensity with increasing the air pressure is displayed. Spectra were acquired at 1.3 mbar, 0.34 bar, 0.68 bar, 1 bar, 1.36 bar, 1.70 bar, 2.04 bar, 2.38 bar, 2.72 bar, and 3.06 bar. Background correction has been performed. 


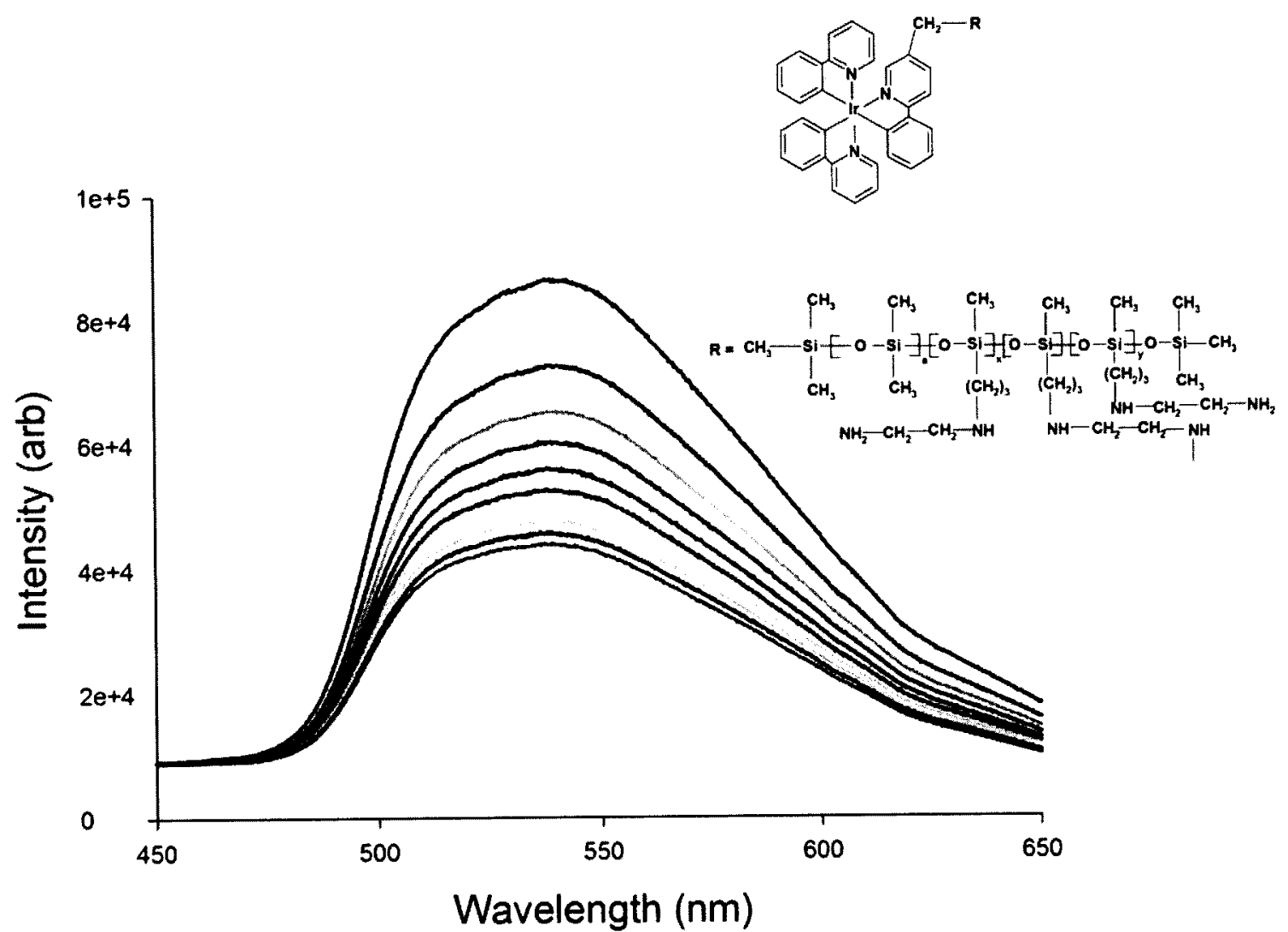

Figure 3.69: Effect of pressure on thin film luminescence of 13 in 1:9 Silamine: MC. The decrease of intensity with increasing the air pressure is displayed. Spectra were acquired at 1.3 mbar, 0.34 bar, 0.68 bar, 1 bar, 1.36 bar, 1.70 bar, 2.04 bar, 2.38 bar, 2.72 bar, and 3.06 bar. Background correction has been performed. 


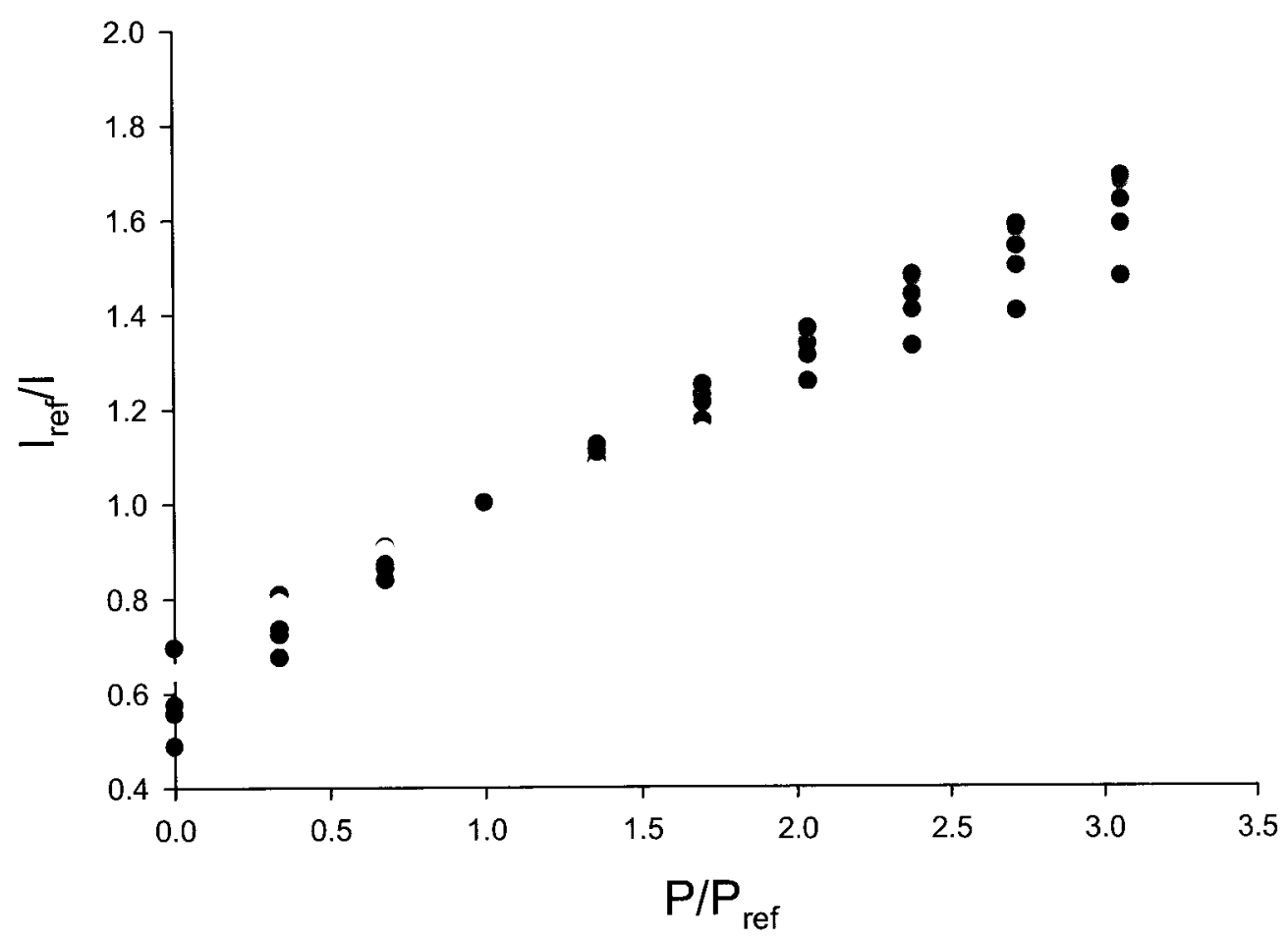

Figure 3.70: Stern-Volmer plots of $\mathbf{5}$ in 2.3:1 Silamine: $\mathrm{MC}, \boldsymbol{\bullet} ; \mathbf{5}$ in 1:1 Silamine: MC, $\bullet \mathbf{1 2}$ in 2.3:1 Silamine: MC, ; 12 in 1:1 Silamine: MC, ; 13 in 2.3:1 Silamine: MC, $\bullet \mathbf{1 3}$ in 1:1 Silamine: $\mathrm{MC}, \bullet$.

Table 3.14: PSP films oxygen sensitivity data for 5,12 , and 13 .

\begin{tabular}{|c|c|c|}
\hline Luminophore & Matrix & $Q_{s}\left(\mathrm{r}^{2}\right)$ \\
\hline 5 & $\begin{array}{c}\text { 2.3:1 Silamine: } \mathrm{MC} \\
\text { 1:1 Silamine: } \mathrm{MC} \\
\text { 1:9 Silamine: } \mathrm{MC}\end{array}$ & $\begin{array}{c}0.35(0.993)^{\mathrm{a}} \\
0.25(0.994)^{\mathrm{a}} \\
0.15(0.950)^{\mathrm{e}}, 0.05(0.980)^{\mathrm{f}}\end{array}$ \\
\hline 12 & $\begin{array}{c}\text { 2.3:1 Silamine: } \mathrm{MC} \\
\text { 1:1 Silamine: } \mathrm{MC} \\
\text { 1:9 Silamine: } \mathrm{MC}\end{array}$ & $\begin{array}{c}0.38(0.988)^{\mathrm{a}} \\
0.24(0.973)^{\mathrm{a}} \\
0.66^{\mathrm{d}}, 0.12(0.966)^{\mathrm{c}}\end{array}$ \\
\hline 13 & $\begin{array}{c}\text { 2.3:1 Silamine }: \mathrm{MC} \\
\text { 1:1 Silamine: } \mathrm{MC} \\
\text { 1:9 Silamine: } \mathrm{MC}\end{array}$ & $\begin{array}{l}0.39(0.989)^{\mathrm{a}} \\
0.32(0.991)^{\mathrm{a}} \\
0.23(0.980)^{\mathrm{a}}\end{array}$ \\
\hline
\end{tabular}

${ }^{\mathrm{a}} 0.007-45 \mathrm{psi} ;{ }^{\mathrm{b}} 5-45 \mathrm{psi} ;{ }^{\mathrm{c}} 10-45 \mathrm{psi} ;{ }^{\mathrm{d}} 0.007-5 \mathrm{psi} ;{ }^{\mathrm{e}} 5-14.7 \mathrm{psi} ;{ }^{\mathrm{f}} 20-45 \mathrm{psi}$

In order to increase the mechanical properties of the film, MC was blended with luminophores which resulted in decrease in oxygen sensitivity compared with the film 
composed of a lower proportion of low $\mathrm{T}_{\mathrm{g}}$ polymer (the effect being the least for 13). This is shown in Figure 3.70 and the data in Table 3.14 in which the optimum PSP formulation is $\mathbf{1 3}$ in 2.3:1 Silamine:MC which gave an oxygen sensitivity of 0.39 , with good linear behavior in a pressure range of 0.007 to $45 \mathrm{psi}$.

The luminescence microscopy of films of $\mathbf{1 3}$ was examined, and the results are shown in Figure 3.71. 

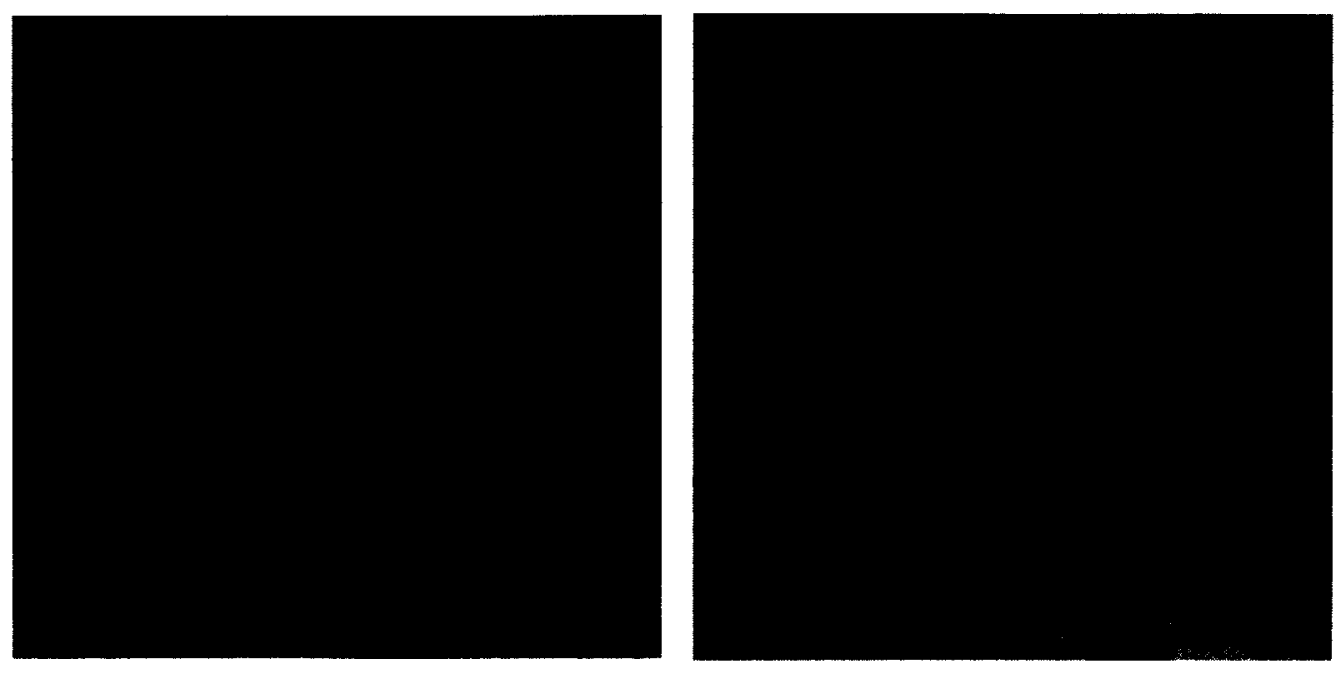

A

B
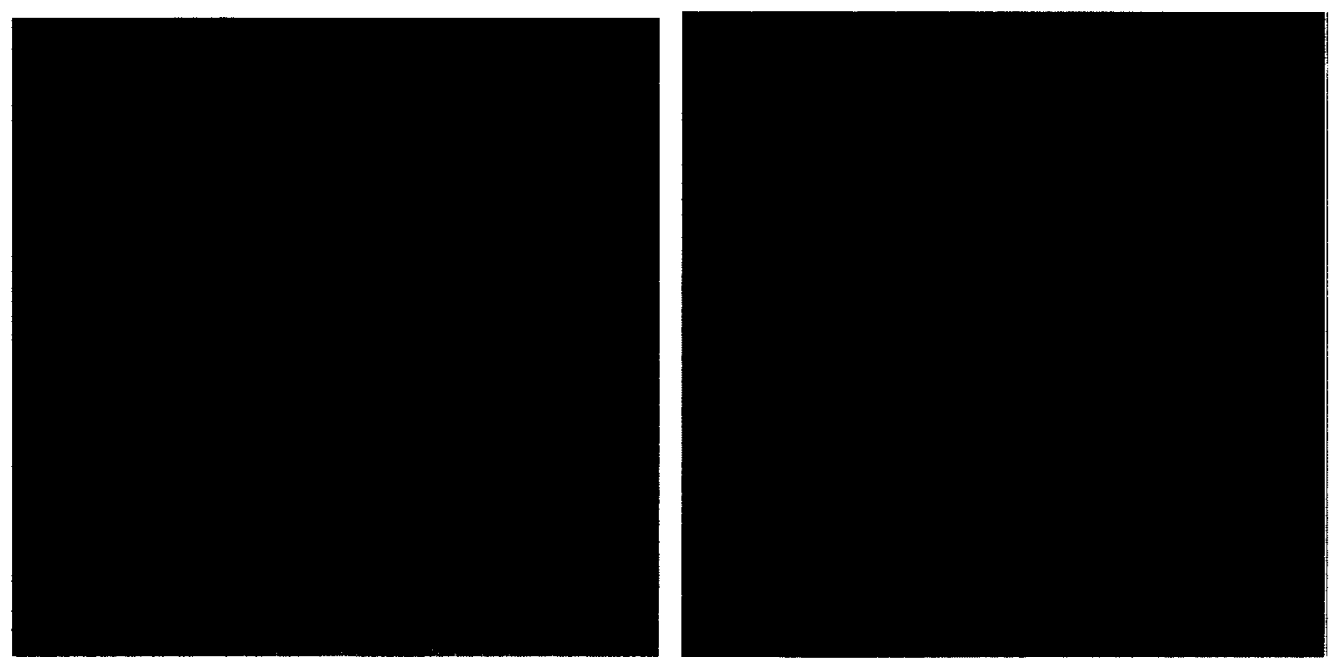

C

D

Figure 3.71: Luminescence microscopy of films of 13 performed under ambient conditions: A, 9:1 Silamine: MC; B, 5:1 Silamine: MC; C, 1:1 Silamine: MC; D, 1:5 Silamine: MC. The scale in red is $10 \mu \mathrm{m}$.

In Figure $3.71 \mathrm{~A}$, luminescence microscopy shows that at low concentration of $\mathrm{MC}$, the luminescence from the film is largely dispersed with little evidence of association around particles of $\mathrm{MC}$ unlike that seen for $\mathbf{8}$ (Figure 3.33). Increasing $\mathrm{MC}$ concentration results in a slight increase in luminescence which suggests that while there 
is a weak association of the luminophore with $\mathrm{MC}$, the resulting increase in lifetime is insufficient to compensate for the decrease in oxygen diffusion.

The temperature dependence of emission intensity of $\mathbf{5}$ and $\mathbf{1 3}$ in 2.3:1 Silamine:MC films were examined in the temperature range of 10 to $40{ }^{\circ} \mathrm{C}$ under 1 bar atmospheric pressure. The emission intensities were normalized to that at $10^{\circ} \mathrm{C}$ and the results plotted in Figure 3.72.

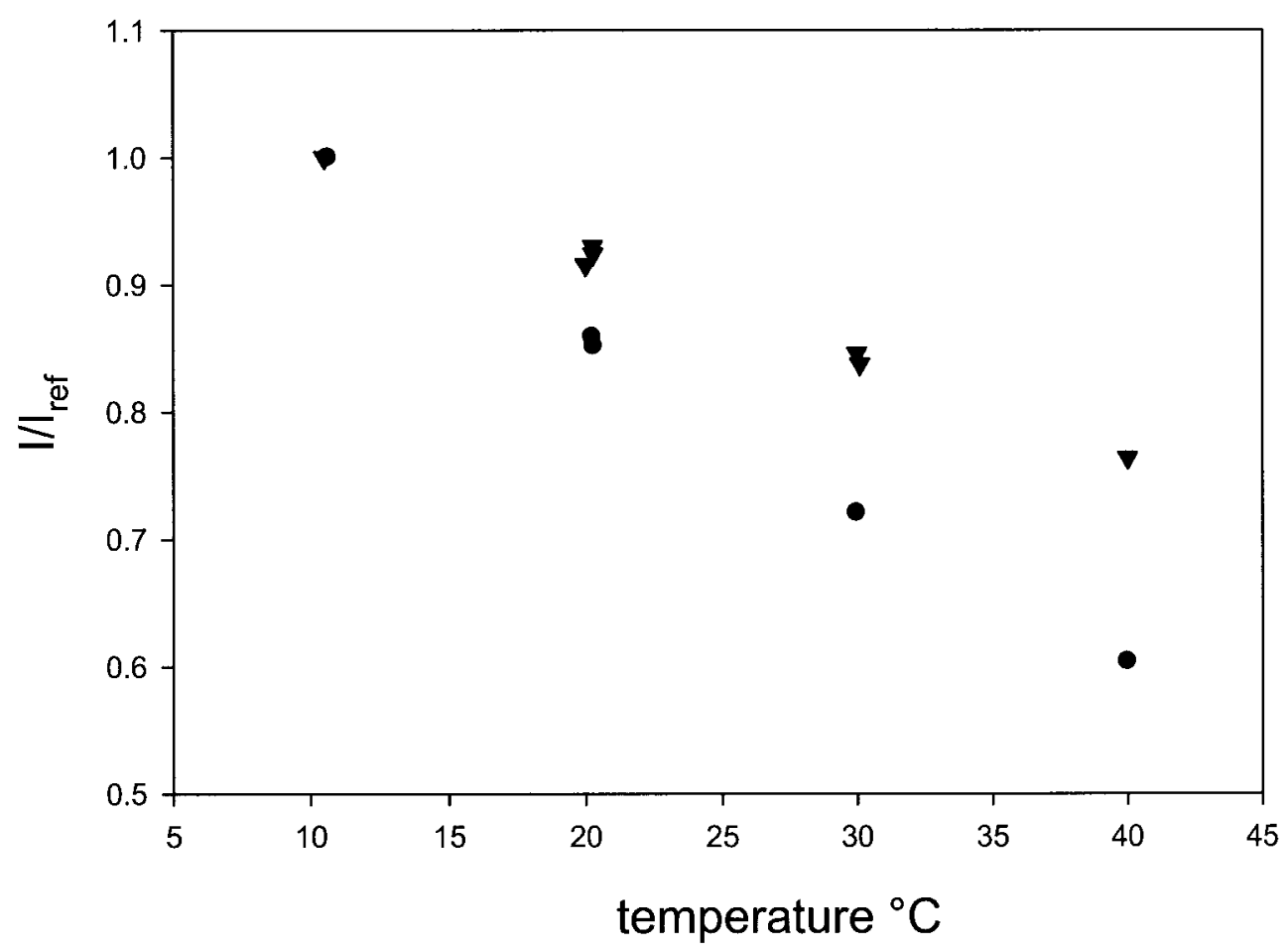

Figure 3.72: Temperature versus emission intensity ratio $\left(\mathrm{I}_{\mathrm{ref}}\right.$ is the intensity at $\left.10^{\circ} \mathrm{C}\right)$ of $\mathbf{5}$ in 2.3:1 Silamine: MC: 1 bar $\bullet 13$ in 2.3:1 Silamine: MC: 1 bar $\boldsymbol{\nabla}$.

In Figure 3.72, the data for the temperature dependence of emission gave good linear least-squares fits passing through or at least closely approaching the normalization value at $10^{\circ} \mathrm{C}$. The temperature sensitivities (percent intensity loss per degree) of the films were determined to be $-1.3 \%{ }^{\circ} \mathrm{C}^{-1}$ for $\mathbf{5}$ and $-0.8 \%{ }^{\circ} \mathrm{C}^{-1}$ for $\mathbf{1 3}$ at 1 bar atmospheric pressure. Although the temperature sensitivity of $\mathbf{1 3}$ in 2.3:1 Silamine: MC film is 
improved compared to $\mathbf{5}$ and $\mathbf{8}$ (see section 3.6), it still must be compensated for by using a temperature sensitive calibrant to obtain correct barometric data.

\subsubsection{PSP 14}

Table 3.15 lists oxygen quenching sensitivity data for $\mathbf{1 4}$ and the effect of air pressure on emission intensity is illustrated in Figures 3.73-3.75. Modified Stern-Volmer plots of $\mathbf{1 4}$ in three Silamine: MC formulations are shown in Figure 3.76.

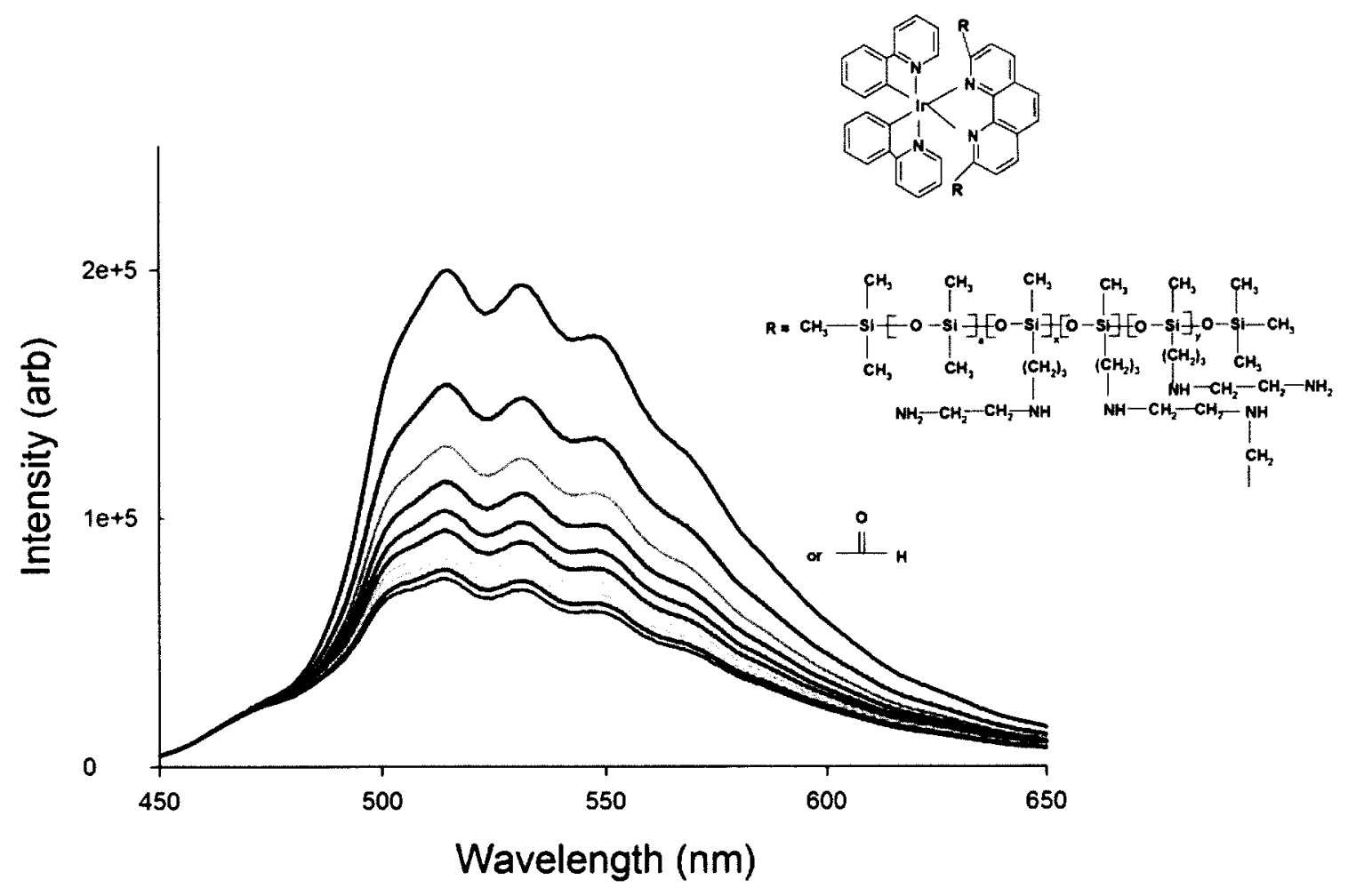

Figure 3.73: Effect of pressure on thin film luminescence of 14 in 5:1 Silamine: MC. The decrease of intensity with increasing the air pressure is displayed. Spectra were acquired at 1.3 mbar, 0.34 bar, 0.68 bar, 1 bar, 1.36 bar, 1.70 bar, 2.04 bar, 2.38 bar, 2.72 bar, and 3.06 bar. Background correction has been performed. 


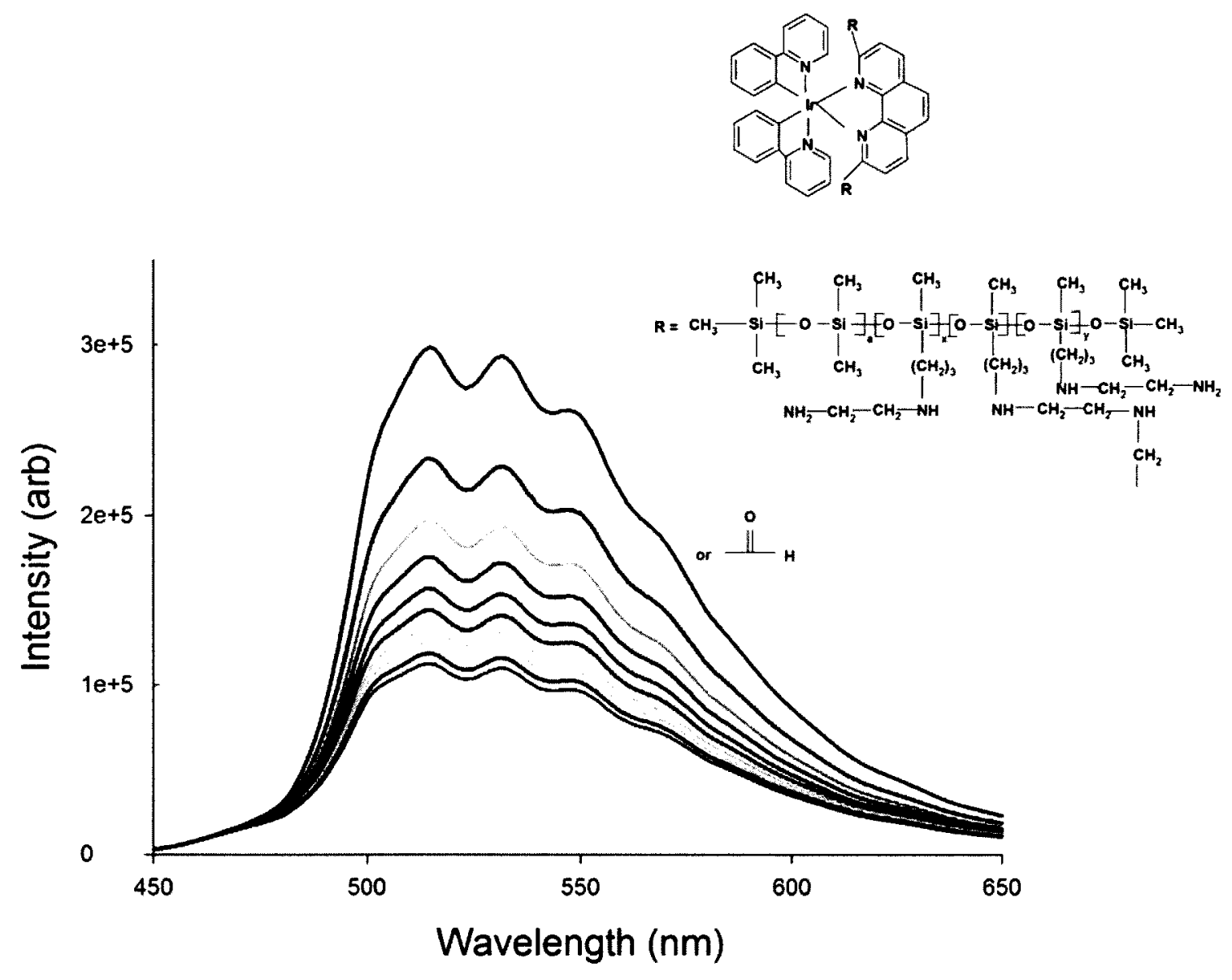

Figure 3.74: Effect of pressure on thin film luminescence of 14 in 1:1 Silamine: MC. The decrease of intensity with increasing the air pressure is displayed. Spectra were acquired at 1.3 mbar, $0.34 \mathrm{bar}, 0.68 \mathrm{bar}, 1 \mathrm{bar}, 1.36 \mathrm{bar}, 1.70 \mathrm{bar}, 2.04 \mathrm{bar}, 2.38 \mathrm{bar}$, $2.72 \mathrm{bar}$, and 3.06 bar. Background correction has been performed. 


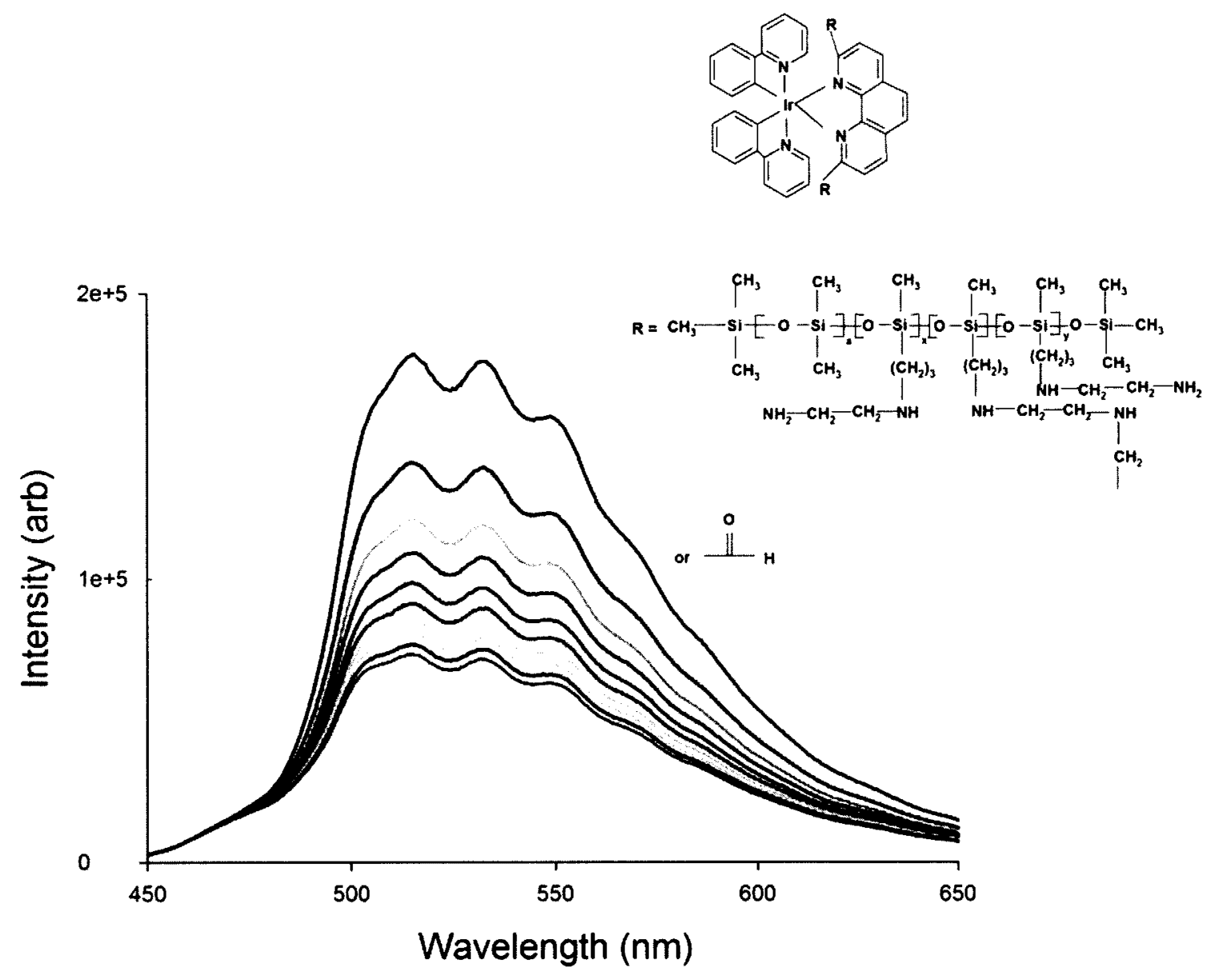

Figure 3.75: Effect of pressure on thin film luminescence of 14 in 1:2.3 Silamine: MC. The decrease of intensity with increasing the air pressure is displayed. Spectra were acquired at 1.3 mbar, 0.34 bar, 0.68 bar, 1 bar, 1.36 bar, 1.70 bar, 2.04 bar, 2.38 bar, 2.72 bar, and 3.06 bar. Background correction has been performed. 


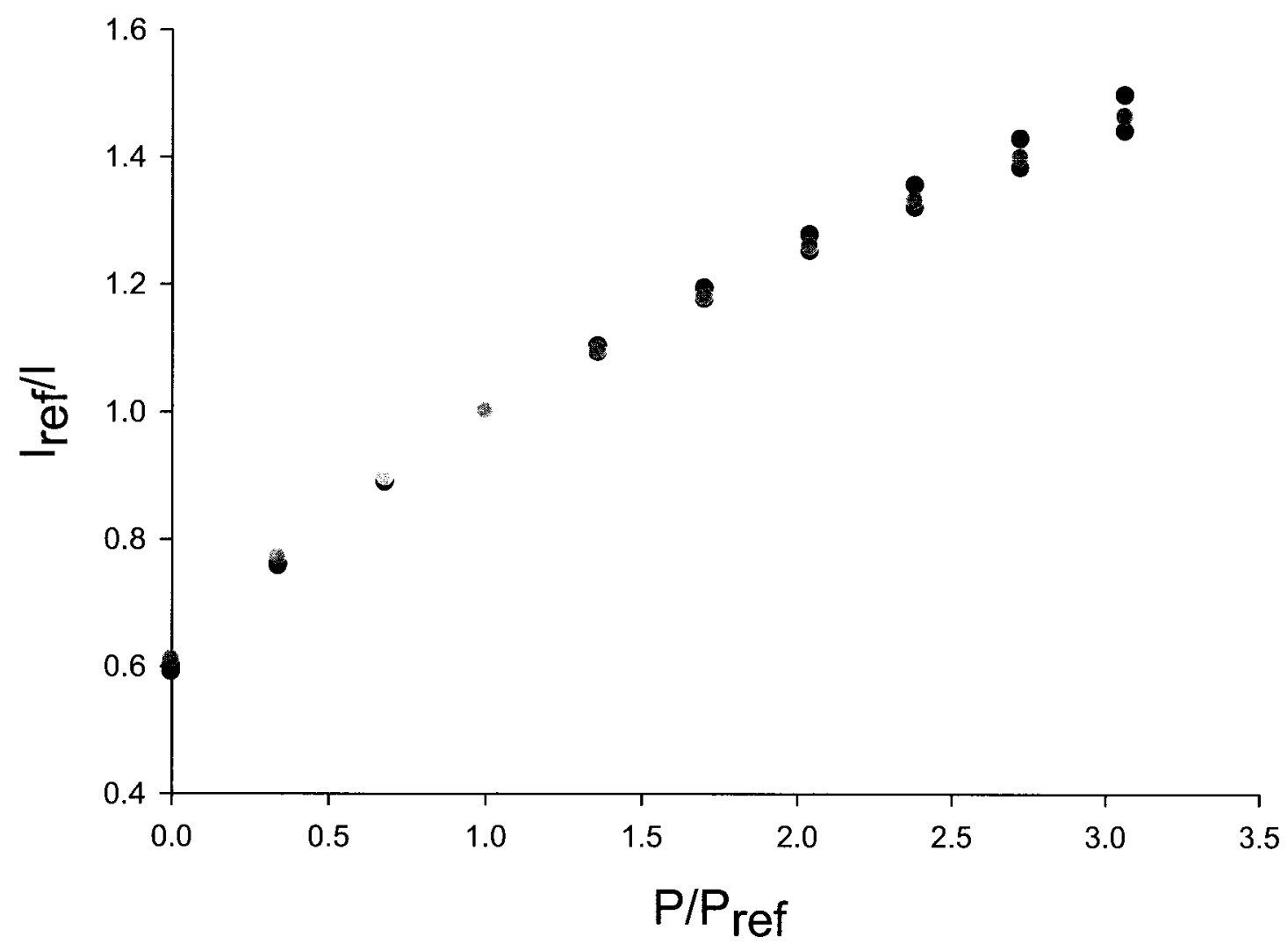

Figure 3.76: Stern-Volmer plots of 14 in 5:1 Silamine: MC, $\bullet$; 14 in 1:1 Silamine: MC, $\bullet$; 14 in 1:2.3 Silamine: MC, .

Table 3.15: PSP films oxygen sensitivity data for 14 .

\begin{tabular}{|c|c|c|}
\hline Luminophore & Matrix & $\boldsymbol{Q}_{\mathbf{s}}\left(\mathbf{r}^{2}\right)$ \\
\hline \multirow{14}{14}{} & 5:1 Silamine $: \mathrm{MC}$ & $0.27(0.969)^{\mathrm{a}}$ \\
& $1: 1$ Silamine: $\mathrm{MC}$ & $0.28(0.981)^{\mathrm{a}}$ \\
& $1: 2.3$ Silamine: $\mathrm{MC}$ & $0.27(0.977)^{\mathrm{a}}$ \\
\hline
\end{tabular}

${ }^{\mathrm{a}} 0.007-45 \mathrm{psi}$

As shown in Figure 3.76 and the data in Table 3.15, blending MC with 14 has a subtle effect on oxygen sensitivity and the PSP formulations exhibit good linear behavior in a pressure range of 0.007 to 45 psi. 
The temperature dependence of emission intensity of $\mathbf{1 4}$ in 5:1 Silamine:MC films was examined in the temperature range of 10 to $40{ }^{\circ} \mathrm{C}$ under vacuum. The emission intensities were normalized to that at $10{ }^{\circ} \mathrm{C}$ and the results plotted in Figure 3.77 .

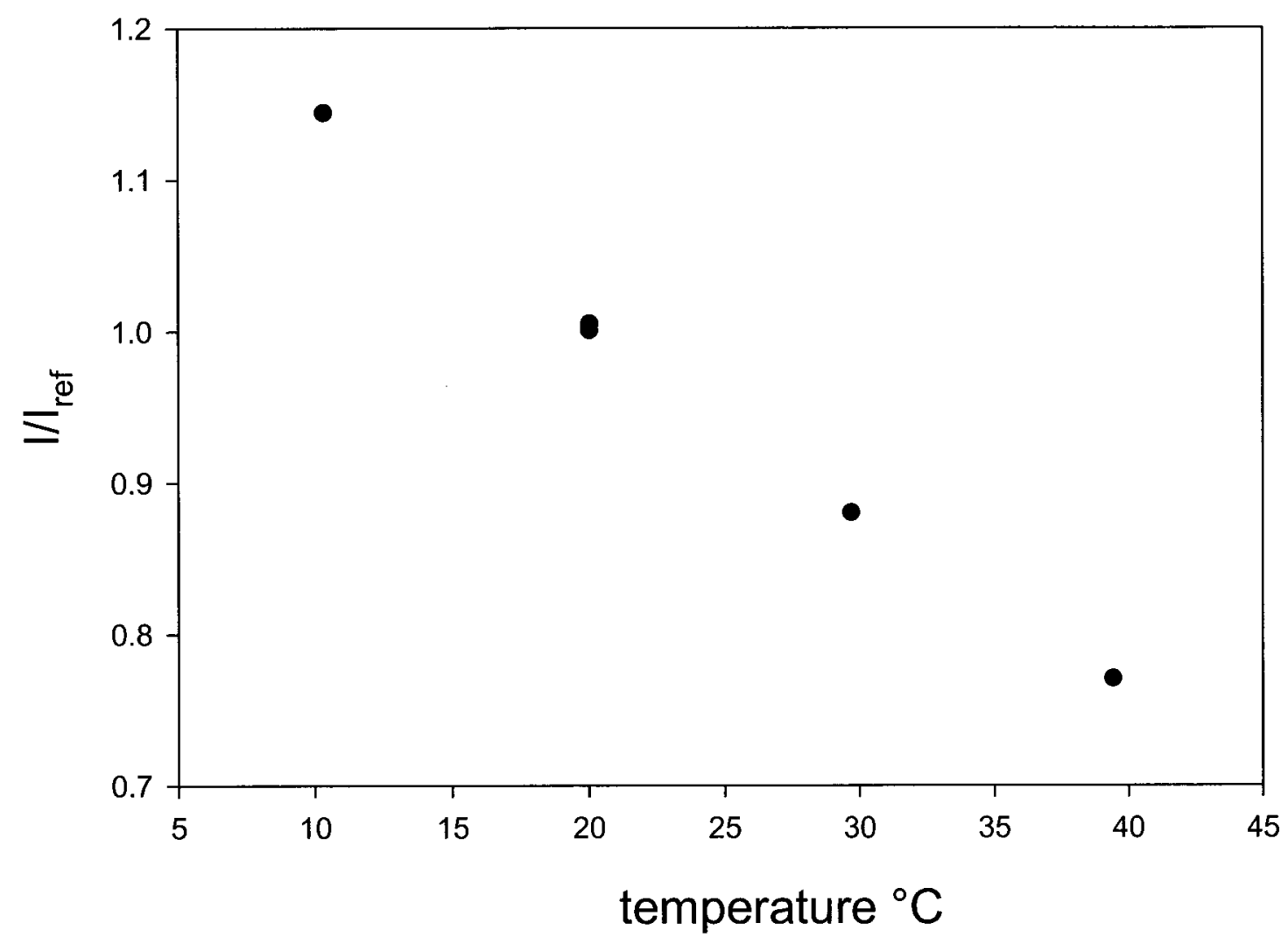

Figure 3.77: Temperature versus emission intensity ratio $\left(\mathrm{I}_{\mathrm{ref}}\right.$ is the intensity at $\left.10^{\circ} \mathrm{C}\right)$ of 14 in 5:1 Silamine: MC: vacuum $\bullet$.

The film was found to have a temperature sensitivity of $-1.3 \%{ }^{\circ} \mathrm{C}^{-1}$ at vacuum. 


\subsubsection{PSP 15}

As illustrated in Figure 3.78, the excimer emission of 15 is insignificantly quenched by oxygen with a pressure sensitivity of $10.6 \% /$ bar in a pressure range of 0.007 to $14.7 \mathrm{psi}$.
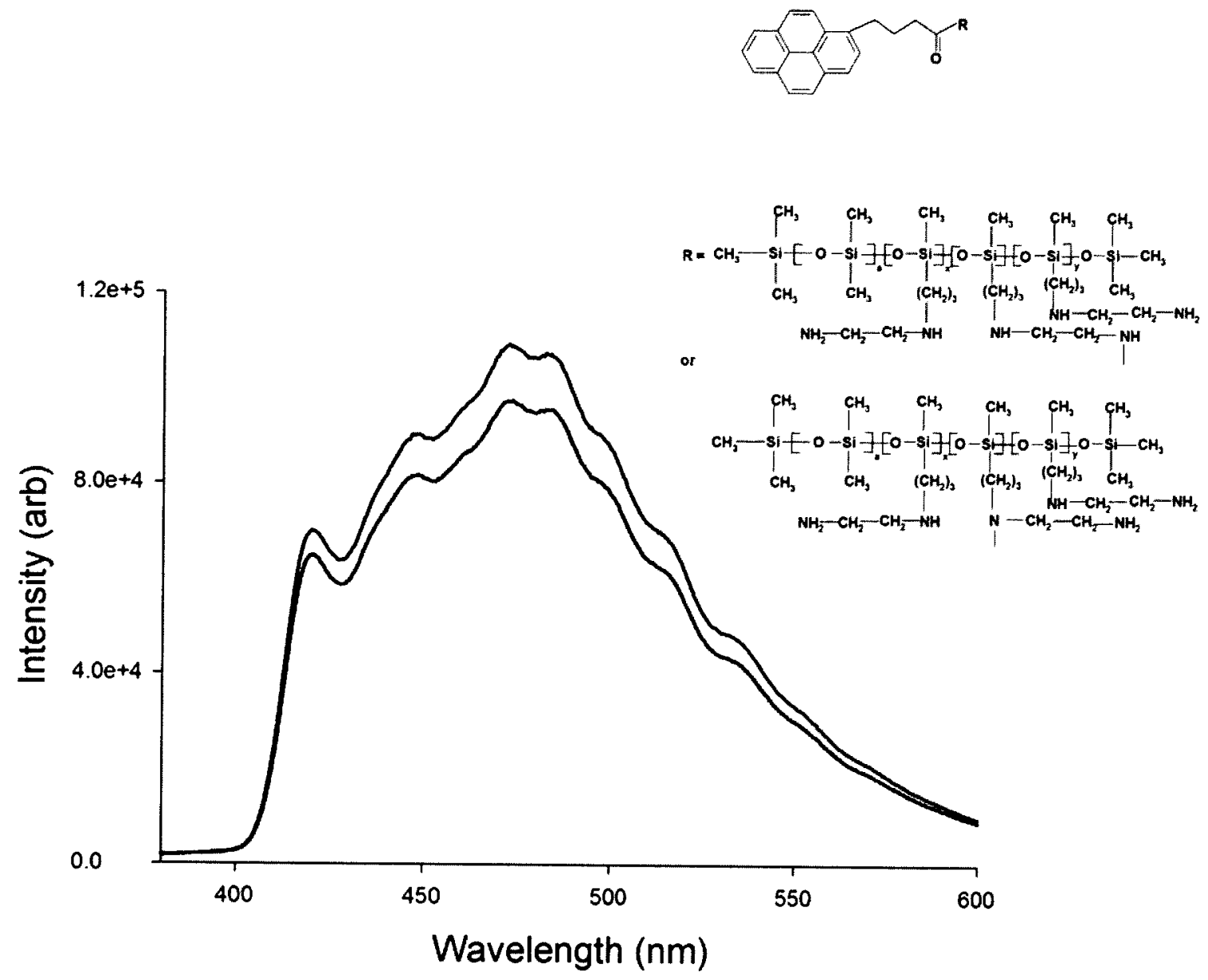

Figure 3.78: Effect of pressure on thin film luminescence of $\mathbf{1 5}$ in 9:1 Silamine: MC. The decrease of intensity with increasing the air pressure is displayed. Spectra were acquired at 1.3 mbar (black) and 1 bar (red).

While some pyrene derivatives (including PBA) exhibit poor oxygen quenching sensitivities in silicon matrices, ${ }^{97}$ there exists promising compound 1-decyl-4-(1-pyrenyl) butanoate with high oxygen sensitivity. Since preparation of a pyrene-based water- 
soluble formulation with appropriate excimer emission intensity was successful, it would be worthwhile to make a new water-soluble pyrene derivative with high oxygen quenching sensitivity. Synthesis of 4-(1-pyrenyl) butanal from PBA and attempt to bound it to Silamine by coupling or reductive attachment is proposed.

\subsubsection{Luminophore 7}

The cationic palladium porphyrin dispersed in PDMS-based Copolymer showed very broad red phosphorescence illustrated in Figure 3.79 the intensity of which was not very sensitive to the air pressure. 


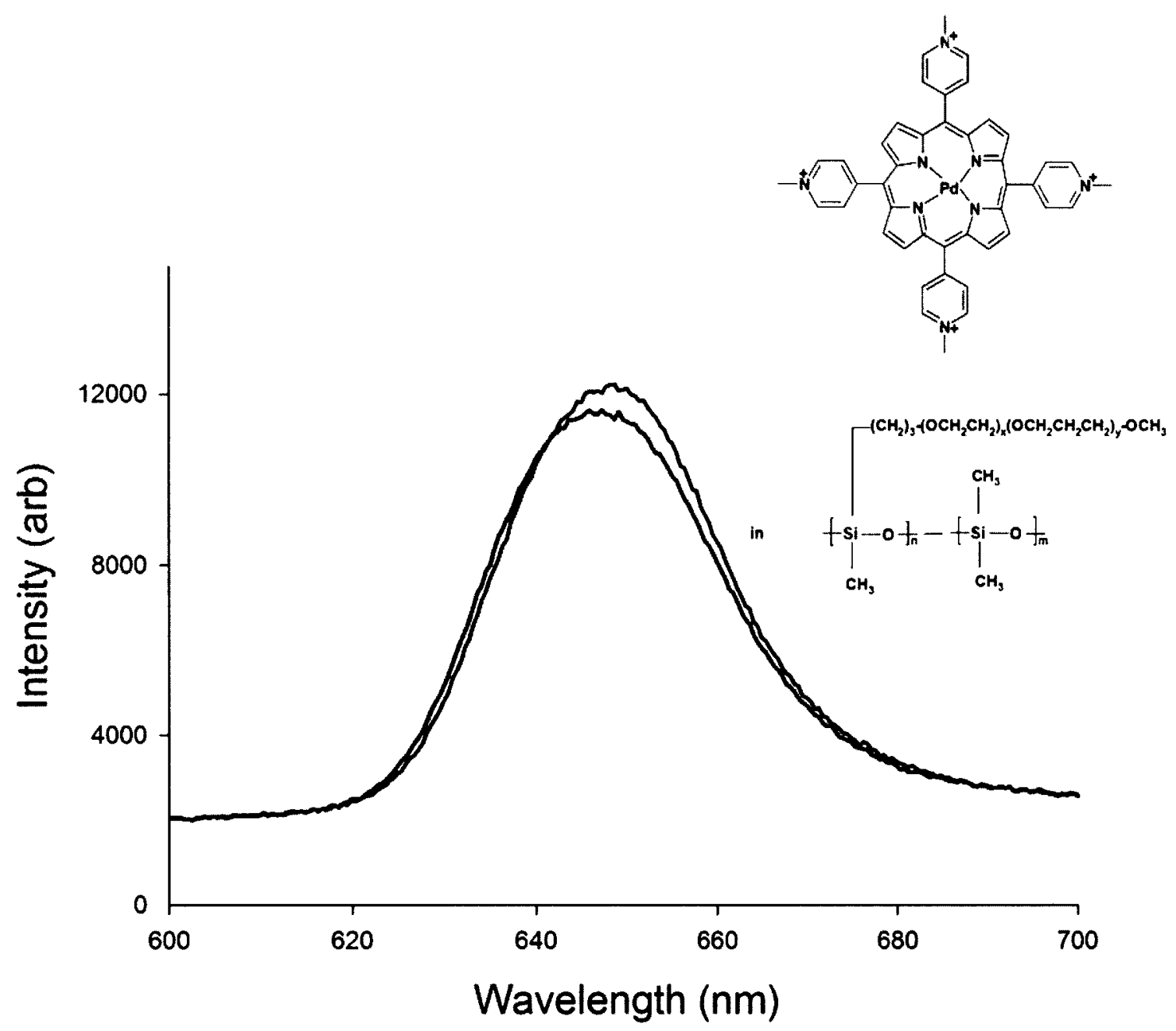

Figure 3.79: Effect of pressure on thin film luminescence of 7 in 9:1 PDMS-based Copolymer: MC. Spectra were acquired at 1.3 mbar (black) and 1 bar (red).

Porphyrins tend to aggregate resulting in oxygen insensitive dimers and higher aggregates $^{98}$ which can be an explanation for the oxygen sensitivity behavior of 7 . 


\section{Chapter 4}

\section{Conclusions and Future Studies}

\subsection{Summary of Water-Soluble PSP films}

Completely water-soluble pressure sensitive paints for visualizing air pressure are presented here. Using inexpensive water-soluble polymers and microcrystalline cellulose, spray-able PSP formulations were prepared. These paints offer an environmentally friendly alternative to non-aqueous PSP formulations and provide improved workplace safety, and lower contamination.

The first challenge was acquiring water-soluble matrices that dissolve luminophores and have good mechanical properties and high oxygen permeabilities. To achieve this goal, polyethylene glycol and water-soluble copolymers of the well known oxygen permeable polymer, polydimethylsiloxane, were used. The mechanical properties of these polymers need to be improved for practical applications. To compensate for the poor mechanical properties of the water-soluble polymers, microcrystalline cellulose was included in the formulations.

Luminescent iridium complexes have been used in PSPs because of their large quantum yields of emission under room temperature and normal pressure, and efficient quenching of the photoexcited triplet state by molecular oxygen. However, their solubility is poor in aqueous solution. The poor solubility was overcome by covalent attachment to water-soluble polymers. 
Producing water-soluble luminescent polymers by covalent attachment to the water-soluble amine-functionalized polymer Silamine D208-EDA by reductive amination was confirmed in $\mathbf{8}, \mathbf{1 3}$, and $\mathbf{1 4}$. In addition, it was shown that utilizing coupling reactions in synthesis of $\mathbf{9}, \mathbf{1 1}, \mathbf{1 2}$, and $\mathbf{1 5}$ is a practical covalent bonding method. Normally, polymer bound oxygen sensors show extended lifetimes compared with original dyes.

Oxygen quenching sensitivities of PSP films composed of 1, 2, 5, 7, and 10 dispersed in water-soluble polymer matrices, and $8,9,11,12,13,14$, and 15 in different film compositions were tested. Blending MC improved both oxygen quenching sensitivities and mechanical properties of the PSP films. It is suggested that this effect originates from a decrease in the luminophores freedom of motion and consequentially an increase in its luminescence lifetime. Moreover, the preferential binding of luminophore to MC could lead to de-aggregation and a decrease in self-quenching.

The optimum PSP formulations are $\mathbf{1 2}$ in 2.3:1 Silamine:MC, $\mathbf{1 3}$ in 2.3:1 Silamine:MC, and 8 in 1:1 Silamine:MC, which gave oxygen sensitivities of 0.379, 0.385 , and 0.502 , respectively, and are appropriate for pressure sensitive paint applications.

\subsection{Future Work}

Among water-soluble PSPs reported in this research, three sensors, 8, 12, and 13, are favourable with potential wind tunnel applications. In order to achieve even more sensitive water-soluble paints, it may be worthwhile to examine 7 , as a sensor with an 
increased lifetime, trying to eliminate aggregation using molecular carriers, such as cyclodextrin or calixarene, which monomerize or solubilize the aggregate and form noncovalent supramolecular complexes with porphyrins. ${ }^{98}$ Synthesis of new water-soluble oxygen permeable polymers (c. g. copolymerizing water-soluble polymer poly(vinyl pyrrolidone) with fluoropolymers) may result in highly sensitive water-soluble PSPs. Fluoropolymers such as poly(hexafluoroisopropyl-co-heptafluoro- $n$-butyl methacrylate) (known as FIB) and poly(2,2,2-trifluoroethyl-co-isobutyl methacrylate) are photostable oxygen permeable polymers. ${ }^{41}$ These copolymers can provide improved mechanical properties as well as oxygen permeability.

Reductive amination and coupling reactions utilized in this work are presented as efficient procedures towards covalent binding of sensors to water-soluble polymers. A pyrene-based water-soluble formulation with appropriate excimer emission intensity was successfully prepared. While some pyrene derivatives (including PBA) exhibit poor oxygen quenching sensitivities in silicon matrices, ${ }^{97}$ there exists promising compound 1decyl-4-(1-pyrenyl) butanoate with high oxygen sensitivity. It would be worthwhile to make a new water-soluble pyrene derivative with high oxygen quenching sensitivity by synthesis of 4-(1-pyrenyl) butanal from PBA and attempt to bound it to Silamine by coupling or reductive attachment.

Response times of pressure sensitive paints to passing shockwaves are important in studying pressure changes in unsteady flows. Including pressure sensitive luminophores in polymeric microspheres could afford fast responding oxygen sensors. Films of a luminescent platinum (II) porphyrin in core-shell particles made of a polystyrene core and a poly(vinyl pyrrolidone) shell were prepared using the aqueous 
dispersion of the sensor. The hydrogen bonds between poly(vinyl pyrrolidone) shell and aluminum surface provide the advantage of omitting the need for a binder polymer and its diffusional barrier, leading to desirable short response times. Response time of the films was approximately measured to be $<1 \mathrm{~s} .{ }^{99}$ Instrumentation for accurate measurements must be developed. Designing core-shell particles with tuned oxygen permeabilities could result in improved response times. Developing porous films would likely be beneficial in unsteady flow measurements.

The morphology and oxygen quenching sensitivities of blend films consisting of a linear poly(dimethylsiloxane) end-functionalized with a luminescent $\operatorname{Ir}(\mathrm{III})$ complex blended with polystyrene have shown the oxygen sensor response of the blend films with micrometer spatial resolution. ${ }^{58}$ Studying the film morphologies in order to map the oxygen permeability of the films and investigating the correlation between morphology and sensitivity of the films would also provide more insight into sensor design. 


\section{References}

1) Clark, L. C. Jnr. Trans. Am. Soc. Artif. Intern. Organs. 1956, 2, 41.

2) Ostermeier, W.; Ratingen, D. E. U. S. patent 4,807,463, 1989.

3) Hendricks H.D. US patent. $3709663,1973$.

4) Freeman T.M., Seitz W.R., Anal. Chem. 1981, 53, 98.

5) Kautsky, H.; Hirsch, A. Z. Anorg. Allg. Chem. 1935, 222, 126.

6) Typical examples include: a) Burke, C. S.; Moore, J. P.; Wencel, D.; McEvoy, A. K.; MacCraith, B. D. J. Biomed. Opt. 2008, 13, 014027/1. b) Ergeneman, O.; Dogangil, G.; Kummer, M. P.; Abbott, J. J.; Nazeeruddin, M. K.; Nelson, B. J. IEEE Sensors J. 2008, 8, 29. c) Papkovsky, D. B.; O'Riordan, T.; Soini, A. Biochem. Soc. Trans. 2000, 28, 74. d) Holekamp N. M.; Shui Y.-B.; Beebe D. Am. J. ophthal. 2006, 141, 1027. e) Holekamp N. M.; Shui Y.-B.; Beebe D. C. Am. J. ophthal.2005, 139, 302.

7) a) Basu, B. J. Sens. Act. B 2007, 123, 568. b) Takeuchi, Y.; Amao, Y. Springer Series Chem. Sens. Biosens. 2005, 3, 303. c) DeRosa, M. C.; Hodgson, D. J.; Enright, G. D.;

Dawson, B.; Evans, C. E. B.; Crutchley, R. J. J. Am. Chem. Soc. 2004, 126, 7619. d) Gregory, J. W.; Asai, K; Kameda, M.; Liu, T.; Sullivan, J. P. Proc. Inst. Mech. Eng. Part G: J. Aerospace Eng. 2007, 222, 249

8) a) Hasumoto, H.; Imazu, T.; Miura, T.; Kogure, K. J. Oceanography 2006, 62, 99. b) Gillanders, R. N.; Tedford, M. C.; Crilly, P. J.; Bailey, R. T. Anal. Chim. Act. 2005, 545, 189. c) Orellana, G.; Garcia-Fresnadillo, D. Springer Series Chem. Sens. Biosens. 2004, 1, 309. d) Hecht, H.; Kolling, M. Environ. Geol. 2002, 42, 800. e) Navarro, F.; 
Villoslada; Orellana, G.; Moreno-Bondi, M. C.; Vick, T.; Driver M.; Hildebrand, G.; Liefeith, K. Anal. Chem. 2001, 73, 5150.

9) a) Guo, L.; Ni, Q.; Li, J.; Zhang, L.; Lin, X.; Xie, Z.; Chen, G. Talanta 2008, 74, 1032. b) Jorge, P.; Caldas, P.; Da Silva, J. C. G.; Rosa, C.; Oliva, A.; Santos, J.; Farahi, F. Fiber Integ. Opt. 2005, 24, 201. c) Glazer, B. T.; Marsh, A. G.; Stierhoff, K.; Luther, G. W. Anal. Chim. Acta 2004, 518, 93.

10) a) O'Mahony, F. C.; O'Riordan, T. C.; Papkovskaia, N.; Kerry, J. P.; Papkovsky, D. B. Food Control 2005, 17, 286. b) O'Mahony, F. C.; O'Riordan, T. C.; Papkovskaia, N.; Ogurtsov, V. I.; Kerry, J. P.; Papkovsky, D. B. Packaging Technology \& Science 2004, 17, 225. c) von Bultzingslowen, C.; McEvoy, A. K.; McDonagh, C.; MacCraith, B. D.; Klimant, I.; Krause, C.; Wolfbeis, O. S. Analyst 2002, 127, 1478. d) Fitzgerald, M.; Papkovsky, D. B.; Smiddy, M.; Kerry, J. P.; O'Sullivan, C. K.; Buckley, D. J.; Guilbault, G. G. J. Food. Sci. 2001, 66, 105.

11) See for example: a) Nock, V.; Blaikie, R. J.; David, T. Proceedings of SPIE 2008, 6799, 67990Y/1. b) Lin, L.; Xiao, L.-L.; Huang, S.; Zhao, L.; Cui, J.-S.; Wang, X.-H.; Chen, X. Biosens. \& Bioelec. 2006, 21, 1703. c) Papkovsky, D. B.; Hynes, J.; Fernandes, R. Proceed. SPIE Int. Soc. Opt. Eng. 2005, 5994, 599406/1. d) Gao, F. G.; Fay, J. M.; Mathew, G.; Jeevarajan, A. S.; Anderson, M. M. J. Biomed. Opt. 2005, 10, 054005/1. e) Kwok, N.-Y.; Dong, S.; Lo, W.; Wong, K -Y. Sens. Act. B 2005, B110, 289. f) Gao, F.G.; Jeevarajan, A. S.; Anderson, M. M. Biotechnol. Bioeng. 2004, 86, 425. g) Tsukada, K.; Sakai, S.; Hase, K.; Minamitani, H. Biosens. Bioelectron. 2003, 18, 1439. h) John, G. T.; Klimant, I.; Wittmann, C.; Heinzle, E. Biotech. Bioeng. 2003, 81, 829. i) Chang-Yen, D. A.; Gale, B. K. IEEE Inter. Conf. Micro Electro Mech. Sys. Tech. Dig. 2002, 15, 574. j) 
O'Riordan, T. C.; Buckley, D.; Ogurtsov, V.; O'Connor, R.; Papkovsky, D. B. Anal. Biochem. 2000, 278, 221. k) Cheng, Y.; Samia, A. C.; Meyers, J. D.; Panagopoulos, I. ; Fei, B.; Burda, C. J. Am. Chem. Soc. 2008, 130, 10643. 1) Dolmans, D. E. J. G. J.; Fukumura, D.; Jain, R. K. Nature Rev. Cancer 2003, 3, 380. m) Brinas, R. P.; Troxler, T.; Hochstrasser, R. M.; Vinogradov, S. A. J. Am. Chem. Soc. 2005, 127, 11851.

12) a) Lewis G.N., Kasha M., J. Am. Chem. Soc. 1944, 66, 2100. b) Lott P.F., Hurtubise R.J., J. Chem. Edu. 1974, 51, A315.

13) Förster, T. Discuss Faraday Soc. 1959, 27, 7.

14) Dexter, D. J. Chem. Phys. 1953, $21,836$.

15) Atkins, P.; DePaula, J. Physical Chemistry, 7th edn. Oxford University Press, 2002, pp 920-934.

16) a) Smoluchowski, M. Z. Phys. Chem. 1917, 92, 129. b) Subczynski, W. K.; Hyde, J. S. Biophys. J. 1984, 45, 743. c) Vanderkooi, J. M.; Maniara, G.; Green, T. J.; Wilson, D. F. J. Biol. Chem. 1987, 262, 5476.

17) a) Valeur, B.; Bronchon, J. C. New Trends in Fluorescence Spectroscopy Application to Chemical and Life Science Springer Series on Fluorescence, Methods and Applications Springer, Berlin Heidelberg, 2001. b) Lakowicz, J. R.; Weber, G. Biochem. 1973, 12, 4161. c) Bowen, E. J. Trans. Farad. Soc. 1954, 50, 97. d) Stern, V.; Volmer, M. Physik Z. 1919, 20, 183. e) Lu, X.; Winnik, M. A. Chem. Mater. 2001, 13, 3449.

18) Gijzeman, O. L. J.; Kaufman, F.; Porter, G. J. Chem. Soc. Faraday Trans. II. 1973, $69,708$. 
19) a) Abdel-Shafi, A. A.; Worrall, D. R.; Ershov, A. Y. Dalton Trans. 2004, 1, 30. b) Abdel-Shafi, A. A.;Beer, P. D.; Mortimer, R. J.; Wilkinson, F. Phys. Chem. Chem. Phys. 2000, 2, 3137. c) Wilkinson, F.; Abdel-Shafi, A. A. J. Phys. Chem. A 1999, I03, 5425. 20) a)Obata, M.; Matsuura, N.; Mitsuo, K.; Nagai, H.; Asai, K.; Harada, M.; Hirohara, S.; Tanihara, M.; Yano, S. J. Polym. Sci. A 2010, 48, 663-670. b) Basu, B. J.; Vasantharajan, N.; Raju, C. Sens. Actuat. B 2009, 138, 283-288. c) Carlson, B.; Bullock, J. P.; Hance, T. M.; Phelan, G. D. Anal. Chem. 2009, 81, 262. d) Saerner, G.; Goeransson, U.; Linden, J.; Richter, M.; Alden, M. Meas. Sci. Tech. 2008, 19, 085307/1.e) Gregory, J. W.; Asai, K.; Kameda, M.; Liu, T.; Sullivan, J. P. Proceed. Ins. Mech. Eng. G J. Aeros. Eng. 2008, 222, 249. f) Huang, C.; Gregory, J. W.; Nagai, H.; Asai, K.; Sullivan, J. P. MicroElectro-Mech. Sys. 2007, 8, 577. g) Lee, S. J.; Kang, J. H. J. Visual. 2006, 9, 137. h) Hrdlovic, P. Polymer News 2002, 27, 350. i) Muller, G.; Krotz, G.; Schalk, J. Physica Status Solidi A Appl. Res.2001, 185, 1. j) Nagai, H.; Naraoka, R.; Sawada, K.; Asai, K. AIAA Journal 2008, 46, 215. k) Guerrero-Viramontes, J. A.; Moreno Hernandez, D.; Mendoza Santoyo, F.; Moran Loza, J. M.; Garcia Arreola, A. Proceed. SPIE Int. Soc. Opt. Eng. 2007, 6422, 64220H/1. 1) Yamashita, T. Y.; Sugiura, H.; Nagai, H.; Asai, K.; Ishida, K. J.Visual. 2007, 10, 289. m) Huang, C.; Gregory, J. W.; Sullivan, J. P. J. Microelectromech. Sys. 2007, 16, 777. n) Matsuda, Y.; Mori, H.; Niimi, T.; Uenishi, H.; Hirako, M. Exp. Fluids 2007, 42, 543. o) Castaldo, A.; Massera, E.; Quercia, L.; Di Francia, G. Sens. Act. B 2006, 118, 328. p) Mitsuo, K.; Asai, K.; Takahashi, A.; Mizushima, H. Meas. Sci. Technol. 2006, 17, 1282. q) Kimura, F.; Khalil, G.; Zettsu, N.; Xia, Y.; Callis, J.; Gouterman, M.; Dalton, L.; Dabiri, D.; Rodriguez, M. Meas. Sci. Technol. 2006, 17, 1254. r) Mori, H.; Niimi, T.; Hirako, M.; Uenishi, H. Meas. Sci. 
Technol. 2006, 17, 1242. s) McGraw, C. M.; Bell, J. H.; Khalil, G.; Callis, J. B. Exp. Fluids 2006, 40, 203. t) Kameda, M.; Tabei, T.; Nakakita, K.; Sakaue, H.; Asai, K. Meas. Sci. Technol. 2005, 16, 2517. u) Köse, M. E.; Omar, A.; Virgin, C. A.; Carroll, B. F.; Schanze, K. S. Langmuir 2005, 21, 9110. v) Koese, M. E.; Carroll, B. F.; Schanze, K. S. Langmuir 2005, 21, 9121. w) Obata, M.; Tanaka, Y.; Araki, N.; Hirohara, S.; Yano, S.; Mitsuo, K.; Asai, K.; Harada, M.; Kakuchi, T.; Outsuki, C. J. Polym. Sci. A Polym. Chem. 2005, 43,2997.

21) a) Gouterman, M.; Callis, J.; Dalton, L; Khalil, G.; Mebarki, Y.; Cooper, K. R.; Grenier, M. Meas. Sci.Technol. 2004, 15, 1986. b) Setnescu, R.; Jipa, S.; Setnescu, T.; Dånet, A. F.; Dondoi, M.; Dumitru, M. J. Optoelect. Adv. Mater. 2006, 8, 682.

22) Mills, A. Sens. Actuat. B 1998, 51, 60.

23) Bell, J. H.; Schairer, E. T.; Hand, L. A.; Mehta, R. D. Annu. Rev. Fluid Mech. 2001, $33,155$.

24) Khalil, G. E.; Costin, C.; Crafton, J.; Jones, G.; Grenoble, S.; Gouterman, M.; Callis, J. B.; Dalton, L. R. Sens. Actuators B 2004, 97, 13.

25) a) Peterson, J. I.; Fitzgerald, R. V. Rev. Sci. Instrum. 1980, 51, 670. b) Sanzmartinez, A.; Rois, A.; Valcarcel, M. Anal. Chim. Acta 1993, 284, 189.

26) a) Bergman, I. Nature, 1968, 218, 396. b) Wolfbeis, O. S.; Offenbacher, H.; Kroneis, H.; Marsoner, H. Mikrochim Acta. 1984, 1, 153. c) Kroneis, H. W.; Marsoner, H. J. Sens. Actuat. 1983, 4, 587. d) Lübbers, D. W.; Opitz, N. Sens. Actuat. 1983, 4, 641. e) Cox, M. E.; Dunn, D. Appl. Optics 1985, 24, 2114. f) Wolfbeis, O. S.; Posch, H. E.; Kroneis, H. Anal. Chem. 1985, 57, 2556. g) Wolfbeis, O. S. Fiber optic chemical sensors and biosensors, vol. 2 CRC Press, Boca Raton FL, 1991. 
27) a) Kalyanasundaram, K. Photochemistry of polypyridine and porphyrin complexes Academic Press, New York, 1992. b) Xu, W. Y.; Kneas, K. A.; Demas, J. N.; DeGraff, B. A. Anal. Chem., 1996, 68, 2605. c) Amao, Y.; Ishikawa, Y.; Okura, I. Anal. Chim. Acta. 2001, 445, 177. d) Vander Donckt, E.; Camerman, B.; Hendrick, F.; Herne, R.; Vandeloise, R. Bull. Soc. Chim. Belg. 1994, 103, 207. e) Di Marco, G.; Lanza, M.; Pieruccini, M.; Campagna, S. Adv. Mater. 1996, 8, 576. f) Di Marco, G.; Lanza, M.; Mamo, A.; Sefio, I.; Di Pietro, C.; Romeo, G.; Campagna, S. Anal. Chem. 1998, 70, 5019. g) Papkovsky, D. B.; Ponomarev, G. V.; Trettnak, W.; O’Leary, P. Anal. Chem. 1995, 67, 4112. h) Lee, S.-K.; Okura, I. Anal. Commun. 1997, 34, 185. i) Hartmann, P.; Trettnak, W. Anal. Chem. 1996, 68, 2615.j) Medina-Castillo, A. L.; Fernandez-Sanchez, J. F.; Klein, C.; Nazeeruddin, M. K.; Segura-Carretero, A.; Fernandez-Gutierrez, A.; Graetzel, M.; Spichiger-Keller, U. E. Analyst 2007, 132, 929.

28) Kneas, K. A.; Xu, W.; Demas, J. N.; DeGraff, B. A. Applied Spectr. 1997, 51, 1346.

29) Hyakutake, T.; Okura, I.; Asai, K.; Nishide, H. J. Mater. Chem. 2008, 18, 917.

30) Demas, J. N.; DeGraff, B. A. Anal. Chem. 1991, 63, 829A.

31) a) Douglas, P.; Eaton, K. Sens. Actuat. B 2002, 82, 200. b) Vasil'ev, V. V.; Borisov, S. M. Sens. Actuat. B 2002, 82, 272. c) DiMarco, G.; Lanza, M. Sens. Actuat. B 2000, 63, 42. d) Mills, A. Lepre, A. Anal. Chem. 1997, 69, 4653. e) Preininger, C.; Klimant, I.; Wolfbeis, O. S. Anal. Chem. 1994, 66, 1841. f) Amao, Y.; Miyahita, T.; Okura, I. Analyst 2000, 125, 871. g) Bacon, J. R.; Demas, J. N. Anal. Chem. 1987, 59, 2780.

32) a) Miller, M. T.; Karpishin, T. M. Sens. Actuat. B 1999, 61, 222. b) Wang, Y.; Li, B.; Liu, Y.; Zhang, L.; Zuo, Q.; Shi, L.; Su, Z. Chem. Commun. 2009, 39, 5868. 
33) Lojkowski, W.; Millers, D.; Fidelus, J.; Grigorieva, L.; Opalinska, A.; Narkiewicz, U.; Strek, W. WO 2007027116, 22pp.

34) a) Amao, Y.; Okura, I.; Miyashita, T. Chem. Lett. 2000, 1286. b) Amao, Y.; Okura, I.; Miyashita, T. Chem. Lett. 2000, 934.c) Amao, Y.; Ishikawa, Y.; Okura, I.; Miyashita, T. Bull. Chem. Soc. Jpn. 2001, 74, 2445.

35) Mills, A.; Lepre, A.; Theobald, B. R. C.; Slade, E.; Burrer, B. A. Anal. Chem. 1997, $69,2842$.

36) a) Yafuso, M.; Korkowski, P. F.; Mader, R. A.; Yan, C.; Carlock, J. T. US Patent 5,296,381, 1994. b) Holloway, R. R.; Kiang, T. T. US Patent 5,070,158, 1991. c) Wang, Z.; McWilliams, A. R.; Evans, C. E. B.; Lu, X.; Chung, S.; Winnik, M. A.; Manners, I. Adv. Func. Mat. 2002, 12, 415. d) DeRosa, M. C.; Mosher, P. J.; Evans, C. E. B.; Crutchley, R. J. Macromol. Symp. 2003, 196, 235. e) DeRosa, M. C.; Mosher, P. J.; Yap, G. P. A.; Focsaneanu, K. S.; Crutchley, R. J.; Evans, C. E. B. Inorg. Chem. 2003, 42, 4864.

37) Chu B. W. K.; Yam, V. W. W. Langmuir 2006, 22, 7437.

38) Hah, H. D.; Sakai, T.; Asai, K.; Nishide, H. Macromol. Symp. 2003, 204, 27.

39) Amao, Y. Microchim. Acta 2003, 143, 1.

40) Amao, Y.; Asai, K.; Miyashita, T.; Okura, I. Polym. Adv. Technol.2000, 11, 705.

41) a) Amao, Y.; Miyashita, T.; Okura, I. J. Fluor. Chem. 2001, 11. b) Amao, Y.; Asai, K.; Miyashita, T.; Okura, I. Polym. J. 1999, 31, 1267. c) Amao, Y.; Miyashita, T.; Okura, I. Anal. Chim. Acta. 2000, 421, 167. d) Amao, Y.; Asai, K.; Miyashita, T.; Okura, I. Chem. Lett. 1999, 1031. e) Amao, Y.; Miyashita, T.; Okura, I. J. Porphyrin. Phthalocyanine 2000, 5, 433. 
42) Puklin, E.; Carlson, B.; Gouin, S.; Costin, C.; Green, E.; Ponomarev, S.; Tanji, H.; Gouterman, M. J. Appl. Polym. Sci. 1999, 77, 2795.

43) Pauly, S. Polymer handbook, 3rd edn., Wiley, New York, 1989.

44) Xu, W. Y.; McDonough, R. C.; Langsdorf, B.; Demas, J. N.; DeGraff, B. A. Anal. Chem. 1994, 66, 4133.

45) a) klimant, I.; Wolfbeis, O. S. Anal. Chem. 1995, 67, 3160. b) Carraway, E. R.; Demas, J. N.; DeGraff, B. A. Anal. Chem. 1991, 63, 322.

46) a) Nagai, K.; Masuda, T.; Nakagawa, T.; Freeman, B. D.; Pinnau, I. Prog. Polym. Sci. 2001, 26,721. b) Masuda, T.; Isobe, E.; Higashimura, T.; Takada, K. J. Am. Chem. Soc. $1983,105,7473$.

47) Avnir, D.; Levy, D.; Reisfeld, R. J. Phys. Chem.1984, 88, 5956.

48) a) Chen, X.; Zhong, Z.; Li, Z.; Jiang, Y.; Wang, X.; Wong, K. Y. Sens. Actuat. B 2002, 87, 233. b) Mackenzie, J. D.; Bescher, E. P. J. Sol-Gel Sci. Tech. 1998, 13, 371. c) Schmidt, H.; Jonschker, G.; Goedicke, S.; Mennig, M. J. Sol-Gel Sci. Tech. 2000, 19, 39.

49) a) Malins, C.; Fanni, S.; Glever, H. G.; Vos, J. G.; MacCraith, B. D. Anal. Commun. 1999, 36, 3. b) Franville, A. C.; Zambon, D.; Mahiou, R. Chem. Mater. 2000, 12, 428.

50) Cot, L.; Ayral, A.; Durand, J.; Guizard, C.; Hovnanian, N.; Julbe, A.; Larbot, A. Solid State Sci. 2000, 2, 313.

51) Lu, X.; Manners, I.; Winnik, M. A. Macromolecules 2001, 34, 1917.

52) a) Lazarin, A. M.; Gushikem, Y.; Castro, S. C. J. Mater. Chem. 2000, 10, 2526. b) Filho, U. P. R.; Gushikem, Y.; Fujiwara, F. Y.; Castro, S. C.; Torriani, I. C. L.; Cavalcanti, L. P. Langmuir 1994, 10, 4357. c) Wara, N. M.; Francis, L. F.; Velamakanni, B. V.J. Membr. Sci. 1995, 104, 43. 
53) Fernández-Sánchez, J. F.; Cannas, R.; Spichiger, S.; Steiger, R.; Spichiger-Keller, U. E. Anal. Chim. Acta 2006, 566, 271.

54) a) Schmidt, H. Mat. Res. Soc. Symp. Proc. 1984, 32, 327. b) McDonagh, C.; MacCraith, B. D.; McEvoy, A. K. Anal. Chem. 1998, 70, 45.

55) a) Bukowski, R. M.; Ciriminna, R.; Pagliaro, M.; Bright, F. V. Anal. Chem. 2005, 77, 2670. b) Chu, C. S.; Lo, Y. L. Sens. Actuators, B 2007, 124,376. c) Campostrini, R.; Ischia, M.; Carturan, G.; Armelao, L. J. Sol-Gel Sci. Technol. 2002, 23, 107.

56) a) Merienne, M.-C.; Le Sant, Y.; Ancelle, J.; Soulevant, D. Meas. Sci. Technol. 2004, 15, 2349. b) Asai, K.; Amao, Y.; Iijima, Y.; Okura, I.; Nishide, H. J. Thermophys. Heat Transfer 2002, 16, 109. c) Kameda, M.; Tezuka, N.; Hangai, T.; Asai, K.; Nakakita, K.; Amao, Y. Meas. Sci. Technol. 2004, 15, 489.

57) Oglesby, D. M.; Ingram, J. L.; Jordan, J. D.; Watkins, A. N.; Leighty, B. D. NASA/TM 2004, 2004-213268,1.

58) Kose, M. E.; Crutchley, R. J. ; DeRosa, M. C.; Ananthakrishnan, N.; Reynolds,J. R.; Schanze, K. S. Langmuir, 2005, 21, 8255.

59) Lin, H.; Freeman, B. D. J. Memb. Sci. 2004, 239, 105.

60) Park, H. J.; Weller, C. L.; Vergano, P. J.; Testin, R. F. J. Food Sci. 1993, 58, 1361.

61) a) Borch, R. F.; Durst, H. D. J. Am. Chem. Soc. 1969, 91, 3996. b) Borch, R. F.; Bernstein, M. D.; Durst, H. D. J. Am. Chem.Soc. 1971, 93, 2897.

62) Bomann, M. D.; Guch, I. C.; DiMare, M. J. Org. Chem. 1995, 60, 5995.

http://www.siltechcorp.com/index.php?option=com_content\&task=view\&id=44\&Itemid $=33$ 
64) Markó, I. E.; Giles, P. R.; Tsukazaki, M.; Brown, S. M.; Urch, C. J. Science, 1996, 274, 2044.

65) Sheehan, J. C.; Hess, G. P. J. Am. Chem. Soc. 1955, 77, 1067.

66) Layer, R. W. Chem. Rev. 1963, 63, 489.

67) a) Lo, K. K.-W.; Chung, C.-K.; Zhu, N. Chem. Eur. J. 2003, 9, 475. b) Lo, K. K.-W.; Lau, J. S.-Y. Inorg. Chem. 2007, 46, 700.

68) Evans, C. E. B.; Crutchley, R. J.; Derosa, M. C.; Mosher, P. J. PCT Int. Appl., 2004, 65 pp. WO 2004031321.

69) a) Lo, K. K.-W.; Ng, D. C. M.; Chung, C. K. Organometallics 2001, 20, 4999. b) Lo, K. K.-W.; Chung, C. K.; Ng, D. C. M.; Zhu, N. Y. New J. Chem. 2002, 26, 81. c) Lo, K. K.-W.; Chung, C. K.; Lee, T. K. M.; Lui, L. H.; Tsang, K. H. K.; Zhu, N. Y. Inorg. Chem. 2003, 42, 6886. d) Lo, K. K.-W.; Zhang, K. Y.; Leung, S. K.; Tang, M. C. Angew. Chem. Int. Ed. 2008, 47,2213. e) Lo, K. K.-W.; Zhang, K. Y.; Chung, C. K.; Kwok, K. Y. Chem. Eur. J. 2007, 13, 7110. f) Lo, K. K.-W.; Chung, C. K.; Zhu, N. Y. Chem. Eur. J. 2006, 12, 1500. g) Lo, K. K.-W.; Hui, W. K.; Chung, C. K.; Tsang, K. H. K.; Ng, D. C. M.; Zhu, N. Y.; Cheung, K. K. Coord. Chem. Rev. 2005, 249, 1434.

70) a) Borisov, S. M.; Klimant, I. Anal. Chem. 2007, 79, 7501. b) Fernandez-Sanchez, J. F.; Roth, T.; Cannas, R.; Nazeeruddin, M. K.; Spichiger, S.; Graetzel, M.; SpichigerKeller, U. E. Talanta 2007, 71, 242.

71) a) Bolink, H. J.; Cappelli, L.; Coronado, E.; Gratzel, M.; Orti, E.; Costa, R. D.; Viruela, P. M.; Nazeeruddin, M. K. J. Am. Chem. Soc. 2006, 128, 14786. b) Bolink, H. J.; Cappelli, L.; Coronado, E.; Parham, A.; Stossel, P. Chem. Mater. 2006, 18, 2778. c) Chen, F. C.; Yang, Y.; Pei, Q. Appl. Phys. Lett. 2002, 81, 4278. d) Su, H. C.; Chen, H. F.; 
Fang, F. C.; Liu, C. C.; Wu, C. C.; Wong, K. T.; Liu, Y. H.; Peng, S. M. J. Am. Chem. Soc. 2008, 130, 3413. e) Zeng, X. S.; Tavasli, M.; Perepichka, I. E.; Batsanov, A. S.; Bryce, M. R.; Chiang, C. J.; Rothe, C.; Monkman, A. P. Chem. Eur. J. 2008, 14, 933. f) Bolink, H. J.; Cappelli, L.; Cheylan, S.; Coronado, E.; Costa, R. D.; Lardies, N.; Nazeeruddin, M. K.; Orti, E. J. Mater. Chem. 2007, 17, 5032. g) Dragonetti, C.; Falciola, L.; Mussini, P.; Righetto, S.; Roberto, D.; Ugo, R.; Valore, A.; De Angelis, F.; Fantacci, S.; Sgamellotti, A.; Ramon, M.; Muccini, M. Inorg. Chem. 2007, 46, 8533. h) Tamayo, A. B.; Garon, S.; Sajoto, T.; Djurovich, P. I.; Tsyba, I. M.; Bau, R.; Thompson, M. E. Inorg. Chem. 2005, 44, 8723. i) Nazeeruddin, M. K.; Wegh, R. T.; Zhou, Z.; Klein, C.; Wang, Q.; De Angelis, F.; Fantacci, S.; Gratzel, M. Inorg. Chem. 2006, 45, 9245.

72) a) Baldo, M. A.; O’Brien, D. F.; You, Y.; Shoustikov, A.; Sibley, S.; Thompson, M. E.; Forrest, S. R. Nature 1998, 395, 151. b) Baldo, M. A.; Lamansky, S.; Burrows, P. E.; Thompson, M. E.; Forrest, S. R. Appl. Phys. Lett. 1999, 75, 4. c) Lamansky, S.; Djurovich, P.; Murphy, D.; Abdel-Razzaq, F.; Lee, H. E.; Adachi, C.; Burrows, P. E.; Forrest, S. R.; Thompson, M. E. J. Am. Chem. Soc. 2001, 123, 4304. d) Tsuboyama, A.; Iwawaki, H.; Furugori, M.; Mukaide, T.; Kamatani, J.; Igawa, S.; Moriyama, T.; Miura, S.; Takiguchi, T.; Okada, S.; Hoshino, M.; Ueno, K. J. Am. Chem. Soc. 2003, 125, 12971. e) Adachi, C.; Baldo, M. A.; Forrest, S. R.; Thompson, M. E. Appl. Phys. Lett. 2000, 77, 904. f) Grushin, V. V.; Herron, N.; LeCloux, D. D.; Marshall, W. J.; Petrov, V. A.; Wang, Y. Chem. Commun. 2001, 1494. g) Adachi, C.; Baldo, M. A.; Forrest, S. R.; Lamansky, S.; Thompson, M. E.; Kwong, R. C. Appl. Phys. Lett. 2001, 78, 1622. h) Adachi, C.; Baldo, M. A.; Thompson, M. E.; Forrest, S. R. J. Appl. Phys. 2001, 90, 5048. i) Duan, J. P.; Sun, P. P.; Cheng, C. H. Adv. Mater. 2003, 15, 224. j) Su, Y. J.; Huang, H. 
L.; Li, C. L.; Chien, C. H.; Tao, Y. T.; Chou, P. T.; Datta, S.; Liu, R. S. Adv. Mater. 2003, 15, 884. k) Nazeeruddin, M. K.; Humphry-Baker, R.; Berner, D.; Rivier, S.; Zuppiroli, L.; Graetzel, M. J. Am. Chem. Soc. 2003, 125, 8790. 1) Inomata, H.; Goushi, K; Masuko, T.; Konno, T.; Imai, T.; Sasabe, H.; Brown, J. J.; Adachi, C. Chem. Mater. 2004, 16, 1285. m) Paulose, B.; Rayabarapu, D. K.; Duan, J. P.; Cheng, C. H. Adv. Mater. 2004, 16, 2003. n) Rayabarapu, D. K.; Paulose, B.; Duan, J. P.; Cheng, C. H. $A d v$. Mater. 2005, 17, 349. o) Yeh, S. J.; Wu, M. F.; Chen, C. T.; Song, Y. H.; Chi, Y.; Ho, M. H.; Hsu, S. F.; Chen, C. H. Adv. Mater. 2005, 17, 285. p) Guan, M.; Chen, Z. Q.; Bian, Z. Q.; Liu, Z. W.; Gong, Z. L.; Baik, W.; Lee, H. J.; Huang, C. H. Org. Electron. 2006, 7, 330. q) Tsuzuki, T.; Tokito, S. Adv. Mater. 2007, 19, 276. r) Ho, C. L.; Wong, W. Y.; Gao, Z. Q.; Chen, C. H.; Cheah, K. W.; Yao, B.; Xie, Z. Y.; Wang, Q.; Ma, D. G.; Wang, L. A.; Yu, X. M.; Kwok, H. S.; Lin, Z. Y. Adv. Funct. Mater. 2008, $18,319$.

73) a) Chen, F. F.; Bian, Z. Q.; Liu, Z. W.; Nie, D. B.; Chen, Z. Q.; Huang, C. H. Inorg. Chem. 2008, 47, 2507. b) Mehlstaubl, M.; Kottas, G. S.; Colella, S.; De Cola, L. Dalton Trans. 2008, 2385. c) Coppo, P.; Duati, M.; Kozhevnikov, V. N.; Hofstraat, J. W.; De Cola, L. Angew. Chem. Int. Ed. 2005, 44, 1806.

74) a) Sprouse, S.; King, K. A.; Spellane, P. J.; Watts, R. J. J. Am. Chem. Soc., 1984, 106, 6647. b) Nonoyama, M. Bull. Chem. Soc. Jpn., 1974, 47, 767.

75) Li, J.; Djurovich, P. I.; Alleyne, B. D.; Yousufuddin, M.; Ho, N. N.; Thomas, J. C.; Peters, J. C.; Bau, R.; Thompson, M. E. Inorg. Chem. 2005, 44, 1713.

76) a) Lafolet, F.; Welter, S.; Popovic', Z.; De Cola, L. J. Mater. Chem. 2005, 15, 2820. b) Huynh, L.; Wang, Z.; Yang, J.; Stoeva, V.; Lough, A.; Manners, I.; Winnik, M. A. Chem. Mater. 2005, 17, 4765. 
77) a) De Gans, B. J.; Duineveld, P. U. S. Schubert, Adv. Mater. 2004, 16, 203. b)

Bedlek-Anslow, J. M.; Hubner, J. P.; Carroll, B. F.; Schanze, K. S. Langmuir 2000, 16, 9137.

78) Lowry, M. S.; Bernhard, S. Chem. Eur. J. 2006, 12, 7970.

79) Chandler, C. J.; Deady, L. W.; Reiss, J. A. J. Heterocyclic Chem.1981, 18, 599.

80) Pasternack, R. F.; Brigandi, R. A.; Abrams, M. J.; Williams, A. P.; Gibbs, E. J. Inorg. Chem. 1990, 29, 4483.

81) Kalyanasundaram, K.; Neumann-Spallart, M. J. Phys. Chem. 1982, 86, 5163.

82) a) Sheldrick, G. SADABS and SHELXTL; Bruker-AXS Inc; Madison, WI, 2001. b) Sheldrick, G. M. Acta Cryst 2008, A64, 112.

83) Spek, A. L. J. Appl. Crystallogr. 2003, 36, 7.

84) Lamansky, S.; Djurovich, P.; Murphy, D.; Abdel-Razzaq, F.; Kwong, R.; Tsyba, I.; Bortz, M.; Mui, B.; Bau, R.; Thompson, M. E. Inorg. Chem. 2001, 40, 1704.

85) Chen, H.; Zhao, Q.; Wu, Y.; Li, F.; Yang, H.; Yi, T.; Huang, C. Inorg. Chem. 2007, 46,11075 .

86) a) Tejel, C.; Ciriano, M. A.; Lopez, J. A.; Lahoz, F. J.; Oro, L. A. Organometallics 2000, 19, 4977. b) Tejel, C.; Ciriano, M. A.; Lahoz, F. J.; Oro, L. A. Organometallics 1998, 17, 1449. c) Dobbs, D. A.; Bergman, R. G. Inorg. Chem. 1994, 33, 5329. d) Mague, J. T. Polyhedron 1992, 11, 677. e) Maverick, A. W.; Smith, T. P.; Maverick, E. F.; Gray, H. B. Inorg. Chem. 1987, 26, 4336.

87) Tamayo, A. B.; Alleyne, B. D.; Djurovich, P. I.; Lamansky, S.; Tsyba, I.; Ho, N. N.; Bau, R.; Thompson, M. E. J. Am. Chem. Soc. 2003, 125, 7377.

88) Qian, G.; Yang, Z.; Yang, C.; Wang, M. J. Appl, Phys. 2000, 88, 2503. 
89) Caron, S.; Massett, S. S.; Bogle, D. E.; Castaldi, M. J.; Braish, T. F. Org. proc. Res. Dev., 2001, 5, 254.

90) Schmid, B.; Garces, F. O.; Watts, R. J. Inorg. Chem., 1994, 33, 9.

91) Colombo, M. G.; Brunold, T. C.; Riedener, T.; Güdel, H. U.; Förtsch, M.; Bürgi, H.B. Inorg. Chem., 1994, 33, 545.

92) a) Ohsawa, Y.; Sprouse, S.; King, K. A.; DeArmond, M. K.; Hanck, K. W.; Watts, R. J. J. Phys. Chem. 1987, 91, 1047. b) Didier, P.; Ortmans, I.; Kirsch-De Mesmaeker, A.; Watts, R. J. Inorg. Chem. 1993, 32, 5239. c) Calogero, G.; Giuffrida, G.; Serroni, S.; Ricevuto, V.; Campagna, S. Inorg. Chem. 1995, 34, 541. d) Neve, F.; Crispini, A.; Campagna, S.; Serroni, S. Inorg. Chem. 1999, 38, 2250. e) van Diemen, J. H.; Hage, R.; Haasnoot, J. G.; Lempers, H. E. B.; Reedijk, J.; Vos, J. G.; De Cola, L.; Barigelletti, F.; Balzani, V. Inorg. Chem. 1992, 31, 3518. f) Serroni, S.; Juris, A.; Campagna, S.; Venturi, M.; Denti, G.; Balzani, V. J. Am. Chem. Soc. 1994, 116, 9086. g) Ortmans, I.; Didier, P.; Kirsch-De Mesmaeker, A. Inorg. Chem. 1995, 34, 3695. h) Maestri, M.; Balzani, V.; Deuschel-Cornioley, C.; von Zelewsky, A. Adv. Photochem. 1992, 17, 1.

93) Wilde, A. P.; King, K. A.; Watts, R. J. J. Phys. Chem. 1991, 95, 629.

94) Gross, E.; Meienhofer, J. The Peptides 1979-1981, Academic Press, New York.

95) Basu, B. J.; Anandan, C.; Rajam, K. S. Sens. Actuat. B 2003, 94, 257.

96) Yang, S.; Wu, X.; Wang, X.; Samuelson, L. A.; Cholli, A. L.; Kumar, J. J. Macromol. Sci. A 2003, 40, 1275.

97) Basu, B. J.; Rajam, K. S. Sens. Actuat. 2004, 99, 459.

98) Mosinger, J.; Janošková, M.; Lang, K.; Kubát, P. J. Photochem. Photobiol. A 2006, $181,283$. 
99) Fischer, L. H.; Borisov, S. M.; Schaeferling, M.; Klimant, I.; Wolfbeis, O. S. Analyst 2010, $135,1224$. 


\section{Appendix A: Crystallographic Data}

Table 1: Crystal data and structure refinement for $2 \cdot 0.25\left(\mathrm{CH}_{2} \mathrm{Cl}_{2}\right)$

Computing details

Data collection: SMART (Bruker, 2002); cell refinement: SAINT (Bruker, 2002); data reduction: SAINT; program(s) used to solve structure: SHELXS97 (Sheldrick, 1990); program(s) used to refine structure: SHELXL97 (Sheldrick, 1997); molecular graphics: SHELXTL (Sheldrick, 2001); software used to prepare material for publication: SHELXTL (Sheldrick, 2001).

\section{Crystal data}

$$
\begin{aligned}
& \mathrm{C}_{35.25} \mathrm{H}_{34.50} \mathrm{Cl}_{0.50} \mathrm{~F}_{3} \mathrm{IrN}_{4} \mathrm{O}_{3} \mathrm{~S} \\
& M_{r}=861.15 \\
& \text { Triclinic, } P 1 \\
& a=15.164(5) \AA \\
& b=17.083(6) \AA \\
& c=17.242(6) \AA \\
& \alpha=117.569(5)^{\mathrm{o}} \\
& \beta=104.876(5)^{\mathrm{o}} \\
& \gamma=100.075(5)^{\mathrm{o}} \\
& V=3595(2) \AA^{3}
\end{aligned}
$$

$$
\begin{aligned}
& Z=4 \\
& F_{000}=1706 \\
& D_{\mathrm{x}}=1.591 \mathrm{Mg} \mathrm{m}^{-3} \\
& \text { Mo } K \alpha \text { radiation } \\
& \lambda=0.71073 \AA \\
& \text { Cell parameters from } 439 \text { reflections } \\
& \theta=1.6-23.4^{\circ} \\
& \mu=3.86 \mathrm{~mm}^{-1} \\
& T=120(2) \mathrm{K} \\
& \text { Plate, yellow } \\
& 0.25 \times 0.17 \times 0.08 \mathrm{~mm}
\end{aligned}
$$

\section{Data collection}

Bruker AXS APEX diffractometer

Radiation source: fine-focus sealed tube Monochromator: graphite

Detector resolution: 836.6 pixels $\mathrm{mm}^{-1}$

$$
T=120(2) \mathrm{K}
$$

$\omega$ scans

Abs. corr.: multi-scan SADABS (Sheldrick, 2003)

$T_{\min }=0.441, T_{\max }=0.745$

38770 measured reflections
12670 independent reflections

9231 reflections with $I>2 \sigma(I)$

$R_{\text {int }}=0.040$

$\theta_{\max }=25.0^{\circ}$

$\theta_{\min }=1.6^{\circ}$

$h=-18 \rightarrow 18$

$k=-20 \rightarrow 20$

$l=-20 \rightarrow 20$ 


\section{Refinement}

Refinement on $F 2$

Least-squares matrix: full

$R\left[F^{2}>2 \sigma\left(F^{2}\right)\right]=0.050$

$w R\left(F^{2}\right)=0.142$

$S=1.06$

12670 reflections

811 parameters

1632 restraints

Primary atom site location: structure-invariant direct methods

\section{Special details}

Experimental. Data collection is performed with four batch runs at $\varphi=0.00^{\circ}(600$ frames), at $\varphi=90.00^{\circ}$ (600 frames), at $\varphi=180^{\circ}$ (600 frames) and at $\varphi=270^{\circ}(600$ frames). Frame width $=0.30 \backslash \&$ in $\omega$. Data is merged, corrected for decay, and treated with multiscan absorption corrections.

Geometry. All e.s.d.'s (except the e.s.d. in the dihedral angle between two 1.s. planes) are estimated using the full covariance matrix. The cell e.s.d.'s are taken into account individually in the estimation of e.s.d.'s in distances, angles and torsion angles; correlations between e.s.d.'s in cell parameters are only used when they are defined by crystal symmetry. An approximate (isotropic) treatment of cell e.s.d.'s is used for estimating e.s.d.'s involving l.s. planes.

Refinement. Refinement of $F^{2}$ against ALL reflections. The weighted $R$-factor $w R$ and goodness of fit $S$ are based on $F^{2}$, conventional $R$-factors $R$ are based on $F$, with $F$ set to zero for negative $F^{2}$. The threshold expression of $F^{2}>\sigma\left(F^{2}\right)$ is used only for calculating $R$-factors(gt) etc. and is not relevant to the choice of reflections for refinement. $R$-factors based on $F^{2}$ are statistically about twice as large as those based on $F$, and $R$ - factors based on ALL data will be even larger.

Table 2: Fractional atomic coordinates and isotropic or equivalent isotropic displacement parameters $\left(\AA^{2}\right)$ for $2 \cdot 0.25\left(\mathrm{CH}_{2} \mathrm{Cl}_{2}\right)$.

$\begin{array}{llllll} & x & y & z & U_{\text {iso }}{ }^{*} / U_{\text {eq }} & \text { Occ. }(<1) \\ \text { Ir1 } & 0.445091(17) & 0.217574(17) & 0.686611(18) & 0.05862(11) \\ \text { Ir2 } & 0.89507(2) & 0.219383(18) & 0.236746(18) & 0.06763(12) \\ \text { N1 } & 0.5671(4) & 0.1807(4) & 0.7003(4) & 0.0726(14) \\ \text { C1 } & 0.6587(5) & 0.2421(7) & 0.7557(6) & 0.097(2) \\ \text { H1A } & 0.6690 & 0.3076 & 0.7882 & 0.117^{*} \\ \text { C2 } & 0.7379(6) & 0.2126(8) & 0.7665(7) & 0.112(3)\end{array}$




\begin{tabular}{|c|c|c|c|c|c|}
\hline $\mathrm{H} 2 \mathrm{~A}$ & 0.8018 & 0.2568 & 0.8058 & $0.135^{*}$ & \\
\hline C3 & $0.7217(6)$ & $0.1187(7)$ & $0.7195(6)$ & $0.101(3)$ & \\
\hline $\mathrm{H} 3 \mathrm{~A}$ & 0.7752 & 0.0973 & 0.7268 & $0.121^{*}$ & \\
\hline $\mathrm{C} 4$ & $0.6299(6)$ & $0.0542(7)$ & $0.6618(6)$ & $0.090(2)$ & \\
\hline $\mathrm{H} 4 \mathrm{~A}$ & 0.6198 & -0.0111 & 0.6283 & $0.108^{*}$ & \\
\hline $\mathrm{C} 5$ & $0.5512(5)$ & $0.0865(5)$ & $0.6529(5)$ & $0.0672(15)$ & \\
\hline C6 & $0.4504(4)$ & $0.0276(4)$ & $0.5957(4)$ & $0.0621(14)$ & \\
\hline $\mathrm{C} 11$ & $0.4199(6)$ & $-0.0718(5)$ & $0.5399(5)$ & $0.0758(18)$ & \\
\hline $\mathrm{H} 11 \mathrm{~A}$ & 0.4667 & -0.1025 & 0.5361 & $0.091 *$ & \\
\hline $\mathrm{C} 10$ & $0.3245(6)$ & $-0.1227(5)$ & $0.4924(5)$ & $0.0802(19)$ & \\
\hline $\mathrm{H} 10 \mathrm{~A}$ & 0.3042 & -0.1894 & 0.4583 & $0.096^{*}$ & \\
\hline C9 & $0.2549(5)$ & $-0.0788(4)$ & $0.4927(5)$ & $0.0744(17)$ & \\
\hline $\mathrm{C} 8$ & $0.2856(5)$ & $0.0204(4)$ & $0.5454(4)$ & $0.0611(15)$ & \\
\hline $\mathrm{H} 8 \mathrm{~A}$ & 0.2385 & 0.0504 & 0.5450 & $0.073^{*}$ & \\
\hline $\mathrm{C} 7$ & $0.3828(4)$ & $0.0747(4)$ & $0.5977(4)$ & $0.0558(13)$ & \\
\hline $\mathrm{C} 12$ & $0.1519(6)$ & $-0.1353(6)$ & $0.4409(6)$ & $0.101(3)$ & \\
\hline $\mathrm{H} 12 \mathrm{~A}$ & 0.1343 & -0.2017 & 0.4100 & $0.121^{*}$ & \\
\hline $\mathrm{O} 1$ & $0.0910(6)$ & $-0.1044(6)$ & $0.4353(6)$ & $0.152(3)$ & \\
\hline $\mathrm{N} 2$ & $0.3128(4)$ & $0.2348(4)$ & $0.6716(4)$ & $0.0659(13)$ & \\
\hline $\mathrm{C} 13$ & $0.2627(5)$ & $0.2537(5)$ & $0.6098(5)$ & $0.0783(19)$ & \\
\hline H13A & 0.2924 & 0.2644 & 0.5718 & $0.094^{*}$ & \\
\hline $\mathrm{C} 14$ & $0.1721(6)$ & $0.2586(6)$ & $0.5985(6)$ & $0.093(2)$ & \\
\hline $\mathrm{H} 14 \mathrm{~A}$ & 0.1391 & 0.2711 & 0.5530 & $0.112^{*}$ & \\
\hline $\mathrm{C} 15$ & $0.1298(6)$ & $0.2448(6)$ & $0.6552(7)$ & $0.099(2)$ & \\
\hline $\mathrm{H} 15 \mathrm{~A}$ & 0.0669 & 0.2486 & 0.6495 & $0.119^{*}$ & \\
\hline $\mathrm{C} 16$ & $0.1778(5)$ & $0.2257(5)$ & $0.7196(6)$ & $0.087(2)$ & \\
\hline $\mathrm{H} 16 \mathrm{~A}$ & 0.1481 & 0.2153 & 0.7578 & $0.104^{*}$ & \\
\hline $\mathrm{C} 17$ & $0.2706(4)$ & $0.2217(4)$ & $0.7287(5)$ & $0.0680(16)$ & \\
\hline $\mathrm{C} 18$ & $0.3318(5)$ & $0.2044(5)$ & $0.7941(4)$ & $0.0663(16)$ & \\
\hline $\mathrm{C} 23$ & $0.3008(6)$ & $0.1882(6)$ & $0.8572(5)$ & $0.089(2)$ & \\
\hline $\mathrm{H} 23 \mathrm{~A}$ & 0.2387 & 0.1896 & 0.8583 & $0.107^{*}$ & \\
\hline $\mathrm{C} 22$ & $0.3600(6)$ & $0.1704(7)$ & $0.9172(6)$ & $0.103(3)$ & \\
\hline $\mathrm{H} 22 \mathrm{~A}$ & 0.3396 & 0.1618 & 0.9612 & $0.124^{*}$ & \\
\hline $\mathrm{C} 21$ & $0.4506(6)$ & $0.1647(6)$ & $0.9143(5)$ & $0.084(2)$ & \\
\hline $\mathrm{C} 20$ & $0.4784(5)$ & $0.1785(5)$ & $0.8497(4)$ & $0.0672(16)$ & \\
\hline $\mathrm{H} 20 \mathrm{~A}$ & 0.5398 & 0.1757 & 0.8475 & $0.081^{*}$ & \\
\hline $\mathrm{C} 19$ & $0.4193(4)$ & $0.1962(4)$ & $0.7882(4)$ & $0.0575(13)$ & \\
\hline $\mathrm{C} 24$ & $0.5094(7)$ & $0.1411(7)$ & $0.9775(6)$ & $0.103(3)$ & \\
\hline $\mathrm{H} 24 \mathrm{~A}$ & 0.4876 & 0.1371 & 1.0230 & $0.123^{*}$ & \\
\hline $\mathrm{O} 2$ & $0.5835(5)$ & $0.1266(5)$ & $0.9742(4)$ & $0.1085(19)$ & \\
\hline N5 & $1.0363(12)$ & $0.293(2)$ & $0.2759(10)$ & $0.081(2)$ & $0.536(8)$ \\
\hline $\mathrm{C} 35$ & $1.0794(13)$ & $0.280(2)$ & $0.2142(12)$ & $0.095(3)$ & $0.536(8)$ \\
\hline H35A & 1.0422 & 0.2345 & 0.1488 & $0.114^{*}$ & $0.536(8)$ \\
\hline $\mathrm{C} 36$ & $1.1761(12)$ & $0.3297(16)$ & $0.2418(13)$ & $0.108(4)$ & $0.536(8)$ \\
\hline H36A & 1.2060 & 0.3163 & 0.1972 & $0.130^{*}$ & $0.536(8)$ \\
\hline $\mathrm{C} 37$ & $1.2274(12)$ & $0.3996(15)$ & $0.3367(13)$ & $0.109(4)$ & $0.536(8)$ \\
\hline $\mathrm{H} 37 \mathrm{~A}$ & 1.2936 & 0.4352 & 0.3581 & $0.130^{*}$ & $0.536(8)$ \\
\hline C38 & $1.1813(12)$ & $0.4178(15)$ & $0.4010(12)$ & $0.100(4)$ & $0.536(8)$ \\
\hline $\mathrm{H} 38 \mathrm{~A}$ & 1.2164 & 0.4654 & 0.4661 & $0.120^{*}$ & $0.536(8)$ \\
\hline $\mathrm{C} 39$ & $1.0845(12)$ & $0.3661(19)$ & $0.3691(12)$ & $0.087(3)$ & $0.536(8)$ \\
\hline $\mathrm{C} 40$ & $1.0280(13)$ & $0.373(2)$ & $0.4271(11)$ & $0.083(3)$ & $0.536(8)$ \\
\hline
\end{tabular}




\begin{tabular}{|c|c|c|c|c|c|}
\hline $\mathrm{C} 45$ & $1.0674(13)$ & $0.4397(18)$ & $0.5256(11)$ & $0.098(4)$ & $0.536(8)$ \\
\hline $\mathrm{H} 45 \mathrm{~A}$ & 1.1327 & 0.4811 & 0.5550 & $0.117^{*}$ & $0.536(8)$ \\
\hline $\mathrm{C} 44$ & $1.0143(13)$ & $0.446(2)$ & $0.5796(12)$ & $0.102(4)$ & $0.536(8)$ \\
\hline $\mathrm{H} 44 \mathrm{~A}$ & 1.0424 & 0.4881 & 0.6464 & $0.123 *$ & $0.536(8)$ \\
\hline $\mathrm{C} 43$ & $0.9162(11)$ & $0.3869(19)$ & $0.5330(10)$ & $0.089(3)$ & $0.536(8)$ \\
\hline $\mathrm{C} 42$ & $0.8774(11)$ & $0.328(2)$ & $0.4343(9)$ & $0.073(3)$ & $0.536(8)$ \\
\hline $\mathrm{H} 42 \mathrm{~A}$ & 0.8092 & 0.2952 & 0.4035 & $0.087^{*}$ & $0.536(8)$ \\
\hline $\mathrm{C} 41$ & $0.9303(16)$ & $0.313(3)$ & $0.3786(12)$ & $0.073(2)$ & $0.536(8)$ \\
\hline $\mathrm{C} 46$ & $0.8574(13)$ & $0.3965(16)$ & $0.5900(11)$ & $0.099(3)$ & $0.536(8)$ \\
\hline $\mathrm{H} 46 \mathrm{~A}$ & 0.8885 & 0.4456 & 0.6550 & $0.119^{*}$ & $0.536(8)$ \\
\hline $\mathrm{O} 3$ & $0.7784(11)$ & $0.3522(9)$ & $0.5660(8)$ & $0.124(3)$ & $0.536(8)$ \\
\hline N5' & $1.0312(13)$ & $0.283(3)$ & $0.2506(12)$ & $0.081(2)$ & $0.464(8)$ \\
\hline C35' & $1.0650(16)$ & $0.262(3)$ & $0.1808(13)$ & $0.095(3)$ & $0.464(8)$ \\
\hline H35B & 1.0216 & 0.2130 & 0.1182 & $0.114^{*}$ & $0.464(8)$ \\
\hline C36' & $1.1587(14)$ & $0.305(2)$ & $0.1935(14)$ & $0.108(4)$ & $0.464(8)$ \\
\hline $\mathrm{H} 36 \mathrm{~B}$ & 1.1784 & 0.2882 & 0.1416 & $0.130^{*}$ & $0.464(8)$ \\
\hline C37' & $1.2215(14)$ & $0.3733(19)$ & $0.2852(14)$ & $0.109(4)$ & $0.464(8)$ \\
\hline H37B & 1.2851 & 0.4079 & 0.2975 & $0.130^{*}$ & $0.464(8)$ \\
\hline $\mathrm{C} 38^{\prime}$ & $1.1914(13)$ & $0.3913(19)$ & $0.3592(14)$ & $0.100(4)$ & $0.464(8)$ \\
\hline H38B & 1.2364 & 0.4350 & 0.4226 & $0.120^{*}$ & $0.464(8)$ \\
\hline C39' & $1.0968(14)$ & $0.347(2)$ & $0.3425(12)$ & $0.087(3)$ & $0.464(8)$ \\
\hline $\mathrm{C}^{\prime} 0^{\prime}$ & $1.0532(15)$ & $0.368(3)$ & $0.4125(12)$ & $0.083(3)$ & $0.464(8)$ \\
\hline $\mathrm{C} 45^{\prime}$ & $1.1026(14)$ & $0.435(2)$ & $0.5108(13)$ & $0.098(4)$ & $0.464(8)$ \\
\hline $\mathrm{H} 45 \mathrm{~B}$ & 1.1699 & 0.4689 & 0.5353 & $0.117^{*}$ & $0.464(8)$ \\
\hline C44' & $1.0549(15)$ & $0.451(2)$ & $0.5712(14)$ & $0.102(4)$ & $0.464(8)$ \\
\hline $\mathrm{H} 44 \mathrm{~B}$ & 1.0883 & 0.4990 & 0.6365 & $0.123^{*}$ & $0.464(8)$ \\
\hline C43' & $0.9569(14)$ & $0.398(2)$ & $0.5368(11)$ & 0.089 & $0.464(8)$ \\
\hline $\mathrm{C} 42^{\prime}$ & $0.9101(13)$ & $0.331(2)$ & $0.4389(11)$ & $0.073(3)$ & $0.464(8)$ \\
\hline $\mathrm{H} 42 \mathrm{~B}$ & 0.8446 & 0.2923 & 0.4151 & $0.087^{*}$ & $0.464(8)$ \\
\hline C41' & $0.9529(19)$ & $0.319(4)$ & $0.3771(13)$ & $0.073(2)$ & $0.464(8)$ \\
\hline $\mathrm{C} 46^{\prime}$ & $0.9045(15)$ & $0.4099(19)$ & $0.5993(11)$ & $0.099(3)$ & $0.464(8)$ \\
\hline H46B & 0.8404 & 0.3658 & 0.5685 & $0.119^{*}$ & $0.464(8)$ \\
\hline O3' & $0.9266(11)$ & $0.4638(10)$ & $0.6803(8)$ & $0.124(3)$ & $0.464(8)$ \\
\hline N6 & $0.7658(4)$ & $0.1534(3)$ & $0.2328(3)$ & $0.0611(12)$ & \\
\hline $\mathrm{C} 47$ & $0.6797(5)$ & $0.1660(5)$ & $0.2035(5)$ & $0.0729(17)$ & \\
\hline $\mathrm{H} 47 \mathrm{~A}$ & 0.6773 & 0.2049 & 0.1783 & $0.087^{*}$ & \\
\hline $\mathrm{C} 48$ & $0.5969(5)$ & $0.1257(5)$ & $0.2084(5)$ & $0.0750(18)$ & \\
\hline $\mathrm{H} 48 \mathrm{~A}$ & 0.5388 & 0.1372 & 0.1880 & $0.090^{*}$ & \\
\hline C49 & $0.5997(6)$ & $0.0683(5)$ & $0.2435(5)$ & $0.0802(19)$ & \\
\hline $\mathrm{H} 49 \mathrm{~A}$ & 0.5431 & 0.0391 & 0.2475 & $0.096^{*}$ & \\
\hline C50 & $0.6866(5)$ & $0.0531(5)$ & $0.2735(5)$ & $0.0707(17)$ & \\
\hline $\mathrm{H} 50 \mathrm{~A}$ & 0.6891 & 0.0138 & 0.2982 & $0.085^{*}$ & \\
\hline C51 & $0.7683(4)$ & $0.0951(4)$ & $0.2671(4)$ & $0.0571(13)$ & \\
\hline C52 & $0.8623(4)$ & $0.0836(4)$ & $0.2922(4)$ & $0.0581(14)$ & \\
\hline $\mathrm{C} 57$ & $0.8803(5)$ & $0.0259(4)$ & $0.3277(5)$ & $0.0659(16)$ & \\
\hline $\mathrm{H} 57 \mathrm{~A}$ & 0.8300 & -0.0069 & 0.3362 & $0.079^{*}$ & \\
\hline C56 & $0.9697(5)$ & $0.0169(5)$ & $0.3499(5)$ & $0.0684(16)$ & \\
\hline H56A & 0.9816 & -0.0221 & 0.3737 & $0.082^{*}$ & \\
\hline C55 & $1.0437(5)$ & $0.0658(4)$ & $0.3372(4)$ & $0.0636(15)$ & \\
\hline C54 & $1.0266(4)$ & $0.1237(4)$ & $0.3036(4)$ & $0.0598(15)$ & \\
\hline H54A & 1.0772 & 0.1567 & 0.2954 & $0.072 *$ & \\
\hline
\end{tabular}




\begin{tabular}{|c|c|c|c|c|c|}
\hline C53 & $0.9366(4)$ & $0.1339(4)$ & $0.2818(4)$ & $0.0577(14)$ & \\
\hline C58 & $1.1387(5)$ & $0.0521(5)$ & $0.3573(5)$ & $0.0751(18)$ & \\
\hline $\mathrm{H} 58 \mathrm{~A}$ & 1.1449 & 0.0094 & 0.3774 & $0.090^{*}$ & \\
\hline O4 & $1.2085(4)$ & $0.0893(4)$ & $0.3510(4)$ & $0.0881(15)$ & \\
\hline N4 & $0.4673(4)$ & $0.2592(4)$ & $0.5293(4)$ & $0.0743(15)$ & \\
\hline N7 & $0.8304(4)$ & $0.0597(5)$ & $0.0212(4)$ & $0.0753(15)$ & \\
\hline N8 & $0.8316(5)$ & $0.3488(4)$ & $0.1651(4)$ & $0.0771(16)$ & \\
\hline N3 & $0.5483(6)$ & $0.4334(5)$ & $0.8495(6)$ & $0.089(2)$ & $0.808(6)$ \\
\hline $\mathrm{C} 25$ & $0.5097(6)$ & $0.3567(6)$ & $0.7895(8)$ & $0.071(2)$ & $0.808(6)$ \\
\hline $\mathrm{C} 26$ & $0.6023(8)$ & $0.5376(8)$ & $0.9202(8)$ & $0.116(3)$ & $0.808(6)$ \\
\hline $\mathrm{C} 27$ & $0.5223(9)$ & $0.5760(8)$ & $0.9472(8)$ & $0.139(4)$ & $0.808(6)$ \\
\hline $\mathrm{H} 27 \mathrm{~A}$ & 0.5499 & 0.6446 & 0.9897 & $0.208^{*}$ & $0.808(6)$ \\
\hline $\mathrm{H} 27 \mathrm{~B}$ & 0.4972 & 0.5485 & 0.9793 & $0.208^{*}$ & $0.808(6)$ \\
\hline $\mathrm{H} 27 \mathrm{C}$ & 0.4693 & 0.5593 & 0.8896 & $0.208^{*}$ & $0.808(6)$ \\
\hline $\mathrm{C} 28$ & $0.6780(8)$ & $0.5473(9)$ & $1.0024(8)$ & $0.142(5)$ & $0.808(6)$ \\
\hline $\mathrm{H} 28 \mathrm{~A}$ & 0.7164 & 0.6139 & 1.0488 & $0.213^{*}$ & $0.808(6)$ \\
\hline $\mathrm{H} 28 \mathrm{~B}$ & 0.7207 & 0.5132 & 0.9800 & $0.213^{*}$ & $0.808(6)$ \\
\hline $\mathrm{H} 28 \mathrm{C}$ & 0.6465 & 0.5211 & 1.0324 & $0.213^{*}$ & $0.808(6)$ \\
\hline $\mathrm{C} 29$ & $0.6489(10)$ & $0.5806(10)$ & $0.8724(11)$ & $0.155(5)$ & $0.808(6)$ \\
\hline $\mathrm{H} 29 \mathrm{~A}$ & 0.6825 & 0.6483 & 0.9174 & $0.233^{*}$ & $0.808(6)$ \\
\hline $\mathrm{H} 29 \mathrm{~B}$ & 0.5981 & 0.5698 & 0.8165 & $0.233^{*}$ & $0.808(6)$ \\
\hline $\mathrm{H} 29 \mathrm{C}$ & 0.6955 & 0.5509 & 0.8529 & $0.233^{*}$ & $0.808(6)$ \\
\hline N3' & 0.6029 (19) & $0.4168(17)$ & $0.814(3)$ & $0.089(2)$ & $0.192(6)$ \\
\hline $\mathrm{C} 25^{\prime}$ & $0.540(3)$ & $0.3495(17)$ & $0.773(3)$ & $0.071(2)$ & $0.192(6)$ \\
\hline $\mathrm{C} 26^{\prime}$ & $0.689(2)$ & $0.5064(18)$ & $0.879(2)$ & $0.116(3)$ & $0.192(6)$ \\
\hline $\mathrm{C} 27^{\prime}$ & $0.654(4)$ & $0.579(4)$ & $0.945(4)$ & $0.139(4)$ & $0.192(6)$ \\
\hline $\mathrm{H} 27 \mathrm{D}$ & 0.7090 & 0.6370 & 0.9921 & $0.208^{*}$ & $0.192(6)$ \\
\hline $\mathrm{H} 27 \mathrm{E}$ & 0.6243 & 0.5542 & 0.9767 & $0.208^{*}$ & $0.192(6)$ \\
\hline $\mathrm{H} 27 \mathrm{~F}$ & 0.6055 & 0.5924 & 0.9071 & $0.208^{*}$ & $0.192(6)$ \\
\hline $\mathrm{C} 28^{\prime}$ & $0.764(3)$ & $0.483(3)$ & $0.935(3)$ & $0.142(5)$ & $0.192(6)$ \\
\hline $\mathrm{H} 28 \mathrm{D}$ & 0.8219 & 0.5394 & 0.9797 & $0.213^{*}$ & $0.192(6)$ \\
\hline $\mathrm{H} 28 \mathrm{E}$ & 0.7823 & 0.4336 & 0.8907 & $0.213^{*}$ & $0.192(6)$ \\
\hline $\mathrm{H} 28 \mathrm{~F}$ & 0.7367 & 0.4610 & 0.9698 & $0.213^{*}$ & $0.192(6)$ \\
\hline $\mathrm{C} 29^{\prime}$ & $0.718(4)$ & $0.529(4)$ & $0.813(3)$ & $0.155(5)$ & $0.192(6)$ \\
\hline $\mathrm{H} 29 \mathrm{D}$ & 0.7798 & 0.5819 & 0.8492 & $0.233^{*}$ & $0.192(6)$ \\
\hline $\mathrm{H} 29 \mathrm{E}$ & 0.6680 & 0.5459 & 0.7822 & $0.233^{*}$ & $0.192(6)$ \\
\hline $\mathrm{H} 29 \mathrm{~F}$ & 0.7264 & 0.4739 & 0.7648 & $0.233^{*}$ & $0.192(6)$ \\
\hline $\mathrm{C} 30$ & $0.4604(5)$ & $0.2418(5)$ & $0.5858(5)$ & $0.0675(16)$ & \\
\hline C31 & $0.4789(6)$ & $0.2933(6)$ & $0.4661(6)$ & $0.0806(19)$ & \\
\hline $\mathrm{C} 32$ & $0.3832(8)$ & $0.3003(10)$ & $0.4236(10)$ & $0.162(5)$ & \\
\hline $\mathrm{H} 32 \mathrm{~A}$ & 0.3877 & 0.3230 & 0.3814 & $0.243^{*}$ & \\
\hline $\mathrm{H} 32 \mathrm{~B}$ & 0.3669 & 0.3443 & 0.4744 & $0.243^{*}$ & \\
\hline $\mathrm{H} 32 \mathrm{C}$ & 0.3326 & 0.2382 & 0.3873 & $0.243^{*}$ & \\
\hline C33 & $0.5489(9)$ & $0.3909(7)$ & $0.5272(8)$ & $0.142(4)$ & \\
\hline $\mathrm{H} 33 \mathrm{~A}$ & 0.5577 & 0.4159 & 0.4876 & $0.212^{*}$ & \\
\hline H33B & 0.6114 & 0.3913 & 0.5619 & $0.212^{*}$ & \\
\hline $\mathrm{H} 33 \mathrm{C}$ & 0.5238 & 0.4302 & 0.5724 & $0.212^{*}$ & \\
\hline C34 & $0.4989(12)$ & $0.2236(9)$ & $0.3904(9)$ & $0.178(6)$ & \\
\hline $\mathrm{H} 34 \mathrm{~A}$ & 0.5063 & 0.2448 & 0.3478 & $0.267^{*}$ & \\
\hline H34B & 0.4449 & 0.1637 & 0.3547 & $0.267^{*}$ & \\
\hline $\mathrm{H} 34 \mathrm{C}$ & 0.5589 & 0.2150 & 0.4176 & $0.267^{*}$ & \\
\hline
\end{tabular}




\begin{tabular}{|c|c|c|c|c|c|}
\hline C59 & $0.8570(5)$ & $0.1189(5)$ & $0.1001(5)$ & $0.0684(16)$ & \\
\hline $\mathrm{C} 60$ & $0.7890(6)$ & $-0.0148(6)$ & $-0.0785(5)$ & $0.087(2)$ & \\
\hline C61 & $0.7076(9)$ & $-0.0919(9)$ & $-0.0929(8)$ & $0.157(5)$ & \\
\hline H61A & 0.7346 & -0.1208 & -0.0593 & $0.235^{*}$ & \\
\hline H61B & 0.6737 & -0.1397 & -0.1608 & $0.235^{*}$ & \\
\hline $\mathrm{H} 61 \mathrm{C}$ & 0.6618 & -0.0651 & -0.0679 & $0.235^{*}$ & \\
\hline $\mathrm{C} 62$ & $0.8690(7)$ & $-0.0411(7)$ & $-0.1092(7)$ & 0.120 & \\
\hline H62A & 0.8997 & -0.0685 & -0.0755 & $0.181^{*}$ & \\
\hline H62B & 0.9176 & 0.0151 & -0.0945 & $0.181^{*}$ & \\
\hline $\mathrm{H} 62 \mathrm{C}$ & 0.8420 & -0.0872 & -0.1777 & $0.181^{*}$ & \\
\hline C63 & $0.7445(7)$ & $0.0266(7)$ & $-0.1340(6)$ & $0.111(3)$ & \\
\hline H63A & 0.6911 & 0.0442 & -0.1167 & $0.166^{*}$ & \\
\hline H63B & 0.7195 & -0.0203 & -0.2021 & $0.166^{*}$ & \\
\hline $\mathrm{H} 63 \mathrm{C}$ & 0.7942 & 0.0824 & -0.1187 & $0.166^{*}$ & \\
\hline C64 & $0.8505(6)$ & $0.3044(5)$ & $0.1938(5)$ & $0.0762(19)$ & \\
\hline $\mathrm{C} 65$ & $0.8122(6)$ & $0.3966(5)$ & $0.1142(5)$ & $0.094(2)$ & \\
\hline C66 & $0.744(2)$ & $0.3290(17)$ & $0.0108(11)$ & $0.110(4)$ & $0.351(16)$ \\
\hline H66A & 0.7821 & 0.3158 & -0.0291 & $0.165^{*}$ & $0.351(16)$ \\
\hline H66B & 0.6985 & 0.3572 & -0.0076 & $0.165^{*}$ & $0.351(16)$ \\
\hline $\mathrm{H} 66 \mathrm{C}$ & 0.7081 & 0.2702 & 0.0024 & $0.165^{*}$ & $0.351(16)$ \\
\hline C67 & $0.9164(13)$ & $0.444(2)$ & $0.124(2)$ & $0.133(4)$ & $0.351(16)$ \\
\hline H67A & 0.9624 & 0.4747 & 0.1893 & $0.199 *$ & $0.351(16)$ \\
\hline H67B & 0.9145 & 0.4912 & 0.1064 & $0.199^{*}$ & $0.351(16)$ \\
\hline $\mathrm{H} 67 \mathrm{C}$ & 0.9371 & 0.3962 & 0.0811 & $0.199^{*}$ & $0.351(16)$ \\
\hline C68 & $0.776(2)$ & 0.4754 (19) & $0.171(2)$ & $0.112(4)$ & $0.351(16)$ \\
\hline H68A & 0.8311 & 0.5280 & 0.2274 & $0.168^{*}$ & $0.351(16)$ \\
\hline H68B & 0.7285 & 0.4511 & 0.1909 & $0.168^{*}$ & $0.351(16)$ \\
\hline $\mathrm{H} 68 \mathrm{C}$ & 0.7454 & 0.4973 & 0.1313 & $0.168^{*}$ & $0.351(16)$ \\
\hline C66' & $0.7018(9)$ & $0.3395(10)$ & $0.0442(10)$ & $0.110(4)$ & $0.649(16)$ \\
\hline H66D & 0.6637 & 0.3292 & 0.0789 & $0.165^{*}$ & $0.649(16)$ \\
\hline $\mathrm{H} 66 \mathrm{E}$ & 0.6964 & 0.2788 & -0.0078 & $0.165^{*}$ & $0.649(16)$ \\
\hline $\mathrm{H} 66 \mathrm{~F}$ & 0.6770 & 0.3753 & 0.0184 & $0.165^{*}$ & $0.649(16)$ \\
\hline $\mathrm{C} 67^{\prime}$ & $0.8798(13)$ & $0.3881(14)$ & $0.0638(13)$ & $0.133(4)$ & $0.649(16)$ \\
\hline H67D & 0.9467 & 0.4253 & 0.1103 & $0.199 *$ & $0.649(16)$ \\
\hline H67E & 0.8633 & 0.4114 & 0.0216 & $0.199^{*}$ & $0.649(16)$ \\
\hline $\mathrm{H} 67 \mathrm{~F}$ & 0.8739 & 0.3220 & 0.0263 & $0.199^{*}$ & $0.649(16)$ \\
\hline C68' & $0.8248(13)$ & $0.4986(9)$ & $0.1843(11)$ & $0.112(4)$ & $0.649(16)$ \\
\hline $\mathrm{H} 68 \mathrm{D}$ & 0.8932 & 0.5330 & 0.2276 & $0.168^{*}$ & $0.649(16)$ \\
\hline $\mathrm{H} 68 \mathrm{E}$ & 0.7846 & 0.4996 & 0.2208 & $0.168^{*}$ & $0.649(16)$ \\
\hline $\mathrm{H} 68 \mathrm{~F}$ & 0.8049 & 0.5284 & 0.1493 & $0.168^{*}$ & $0.649(16)$ \\
\hline
\end{tabular}

Table 3: Atomic displacement parameters $\left(\AA^{2}\right)$ for $2 \cdot 0.25\left(\mathrm{CH}_{2} \mathrm{Cl}_{2}\right)$.

$\begin{array}{lllllll} & U^{11} & U^{22} & U^{33} & U^{12} & U^{13} & U^{23} \\ \text { Ir1 } & 0.05016(16) & 0.05415(17) & 0.06077(18) & 0.01560(12) & 0.00840(12) 0.03014(14) \\ \text { Ir2 } & 0.0775(2) & 0.05193(17) & 0.05900(18) & 0.01162(14) & 0.00037(14) 0.03582(14) \\ \text { N1 } & 0.064(3) & 0.093(4) & 0.069(4) & 0.031(3) & 0.023(3) & 0.049(3)\end{array}$




\begin{tabular}{|c|c|c|c|c|c|c|}
\hline $\mathrm{Cl}$ & $0.053(4)$ & $0.123(6)$ & $0.095(6)$ & $0.025(4)$ & $0.013(4)$ & $0.053(5)$ \\
\hline $\mathrm{C} 2$ & $0.066(5)$ & $0.169(7)$ & $0.099(7)$ & $0.046(5)$ & $0.022(4)$ & $0.072(6)$ \\
\hline $\mathrm{C} 3$ & $0.082(5)$ & $0.170(8)$ & $0.098(6)$ & $0.074(5)$ & $0.043(5)$ & $0.091(6)$ \\
\hline $\mathrm{C} 4$ & $0.095(5)$ & $0.144(6)$ & $0.093(5)$ & $0.078(5)$ & $0.052(4)$ & $0.088(5)$ \\
\hline $\mathrm{C} 5$ & $0.075(4)$ & $0.095(4)$ & $0.062(4)$ & $0.046(3)$ & $0.033(3)$ & $0.055(3)$ \\
\hline C6 & $0.081(4)$ & $0.071(3)$ & $0.055(4)$ & $0.038(3)$ & 0.029 & $0.044(3)$ \\
\hline $\mathrm{C} 11$ & $0.106(5)$ & $0.075(4)$ & $0.073(5)$ & $0.050(4)$ & $0.039(4)$ & $0.050(4)$ \\
\hline $\mathrm{C} 10$ & $0.122(5)$ & $0.056(4)$ & $0.076(5)$ & $0.034(4)$ & $0.039(4)$ & $0.043(4)$ \\
\hline C9 & $0.101(4)$ & $0.057(4)$ & $0.059(4)$ & $0.022(3)$ & $0.017(4)$ & $0.036(3)$ \\
\hline $\mathrm{C} 8$ & $0.075(4)$ & $0.058(3)$ & $0.047(3)$ & 0.023 & $0.010(3)$ & $0.033(3)$ \\
\hline $\mathrm{C} 7$ & $0.073(3)$ & $0.057(3)$ & $0.048(3)$ & $0.029(3)$ & $0.021(3)$ & $0.035(3)$ \\
\hline $\mathrm{C} 12$ & $0.111(5)$ & $0.075(5)$ & $0.076(5)$ & $0.007(4)$ & $-0.009(5)$ & $0.042(4)$ \\
\hline $\mathrm{O} 1$ & $0.119(5)$ & $0.120(6)$ & $0.161(7)$ & $0.000(4)$ & $-0.021(5)$ & $0.087(6)$ \\
\hline N2 & $0.055(3)$ & 0.049 & $0.071(3)$ & $0.018(2)$ & $0.008(2)$ & $0.024(3)$ \\
\hline $\mathrm{C} 13$ & $0.070(4)$ & $0.072(5)$ & $0.080(5)$ & $0.028(4)$ & $0.011(4)$ & $0.039(4)$ \\
\hline C14 & $0.075(5)$ & $0.075(5)$ & $0.110(6)$ & $0.036(4)$ & 0.010 & $0.044(5)$ \\
\hline $\mathrm{C} 15$ & $0.062(5)$ & $0.080(5)$ & $0.121(7)$ & $0.031(4)$ & $0.013(4)$ & $0.038(5)$ \\
\hline $\mathrm{C} 16$ & $0.061(4)$ & $0.079(5)$ & $0.095(6)$ & $0.022(4)$ & $0.020(4)$ & $0.033(4)$ \\
\hline $\mathrm{C} 17$ & $0.053(3)$ & $0.050(4)$ & $0.077(4)$ & $0.015(3)$ & $0.017(3)$ & $0.021(3)$ \\
\hline C18 & $0.067(4)$ & $0.056(4)$ & $0.053(4)$ & $0.025(3)$ & $0.023(3)$ & $0.011(3)$ \\
\hline $\mathrm{C} 23$ & $0.080(5)$ & $0.094(6)$ & $0.081(5)$ & $0.032(4)$ & $0.032(4)$ & $0.036(4)$ \\
\hline $\mathrm{C} 22$ & $0.100(6)$ & $0.134(8)$ & $0.077(5)$ & $0.043(5)$ & $0.041(5)$ & $0.052(5)$ \\
\hline $\mathrm{C} 21$ & $0.095(5)$ & $0.096(6)$ & $0.059(4)$ & $0.037(4)$ & $0.029(4)$ & $0.039(4)$ \\
\hline $\mathrm{C} 20$ & $0.065(4)$ & $0.068(4)$ & $0.048(4)$ & 0.021 & $0.014(3)$ & $0.021(3)$ \\
\hline C19 & 0.063 & $0.043(3)$ & 0.048 & $0.015(3)$ & $0.009(3)$ & $0.018(3)$ \\
\hline $\mathrm{C} 24$ & $0.114(7)$ & $0.138(8)$ & $0.066(5)$ & $0.052(6)$ & $0.036(5)$ & $0.058(5)$ \\
\hline $\mathrm{O} 2$ & $0.111(5)$ & $0.140(5)$ & $0.084(4)$ & $0.053(4)$ & $0.024(4)$ & $0.070(4)$ \\
\hline N5 & $0.082(3)$ & $0.073(6)$ & $0.089(7)$ & $0.012(3)$ & $0.001(5)$ & $0.064(6)$ \\
\hline $\mathrm{C} 35$ & $0.075(6)$ & $0.099(10)$ & $0.105(8)$ & $0.011(5)$ & $0.008(7)$ & $0.071(9)$ \\
\hline $\mathrm{C} 36$ & $0.085(7)$ & $0.106(10)$ & $0.114(9)$ & $-0.005(6)$ & $0.004(8)$ & $0.075(10)$ \\
\hline C37 & $0.094(7)$ & $0.095(10)$ & $0.118(11)$ & $-0.003(6)$ & $0.003(7)$ & $0.074(10)$ \\
\hline C38 & $0.092(5)$ & $0.077(10)$ & $0.113(9)$ & $0.010(6)$ & $0.000(6)$ & $0.064(8)$ \\
\hline C39 & $0.076(5)$ & $0.067(9)$ & $0.098(7)$ & $0.007(5)$ & $-0.011(4)$ & $0.058(6)$ \\
\hline $\mathrm{C} 40$ & $0.085(7)$ & $0.049(5)$ & $0.084(5)$ & $0.007(8)$ & $-0.014(4)$ & $0.042(5)$ \\
\hline $\mathrm{C} 45$ & $0.105(9)$ & $0.050(5)$ & $0.087(5)$ & $0.017(9)$ & $-0.021(5)$ & $0.032(5)$ \\
\hline $\mathrm{C} 44$ & $0.119(10)$ & $0.058(6)$ & 0.070 & $0.016(10)$ & $-0.025(5)$ & $0.027(5)$ \\
\hline $\mathrm{C} 43$ & $0.114(9)$ & $0.057(6)$ & $0.054(4)$ & $0.030(9)$ & $-0.017(5)$ & 0.023 \\
\hline $\mathrm{C} 42$ & $0.081(8)$ & $0.047(4)$ & $0.056(3)$ & $0.027(8)$ & $-0.014(5)$ & 0.022 \\
\hline $\mathrm{C} 41$ & $0.083(8)$ & $0.045(6)$ & $0.066(3)$ & $0.012(7)$ & $-0.007(4)$ & 0.034 \\
\hline $\mathrm{C} 46$ & $0.122(10)$ & $0.083(7)$ & $0.053(5)$ & $0.068(9)$ & $-0.009(7)$ & 0.017 \\
\hline $\mathrm{O} 3$ & $0.155(8)$ & $0.108(7)$ & $0.068(5)$ & $0.057(6)$ & $0.015(5)$ & $0.028(5)$ \\
\hline N5' & $0.082(3)$ & $0.073(6)$ & $0.089(7)$ & $0.012(3)$ & $0.001(5)$ & $0.064(6)$ \\
\hline C35' & $0.075(6)$ & $0.099(10)$ & $0.105(8)$ & $0.011(5)$ & $0.008(7)$ & $0.071(9)$ \\
\hline C36' & $0.085(7)$ & $0.106(10)$ & $0.114(9)$ & $-0.005(6)$ & $0.004(8)$ & $0.075(10)$ \\
\hline C $37^{\prime}$ & $0.094(7)$ & $0.095(10)$ & $0.118(11)$ & $-0.003(6)$ & $0.003(7)$ & $0.074(10)$ \\
\hline C38' & $0.092(5)$ & $0.077(10)$ & $0.113(9)$ & $0.010(6)$ & $0.000(6)$ & $0.064(8)$ \\
\hline C39' & $0.076(5)$ & $0.067(9)$ & $0.098(7)$ & $0.007(5)$ & $-0.011(4)$ & $0.058(6)$ \\
\hline $\mathrm{C} 40^{\prime}$ & $0.085(7)$ & $0.049(5)$ & $0.084(5)$ & $0.007(8)$ & $-0.014(4)$ & $0.042(5)$ \\
\hline $\mathrm{C} 45^{\prime}$ & $0.105(9)$ & $0.050(5)$ & $0.087(5)$ & $0.017(9)$ & $-0.021(5)$ & $0.032(5)$ \\
\hline C44' & $0.119(10)$ & $0.058(6)$ & $0.070(5)$ & $0.016(10)$ & $-0.025(5)$ & $0.027(5)$ \\
\hline $\mathrm{C} 43^{\prime}$ & $0.114(9)$ & $0.057(6)$ & $0.054(4)$ & $0.030(9)$ & $-0.017(5)$ & 0.023 \\
\hline
\end{tabular}




\begin{tabular}{|c|c|c|c|c|c|c|}
\hline $\mathrm{C} 42^{\prime}$ & $0.081(8)$ & $0.047(4)$ & $0.056(3)$ & $0.027(8)$ & $-0.014(5)$ & $0.022(3)$ \\
\hline $\mathrm{C} 41^{\prime}$ & $0.083(8)$ & $0.045(6)$ & $0.066(3)$ & $0.012(7)$ & $-0.007(4)$ & $0.034(3)$ \\
\hline $\mathrm{C} 46^{\prime}$ & $0.122(10)$ & $0.083(7)$ & $0.053(5)$ & $0.068(9)$ & $-0.009(7)$ & $0.017(5)$ \\
\hline O3' & $0.155(8)$ & $0.108(7)$ & $0.068(5)$ & $0.057(6)$ & $0.015(5)$ & $0.028(5)$ \\
\hline N6 & $0.079(3)$ & $0.048(3)$ & $0.039(3)$ & $0.019(2)$ & $0.008(2)$ & $0.018(2)$ \\
\hline $\mathrm{C} 47$ & $0.086(4)$ & $0.071(4)$ & $0.054(4)$ & $0.034(4)$ & $0.008(3)$ & $0.035(3)$ \\
\hline $\mathrm{C} 48$ & $0.083(4)$ & $0.089(5)$ & $0.063(4)$ & $0.039(4)$ & $0.025(4)$ & $0.048(4)$ \\
\hline $\mathrm{C} 49$ & $0.090(5)$ & $0.085(5)$ & $0.076(5)$ & $0.041(4)$ & $0.037(4)$ & 0.044 (4) \\
\hline $\mathrm{C} 50$ & $0.092(4)$ & $0.063(4)$ & $0.065(4)$ & $0.033(4)$ & $0.028(4)$ & 0.039 (3) \\
\hline $\mathrm{C} 51$ & $0.075(3)$ & $0.043(3)$ & $0.036(3)$ & $0.015(3)$ & $0.006(3)$ & $0.018(3)$ \\
\hline C52 & $0.077(3)$ & $0.046(3)$ & $0.043(3)$ & $0.016(3)$ & $0.013(3)$ & $0.023(3)$ \\
\hline C57 & $0.079(4)$ & $0.058(4)$ & $0.064(4)$ & $0.023(3)$ & $0.021(3)$ & $0.038(3)$ \\
\hline C56 & $0.081(4)$ & $0.059(4)$ & $0.060(4)$ & $0.019(3)$ & $0.013(3)$ & $0.037(3)$ \\
\hline C55 & $0.079(4)$ & $0.048(3)$ & $0.046(3)$ & $0.017(3)$ & $0.008(3)$ & $0.022(3)$ \\
\hline C54 & $0.066(3)$ & $0.049(3)$ & $0.051(3)$ & $0.008(3)$ & $0.008(3)$ & $0.027(3)$ \\
\hline C53 & $0.070(3)$ & $0.046(3)$ & $0.040(3)$ & $0.011(3)$ & $0.005(3)$ & $0.022(3)$ \\
\hline C58 & $0.078(4)$ & $0.074(5)$ & $0.066(4)$ & $0.023(4)$ & $0.009(4)$ & $0.043(4)$ \\
\hline $\mathrm{O} 4$ & $0.076(3)$ & $0.100(4)$ & $0.094(4)$ & $0.028(3)$ & $0.018(3)$ & $0.065(3)$ \\
\hline N4 & $0.077(4)$ & $0.068(4)$ & $0.080(4)$ & $0.024(3)$ & $0.020(3)$ & $0.046(3)$ \\
\hline N7 & $0.075(4)$ & $0.081(4)$ & $0.066(3)$ & $0.022(3)$ & $0.016(3)$ & $0.045(3)$ \\
\hline N8 & $0.099(4)$ & $0.068(4)$ & $0.059(3)$ & $0.028(3)$ & $0.009(3)$ & $0.040(3)$ \\
\hline N3 & $0.062(4)$ & $0.063(3)$ & $0.091(5)$ & $0.023(3)$ & -0.004 & $0.022(3)$ \\
\hline $\mathrm{C} 25$ & $0.050(5)$ & 0.062 & $0.080(5)$ & $0.013(3)$ & $0.005(4)$ & $0.035(3)$ \\
\hline $\mathrm{C} 26$ & $0.095(6)$ & $0.065(5)$ & $0.115(8)$ & $0.014(4)$ & $-0.013(5)$ & 0.026 \\
\hline $\mathrm{C} 27$ & $0.141(9)$ & $0.072(6)$ & $0.110(8)$ & $0.047(6)$ & $-0.016(6)$ & $0.010(6)$ \\
\hline $\mathrm{C} 28$ & $0.101(8)$ & $0.085(8)$ & $0.128(9)$ & $0.018(6)$ & $-0.034(6)$ & $0.020(6)$ \\
\hline $\mathrm{C} 29$ & $0.126(10)$ & $0.091(9)$ & $0.175(11)$ & $-0.008(7)$ & $0.010(8)$ & $0.058(8)$ \\
\hline N3' & $0.062(4)$ & $0.063(3)$ & $0.091(5)$ & 0.023 & $-0.004(3)$ & $0.022(3)$ \\
\hline $\mathrm{C} 25^{\prime}$ & $0.050(5)$ & $0.062(3)$ & $0.080(5)$ & $0.013(3)$ & $0.005(4)$ & 0.035 \\
\hline $\mathrm{C} 26^{\prime}$ & $0.095(6)$ & $0.065(5)$ & $0.115(8)$ & $0.014(4)$ & $-0.013(5)$ & $0.026(5)$ \\
\hline $\mathrm{C} 27^{\prime}$ & $0.141(9)$ & $0.072(6)$ & $0.110(8)$ & $0.047(6)$ & $-0.016(6)$ & $0.010(6)$ \\
\hline C28' & $0.101(8)$ & $0.085(8)$ & $0.128(9)$ & $0.018(6)$ & $-0.034(6)$ & $0.020(6)$ \\
\hline $\mathrm{C} 29^{\prime}$ & $0.126(10)$ & $0.091(9)$ & $0.175(11)$ & $-0.008(7)$ & $0.010(8)$ & $0.058(8)$ \\
\hline C30 & $0.056(4)$ & $0.057(4)$ & $0.072(4)$ & $0.012(3)$ & $0.011(3)$ & 0.032 \\
\hline C31 & $0.100(5)$ & $0.076(4)$ & $0.084(5)$ & $0.033(4)$ & $0.032(4)$ & $0.056(4)$ \\
\hline C32 & $0.116(7)$ & $0.222(14)$ & $0.210(13)$ & $0.054(7)$ & $0.033(7)$ & $0.177(12)$ \\
\hline $\mathrm{C} 33$ & $0.155(9)$ & $0.104(6)$ & $0.153(9)$ & $0.005(6)$ & $0.032(7)$ & $0.087(6)$ \\
\hline C34 & $0.361(19)$ & $0.142(8)$ & $0.144(9)$ & $0.140(11)$ & $0.155(12)$ & $0.112(7)$ \\
\hline C59 & $0.073(4)$ & $0.075(4)$ & $0.062(3)$ & $0.023(3)$ & $0.016(3)$ & $0.046(3)$ \\
\hline C60 & $0.083(5)$ & $0.087(5)$ & $0.063(4)$ & $0.013(4)$ & $0.011(4)$ & $0.035(4)$ \\
\hline C61 & $0.156(9)$ & $0.142(9)$ & $0.091(7)$ & $-0.047(7)$ & $0.007(7)$ & $0.056(6)$ \\
\hline C62 & $0.125(7)$ & $0.106(7)$ & $0.089(6)$ & $0.054(6)$ & $0.031(6)$ & $0.023(5)$ \\
\hline C63 & $0.118(7)$ & $0.139(8)$ & $0.070(5)$ & $0.047(6)$ & $0.018(5)$ & $0.061(6)$ \\
\hline C64 & $0.087(5)$ & $0.067(4)$ & $0.067(4)$ & $0.023(4)$ & $0.010(4)$ & $0.042(4)$ \\
\hline C65 & $0.131(6)$ & $0.079(5)$ & $0.060(4)$ & $0.033(4)$ & $0.000(4)$ & $0.048(4)$ \\
\hline C66 & $0.149(8)$ & $0.084(6)$ & $0.062(6)$ & $0.021(7)$ & $-0.009(6)$ & 0.045 \\
\hline C67 & $0.167(9)$ & $0.146(13)$ & $0.102(11)$ & $0.039(7)$ & $0.038(8)$ & $0.091(9)$ \\
\hline C68 & $0.139(11)$ & $0.062(6)$ & $0.081(7)$ & $0.025(7)$ & $-0.020(9)$ & $0.034(5)$ \\
\hline C66 $6^{\prime}$ & $0.149(8)$ & $0.084(6)$ & $0.062(6)$ & $0.021(7)$ & $-0.009(6)$ & $0.045(5)$ \\
\hline C67' & $0.167(9)$ & $0.146(13)$ & $0.102(11)$ & $0.039(7)$ & $0.038(8)$ & $0.091(9)$ \\
\hline C68' & 0.139 (11) & $0.062(6)$ & $0.081(7)$ & $0.025(7)$ & $-0.020(9)$ & $0.034(5)$ \\
\hline
\end{tabular}


Table 4: Geometric parameters $\left(\AA,{ }^{\circ}\right)$ for $2 \cdot 0.25\left(\mathrm{CH}_{2} \mathrm{Cl}_{2}\right)$.

\begin{tabular}{|c|c|c|c|}
\hline $\mathrm{Ir} 1-\mathrm{C} 25^{\prime}$ & $2.009(17)$ & $\mathrm{C} 46-\mathrm{O} 3$ & $1.148(14)$ \\
\hline $\mathrm{Ir} 1-\mathrm{C} 30$ & $2.022(8)$ & $\mathrm{N} 5^{\prime}-\mathrm{C} 35^{\prime}$ & $1.349(13)$ \\
\hline $\operatorname{Ir} 1--\mathrm{C} 25$ & $2.037(8)$ & $\mathrm{N} 5^{\prime}-\mathrm{C} 39^{\prime}$ & $1.378(12)$ \\
\hline $\mathrm{Ir} 1-\mathrm{C} 7$ & $2.046(6)$ & $\mathrm{C} 35^{\prime}-\mathrm{C} 36^{\prime}$ & $1.392(13)$ \\
\hline $\mathrm{Ir} 1-\mathrm{N} 2$ & $2.047(5)$ & $\mathrm{C} 36^{\prime}-\mathrm{C} 37^{\prime}$ & $1.378(14)$ \\
\hline Ir1-N1 & $2.049(5)$ & $\mathrm{C} 37^{\prime}-\mathrm{C} 38^{\prime}$ & $1.384(14)$ \\
\hline $\operatorname{Ir} 1-\mathrm{C} 19$ & $2.061(6)$ & $\mathrm{C} 38^{\prime}-\mathrm{C} 39^{\prime}$ & $1.387(13)$ \\
\hline Ir $2-$ C59 & $2.010(8)$ & $\mathrm{C} 39^{\prime}-\mathrm{C} 40^{\prime}$ & $1.454(12)$ \\
\hline $\mathrm{Ir} 2-\mathrm{C} 41^{\prime}$ & $2.026(12)$ & $\mathrm{C} 40^{\prime}-\mathrm{C} 41^{\prime}$ & $1.408(12)$ \\
\hline Ir $2-\mathrm{N} 5$ & $2.039(10)$ & $\mathrm{C} 40^{\prime}-\mathrm{C} 45^{\prime}$ & $1.414(12)$ \\
\hline Ir $2--$ C64 & $2.049(7)$ & $\mathrm{C} 45^{\prime}-\mathrm{C} 44^{\prime}$ & $1.368(14)$ \\
\hline Ir2--N6 & $2.051(5)$ & $\mathrm{C} 44^{\prime}-\mathrm{C} 43^{\prime}$ & $1.402(13)$ \\
\hline $\operatorname{Ir} 2-\mathrm{N} 5^{\prime}$ & $2.056(11)$ & $\mathrm{C} 43^{\prime}-\mathrm{C} 42^{\prime}$ & $1.404(12)$ \\
\hline $\mathrm{Ir} 2-\mathrm{C} 41$ & $2.057(11)$ & $\mathrm{C} 43^{\prime}-\mathrm{C} 46^{\prime}$ & $1.458(14)$ \\
\hline $\operatorname{Ir} 2-\mathrm{C} 53$ & $2.062(6)$ & $\mathrm{C} 42^{\prime}-\mathrm{C} 41^{\prime}$ & $1.343(13)$ \\
\hline $\mathrm{N} 1-\mathrm{C} 1$ & $1.350(8)$ & $\mathrm{C} 46^{\prime}-\mathrm{O} 3^{\prime}$ & $1.172(13)$ \\
\hline $\mathrm{N} 1-\mathrm{C} 5$ & $1.364(8)$ & N6-C47 & $1.365(7)$ \\
\hline $\mathrm{C} 1-\mathrm{C} 2$ & $1.384(10)$ & N6-C51 & $1.375(7)$ \\
\hline $\mathrm{C} 2-\mathrm{C} 3$ & $1.358(11)$ & $\mathrm{C} 47-\mathrm{C} 48$ & $1.365(9)$ \\
\hline $\mathrm{C} 3-\mathrm{C} 4$ & $1.370(10)$ & $\mathrm{C} 48-\mathrm{C} 49$ & $1.370(9)$ \\
\hline $\mathrm{C} 4-\mathrm{C} 5$ & $1.402(8)$ & $\mathrm{C} 49-\mathrm{C} 50$ & $1.402(8)$ \\
\hline $\mathrm{C} 5-\mathrm{C} 6$ & $1.446(8)$ & $\mathrm{C} 50-\mathrm{C} 51$ & $1.376(8)$ \\
\hline $\mathrm{C} 6-\mathrm{C} 7$ & $1.409(7)$ & $\mathrm{C} 51-\mathrm{C} 52$ & $1.455(7)$ \\
\hline $\mathrm{C} 6-\mathrm{C} 11$ & $1.417(8)$ & $\mathrm{C} 52-\mathrm{C} 53$ & $1.396(7)$ \\
\hline $\mathrm{C} 11-\mathrm{C} 10$ & $1.347(9)$ & $\mathrm{C} 52-\mathrm{C} 57$ & $1.410(7)$ \\
\hline $\mathrm{C} 10-\mathrm{C} 9$ & $1.398(9)$ & $\mathrm{C} 57-\mathrm{C} 56$ & $1.367(8)$ \\
\hline $\mathrm{C} 9-\mathrm{C} 8$ & $1.412(8)$ & $\mathrm{C} 56-\mathrm{C} 55$ & $1.405(8)$ \\
\hline $\mathrm{C} 9-\mathrm{C} 12$ & $1.459(10)$ & $\mathrm{C} 55-\mathrm{C} 54$ & $1.387(7)$ \\
\hline $\mathrm{C} 8-\mathrm{C} 7$ & $1.382(8)$ & $\mathrm{C} 55-\mathrm{C} 58$ & $1.479(8)$ \\
\hline $\mathrm{C} 12-\mathrm{O} 1$ & $1.147(9)$ & $\mathrm{C} 54-\mathrm{C} 53$ & $1.383(8)$ \\
\hline $\mathrm{N} 2-\mathrm{C} 13$ & $1.344(8)$ & $\mathrm{C} 58-\mathrm{O} 4$ & $1.193(7)$ \\
\hline $\mathrm{N} 2-\mathrm{C} 17$ & $1.381(8)$ & $\mathrm{N} 4-\mathrm{C} 30$ & $1.164(8)$ \\
\hline $\mathrm{C} 13-\mathrm{C} 14$ & $1.360(9)$ & $\mathrm{N} 4-\mathrm{C} 31$ & $1.487(9)$ \\
\hline $\mathrm{C} 14-\mathrm{C} 15$ & $1.380(10)$ & $\mathrm{N} 7-\mathrm{C} 59$ & $1.157(8)$ \\
\hline $\mathrm{C} 15-\mathrm{C} 16$ & $1.369(10)$ & $\mathrm{N} 7-\mathrm{C} 60$ & $1.453(10)$ \\
\hline $\mathrm{C} 16-\mathrm{C} 17$ & $1.392(8)$ & N8-C64 & $1.118(8)$ \\
\hline $\mathrm{C} 17-\mathrm{C} 18$ & $1.444(8)$ & $\mathrm{N} 8-\mathrm{C} 65$ & $1.464(8)$ \\
\hline $\mathrm{C} 18-\mathrm{C} 19$ & $1.383(8)$ & $\mathrm{N} 3-\mathrm{C} 25$ & $1.138(10)$ \\
\hline $\mathrm{C} 18-\mathrm{C} 23$ & $1.406(9)$ & $\mathrm{N} 3-\mathrm{C} 26$ & $1.515(13)$ \\
\hline $\mathrm{C} 23-\mathrm{C} 22$ & $1.371(10)$ & $\mathrm{C} 26-\mathrm{C} 28$ & $1.496(12)$ \\
\hline $\mathrm{C} 22-\mathrm{C} 21$ & $1.406(9)$ & $\mathrm{C} 26-\mathrm{C} 29$ & $1.548(13)$ \\
\hline $\mathrm{C} 21-\mathrm{C} 20$ & $1.386(8)$ & $\mathrm{C} 26-\mathrm{C} 27$ & $1.550(12)$ \\
\hline $\mathrm{C} 21-\mathrm{C} 24$ & $1.476(9)$ & $\mathrm{N} 3{ }^{\prime}-\mathrm{C} 25^{\prime}$ & $1.114(18)$ \\
\hline $\mathrm{C} 20-\mathrm{C} 19$ & $1.387(8)$ & $\mathrm{N} 3{ }^{\prime}-\mathrm{C} 26^{\prime}$ & $1.50(2)$ \\
\hline $\mathrm{C} 24-\mathrm{O} 2$ & $1.202(8)$ & $\mathrm{C} 26^{\prime}-\mathrm{C} 29^{\prime}$ & $1.505(16)$ \\
\hline
\end{tabular}




\begin{tabular}{|c|c|c|c|}
\hline $\mathrm{N} 5-\mathrm{C} 35$ & $1.340(12)$ & $\mathrm{C} 26^{\prime}-\mathrm{C} 27^{\prime}$ & $1.527(16)$ \\
\hline N5-C39 & $1.376(12)$ & $\mathrm{C} 26^{\prime}-\mathrm{C} 28^{\prime}$ & $1.528(16)$ \\
\hline $\mathrm{C} 35-\mathrm{C} 36$ & $1.390(13)$ & $\mathrm{C} 31-\mathrm{C} 34$ & $1.450(13)$ \\
\hline $\mathrm{C} 36-\mathrm{C} 37$ & $1.386(14)$ & $\mathrm{C} 31-\mathrm{C} 33$ & $1.483(12)$ \\
\hline $\mathrm{C} 37-\mathrm{C} 38$ & $1.403(14)$ & $\mathrm{C} 31-\mathrm{C} 32$ & $1.501(12)$ \\
\hline $\mathrm{C} 38-\mathrm{C} 39$ & $1.386(12)$ & $\mathrm{C} 60-\mathrm{C} 62$ & $1.502(12)$ \\
\hline $\mathrm{C} 39-\mathrm{C} 40$ & $1.454(12)$ & $\mathrm{C} 60-\mathrm{C} 61$ & $1.514(12)$ \\
\hline $\mathrm{C} 40-\mathrm{C} 45$ & $1.408(12)$ & $\mathrm{C} 60-\mathrm{C} 63$ & $1.528(11)$ \\
\hline $\mathrm{C} 40-\mathrm{C} 41$ & $1.412(12)$ & $\mathrm{C} 65-\mathrm{C} 67^{\prime}$ & $1.490(11)$ \\
\hline $\mathrm{C} 45-\mathrm{C} 44$ & $1.362(13)$ & $\mathrm{C} 65-\mathrm{C} 66$ & $1.519(12)$ \\
\hline $\mathrm{C} 44-\mathrm{C} 43$ & $1.408(13)$ & $\mathrm{C} 65-\mathrm{C} 68^{\prime}$ & $1.532(11)$ \\
\hline $\mathrm{C} 43-\mathrm{C} 42$ & $1.397(11)$ & $\mathrm{C} 65-\mathrm{C} 68$ & $1.546(13)$ \\
\hline $\mathrm{C} 43-\mathrm{C} 46$ & $1.463(13)$ & $\mathrm{C} 65-\mathrm{C} 67$ & $1.575(13)$ \\
\hline $\mathrm{C} 42-\mathrm{C} 41$ & $1.368(12)$ & $\mathrm{C} 65-\mathrm{C} 66^{\prime}$ & $1.585(11)$ \\
\hline $\mathrm{C} 25^{\prime}-\mathrm{Ir} 1-\mathrm{C} 30$ & $81.8(17)$ & $\mathrm{C} 42-\mathrm{C} 43-\mathrm{C} 44$ & $119.0(12)$ \\
\hline $\mathrm{C} 25^{\prime}-\mathrm{Ir} 1-\mathrm{C} 25$ & $17.4(14)$ & $\mathrm{C} 42-\mathrm{C} 43-\mathrm{C} 46$ & $122.8(11)$ \\
\hline $\mathrm{C} 30-\operatorname{Ir} 1-\mathrm{C} 25$ & $91.9(4)$ & $\mathrm{C} 44-\mathrm{C} 43-\mathrm{C} 46$ & $118.0(12)$ \\
\hline $\mathrm{C} 25^{\prime}-\mathrm{Ir} 1-\mathrm{C} 7$ & $163.8(13)$ & $\mathrm{C} 41-\mathrm{C} 42-\mathrm{C} 43$ & $124.8(12)$ \\
\hline $\mathrm{C} 30-\mathrm{Ir} 1-\mathrm{C} 7$ & $95.5(2)$ & $\mathrm{C} 42-\mathrm{C} 41-\mathrm{C} 40$ & $114.3(10)$ \\
\hline $\mathrm{C} 25--\operatorname{Ir} 1-\mathrm{C} 7$ & $172.4(4)$ & $\mathrm{C} 42-\mathrm{C} 41-\mathrm{Ir} 2$ & $132.5(9)$ \\
\hline $\mathrm{C} 25^{\prime}-\mathrm{Ir} 1-\mathrm{N} 2$ & $103.8(13)$ & $\mathrm{C} 40-\mathrm{C} 41-\mathrm{Ir} 2$ & $113.2(9)$ \\
\hline $\mathrm{C} 30-\mathrm{Ir} 1-\mathrm{N} 2$ & $94.9(2)$ & $\mathrm{O} 3-\mathrm{C} 46-\mathrm{C} 43$ & $128.1(15)$ \\
\hline $\mathrm{C} 25-\mathrm{Ir} 1-\mathrm{N} 2$ & $88.9(3)$ & $\mathrm{C} 35^{\prime}-\mathrm{N} 5^{\prime}-\mathrm{C} 39^{\prime}$ & $117.4(11)$ \\
\hline $\mathrm{C} 7-\mathrm{Ir} 1-\mathrm{N} 2$ & $92.3(2)$ & $\mathrm{C} 35^{\prime}-\mathrm{N} 5^{\prime}-\mathrm{Ir} 2$ & $126.7(11)$ \\
\hline $\mathrm{C} 25^{\prime}-\mathrm{Ir} 1-\mathrm{N} 1$ & $84.0(13)$ & $\mathrm{C} 39^{\prime}-\mathrm{N} 55^{\prime}-\mathrm{Ir} 2$ & $115.3(10)$ \\
\hline $\mathrm{C} 30-\operatorname{Ir} 1-\mathrm{N} 1$ & $91.3(2)$ & $\mathrm{N} 5^{\prime}-\mathrm{C} 35^{\prime}-\mathrm{C} 36^{\prime}$ & $125.0(14)$ \\
\hline $\mathrm{C} 25-\mathrm{Ir} 1-\mathrm{N} 1$ & $97.9(3)$ & $\mathrm{C} 37^{\prime}-\mathrm{C} 36^{\prime}-\mathrm{C} 35^{\prime}$ & $116.6(15)$ \\
\hline $\mathrm{C} 7-\mathrm{Ir} 1-\mathrm{N} 1$ & $80.1(2)$ & $\mathrm{C} 36^{\prime}-\mathrm{C} 37^{\prime}-\mathrm{C} 38^{\prime}$ & $119.7(14)$ \\
\hline $\mathrm{N} 2-\mathrm{Ir} 1-\mathrm{N} 1$ & $170.7(2)$ & $\mathrm{C} 37^{\prime}-\mathrm{C} 38^{\prime}-\mathrm{C} 39^{\prime}$ & $121.2(14)$ \\
\hline $\mathrm{C} 25^{\prime}-\operatorname{Ir} 1-\mathrm{C} 19$ & $98.7(17)$ & $\mathrm{N} 5^{\prime}-\mathrm{C} 39^{\prime}-\mathrm{C} 38^{\prime}$ & $119.6(12)$ \\
\hline $\mathrm{C} 30-\mathrm{Ir} 1-\mathrm{C} 19$ & $175.2(2)$ & $\mathrm{N} 5^{\prime}-\mathrm{C} 39^{\prime}-\mathrm{C} 40^{\prime}$ & $113.5(11)$ \\
\hline $\mathrm{C} 25-\mathrm{Ir} 1-\mathrm{C} 19$ & $87.4(4)$ & $\mathrm{C} 38^{\prime}-\mathrm{C} 39^{\prime}-\mathrm{C} 40^{\prime}$ & $126.5(13)$ \\
\hline $\mathrm{C} 7-\mathrm{Ir} 1-\mathrm{C} 19$ & $85.4(2)$ & $\mathrm{C} 41^{\prime}-\mathrm{C} 40^{\prime}-\mathrm{C} 45^{\prime}$ & $118.7(12)$ \\
\hline $\mathrm{N} 2-\mathrm{Ir} 1-\mathrm{C} 19$ & $80.3(2)$ & $\mathrm{C} 41^{\prime}-\mathrm{C} 40^{\prime}-\mathrm{C} 39^{\prime}$ & $116.2(11)$ \\
\hline $\mathrm{N} 1-\mathrm{Ir} 1-\mathrm{C} 19$ & $93.5(2)$ & $\mathrm{C} 45^{\prime}-\mathrm{C} 40^{\prime}-\mathrm{C} 39^{\prime}$ & $125.1(12)$ \\
\hline $\mathrm{C} 59-\mathrm{Ir} 2-\mathrm{C} 41^{\prime}$ & $171.7(13)$ & $\mathrm{C} 44^{\prime}-\mathrm{C} 45^{\prime}-\mathrm{C} 40^{\prime}$ & $121.0(14)$ \\
\hline C59--Ir2-N5 & $102.9(5)$ & $\mathrm{C} 45^{\prime}-\mathrm{C} 44^{\prime}-\mathrm{C} 43^{\prime}$ & $120.1(13)$ \\
\hline $\mathrm{C} 41^{\prime}-\mathrm{Ir} 2-\mathrm{N} 5$ & $70.1(6)$ & $\mathrm{C} 44^{\prime}-\mathrm{C} 43^{\prime}-\mathrm{C} 42^{\prime}$ & $117.5(12)$ \\
\hline $\mathrm{C} 59-\mathrm{Ir} 2-\mathrm{C} 64$ & $89.1(3)$ & $\mathrm{C} 44^{\prime}-\mathrm{C} 43^{\prime}-\mathrm{C} 46^{\prime}$ & $121.9(12)$ \\
\hline $\mathrm{C} 41^{\prime}-\mathrm{Ir} 2-\mathrm{C} 64$ & $95(2)$ & $\mathrm{C} 42^{\prime}-\mathrm{C} 43^{\prime}-\mathrm{C} 46^{\prime}$ & $120.6(13)$ \\
\hline N5-Ir2-C64 & $90.1(12)$ & $\mathrm{C} 41^{\prime}-\mathrm{C} 42^{\prime}-\mathrm{C} 43^{\prime}$ & $123.4(13)$ \\
\hline $\mathrm{C} 59-\mathrm{Ir} 2-\mathrm{N} 6$ & $89.4(2)$ & $\mathrm{C} 42^{\prime}-\mathrm{C} 41^{\prime}-\mathrm{C} 40^{\prime}$ & $118.8(13)$ \\
\hline $\mathrm{C} 41^{\prime}-\mathrm{Ir} 2-\mathrm{N} 6$ & $97.0(6)$ & $\mathrm{C} 42^{\prime}-\mathrm{C} 41^{\prime}-\mathrm{Ir} 2$ & $126.3(12)$ \\
\hline N5-Ir2-N6 & $165.2(6)$ & $\mathrm{C} 40^{\prime}-\mathrm{C} 41^{\prime}-\mathrm{Ir} 2$ & $113.8(10)$ \\
\hline $\mathrm{C} 64-\mathrm{Ir} 2-\mathrm{N} 6$ & $98.5(3)$ & $\mathrm{O} 3^{\prime}-\mathrm{C} 46^{\prime}-\mathrm{C} 43^{\prime}$ & $132.1(19)$ \\
\hline $\mathrm{C} 59-\mathrm{Ir} 2-\mathrm{N} 5^{\prime}$ & $93.3(5)$ & $\mathrm{C} 47-\mathrm{N} 6-\mathrm{C} 51$ & $117.9(6)$ \\
\hline $\mathrm{C} 41^{\prime}-\mathrm{Ir} 2-\mathrm{N} 5^{\prime}$ & $80.0(6)$ & $\mathrm{C} 47-\mathrm{N} 6-\mathrm{Ir} 2$ & $125.7(4)$ \\
\hline N5-Ir2-N5' & $10.3(8)$ & $\mathrm{C} 51-\mathrm{N} 6-\mathrm{Ir} 2$ & $116.3(4)$ \\
\hline $\mathrm{C} 64-\mathrm{Ir} 2-\mathrm{N} 5$ & $86.4(14)$ & $\mathrm{C} 48-\mathrm{C} 47-\mathrm{N} 6$ & $123.8(6)$ \\
\hline $\mathrm{N} 6-\mathrm{Ir} 2-\mathrm{N} 5{ }^{\prime}$ & $174.5(12)$ & $\mathrm{C} 47-\mathrm{C} 48-\mathrm{C} 49$ & $118.3(7)$ \\
\hline $\mathrm{C} 59-\mathrm{Ir} 2-\mathrm{C} 41$ & $174.8(15)$ & $\mathrm{C} 48-\mathrm{C} 49-\mathrm{C} 50$ & $119.5(7)$ \\
\hline
\end{tabular}




\begin{tabular}{|c|c|c|c|}
\hline $\mathrm{C} 41^{\prime}-\operatorname{Ir} 2-\mathrm{C} 41$ & $9.9(7)$ & $\mathrm{C} 51-\mathrm{C} 50-\mathrm{C} 49$ & $120.2(6)$ \\
\hline N5-Ir2-C41 & $80.0(5)$ & N6-C51-C50 & $120.3(5)$ \\
\hline C64-Ir2-C41 & $95.2(18)$ & $\mathrm{N} 6-\mathrm{C} 51-\mathrm{C} 52$ & $113.2(5)$ \\
\hline $\mathrm{N} 6-\mathrm{Ir} 2-\mathrm{C} 41$ & $87.2(5)$ & $\mathrm{C} 50-\mathrm{C} 51-\mathrm{C} 52$ & $126.5(5)$ \\
\hline $\mathrm{N} 5{ }^{\prime}-\mathrm{Ir} 2-\mathrm{C} 41$ & $89.9(6)$ & $\mathrm{C} 53-\mathrm{C} 52-\mathrm{C} 57$ & $120.1(6)$ \\
\hline C59-Ir2-C53 & $91.7(3)$ & $\mathrm{C} 53-\mathrm{C} 52-\mathrm{C} 51$ & $117.5(5)$ \\
\hline $\mathrm{C} 41^{\prime}-\mathrm{Ir} 2-\mathrm{C} 53$ & $84(2)$ & $\mathrm{C} 57-\mathrm{C} 52-\mathrm{C} 51$ & $122.5(5)$ \\
\hline $\mathrm{N} 5-\mathrm{Ir} 2-\mathrm{C} 53$ & $91.3(12)$ & $\mathrm{C} 56-\mathrm{C} 57-\mathrm{C} 52$ & $120.4(6)$ \\
\hline C64-Ir2-C53 & $178.2(3)$ & $\mathrm{C} 57-\mathrm{C} 56-\mathrm{C} 55$ & $119.4(6)$ \\
\hline N6--Ir2-C53 & $79.9(2)$ & $\mathrm{C} 54-\mathrm{C} 55-\mathrm{C} 56$ & $120.3(6)$ \\
\hline N5'--Ir2-C53 & $95.2(14)$ & $\mathrm{C} 54-\mathrm{C} 55-\mathrm{C} 58$ & $120.8(6)$ \\
\hline $\mathrm{C} 41-\mathrm{Ir} 2-\mathrm{C} 53$ & $83.9(18)$ & $\mathrm{C} 56-\mathrm{C} 55-\mathrm{C} 58$ & $118.9(6)$ \\
\hline $\mathrm{C} 1-\mathrm{N} 1-\mathrm{C} 5$ & $119.6(6)$ & $\mathrm{C} 53-\mathrm{C} 54-\mathrm{C} 55$ & $120.6(6)$ \\
\hline $\mathrm{C} 1-\mathrm{N} 1-\mathrm{Ir} 1$ & $124.6(5)$ & $\mathrm{C} 54-\mathrm{C} 53-\mathrm{C} 52$ & $119.2(5)$ \\
\hline $\mathrm{C} 5-\mathrm{N} 1-\mathrm{Ir} 1$ & $115.7(4)$ & $\mathrm{C} 54-\mathrm{C} 53-\mathrm{Ir} 2$ & $128.0(4)$ \\
\hline $\mathrm{N} 1--\mathrm{C} 1-\mathrm{C} 2$ & $122.0(8)$ & $\mathrm{C} 52-\mathrm{C} 53-\mathrm{Ir} 2$ & $112.8(4)$ \\
\hline $\mathrm{C} 3-\mathrm{C} 2-\mathrm{C} 1$ & $118.2(8)$ & $\mathrm{O} 4-\mathrm{C} 58-\mathrm{C} 55$ & $126.7(7)$ \\
\hline $\mathrm{C} 2-\mathrm{C} 3-\mathrm{C} 4$ & $121.5(8)$ & $\mathrm{C} 30-\mathrm{N} 4-\mathrm{C} 31$ & $173.1(7)$ \\
\hline $\mathrm{C} 3-\mathrm{C} 4-\mathrm{C} 5$ & $118.8(8)$ & $\mathrm{C} 59-\mathrm{N} 7-\mathrm{C} 60$ & $175.3(8)$ \\
\hline $\mathrm{N} 1-\mathrm{C} 5-\mathrm{C} 4$ & $119.9(7)$ & $\mathrm{C} 64-\mathrm{N} 8-\mathrm{C} 65$ & $169.8(8)$ \\
\hline $\mathrm{N} 1-\mathrm{C} 5-\mathrm{C} 6$ & $114.7(5)$ & $\mathrm{C} 25-\mathrm{N} 3-\mathrm{C} 26$ & $172.9(12)$ \\
\hline $\mathrm{C} 4-\mathrm{C} 5-\mathrm{C} 6$ & $125.5(7)$ & $\mathrm{N} 3-\mathrm{C} 25-\mathrm{Ir} 1$ & $176.9(10)$ \\
\hline $\mathrm{C} 7-\mathrm{C} 6-\mathrm{C} 11$ & $121.1(6)$ & $\mathrm{C} 28-\mathrm{C} 26-\mathrm{N} 3$ & $107.0(9)$ \\
\hline $\mathrm{C} 7-\mathrm{C} 6-\mathrm{C} 5$ & $116.1(5)$ & $\mathrm{C} 28-\mathrm{C} 26-\mathrm{C} 29$ & $111.2(9)$ \\
\hline $\mathrm{C} 11-\mathrm{C} 6-\mathrm{C} 5$ & $122.9(6)$ & $\mathrm{N} 3-\mathrm{C} 26-\mathrm{C} 29$ & $109.2(10)$ \\
\hline $\mathrm{C} 10-\mathrm{C} 11-\mathrm{C} 6$ & $119.8(6)$ & $\mathrm{C} 28-\mathrm{C} 26-\mathrm{C} 27$ & $112.4(9)$ \\
\hline $\mathrm{C} 11-\mathrm{C} 10-\mathrm{C} 9$ & $120.9(6)$ & $\mathrm{N} 3-\mathrm{C} 26-\mathrm{C} 27$ & $103.7(8)$ \\
\hline $\mathrm{C} 10-\mathrm{C} 9-\mathrm{C} 8$ & $119.2(6)$ & $\mathrm{C} 29-\mathrm{C} 26-\mathrm{C} 27$ & $112.9(9)$ \\
\hline $\mathrm{C} 10-\mathrm{C} 9-\mathrm{C} 12$ & $119.9(7)$ & $\mathrm{C} 25^{\prime}-\mathrm{N} 3^{\prime}-\mathrm{C} 26^{\prime}$ & $173(5)$ \\
\hline $\mathrm{C} 8-\mathrm{C} 9-\mathrm{C} 12$ & $120.8(7)$ & $\mathrm{N} 3{ }^{\prime}-\mathrm{C} 25^{\prime}-\operatorname{Ir} 1$ & $169(4)$ \\
\hline $\mathrm{C} 7-\mathrm{C} 8-\mathrm{C} 9$ & $121.4(6)$ & $\mathrm{N} 3^{\prime}-\mathrm{C} 26^{\prime}-\mathrm{C} 29^{\prime}$ & $104(3)$ \\
\hline $\mathrm{C} 8-\mathrm{C} 7-\mathrm{C} 6$ & $117.5(5)$ & $\mathrm{N} 3^{\prime}-\mathrm{C} 26^{\prime}-\mathrm{C} 27^{\prime}$ & $107(3)$ \\
\hline $\mathrm{C} 8-\mathrm{C} 7-\mathrm{Ir} 1$ & $129.1(4)$ & $\mathrm{C} 29^{\prime}-\mathrm{C} 26^{\prime}-\mathrm{C} 27^{\prime}$ & $114.0(14)$ \\
\hline $\mathrm{C} 6-\mathrm{C} 7-\mathrm{Ir} 1$ & $113.3(4)$ & $\mathrm{N} 3^{\prime}-\mathrm{C} 26^{\prime}-\mathrm{C} 28^{\prime}$ & $106(3)$ \\
\hline $\mathrm{O} 1-\mathrm{C} 12-\mathrm{C} 9$ & $123.9(9)$ & $\mathrm{C} 29^{\prime}-\mathrm{C} 26^{\prime}-\mathrm{C} 28^{\prime}$ & $114.0(14)$ \\
\hline $\mathrm{C} 13-\mathrm{N} 2-\mathrm{C} 17$ & $117.9(6)$ & $\mathrm{C} 27^{\prime}-\mathrm{C} 26^{\prime}-\mathrm{C} 28^{\prime}$ & $111.4(13)$ \\
\hline $\mathrm{C} 13-\mathrm{N} 2-\mathrm{Ir} 1$ & $126.4(5)$ & $\mathrm{N} 4-\mathrm{C} 30-\mathrm{Ir} 1$ & $176.8(6)$ \\
\hline $\mathrm{C} 17-\mathrm{N} 2-\mathrm{Ir} 1$ & $115.6(4)$ & $\mathrm{C} 34-\mathrm{C} 31-\mathrm{C} 33$ & $119.1(10)$ \\
\hline $\mathrm{N} 2-\mathrm{C} 13-\mathrm{C} 14$ & $124.1(7)$ & $\mathrm{C} 34-\mathrm{C} 31-\mathrm{N} 4$ & $108.5(7)$ \\
\hline $\mathrm{C} 13-\mathrm{C} 14-\mathrm{C} 15$ & $117.8(8)$ & $\mathrm{C} 33-\mathrm{C} 31-\mathrm{N} 4$ & $107.3(7)$ \\
\hline $\mathrm{C} 16-\mathrm{C} 15-\mathrm{Cl} 4$ & $120.4(7)$ & $\mathrm{C} 34-\mathrm{C} 31-\mathrm{C} 32$ & $108.6(10)$ \\
\hline $\mathrm{C} 15-\mathrm{C} 16-\mathrm{C} 17$ & $119.6(8)$ & $\mathrm{C} 33-\mathrm{C} 31-\mathrm{C} 32$ & $105.9(9)$ \\
\hline $\mathrm{N} 2-\mathrm{C} 17-\mathrm{C} 16$ & $120.1(6)$ & $\mathrm{N} 4-\mathrm{C} 31-\mathrm{C} 32$ & $106.7(7)$ \\
\hline $\mathrm{N} 2-\mathrm{C} 17-\mathrm{C} 18$ & $113.2(5)$ & N7-C59-Ir2 & $176.6(7)$ \\
\hline $\mathrm{C} 16-\mathrm{C} 17-\mathrm{C} 18$ & $126.7(7)$ & $\mathrm{N} 7-\mathrm{C} 60-\mathrm{C} 62$ & $109.4(7)$ \\
\hline $\mathrm{C} 19-\mathrm{C} 18-\mathrm{C} 23$ & $119.9(6)$ & N7--C60--C61 & $108.0(7)$ \\
\hline $\mathrm{C} 19-\mathrm{C} 18-\mathrm{C} 17$ & $118.4(6)$ & $\mathrm{C} 62-\mathrm{C} 60-\mathrm{C} 61$ & $117.7(10)$ \\
\hline $\mathrm{C} 23-\mathrm{C} 18-\mathrm{C} 17$ & $121.5(6)$ & $\mathrm{N} 7-\mathrm{C} 60-\mathrm{C} 63$ & $106.8(7)$ \\
\hline $\mathrm{C} 22-\mathrm{C} 23-\mathrm{C} 18$ & $120.0(7)$ & $\mathrm{C} 62-\mathrm{C} 60-\mathrm{C} 63$ & $106.5(8)$ \\
\hline $\mathrm{C} 23-\mathrm{C} 22-\mathrm{C} 21$ & $120.8(7)$ & $\mathrm{C} 61-\mathrm{C} 60-\mathrm{C} 63$ & $107.9(8)$ \\
\hline $\mathrm{C} 20-\mathrm{C} 21-\mathrm{C} 22$ & $118.0(7)$ & N8 $-\mathrm{C} 64-\mathrm{Ir} 2$ & $173.1(8)$ \\
\hline
\end{tabular}




\begin{tabular}{|c|c|c|c|}
\hline $\mathrm{C} 20-\mathrm{C} 21-\mathrm{C} 24$ & $123.5(7)$ & $\mathrm{N} 8-\mathrm{C} 65-\mathrm{C} 67^{\prime}$ & $106.6(9)$ \\
\hline $\mathrm{C} 22-\mathrm{C} 21-\mathrm{C} 24$ & $118.4(7)$ & N8-C65-C66 & $112.5(13)$ \\
\hline $\mathrm{C} 19-\mathrm{C} 20-\mathrm{C} 21$ & $122.0(6)$ & C67'-C65-C66 & $77.4(11)$ \\
\hline $\mathrm{C} 18-\mathrm{C} 19-\mathrm{C} 20$ & $119.1(6)$ & N8-C $65-\mathrm{C} 68^{\prime}$ & $109.6(9)$ \\
\hline $\mathrm{C} 18-\mathrm{C} 19-\mathrm{Ir} 1$ & $112.3(4)$ & $\mathrm{C} 67^{\prime}-\mathrm{C} 65-\mathrm{C} 68^{\prime}$ & $113.5(9)$ \\
\hline $\mathrm{C} 20-\mathrm{C} 19-\mathrm{Ir} 1$ & $128.6(5)$ & $\mathrm{C} 66-\mathrm{C} 65-\mathrm{C} 68^{\prime}$ & $130.8(15)$ \\
\hline $\mathrm{O} 2-\mathrm{C} 24-\mathrm{C} 21$ & $123.1(8)$ & $\mathrm{N} 8-\mathrm{C} 65-\mathrm{C} 68$ & $107.3(17)$ \\
\hline $\mathrm{C} 35-\mathrm{N} 5-\mathrm{C} 39$ & $119.8(11)$ & $\mathrm{C} 67-\mathrm{C} 65-\mathrm{C} 68$ & $135.5(18)$ \\
\hline C35-N5-Ir2 & $123.3(10)$ & $\mathrm{C} 66-\mathrm{C} 65-\mathrm{C} 68$ & $114.2(11)$ \\
\hline C39--N5-Ir2 & $116.6(9)$ & $\mathrm{C} 68^{\prime}-\mathrm{C} 65-\mathrm{C} 68$ & $26.2(12)$ \\
\hline $\mathrm{N} 5-\mathrm{C} 35-\mathrm{C} 36$ & $122.8(13)$ & N8-C65-C67 & $102.8(13)$ \\
\hline $\mathrm{C} 37-\mathrm{C} 36-\mathrm{C} 35$ & $117.7(14)$ & $\mathrm{C} 67^{\prime}-\mathrm{C} 65-\mathrm{C} 67$ & $35.8(11)$ \\
\hline $\mathrm{C} 36-\mathrm{C} 37-\mathrm{C} 38$ & $119.9(13)$ & $\mathrm{C} 66-\mathrm{C} 65-\mathrm{C} 67$ & $111.3(10)$ \\
\hline $\mathrm{C} 39-\mathrm{C} 38-\mathrm{C} 37$ & $119.8(13)$ & $\mathrm{C} 68^{\prime}-\mathrm{C} 65-\mathrm{C} 67$ & $82.3(13)$ \\
\hline N5-C39-C38 & $119.6(11)$ & $\mathrm{C} 68-\mathrm{C} 65-\mathrm{C} 67$ & $108.0(11)$ \\
\hline $\mathrm{N} 5-\mathrm{C} 39-\mathrm{C} 40$ & $113.7(10)$ & $\mathrm{N} 8-\mathrm{C} 65-\mathrm{C} 66^{\prime}$ & $103.3(7)$ \\
\hline $\mathrm{C} 38-\mathrm{C} 39-\mathrm{C} 40$ & $126.3(12)$ & $\mathrm{C} 67^{\prime}-\mathrm{C} 65-\mathrm{C} 66^{\prime}$ & $113.1(7)$ \\
\hline $\mathrm{C} 45-\mathrm{C} 40-\mathrm{C} 41$ & $121.9(11)$ & $\mathrm{C} 66-\mathrm{C} 65-\mathrm{C} 66^{\prime}$ & $35.8(11)$ \\
\hline $\mathrm{C} 45-\mathrm{C} 40-\mathrm{C} 39$ & $121.9(11)$ & $\mathrm{C} 68^{\prime}-\mathrm{C} 65-\mathrm{C} 66^{\prime}$ & $110.2(8)$ \\
\hline $\mathrm{C} 41-\mathrm{C} 40-\mathrm{C} 39$ & $116.1(10)$ & $\mathrm{C} 68-\mathrm{C} 65-\mathrm{C} 66^{\prime}$ & $86.0(11)$ \\
\hline $\mathrm{C} 44-\mathrm{C} 45-\mathrm{C} 40$ & $121.5(13)$ & $\mathrm{C} 67-\mathrm{C} 65-\mathrm{C} 66^{\prime}$ & $144.9(12)$ \\
\hline $\mathrm{C} 45-\mathrm{C} 44-\mathrm{C} 43$ & $117.8(12)$ & & \\
\hline $\mathrm{C} 25^{\prime}-\mathrm{Ir} 1-\mathrm{N} 1-\mathrm{C} 1$ & $3.0(18)$ & $\mathrm{C} 64-\mathrm{Ir} 2-\mathrm{N} 5^{\prime}-\mathrm{C} 35^{\prime}$ & $-82(4)$ \\
\hline $\mathrm{C} 30-\mathrm{Ir} 1-\mathrm{N} 1-\mathrm{C} 1$ & $84.6(7)$ & $\mathrm{N} 6-\mathrm{Ir} 2-\mathrm{N} 5^{\prime}-\mathrm{C} 35^{\prime}$ & $125(8)$ \\
\hline $\mathrm{C} 25-\mathrm{Ir} 1-\mathrm{N} 1-\mathrm{C} 1$ & $-7.5(7)$ & $\mathrm{C} 41-\mathrm{Ir} 2-\mathrm{N} 5^{\prime}-\mathrm{C} 35^{\prime}$ & $-177(5)$ \\
\hline $\mathrm{C} 7-\mathrm{Ir} 1-\mathrm{N} 1-\mathrm{C} 1$ & $179.9(7)$ & $\mathrm{C} 53-\mathrm{Ir} 2-\mathrm{N} 5^{\prime}-\mathrm{C} 35^{\prime}$ & $99(4)$ \\
\hline $\mathrm{N} 2-\mathrm{Ir} 1-\mathrm{N} 1-\mathrm{C} 1$ & $-143.9(12)$ & $\mathrm{C} 59-\mathrm{Ir} 2-\mathrm{N} 5^{\prime}-\mathrm{C} 39^{\prime}$ & $-165(3)$ \\
\hline $\mathrm{C} 19-\mathrm{Ir} 1-\mathrm{N} 1-\mathrm{C} 1$ & $-95.4(6)$ & $\mathrm{C} 41^{\prime}-\operatorname{Ir} 2-\mathrm{N} 5^{\prime}-\mathrm{C} 39^{\prime}$ & $10(4)$ \\
\hline $\mathrm{C} 25^{\prime}-\mathrm{Ir} 1-\mathrm{N} 1-\mathrm{C} 5$ & $179.4(18)$ & N5-Ir2-N5'-C39' & $-5(12)$ \\
\hline $\mathrm{C} 30-\operatorname{Ir} 1-\mathrm{N} 1-\mathrm{C} 5$ & $-99.0(5)$ & $\mathrm{C} 64-\mathrm{Ir} 2-\mathrm{N} 5^{\prime}-\mathrm{C} 39^{\prime}$ & $106(3)$ \\
\hline $\mathrm{C} 25-\mathrm{Ir} 1-\mathrm{N} 1-\mathrm{C} 5$ & $168.9(5)$ & $\mathrm{N} 6-\mathrm{Ir} 2-\mathrm{N} 5-\mathrm{C} 39^{\prime}$ & $-46(13)$ \\
\hline $\mathrm{C} 7-\operatorname{Ir} 1-\mathrm{N} 1-\mathrm{C} 5$ & $-3.7(5)$ & $\mathrm{C} 41-\mathrm{Ir} 2-\mathrm{N} 5^{\prime}-\mathrm{C} 39^{\prime}$ & $11(4)$ \\
\hline $\mathrm{N} 2-\operatorname{Ir} 1-\mathrm{N} 1-\mathrm{C} 5$ & $32.5(16)$ & $\mathrm{C} 53-\operatorname{Ir} 2-\mathrm{N} 5^{\prime}-\mathrm{C} 39^{\prime}$ & $-73(3)$ \\
\hline $\mathrm{C} 19-\mathrm{Ir} 1-\mathrm{N} 1-\mathrm{C} 5$ & $81.0(5)$ & $\mathrm{C} 39^{\prime}-\mathrm{N} 5^{\prime}-\mathrm{C} 35^{\prime}-\mathrm{C} 36^{\prime}$ & $-7(7)$ \\
\hline $\mathrm{C} 5-\mathrm{N} 1-\mathrm{C} 1-\mathrm{C} 2$ & $-0.3(12)$ & $\mathrm{Ir} 2-\mathrm{N} 5^{\prime}-\mathrm{C} 35^{\prime}-\mathrm{C} 36^{\prime}$ & $-178(4)$ \\
\hline $\mathrm{Ir} 1-\mathrm{N} 1-\mathrm{C} 1-\mathrm{C} 2$ & $176.0(7)$ & $\mathrm{N} 5^{\prime}-\mathrm{C} 35^{\prime}-\mathrm{C} 36^{\prime}-\mathrm{C} 37^{\prime}$ & $2(6)$ \\
\hline $\mathrm{N} 1-\mathrm{C} 1-\mathrm{C} 2-\mathrm{C} 3$ & $0.0(14)$ & $\mathrm{C} 35^{\prime}-\mathrm{C} 36^{\prime}-\mathrm{C} 37^{\prime}-\mathrm{C} 38^{\prime}$ & $4(5)$ \\
\hline $\mathrm{C} 1-\mathrm{C} 2-\mathrm{C} 3-\mathrm{C} 4$ & $0.8(14)$ & $\mathrm{C} 36^{\prime}-\mathrm{C} 37^{\prime}-\mathrm{C} 38^{\prime}-\mathrm{C} 39^{\prime}$ & $-5(5)$ \\
\hline $\mathrm{C} 2-\mathrm{C} 3-\mathrm{C} 4-\mathrm{C} 5$ & $-1.3(13)$ & $\mathrm{C} 35^{\prime}-\mathrm{N} 5^{\prime}-\mathrm{C} 39^{\prime}-\mathrm{C} 38^{\prime}$ & $6(6)$ \\
\hline $\mathrm{C} 1-\mathrm{N} 1-\mathrm{C} 5-\mathrm{C} 4$ & $-0.2(10)$ & Ir $2-\mathrm{N} 5^{\prime}-\mathrm{C} 39^{\prime}-\mathrm{C} 38^{\prime}$ & $178(3)$ \\
\hline $\mathrm{Ir} 1-\mathrm{N} 1-\mathrm{C} 5-\mathrm{C} 4$ & $-176.8(5)$ & $\mathrm{C} 35^{\prime}-\mathrm{N} 5^{\prime}-\mathrm{C} 39^{\prime}-\mathrm{C} 40^{\prime}$ & $178(4)$ \\
\hline $\mathrm{C} 1-\mathrm{N} 1-\mathrm{C} 5-\mathrm{C} 6$ & $-179.9(6)$ & $\mathrm{Ir} 2-\mathrm{N} 5^{\prime}-\mathrm{C} 39^{\prime}-\mathrm{C} 40^{\prime}$ & $-9(5)$ \\
\hline $\mathrm{Ir} 1-\mathrm{N} 1-\mathrm{C} 5-\mathrm{C} 6$ & $3.5(7)$ & $\mathrm{C} 37^{\prime}-\mathrm{C} 38^{\prime}-\mathrm{C} 39^{\prime}-\mathrm{N} 5^{\prime}$ & $0(5)$ \\
\hline $\mathrm{C} 3-\mathrm{C} 4-\mathrm{C} 5-\mathrm{N} 1$ & $1.0(10)$ & $\mathrm{C} 37^{\prime}-\mathrm{C} 38^{\prime}-\mathrm{C} 39^{\prime}-\mathrm{C} 40^{\prime}$ & $-172(4)$ \\
\hline $\mathrm{C} 3-\mathrm{C} 4-\mathrm{C} 5-\mathrm{C} 6$ & $-179.4(7)$ & $\mathrm{N} 5{ }^{\prime}-\mathrm{C} 39^{\prime}-\mathrm{C} 40^{\prime}-\mathrm{C} 41^{\prime}$ & $1(6)$ \\
\hline $\mathrm{N} 1-\mathrm{C} 5-\mathrm{C} 6-\mathrm{C} 7$ & $-0.9(8)$ & $\mathrm{C} 38^{\prime}-\mathrm{C} 39^{\prime}-\mathrm{C} 40^{\prime}-\mathrm{C} 41^{\prime}$ & $174(5)$ \\
\hline $\mathrm{C} 4-\mathrm{C} 5-\mathrm{C} 6-\mathrm{C} 7$ & $179.5(6)$ & $\mathrm{N} 5^{\prime}-\mathrm{C} 39^{\prime}-\mathrm{C} 40^{\prime}-\mathrm{C} 45^{\prime}$ & $-175(4)$ \\
\hline $\mathrm{N} 1-\mathrm{C} 5-\mathrm{C} 6-\mathrm{C} 11$ & $178.7(6)$ & $\mathrm{C} 38^{\prime}-\mathrm{C} 39^{\prime}-\mathrm{C} 40^{\prime}-\mathrm{C} 45^{\prime}$ & $-3(7)$ \\
\hline $\mathrm{C} 4-\mathrm{C} 5-\mathrm{C} 6-\mathrm{C} 11$ & $-0.9(10)$ & $\mathrm{C} 41^{\prime}-\mathrm{C} 40^{\prime}-\mathrm{C} 45^{\prime}-\mathrm{C} 44^{\prime}$ & $2(7)$ \\
\hline $\mathrm{C} 7-\mathrm{C} 6-\mathrm{C} 11-\mathrm{C} 10$ & $-4.1(10)$ & $\mathrm{C} 39^{\prime}-\mathrm{C} 40^{\prime}-\mathrm{C} 45^{\prime}-\mathrm{C} 44^{\prime}$ & $178(4)$ \\
\hline $\mathrm{C} 5-\mathrm{C} 6-\mathrm{C} 11-\mathrm{C} 10$ & $176.3(6)$ & $\mathrm{C} 40^{\prime}-\mathrm{C} 45^{\prime}-\mathrm{C} 44^{\prime}-\mathrm{C} 43^{\prime}$ & $3(6)$ \\
\hline
\end{tabular}




\begin{tabular}{|c|c|c|c|}
\hline $\mathrm{C} 6-\mathrm{C} 11-\mathrm{C} 10-\mathrm{C} 9$ & $3.9(11)$ & $\mathrm{C} 45^{\prime}-\mathrm{C} 44^{\prime}-\mathrm{C} 43^{\prime}-\mathrm{C} 42^{\prime}$ & $-3(5)$ \\
\hline $\mathrm{C} 11-\mathrm{C} 10-\mathrm{C} 9-\mathrm{C} 8$ & $-1.5(11)$ & $\mathrm{C} 45^{\prime}-\mathrm{C} 44^{\prime}-\mathrm{C} 43^{\prime}-\mathrm{C} 46^{\prime}$ & $177(3)$ \\
\hline $\mathrm{C} 11-\mathrm{C} 10-\mathrm{C} 9-\mathrm{C} 12$ & $-179.8(7)$ & $\mathrm{C} 44^{\prime}-\mathrm{C} 43^{\prime}-\mathrm{C} 42^{\prime}-\mathrm{C} 41^{\prime}$ & $-3(6)$ \\
\hline $\mathrm{C} 10-\mathrm{C} 9-\mathrm{C} 8-\mathrm{C} 7$ & $-0.8(10)$ & $\mathrm{C} 46^{\prime}-\mathrm{C} 43^{\prime}-\mathrm{C} 42^{\prime}-\mathrm{C} 41^{\prime}$ & $177(5)$ \\
\hline $\mathrm{C} 12-\mathrm{C} 9-\mathrm{C} 8-\mathrm{C} 7$ & $177.5(7)$ & $\mathrm{C} 43^{\prime}-\mathrm{C} 42^{\prime}-\mathrm{C} 41^{\prime}-\mathrm{C} 40^{\prime}$ & $8(8)$ \\
\hline $\mathrm{C} 9-\mathrm{C} 8-\mathrm{C} 7-\mathrm{C} 6$ & $0.6(9)$ & $\mathrm{C} 43^{\prime}-\mathrm{C} 42^{\prime}-\mathrm{C} 41^{\prime}-\operatorname{Ir} 2$ & $175(4)$ \\
\hline $\mathrm{C} 9-\mathrm{C} 8-\mathrm{C} 7-\mathrm{Ir} 1$ & $-175.2(5)$ & $\mathrm{C} 45^{\prime}-\mathrm{C} 40^{\prime}-\mathrm{C} 41^{\prime}-\mathrm{C} 42^{\prime}$ & $-8(8)$ \\
\hline $\mathrm{C} 11-\mathrm{C} 6-\mathrm{C} 7-\mathrm{C} 8$ & $1.8(9)$ & $\mathrm{C} 39^{\prime}-\mathrm{C} 40^{\prime}-\mathrm{C} 41^{\prime}-\mathrm{C} 42^{\prime}$ & $176(5)$ \\
\hline $\mathrm{C} 5-\mathrm{C} 6-\mathrm{C} 7-\mathrm{C} 8$ & $-178.6(5)$ & $\mathrm{C} 45^{\prime}-\mathrm{C} 40^{\prime}-\mathrm{C} 41^{\prime}-\mathrm{Ir} 2$ & $-176(4)$ \\
\hline $\mathrm{C} 11-\mathrm{C} 6-\mathrm{C} 7-\mathrm{Ir} 1$ & $178.3(5)$ & $\mathrm{C} 39^{\prime}-\mathrm{C} 40^{\prime}-\mathrm{C} 41^{\prime}-\mathrm{Ir} 2$ & $7(6)$ \\
\hline $\mathrm{C} 5-\mathrm{C} 6-\mathrm{C} 7-\mathrm{Ir} 1$ & $-2.1(7)$ & $\mathrm{C} 59-\mathrm{Ir} 2-\mathrm{C} 41^{\prime}-\mathrm{C} 42^{\prime}$ & $-140(8)$ \\
\hline $\mathrm{C} 25^{\prime}-\mathrm{Ir} 1-\mathrm{C} 7-\mathrm{C} 8$ & $-170(6)$ & $\mathrm{N} 5-\mathrm{Ir} 2-\mathrm{C} 41^{\prime}-\mathrm{C} 42^{\prime}$ & $-174(6)$ \\
\hline $\mathrm{C} 30-\mathrm{Ir} 1-\mathrm{C} 7-\mathrm{C} 8$ & $-90.7(6)$ & $\mathrm{C} 64-\mathrm{Ir} 2-\mathrm{C} 41^{\prime}-\mathrm{C} 42^{\prime}$ & $98(6)$ \\
\hline $\mathrm{C} 25-\mathrm{Ir} 1-\mathrm{C} 7-\mathrm{C} 8$ & $104(2)$ & $\mathrm{N} 6-\mathrm{Ir} 2-\mathrm{C} 41^{\prime}-\mathrm{C} 42^{\prime}$ & $-1(6)$ \\
\hline $\mathrm{N} 2-\mathrm{Ir} 1-\mathrm{C} 7-\mathrm{C} 8$ & $4.5(6)$ & $\mathrm{N} 5^{\prime}-\mathrm{Ir} 2-\mathrm{C} 41^{\prime}-\mathrm{C} 42^{\prime}$ & $-177(6)$ \\
\hline $\mathrm{N} 1-\mathrm{Ir} 1-\mathrm{C} 7-\mathrm{C} 8$ & $179.0(6)$ & $\mathrm{C} 41-\mathrm{Ir} 2-\mathrm{C} 41^{\prime}-\mathrm{C} 42^{\prime}$ & $8(18)$ \\
\hline $\mathrm{C} 19-\mathrm{Ir} 1-\mathrm{C} 7-\mathrm{C} 8$ & $84.6(6)$ & $\mathrm{C} 53-\mathrm{Ir} 2-\mathrm{C} 41^{\prime}-\mathrm{C} 42^{\prime}$ & $-80(6)$ \\
\hline $\mathrm{C} 25^{\prime}-\mathrm{Ir} 1-\mathrm{C} 7-\mathrm{C} 6$ & $14(6)$ & $\mathrm{C} 59-\mathrm{Ir} 2-\mathrm{C} 41^{\prime}-\mathrm{C} 40^{\prime}$ & $27(17)$ \\
\hline $\mathrm{C} 30-\mathrm{Ir} 1-\mathrm{C} 7-\mathrm{C} 6$ & $93.4(4)$ & $\mathrm{N} 5-\mathrm{Ir} 2-\mathrm{C} 41^{\prime}-\mathrm{C} 40^{\prime}$ & $-6(4)$ \\
\hline $\mathrm{C} 25-\mathrm{Ir} 1-\mathrm{C} 7-\mathrm{C} 6$ & $-72(2)$ & $\mathrm{C} 64-\mathrm{Ir} 2-\mathrm{C} 41^{\prime}-\mathrm{C} 40^{\prime}$ & $-95(5)$ \\
\hline $\mathrm{N} 2-\operatorname{Ir} 1-\mathrm{C} 7-\mathrm{C} 6$ & $-171.5(4)$ & $\mathrm{N} 6-\mathrm{Ir} 2-\mathrm{C} 41^{\prime}-\mathrm{C} 40^{\prime}$ & $166(4)$ \\
\hline $\mathrm{N} 1-\mathrm{Ir} 1-\mathrm{C} 7-\mathrm{C} 6$ & $3.0(4)$ & $\mathrm{N} 5{ }^{\prime}-\mathrm{Ir} 2-\mathrm{C} 41^{\prime}-\mathrm{C} 40^{\prime}$ & $-9(5)$ \\
\hline $\mathrm{C} 19-\mathrm{Ir} 1-\mathrm{C} 7-\mathrm{C} 6$ & $-91.4(4)$ & $\mathrm{C} 41-\mathrm{Ir} 2-\mathrm{C} 41^{\prime}-\mathrm{C} 40^{\prime}$ & $175(27)$ \\
\hline $\mathrm{C} 10-\mathrm{C} 9-\mathrm{C} 12-\mathrm{O} 1$ & $-177.3(10)$ & $\mathrm{C} 53-\mathrm{Ir} 2-\mathrm{C} 41^{\prime}-\mathrm{C} 40^{\prime}$ & $87(5)$ \\
\hline $\mathrm{C} 8-\mathrm{C} 9-\mathrm{C} 12-\mathrm{O} 1$ & $4.4(14)$ & $\mathrm{C} 44^{\prime}-\mathrm{C} 43^{\prime}-\mathrm{C} 46^{\prime}-\mathrm{O}^{\prime}$ & $5(5)$ \\
\hline $\mathrm{C} 25-\operatorname{Ir} 1-\mathrm{N} 2-\mathrm{C} 13$ & $83.9(18)$ & $\mathrm{C} 42^{\prime}-\mathrm{C} 43^{\prime}-\mathrm{C} 46^{\prime}-\mathrm{O} 3^{\prime}$ & $-174(3)$ \\
\hline $\mathrm{C} 30-\mathrm{Ir} 1-\mathrm{N} 2-\mathrm{C} 13$ & $1.2(6)$ & $\mathrm{C} 59-\mathrm{Ir} 2-\mathrm{N} 6-\mathrm{C} 47$ & $-86.0(5)$ \\
\hline $\mathrm{C} 25-\mathrm{Ir} 1-\mathrm{N} 2-\mathrm{C} 13$ & $92.9(7)$ & $\mathrm{C} 41^{\prime}-\mathrm{Ir} 2-\mathrm{N} 6-\mathrm{C} 47$ & $99(2)$ \\
\hline $\mathrm{C} 7-\mathrm{Ir} 1-\mathrm{N} 2-\mathrm{C} 13$ & $-94.6(6)$ & $\mathrm{N} 5-\mathrm{Ir} 2-\mathrm{N} 6-\mathrm{C} 47$ & $128(4)$ \\
\hline $\mathrm{N} 1-\mathrm{Ir} 1-\mathrm{N} 2-\mathrm{C} 13$ & $-130.1(13)$ & $\mathrm{C} 64-\mathrm{Ir} 2-\mathrm{N} 6-\mathrm{C} 47$ & $3.0(6)$ \\
\hline $\mathrm{C} 19-\mathrm{Ir} 1-\mathrm{N} 2-\mathrm{C} 13$ & $-179.5(6)$ & $\mathrm{N} 5-\mathrm{I} 2-\mathrm{N} 6-\mathrm{C} 47$ & $155(10)$ \\
\hline $\mathrm{C} 25^{\prime}-\operatorname{Ir} 1-\mathrm{N} 2-\mathrm{C} 17$ & $-98.8(18)$ & $\mathrm{C} 41-\mathrm{Ir} 2-\mathrm{N} 6-\mathrm{C} 47$ & $97.8(19)$ \\
\hline $\mathrm{C} 30-\mathrm{Ir} 1-\mathrm{N} 2-\mathrm{C} 17$ & $178.5(5)$ & $\mathrm{C} 53-\mathrm{Ir} 2-\mathrm{N} 6-\mathrm{C} 47$ & $-177.9(5)$ \\
\hline $\mathrm{C} 25-\mathrm{Ir} 1-\mathrm{N} 2-\mathrm{C} 17$ & $-89.7(6)$ & $\mathrm{C} 59-\mathrm{Ir} 2-\mathrm{N} 6-\mathrm{C} 51$ & $97.2(4)$ \\
\hline $\mathrm{C} 7-\mathrm{Ir} 1-\mathrm{N} 2-\mathrm{C} 17$ & $82.8(5)$ & $\mathrm{C} 41^{\prime}-\mathrm{Ir} 2-\mathrm{N} 6-\mathrm{C} 51$ & $-77(2)$ \\
\hline $\mathrm{N} 1-\mathrm{Ir} 1-\mathrm{N} 2-\mathrm{C} 17$ & $47.2(15)$ & $\mathrm{N} 5-\mathrm{Ir} 2-\mathrm{N} 6-\mathrm{C} 51$ & $-49(4)$ \\
\hline $\mathrm{C} 19-\mathrm{Ir} 1-\mathrm{N} 2-\mathrm{C} 17$ & $-2.1(4)$ & $\mathrm{C} 64-\mathrm{Ir} 2-\mathrm{N} 6-\mathrm{C} 51$ & $-173.7(4)$ \\
\hline $\mathrm{C} 17-\mathrm{N} 2-\mathrm{C} 13-\mathrm{C} 14$ & $-1.7(11)$ & $\mathrm{N} 5-\mathrm{Ir} 2-\mathrm{N} 6-\mathrm{C} 51$ & $-21(10)$ \\
\hline $\mathrm{Ir} 1-\mathrm{N} 2-\mathrm{C} 13-\mathrm{C} 14$ & $175.6(6)$ & $\mathrm{C} 41-\mathrm{Ir} 2-\mathrm{N} 6-\mathrm{C} 51$ & $-78.9(19)$ \\
\hline $\mathrm{N} 2-\mathrm{C} 13-\mathrm{C} 14-\mathrm{C} 15$ & $1.2(12)$ & $\mathrm{C} 53-\mathrm{Ir} 2-\mathrm{N} 6-\mathrm{C} 51$ & $5.4(4)$ \\
\hline $\mathrm{C} 13-\mathrm{C} 14-\mathrm{C} 15-\mathrm{C} 16$ & $-0.8(13)$ & $\mathrm{C} 51-\mathrm{N} 6-\mathrm{C} 47-\mathrm{C} 48$ & $1.6(10)$ \\
\hline $\mathrm{C} 14-\mathrm{C} 15-\mathrm{C} 16-\mathrm{C} 17$ & $1.0(13)$ & Ir2-N6-C47-C48 & $-175.1(5)$ \\
\hline $\mathrm{C} 13-\mathrm{N} 2-\mathrm{C} 17-\mathrm{C} 16$ & $1.8(10)$ & $\mathrm{N} 6-\mathrm{C} 47-\mathrm{C} 48-\mathrm{C} 49$ & $-0.9(11)$ \\
\hline $\mathrm{Ir} 1-\mathrm{N} 2-\mathrm{C} 17-\mathrm{C} 16$ & $-175.8(5)$ & $\mathrm{C} 47-\mathrm{C} 48-\mathrm{C} 49-\mathrm{C} 50$ & $0.3(11)$ \\
\hline $\mathrm{C} 13-\mathrm{N} 2-\mathrm{C} 17-\mathrm{C} 18$ & $-178.3(6)$ & $\mathrm{C} 48-\mathrm{C} 49-\mathrm{C} 50-\mathrm{C} 51$ & $-0.4(11)$ \\
\hline $\mathrm{Ir} 1-\mathrm{N} 2-\mathrm{C} 17-\mathrm{C} 18$ & $4.1(7)$ & $\mathrm{C} 47-\mathrm{N} 6-\mathrm{C} 51-\mathrm{C} 50$ & $-1.6(8)$ \\
\hline $\mathrm{C} 15-\mathrm{C} 16-\mathrm{C} 17-\mathrm{N} 2$ & $-1.5(11)$ & $\operatorname{Ir} 2-\mathrm{N} 6-\mathrm{C} 51-\mathrm{C} 50$ & $175.4(5)$ \\
\hline $\mathrm{C} 15-\mathrm{C} 16-\mathrm{C} 17-\mathrm{C} 18$ & $178.6(7)$ & $\mathrm{C} 47-\mathrm{N} 6-\mathrm{C} 51-\mathrm{C} 52$ & $177.5(5)$ \\
\hline $\mathrm{N} 2-\mathrm{C} 17-\mathrm{C} 18-\mathrm{C} 19$ & $-4.6(9)$ & Ir2-N6- C51-C52 & $-5.5(6)$ \\
\hline $\mathrm{C} 16-\mathrm{C} 17-\mathrm{C} 18-\mathrm{C} 19$ & $175.4(7)$ & $\mathrm{C} 49-\mathrm{C} 50-\mathrm{C} 51-\mathrm{N} 6$ & $1.1(9)$ \\
\hline $\mathrm{N} 2-\mathrm{C} 17-\mathrm{C} 18-\mathrm{C} 23$ & $-179.2(6)$ & $\mathrm{C} 49-\mathrm{C} 50-\mathrm{C} 51-\mathrm{C} 52$ & $-177.9(6)$ \\
\hline
\end{tabular}




\begin{tabular}{|c|c|c|c|}
\hline $\mathrm{C} 16-\mathrm{C} 17-\mathrm{C} 18-\mathrm{C} 23$ & $0.8(11)$ & $\mathrm{N} 6-\mathrm{C} 51-\mathrm{C} 52-\mathrm{C} 53$ & $2.0(7)$ \\
\hline $\mathrm{C} 19-\mathrm{C} 18-\mathrm{C} 23-\mathrm{C} 22$ & $4.3(12)$ & $\mathrm{C} 50-\mathrm{C} 51-\mathrm{C} 52-\mathrm{C} 53{ }^{\prime}$ & $-179.0(6)$ \\
\hline $\mathrm{C} 17-\mathrm{C} 18-\mathrm{C} 23-\mathrm{C} 22$ & $178.8(8)$ & N6-C51-C52-C57 & $-179.2(6)$ \\
\hline $\mathrm{C} 18-\mathrm{C} 23-\mathrm{C} 22-\mathrm{C} 21$ & $-2.3(14)$ & $\mathrm{C} 50-\mathrm{C} 51-\mathrm{C} 52-\mathrm{C} 57$ & $-0.2(10)$ \\
\hline $\mathrm{C} 23-\mathrm{C} 22-\mathrm{C} 21-\mathrm{C} 20$ & $0.5(14)$ & $\mathrm{C} 53-\mathrm{C} 52-\mathrm{C} 57-\mathrm{C} 56$ & $-1.6(9)$ \\
\hline $\mathrm{C} 23-\mathrm{C} 22-\mathrm{C} 21-\mathrm{C} 24$ & $-177.3(9)$ & $\mathrm{C} 51-\mathrm{C} 52-\mathrm{C} 57-\mathrm{C} 56$ & $179.6(6)$ \\
\hline $\mathrm{C} 22-\mathrm{C} 21-\mathrm{C} 20-\mathrm{C} 19$ & $-0.9(12)$ & $\mathrm{C} 52-\mathrm{C} 57-\mathrm{C} 56-\mathrm{C} 55$ & $0.1(10)$ \\
\hline $\mathrm{C} 24-\mathrm{C} 21-\mathrm{C} 20-\mathrm{C} 19$ & $176.8(8)$ & $\mathrm{C} 57-\mathrm{C} 56-\mathrm{C} 55-\mathrm{C} 54$ & $0.8(9)$ \\
\hline $\mathrm{C} 23-\mathrm{C} 18-\mathrm{C} 19-\mathrm{C} 20$ & $-4.6(10)$ & $\mathrm{C} 57-\mathrm{C} 56-\mathrm{C} 55-\mathrm{C} 58$ & $-177.3(6)$ \\
\hline $\mathrm{C} 17-\mathrm{C} 18-\mathrm{C} 19-\mathrm{C} 20$ & $-179.2(6)$ & $\mathrm{C} 56-\mathrm{C} 55-\mathrm{C} 54-\mathrm{C} 53$ & $-0.2(9)$ \\
\hline $\mathrm{C} 23-\mathrm{C} 18-\mathrm{C} 19-\mathrm{Ir} 1$ & $177.4(5)$ & $\mathrm{C} 58-\mathrm{C} 55-\mathrm{C} 54-\mathrm{C} 53$ & $177.9(6)$ \\
\hline $\mathrm{C} 17-\mathrm{C} 18-\mathrm{C} 19-\mathrm{Ir} 1$ & $2.8(7)$ & $\mathrm{C} 55-\mathrm{C} 54-\mathrm{C} 53-\mathrm{C} 52$ & $-1.3(9)$ \\
\hline $\mathrm{C} 21-\mathrm{C} 20-\mathrm{C} 19-\mathrm{C} 18$ & $2.9(10)$ & $\mathrm{C} 55-\mathrm{C} 54-\mathrm{C} 53-\mathrm{Ir} 2$ & $177.1(5)$ \\
\hline $\mathrm{C} 21-\mathrm{C} 20-\mathrm{C} 19-\mathrm{Ir} 1$ & $-179.5(5)$ & $\mathrm{C} 57-\mathrm{C} 52-\mathrm{C} 53-\mathrm{C} 54$ & $2.2(9)$ \\
\hline $\mathrm{C} 25^{\prime}-\mathrm{Ir} 1-\mathrm{C} 19-\mathrm{C} 18$ & $102.3(14)$ & $\mathrm{C} 51-\mathrm{C} 52-\mathrm{C} 53-\mathrm{C} 54$ & $-179.0(5)$ \\
\hline $\mathrm{C} 30-\mathrm{Ir} 1-\mathrm{C} 19-\mathrm{C} 18$ & $8(3)$ & $\mathrm{C} 57-\mathrm{C} 52-\mathrm{C} 53-\mathrm{Ir} 2$ & $-176.4(5)$ \\
\hline $\mathrm{C} 25-\mathrm{Ir} 1-\mathrm{C} 19-\mathrm{C} 18$ & $89.0(5)$ & $\mathrm{C} 51-\mathrm{C} 52-\mathrm{C} 53-\mathrm{Ir} 2$ & $2.4(7)$ \\
\hline $\mathrm{C} 7-\mathrm{Ir} 1-\mathrm{C} 19-\mathrm{C} 18$ & $-93.5(5)$ & $\mathrm{C} 59-\mathrm{Ir} 2-\mathrm{C} 53-\mathrm{C} 54$ & $88.4(6)$ \\
\hline $\mathrm{N} 2-\mathrm{Ir} 1-\mathrm{C} 19-\mathrm{C} 18$ & $-0.4(4)$ & $\mathrm{C} 41^{\prime}-\mathrm{Ir} 2-\mathrm{C} 53-\mathrm{C} 54$ & $-84.4(7)$ \\
\hline $\mathrm{N} 1-\mathrm{Ir} 1-\mathrm{C} 19-\mathrm{C} 18$ & $-173.3(5)$ & N5-Ir2-C53-C54 & $-14.5(7)$ \\
\hline $\mathrm{C} 25^{\prime}-\mathrm{Ir} 1-\mathrm{C} 19-\mathrm{C} 20$ & $-75.5(15)$ & C64-Ir2-C53-C54 & $-154(8)$ \\
\hline $\mathrm{C} 30-\mathrm{Ir} 1-\mathrm{C} 19-\mathrm{C} 20$ & $-170(3)$ & N6-Ir2-C53-C54 & $177.5(6)$ \\
\hline $\mathrm{C} 25-\mathrm{Ir} 1-\mathrm{C} 19-\mathrm{C} 20$ & $-88.8(6)$ & N5'-Ir2-C53-C54 & $-5.0(8)$ \\
\hline $\mathrm{C} 7-\mathrm{Ir} 1-\mathrm{C} 19-\mathrm{C} 20$ & $88.8(6)$ & $\mathrm{C} 41-\mathrm{Ir} 2-\mathrm{C} 53-\mathrm{C} 54$ & $-94.3(7)$ \\
\hline $\mathrm{N} 2-\mathrm{Ir} 1-\mathrm{C} 19-\mathrm{C} 20$ & $-178.1(6)$ & $\mathrm{C} 59-\mathrm{Ir} 2-\mathrm{C} 53-\mathrm{C} 52$ & $-93.1(5)$ \\
\hline $\mathrm{N} 1-\mathrm{Ir} 1-\mathrm{C} 19-\mathrm{C} 20$ & $9.0(6)$ & $\mathrm{C} 41^{\prime}-\mathrm{Ir} 2-\mathrm{C} 53-\mathrm{C} 52$ & $94.1(6)$ \\
\hline $\mathrm{C} 20-\mathrm{C} 21-\mathrm{C} 24-\mathrm{O} 2$ & $-4.4(16)$ & $\mathrm{N} 5-\mathrm{Ir} 2-\mathrm{C} 53-\mathrm{C} 52$ & $164.0(6)$ \\
\hline $\mathrm{C} 22-\mathrm{C} 21-\mathrm{C} 24-\mathrm{O} 2$ & $173.3(10)$ & $\mathrm{C} 64-\mathrm{Ir} 2-\mathrm{C} 53-\mathrm{C} 52$ & $24(8)$ \\
\hline $\mathrm{C} 59-\mathrm{Ir} 2-\mathrm{N} 5-\mathrm{C} 35$ & $9(4)$ & $\mathrm{N} 6-\mathrm{Ir} 2-\mathrm{C} 53-\mathrm{C} 52$ & $-4.0(4)$ \\
\hline $\mathrm{C} 41^{\prime}-\mathrm{Ir} 2-\mathrm{N} 5-\mathrm{C} 35$ & $-175(4)$ & $\mathrm{N} 5-\mathrm{Ir} 2-\mathrm{C} 53-\mathrm{C} 52$ & $173.5(7)$ \\
\hline $\mathrm{C} 64-\mathrm{Ir} 2-\mathrm{N} 5-\mathrm{C} 35$ & $-80(3)$ & $\mathrm{C} 41-\mathrm{Ir} 2-\mathrm{C} 53-\mathrm{C} 52$ & $84.2(6)$ \\
\hline N6-Ir2-N5-C35 & $154(3)$ & $\mathrm{C} 54-\mathrm{C} 55-\mathrm{C} 58-\mathrm{O} 4$ & $2.8(11)$ \\
\hline $\mathrm{N} 5-\mathrm{Ir} 2-\mathrm{N} 5-\mathrm{C} 35$ & $-11(12)$ & $\mathrm{C} 56-\mathrm{C} 55-\mathrm{C} 58-\mathrm{O} 4$ & $-179.1(7)$ \\
\hline $\mathrm{C} 41-\mathrm{Ir} 2-\mathrm{N} 5-\mathrm{C} 35$ & $-175(4)$ & $\mathrm{C} 26-\mathrm{N} 3-\mathrm{C} 25-\mathrm{Ir} 1$ & $128(17)$ \\
\hline $\mathrm{C} 53-\mathrm{Ir} 2-\mathrm{N} 5-\mathrm{C} 35$ & $101(3)$ & $\mathrm{C} 25-\mathrm{Ir} 1-\mathrm{C} 25-\mathrm{N} 3$ & $-79(18)$ \\
\hline $\mathrm{C} 59-\mathrm{Ir} 2-\mathrm{N} 5-\mathrm{C} 39$ & $-177(3)$ & $\mathrm{C} 30-\operatorname{Ir} 1-\mathrm{C} 25-\mathrm{N} 3$ & $-133(18)$ \\
\hline $\mathrm{C} 41^{\prime}-\mathrm{Ir} 2-\mathrm{N} 5-\mathrm{C} 39$ & $-2(3)$ & $\mathrm{C} 7-\mathrm{Ir} 1-\mathrm{C} 25-\mathrm{N} 3$ & $33(20)$ \\
\hline $\mathrm{C} 64-\mathrm{Ir} 2-\mathrm{N} 5-\mathrm{C} 39$ & $93(3)$ & $\mathrm{N} 2-\mathrm{Ir} 1-\mathrm{C} 25-\mathrm{N} 3$ & $132(18)$ \\
\hline N6-Ir2-N5-C39 & $-32(7)$ & $\mathrm{N} 1-\mathrm{Ir} 1-\mathrm{C} 25-\mathrm{N} 3$ & $-42(18)$ \\
\hline $\mathrm{N} 5-\mathrm{Ir} 2-\mathrm{N} 5-\mathrm{C} 39$ & $162(17)$ & $\mathrm{C} 19-\mathrm{Ir} 1-\mathrm{C} 25-\mathrm{N} 3$ & $52(18)$ \\
\hline $\mathrm{C} 41-\mathrm{Ir} 2-\mathrm{N} 5-\mathrm{C} 39$ & $-2(3)$ & $\mathrm{C} 25-\mathrm{N} 3-\mathrm{C} 26-\mathrm{C} 28$ & $-128(7)$ \\
\hline $\mathrm{C} 53-\mathrm{Ir} 2-\mathrm{N} 5-\mathrm{C} 39$ & $-85(3)$ & $\mathrm{C} 25-\mathrm{N} 3-\mathrm{C} 26-\mathrm{C} 29$ & $-7(8)$ \\
\hline $\mathrm{C} 39-\mathrm{N} 5-\mathrm{C} 35-\mathrm{C} 36$ & $8(6)$ & $\mathrm{C} 25-\mathrm{N} 3-\mathrm{C} 26-\mathrm{C} 27$ & $113(7)$ \\
\hline Ir $2-\mathrm{N} 5-\mathrm{C} 35-\mathrm{C} 36$ & $-179(3)$ & $\mathrm{C} 26^{\prime}-\mathrm{N} 3^{\prime}-\mathrm{C} 25^{\prime}-\mathrm{Ir} 1$ & $-105(44)$ \\
\hline $\mathrm{N} 5-\mathrm{C} 35-\mathrm{C} 36-\mathrm{C} 37$ & $-4(5)$ & $\mathrm{C} 30-\mathrm{Ir} 1-\mathrm{C} 25^{\prime}-\mathrm{N} 3^{\prime}$ & $-63(27)$ \\
\hline $\mathrm{C} 35-\mathrm{C} 36-\mathrm{C} 37-\mathrm{C} 38$ & $0(4)$ & $\mathrm{C} 25-\mathrm{Ir} 1-\mathrm{C} 25^{\prime}-\mathrm{N} 3{ }^{\prime}$ & $172(33)$ \\
\hline $\mathrm{C} 36-\mathrm{C} 37-\mathrm{C} 38-\mathrm{C} 39$ & $0(4)$ & $\mathrm{C} 7-\mathrm{Ir} 1-\mathrm{C} 25^{\prime}-\mathrm{N} 3^{\prime}$ & $18(33)$ \\
\hline $\mathrm{C} 35-\mathrm{N} 5-\mathrm{C} 39-\mathrm{C} 38$ & $-8(5)$ & $\mathrm{N} 2-\operatorname{Ir} 1-\mathrm{C} 25^{\prime}-\mathrm{N} 3^{\prime}$ & $-156(27)$ \\
\hline Ir $2-\mathrm{N} 5-\mathrm{C} 39-\mathrm{C} 38$ & $179(3)$ & $\mathrm{N} 1-\mathrm{Ir} 1-\mathrm{C} 25^{\prime}-\mathrm{N} 3^{\prime}$ & $29(27)$ \\
\hline $\mathrm{C} 35-\mathrm{N} 5-\mathrm{C} 39-\mathrm{C} 40$ & $178(4)$ & $\mathrm{C} 19-\mathrm{Ir} 1-\mathrm{C} 25^{\prime}-\mathrm{N} 3{ }^{\prime}$ & $122(27)$ \\
\hline $\mathrm{Ir} 2-\mathrm{N} 5-\mathrm{C} 39-\mathrm{C} 40$ & $5(4)$ & $\mathrm{C} 25^{\prime}-\mathrm{N} 3^{\prime}-\mathrm{C} 26^{\prime}-\mathrm{C} 29^{\prime}$ & $164(37)$ \\
\hline
\end{tabular}




\begin{tabular}{|c|c|c|c|}
\hline $\mathrm{C} 37-\mathrm{C} 38-\mathrm{C} 39-\mathrm{N} 5$ & $4(4)$ & $\mathrm{C} 25^{\prime}-\mathrm{N} 3^{\prime}-\mathrm{C} 26^{\prime}-\mathrm{C} 27^{\prime}$ & $-75(38)$ \\
\hline $\mathrm{C} 37-\mathrm{C} 38-\mathrm{C} 39-\mathrm{C} 40$ & $177(3)$ & $\mathrm{C} 25^{\prime}-\mathrm{N} 3^{\prime}-\mathrm{C} 26^{\prime}-\mathrm{C} 28^{\prime}$ & $43(38)$ \\
\hline $\mathrm{N} 5-\mathrm{C} 39-\mathrm{C} 40-\mathrm{C} 45$ & $176(4)$ & $\mathrm{C} 31-\mathrm{N} 4-\mathrm{C} 30-\mathrm{Ir} 1$ & $36(15)$ \\
\hline $\mathrm{C} 38-\mathrm{C} 39-\mathrm{C} 40-\mathrm{C} 45$ & $3(6)$ & $\mathrm{C} 25^{\prime}-\operatorname{Ir} 1-\mathrm{C} 30-\mathrm{N} 4$ & $-63(11)$ \\
\hline $\mathrm{N} 5-\mathrm{C} 39-\mathrm{C} 40-\mathrm{C} 41$ & $-6(5)$ & $\mathrm{C} 25-\mathrm{Ir} 1-\mathrm{C} 30-\mathrm{N} 4$ & $-49(11)$ \\
\hline $\mathrm{C} 38-\mathrm{C} 39-\mathrm{C} 40-\mathrm{C} 41$ & $-180(4)$ & $\mathrm{C} 7-\mathrm{Ir} 1-\mathrm{C} 30-\mathrm{N} 4$ & $133(11)$ \\
\hline $\mathrm{C} 41-\mathrm{C} 40-\mathrm{C} 45-\mathrm{C} 44$ & $3(6)$ & $\mathrm{N} 2-\mathrm{Ir} 1-\mathrm{C} 30-\mathrm{N} 4$ & $40(11)$ \\
\hline $\mathrm{C} 39-\mathrm{C} 40-\mathrm{C} 45-\mathrm{C} 44$ & $-180(4)$ & $\mathrm{N} 1-\mathrm{Ir} 1-\mathrm{C} 30-\mathrm{N} 4$ & $-147(11)$ \\
\hline $\mathrm{C} 40-\mathrm{C} 45-\mathrm{C} 44-\mathrm{C} 43$ & $-3(5)$ & $\mathrm{C} 19-\mathrm{Ir} 1-\mathrm{C} 30-\mathrm{N} 4$ & $32(13)$ \\
\hline $\mathrm{C} 45-\mathrm{C} 44-\mathrm{C} 43-\mathrm{C} 42$ & $-2(4)$ & $\mathrm{C} 30-\mathrm{N} 4-\mathrm{C} 31-\mathrm{C} 34$ & $158(6)$ \\
\hline $\mathrm{C} 45-\mathrm{C} 44-\mathrm{C} 43-\mathrm{C} 46$ & $-177(3)$ & $\mathrm{C} 30-\mathrm{N} 4-\mathrm{C} 31-\mathrm{C} 33$ & $29(6)$ \\
\hline $\mathrm{C} 44-\mathrm{C} 43-\mathrm{C} 42-\mathrm{C} 41$ & $9(5)$ & $\mathrm{C} 30-\mathrm{N} 4-\mathrm{C} 31-\mathrm{C} 32$ & $-85(6)$ \\
\hline $\mathrm{C} 46-\mathrm{C} 43-\mathrm{C} 42-\mathrm{C} 41$ & $-176(4)$ & $\mathrm{C} 60-\mathrm{N} 7-\mathrm{C} 59-\mathrm{Ir} 2$ & $16(18)$ \\
\hline $\mathrm{C} 43-\mathrm{C} 42-\mathrm{C} 41-\mathrm{C} 40$ & $-9(6)$ & $\mathrm{C} 41^{\prime}-\mathrm{Ir} 2-\mathrm{C} 59-\mathrm{N} 7$ & $155(16)$ \\
\hline $\mathrm{C} 43-\mathrm{C} 42-\mathrm{C} 41-\mathrm{Ir} 2$ & $172(4)$ & $\mathrm{N} 5-\mathrm{Ir} 2-\mathrm{C} 59-\mathrm{N} 7$ & $-173(10)$ \\
\hline $\mathrm{C} 45-\mathrm{C} 40-\mathrm{C} 41-\mathrm{C} 42$ & $3(7)$ & $\mathrm{C} 64-\mathrm{Ir} 2-\mathrm{C} 59-\mathrm{N} 7$ & $-83(10)$ \\
\hline $\mathrm{C} 39-\mathrm{C} 40-\mathrm{C} 41-\mathrm{C} 42$ & $-174(4)$ & $\mathrm{N} 6-\mathrm{Ir} 2-\mathrm{C} 59-\mathrm{N} 7$ & $15(10)$ \\
\hline $\mathrm{C} 45-\mathrm{C} 40-\mathrm{C} 41-\mathrm{Ir} 2$ & $-177(3)$ & $\mathrm{N} 5-\mathrm{Ir} 2-\mathrm{C} 59-\mathrm{N} 7$ & $-169(11)$ \\
\hline $\mathrm{C} 39-\mathrm{C} 40-\mathrm{C} 41-\mathrm{Ir} 2$ & $5(6)$ & $\mathrm{C} 41-\mathrm{Ir} 2-\mathrm{C} 59-\mathrm{N} 7$ & $63(17)$ \\
\hline $\mathrm{C} 59-\mathrm{Ir} 2-\mathrm{C} 41-\mathrm{C} 42$ & $-58(17)$ & $\mathrm{C} 53-\mathrm{Ir} 2-\mathrm{C} 59-\mathrm{N} 7$ & $95(10)$ \\
\hline $\mathrm{C} 41^{\prime}-\mathrm{Ir} 2-\mathrm{C} 41-\mathrm{C} 42$ & $179(28)$ & $\mathrm{C} 59-\mathrm{N} 7-\mathrm{C} 60-\mathrm{C} 62$ & $-179(100)$ \\
\hline $\mathrm{N} 5-\mathrm{Ir} 2-\mathrm{C} 41-\mathrm{C} 42$ & $177(6)$ & $\mathrm{C} 59-\mathrm{N} 7-\mathrm{C} 60-\mathrm{C} 61$ & $-50(9)$ \\
\hline $\mathrm{C} 64-\mathrm{Ir} 2-\mathrm{C} 41-\mathrm{C} 42$ & $88(5)$ & $\mathrm{C} 59-\mathrm{N} 7-\mathrm{C} 60-\mathrm{C} 63$ & $66(9)$ \\
\hline $\mathrm{N} 6-\mathrm{Ir} 2-\mathrm{C} 41-\mathrm{C} 42$ & $-10(5)$ & $\mathrm{C} 65-\mathrm{N} 8-\mathrm{C} 64-\mathrm{Ir} 2$ & $21(9)$ \\
\hline $\mathrm{N} 5{ }^{\prime}-\mathrm{Ir} 2-\mathrm{C} 41-\mathrm{C} 42$ & $175(5)$ & $\mathrm{C} 59-\mathrm{Ir} 2-\mathrm{C} 64-\mathrm{N} 8$ & $-50(6)$ \\
\hline $\mathrm{C} 53-\mathrm{Ir} 2-\mathrm{C} 41-\mathrm{C} 42$ & $-90(5)$ & $\mathrm{C} 41^{\prime}-\mathrm{Ir} 2-\mathrm{C} 64-\mathrm{N} 8$ & $123(6)$ \\
\hline $\mathrm{C} 59-\mathrm{Ir} 2-\mathrm{C} 41-\mathrm{C} 40$ & $123(9)$ & $\mathrm{N} 5-\mathrm{Ir} 2-\mathrm{C} 64-\mathrm{N} 8$ & $53(6)$ \\
\hline $\mathrm{C} 41^{\prime}-\mathrm{Ir} 2-\mathrm{C} 41-\mathrm{C} 40$ & $0(20)$ & $\mathrm{N} 6-\mathrm{Ir} 2-\mathrm{C} 64-\mathrm{N} 8$ & $-140(6)$ \\
\hline $\mathrm{N} 5-\mathrm{Ir} 2-\mathrm{C} 41-\mathrm{C} 40$ & $-2(4)$ & $\mathrm{N} 5{ }^{\prime}-\mathrm{Ir} 2-\mathrm{C} 64-\mathrm{N} 8$ & $43(6)$ \\
\hline $\mathrm{C} 64-\mathrm{Ir} 2-\mathrm{C} 41-\mathrm{C} 40$ & $-91(4)$ & $\mathrm{C} 41-\mathrm{Ir} 2-\mathrm{C} 64-\mathrm{N} 8$ & $132(6)$ \\
\hline $\mathrm{N} 6-\mathrm{Ir} 2-\mathrm{C} 41-\mathrm{C} 40$ & $171(4)$ & $\mathrm{C} 53-\mathrm{Ir} 2-\mathrm{C} 64-\mathrm{N} 8$ & $-168(6)$ \\
\hline $\mathrm{N} 5-\mathrm{Ir} 2-\mathrm{C} 41-\mathrm{C} 40$ & $-5(4)$ & $\mathrm{C} 64-\mathrm{N} 8-\mathrm{C} 65-\mathrm{C} 67^{\prime}$ & $-28(5)$ \\
\hline $\mathrm{C} 53-\mathrm{Ir} 2-\mathrm{C} 41-\mathrm{C} 40$ & $91(4)$ & $\mathrm{C} 64-\mathrm{N} 8-\mathrm{C} 65-\mathrm{C} 66$ & $55(5)$ \\
\hline $\mathrm{C} 42-\mathrm{C} 43-\mathrm{C} 46-\mathrm{O} 3$ & $9(4)$ & $\mathrm{C} 64-\mathrm{N} 8-\mathrm{C} 65-\mathrm{C} 68^{\prime}$ & $-151(4)$ \\
\hline $\mathrm{C} 44-\mathrm{C} 43-\mathrm{C} 46-\mathrm{O} 3$ & $-176(3)$ & $\mathrm{C} 64-\mathrm{N} 8-\mathrm{C} 65-\mathrm{C} 68$ & $-179(100)$ \\
\hline $\mathrm{C} 59-\mathrm{Ir} 2-\mathrm{N} 5^{\prime}-\mathrm{C} 35^{\prime}$ & $7(4)$ & C64-N8-C65-C67 & $-65(5)$ \\
\hline $\mathrm{C} 41^{\prime}-\mathrm{Ir} 2-\mathrm{N} 5^{\prime}-\mathrm{C} 35^{\prime}$ & $-178(5)$ & $\mathrm{C} 64-\mathrm{N} 8-\mathrm{C} 65-\mathrm{C} 66^{\prime}$ & $91(4)$ \\
\hline $\mathrm{N} 5-\mathrm{Ir} 2-\mathrm{N} 5^{\prime}-\mathrm{C} 35^{\prime}$ & $166(18)$ & & \\
\hline
\end{tabular}

Table 5: Crystal data and structure refinement for $\mathbf{4} \cdot \mathrm{CH}_{2} \mathrm{Cl}_{2}$

$\mathrm{C}_{35} \mathrm{H}_{30} \mathrm{Cl}_{3} \mathrm{IrN}_{4}$

$M_{r}=805.18$

Monoclinic, $P 2_{1} / c$

$a=10.4363$ (15) $\AA$

$b=25.923(4) \AA$

$c=11.7427(17) \AA$

$\beta=100.315(2)^{\circ}$
$F(000)=1584$

$D_{\mathrm{x}}=1.711 \mathrm{Mg} \mathrm{m}^{-3}$

Mo $K \alpha$ radiation, $\lambda=0.71073 \AA$

Cell parameters from 1021 reflections

$\theta=2.5-25.6^{\circ}$

$\mu=4.56 \mathrm{~mm}^{-1}$

$T=200 \mathrm{~K}$ 
$V=3125.5(8) \AA^{3}$

$Z=4$

Data collection

Bruker AXS APEX diffractometer

Radiation source: fine-focus sealed tube graphite

Detector resolution: 836.6 pixels $\mathrm{mm}^{-1}$

$\omega$ scans

Abs. corr.: Multi-scan SADABS (Sheldrick, 2003)

$T_{\min }=0.533, T_{\max }=0.712$

42578 measured reflections
Block, Yellow

$0.16 \times 0.12 \times 0.08 \mathrm{~mm}$

\section{Refinement}

7764 independent reflections

6685 reflections with $I>2 \sigma(I)$

$R_{\text {int }}=0.042$

$\theta_{\max }=28.3^{\circ}, \theta_{\min }=1.9^{\circ}$

$h=-13 \rightarrow 13$

$k=-34 \rightarrow 34$

$l=-15 \rightarrow 15$

Refinement on $F^{2} \quad$ Primary atom site location: Structure-invariant direct methods Least-squares matrix: Full Secondary atom site location: Difference Fourier map $R\left[F^{2}>2 \sigma\left(F^{2}\right)\right]=0.031 \quad$ Hydrogen site location: Inferred from neighbouring sites $w R\left(F^{2}\right)=0.072 \quad \mathrm{H}$ atoms treated by a mixture of independent and constrained $S=1.01$ refinement

7764 reflections $w=1 /\left[\sigma^{2}\left(F_{0}^{2}\right)+(0.0362 P)^{2}+2.425 P\right]$

395 parameters where $P=\left(F_{\mathrm{o}}^{2}+2 F_{\mathrm{c}}^{2}\right) / 3$

2 restraints $(\Delta / \sigma)_{\max }=0.002$ $\Delta \rho_{\max }=1.42 \mathrm{e} \AA^{-3}$ $\Delta \rho_{\min }=-0.44 \mathrm{e} \AA^{-3}$

Special details

Experimental. Data collection is performed with four batch runs at $\varphi=0.00^{\circ}(600$ frames), at $\varphi=90.00^{\circ}$ (600 frames), at $\varphi=180^{\circ}$ (600 frames) and at $\varphi=270^{\circ}(600$ frames). Frame width $=0.30 \backslash \&$ in $\omega$. Data is merged, corrected for decay, and treated with multi-scan absorption corrections.

Geometry. All esds (except the esd in the dihedral angle between two 1.s. planes) are estimated using the full covariance matrix. The cell esds are taken into account individually in the estimation of esds in distances, angles and torsion angles; correlations between esds in cell parameters are only used when they are defined by crystal symmetry. 
An approximate (isotropic) treatment of cell esds is used for estimating esds involving l.s. planes.

Refinement. Refinement of $\mathrm{F}^{2}$ against ALL reflections. The weighted R-factor $\mathrm{w}$ and goodness of fit $\mathrm{S}$ are based on $\mathrm{F}^{2}$, conventional $\mathrm{R}$-factors $\mathrm{R}$ are based on $\mathrm{F}$, with $\mathrm{F}$ set to zero for negative $F^{2}$. The threshold expression of $F^{2}>2 \operatorname{sigma}\left(F^{2}\right)$ is used only for calculating R-factors(gt) etc. and is not relevant to the choice of reflections for refinement. R-factors based on $\mathrm{F}^{2}$ are statistically about twice as large as those based on $\mathrm{F}$, and R- factors based on ALL data will be even larger.

Table 6: Fractional atomic coordinates and isotropic or equivalent isotropic displacement parameters $\left(\AA^{2}\right)$ for $4 \cdot \mathrm{CH}_{2} \mathrm{Cl}_{2}$

$\begin{array}{lllll} & x & y & z & U_{\text {iso }}^{*} / U_{\text {eq }} \\ \text { Ir } & -0.029066(12) & 0.116314(5) & 0.520128(10) & 0.02764(5) \\ \text { C11 } & -0.20236(9) & 0.05028(3) & 0.51964(8) & 0.03644(19) \\ \text { C12 } & 0.41938(12) & 0.05228(5) & 0.41176(12) & 0.0637(3) \\ \text { C13 } & 0.38311(16) & 0.13280(6) & 0.23797(13) & 0.0770(4) \\ \text { N1 } & -0.0424(3) & 0.14712(11) & 0.6775(2) & 0.0325(6) \\ \text { N2 } & -0.0165(3) & 0.09606(11) & 0.3540(2) & 0.0317(6) \\ \text { N3 } & 0.2863(4) & 0.00873(17) & 0.9927(4) & 0.0674(12) \\ \text { N4 } & 0.0952(3) & 0.05553(12) & 0.6173(3) & 0.0358(7) \\ \text { H4 } & 0.116(4) & 0.0264(11) & 0.576(3) & 0.043^{*} \\ \text { H5 } & 0.045(3) & 0.0426(15) & 0.671(3) & 0.043^{*} \\ \text { C1 } & -0.1251(4) & 0.13120(16) & 0.7454(3) & 0.0405(9) \\ \text { H1A } & -0.1798 & 0.1025 & 0.7214 & 0.049^{*} \\ \text { C2 } & -0.1335(4) & 0.15501(19) & 0.8487(4) & 0.0517(11) \\ \text { H2A } & -0.1933 & 0.1429 & 0.8949 & 0.062^{*} \\ \text { C3 } & -0.0548(5) & 0.19644(19) & 0.8841(4) & 0.0572(12) \\ \text { H3A } & -0.0586 & 0.2134 & 0.9551 & 0.069^{*} \\ \text { C4 } & 0.0289(4) & 0.21282(17) & 0.8154(4) & 0.0517(11) \\ \text { H4A } & 0.0838 & 0.2415 & 0.8392 & 0.062^{*} \\ \text { C5 } & 0.0359(4) & 0.18851(14) & 0.7110(3) & 0.0350(8) \\ \text { C6 } & 0.1243(4) & 0.20093(14) & 0.6319(3) & 0.0360(8) \\ \text { C7 } & 0.2183(4) & 0.24000(15) & 0.6518(4) & 0.0463(10) \\ \text { H7A } & 0.2229 & 0.2619 & 0.7173 & 0.056^{*} \\ \text { C8 } & 0.3040(4) & 0.24680(15) & 0.5767(4) & 0.0458(10) \\ \text { H8A } & 0.3677 & 0.2734 & 0.5901 & 0.055^{*} \\ \text { C9 } & 0.2972(4) & 0.21486(15) & 0.4815(4) & 0.0438(9) \\ \text { H9A } & 0.3575 & 0.2192 & 0.4305 & 0.053^{*} \\ \text { C10 } & 0.2024(4) & 0.17623(14) & 0.4596(3) & 0.0386(8) \\ \text { H10A } & 0.1990 & 0.1548 & 0.3935 & 0.046^{*} \\ \text { C11 } & 0.1130(3) & 0.16859(13) & 0.5328(3) & 0.0301(7) \\ \text { C12 } & 0.0633(4) & 0.06022(15) & 0.3235(3) & 0.0397(9) \\ & & & & \end{array}$




\begin{tabular}{|c|c|c|c|c|}
\hline $\mathrm{H} 12 \mathrm{~A}$ & 0.1182 & 0.0417 & 0.3828 & $0.048^{*}$ \\
\hline $\mathrm{C} 13$ & $0.0694(5)$ & $0.04901(19)$ & $0.2102(4)$ & $0.0560(12)$ \\
\hline $\mathrm{H} 13 \mathrm{~A}$ & 0.1269 & 0.0232 & 0.1913 & $0.067^{*}$ \\
\hline $\mathrm{C} 14$ & $-0.0114(5)$ & $0.0767(2)$ & $0.1235(4)$ & $0.0644(14)$ \\
\hline H14A & -0.0108 & 0.0695 & 0.0443 & $0.077^{*}$ \\
\hline $\mathrm{C} 15$ & $-0.0903(5)$ & $0.11359(19)$ & $0.1534(4)$ & $0.0587(12)$ \\
\hline H15A & -0.1437 & 0.1330 & 0.0947 & $0.070^{*}$ \\
\hline $\mathrm{C} 16$ & $-0.0945(4)$ & $0.12355(15)$ & $0.2698(3)$ & $0.0397(9)$ \\
\hline $\mathrm{C} 17$ & $-0.1748(4)$ & $0.16218(15)$ & $0.3129(3)$ & $0.0418(9)$ \\
\hline $\mathrm{C} 18$ & $-0.2668(4)$ & $0.19326(18)$ & $0.2407(4)$ & $0.0549(12)$ \\
\hline H18A & -0.2774 & 0.1903 & 0.1589 & $0.066^{*}$ \\
\hline C19 & $-0.3412(5)$ & $0.22792(18)$ & $0.2902(5)$ & $0.0645(14)$ \\
\hline H19A & -0.4025 & 0.2492 & 0.2422 & $0.077^{*}$ \\
\hline $\mathrm{C} 20$ & $-0.3267(5)$ & $0.23166(18)$ & $0.4085(5)$ & $0.0609(13)$ \\
\hline $\mathrm{H} 20 \mathrm{~A}$ & -0.3788 & 0.2554 & 0.4416 & $0.073^{*}$ \\
\hline $\mathrm{C} 21$ & $-0.2374(4)$ & $0.20141(15)$ & $0.4799(4)$ & $0.0453(10)$ \\
\hline $\mathrm{H} 21 \mathrm{~A}$ & -0.2291 & 0.2046 & 0.5615 & $0.054 *$ \\
\hline $\mathrm{C} 22$ & $-0.1578(3)$ & $0.16568(13)$ & $0.4334(3)$ & $0.0346(8)$ \\
\hline $\mathrm{C} 23$ & $0.2425(5)$ & $0.0421(2)$ & $0.9032(4)$ & $0.0633(14)$ \\
\hline $\mathrm{H} 23 \mathrm{~A}$ & 0.1975 & 0.0724 & 0.9189 & $0.076^{*}$ \\
\hline $\mathrm{C} 24$ & $0.2609(4)$ & $0.03336(15)$ & $0.7922(3)$ & $0.0428(9)$ \\
\hline $\mathrm{C} 25$ & $0.3255(5)$ & $-0.01098(18)$ & $0.7753(4)$ & $0.0546(11)$ \\
\hline $\mathrm{H} 25 \mathrm{~A}$ & 0.3396 & -0.0190 & 0.6996 & $0.066^{*}$ \\
\hline $\mathrm{C} 26$ & $0.3699(4)$ & $-0.04387(15)$ & $0.8627(3)$ & $0.0433(10)$ \\
\hline $\mathrm{H} 26 \mathrm{~A}$ & 0.4148 & -0.0743 & 0.8477 & $0.052 *$ \\
\hline $\mathrm{C} 27$ & $0.3511(3)$ & $-0.03381(15)$ & $0.9703(3)$ & $0.0355(8)$ \\
\hline $\mathrm{C} 28$ & $0.2155(4)$ & $0.07060(16)$ & $0.6936(4)$ & $0.0494(10)$ \\
\hline $\mathrm{H} 28 \mathrm{~A}$ & 0.2027 & 0.1050 & 0.7266 & $0.059^{*}$ \\
\hline $\mathrm{H} 28 \mathrm{~B}$ & 0.2851 & 0.0740 & 0.6468 & $0.059^{*}$ \\
\hline $\mathrm{C} 29$ & $0.4680(4)$ & $-0.11446(15)$ & $1.0485(4)$ & $0.0465(10)$ \\
\hline $\mathrm{H} 29 \mathrm{~A}$ & 0.4780 & -0.1232 & 0.9720 & $0.056^{*}$ \\
\hline $\mathrm{C} 30$ & $0.5178(5)$ & $-0.14675(17)$ & $1.1397(4)$ & $0.0592(12)$ \\
\hline $\mathrm{H} 30 \mathrm{~A}$ & 0.5628 & -0.1773 & 1.1253 & $0.071^{*}$ \\
\hline $\mathrm{C} 31$ & $0.5031(5)$ & $-0.13524(19)$ & $1.2499(4)$ & $0.0599(13)$ \\
\hline $\mathrm{H} 31 \mathrm{~A}$ & 0.5356 & -0.1580 & 1.3118 & $0.072^{*}$ \\
\hline C32 & $0.4413(5)$ & $-0.0906(2)$ & $1.2707(4)$ & $0.0589(13)$ \\
\hline $\mathrm{H} 32 \mathrm{~A}$ & 0.4324 & -0.0821 & 1.3476 & $0.071^{*}$ \\
\hline $\mathrm{C} 33$ & $0.3915(4)$ & $-0.05781(18)$ & $1.1807(4)$ & $0.0489(10)$ \\
\hline H33A & 0.3485 & -0.0270 & 1.1965 & $0.059^{*}$ \\
\hline $\mathrm{C} 34$ & $0.4033(4)$ & $-0.06922(15)$ & $1.0680(3)$ & $0.0375(8)$ \\
\hline $\mathrm{C} 35$ & $0.4843(6)$ & $0.1089(2)$ & $0.3663(6)$ & $0.0815(19)$ \\
\hline H35A & 0.5729 & 0.1022 & 0.3507 & $0.098^{*}$ \\
\hline H35B & 0.4911 & 0.1352 & 0.4283 & $0.098^{*}$ \\
\hline
\end{tabular}


Table 7: Atomic displacement parameters $\left(\AA^{2}\right)$ for $4 \cdot \mathrm{CH}_{2} \mathrm{Cl}_{2}$.

\begin{tabular}{|c|c|c|c|c|c|c|}
\hline & $U^{11}$ & $U^{22}$ & $U^{33}$ & $U^{12}$ & $U^{13}$ & $U^{23}$ \\
\hline Ir & $0.03067(8)$ & $0.02483(7)$ & $0.02505(7)$ & $0.00162(5)$ & $-0.00140(5)$ & -0.00120 \\
\hline Cl1 & $0.0382(5)$ & $0.0310(4)$ & $0.0391(5)$ & $-0.0031(3)$ & $0.0041(4)$ & $-0.0046(3)$ \\
\hline $\mathrm{Cl} 2$ & $0.0617(7)$ & $0.0543(7)$ & $0.0715(8)$ & $0.0009(6)$ & $0.0024(6)$ & $0.0075(6)$ \\
\hline $\mathrm{Cl} 3$ & $0.0902(10)$ & $0.0703(8)$ & $0.0801(9)$ & $0.0238(7)$ & $0.0413(8)$ & $0.0198(7)$ \\
\hline N1 & $0.0324(15)$ & $0.0328(15)$ & $0.0302(15)$ & $0.0031(12)$ & $0.0002(12)$ & $-0.0041(12)$ \\
\hline N2 & $0.0341(16)$ & $0.0317(15)$ & $0.0282(15)$ & $-0.0067(12)$ & $0.0023(12)$ & $-0.0031(12)$ \\
\hline N3 & $0.069(3)$ & $0.082(3)$ & $0.051(2)$ & $0.026(2)$ & $0.009(2)$ & $0.001(2)$ \\
\hline N4 & $0.0394(18)$ & $0.0343(16)$ & $0.0312(16)$ & $0.0076(13)$ & $-0.0003(13)$ & 0.0004 \\
\hline $\mathrm{C} 1$ & $0.040(2)$ & $0.046(2)$ & $0.037(2)$ & $-0.0030(17)$ & $0.0072(16)$ & $-0.0057(17)$ \\
\hline $\mathrm{C} 2$ & $0.047(2)$ & $0.069(3)$ & $0.040(2)$ & $-0.006(2)$ & $0.0113(19)$ & $-0.013(2)$ \\
\hline $\mathrm{C} 3$ & $0.055(3)$ & $0.071(3)$ & $0.048(3)$ & $-0.004(2)$ & $0.014(2)$ & $-0.028(2)$ \\
\hline $\mathrm{C} 4$ & $0.049(3)$ & $0.051(3)$ & $0.053(3)$ & $-0.009(2)$ & $0.006(2)$ & $-0.023(2)$ \\
\hline $\mathrm{C} 5$ & $0.0342(19)$ & $0.0334(18)$ & $0.0346(19)$ & $0.0023(15)$ & $-0.0013(15)$ & $-0.0049(15)$ \\
\hline C6 & $0.037(2)$ & $0.0309(18)$ & $0.0364(19)$ & $0.0030(15)$ & $-0.0028(15)$ & $-0.0040(15)$ \\
\hline $\mathrm{C} 7$ & $0.054(3)$ & $0.035(2)$ & $0.046(2)$ & $-0.0079(18)$ & -0 & $-0.0085(17)$ \\
\hline $\mathrm{C} 8$ & $0.046(2)$ & $0.033(2)$ & $0.055(3)$ & $-0.0112(17)$ & $-0.0027(19)$ & 0.0000 \\
\hline C9 & $0.040(2)$ & $0.041(2)$ & $0.048(2)$ & $-0.0021(17)$ & $0.0018(18)$ & $0.0124(18)$ \\
\hline $\mathrm{C} 10$ & $0.042(2)$ & $0.0344(19)$ & $0.0358(19)$ & $0.0013(16)$ & $-0.0029(16)$ & $0.0019(15)$ \\
\hline $\mathrm{C} 11$ & $0.0287(17)$ & $0.0293(16)$ & $0.0288(17)$ & $0.0041(13)$ & $-0.0041(14)$ & 0.0018 \\
\hline $\mathrm{C} 12$ & $0.042(2)$ & $0.039(2)$ & $0.038(2)$ & $-0.0018(17)$ & $0.0094(17)$ & $-0.0049(16)$ \\
\hline $\mathrm{C} 13$ & $0.055(3)$ & $0.068(3)$ & $0.047(3)$ & $-0.004(2)$ & $0.015(2)$ & $-0.020(2)$ \\
\hline C14 & $0.067(3)$ & $0.097(4)$ & $0.032(2)$ & $-0.012(3)$ & $0.015(2)$ & $-0.015(2)$ \\
\hline C15 & $0.063(3)$ & $0.075(3)$ & $0.034(2)$ & $3(2)$ & $0.001(2)$ & $0.004(2)$ \\
\hline C16 & $0.043(2)$ & $0.043(2)$ & $0.0290(18)$ & $-0.0092(17)$ & $-0.0036(16)$ & 0.0029 \\
\hline $\mathrm{C} 17$ & $0.042(2)$ & $0.038(2)$ & $0.039(2)$ & $-0.0042(16)$ & $-0.0096(17)$ & $0.0066(16)$ \\
\hline C18 & $0.055(3)$ & $0.056(3)$ & $0.043(2)$ & $-0.003(2)$ & $-0.020(2)$ & $0.009(2)$ \\
\hline $\mathrm{C} 19$ & $0.052(3)$ & $0.048(3)$ & $0.081(4)$ & $0.009(2)$ & $-0.022(3)$ & $0.012(2)$ \\
\hline $\mathrm{C} 20$ & 0.048 & $0.044(2)$ & $0.083(4)$ & $0.011(2)$ & $-0.008(2)$ & $0.001(2)$ \\
\hline $\mathrm{C} 21$ & $0.038(2)$ & $0.035(2)$ & $0.058(3)$ & $0.0044(17)$ & $-0.0016(19)$ & $-0.0021(18)$ \\
\hline $\mathrm{C} 22$ & $0.0337(19)$ & $0.0271(17)$ & $0.039(2)$ & $-0.0018(14)$ & $-0.0048(15)$ & $0.0026(14)$ \\
\hline $\mathrm{C} 23$ & $0.057(3)$ & $0.076(3)$ & $0.054(3)$ & $0.032(3)$ & $0.002(2)$ & $-0.003(2)$ \\
\hline $\mathrm{C} 24$ & $0.040(2)$ & $0.038(2)$ & $0.043(2)$ & $0.0021(17)$ & $-0.0136(17)$ & $-0.0048(17)$ \\
\hline $\mathrm{C} 25$ & $0.071(3)$ & $0.052(3)$ & $0.036(2)$ & $0.013(2)$ & $-0.004(2)$ & $-0.0002(19)$ \\
\hline $\mathrm{C} 26$ & $0.059(3)$ & $0.036(2)$ & $0.0306(19)$ & $0.0182(18)$ & $-0.0018(18)$ & $-0.0001(15)$ \\
\hline $\mathrm{C} 27$ & $0.0279(18)$ & $0.042(2)$ & $0.0338(18)$ & $-0.0001(15)$ & $-0.0017(14)$ & $-0.0017(15)$ \\
\hline $\mathrm{C} 28$ & $0.042(2)$ & $0.044(2)$ & $0.055(3)$ & $-0.0040(18)$ & $-0.0129(19)$ & $0.0041(19)$ \\
\hline $\mathrm{C} 29$ & $0.051(3)$ & $0.042(2)$ & $0.045(2)$ & $-0.0009(18)$ & $0.0025(19)$ & $-0.0013(17)$ \\
\hline $\mathrm{C} 30$ & $0.063(3)$ & $0.038(2)$ & $0.071(3)$ & $-0.001(2)$ & $-0.002(2)$ & $0.010(2)$ \\
\hline C31 & $0.058(3)$ & $0.057(3)$ & $0.058(3)$ & $-0.013(2)$ & $-0.009(2)$ & $0.024(2)$ \\
\hline $\mathrm{C} 32$ & $0.053(3)$ & $0.083(4)$ & $0.037(2)$ & $-0.013(3)$ & $-0.003(2)$ & $0.010(2)$ \\
\hline C33 & $0.045(2)$ & $0.062(3)$ & $0.038(2)$ & $-0.001(2)$ & $0.0027(18)$ & $-0.0012(19)$ \\
\hline C34 & $0.035(2)$ & $0.040(2)$ & $0.0337(19)$ & $-0.0045(16)$ & $-0.0021(15)$ & $0.0002(16)$ \\
\hline C35 & $0.059(3)$ & $0.052(3)$ & $0.128(6)$ & $0.002(2)$ & $0.002(3)$ & $0.020(3)$ \\
\hline
\end{tabular}


Table 8: Geometric parameters $\left(\AA,{ }^{\circ}\right)$ for $\mathbf{4} \cdot \mathrm{CH}_{2} \mathrm{Cl}_{2}$.

\begin{tabular}{|c|c|c|c|}
\hline $\mathrm{Ir}-\mathrm{C} 11$ & $1.994(3)$ & $\mathrm{C} 10-\mathrm{C} 11$ & $1.391(5)$ \\
\hline $\mathrm{Ir}-\mathrm{C} 22$ & $1.997(3)$ & $\mathrm{C} 12-\mathrm{C} 13$ & $1.375(6)$ \\
\hline $\mathrm{Ir}-\mathrm{-N} 1$ & $2.040(3)$ & $\mathrm{C} 13-\mathrm{C} 14$ & $1.397(7)$ \\
\hline $\mathrm{Ir}-\mathrm{N} 2$ & $2.047(3)$ & $\mathrm{C} 14-\mathrm{C} 15$ & $1.350(7)$ \\
\hline $\mathrm{Ir}-\mathrm{N} 4$ & $2.222(3)$ & $\mathrm{C} 15-\mathrm{C} 16$ & $1.399(6)$ \\
\hline $\mathrm{Ir}-\mathrm{Cl} 1$ & $2.4894(9)$ & $\mathrm{C} 16-\mathrm{C} 17$ & $1.454(6)$ \\
\hline $\mathrm{C} 12-\mathrm{C} 35$ & $1.741(5)$ & $\mathrm{C} 17-\mathrm{C} 22$ & $1.397(5)$ \\
\hline $\mathrm{Cl} 3-\mathrm{C} 35$ & $1.789(6)$ & $\mathrm{C} 17-\mathrm{C} 18$ & $1.414(5)$ \\
\hline $\mathrm{N} 1-\mathrm{Cl}$ & $1.341(5)$ & $\mathrm{C} 18-\mathrm{C} 19$ & $1.381(7)$ \\
\hline $\mathrm{N} 1-\mathrm{C} 5$ & $1.363(5)$ & $\mathrm{C} 19-\mathrm{C} 20$ & $1.374(7)$ \\
\hline $\mathrm{N} 2-\mathrm{C} 12$ & $1.339(5)$ & $\mathrm{C} 20-\mathrm{C} 21$ & $1.381(6)$ \\
\hline $\mathrm{N} 2-\mathrm{C} 16$ & $1.364(5)$ & $\mathrm{C} 21-\mathrm{C} 22$ & $1.417(5)$ \\
\hline $\mathrm{N} 3-\mathrm{C} 27$ & $1.344(5)$ & $\mathrm{C} 23-\mathrm{C} 24$ & $1.370(6)$ \\
\hline $\mathrm{N} 3-\mathrm{C} 23$ & $1.375(6)$ & $\mathrm{C} 24-\mathrm{C} 25$ & $1.365(6)$ \\
\hline $\mathrm{N} 4-\mathrm{C} 28$ & $1.459(5)$ & $\mathrm{C} 24-\mathrm{C} 28$ & $1.517(5)$ \\
\hline $\mathrm{C} 1-\mathrm{C} 2$ & $1.378(5)$ & $\mathrm{C} 25-\mathrm{C} 26$ & $1.351(5)$ \\
\hline $\mathrm{C} 2-\mathrm{C} 3$ & $1.371(6)$ & $\mathrm{C} 26-\mathrm{C} 27$ & $1.338(5)$ \\
\hline $\mathrm{C} 3-\mathrm{C} 4$ & $1.358(6)$ & $\mathrm{C} 27-\mathrm{C} 34$ & $1.494(5)$ \\
\hline $\mathrm{C} 4-\mathrm{C} 5$ & $1.392(5)$ & $\mathrm{C} 29-\mathrm{C} 30$ & $1.385(6)$ \\
\hline $\mathrm{C} 5-\mathrm{C} 6$ & $1.458(5)$ & $\mathrm{C} 29-\mathrm{C} 34$ & $1.392(6)$ \\
\hline $\mathrm{C} 6-\mathrm{C} 7$ & $1.400(5)$ & $\mathrm{C} 30-\mathrm{C} 31$ & $1.363(7)$ \\
\hline $\mathrm{C} 6-\mathrm{C} 11$ & $1.422(5)$ & C31-C32 & $1.368(7)$ \\
\hline $\mathrm{C} 7-\mathrm{C} 8$ & $1.375(6)$ & $\mathrm{C} 32-\mathrm{C} 33$ & $1.384(6)$ \\
\hline $\mathrm{C} 8-\mathrm{C} 9$ & $1.383(6)$ & $\mathrm{C} 33-\mathrm{C} 34$ & $1.382(5)$ \\
\hline $\mathrm{C} 9-\mathrm{C} 10$ & $1.398(5)$ & & \\
\hline $\mathrm{C} 11-\mathrm{Ir}-\mathrm{C} 22$ & $91.49(14)$ & $\mathrm{C} 10-\mathrm{C} 11-\mathrm{Ir}$ & $128.5(3)$ \\
\hline $\mathrm{C} 11-\mathrm{Ir}-\mathrm{N} 1$ & $80.62(13)$ & $\mathrm{C} 6-\mathrm{C} 11-\mathrm{Ir}$ & $114.4(3)$ \\
\hline $\mathrm{C} 22--\mathrm{Ir}-\mathrm{N} 1$ & $93.55(14)$ & $\mathrm{N} 2-\mathrm{C} 12-\mathrm{C} 13$ & $123.0(4)$ \\
\hline $\mathrm{C} 11-\mathrm{Ir}-\mathrm{N} 2$ & $94.09(13)$ & $\mathrm{C} 12-\mathrm{C} 13-\mathrm{C} 14$ & $118.0(4)$ \\
\hline $\mathrm{C} 22-\mathrm{Ir}-\mathrm{N} 2$ & $80.27(14)$ & $\mathrm{C} 15-\mathrm{C} 14-\mathrm{C} 13$ & $119.4(4)$ \\
\hline $\mathrm{N} 1-\mathrm{Ir}-\mathrm{N} 2$ & $171.81(11)$ & $\mathrm{C} 14-\mathrm{C} 15-\mathrm{C} 16$ & $120.8(4)$ \\
\hline $\mathrm{C} 11-\mathrm{Ir}--\mathrm{N} 4$ & $94.93(13)$ & $\mathrm{N} 2-\mathrm{C} 16-\mathrm{C} 15$ & $119.6(4)$ \\
\hline $\mathrm{C} 22-\mathrm{Ir}-\mathrm{N} 4$ & $173.52(13)$ & $\mathrm{N} 2-\mathrm{C} 16-\mathrm{C} 17$ & $114.4(3)$ \\
\hline $\mathrm{N} 1-\mathrm{Ir}-\mathrm{N} 4$ & $86.61(12)$ & $\mathrm{C} 15-\mathrm{C} 16-\mathrm{C} 17$ & $126.0(4)$ \\
\hline $\mathrm{N} 2-\mathrm{Ir}-\mathrm{N} 4$ & $100.14(12)$ & $\mathrm{C} 22-\mathrm{C} 17-\mathrm{C} 18$ & $121.3(4)$ \\
\hline $\mathrm{C} 11-\mathrm{Ir}-\mathrm{Cl1}$ & $175.90(10)$ & $\mathrm{C} 22-\mathrm{C} 17-\mathrm{C} 16$ & $114.9(3)$ \\
\hline $\mathrm{C} 22-\mathrm{Ir}-\mathrm{Cl1}$ & $90.98(10)$ & $\mathrm{C} 18-\mathrm{C} 17-\mathrm{C} 16$ & $123.7(4)$ \\
\hline $\mathrm{N} 1-\mathrm{Ir}-\mathrm{C} 11$ & $95.97(9)$ & $\mathrm{C} 19-\mathrm{C} 18-\mathrm{C} 17$ & $119.4(4)$ \\
\hline $\mathrm{N} 2-\mathrm{Ir}-\mathrm{Cl} 1$ & $89.55(8)$ & $\mathrm{C} 20-\mathrm{C} 19-\mathrm{C} 18$ & $120.2(4)$ \\
\hline $\mathrm{N} 4-\mathrm{Ir}-\mathrm{Cl} 1$ & $82.56(9)$ & $\mathrm{C} 19-\mathrm{C} 20-\mathrm{C} 21$ & $121.0(5)$ \\
\hline $\mathrm{C} 1-\mathrm{N} 1-\mathrm{C} 5$ & $119.1(3)$ & $\mathrm{C} 20-\mathrm{C} 21-\mathrm{C} 22$ & $121.0(4)$ \\
\hline $\mathrm{C} 1-\mathrm{N} 1-\mathrm{Ir}$ & $125.0(3)$ & $\mathrm{C} 17-\mathrm{C} 22-\mathrm{C} 21$ & $117.1(3)$ \\
\hline
\end{tabular}




\begin{tabular}{|c|c|c|c|}
\hline $\mathrm{C} 5-\mathrm{N} 1-\mathrm{Ir}$ & $115.8(2)$ & $\mathrm{C} 17-\mathrm{C} 22-\mathrm{Ir}$ & $115.2(3)$ \\
\hline $\mathrm{C} 12-\mathrm{N} 2-\mathrm{C} 16$ & $119.2(3)$ & $\mathrm{C} 21-\mathrm{C} 22-\mathrm{Ir}$ & $127.6(3)$ \\
\hline $\mathrm{C} 12-\mathrm{N} 2-\mathrm{Ir}$ & $125.6(3)$ & $\mathrm{C} 24-\mathrm{C} 23-\mathrm{N} 3$ & $122.7(4)$ \\
\hline $\mathrm{C} 16-\mathrm{N} 2-\mathrm{Ir}$ & $115.2(3)$ & $\mathrm{C} 25-\mathrm{C} 24-\mathrm{C} 23$ & $115.7(4)$ \\
\hline $\mathrm{C} 27-\mathrm{N} 3-\mathrm{C} 23$ & $118.4(4)$ & $\mathrm{C} 25-\mathrm{C} 24-\mathrm{C} 28$ & $121.4(4)$ \\
\hline $\mathrm{C} 28-\mathrm{N} 4-\mathrm{Ir}$ & $119.0(2)$ & $\mathrm{C} 23-\mathrm{C} 24-\mathrm{C} 28$ & $122.9(4)$ \\
\hline $\mathrm{N} 1-\mathrm{C} 1-\mathrm{C} 2$ & $122.4(4)$ & $\mathrm{C} 26-\mathrm{C} 25-\mathrm{C} 24$ & $122.2(4)$ \\
\hline $\mathrm{C} 3-\mathrm{C} 2-\mathrm{C} 1$ & $119.2(4)$ & $\mathrm{C} 27-\mathrm{C} 26-\mathrm{C} 25$ & $120.4(4)$ \\
\hline $\mathrm{C} 4-\mathrm{C} 3-\mathrm{C} 2$ & $118.7(4)$ & $\mathrm{C} 26-\mathrm{C} 27-\mathrm{N} 3$ & $120.6(4)$ \\
\hline $\mathrm{C} 3-\mathrm{C} 4-\mathrm{C} 5$ & $121.5(4)$ & $\mathrm{C} 26-\mathrm{C} 27-\mathrm{C} 34$ & $120.7(3)$ \\
\hline $\mathrm{N} 1-\mathrm{C} 5-\mathrm{C} 4$ & $119.2(4)$ & N3 $-\mathrm{C} 27-\mathrm{C} 34$ & $118.7(3)$ \\
\hline $\mathrm{N} 1-\mathrm{C} 5-\mathrm{C} 6$ & $113.8(3)$ & $\mathrm{N} 4-\mathrm{C} 28-\mathrm{C} 24$ & $114.5(3)$ \\
\hline $\mathrm{C} 4-\mathrm{C} 5-\mathrm{C} 6$ & $126.9(4)$ & $\mathrm{C} 30-\mathrm{C} 29-\mathrm{C} 34$ & $120.5(4)$ \\
\hline $\mathrm{C} 7-\mathrm{C} 6-\mathrm{C} 11$ & $121.1(4)$ & $\mathrm{C} 31-\mathrm{C} 30-\mathrm{C} 29$ & $120.7(5)$ \\
\hline $\mathrm{C} 7-\mathrm{C} 6-\mathrm{C} 5$ & $123.9(3)$ & $\mathrm{C} 30-\mathrm{C} 31-\mathrm{C} 32$ & $119.4(4)$ \\
\hline $\mathrm{C} 11-\mathrm{C} 6-\mathrm{C} 5$ & $115.0(3)$ & $\mathrm{C} 31-\mathrm{C} 32-\mathrm{C} 33$ & $120.5(5)$ \\
\hline $\mathrm{C} 8-\mathrm{C} 7-\mathrm{C} 6$ & $120.2(4)$ & C34-C33-C32 & $120.9(4)$ \\
\hline $\mathrm{C} 7-\mathrm{C} 8-\mathrm{C} 9$ & $119.7(4)$ & $\mathrm{C} 33-\mathrm{C} 34-\mathrm{C} 29$ & $117.8(4)$ \\
\hline $\mathrm{C} 8-\mathrm{C} 9-\mathrm{C} 10$ & $120.7(4)$ & $\mathrm{C} 33-\mathrm{C} 34-\mathrm{C} 27$ & $121.4(4)$ \\
\hline $\mathrm{C} 11-\mathrm{C} 10-\mathrm{C} 9$ & $121.3(4)$ & $\mathrm{C} 29-\mathrm{C} 34-\mathrm{C} 27$ & $120.8(4)$ \\
\hline $\mathrm{C} 10-\mathrm{C} 11-\mathrm{C} 6$ & $117.1(3)$ & $\mathrm{Cl} 2-\mathrm{C} 35-\mathrm{Cl} 3$ & $110.3(3)$ \\
\hline $\mathrm{C} 11-\mathrm{Ir}-\mathrm{N} 1-\mathrm{Cl}$ & $-177.8(3)$ & $\mathrm{C} 16-\mathrm{N} 2-\mathrm{C} 12-\mathrm{C} 13$ & $-1.0(6)$ \\
\hline $\mathrm{C} 22-\mathrm{Ir}-\mathrm{N} 1-\mathrm{C} 1$ & $91.3(3)$ & $\mathrm{Ir}-\mathrm{N} 2-\mathrm{C} 12-\mathrm{C} 13$ & $-178.6(3)$ \\
\hline $\mathrm{N} 2-\mathrm{Ir}-\mathrm{N} 1-\mathrm{C} 1$ & $132.1(7)$ & $\mathrm{N} 2-\mathrm{C} 12-\mathrm{C} 13-\mathrm{C} 14$ & $0.2(7)$ \\
\hline $\mathrm{N} 4-\mathrm{Ir}-\mathrm{N} 1-\mathrm{C} 1$ & $-82.2(3)$ & $\mathrm{C} 12-\mathrm{C} 13-\mathrm{C} 14-\mathrm{C} 15$ & $1.1(7)$ \\
\hline $\mathrm{Cl} 1-\mathrm{Ir}-\mathrm{N} 1-\mathrm{Cl}$ & $0.0(3)$ & $\mathrm{C} 13-\mathrm{C} 14-\mathrm{C} 15-\mathrm{C} 16$ & $-1.6(8)$ \\
\hline $\mathrm{C} 11-\mathrm{Ir}-\mathrm{N} 1-\mathrm{C} 5$ & $5.4(2)$ & $\mathrm{C} 12-\mathrm{N} 2-\mathrm{C} 16-\mathrm{C} 15$ & $0.4(5)$ \\
\hline $\mathrm{C} 22-\mathrm{Ir}-\mathrm{N} 1-\mathrm{C} 5$ & $-85.5(3)$ & $\mathrm{Ir}-\mathrm{N} 2-\mathrm{C} 16-\mathrm{C} 15$ & $178.3(3)$ \\
\hline $\mathrm{N} 2-\mathrm{Ir}-\mathrm{N} 1-\mathrm{C} 5$ & $-44.8(9)$ & $\mathrm{C} 12-\mathrm{N} 2-\mathrm{C} 16-\mathrm{C} 17$ & $-178.7(3)$ \\
\hline $\mathrm{N} 4-\mathrm{Ir}-\mathrm{N} 1-\mathrm{C} 5$ & $101.0(3)$ & $\mathrm{Ir}-\mathrm{N} 2-\mathrm{C} 16-\mathrm{C} 17$ & $-0.8(4)$ \\
\hline $\mathrm{C} 11-\mathrm{Ir}-\mathrm{N} 1-\mathrm{C} 5$ & $-176.9(2)$ & $\mathrm{C} 14-\mathrm{C} 15-\mathrm{C} 16-\mathrm{N} 2$ & $0.9(7)$ \\
\hline $\mathrm{C} 11-\mathrm{Ir}-\mathrm{N} 2-\mathrm{C} 12$ & $87.2(3)$ & $\mathrm{C} 14-\mathrm{C} 15-\mathrm{C} 16-\mathrm{C} 17$ & $179.9(4)$ \\
\hline $\mathrm{C} 22-\mathrm{Ir}-\mathrm{N} 2-\mathrm{C} 12$ & $178.0(3)$ & $\mathrm{N} 2-\mathrm{C} 16-\mathrm{C} 17-\mathrm{C} 22$ & $1.1(5)$ \\
\hline $\mathrm{N} 1-\mathrm{Ir}-\mathrm{N} 2-\mathrm{C} 12$ & $136.6(7)$ & $\mathrm{C} 15-\mathrm{C} 16-\mathrm{C} 17-\mathrm{C} 22$ & $-178.0(4)$ \\
\hline $\mathrm{N} 4-\mathrm{Ir}-\mathrm{N} 2-\mathrm{C} 12$ & $-8.6(3)$ & $\mathrm{N} 2-\mathrm{C} 16-\mathrm{C} 17-\mathrm{C} 18$ & $-177.0(4)$ \\
\hline $\mathrm{C} 11-\mathrm{Ir}-\mathrm{N} 2-\mathrm{C} 12$ & $-90.9(3)$ & $\mathrm{C} 15-\mathrm{C} 16-\mathrm{C} 17-\mathrm{C} 18$ & $3.9(7)$ \\
\hline $\mathrm{C} 11-\mathrm{Ir}-\mathrm{N} 2-\mathrm{C} 16$ & $-90.5(3)$ & $\mathrm{C} 22-\mathrm{C} 17-\mathrm{C} 18-\mathrm{C} 19$ & $0.2(6)$ \\
\hline $\mathrm{C} 22-\mathrm{Ir}-\mathrm{N} 2-\mathrm{C} 16$ & $0.3(3)$ & $\mathrm{C} 16-\mathrm{C} 17-\mathrm{C} 18-\mathrm{C} 19$ & $178.2(4)$ \\
\hline $\mathrm{N} 1-\mathrm{Ir}-\mathrm{N} 2-\mathrm{C} 16$ & $-41.1(9)$ & $\mathrm{C} 17-\mathrm{C} 18-\mathrm{C} 19-\mathrm{C} 20$ & $-0.7(7)$ \\
\hline $\mathrm{N} 4-\mathrm{Ir}-\mathrm{N} 2-\mathrm{C} 16$ & $173.8(3)$ & $\mathrm{C} 18-\mathrm{C} 19-\mathrm{C} 20-\mathrm{C} 21$ & $0.6(8)$ \\
\hline $\mathrm{C} 11-\mathrm{Ir}-\mathrm{N} 2-\mathrm{C} 16$ & $91.4(2)$ & $\mathrm{C} 19-\mathrm{C} 20-\mathrm{C} 21-\mathrm{C} 22$ & $0.1(7)$ \\
\hline $\mathrm{C} 11-\mathrm{Ir}-\mathrm{N} 4-\mathrm{C} 28$ & $20.6(3)$ & $\mathrm{C} 18-\mathrm{C} 17-\mathrm{C} 22-\mathrm{C} 21$ & $0.4(6)$ \\
\hline $\mathrm{C} 22-\mathrm{Ir}-\mathrm{N} 4-\mathrm{C} 28$ & $-151.2(11)$ & $\mathrm{C} 16-\mathrm{C} 17-\mathrm{C} 22-\mathrm{C} 21$ & $-177.7(3)$ \\
\hline $\mathrm{N} 1-\mathrm{Ir}-\mathrm{N} 4-\mathrm{C} 28$ & $-59.6(3)$ & $\mathrm{C} 18-\mathrm{C} 17-\mathrm{C} 22-\mathrm{Ir}$ & $177.3(3)$ \\
\hline $\mathrm{N} 2-\mathrm{Ir}-\mathrm{N} 4-\mathrm{C} 28$ & $115.7(3)$ & $\mathrm{C} 16-\mathrm{C} 17-\mathrm{C} 22-\mathrm{Ir}$ & $-0.8(4)$ \\
\hline $\mathrm{C} 11-\mathrm{Ir}-\mathrm{N} 4-\mathrm{C} 28$ & $-156.1(3)$ & $\mathrm{C} 20-\mathrm{C} 21-\mathrm{C} 22-\mathrm{C} 17$ & $-0.6(6)$ \\
\hline
\end{tabular}




\begin{tabular}{|c|c|c|c|}
\hline $\mathrm{C} 5-\mathrm{N} 1-\mathrm{C} 1-\mathrm{C} 2$ & $-0.4(6)$ & $\mathrm{C} 20-\mathrm{C} 21-\mathrm{C} 22-\mathrm{Ir}$ & $-177.1(3)$ \\
\hline $\mathrm{Ir}-\mathrm{N} 1-\mathrm{C} 1-\mathrm{C} 2$ & $-177.1(3)$ & $\mathrm{C} 11-\mathrm{Ir}-\mathrm{C} 22-\mathrm{C} 17$ & $94.2(3)$ \\
\hline $\mathrm{N} 1-\mathrm{C} 1-\mathrm{C} 2-\mathrm{C} 3$ & $-0.1(7)$ & $\mathrm{N} 1-\mathrm{Ir}-\mathrm{C} 22-\mathrm{C} 17$ & $174.9(3)$ \\
\hline $\mathrm{C} 1-\mathrm{C} 2-\mathrm{C} 3-\mathrm{C} 4$ & $0.3(7)$ & $\mathrm{N} 2-\mathrm{Ir}-\mathrm{C} 22-\mathrm{C} 17$ & $0.3(3)$ \\
\hline $\mathrm{C} 2-\mathrm{C} 3-\mathrm{C} 4-\mathrm{C} 5$ & $0.0(7)$ & $\mathrm{N} 4-\mathrm{Ir}-\mathrm{C} 22-\mathrm{C} 17$ & $-93.9(12)$ \\
\hline $\mathrm{C} 1-\mathrm{N} 1-\mathrm{C} 5-\mathrm{C} 4$ & $0.6(5)$ & $\mathrm{C} 11-\mathrm{Ir}-\mathrm{C} 22-\mathrm{C} 17$ & $-89.1(3)$ \\
\hline $\mathrm{Ir}-\mathrm{N} 1-\mathrm{C} 5-\mathrm{C} 4$ & $177.7(3)$ & $\mathrm{C} 11-\mathrm{Ir}-\mathrm{C} 22-\mathrm{C} 21$ & $-89.3(3)$ \\
\hline $\mathrm{C} 1-\mathrm{N} 1-\mathrm{C} 5-\mathrm{C} 6$ & $178.1(3)$ & $\mathrm{N} 1-\mathrm{Ir}-\mathrm{C} 22-\mathrm{C} 21$ & $-8.6(3)$ \\
\hline $\mathrm{Ir}-\mathrm{N} 1-\mathrm{C} 5-\mathrm{C} 6$ & $-4.9(4)$ & $\mathrm{N} 2-\mathrm{Ir}-\mathrm{C} 22-\mathrm{C} 21$ & $176.8(4)$ \\
\hline $\mathrm{C} 3-\mathrm{C} 4-\mathrm{C} 5-\mathrm{N} 1$ & $-0.4(7)$ & $\mathrm{N} 4-\mathrm{Ir}-\mathrm{C} 22-\mathrm{C} 21$ & $82.6(12)$ \\
\hline $\mathrm{C} 3-\mathrm{C} 4-\mathrm{C} 5-\mathrm{C} 6$ & $-177.5(4)$ & $\mathrm{C} 11-\mathrm{Ir}-\mathrm{C} 22-\mathrm{C} 21$ & $87.4(3)$ \\
\hline $\mathrm{N} 1-\mathrm{C} 5-\mathrm{C} 6-\mathrm{C} 7$ & $-176.4(3)$ & $\mathrm{C} 27-\mathrm{N} 3-\mathrm{C} 23-\mathrm{C} 24$ & $0.8(8)$ \\
\hline $\mathrm{C} 4-\mathrm{C} 5-\mathrm{C} 6-\mathrm{C} 7$ & $0.8(6)$ & $\mathrm{N} 3-\mathrm{C} 23-\mathrm{C} 24-\mathrm{C} 25$ & $0.3(7)$ \\
\hline $\mathrm{N} 1-\mathrm{C} 5-\mathrm{C} 6-\mathrm{C} 11$ & $0.9(5)$ & $\mathrm{N} 3-\mathrm{C} 23-\mathrm{C} 24-\mathrm{C} 28$ & $-178.5(4)$ \\
\hline $\mathrm{C} 4-\mathrm{C} 5-\mathrm{C} 6-\mathrm{C} 11$ & $178.1(4)$ & $\mathrm{C} 23-\mathrm{C} 24-\mathrm{C} 25-\mathrm{C} 26$ & $-0.8(7)$ \\
\hline $\mathrm{C} 11-\mathrm{C} 6-\mathrm{C} 7-\mathrm{C} 8$ & $-1.8(6)$ & $\mathrm{C} 28-\mathrm{C} 24-\mathrm{C} 25-\mathrm{C} 26$ & $178.0(4)$ \\
\hline $\mathrm{C} 5-\mathrm{C} 6-\mathrm{C} 7-\mathrm{C} 8$ & $175.4(4)$ & $\mathrm{C} 24-\mathrm{C} 25-\mathrm{C} 26-\mathrm{C} 27$ & $0.3(7)$ \\
\hline $\mathrm{C} 6-\mathrm{C} 7-\mathrm{C} 8-\mathrm{C} 9$ & $-0.1(6)$ & $\mathrm{C} 25-\mathrm{C} 26-\mathrm{C} 27-\mathrm{N} 3$ & $0.9(7)$ \\
\hline $\mathrm{C} 7-\mathrm{C} 8-\mathrm{C} 9-\mathrm{C} 10$ & $1.2(6)$ & $\mathrm{C} 25-\mathrm{C} 26-\mathrm{C} 27-\mathrm{C} 34$ & $-178.3(4)$ \\
\hline $\mathrm{C} 8-\mathrm{C} 9-\mathrm{C} 10-\mathrm{C} 11$ & $-0.4(6)$ & $\mathrm{C} 23-\mathrm{N} 3-\mathrm{C} 27-\mathrm{C} 26$ & $-1.4(7)$ \\
\hline $\mathrm{C} 9-\mathrm{C} 10-\mathrm{C} 11-\mathrm{C} 6$ & $-1.4(5)$ & $\mathrm{C} 23-\mathrm{N} 3-\mathrm{C} 27-\mathrm{C} 34$ & $177.8(4)$ \\
\hline $\mathrm{C} 9-\mathrm{C} 10-\mathrm{C} 11-\mathrm{Ir}$ & $-179.6(3)$ & $\mathrm{Ir}-\mathrm{N} 4-\mathrm{C} 28-\mathrm{C} 24$ & $154.1(3)$ \\
\hline $\mathrm{C} 7-\mathrm{C} 6-\mathrm{C} 11-\mathrm{C} 10$ & $2.5(5)$ & $\mathrm{C} 25-\mathrm{C} 24-\mathrm{C} 28-\mathrm{N} 4$ & $79.8(5)$ \\
\hline $\mathrm{C} 5-\mathrm{C} 6-\mathrm{C} 11-\mathrm{C} 10$ & $-174.9(3)$ & $\mathrm{C} 23-\mathrm{C} 24-\mathrm{C} 28-\mathrm{N} 4$ & $-101.4(5)$ \\
\hline $\mathrm{C} 7-\mathrm{C} 6-\mathrm{C} 11-\mathrm{Ir}$ & $-179.1(3)$ & $\mathrm{C} 34-\mathrm{C} 29-\mathrm{C} 30-\mathrm{C} 31$ & $0.8(7)$ \\
\hline $\mathrm{C} 5-\mathrm{C} 6-\mathrm{C} 11-\mathrm{Ir}$ & $3.6(4)$ & $\mathrm{C} 29-\mathrm{C} 30-\mathrm{C} 31-\mathrm{C} 32$ & $-1.6(7)$ \\
\hline $\mathrm{C} 22-\mathrm{Ir}-\mathrm{C} 11-\mathrm{C} 10$ & $-93.1(3)$ & $\mathrm{C} 30-\mathrm{C} 31-\mathrm{C} 32-\mathrm{C} 33$ & $1.3(7)$ \\
\hline $\mathrm{N} 1-\mathrm{Ir}-\mathrm{C} 11-\mathrm{C} 10$ & $173.5(3)$ & $\mathrm{C} 31-\mathrm{C} 32-\mathrm{C} 33-\mathrm{C} 34$ & $-0.2(7)$ \\
\hline $\mathrm{N} 2-\mathrm{Ir}-\mathrm{C} 11-\mathrm{C} 10$ & $-12.8(3)$ & $\mathrm{C} 32-\mathrm{C} 33-\mathrm{C} 34-\mathrm{C} 29$ & $-0.6(6)$ \\
\hline $\mathrm{N} 4-\mathrm{Ir}-\mathrm{C} 11-\mathrm{C} 10$ & $87.8(3)$ & $\mathrm{C} 32-\mathrm{C} 33-\mathrm{C} 34-\mathrm{C} 27$ & $-179.4(4)$ \\
\hline $\mathrm{C} 11-\mathrm{Ir}-\mathrm{C} 11-\mathrm{C} 10$ & $139.8(12)$ & $\mathrm{C} 30-\mathrm{C} 29-\mathrm{C} 34-\mathrm{C} 33$ & $0.3(6)$ \\
\hline $\mathrm{C} 22-\mathrm{Ir}-\mathrm{C} 11-\mathrm{C} 6$ & $88.6(3)$ & $\mathrm{C} 30-\mathrm{C} 29-\mathrm{C} 34-\mathrm{C} 27$ & $179.0(4)$ \\
\hline $\mathrm{N} 1-\mathrm{Ir}-\mathrm{C} 11-\mathrm{C} 6$ & $-4.7(2)$ & $\mathrm{C} 26-\mathrm{C} 27-\mathrm{C} 34-\mathrm{C} 33$ & $176.1(4)$ \\
\hline $\mathrm{N} 2-\mathrm{Ir}-\mathrm{C} 11-\mathrm{C} 6$ & $169.0(3)$ & $\mathrm{N} 3-\mathrm{C} 27-\mathrm{C} 34-\mathrm{C} 33$ & $-3.1(6)$ \\
\hline $\mathrm{N} 4-\mathrm{Ir}-\mathrm{C} 11-\mathrm{C} 6$ & $-90.4(3)$ & $\mathrm{C} 26-\mathrm{C} 27-\mathrm{C} 34-\mathrm{C} 29$ & $-2.6(6)$ \\
\hline $\mathrm{Cl1}-\mathrm{Ir}-\mathrm{C} 11-\mathrm{C} 6$ & $-38.4(15)$ & $\mathrm{N} 3-\mathrm{C} 27-\mathrm{C} 34-\mathrm{C} 29$ & $178.2(4)$ \\
\hline
\end{tabular}

